\title{
Enantioselective Copper-Catalyzed Radical Cyanation of Propargylic C - H Bonds: Easy Access to Chiral Allenyl Nitriles
}

Ronghua Lu, ${ }^{\dagger a}$ Tilong Yang, ${ }^{\dagger b}$ Xin Chen, ${ }^{c}$ Wenzheng Fan, ${ }^{a}$ Pinhong Chen, ${ }^{a}$ Zhenyang Lin, ${ }^{* b}$ and Guosheng Liu*a,c

a State Key Laboratory of Organometallic Chemistry and Shanghai Hongkong Joint Laboratory in Chemical Synthesis, Center for Excellence in Molecular Synthesis, Shanghai Institute of Organic Chemistry, University of Chinese Academy of Sciences, Chinese Academy of Sciences, 345 Lingling Road, Shanghai, China, 200032

${ }^{b}$ Department of Chemistry, The Hong Kong University of Science and Technology, Clear Water Bay, Kowloon, Hong Kong, China.

${ }^{c}$ Chang-Kung Chuang Institute, East China Normal University, 3663 North Zhongshan Road, Shanghai 200062, China.

Email: gliu@mail.sioc.ac.cn

${ }^{\dagger}$ Equal Contribution

Table of Contents

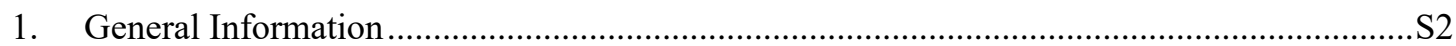

2. General Procedure for Enantioselective Cyanation of Propargylic C-H Bonds ....................S3

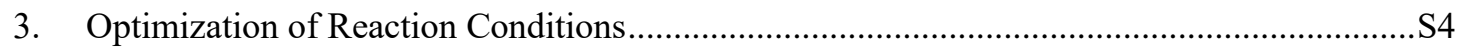

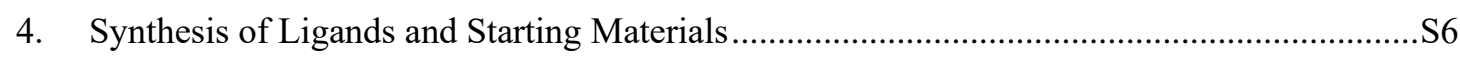

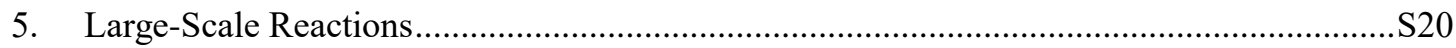

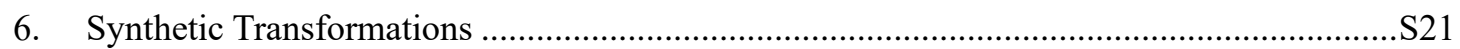

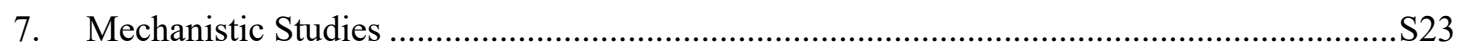

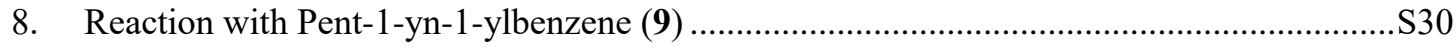

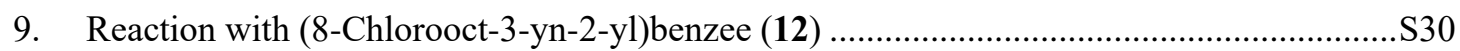

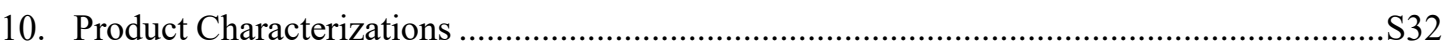

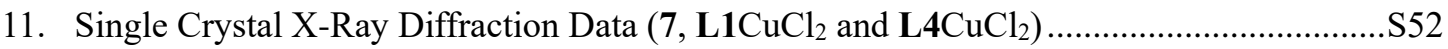

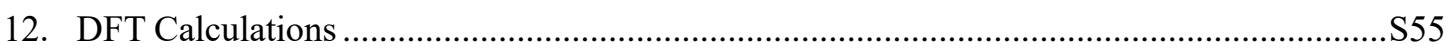

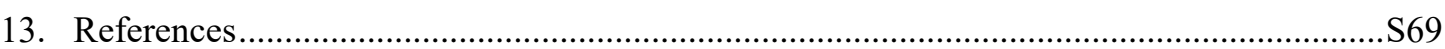

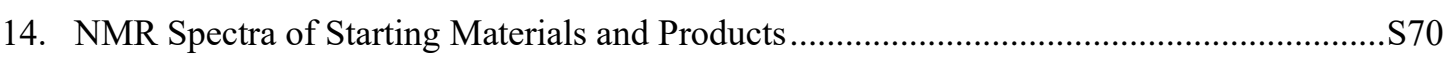

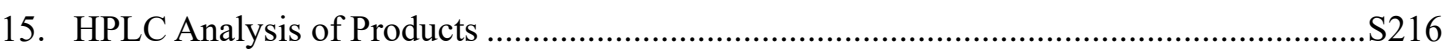




\section{General Information}

All commercially available compounds were purchased from Aldrich, Alfa Aesar, TCI or Adamas and used without further purification, unless otherwise stated. NMR spectra were recorded on Varian Inova 400, Agilent 400 or Bruker 400 (400 MHz for ${ }^{1} \mathrm{H}, 376 \mathrm{MHz}$ for ${ }^{19} \mathrm{~F}, 100 \mathrm{MHz}$ for ${ }^{13} \mathrm{C}$ ) spectrometer. The chemical shifts $(\delta)$ are given in parts per million relative to $\mathrm{CDCl}_{3}\left(7.26\right.$ ppm for $\left.{ }^{1} \mathrm{H}\right)$ or TMS $\left(0\right.$ ppm for $\left.{ }^{1} \mathrm{H}\right)$ and $\mathrm{CDCl}_{3}\left(77.0 \mathrm{ppm}\right.$ for $\left.{ }^{13} \mathrm{C}\right)$. and ${ }^{19} \mathrm{~F}$ NMR chemical shifts were determined relative to $\mathrm{CFCl}_{3}$ as outside standard and low field is positive. ${ }^{1} \mathrm{H}$ and ${ }^{19} \mathrm{~F}$ multiplicities are reported as follows: singlet (s), doublet (d), triplet (t), doublet of doublets (dd), quartet (q), multiple (m), and broad resonance (br). High performance liquid chromatography was performed on Waters 2487-600E, Waters ACQUITY UPC2, and Ultimate 3000 Series HPLC, using AD-H, OD-H, IG, IH, OJ-H, AY-3, AS-RH and AD-RH chiral column eluted with a mixture of hexane and isopropyl alcohol or water and acetonitrile. Optical rotation was measured on a Rudolph-Autopol I using $10 \mathrm{~cm}$ glass cells with a sodium $589 \mathrm{~nm}$ filter. High Resolution Mass spectral data were obtained on an Aglient Technologies 7250 GCQTOF spectrometer in EI mode or an Agilent Technologies 6224 TOF LC MS spectrometer in ESI mode or a Thermo Fisher Scientific LTQ FTICR-MS in DART mode or a JEOL AccuTOF GCv4G GCT MS in FI mode. Flash column chromatography was performed on silica gel (particle size 200-300 mesh, purchased from Canada) and eluted with petroleum ether/ethyl acetate. Solvent was purified according to the procedure from the book named "Purification of Laboratory Chemicals". $\mathrm{PhF}_{6}$ and $\mathrm{PhCF}_{3}$ were deoxygenated with anhydrous $\mathrm{Ar}$ bubble for at least $30 \mathrm{~min}$ before use. 


\section{General Procedure for Enantioselective Cyanation of Propargylic $\mathrm{C}-\mathrm{H}$}

\section{Bonds}

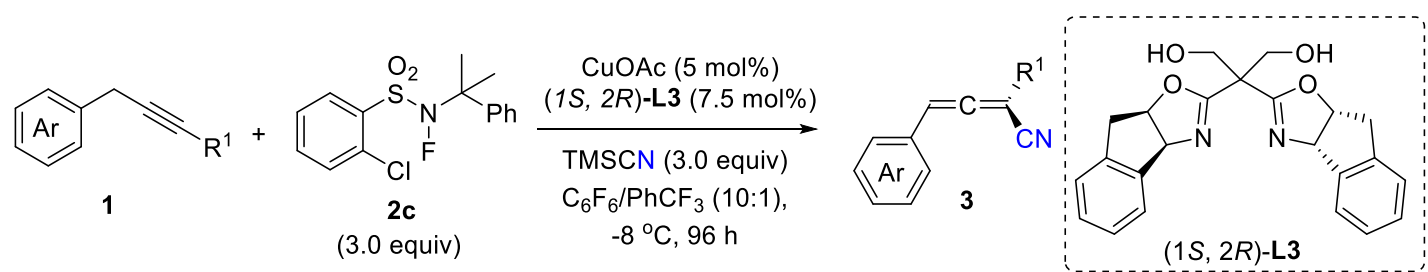

In a dried sealed $10 \mathrm{~mL}$ Schlenk tube, CuOAc (0.01 mmol, $5 \mathrm{~mol} \%)$, chiral bisoxazoline ligand (1S,2R)-L3 (0.015 mmol, $7.5 \mathrm{~mol} \%$ ), and NFAS 2c (196 mg, 0.6 mmol, 3.0 equiv.) were dissolved in a mixed solvent of $\mathrm{C}_{6} \mathrm{~F}_{6}(1.0 \mathrm{~mL})$ and $\mathrm{PhCF}_{3}(0.1$ $\mathrm{mL}$ ) under a $N_{2}$ atmosphere, and the mixture was stirred for $30 \mathrm{~min}$. The reaction mixture was cooled down to $-30{ }^{\circ} \mathrm{C}$, then substrate 1 ( $0.2 \mathrm{mmol}, 1.0$ equiv.), and TMSCN ( $80 \mu \mathrm{L}, 0.6$ mmol, 3.0 equiv.) were added sequentially into the above solution. The tube was sealed with Teflon septum and the reaction mixture was stirred at $-8{ }^{\circ} \mathrm{C}$ for another 96 hours. After the reaction was completed, the mixture was quenched by a short pad of silica gel with a gradient eluent of petroleum ether and ethyl acetate, solvent was removed under vacuum, and the residue was purified by column chromatography on silica gel with a gradient eluent of petroleum ether and ethyl acetate to give the desired product 3 . These results were summaried in Table 2. Note: Most of the neat allenyl nitriles are unstable, but they are quite stable in solution. It is recommended that they are stored as solution, such as in $\mathrm{CH}_{2} \mathrm{Cl}_{2}$ or acetone. 


\section{Optimization of Reaction Conditions}

Table S1. Optimization of primary solvents with NFAS 2 a and Ligand L1 ${ }^{a}$<smiles>CCCCC#CCc1ccc(Br)cc1</smiles>

(3.0 equiv)

\begin{tabular}{cccl}
\hline entry & solvent & Yield of $3 \mathbf{a}^{b}$ & $\mathrm{ee}^{\mathrm{c}}$ \\
\hline 1 & $\mathrm{C}_{6} \mathrm{~F}_{6}$ & $60 \%$ & $42 \%$ \\
2 & Toluene & $0 \%$ & n.d. \\
3 & Benzene & trace & n.d. \\
4 & $\mathrm{PhCl}$ & $10 \%$ & $40 \%$ \\
5 & $\mathrm{EA}$ & $3 \%$ & $35 \%$ \\
6 & $\mathrm{CH}_{3} \mathrm{CN}$ & trace & n.d. \\
7 & $\mathrm{dioxane}$ & trace & n.d. \\
8 & $\mathrm{THF}$ & trace & n.d. \\
9 & $\mathrm{DCM}$ & $11 \%$ & $38 \%$ \\
\hline
\end{tabular}

${ }^{a}$ All reactions were run on $0.1 \mathrm{mmol}$ scale in $\mathrm{C}_{6} \mathrm{~F}_{6}(0.5 \mathrm{~mL})$ at room temperature under nitrogen atmosphere. ${ }^{b}$ Yields determined by crude ${ }^{1} \mathrm{H}$ NMR with $\mathrm{CF}_{3}$-DMA as internal standard. ${ }^{c}$ Enantiomeric excess (ee) values determined by HPLC on a chiral stationary phase.

Table S2. Optimization of ligands with NFAS 2a ${ }^{a, b, c}$

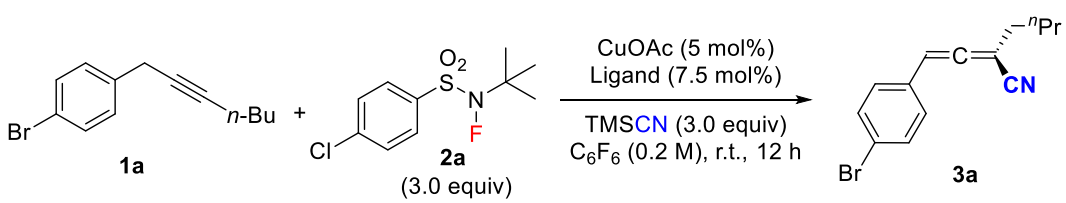

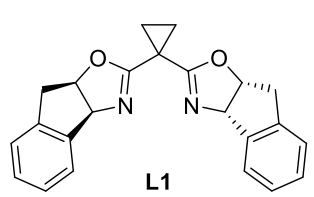

$60 \%(42 \%$ ee $)$

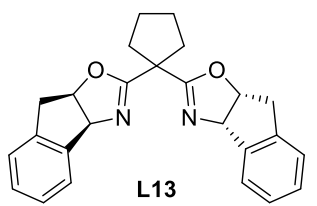

$52 \%(56 \%$ ee $)$

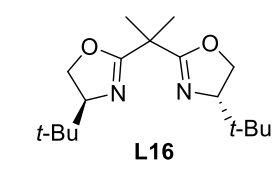

$0 \%$ (n.d.)

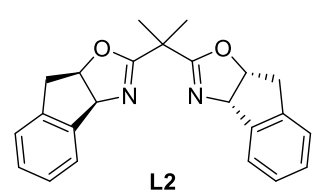

$47 \%(56 \%$ ee $)$

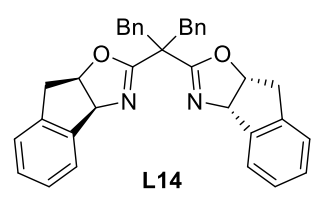

$12 \%(55 \%$ ee $)$

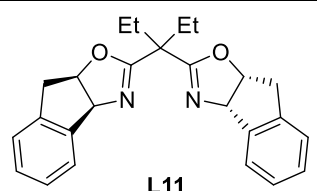

$23 \%(58 \%$ ee $)$

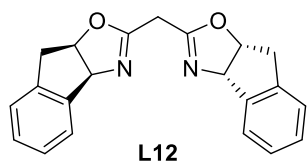

$31 \%(16 \%$ ee $)$

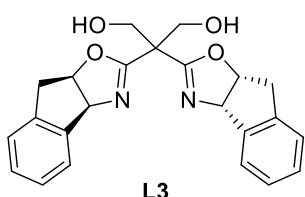

$23 \%(69 \%$ ee $)$

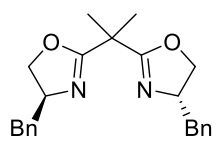

L15

$43 \%(56 \%$ ee $)$
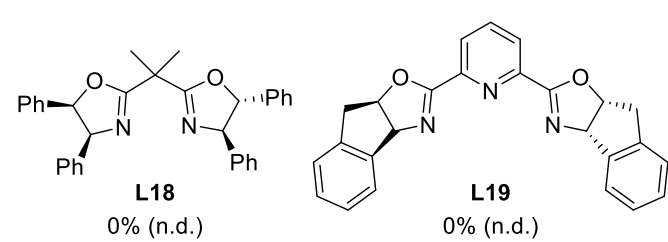

${ }^{a}$ All reactions were run on $0.1 \mathrm{mmol}$ scale in $\mathrm{C}_{6} \mathrm{~F}_{6}(0.5 \mathrm{~mL})$ at room temperature under nitrogen atmosphere. ${ }^{b}$ Yields determined by crude ${ }^{1} \mathrm{H}$ NMR with $\mathrm{CF}_{3}$-DMAc as internal standard. ${ }^{c}$ Enantiomeric excess (ee) values determined by $\mathrm{HPLC}$ on a chiral stationary phase.

Table S3. Optimization of NFAS with Ligand $\mathbf{L 1}$ or $\mathbf{L} 3^{a, b, c}$ 


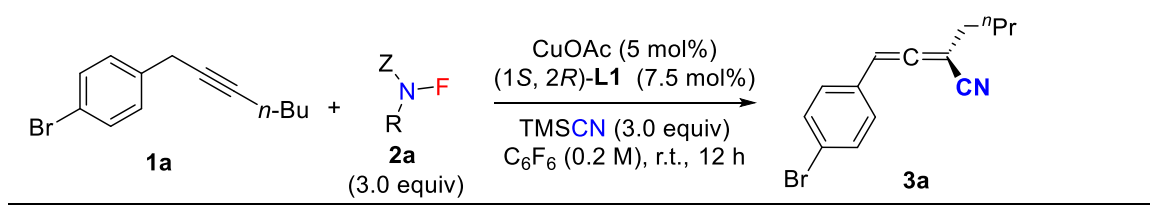<smiles>CC(C)(C)N(F)S(=O)c1ccc(Cl)cc1</smiles>

2a

$60 \%(42 \%$ ee)

$23 \%(69 \% \text { ee })^{d}$<smiles>CC(C)(C)N(F)[N+](=O)c1ccccc1Cl</smiles>

2b

$65 \%$ (55\% ee) $31 \%(75 \% \text { ee })^{d}$<smiles>CC(C)(c1ccccc1)N(F)S(=O)c1ccccc1Cl</smiles>

2c

$62 \%(57 \%$ ee $)$ $45 \%(78 \% \text { ee })^{d}$

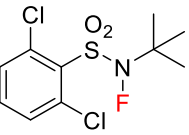

2d $9 \%(72 \% \text { ee })^{d}$

${ }^{a}$ All reactions were run on $0.1 \mathrm{mmol}$ scale in $\mathrm{C}_{6} \mathrm{~F}_{6}(0.5 \mathrm{~mL})$ at room temperature under nitrogen atmosphere. ${ }^{b}$ Yields determined by crude ${ }^{1} \mathrm{H}$ NMR with $\mathrm{CF}_{3}$-DMAc as internal standard. ${ }^{c}$ Enantiomeric excess (ee) values determined by HPLC on a chiral stationary phase. ${ }^{d}(1 S, 2 R)$-L3

Table S4. Optimization of solvents with NFAS $\mathbf{2 c}$ and $\mathbf{L 3}{ }^{a}$

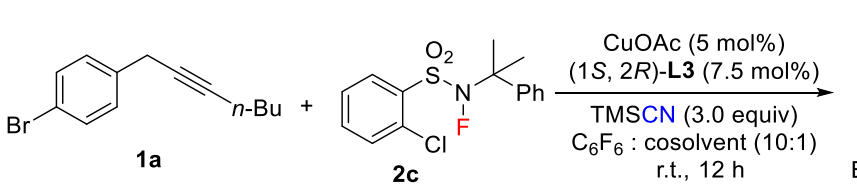<smiles>CC(C)CC(C#N)C=Cc1ccc(Br)cc1</smiles>
(3.0 equiv)

\begin{tabular}{cccc}
\hline entry & cosolvent & ${\text { Yield of } \mathbf{3} \mathbf{a}^{b}}$ & $\mathrm{ee}^{\mathrm{c}}$ \\
\hline 1 & none & $45 \%$ & $78 \%$ \\
2 & $\mathrm{EA}$ & $46 \%$ & $75 \%$ \\
3 & DMSO & $32 \%$ & $64 \%$ \\
4 & dioxane & $40 \%$ & $77 \%$ \\
5 & $\mathrm{DCM}$ & $41 \%$ & $75 \%$ \\
6 & $\mathrm{Acetone}$ & $21 \%$ & $76 \%$ \\
7 & $\mathrm{CH}_{3} \mathrm{CN}$ & $38 \%$ & $75 \%$ \\
8 & $\mathrm{THF}$ & $10 \%$ & $78 \%$ \\
9 & $\mathrm{PhCF}_{3}$ & $55 \%$ & $79 \%$
\end{tabular}

${ }^{a} \mathrm{All}$ reactions were run on $0.1 \mathrm{mmol}$ scale in $\mathrm{C}_{6} \mathrm{~F}_{6}(0.5 \mathrm{~mL})$ and coslovent $(50 \mu \mathrm{L})$ at room temperature under nitrogen atmosphere. ${ }^{b}$ Yields determined by crude ${ }^{1} \mathrm{H} \mathrm{NMR}$ with $\mathrm{CF}_{3}-\mathrm{DMA}$ as internal standard. ${ }^{c}$ Enantiomeric excess (ee) values determined by HPLC on a chiral stationary phase.

Table S5. Optimization of reaction temperature ${ }^{a}$

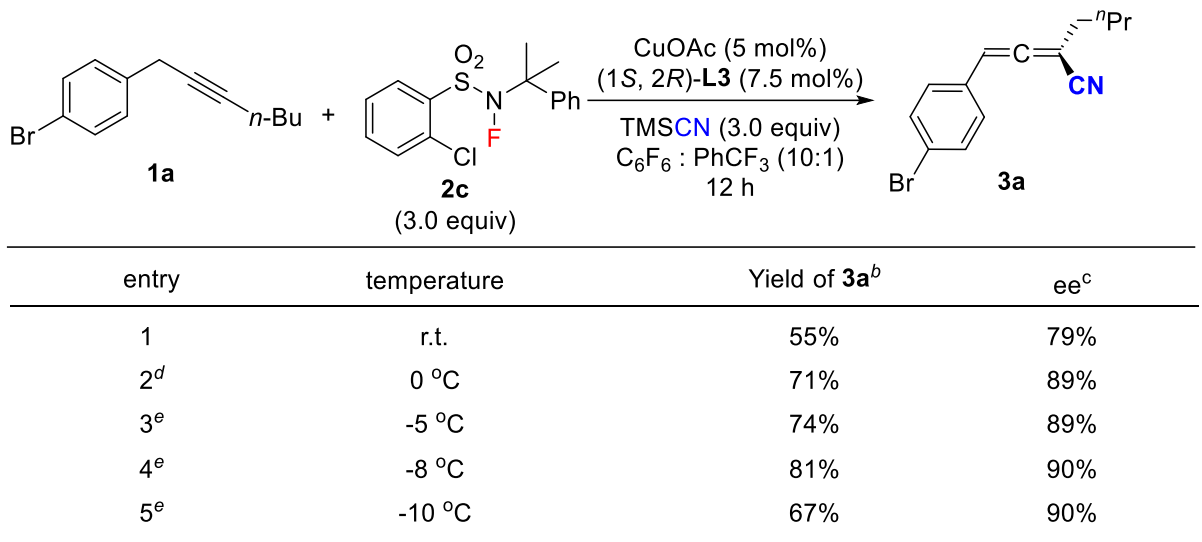

${ }^{a}$ All reactions were run on $0.1 \mathrm{mmol}$ scale in $\mathrm{C}_{6} \mathrm{~F}_{6}(0.5 \mathrm{~mL})$ and $\mathrm{PhCF}_{3}(50 \mu \mathrm{L})$ under nitrogen atmosphere. ${ }^{b}$ Yields determined by crude ${ }^{1} \mathrm{H}$ NMR with $\mathrm{CF}_{3}$-DMA as an internal standard. ${ }^{c}$ Enantiomeric excess (ee) values determined by HPLC on a chiral stationary phase. ${ }^{d} 48 \mathrm{~h} .{ }^{e} 96 \mathrm{~h}$

Table S6. Optimization of Copper catalyst ${ }^{a}$ 


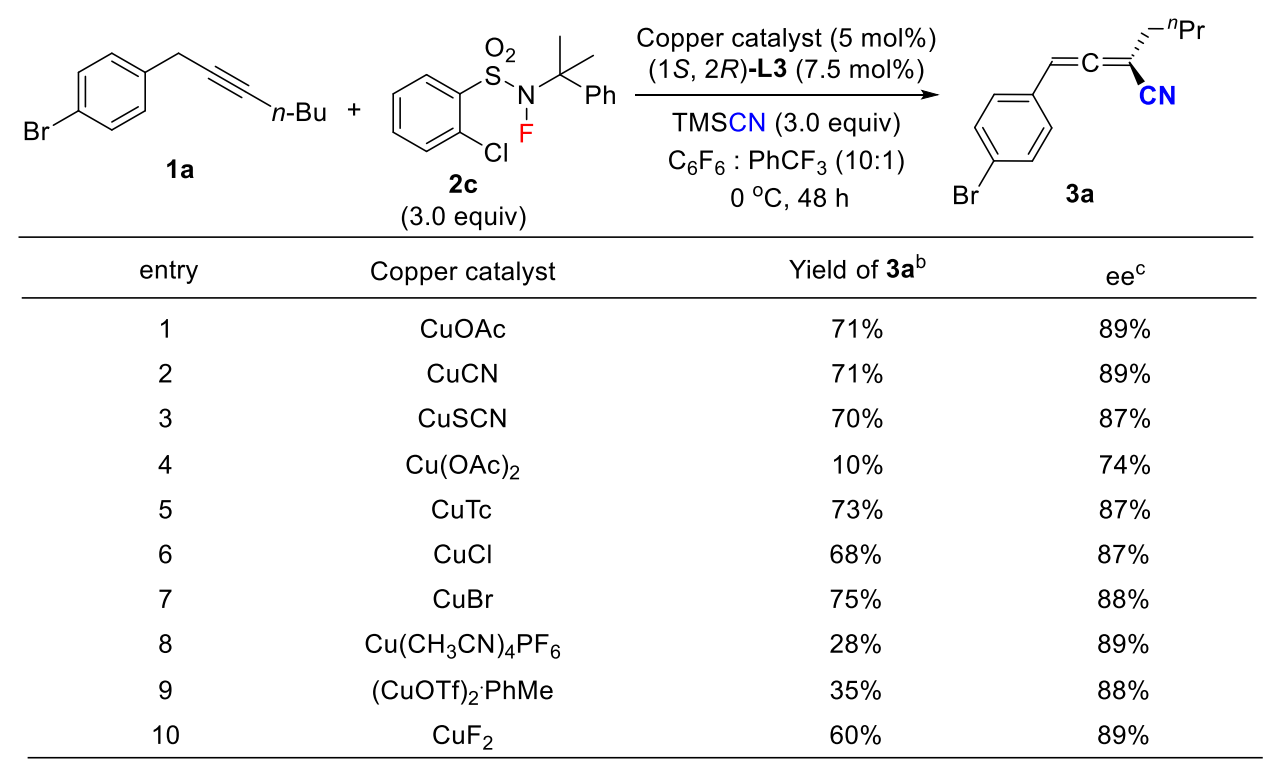

${ }^{a}$ All reactions were run on $0.1 \mathrm{mmol}$ scale in $\mathrm{C}_{6} \mathrm{~F}_{6}(0.5 \mathrm{~mL})$ and $\mathrm{PhCF}_{3}(50 \mu \mathrm{L})$ under nitrogen atmosphere at $0{ }^{\circ} \mathrm{C}$. ${ }^{b}$ Yields determined by crude ${ }^{1} \mathrm{H}$ NMR with $\mathrm{CF}_{3}$-DMA as an internal standard. ${ }^{c}$ Enantiomeric excess (ee) values determined by HPLC on a chiral stationary phase.

\section{Synthesis of Ligands and Starting Materials}

\subsection{Procedure for the Synthesis of Ligands}

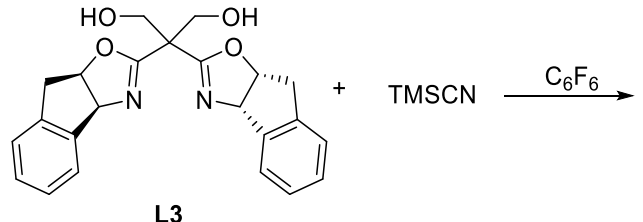

L3

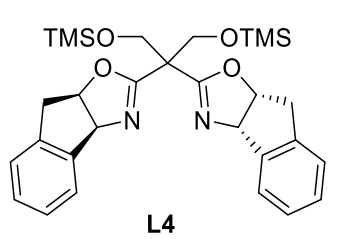

L4

To a stirred solution of $\mathbf{L 3}(195 \mathrm{mg}, 0.5 \mathrm{mmol})$ in $\mathrm{C}_{6} \mathrm{~F}_{6}(20 \mathrm{~mL})$ was added TMSCN (1 mL,7.5 mmol) and the mixture was stirred at room temperature for $4 \mathrm{~h}$ until the solid was completely dissolved. The mixture was then concentrated under reduced pressure, the residue was purified by flash chromatography on silica gel $(\mathrm{PE} /$ Acetone $=10: 1)$ to give the product $\mathbf{L 4}(260 \mathrm{mg}, 97 \%$ yield $)$ as a white solid. M.p. $132.6-134.5^{\circ} \mathrm{C}$.

$[\alpha]_{\mathrm{D}}^{24.2}-295.31\left(c \mathrm{1.00}, \mathrm{CHCl}_{3}\right)$.

${ }^{1} \mathrm{H}$ NMR (400 MHz, $\left.\mathrm{CDCl}_{3}\right) \delta 7.50-7.48(\mathrm{~m}, 2 \mathrm{H}), 7.24-7.22(\mathrm{~m}, 4 \mathrm{H}), 7.20-7.18$ (m, 2H), $5.51(\mathrm{~d}, J=7.6 \mathrm{~Hz}, 2 \mathrm{H}), 5.17(\mathrm{td}, J=6.8,1.6 \mathrm{~Hz}, 2 \mathrm{H}), 4.02(\mathrm{~d}, J=9.2 \mathrm{~Hz}$, 2H), $3.98(\mathrm{~d}, J=9.2 \mathrm{~Hz}, 2 \mathrm{H}), 3.24(\mathrm{dd}, J=18.0,6.8 \mathrm{~Hz}, 2 \mathrm{H}), 2.84(\mathrm{~d}, J=17.6 \mathrm{~Hz}$, $2 \mathrm{H}),-0.11(\mathrm{~s}, 18 \mathrm{H})$. 
${ }^{13} \mathrm{C} \mathrm{NMR}\left(100 \mathrm{MHz}, \mathrm{CDCl}_{3}\right) \delta 164.2,141.9,139.7,128.2,127.2,125.6,124.9,82.1$, $76.5,59.7,49.7,39.5,-0.8$.

IR (neat): 2958, 1649, 1249, 1128, 1074, 1055, 877, 837, $744 \mathrm{~cm}^{-1}$.

HRMS: $m / z$ (ESI) calculated for $\mathrm{C}_{2} \mathrm{H}_{39} \mathrm{~N}_{2} \mathrm{O}_{4} \mathrm{Si}_{2}{ }^{+}[\mathrm{M}+\mathrm{H}]^{+}:$535.2443, found: 535.2432.

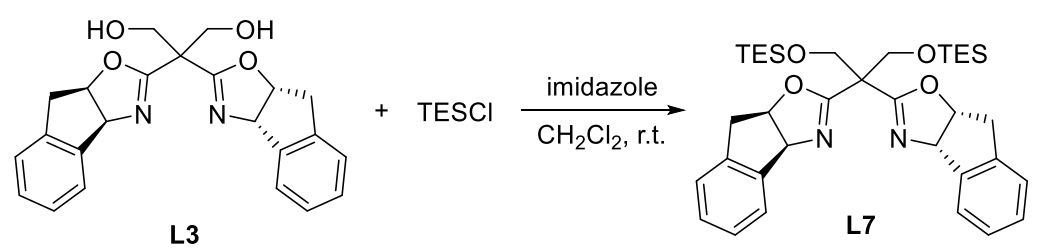

To a stirred solution of $\mathbf{L 3}$ (195 mg, $0.5 \mathrm{mmol}$ ) and imidazole (340 mg, $5 \mathrm{mmol}$ ) in $\mathrm{CH}_{2} \mathrm{Cl}_{2}(10 \mathrm{~mL})$ was added TESCl $(753 \mathrm{mg}, 5 \mathrm{mmol})$ and the mixture was stirred at room temperature overnight until the reaction was complete as monitored by TLC plate. The mixture was then concentrated under reduced pressure, the residue was purified by flash chromatography on silica gel (PE/Acetone $=10: 1)$ to give the product L7 (278 mg, 90\% yield) as a white solid.

M.p. $100.2-101.5^{\circ} \mathrm{C}$.

$[\alpha]_{\mathrm{D}}^{24.7}-187.89\left(c \mathrm{1} .00, \mathrm{CHCl}_{3}\right)$.

${ }^{1} \mathrm{H}$ NMR (400 MHz, $\left.\mathrm{CDCl}_{3}\right) \delta 7.49$ - 7.47 (m, 2H), $7.24-7.22(\mathrm{~m}, 4 \mathrm{H}), 7.20$ - 7.17 (m, 2H), $5.51(\mathrm{~d}, J=8.0 \mathrm{~Hz}, 2 \mathrm{H}), 5.17$ (t, $J=7.2 \mathrm{~Hz}, 2 \mathrm{H}), 4.06(\mathrm{~s}, 4 \mathrm{H}), 3.27(\mathrm{dd}, J=$ 18.0, $6.8 \mathrm{~Hz}, 2 \mathrm{H}), 2.92(\mathrm{~d}, J=18.0 \mathrm{~Hz}, 2 \mathrm{H}), 0.76(\mathrm{t}, J=7.6 \mathrm{~Hz}, 18 \mathrm{H}), 0.36(\mathrm{qd}, J=$ 8.2, $1.6 \mathrm{~Hz}, 12 \mathrm{H})$.

${ }^{13} \mathrm{C}$ NMR $\left(100 \mathrm{MHz}, \mathrm{CDCl}_{3}\right) \delta 164.3,141.8,139.7,128.2,127.2,125.6,124.9,82.3$, $76.6,59.9,50.2,39.5,6.6,4.1$.

IR (neat): 2949, 2873, 1652, 1460, 1112, 1080, 1014, 820, $728 \mathrm{~cm}^{-1}$.

HRMS: $m / z$ (ESI) calculated for $\mathrm{C}_{35} \mathrm{H}_{51} \mathrm{~N}_{2} \mathrm{O}_{4} \mathrm{Si}_{2}{ }^{+}[\mathrm{M}+\mathrm{H}]^{+}:$619.3382, found: 619.3376 .

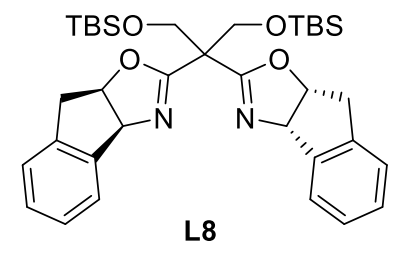

(247 mg, 80\% yield, white solid).

M.p. $114.5-115.8^{\circ} \mathrm{C}$. 
$[\alpha]_{\mathrm{D}}^{25.1}-116.55\left(c \mathrm{1} .79, \mathrm{CHCl}_{3}\right)$.

${ }^{1} \mathrm{H}$ NMR (400 MHz, $\left.\mathrm{CDCl}_{3}\right) \delta 7.49$ - 7.47 (m, 2H), 7.23 - $7.22(\mathrm{~m}, 4 \mathrm{H}), 7.20$ - 7.16 $(\mathrm{m}, 2 \mathrm{H}), 5.51(\mathrm{~d}, J=8.4 \mathrm{~Hz}, 2 \mathrm{H}), 5.18(\mathrm{t}, J=7.6 \mathrm{~Hz}, 2 \mathrm{H}), 4.06(\mathrm{~s}, 4 \mathrm{H}), 3.26(\mathrm{dd}, J=$ 18.0, 7.2 Hz, 2H), 2.89 (d, $J=18.4 \mathrm{~Hz}, 2 \mathrm{H}), 0.64(\mathrm{~s}, 18 \mathrm{H}),-0.09$ (s, 6H), -0.14 (s, $6 \mathrm{H})$.

${ }^{13} \mathrm{C} \mathrm{NMR}\left(100 \mathrm{MHz}, \mathrm{CDCl}_{3}\right) \delta 164.2,141.7,139.6,128.3,127.3,125.6,125.0,82.2$, $76.6,60.1,50.2,39.5,25.5,17.8,-5.8$.

IR (neat): 2929, 2855, 1648, 1464, 1251, 1079, 1058, 834, 775, $754 \mathrm{~cm}^{-1}$.

HRMS: $m / z$ (ESI) calculated for $\mathrm{C}_{35} \mathrm{H}_{51} \mathrm{~N}_{2} \mathrm{O}_{4} \mathrm{Si}_{2}{ }^{+}[\mathrm{M}+\mathrm{H}]^{+}:$619.3382, found: 619.3376 .
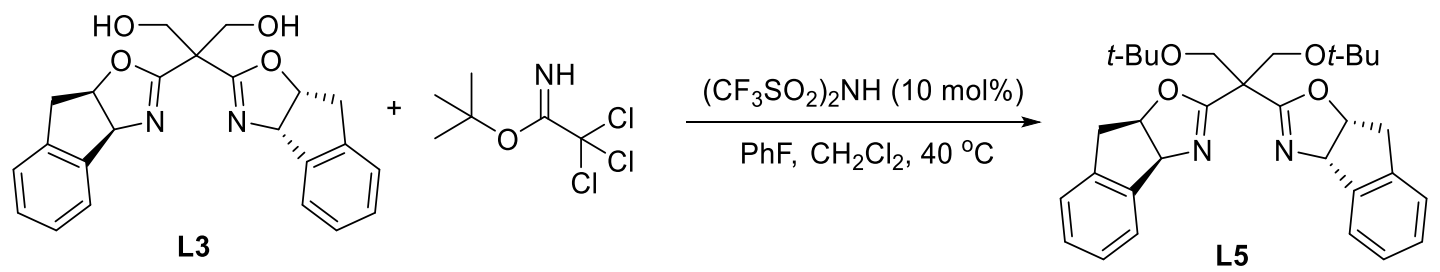

To a stirred solution of $\mathbf{L 3}$ (195 $\mathrm{mg}, 0.5 \mathrm{mmol}$ ) in fluorobenzene (6 $\mathrm{mL})$ was added bis(trifluoromethanesulfonyl)amide (28 mg, $0.1 \mathrm{mmol})$ in $\mathrm{CH}_{2} \mathrm{Cl}_{2}(1 \mathrm{~mL})$ and the mixture was heated to $40{ }^{\circ} \mathrm{C}$. Tert-butyl 2,2,2-trichloroacetimidate (1.09 g, $\left.5 \mathrm{mmol}\right)$ was then added and the mixture was heated for $20 \mathrm{~h}$. The mixture was then diluted with hexanes $(25 \mathrm{~mL})$ and solids were filtered off and washed with hexanes. The organic layer was washed with water, dried over $\mathrm{Na}_{2} \mathrm{SO}_{4}$, filtered, and concentrated under reduced pressure, the residue was purified by column chromatography on silica gel $(\mathrm{PE} /$ Acetone $=10: 1)$ to give the product $(200 \mathrm{mg}, 80 \%$ yield $)$ as a white solid. M.p. $124.5-125.7^{\circ} \mathrm{C}$. $[\alpha]_{\mathrm{D}}^{25.1}-164.71\left(c 1.00, \mathrm{CHCl}_{3}\right)$.

${ }^{1} \mathrm{H}$ NMR $\left(400 \mathrm{MHz}, \mathrm{CDCl}_{3}\right) \delta 7.50-7.48(\mathrm{~m}, 2 \mathrm{H}), 7.24-7.22(\mathrm{~m}, 4 \mathrm{H}), 7.20-7.18$ (m, 2H), $5.51(\mathrm{~d}, J=8.0 \mathrm{~Hz}, 2 \mathrm{H}), 5.19$ (t, $J=6.4 \mathrm{~Hz}, 2 \mathrm{H}), 3.75$ (d, $J=11.6 \mathrm{~Hz}, 2 \mathrm{H})$, $3.73(\mathrm{~d}, J=11.6 \mathrm{~Hz}, 2 \mathrm{H}), 3.26(\mathrm{dd}, J=18.0,6.8 \mathrm{~Hz}, 2 \mathrm{H}), 2.92(\mathrm{~d}, J=18.0 \mathrm{~Hz}, 2 \mathrm{H})$, $0.91(\mathrm{~s}, 18 \mathrm{H})$.

${ }^{13} \mathrm{C} \mathrm{NMR}\left(100 \mathrm{MHz}, \mathrm{CDCl}_{3}\right) \delta 164.8,142.0,139.7,128.1,127.2,125.7,124.8,82.2$, 
$76.4,72.2,59.2,47.8,39.5,27.2$.

IR (neat): 2968, 1657, 1457, 1260, 1193, 1059, 1019, 988, 802, $755 \mathrm{~cm}^{-1}$.

HRMS: $m / z$ (ESI) calculated for $\mathrm{C}_{31} \mathrm{H}_{39} \mathrm{~N}_{2} \mathrm{O}_{4}{ }^{+}[\mathrm{M}+\mathrm{H}]^{+}$: 503.2904, found: 503.2908.

\subsection{Synthesis of $N$-Fluoro- $N$-Alkylbenzenesulfonamides (NFAS)}

The preparation of $\mathbf{2 a - 2 d}$ was performed according to the reported procedure ${ }^{1}$. And the data of $N$-fluoro- $N$-alkylbenzenesulfonamides 2a were consistent with the reported literature ${ }^{1}$.

$\mathbf{2 b}$ was selected as an example for the operation in details.<smiles>CC(C)(C)NS(=O)(=O)c1ccccc1Cl</smiles>

s1

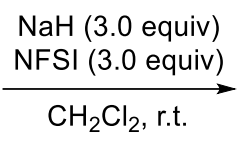

$\mathrm{CH}_{2} \mathrm{Cl}_{2}$, r.t.<smiles>CC(C)(C)N(F)S(=O)c1ccccc1Cl</smiles>

2b

In a $500 \mathrm{~mL}$ round-bottomed flask, to a stirred suspension of $\mathrm{NaH}(3.6 \mathrm{~g}, 90 \mathrm{mmol}$, $60 \mathrm{wt} \%$ in mineral oil) in anhydrous $\mathrm{CH}_{2} \mathrm{Cl}_{2}(250 \mathrm{~mL})$, a solution of $N$-(tert-butyl)-2-chlorobenzenesulfonamide $\mathbf{S 1}(7.43 \mathrm{~g}, 30 \mathrm{mmol})$ in anhydrous $\mathrm{CH}_{2} \mathrm{Cl}_{2}(50 \mathrm{~mL})$ was slowly added at room temperature under a nitrogen atmosphere. After stirring for $30 \mathrm{~min}, \mathrm{~N}$-fluoro-benzenesulfonimide (NFSI, $28.4 \mathrm{~g}, 90 \mathrm{mmol}$ ) was added. The reaction mixture was stirred for another $6 \mathrm{~h}$, then quenched with water slowly. The reaction mixture was extracted with $\mathrm{CH}_{2} \mathrm{Cl}_{2}(200 \mathrm{~mL} \times 3)$. The organic layers were combined and dried over anhydrous $\mathrm{MgSO}_{4}$, and filtered through a pad of Celite. The organic solvent was removed under vaccum, and the residue was purified by column chromatography on silica gel (petroleum ether/ethyl acetate 20:1) to afford the product NFAS $\mathbf{2} \mathbf{b}$ as a white solid, which was further purified by recrystallization from hexanes (4.93 g, 62\% yield).

$N$-(tert-butyl)-2-chloro- $N$-fluorobenzenesulfonamide (2b).

M.p. $39.2-40.1^{\circ} \mathrm{C}$.

${ }^{1} \mathrm{H}$ NMR $\left(400 \mathrm{MHz}, \mathrm{CDCl}_{3}\right) \delta 8.13(\mathrm{~d}, J=8.0 \mathrm{~Hz}, 1 \mathrm{H}), 7.58-7.55(\mathrm{~m}, 2 \mathrm{H}), 7.46-$ $7.17(\mathrm{~m}, 1 \mathrm{H}), 1.56(\mathrm{~s}, 9 \mathrm{H})$.

${ }^{13} \mathrm{C} \mathrm{NMR}\left(100 \mathrm{MHz}, \mathrm{CDCl}_{3}\right) \delta 135.1,134.6,133.9,133.0,132.2,126.9,67.3(\mathrm{~d}, J=$ 
$12.8 \mathrm{~Hz}), 27.5(\mathrm{~d}, J=6.0 \mathrm{~Hz})$.

${ }^{19} \mathrm{~F}$ NMR (376 MHz, $\left.\mathrm{CDCl}_{3}\right) \delta-61.0(\mathrm{~s})$.

IR (neat): 2981, 1573, 1353, 1171, 1044, 894, 757, 610, 555, 529, $470 \mathrm{~cm}^{-1}$.

HRMS: $m / z$ (DART) calculated for $\mathrm{C}_{10} \mathrm{H}_{14} \mathrm{NO}_{2} \mathrm{ClFS}^{+}[\mathrm{M}+\mathrm{H}]^{+}: 266.0412$, found: 266.0412 .<smiles>CC(C)(c1ccccc1)N(F)S(=O)c1ccccc1Cl</smiles>

2c

2-chloro- $N$-fluoro- $N$-(2-phenylpropan-2-yl)benzenesulfonamide (2c).

M.p. $50.9-52.7^{\circ} \mathrm{C}$.

${ }^{1} \mathrm{H}$ NMR $\left(400 \mathrm{MHz}, \mathrm{CDCl}_{3}\right) \delta 8.08(\mathrm{~d}, J=8.0 \mathrm{~Hz}, 1 \mathrm{H}), 7.59-7.57(\mathrm{~m}, 4 \mathrm{H}), 7.43-$ 7.39 (m, 1H), $7.37-7.33(\mathrm{~m}, 2 \mathrm{H}), 7.31-7.26(\mathrm{~m}, 1 \mathrm{H}), 1.98$ (s, 6H).

${ }^{13} \mathrm{C}$ NMR $\left(100 \mathrm{MHz}, \mathrm{CDCl}_{3}\right) \delta 143.2(\mathrm{~d}, J=2.7 \mathrm{~Hz}), 135.1,134.6,133.9,133.1$, $132.3,128.2,127.8,126.9,126.0,70.7$ (d, $J=13.3 \mathrm{~Hz}), 26.5$ (d, $J=6.2 \mathrm{~Hz})$.

${ }^{19} \mathrm{~F} \mathrm{NMR}\left(376 \mathrm{MHz}, \mathrm{CDCl}_{3}\right) \delta-56.2(\mathrm{~s})$.

IR (neat): 3004, 1574, 1453, 1356, 1254, 1173, 1153, 1110, 1041, 897, 768, 696, 565, $545,467 \mathrm{~cm}^{-1}$.

Anal. Calcd. for $\mathrm{C}_{15} \mathrm{H}_{15} \mathrm{NO}_{2} \mathrm{ClFS}$ : C 54.96, H 4.61, N 4.27, found: C 54.72, H 4.57, N 4.14 .

HRMS: $m / z$ (ESI) calculated for $\mathrm{C}_{15} \mathrm{H}_{15} \mathrm{NO}_{2} \mathrm{ClFSNa}^{+}[\mathrm{M}+\mathrm{Na}]^{+}: 350.0388$, found: 350.0389;<smiles>CC(C)(C)N(F)S(=O)c1c(Cl)cccc1Cl</smiles>

2d

$N$-(tert-butyl)-2,6-dichloro- $N$-fluorobenzenesulfonamide (2d).

M.p. $79.0-79.5^{\circ} \mathrm{C}$.

${ }^{1} \mathrm{H}$ NMR (400 MHz, $\left.\mathrm{CDCl}_{3}\right) \delta 7.50-7.48(\mathrm{~m}, 2 \mathrm{H}), 7.42-7.38(\mathrm{~m}, 1 \mathrm{H}), 1.57(\mathrm{~s}, 9 \mathrm{H})$.

${ }^{13} \mathrm{C}$ NMR $\left(100 \mathrm{MHz}, \mathrm{CDCl}_{3}\right) \delta 137.4,133.9,132.4,131.7,67.8(\mathrm{~d}, J=11.6 \mathrm{~Hz}), 27.5$ $(\mathrm{d}, J=6.0 \mathrm{~Hz})$. 
${ }^{19} \mathrm{~F}$ NMR $\left(376 \mathrm{MHz}, \mathrm{CDCl}_{3}\right) \delta-62.2(\mathrm{~s})$.

IR (neat): 2978, 1559, 1422, 1363, 1235, 1172, 893, 801, 783, 632, 611, 564, 454

$\mathrm{cm}^{-1}$.

HRMS: $m / z$ (DART) calculated for $\mathrm{C}_{10} \mathrm{H}_{13} \mathrm{NO}_{2} \mathrm{Cl}_{2} \mathrm{FS}^{+}[\mathrm{M}+\mathrm{H}]^{+}:$300.0023, found: 300.0022 .

\subsection{Procedure for Synthesis of Starting Materials}

The preparation of 1a-1z, 1aa-1ae was performed according to the reported procedure ${ }^{2,3}$. And the data of $\mathbf{1} \mathbf{c}^{2}, \mathbf{1 d}^{4}, \mathbf{1 e}^{5}, \mathbf{1 g}^{6}, \mathbf{1 a e}^{5}$ were consistent with the reported literatures.

\subsubsection{General Procedure 1 for the Preparation of Benzyl Alkynes}

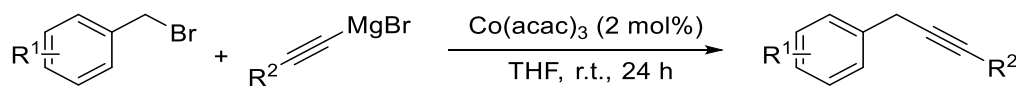

To a solution of terminal alkynes $(30 \mathrm{mmol})$ in dry THF $(60 \mathrm{~mL})$ was slowly added a solution of ethylmagnesium bromide $\left(30 \mathrm{ml}, 1.0 \mathrm{M}\right.$ in THF) at $0{ }^{\circ} \mathrm{C}$ under a nitrogen atmosphere, and then the mixture was heated up to $40{ }^{\circ} \mathrm{C}$ and stirred for $2 \mathrm{~h}$. After cooling down to room temperature, a mixture of benzyl bromide $(20 \mathrm{mmol})$ and $\mathrm{Co}(\operatorname{acac})_{3}(2 \mathrm{~mol} \%)$ in dry THF $(40 \mathrm{~mL})$ was added into the reaction mixture at $0{ }^{\circ} \mathrm{C}$ and the mixture was stirred under room temperature for $24 \mathrm{~h}$. After addition of sat. aq $\mathrm{NH}_{4} \mathrm{Cl}$, the aqueous layer was extracted with diethyl ether for three times. The combined organic layer was then washed by brine, dried over $\mathrm{Na}_{2} \mathrm{SO}_{4}$, filtered, and concentrated under reduced pressure, the residue was purified by column chromatography on silica gel or distillation under high vacuum to afford the corresponding products ${ }^{2}$.

\subsubsection{General Procedure 2 for the Preparation of Benzyl Alkynes}



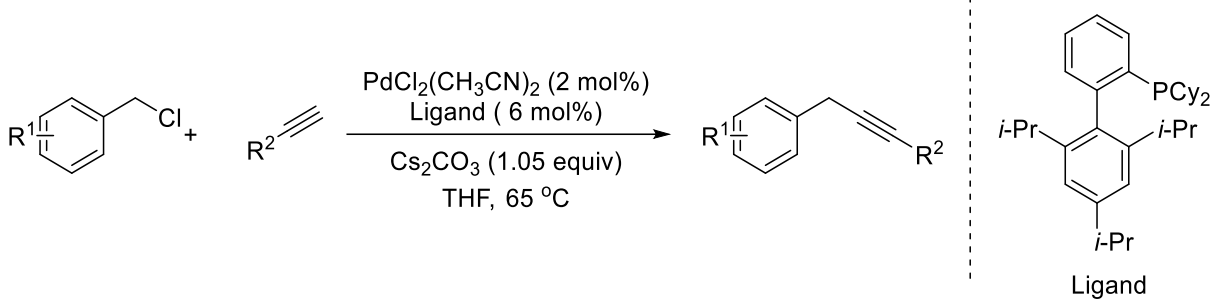

A Schlenk tube equipped with a stirring bar was charged with $\mathrm{PdCl}_{2}\left(\mathrm{CH}_{3} \mathrm{CN}\right)_{2}(0.4$ mmol, 2 mol\%), 2-dicyclohexylphosphino-2',4',6'-triisopropylbiphenyl (1.2 mmol, 6 mol\%), and $\mathrm{Cs}_{2} \mathrm{CO}_{3}(342 \mathrm{mg}, 21 \mathrm{mmol})$. The tube was evacuated and back-filled with argon for three times. Dry THF $(50 \mathrm{~mL})$ and benzyl chloride $(20 \mathrm{mmol})$ were added and bubbled for $10 \mathrm{~min}$. Terminal alkynes $(30 \mathrm{mmol})$ were then added and the mixture was heated to $65{ }^{\circ} \mathrm{C}$ until the reaction was completed as monitored by TLC plate. After cooling down to room temperature, filtered off insoluble solids, and concentrated under reduced pressure. The residue was purified by column chromatography on silica gel or distillation under high vacuum to afford the corresponding products ${ }^{3}$. (Note: neat products are stored at $-30{ }^{\circ} \mathrm{C}$ in glove box refrigerator).<smiles>CC(C)CC#CCc1ccc(Br)cc1</smiles>

${ }^{1} \mathrm{H}$ NMR (400 MHz, $\left.\mathrm{CDCl}_{3}\right) \delta 7.45-7.42(\mathrm{~m}, 2 \mathrm{H}), 7.24-7.21(\mathrm{~m}, 2 \mathrm{H}), 3.53(\mathrm{t}, J=$ $2.0 \mathrm{~Hz}, 2 \mathrm{H}), 2.25-2.21(\mathrm{~m}, 2 \mathrm{H}), 1.56-1.49(\mathrm{~m}, 2 \mathrm{H}), 1.46-1.39(\mathrm{~m}, 2 \mathrm{H}), 0.93(\mathrm{t}, J$ $=7.2 \mathrm{~Hz}, 3 \mathrm{H})$.

${ }^{13} \mathrm{C}$ NMR $\left(100 \mathrm{MHz}, \mathrm{CDCl}_{3}\right) \delta 136.8,131.6,129.8,120.3,83.3,77.5,31.2,24.8,22.2$, $18.7,13.8$.

IR (neat): 2957, 2930, 1710, 1486, 1404, 1070, 1011, 795, 634, $477 \mathrm{~cm}^{-1}$.

HRMS: $m / z$ (EI) calculated for $\mathrm{C}_{13} \mathrm{H}_{15} \mathrm{Br}^{+}[\mathrm{M}]^{+}:$250.0352, found: 250.0361 .<smiles>CCCC#CCc1ccccc1</smiles>

1b

${ }^{1} \mathrm{H}$ NMR (400 MHz, $\left.\mathrm{CDCl}_{3}\right) \delta 7.36-7.28(\mathrm{~m}, 4 \mathrm{H}), 7.23-7.21(\mathrm{~m}, 1 \mathrm{H}), 3.58(\mathrm{t}, J=$ $2.4 \mathrm{~Hz}, 2 \mathrm{H}), 2.20-2.17(\mathrm{~m}, 2 \mathrm{H}), 1.58-1.50(\mathrm{~m}, 2 \mathrm{H}), 1.00(\mathrm{t}, J=7.2 \mathrm{~Hz}, 3 \mathrm{H})$. 
${ }^{13} \mathrm{C} \mathrm{NMR}\left(100 \mathrm{MHz}, \mathrm{CDCl}_{3}\right) \delta 137.6,128.4,127.8,126.3,82.5,77.6,25.1,22.4,20.8$, 13.5 .

IR (neat): 3029, 2962, 1603, 1494, 1453, 1336, 1072, 725, 695, 596, $459 \mathrm{~cm}^{-1}$.

HRMS: $m / z$ (EI) calculated for $\mathrm{C}_{12} \mathrm{H}_{14}{ }^{+}[\mathrm{M}]^{+}:$158.1090, found: 158.1095 .<smiles>C(#CC1CCCCC1)Cc1ccccc1</smiles>

(containing 14\% 1,2-diphenylethane)

${ }^{1} \mathrm{H}$ NMR (400 MHz, $\left.\mathrm{CDCl}_{3}\right) \delta 7.38$ - $7.19(\mathrm{~m}, 5 \mathrm{H}), 3.61$ (s, 2H), $2.41-2.42(\mathrm{~m}, 1 \mathrm{H})$, $1.85-1.82(\mathrm{~m}, 2 \mathrm{H}), 1.74-1.71(\mathrm{~m}, 2 \mathrm{H}), 1.54-1.27(\mathrm{~m}, 6 \mathrm{H})$.

${ }^{13} \mathrm{C}$ NMR $\left(100 \mathrm{MHz}, \mathrm{CDCl}_{3}\right) \delta 137.7,128.3,127.8,126.3,87.1,77.3,33.0,29.2,25.9$, 25.1, 25.0 .

IR (neat): 2927, 2853, 1602, 1494, 1450, 1072, 1029, 908, 726, 695, 605, $459 \mathrm{~cm}^{-1}$. HRMS: $m / z$ (EI) calculated for $\mathrm{C}_{15} \mathrm{H}_{18}{ }^{+}[\mathrm{M}]^{+}:$198.1403, found: 198.1403.<smiles>Cc1ccc(CC#CCC(C)C)cc1</smiles>

${ }^{1} \mathrm{H}$ NMR (400 MHz, $\left.\mathrm{CDCl}_{3}\right) \delta 7.22(\mathrm{~d}, J=8.0 \mathrm{~Hz}, 2 \mathrm{H}), 7.11(\mathrm{~d}, J=7.6 \mathrm{~Hz}, 2 \mathrm{H}), 3.52$ $(\mathrm{t}, J=2.3 \mathrm{~Hz}, 2 \mathrm{H}), 2.32(\mathrm{~s}, 3 \mathrm{H}), 2.23-2.19(\mathrm{~m}, 2 \mathrm{H}), 1.52-1.47(\mathrm{~m}, 2 \mathrm{H}), 1.45-1.41$ (m, 2H), $0.91(\mathrm{t}, J=7.2 \mathrm{~Hz}, 3 \mathrm{H})$.

${ }^{13} \mathrm{C}$ NMR $\left(100 \mathrm{MHz}, \mathrm{CDCl}_{3}\right) \delta 135.8,134.6,129.1,127.7,82.4,77.7,31.1,24.7,22.0$, 21.0, 18.5, 13.6.

IR (neat): 2957, 2929, 2862, 1513, 1458, 1425, 792, 744, $476 \mathrm{~cm}^{-1}$.

HRMS: $m / z$ (EI) calculated for $\mathrm{C}_{14} \mathrm{H}_{18}{ }^{+}[\mathrm{M}]^{+}:$186.1403, found: 186.1409 .<smiles>COc1ccc(CC#CCC(C)C)cc1</smiles>

1j

${ }^{1} \mathrm{H}$ NMR (400 MHz, $\left.\mathrm{CDCl}_{3}\right) \delta 7.26-7.24(\mathrm{~m}, 2 \mathrm{H}), 6.86-6.83(\mathrm{~m}, 2 \mathrm{H}), 3.78(\mathrm{~s}, 3 \mathrm{H})$, $3.51(\mathrm{t}, J=2.4 \mathrm{~Hz}, 2 \mathrm{H}), 2.23-2.19(\mathrm{~m}, 2 \mathrm{H}), 1.53-1.49(\mathrm{~m}, 2 \mathrm{H}), 1.45-1.41(\mathrm{~m}, 2 \mathrm{H})$, $0.91(\mathrm{t}, J=7.2 \mathrm{~Hz}, 3 \mathrm{H})$.

${ }^{13} \mathrm{C}$ NMR $\left(100 \mathrm{MHz}, \mathrm{CDCl}_{3}\right) \delta$ 158.2, 129.7, 128.7, 113.8, 82.3, 77.9, 55.2, 31.1, 24.2, S13 
$21.9,18.5,13.6$.

IR (neat): 2931, 1610, 1510, 1462, 1244, 1174, 1105, 1035, 815, $510 \mathrm{~cm}^{-1}$.

HRMS: $m / z$ (EI) calculated for $\mathrm{C}_{14} \mathrm{H}_{18} \mathrm{O}^{+}[\mathrm{M}]^{+}: 202.1352$, found: 202.1359 .<smiles>CCCC#CCc1ccc(F)cc1</smiles>

${ }^{1} \mathrm{H}$ NMR $\left(400 \mathrm{MHz}, \mathrm{CDCl}_{3}\right) \delta 7.32-7.29(\mathrm{~m}, 2 \mathrm{H}), 7.02-6.98(\mathrm{~m}, 2 \mathrm{H}), 3.55(\mathrm{t}, J=$ $2.0 \mathrm{~Hz}, 2 \mathrm{H}), 2.26-2.21(\mathrm{~m}, 2 \mathrm{H}), 1.55-1.49(\mathrm{~m}, 2 \mathrm{H}), 1.47-1.41(\mathrm{~m}, 2 \mathrm{H}), 0.93(\mathrm{t}, J$ $=7.6 \mathrm{~Hz}, 3 \mathrm{H})$.

${ }^{13} \mathrm{C} \mathrm{NMR}\left(100 \mathrm{MHz}, \mathrm{CDCl}_{3}\right) \delta 161.6(\mathrm{~d}, J=242.4 \mathrm{~Hz}), 133.2(\mathrm{~d}, J=2.8 \mathrm{~Hz}), 129.2$ (d, $J=8.8 \mathrm{~Hz}), 115.1(\mathrm{~d}, J=21.5 \mathrm{~Hz}), 82.8,77.3,31.1,24.4,22.0,18.5,13.6$.

${ }^{19} \mathrm{~F}$ NMR $\left(376 \mathrm{MHz}, \mathrm{CDCl}_{3}\right) \delta-117.1(\mathrm{~m})$.

IR (neat): 2932, 1605, 1508, 1221, 1156, 814, 750, $496 \mathrm{~cm}^{-1}$.

HRMS: $m / z$ (EI) calculated for $\mathrm{C}_{13} \mathrm{H}_{15} \mathrm{~F}^{+}[\mathrm{M}]^{+}:$190.1152, found: 190.1159 .<smiles>CC#CCc1ccc(Br)cc1</smiles>

${ }^{1} \mathrm{H}$ NMR (400 MHz, $\left.\mathrm{CDCl}_{3}\right) \delta 7.41(\mathrm{~d}, J=8.4 \mathrm{~Hz}, 2 \mathrm{H}), 7.19(\mathrm{~d}, J=8.4 \mathrm{~Hz}, 2 \mathrm{H}), 3.48$ (q, $J=2.4 \mathrm{~Hz}, 2 \mathrm{H}), 1.84(\mathrm{t}, J=2.4 \mathrm{~Hz}, 3 \mathrm{H})$.

${ }^{13} \mathrm{C}$ NMR $\left(100 \mathrm{MHz}, \mathrm{CDCl}_{3}\right) \delta 136.5,131.4,129.6,120.2,78.3,76.1,24.6,3.5$.

IR (neat): 2917, 1485, 1422, 1403, 1069, 1011, 797, 633, $478 \mathrm{~cm}^{-1}$.

HRMS: $m / z$ (EI) calculated for $\mathrm{C}_{10} \mathrm{H}_{9} \mathrm{Br}^{+}[\mathrm{M}]^{+}:$207.9882, found: 207.9890 .<smiles>CC(C)CC#CCc1ccc(I)cc1</smiles>

$1 \mathrm{~m}$

${ }^{1} \mathrm{H}$ NMR (400 MHz, $\left.\mathrm{CDCl}_{3}\right) \delta 7.63(\mathrm{~d}, J=8.0 \mathrm{~Hz}, 2 \mathrm{H}), 7.10(\mathrm{~d}, J=8.0 \mathrm{~Hz}, 2 \mathrm{H}), 3.51$ $(\mathrm{t}, J=2.4 \mathrm{~Hz}, 2 \mathrm{H}), 2.24-2.20(\mathrm{~m}, 2 \mathrm{H}), 1.55-1.40(\mathrm{~m}, 4 \mathrm{H}), 0.92(\mathrm{t}, J=7.6 \mathrm{~Hz}, 3 \mathrm{H})$. ${ }^{13} \mathrm{C} \mathrm{NMR}\left(100 \mathrm{MHz}, \mathrm{CDCl}_{3}\right) \delta 137.4,137.3,129.9,91.5,83.1,76.7,31.0,24.8,22.0$, $18.5,13.6$.

IR (neat): 2955, 2927, 1706, 1482, 1398, 1185, 1006, 790, 624, $472 \mathrm{~cm}^{-1}$.

HRMS: $m / z$ (EI) calculated for $\mathrm{C}_{13} \mathrm{H}_{15} \mathrm{I}^{+}[\mathrm{M}]^{+}: 298.0213$, found: 298.0219 . 
<smiles>CCCC#CCc1ccc(C(F)(F)F)cc1</smiles>

${ }^{1} \mathrm{H}$ NMR (400 MHz, $\left.\mathrm{CDCl}_{3}\right) \delta 7.57(\mathrm{~d}, J=8.4 \mathrm{~Hz}, 2 \mathrm{H}), 7.47(\mathrm{~d}, J=8.0 \mathrm{~Hz}, 2 \mathrm{H}), 3.64$ $(\mathrm{t}, J=2.4 \mathrm{~Hz}, 2 \mathrm{H}), 2.26-2.23(\mathrm{~m}, 2 \mathrm{H}), 1.55-1.51(\mathrm{~m}, 2 \mathrm{H}), 1.47-1.41(\mathrm{~m}, 2 \mathrm{H})$, $0.93(\mathrm{t}, J=7.6 \mathrm{~Hz}, 3 \mathrm{H})$.

${ }^{13} \mathrm{C} \mathrm{NMR}\left(100 \mathrm{MHz}, \mathrm{CDCl}_{3}\right) \delta 141.8,128.8(\mathrm{q}, J=32.0 \mathrm{~Hz}), 128.2,125.3(\mathrm{q}, J=3.7$ Hz), 124.3 (q, $J=270.0 \mathrm{~Hz}), 83.5,76.4,31.0,25.1,22.0,18.5,13.6$.

${ }^{19} \mathrm{~F}$ NMR (376 MHz, $\left.\mathrm{CDCl}_{3}\right) \delta-62.4(\mathrm{~s})$.

IR (neat): 2933, 1620, 1419, 1323, 1162, 1121,1065, 922, $816 \mathrm{~cm}^{-1}$.

HRMS: $m / z$ (EI) calculated for $\mathrm{C}_{14} \mathrm{H}_{15} \mathrm{~F}_{3}{ }^{+}[\mathrm{M}]^{+}:$240.1120, found: 240.1126.<smiles>CCCC#CCc1cccc(Br)c1</smiles>

${ }^{1} \mathrm{H}$ NMR $\left(400 \mathrm{MHz}, \mathrm{CDCl}_{3}\right) \delta 7.50(\mathrm{~s}, 1 \mathrm{H}), 7.34-7.32(\mathrm{~m}, 1 \mathrm{H}), 7.26-7.23(\mathrm{~m}, 1 \mathrm{H})$, $7.16-7.12(\mathrm{~m}, 1 \mathrm{H}), 3.54(\mathrm{t}, J=2.4 \mathrm{~Hz}, 2 \mathrm{H}), 2.22-2.17(\mathrm{~m}, 2 \mathrm{H}), 1.57-1.52(\mathrm{~m}, 2 \mathrm{H})$, $0.99(\mathrm{t}, J=7.6 \mathrm{~Hz}, 3 \mathrm{H})$.

${ }^{13} \mathrm{C} \mathrm{NMR}\left(100 \mathrm{MHz}, \mathrm{CDCl}_{3}\right) \delta 139.9,130.9,129.8,129.5,126.4,122.4,83.2,76.7$, $24.8,22.3,20.8,13.5$.

IR (neat): 2961, 2931, 1594, 1569, 1470, 1426, 1069, 839, 768, 681, 666, $429 \mathrm{~cm}^{-1}$; HRMS: $m / z$ (EI) calculated for $\mathrm{C}_{12} \mathrm{H}_{13} \mathrm{Br}^{+}[\mathrm{M}]^{+}:$236.0195, found: 236.0198.<smiles>CC(C)CC#CCc1ccccc1Cl</smiles>

${ }^{1} \mathrm{H}$ NMR $\left(400 \mathrm{MHz}, \mathrm{CDCl}_{3}\right) \delta 7.62-7.60(\mathrm{~m}, 1 \mathrm{H}), 7.32$ - $7.30(\mathrm{~m}, 1 \mathrm{H}), 7.26-7.22$ (m, 1H), $7.18-7.16(\mathrm{~m}, 1 \mathrm{H}), 3.63$ (t, $J=2.4 \mathrm{~Hz}, 2 \mathrm{H}), 2.25-2.20(\mathrm{~m}, 2 \mathrm{H}), 1.53-$ $1.48(\mathrm{~m}, 2 \mathrm{H}), 1.45-1.39(\mathrm{~m}, 2 \mathrm{H}), 0.90(\mathrm{t}, J=7.2 \mathrm{~Hz}, 3 \mathrm{H})$.

${ }^{13} \mathrm{C}$ NMR $\left(100 \mathrm{MHz}, \mathrm{CDCl}_{3}\right) \delta 135.2,133.4,129.5,129.0,127.8,126.8,83.7,76.0$, 31.1, 23.3, 22.0, 18.5, 13.6.

IR (neat): 2931, 1573, 1468, 1443, 1323, 1124, 1037, 746, 696, $433 \mathrm{~cm}^{-1}$. 
HRMS: $m / z$ (EI) calculated for $\mathrm{C}_{13} \mathrm{H}_{15} \mathrm{Cl}^{+}[\mathrm{M}]^{+}:$206.0857, found: 206.0863.<smiles>CC(C)(C)CC#CCc1ccc2c(c1)OC(F)(F)O2</smiles>

${ }^{1} \mathrm{H}$ NMR $\left(400 \mathrm{MHz}, \mathrm{CDCl}_{3}\right) \delta 7.11(\mathrm{~s}, 1 \mathrm{H}), 7.03-7.01(\mathrm{~m}, 1 \mathrm{H}), 6.98-6.96(\mathrm{~m}, 1 \mathrm{H})$, $3.56(\mathrm{t}, J=2.4 \mathrm{~Hz}, 2 \mathrm{H}), 2.24-2.19(\mathrm{~m}, 2 \mathrm{H}), 1.57-1.50(\mathrm{~m}, 2 \mathrm{H}), 1.42-1.26(\mathrm{~m}, 4 \mathrm{H})$, $0.91(\mathrm{t}, J=7.2 \mathrm{~Hz}, 3 \mathrm{H})$.

${ }^{13} \mathrm{C} \mathrm{NMR}\left(100 \mathrm{MHz}, \mathrm{CDCl}_{3}\right) \delta 143.9,142.3,133.8,131.7$ (t, $\left.J=252.9 \mathrm{~Hz}\right), 122.6$, 109.3, 109.0, 83.5, 76.8, 31.1, 28.6, 25.0, 22.2, 18.7, 14.0.

${ }^{19} \mathrm{~F} \mathrm{NMR}\left(376 \mathrm{MHz}, \mathrm{CDCl}_{3}\right) \delta-50.0(\mathrm{~s})$.

IR (neat): 2932, 1497, 1448, 1233, 1149, 1035, 948, 801, $702 \mathrm{~cm}^{-1}$.

HRMS: $m / z$ (EI) calculated for $\mathrm{C}_{15} \mathrm{H}_{16} \mathrm{~F}_{2} \mathrm{O}_{2}{ }^{+}[\mathrm{M}]^{+}:$266.1113, found: 266.1122 .<smiles>CC(C)CC#CCc1cc(C(C)(C)C)cc(C(C)(C)C)c1</smiles>

${ }^{1} \mathrm{H}$ NMR (400 MHz, $\left.\mathrm{CDCl}_{3}\right) \delta 7.29(\mathrm{~s}, 1 \mathrm{H}), 7.21-7.20(\mathrm{~m}, 2 \mathrm{H}), 3.57(\mathrm{t}, J=2.0 \mathrm{~Hz}$, 2H), $2.26-2.22(\mathrm{~m}, 2 \mathrm{H}), 1.52-1.46(\mathrm{~m}, 4 \mathrm{H}), 1.33(\mathrm{~s}, 18 \mathrm{H}), 0.92(\mathrm{t}, J=7.2 \mathrm{~Hz}, 3 \mathrm{H})$. ${ }^{13} \mathrm{C} \mathrm{NMR}\left(100 \mathrm{MHz}, \mathrm{CDCl}_{3}\right) \delta 150.7,136.6,122.1,120.4,82.4,78.0,34.8,31.5,31.3$, $25.5,21.9,18.5,13.6$.

IR (neat): 2956, 2866, 1599, 1462, 1362, 1248, 851, $711 \mathrm{~cm}^{-1}$.

HRMS: $m / z$ (EI) calculated for $\mathrm{C}_{21} \mathrm{H}_{32}{ }^{+}[\mathrm{M}]^{+}:$284.2499, found: 284.2502.<smiles>ClCCCC#CCc1ccccc1</smiles>

$1 \mathrm{t}$

${ }^{1} \mathrm{H}$ NMR $\left(400 \mathrm{MHz}, \mathrm{CDCl}_{3}\right) \delta 7.33-7.28(\mathrm{~m}, 4 \mathrm{H}), 7.24-7.22(\mathrm{~m}, 1 \mathrm{H}), 3.65(\mathrm{t}, J=$ $6.4 \mathrm{~Hz}, 2 \mathrm{H}), 3.57$ (t, $J=2.8 \mathrm{~Hz}, 2 \mathrm{H}), 2.43-2.39$ (m, 2H), $1.99-1.92$ (m, 2H).

${ }^{13} \mathrm{C} \mathrm{NMR}\left(100 \mathrm{MHz}, \mathrm{CDCl}_{3}\right) \delta 137.2,128.4,127.8,126.4,80.4,78.7,43.8,31.6,25.1$, 16.2 .

IR (neat): 2958, 1603, 1494, 1289, 1072, 1028, 724, 695, 653, $460 \mathrm{~cm}^{-1}$. HRMS: $m / z$ (EI) calculated for $\mathrm{C}_{12} \mathrm{H}_{13} \mathrm{Cl}^{+}[\mathrm{M}]^{+}:$192.0700, found: 192.0707. 


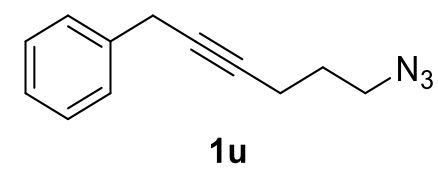

${ }^{1} \mathrm{H}$ NMR $\left(400 \mathrm{MHz}, \mathrm{CDCl}_{3}\right) \delta 7.36-7.25(\mathrm{~m}, 4 \mathrm{H}), 7.26-7.25(\mathrm{~m}, 1 \mathrm{H}), 3.60(\mathrm{t}, J=$ $2.4 \mathrm{~Hz}, 2 \mathrm{H}), 3.44(\mathrm{t}, J=6.8 \mathrm{~Hz}, 2 \mathrm{H}), 2.39-2.34(\mathrm{~m}, 2 \mathrm{H}), 1.84-1.78(\mathrm{~m}, 2 \mathrm{H})$.

${ }^{13} \mathrm{C}$ NMR $\left(100 \mathrm{MHz} \mathrm{CDCl}_{3}\right) \delta 137.2,128.4,127.8,126.5,80.5,78.9,50.2,28.1,25.0$, 16.1.

IR (neat): 2930, 2091, 1494, 1452, 1254, 725, 695, $460 \mathrm{~cm}^{-1}$. HRMS: $m / z$ (EI) calculated for $\mathrm{C}_{12} \mathrm{H}_{12} \mathrm{~N}^{+}\left[\mathrm{M}-\mathrm{N}_{2}-\mathrm{H}\right]^{+}: 170.0964$, found: 170.0970 .<smiles>OCCC#CCc1ccccc1</smiles>

${ }^{1} \mathrm{H}$ NMR (400 MHz, $\left.\mathrm{CDCl}_{3}\right) \delta 7.34-7.22(\mathrm{~m}, 5 \mathrm{H}), 3.72(\mathrm{t}, J=6.0 \mathrm{~Hz}, 2 \mathrm{H}), 3.60(\mathrm{t}, J$ $=2.4 \mathrm{~Hz}, 2 \mathrm{H}), 2.52-2.49(\mathrm{~m}, 2 \mathrm{H}), 1.96(\mathrm{br}, 1 \mathrm{H})$.

${ }^{13} \mathrm{C}$ NMR $\left(100 \mathrm{MHz}, \mathrm{CDCl}_{3}\right) \delta 137.0,128.5,127.8,126.5,79.9,78.8,61.3,25.1,23.2$. IR (neat): 3332, 2884, 1601, 1494, 1452, 1420, 1336, 1041, 725, 694, $459 \mathrm{~cm}^{-1}$. HRMS: $m / z$ (EI) calculated for $\mathrm{C}_{11} \mathrm{H}_{12} \mathrm{O}^{+}[\mathrm{M}]^{+}: 160.0883$, found: 160.0881 .<smiles>OCCCC#CCc1ccccc1</smiles>

${ }^{1} \mathrm{H}$ NMR (400 MHz, $\left.\mathrm{CDCl}_{3}\right) \delta 7.36-7.22(\mathrm{~m}, 5 \mathrm{H}), 3.75(\mathrm{t}, J=6.0 \mathrm{~Hz}, 2 \mathrm{H}), 3.58(\mathrm{t}, J$ $=2.4 \mathrm{~Hz}, 2 \mathrm{H}), 2.37-2.33(\mathrm{~m}, 2 \mathrm{H}), 2.13(\mathrm{br}, 1 \mathrm{H}), 1.81-1.74(\mathrm{~m}, 2 \mathrm{H})$.

${ }^{13} \mathrm{C}$ NMR $\left(100 \mathrm{MHz}, \mathrm{CDCl}_{3}\right) \delta 137.3,128.4,127.7,126.4,81.6,78.2,61.7,31.4,25.0$, 15.3.

IR (neat): 3321, 2884, 1601, 1494, 1452, 1421, 1040, 725, 695, 597, $459 \mathrm{~cm}^{-1}$. HRMS: $m / z$ (EI) calculated for $\mathrm{C}_{12} \mathrm{H}_{14} \mathrm{O}^{+}[\mathrm{M}]^{+}:$174.1039, found: 174.1040 .<smiles>[X]CCCC#CCc1ccccc1</smiles>

${ }^{1} \mathrm{H}$ NMR $\left(400 \mathrm{MHz}, \mathrm{CDCl}_{3}\right) \delta 7.79(\mathrm{~d}, J=8.0 \mathrm{~Hz}, 2 \mathrm{H}), 7.33-7.23(\mathrm{~m}, 7 \mathrm{H}), 4.16(\mathrm{t}, J$ $=6.4 \mathrm{~Hz}, 2 \mathrm{H}), 3.49(\mathrm{t}, J=2.4 \mathrm{~Hz}, 2 \mathrm{H}), 2.41(\mathrm{~s}, 3 \mathrm{H}), 2.32-2.27(\mathrm{~m}, 2 \mathrm{H}), 1.89-1.84$ 
(m, 2H).

${ }^{13} \mathrm{C} \mathrm{NMR}\left(100 \mathrm{MHz}, \mathrm{CDCl}_{3}\right) \delta 144.7,137.1,133.0,129.8,128.4,127.9,127.7,126.5$, 80.1, 78.9, 69.1, 28.1, 25.0, 21.6, 15.1.

IR (neat): 2923, 1598, 1494, 1452, 1357, 1173, 1096, 927, 813, 728, 662, 574, 552 $\mathrm{cm}^{-1}$.

HRMS: $m / z$ (ESI) calculated for $\mathrm{C}_{19} \mathrm{H}_{21} \mathrm{O}_{3} \mathrm{~S}^{+}[\mathrm{M}+\mathrm{H}]^{+}$: 329.1206, found: 329.1202 .<smiles>O=C1c2ccccc2C(=O)N1CCCC#CCc1ccccc1</smiles>

(light yellow soild)

M.p. $79.4-81.8{ }^{\circ} \mathrm{C}$.

${ }^{1} \mathrm{H}$ NMR $\left(400 \mathrm{MHz}, \mathrm{CDCl}_{3}\right) \delta 7.83-7.81(\mathrm{~m}, 2 \mathrm{H}), 7.71-7.68(\mathrm{~m}, 2 \mathrm{H}), 7.30-7.28$ (m, 4H), $7.23-7.19(\mathrm{~m}, 1 \mathrm{H}), 3.82(\mathrm{t}, J=6.8 \mathrm{~Hz}, 2 \mathrm{H}), 3.43(\mathrm{t}, J=2.4 \mathrm{~Hz}, 2 \mathrm{H}), 2.34-$ $2.29(\mathrm{~m}, 2 \mathrm{H}), 1.97-1.90(\mathrm{~m}, 2 \mathrm{H})$.

${ }^{13} \mathrm{C}$ NMR $\left(100 \mathrm{MHz}, \mathrm{CDCl}_{3}\right) \delta 168.4,137.2,133.8,132.2,128.4,127.8,126.4,123.2$, 81.1, 78.4, 37.4, 27.6, 25.0, 16.7 .

IR (neat): 2947, 1768, 1704, 1454, 1432, 1373, 1110, 1028, 707, $528 \mathrm{~cm}^{-1}$.

HRMS: $m / z$ (EI) calculated for $\mathrm{C}_{20} \mathrm{H}_{17} \mathrm{NO}_{2}{ }^{+}[\mathrm{M}]^{+}: 303.1254$, found: 303.1261 .

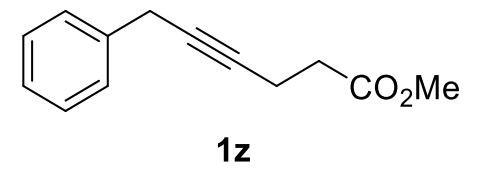

${ }^{1} \mathrm{H}$ NMR (400 MHz, $\left.\mathrm{CDCl}_{3}\right) \delta 7.34-7.24(\mathrm{~m}, 5 \mathrm{H}), 3.70(\mathrm{~s}, 3 \mathrm{H}), 3.58$ (t, $J=2.4 \mathrm{~Hz}$, $2 \mathrm{H}), 2.58-2.56(\mathrm{~m}, 4 \mathrm{H})$.

${ }^{13} \mathrm{C}$ NMR $\left(100 \mathrm{MHz}, \mathrm{CDCl}_{3}\right) \delta 172.4,137.0,128.3,127.7,126.3,80.3,78.4,51.6$, $33.6,24.9,14.7$.

IR (neat): 1739, 1526, 1438, 1364, 1165, 1013, 991, 728, $696 \mathrm{~cm}^{-1}$.

HRMS: $m / z$ (EI) calculated for $\mathrm{C}_{13} \mathrm{H}_{14} \mathrm{O}_{2}{ }^{+}[\mathrm{M}]^{+}: 202.0988$, found: 202.0992 .<smiles>CCCC#CCc1ccsc1</smiles> 
${ }^{1} \mathrm{H}$ NMR (400 MHz, $\left.\mathrm{CDCl}_{3}\right) \delta 7.28-7.26(\mathrm{~m}, 1 \mathrm{H}), 7.14(\mathrm{~s}, 1 \mathrm{H}), 7.01(\mathrm{~d}, J=4.8 \mathrm{~Hz}$, $1 \mathrm{H}), 3.55(\mathrm{~s}, 2 \mathrm{H}), 2.24-2.20(\mathrm{~m}, 2 \mathrm{H}), 1.56-1.41(\mathrm{~m}, 4 \mathrm{H}), 0.92(\mathrm{t}, J=7.6 \mathrm{~Hz}, 3 \mathrm{H})$. ${ }^{13} \mathrm{C} \mathrm{NMR}\left(100 \mathrm{MHz}, \mathrm{CDCl}_{3}\right) \delta 138.0,127.7,125.7,120.9,82.0,77.3,31.1,22.0,20.3$, $18.5,13.6$.

IR (neat): $2957,1425,1300,1078,832,763,683 \mathrm{~cm}^{-1}$.

HRMS: $m / z$ (EI) calculated for $\mathrm{C}_{11} \mathrm{H}_{14} \mathrm{~S}^{+}[\mathrm{M}]^{+}: 178.0811$, found: 178.0816 .<smiles>COc1ccc(CC#CCC(C)C)cn1</smiles>

${ }^{1} \mathrm{H}$ NMR (400 MHz, $\left.\mathrm{CDCl}_{3}\right) \delta 8.06(\mathrm{~s}, 1 \mathrm{H}), 7.50(\mathrm{~d}, J=8.8 \mathrm{~Hz}, 1 \mathrm{H}), 6.65(\mathrm{~d}, J=8.4$ $\mathrm{Hz}, 1 \mathrm{H}), 3.87$ (s, 3H), 3.43 (s, 2H), 2.16 (t, $J=6.0 \mathrm{~Hz}, 2 \mathrm{H}), 1.47-1.34$ (m, 4H), 0.86 (t, $J=7.2 \mathrm{~Hz}, 3 \mathrm{H})$.

${ }^{13} \mathrm{C} \mathrm{NMR}\left(100 \mathrm{MHz}, \mathrm{CDCl}_{3}\right) \delta 162.9,145.5,138.3,125.7,110.4,82.7,76.7,53.2$, $30.9,21.9,21.6,18.3,13.5$.

IR (neat): 2932, 1608, 1573, 1490, 1390, 1281, 1254, 1123, 1026, $825 \mathrm{~cm}^{-1}$.

HRMS: $m / z$ (EI) calculated for $\mathrm{C}_{13} \mathrm{H}_{17} \mathrm{NO}^{+}[\mathrm{M}]^{+}: 203.1305$, found: 203.1310 .

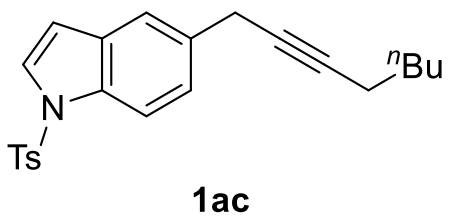

${ }^{1} \mathrm{H}$ NMR (400 MHz, $\left.\mathrm{CDCl}_{3}\right) \delta 8.01(\mathrm{~s}, 1 \mathrm{H}), 7.77(\mathrm{~d}, J=8.0 \mathrm{~Hz}, 2 \mathrm{H}), 7.52(\mathrm{~d}, J=3.6$ $\mathrm{Hz}, 1 \mathrm{H}), 7.44$ (d, $J=8.0 \mathrm{~Hz}, 1 \mathrm{H}), 7.21$ (d, $J=8.0 \mathrm{~Hz}, 3 \mathrm{H}), 6.61(\mathrm{~d}, J=4.0 \mathrm{~Hz}, 1 \mathrm{H})$, $3.70(\mathrm{~s}, 2 \mathrm{H}), 2.33(\mathrm{~s}, 3 \mathrm{H}), 2.26(\mathrm{t}, J=7.2 \mathrm{~Hz}, 2 \mathrm{H}), 1.60-1.55(\mathrm{~m}, 2 \mathrm{H}), 1.45-1.32$ (m, 4H), $0.91(\mathrm{t}, J=7.2 \mathrm{~Hz}, 3 \mathrm{H})$.

${ }^{13} \mathrm{C}$ NMR $\left(100 \mathrm{MHz}, \mathrm{CDCl}_{3}\right) \delta 144.8,135.2,135.0,134.5,129.8,129.2,126.8,126.1$, $123.5,121.0,112.7,108.8,83.0,77.6,31.1,28.8,25.4,22.2,21.5,18.8,14.0$.

IR (neat): 2928, 1596, 1427, 1370, 1268, 1169, 1135, 1114, 1000, 809, 672, 577, 537 $\mathrm{cm}^{-1}$.

HRMS: $m / z$ (EI) calculated for $\mathrm{C}_{23} \mathrm{H}_{25} \mathrm{NO}_{2} \mathrm{~S}^{+}[\mathrm{M}]^{+}: 379.1601$, found: 379.1610 . 


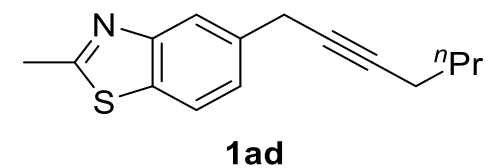

${ }^{1} \mathrm{H}$ NMR (400 MHz, $\left.\mathrm{CDCl}_{3}\right) \delta 7.98(\mathrm{~s}, 1 \mathrm{H}), 7.73(\mathrm{~d}, J=8.4 \mathrm{~Hz}, 1 \mathrm{H}), 7.31(\mathrm{~d}, J=8.4$ $\mathrm{Hz}, 1 \mathrm{H}), 3.70$ (s, 2H), 2.82 (s, 3H), 2.23 (t, $J=6.8 \mathrm{~Hz}, 2 \mathrm{H}), 1.53-1.39$ (m, 4H), 0.91 (t, $J=7.2 \mathrm{~Hz}, 3 \mathrm{H})$.

${ }^{13} \mathrm{C} \mathrm{NMR}\left(100 \mathrm{MHz}, \mathrm{CDCl}_{3}\right) \delta 167.3,153.6,135.8,133.5,124.8,121.3,121.0,83.1$, 77.1, 31.0, 25.0, 22.0, 20.1, 18.5, 13.6.

IR (neat): 2929, 1524, 1456, 1421, 1375, 1326, 1249, 1172, 1152, 908, 798, 730, 643 $\mathrm{cm}^{-1}$.

HRMS: $m / z$ (EI) calculated for $\mathrm{C}_{15} \mathrm{H}_{17} \mathrm{NS}^{+}[\mathrm{M}]^{+}: 243.1076$, found: 243.1078 .

\section{Large-Scale Reactions}
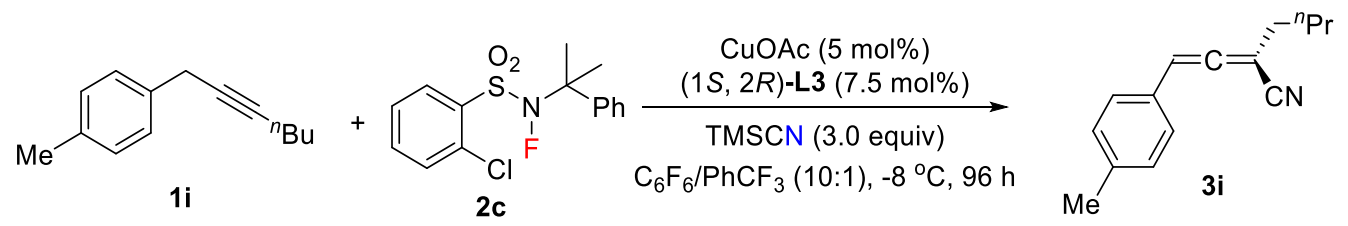

In a dried sealed $100 \mathrm{~mL}$ Schlenk tube, (1S,2R)-L3 (234 mg, $0.6 \mathrm{mmol}, 7.5 \mathrm{~mol} \%)$, CuOAc (49.0 mg, $0.4 \mathrm{mmol}, 5 \mathrm{~mol} \%$ ) and NFAS 2c (7.8 g, $12 \mathrm{mmol})$ were dissolved in a mixed solvent of $\mathrm{C}_{6} \mathrm{~F}_{6}$ and $\mathrm{PhCF}_{3}(44 \mathrm{~mL}, v / v=10: 1)$ under $\mathrm{N}_{2}$, and the mixture was stirred for $30 \mathrm{~min}$ and cooled down to $-30{ }^{\circ} \mathrm{C}$. Then, 1-(hept-2-yn-1-yl)-4-methylbenzene 1i (1.5 g, 8.0 mmol, 1.0 equiv.) and TMSCN (3.2 $\mathrm{mL}, 24 \mathrm{mmol}, 3.0$ equiv.) were added sequentially, and the tube was sealed with Teflon septum. The reaction mixture was warmed up to $-8{ }^{\circ} \mathrm{C}$ and stirred vigorously for $96 \mathrm{~h}$. According to the same workup procedure, the residue was purified by flash chromatography $(\mathrm{PE} / \mathrm{EA}=40: 1)$ to give product $3 \mathbf{i}(1.18 \mathrm{~g}, 70 \%$ yield, $91 \%$ ee $)$ as colorless oil. 


\section{Synthetic Transformations}

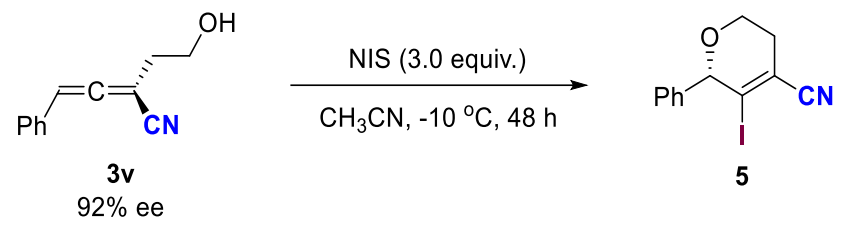

In a dried sealed $10 \mathrm{~mL}$ Schlenk tube, allene 3v (37.0 mg, $0.2 \mathrm{mmol}, 1.0$ equiv), NIS (134 mg, 0.6 mmol, 3.0 equiv) were dissolved in $\mathrm{CH}_{3} \mathrm{CN}(2 \mathrm{~mL})$ under $\mathrm{N}_{2}$. The resulting suspension was stirred at $-10{ }^{\circ} \mathrm{C}$ for 48 hours. Upon completion of the reaction as monitored by TLC plate, the solvent was concentrated under vacuum. The crude residue was purified by flash column chromatography on silica gel to give the desired product 5 (47.5 mg, 76\% yield, 92\% ee) as light yellow oil.

$[\alpha]_{\mathrm{D}}^{24.4}-34.43\left(c 0.85, \mathrm{CHCl}_{3}\right)$

${ }^{1} \mathrm{H}$ NMR $\left(400 \mathrm{MHz}, \mathrm{CDCl}_{3}\right) \delta 7.41-7.40(\mathrm{~m}, 3 \mathrm{H}), 7.31-7.29(\mathrm{~m}, 2 \mathrm{H}), 5.22(\mathrm{t}, J=$ $2.0 \mathrm{~Hz}, 1 \mathrm{H}), 4.08-4.03(\mathrm{~m}, 1 \mathrm{H}), 3.88-3.82(\mathrm{~m}, 1 \mathrm{H}), 2.75-2.68(\mathrm{~m}, 1 \mathrm{H}), 2.55-$ $2.48(\mathrm{~m}, 1 \mathrm{H})$.

${ }^{13} \mathrm{C}$ NMR $\left(100 \mathrm{MHz}, \mathrm{CDCl}_{3}\right) \delta 137.6,129.3,128.9,128.7,120.6,118.9,117.9,83.6$, $61.0,30.9$

IR (neat): 2923, 2219, 1453, 1370, 1256, 1098, 1061, 985, 756, $699 \mathrm{~cm}^{-1}$.

HRMS: $m / z$ (EI) calculated for $\mathrm{C}_{12} \mathrm{H}_{10} \mathrm{INO}^{+}[\mathrm{M}]^{+}: 310.9802$, found: 310.9799 .

HPLC (OD-H, 0.46*25 cm, $5 \mu \mathrm{m}$, hexane/isopropanol $=90 / 10$, flow $0.7 \mathrm{~mL} / \mathrm{min}$, detection at $214 \mathrm{~nm}$ ) retention time $=16.12 \mathrm{~min}$ (major) and $29.82 \mathrm{~min}$ (minor).

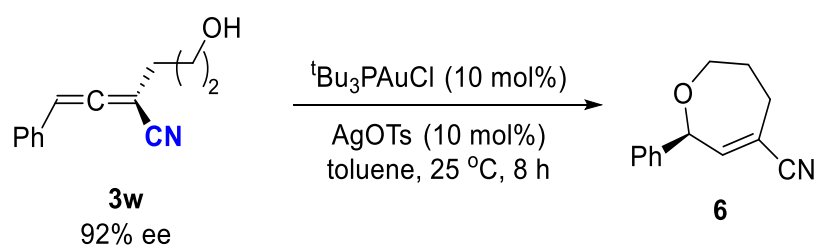

In a dried sealed $10 \mathrm{~mL}$ Schlenk tube, silver(I) tosylate ( $0.02 \mathrm{mmol}, 0.1$ equiv), and chlorotri-t-butylphosphinegold(I) (0.02 mmol, 0.1 equiv) was dissolved in toluene (1 $\mathrm{mL}$ ) under $\mathrm{N}_{2}$. The resulting suspension was allowed to stir at room temperature for 20 minutes. After that, allene $\mathbf{3 w}(40.0 \mathrm{mg}, 0.2 \mathrm{mmol})$ was added and the mixture was stirred until completion of the reaction judged by TLC plate, the solvent was 
concentrated under vacuum. The crude residue was purified by flash column chromatography on silica gel to give the desired product 6 (40.5 mg, 99\% yield, 92\% ee) as light yellow oil.

$[\alpha]_{\mathrm{D}}^{25.0}+46.62\left(c 1.37, \mathrm{CHCl}_{3}\right)$.

${ }^{1} \mathrm{H}$ NMR (400 MHz, $\left.\mathrm{CDCl}_{3}\right) \delta 7.41-7.37(\mathrm{~m}, 2 \mathrm{H}), 7.34-7.32(\mathrm{~m}, 3 \mathrm{H}), 6.61(\mathrm{~d}, J=$ $1.2 \mathrm{~Hz}, 1 \mathrm{H}), 5.20(\mathrm{~s}, 1 \mathrm{H}), 4.09-4.03(\mathrm{~m}, 1 \mathrm{H}), 3.87-3.81(\mathrm{~m}, 1 \mathrm{H}), 2.83-2.77(\mathrm{~m}$, $1 \mathrm{H}), 2.57-2.50(\mathrm{~m}, 1 \mathrm{H}), 2.07-2.01(\mathrm{~m}, 2 \mathrm{H})$.

${ }^{13} \mathrm{C}$ NMR $\left(100 \mathrm{MHz}, \mathrm{CDCl}_{3}\right) \delta 149.9,139.5,128.8,128.4,126.7,119.9,116.4,80.3$, $69.4,28.5,28.3$.

IR (neat): 2942, 2216, 1494, 1451, 1139, 1085, 751, $699 \mathrm{~cm}^{-1}$.

HRMS: $m / z$ (EI) calculated for $\mathrm{C}_{13} \mathrm{H}_{13} \mathrm{NO}^{+}[\mathrm{M}]^{+}:$199.0992, found: 199.0990.

HPLC (OD-H, 0.46*25 cm, $5 \mu \mathrm{m}$, hexane/isopropanol $=95 / 5$, flow $0.7 \mathrm{~mL} / \mathrm{min}$, detection at $214 \mathrm{~nm}$ ) retention time $=17.48 \mathrm{~min}$ (major) and $23.65 \mathrm{~min}$ (minor).
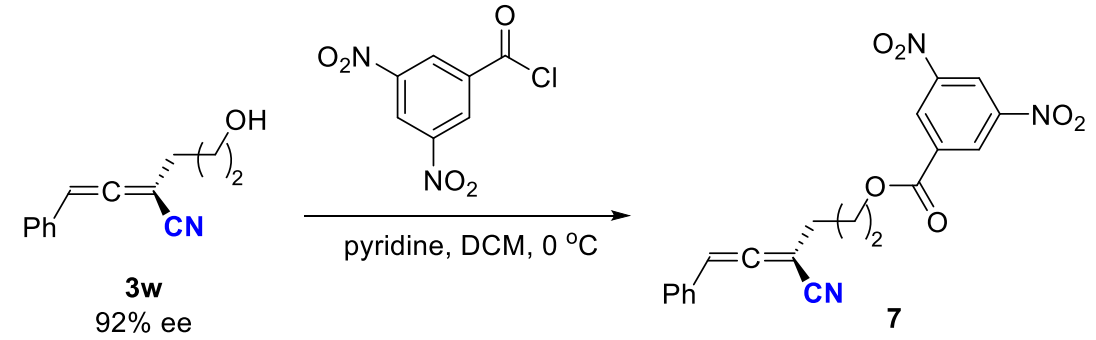

In a dried sealed $10 \mathrm{~mL}$ round bottom flask equipped with a stirring bar, allene $\mathbf{3 w}$ (40 mg, $0.2 \mathrm{mmol}, 1$ equiv) and 3,5-dinitrobenzoyl chloride (55 mg, $0.24 \mathrm{mmol}, 1.2$ equiv) were dissolved in $2 \mathrm{~mL}$ of dry dichloromethane under a nitrogen atmosphere. The solution was added pyridine $\left(33 \mu \mathrm{L}, 0.4 \mathrm{mmol}, 2.0\right.$ equiv) dropwise at $0{ }^{\circ} \mathrm{C}$. The resulting mixture was continued to stir for 2 hours at $0{ }^{\circ} \mathrm{C}$, the final solution was directly subjected to column chromatography on silica gel (petroleum ether/ethyl acetate 5:1) to give the desired product 7 (69.6 $\mathrm{mg}, 88 \%$ yield, $90 \%$ ee) as a white soild.

M.p. $78.1-82.8^{\circ} \mathrm{C}$.

$[\alpha]_{\mathrm{D}}^{24.0}-25.07\left(c\right.$ 1.5, $\left.\mathrm{CHCl}_{3}\right)$.

${ }^{1} \mathrm{H}$ NMR (400 MHz, $\left.\mathrm{CDCl}_{3}\right) \delta 9.20(\mathrm{t}, J=2.2 \mathrm{~Hz}, 1 \mathrm{H}), 9.10(\mathrm{~d}, J=1.2 \mathrm{~Hz}, 2 \mathrm{H}), 7.36$ $-7.23(\mathrm{~m}, 5 \mathrm{H}), 6.70(\mathrm{t}, J=2.9 \mathrm{~Hz}, 1 \mathrm{H}), 4.59-4.52(\mathrm{~m}, 2 \mathrm{H}), 2.58-2.54(\mathrm{~m}, 2 \mathrm{H})$, 
$2.20-2.13(\mathrm{~m}, 2 \mathrm{H})$

${ }^{13} \mathrm{C}$ NMR $\left(100 \mathrm{MHz}, \mathrm{CDCl}_{3}\right) \delta 213.3,162.3,148.6,133.5,130.1,129.3,129.1,129.0$, $127.7,122.4,114.3,100.6,84.9,65.1,28.3,26.4$.

IR (neat): 2928, 2220, 1943, 1731, 1629, 1545, 1460, 1372, 1344, 1274, 1241, 1164 , $1075,917,722,694 \mathrm{~cm}^{-1}$.

HRMS: $m / z$ (ESI) calculated for $\mathrm{C}_{20} \mathrm{H}_{15} \mathrm{~N}_{3} \mathrm{O}_{6} \mathrm{Na}^{+}[\mathrm{M}+\mathrm{Na}]^{+}:$416.0853, found: 416.0860 .

HPLC $\left(\mathrm{IH}-3,0.46^{*} 15 \mathrm{~cm}, 3 \mu \mathrm{m}\right.$, hexane/isopropanol $=80 / 20$, flow $0.7 \mathrm{~mL} / \mathrm{min}$, detection at $214 \mathrm{~nm}$ ) retention time $=27.75 \mathrm{~min}$ (minor) and 31.09 min (major).

\section{Mechanistic Studies}

\subsection{L4 as true ligand in the case of L3.}

Ligand $\mathrm{Box}^{\mathrm{OH}} \mathbf{L 3}$ was insoluble in solvent $\mathrm{C}_{6} \mathrm{~F}_{6}$, while the mixture becomes clear after addition of TMSCN. Analysis of ${ }^{1} \mathrm{H}$ NMR spectra revealed that the hydroxy group in $\mathbf{L 3}$ was protected by trimethylsilyl group in the presence of TMSCN, which is identical to the spectra of $\mathbf{L} \mathbf{4}$ (see Figure $\mathbf{S 1}$ ).

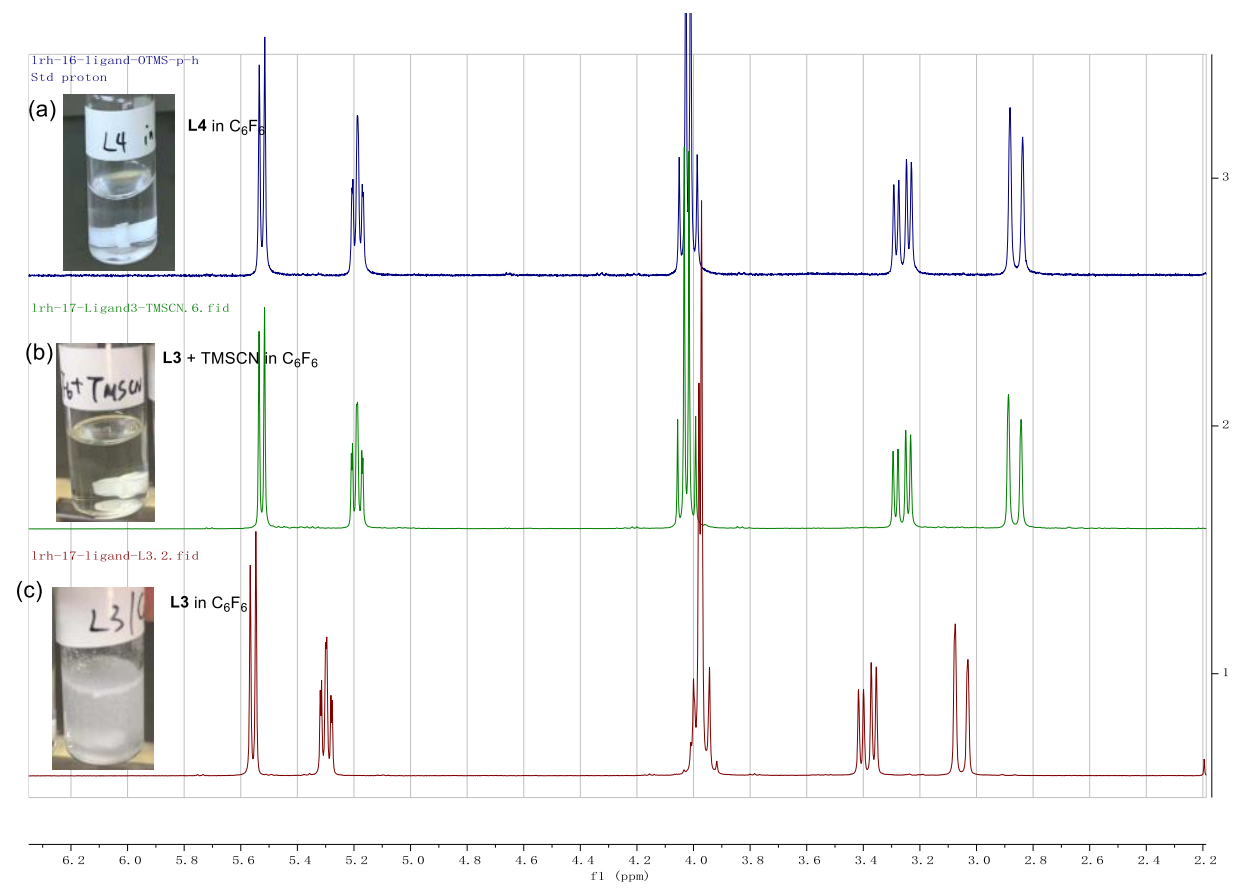

Figure S1. (a) ${ }^{1} \mathrm{H}$ NMR of L4; (b) Crude ${ }^{1} \mathrm{H}$ NMR of $\mathbf{L} 3$ and TMSCN in $\mathrm{C}_{6} \mathrm{~F}_{6}$; (c) ${ }^{1} \mathrm{H}$ NMR of $\mathbf{L 3}$. 


\subsection{With TEMPO as Radical Scavengers}

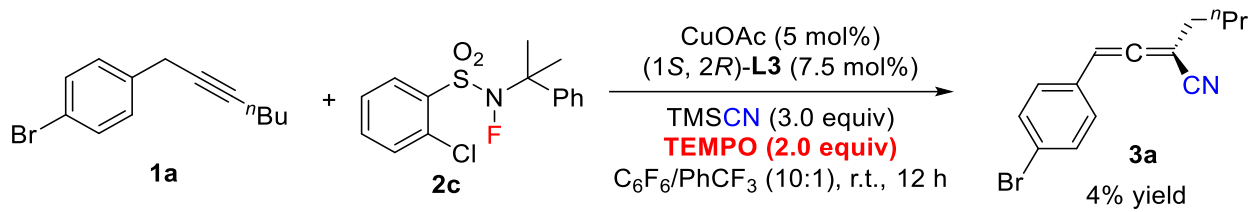

In a dried sealed $10 \mathrm{~mL}$ Schlenk tube, CuOAc (0.005 mmol, $5 \mathrm{~mol} \%)$, chiral bisoxazoline ligand ( $1 S, 2 R)$-L3 (0.0075 mmol, $7.5 \mathrm{~mol} \%$ ), and NFAS (98 $\mathrm{mg}, 0.3$ mmol, 3.0 equiv.) were dissolved in a mixed solvent of $\mathrm{C}_{6} \mathrm{~F}_{6}(0.5 \mathrm{~mL})$ and $\mathrm{PhCF}_{3}(50$ $\mu \mathrm{L})$ under a $N_{2}$ atmosphere, and the mixture was stirred for $30 \mathrm{~min}$. Then $1 \mathrm{a}(0.1$ mmol, 1.0 equiv), TEMPO (0.2 mmol, 2.0 equiv), TMSCN ( $0.3 \mathrm{mmol}, 3.0$ equiv) were sequentially added. The reaction mixture was stirred at room temperature for 12 hours. After the reaction was completed, the mixture was analyzed by ${ }^{1} \mathrm{H}-\mathrm{NMR}$ with $\mathrm{CF}_{3}$-DMAc $(0.03 \mathrm{mmol})$ as an internal standard. We found that only $4 \%$ yield of product 3a was obtained, along with $92 \%$ of 1a remained, thus the addition of 2.0 equivalents of TEMPO could inhibit the reaction, which indicated that a radical intermediate might be involved in the enantioselective cyanation process.

\subsection{With BHT as Radical Scavengers}

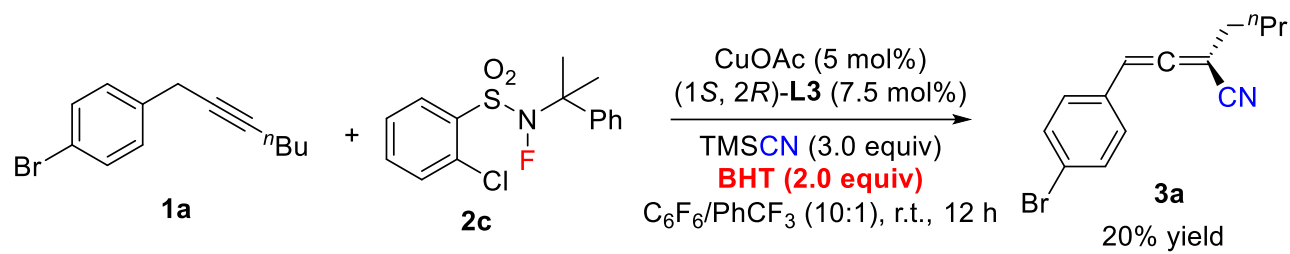

In a dried sealed $10 \mathrm{~mL}$ Schlenk tube, CuOAc (0.005 mmol, $5 \mathrm{~mol} \%)$, chiral bisoxazoline ligand ( $1 S, 2 R)$-L3 (0.0075 mmol, $7.5 \mathrm{~mol} \%$ ), and NFAS (98 mg, 0.3 mmol, 3.0 equiv) were dissolved in a mixed solvent of $\mathrm{C}_{6} \mathrm{~F}_{6}(0.5 \mathrm{~mL})$ and $\mathrm{PhCF}_{3}(50$ $\mu \mathrm{L})$ under a $N_{2}$ atmosphere, and the mixture was stirred for $30 \mathrm{~min}$. Then $1 \mathrm{a}(0.1$ mmol, 1.0 equiv), BHT (0.2 mmol, 2.0 equiv), TMSCN (0.3 mmol, 3.0 equiv) were sequentially added. The reaction mixture was stirred at room temperature for 12 hours. After the reaction was completed, the mixture was analyzed by ${ }^{1} \mathrm{H}-\mathrm{NMR}$ with $\mathrm{CF}_{3}$-DMAc $(0.03 \mathrm{mmol})$ as an internal standard. We found that only $20 \%$ yield of product 3a was obtained along with 52\% 1a remained, thus the addition of 2.0 
equivalents of BHT could also inhibit the reaction, which indicated that a radical intermediate might be involved in the enantioselective cyanation process.

\subsection{Electronic Effect of Benzyl Alkynes}
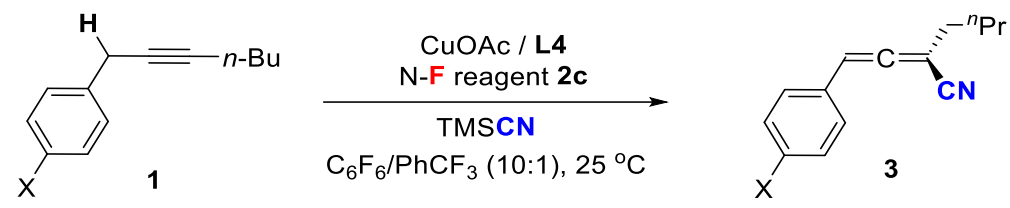

The reactions were conducted according to the general procedure in a NMR tube at $25{ }^{\circ} \mathrm{C}$. In a glove box, a homogenous solution of $\mathrm{CuOAc}(6.3 \mathrm{mg}, 0.05 \mathrm{mmol})$ and $\mathbf{L 4}$ $(40.1 \mathrm{mg}, 0.075 \mathrm{mmol})$ in dried and degassed $\mathrm{C}_{6} \mathrm{~F}_{6}(5 \mathrm{~mL})$ prepared in a $10 \mathrm{~mL}$ volumetric flask. Then, substrate 1 (0.1 mmol), NFAS (98 mg, $0.3 \mathrm{mmol}), \mathrm{PhCF}_{3}(50$ $\mu \mathrm{L}), \mathrm{CF}_{3}-\mathrm{DMAc}(0.03 \mathrm{mmol})$ and $500 \mu \mathrm{L}$ of the solution of $\mathrm{CuOAc} / \mathrm{L} 4$ were added into a dry NMR tube in the glove box. The NMR tube was sealed with rubber stopper and removed out of the glove box. After that, TMSCN (40 $\mu \mathrm{L}, 0.3 \mathrm{mmol})$ was added by syringe. The mixture was monitored by ${ }^{1} \mathrm{H}$ NMR immediately, the results and Hammett plots were shown in Figure S2.

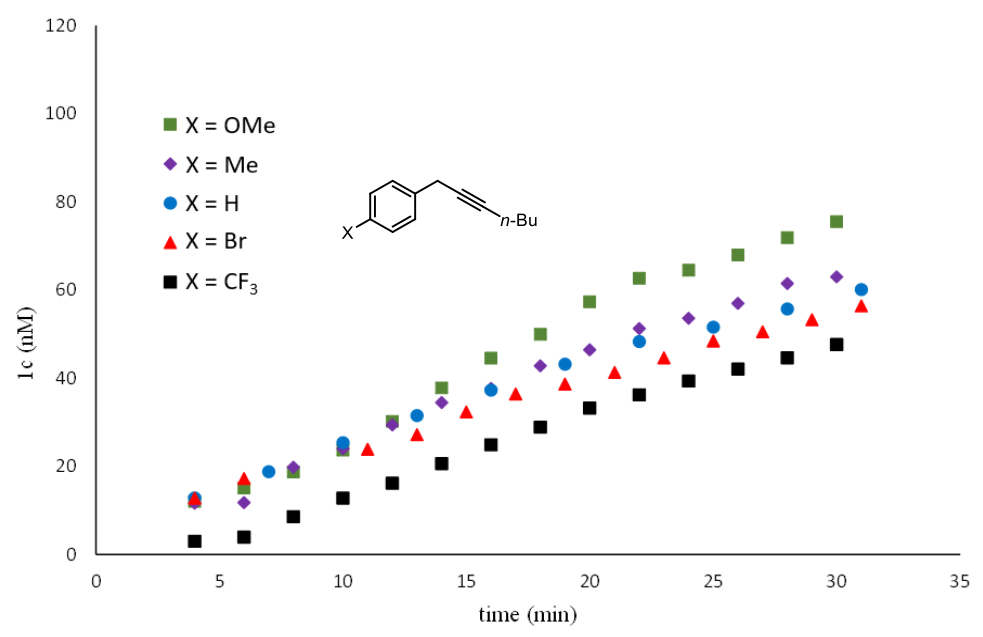




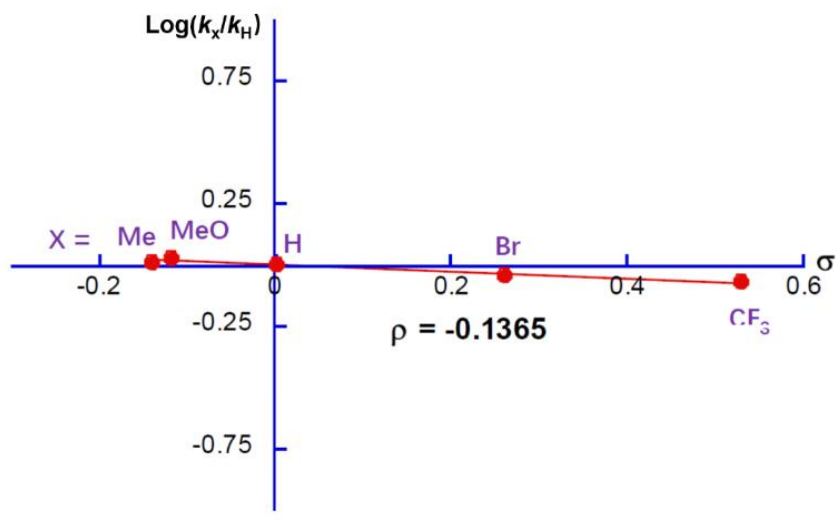

Figure S2. Electronic effect of substrate 1 with substituted benzyl alkynes.

\subsection{Independent-Rate KIE Experiments}

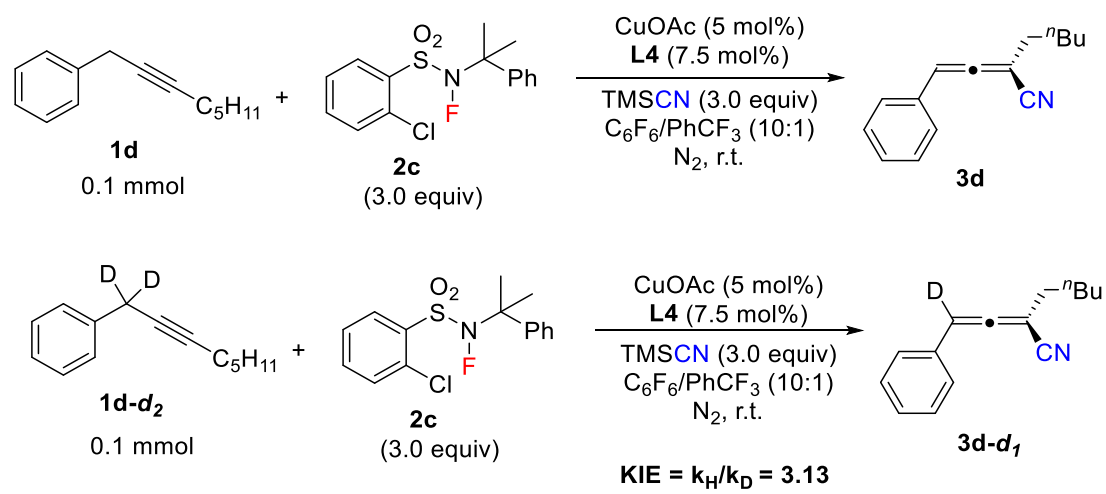

The reaction of $\mathbf{1 d}$ and the reaction of $\mathbf{1 d}-d_{2}$ under the standard conditions were carried out independently and stopped at different times. Since L3 was insoluble in $\mathrm{C}_{6} \mathrm{~F}_{6}$ without $\mathrm{TMSCN}, \mathbf{L} 4$ was used instead. In a dried sealed $25 \mathrm{~mL}$ Schlenk tube, CuOAc (12.3 mg, $0.1 \mathrm{mmol})$ and $\mathbf{L 4}(80.1 \mathrm{mg}, 0.15 \mathrm{mmol})$ were dissolved in a mixed solvent of $\mathrm{C}_{6} \mathrm{~F}_{6}(10 \mathrm{~mL})$ and $\mathrm{PhCF}_{3}(1 \mathrm{~mL})$ under an $\mathrm{Ar}$ atmosphere, and the mixture was stirred for $30 \mathrm{~min}$. To a sealed tube containing NFAS $2 \mathbf{c}(98 \mathrm{mg}, 0.3 \mathrm{mmol}), 0.55$ $\mathrm{mL}$ of the solution of $\mathrm{CuOAc} / \mathbf{L} 4,1 d(18.6 \mathrm{mg}, 0.1 \mathrm{mmol})$ and TMSCN (40 $\mu \mathrm{L}, 0.3$ mmol) were sequentially added under an Ar atmosphere. The tube was sealed with Teflon-septum, the reaction mixture was stirred at room temperature for the indicated time. All the reactions were quenched by addition of $0.5 \mathrm{~mL} \mathrm{CDCl}_{3}$ and $\mathrm{CF}_{3}-\mathrm{DMAc}$ $(0.03 \mathrm{mmol})$ as an internal standard and analyzed by ${ }^{1} \mathrm{H}-\mathrm{NMR}$ as soon as possible. These studies revealed an independent KIE of 3.13, suggesting that the $\mathrm{C}-\mathrm{H}$ bond cleavage is only partially rate-determining. 


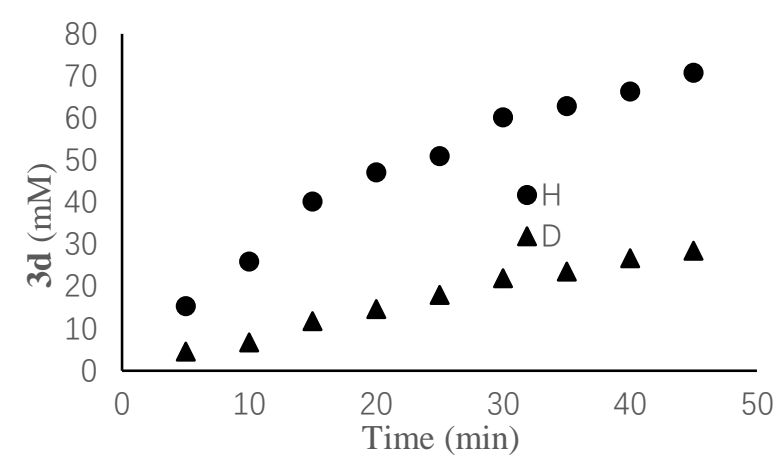

Figure S3. Kinetic isotopic effect experiments

\subsection{Analysis of Bite-Angle of Chiral $\mathrm{L}^{*} \mathrm{Cu}(\mathrm{CN})_{2}$ by DFT Calculation and X-ray.}

To understand how the bite-angle correlates with ee values, we calculated the bite angles of various $\left(\mathrm{L}^{*}\right) \mathrm{Cu}(\mathrm{CN})_{2}$ using the method reported in Bao's work. ${ }^{8}$ As shown below, decreasing the bite angle of $\left(\mathrm{L}^{*}\right) \mathrm{Cu}(\mathrm{CN})_{2}$ could increase the enantioselectivity $(\mathbf{L 1} \rightarrow \mathbf{L 2} \rightarrow \mathbf{L 4})$. In addition, the trend of the calculated bite angles of $(\mathbf{L 1}) \mathrm{Cu}(\mathrm{CN})_{2}$ and $(\mathbf{L 4}) \mathrm{Cu}(\mathrm{CN})_{2}$ is consistent with the trend of the bite angles in the crystal structures of the $(\mathbf{L 1}) \mathrm{CuCl}_{2}$ and $(\mathbf{L 4}) \mathrm{CuCl}_{2}$ complexes (see Figure S4). Here, we reasoned that, the small bite angle in $(\mathrm{L4}) \mathrm{Cu}(\mathrm{CN})_{2}$ is possibly attributed to steric effect as well, where the larger steric bulky -OTMS group pushes two oxazoline rings close to each other.

These calculations were performed at the same level of latter calculations about Cu-catalyzed pathway. SambVca 2.1 was used to visualize the catalytic pockets. ${ }^{9}$. Calculated cartesian coordinates were showed below. 


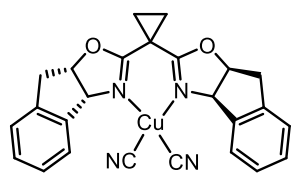

(L1) $\mathrm{Cu}(\mathrm{CN})_{2}$

$\angle \mathrm{N}-\mathrm{C}-\mathrm{C}-\mathrm{N}=3.1^{\circ}$

3a: $56 \%$ ee

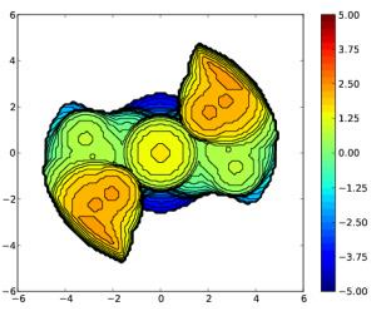

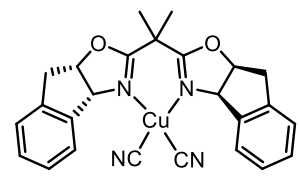

(L2) $\mathrm{Cu}(\mathrm{CN})_{2}$

$\angle \mathrm{N}-\mathrm{C}-\mathrm{C}-\mathrm{N}=1.8^{\circ}$

3a: $65 \%$ ee

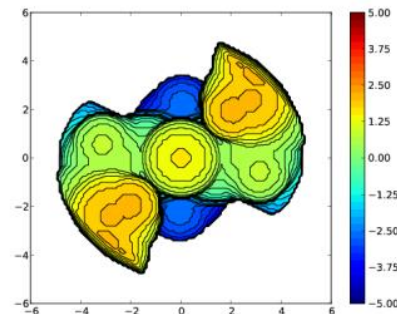

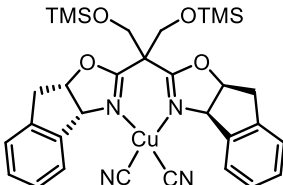

(L4) $\mathrm{Cu}(\mathrm{CN})_{2}$

$\angle \mathrm{N}-\mathrm{C}-\mathrm{C}-\mathrm{N}=0.6^{\circ}$

3a: $78 \%$ ee

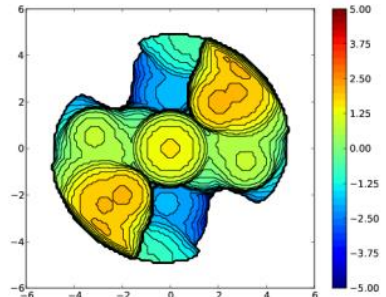

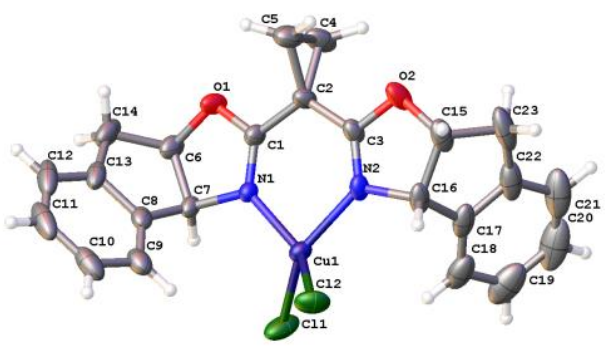

(L1) $\mathrm{CuCl}_{2}$

$\mathrm{N} 1-\mathrm{C} 1-\mathrm{C} 3-\mathrm{N} 2=2.060^{\circ}$

CCDC: 2104644

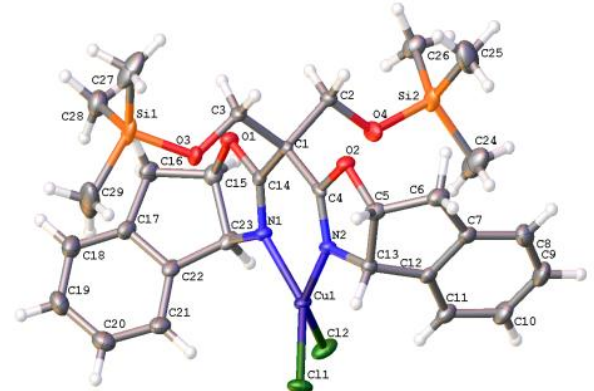

(L4) $\mathrm{CuCl}_{2}$

N2-C4-C14-N1 $=0.397^{\circ}$

CCDC: $\mathbf{2 1 0 4 6 3 7}$

Figure S4. DFT calculations of $\left(\mathrm{L}^{*}\right) \mathrm{Cu}(\mathrm{CN})_{2}$ (top) and X-Ray Crystal structures of ( $\left.\mathrm{L}^{*}\right) \mathrm{CuCl}_{2}$ (bottom).

Table S7. Computational Details $(\mathrm{L} 1) \mathrm{Cu}(\mathrm{CN})_{2}$

$$
\begin{array}{r}
02 \\
\mathrm{C} \\
\mathrm{C} \\
\mathrm{C} \\
\mathrm{C} \\
\mathrm{C} \\
\mathrm{O} \\
\mathrm{N} \\
\mathrm{N} \\
\mathrm{C} \\
\mathrm{C} \\
\mathrm{O} \\
\mathrm{C} \\
\mathrm{C} \\
\mathrm{C} \\
\mathrm{C} \\
\mathrm{C} \\
\mathrm{C} \\
\mathrm{C} \\
\mathrm{C} \\
\mathrm{C} \\
\mathrm{C} \\
\mathrm{C} \\
\mathrm{C} \\
\mathrm{C} \\
\mathrm{C} \\
\mathrm{C} \\
\mathrm{C} \\
\mathrm{H} \\
\mathrm{H}
\end{array}
$$

$\begin{array}{ccc} & & \\ 0.00002900 & -2.24094300 & 0.00016700 \\ 1.10289900 & -1.48072700 & 0.60260500 \\ -1.10276700 & -1.48073400 & -0.60241900 \\ -0.35197000 & -3.58430800 & 0.64506800 \\ 0.35187400 & -3.58447900 & -0.64444500 \\ 2.09577000 & -2.21907300 & 1.10014800 \\ 1.20707500 & -0.20492200 & 0.69856500 \\ -1.20694500 & -0.20493000 & -0.69838600 \\ -2.53898200 & 0.07185700 & -1.27825600 \\ -3.03670300 & -1.31084300 & -1.74123000 \\ -2.09557100 & -2.21908900 & -1.10008400 \\ -3.53276900 & 0.48059500 & -0.22187100 \\ -4.57715800 & -0.43991400 & -0.13867800 \\ -4.43890500 & -1.51811500 & -1.17863300 \\ -3.48386600 & 1.58950600 & 0.61595900 \\ -4.49869500 & 1.76081200 & 1.55262300 \\ -5.54225400 & 0.83885500 & 1.64387900 \\ -5.59068600 & -0.26546900 & 0.79716300 \\ 3.03703300 & -1.31081900 & 1.74108600 \\ 2.53920000 & 0.07188300 & 1.27822800 \\ 4.43911700 & -1.51808000 & 1.17817500 \\ 4.57716000 & -0.43984100 & 0.13823000 \\ 3.53278900 & 0.48066300 & 0.22167300 \\ 5.59048800 & -0.26536100 & -0.79782300 \\ 5.54186900 & 0.83898800 & -1.64449700 \\ 4.49832700 & 1.76093800 & -1.55298900 \\ 3.48370300 & 1.58959900 & -0.61611200 \\ 0.20745000 & -3.82505600 & 1.54511100 \\ -1.41083400 & -3.82726400 & 0.66466000\end{array}$

$-0.20757500$

1.41070800

$-2.43106000$

$-2.95533900$

$-5.18464700$

$-4.55629900$

$-2.67383300$

$-4.47776600$

$-6.32854600$

$-6.41060100$

2.95591600

2.43138300

5.18503700

4.55640700

6.41039000

6.32800100

4.47725100

2.67368400

0.00000800

0.76571700

$-0.76578100$

1.26195600

$-1.26202600$

$(\mathrm{L} 2) \mathrm{Cu}(\mathrm{CN})_{2}$

02

C

C

C

C

$\mathrm{O}$

$\begin{array}{cc}-3.82535800 & -1.54443400 \\ -3.82756500 & -0.66398200 \\ 0.81239100 & -2.07898500 \\ -1.49415000 & -2.81762100 \\ -1.40140500 & -1.97812600 \\ -2.53667000 & -0.78526600 \\ 2.31402000 & 0.53212600 \\ 2.62236300 & 2.21728700 \\ 0.98651500 & 2.38244600 \\ -0.98004900 & 0.86687300 \\ -1.49412700 & 2.81749400 \\ 0.81239800 & 2.07898900 \\ -1.40142000 & 1.97750900 \\ -2.53662000 & 0.78473700 \\ -0.97993600 & -0.86773200 \\ 0.98667000 & -2.38323000 \\ 2.62250800 & -2.21762500 \\ 2.31410400 & -0.53208900 \\ 1.25951800 & 0.00019200 \\ 2.55309800 & 1.23358200 \\ 2.55318200 & -1.23306400 \\ 3.25741800 & 2.02437000 \\ 3.25756100 & -2.02379700 \\ & \\ 2.24047000 & 0.00006100 \\ 1.41257300 & -0.62488700 \\ 1.41264000 & 0.62484100 \\ 3.12459600 & -1.08806700 \\ 3.12413400 & 1.08844800 \\ 2.14645200 & -1.18119700\end{array}$

$-3.82535800 \quad-1.54443400$

$\begin{array}{ll}-3.82756500 & -0.66398200 \\ 0.81239100 & -2.07898500\end{array}$

$-1.49415000 \quad-2.81762100$

$-0.78526600$

0.53212600

2.21728700

2.81749400

.07898900

0.78473700

$-2.21762500$

0.53208900

1.23358200

$-1.23306400$

2.02437000

$0.00008100 \quad 2.24047000-0.00006100$

$\begin{array}{rrr}1.08628100 & 1.41257300 & -0.62488700\end{array}$

$\begin{array}{lrr}-1.08626800 & 1.41264000 & 0.62484100\end{array}$

$\begin{array}{rrr}-0.63860200 & 3.12459600 & -1.08806700 \\ 0.63898500 & 3.12413400 & 1.08844800\end{array}$

$\begin{array}{lll}0.04991100 & 2.14645200 & -1.18119700\end{array}$ 


\begin{tabular}{|c|c|c|c|c|c|c|c|}
\hline $\mathrm{N}$ & 1.21283900 & 0.13785000 & -0.67449800 & $\mathrm{H}$ & 5.10723700 & 3.18670600 & -0.78996200 \\
\hline $\mathrm{N}$ & -1.21287000 & 0.13792300 & 0.67450500 & $\mathrm{H}$ & 6.84799800 & 1.54128000 & -0.16301900 \\
\hline $\mathrm{C}$ & -2.54340800 & -0.13281400 & 1.26912900 & $\mathrm{H}$ & 6.32817500 & -0.29619300 & 1.42955600 \\
\hline $\mathrm{C}$ & -2.98701200 & 1.23445400 & 1.81881100 & $\mathrm{H}$ & -1.75442200 & -0.60209400 & -3.67379800 \\
\hline $\mathrm{O}$ & -2.04991800 & 2.14657100 & 1.18104900 & $\mathrm{H}$ & -1.69232900 & 1.69194300 & -2.72126400 \\
\hline $\mathrm{C}$ & -3.56704100 & -0.44434100 & 0.20711200 & $\mathrm{H}$ & -4.14819600 & -0.55252300 & -3.7028880 \\
\hline $\mathrm{C}$ & -4.58583500 & 0.50851400 & 0.20867300 & $\mathrm{H}$ & -3.94654300 & -1.80069400 & -2.47489300 \\
\hline $\mathrm{C}$ & -4.40138500 & 1.50366900 & 1.32085300 & $\mathrm{H}$ & -6.30544700 & -0.46134600 & -1.40600600 \\
\hline $\mathrm{C}$ & -3.56645900 & -1.49153300 & -0.70823100 & $\mathrm{H}$ & -6.86997200 & 1.39842500 & 0.14628300 \\
\hline $\mathrm{C}$ & -4.60043700 & -1.56347100 & -1.63759800 & $\mathrm{H}$ & -5.17981300 & 3.11770500 & 0.70912700 \\
\hline $\mathrm{C}$ & -5.61598000 & -0.60631500 & -1.64514200 & $\mathrm{H}$ & -2.88966200 & 2.99470200 & -0.25636300 \\
\hline $\mathrm{C}$ & -5.61810900 & 0.43338500 & -0.71927300 & $\mathrm{Cu}$ & -0.02699400 & 2.12087900 & -0.01668 \\
\hline $\mathrm{C}$ & 2.98693900 & 1.23427500 & -1.81897100 & $\mathrm{C}$ & -0.52209600 & 3.43416700 & -1.36895000 \\
\hline $\mathrm{C}$ & 2.54334200 & -0.13294800 & -1.26917500 & $\mathrm{C}$ & 0.44611000 & 3.44421700 & 1.33367400 \\
\hline $\mathrm{C}$ & 4.40134900 & 1.50349200 & -1.32112000 & $\mathrm{~N}$ & -0.83918000 & 4.16196900 & -2.22783500 \\
\hline $\mathrm{C}$ & 4.58582500 & 0.50843800 & -0.20885400 & $\mathrm{~N}$ & 0.75089800 & 4.17835300 & 2.19160400 \\
\hline $\mathrm{C}$ & 3.56701600 & -0.44440100 & -0.20717400 & $\mathrm{C}$ & 0.97028500 & -2.32856200 & -0.81526800 \\
\hline $\mathrm{C}$ & 5.61812500 & 0.43337600 & 0.71906900 & $\mathrm{H}$ & 1.45980400 & -3.02931200 & -0.11701100 \\
\hline $\mathrm{C}$ & 5.61600700 & -0.60623900 & 1.64503300 & $\mathrm{H}$ & 0.38964500 & -2.92136700 & -1.54055800 \\
\hline $\mathrm{C}$ & 4.60044900 & -1.56338000 & 1.63760600 & $\mathrm{C}$ & -0.96280600 & -2.30607100 & 0.82049600 \\
\hline $\mathrm{C}$ & 3.56644500 & -1.49150900 & 0.70826400 & $\mathrm{H}$ & -1.47837400 & -3.00452600 & 0.13831600 \\
\hline $\mathrm{H}$ & 0.13113700 & 3.74593100 & -1.55658100 & $\mathrm{H}$ & -0.37712000 & -2.90188800 & 1.53979000 \\
\hline $\mathrm{H}$ & -1.11892700 & 2.51481300 & -1.86335400 & $\mathrm{O}$ & 1.88170100 & -1.48594400 & -1.45102500 \\
\hline $\mathrm{H}$ & -0.13061000 & 3.74549700 & 1.55716400 & $\mathrm{O}$ & -1.84896400 & -1.44442800 & 1.46534900 \\
\hline $\mathrm{H}$ & 1.11919500 & 2.51400500 & 1.86353500 & $\mathrm{Si}$ & 3.39974200 & -2.02402800 & -1.95368800 \\
\hline $\mathrm{H}$ & -2.44426700 & -0.92212500 & 2.02209800 & $\mathrm{Si}$ & -3.34857400 & -1.96959700 & 2.03358000 \\
\hline $\mathrm{H}$ & -2.85877700 & 1.36409200 & 2.89866000 & $\mathrm{C}$ & 4.24028100 & -0.49812500 & -2.60911200 \\
\hline $\mathrm{H}$ & -5.12322600 & 1.33412400 & 2.13275100 & $\mathrm{H}$ & 4.25098000 & 0.28681100 & -1.83944900 \\
\hline $\mathrm{H}$ & -4.51611600 & 2.55044300 & 1.00976400 & $\mathrm{H}$ & 5.28125200 & -0.70573300 & -2.88947400 \\
\hline $\mathrm{H}$ & -2.77953800 & -2.24557800 & -0.68800800 & $\mathrm{H}$ & 3.72477900 & -0.09936300 & -3.49143000 \\
\hline $\mathrm{H}$ & -4.61668800 & -2.37525800 & -2.36240200 & $\mathrm{C}$ & 3.15822300 & -3.35017900 & -3.25609300 \\
\hline $\mathrm{H}$ & -6.41654400 & -0.67613800 & -2.37983400 & $\mathrm{H}$ & 2.61525500 & -4.21592900 & -2.85191900 \\
\hline $\mathrm{H}$ & -6.41629400 & 1.17539400 & -0.72233300 & $\mathrm{H}$ & 2.59033100 & -2.96770200 & -4.11386500 \\
\hline $\mathrm{H}$ & 2.85863300 & 1.36385500 & -2.89881900 & $\mathrm{H}$ & 4.12340600 & -3.71646200 & -3.63010400 \\
\hline $\mathrm{H}$ & 2.44415700 & -0.92231300 & -2.02208000 & $\mathrm{C}$ & 4.31827800 & -2.71929000 & -0.47611100 \\
\hline $\mathrm{H}$ & 5.12313500 & 1.33384000 & -2.13304300 & $\mathrm{H}$ & 5.30960000 & -3.08359900 & -0.77761800 \\
\hline $\mathrm{H}$ & 4.51613600 & 2.55029300 & -1.01014200 & $\mathrm{H}$ & 4.46519400 & -1.92634900 & 0.26961200 \\
\hline $\mathrm{H}$ & 6.41632100 & 1.17537500 & 0.72203900 & $\mathrm{H}$ & 3.79403400 & -3.55505800 & 0.00631400 \\
\hline $\mathrm{H}$ & 6.41659100 & -0.67600500 & 2.37970900 & $\mathrm{C}$ & -4.19504300 & -0.41704900 & 2.61105400 \\
\hline $\mathrm{H}$ & 4.61671000 & -2.37510100 & 2.36248500 & $\mathrm{H}$ & -4.22704400 & 0.32288800 & 1.79972800 \\
\hline $\mathrm{H}$ & 2.77951100 & -2.24554200 & 0.68813400 & $\mathrm{H}$ & -5.22813800 & -0.61782100 & 2.92364100 \\
\hline $\mathrm{Cu}$ & -0.00003500 & -1.33741800 & 0.00005400 & $\mathrm{H}$ & -3.66601900 & 0.03523700 & 3.45904000 \\
\hline $\mathrm{C}$ & 0.89074900 & -2.65109500 & -1.12555600 & $\mathrm{C}$ & -3.07113200 & -3.20083300 & 3.41905700 \\
\hline $\mathrm{C}$ & -0.89086000 & -2.65099600 & 1.12574900 & $\mathrm{H}$ & -2.50783900 & -4.07818500 & 3.07161200 \\
\hline $\mathrm{N}$ & 1.45326700 & -3.38167600 & -1.84494500 & $\mathrm{H}$ & -2.51318000 & -2.74867400 & 4.24890500 \\
\hline $\mathrm{N}$ & -1.45340600 & -3.38151100 & 1.84518300 & $\mathrm{H}$ & -4.02704200 & -3.56500000 & 3.81827500 \\
\hline $\mathrm{H}$ & -1.39442200 & 3.77692700 & -0.63957900 & $\mathrm{C}$ & -4.25957400 & -2.78243100 & 0.61008100 \\
\hline $\mathrm{H}$ & 1.39493800 & 3.77643500 & 0.64013800 & $\mathrm{H}$ & -5.33886700 & -2.82110000 & 0.80902900 \\
\hline$(\mathrm{L} 4) \mathrm{Cu}(\mathrm{CN})_{2}$ & & & & $\mathrm{H}$ & -4.11238700 & -2.19172700 & -0.30438100 \\
\hline 02 & & & & $\mathrm{H}$ & -3.91917800 & -3.80796500 & 0.41584600 \\
\hline $\mathrm{C}$ & 0.00507800 & -1.44413100 & -0.00919300 & & & & \\
\hline $\mathrm{C}$ & -0.82352900 & -0.62903200 & -0.95715000 & & & & \\
\hline $\mathrm{C}$ & 0.83418100 & -0.61208200 & 0.92266800 & & & & \\
\hline $\mathrm{O}$ & -1.56161800 & -1.37574000 & -1.78127400 & & & & \\
\hline $\mathrm{N}$ & -0.93405900 & 0.64214300 & -1.05466500 & & & & \\
\hline $\mathrm{N}$ & 0.91237200 & 0.66147100 & 1.01954900 & & & & \\
\hline $\mathrm{C}$ & 1.98797000 & 0.94150200 & 1.99455300 & & & & \\
\hline $\mathrm{C}$ & 2.24242900 & -0.41811200 & 2.66375600 & & & & \\
\hline $\mathrm{O}$ & 1.59915100 & -1.34006500 & 1.73928400 & & & & \\
\hline $\mathrm{C}$ & 3.29500600 & 1.25198800 & 1.30976700 & & & & \\
\hline $\mathrm{C}$ & 4.27539800 & 0.32912800 & 1.67538700 & & & & \\
\hline $\mathrm{C}$ & 3.74661800 & -0.65201100 & 2.68643100 & & & & \\
\hline $\mathrm{C}$ & 3.57925200 & 2.28702400 & 0.42568100 & & & & \\
\hline $\mathrm{C}$ & 4.86595600 & 2.38197400 & -0.09801500 & & & & \\
\hline $\mathrm{C}$ & 5.84746500 & 1.45525400 & 0.25776600 & & & & \\
\hline $\mathrm{C}$ & 5.55946000 & 0.42404700 & 1.14811300 & & & & \\
\hline $\mathrm{C}$ & -2.23725500 & -0.47233400 & -2.69946200 & & & & \\
\hline $\mathrm{C}$ & -2.01009500 & 0.89707200 & -2.03771800 & & & & \\
\hline $\mathrm{C}$ & -3.73537800 & -0.74636700 & -2.70210500 & & & & \\
\hline $\mathrm{C}$ & -4.27940000 & 0.22883400 & -1.69385600 & & & & \\
\hline $\mathrm{C}$ & -3.32396600 & 1.18823700 & -1.35700200 & & & & \\
\hline $\mathrm{C}$ & -5.55889500 & 0.29138300 & -1.15140800 & & & & \\
\hline $\mathrm{C}$ & -5.87185000 & 1.33503100 & -0.28426800 & & & & \\
\hline $\mathrm{C}$ & -4.91849000 & 2.30324500 & 0.03604500 & & & & \\
\hline $\mathrm{C}$ & -3.63335200 & 2.23528900 & -0.49560800 & & & & \\
\hline $\mathrm{H}$ & 1.65991900 & 1.73506800 & 2.67489400 & & & & \\
\hline $\mathrm{H}$ & 1.75067100 & -0.55737500 & 3.63247200 & & & & \\
\hline $\mathrm{H}$ & 4.14147300 & -0.44080900 & 3.69083800 & & & & \\
\hline $\mathrm{H}$ & 3.98705300 & -1.70163300 & 2.46686100 & & & & \\
\hline $\mathrm{H}$ & 2.81409700 & 3.01607400 & 0.16083600 & & & & \\
\hline
\end{tabular}




\section{Reaction with Pent-1-yn-1-ylbenzene (9)}
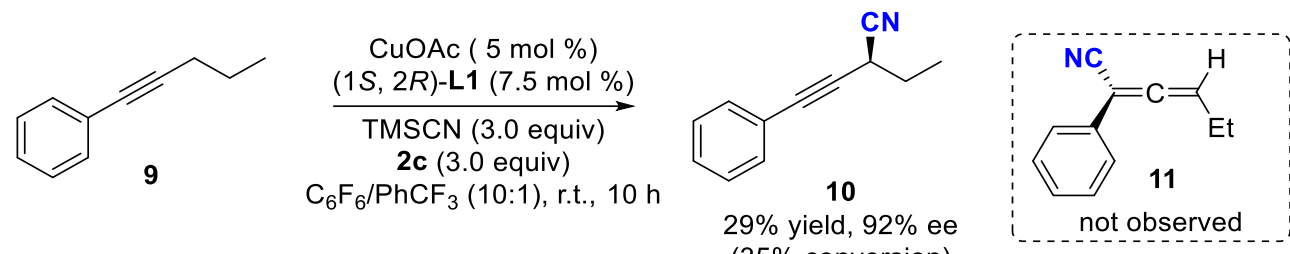

(35\% conversion)

In a dried sealed $10 \mathrm{~mL}$ Schlenk tube, CuOAc (0.01 mmol, $5 \mathrm{~mol} \%)$, chiral bisoxazoline ligand $(1 S, 2 R)-\mathbf{L 1}(0.015 \mathrm{mmol}, 7.5 \mathrm{~mol} \%)$, and NFAS $2 \mathrm{c}(196 \mathrm{mg}, 0.3$ mmol, 3.0 equiv) were dissolved in a mixed solvent of $\mathrm{C}_{6} \mathrm{~F}_{6}(1.0 \mathrm{~mL})$ and $\mathrm{PhCF}_{3}(100$ $\mu \mathrm{L}$ ) under a nitrogen atmosphere, and the mixture was stirred for $30 \mathrm{~min}$. Then, pent-1-yn-1-ylbenzene 9 (0.2 mmol, 1.0 equiv), TMSCN (0.6 mmol, 3.0 equiv) were sequentially added. The reaction mixture was stirred at room temperature for 10 hours. After the reaction was completed, the mixture was analyzed by ${ }^{1} \mathrm{H}-\mathrm{NMR}$ with $\mathrm{CF}_{3}$-DMAc $(0.03 \mathrm{mmol})$ as an internal standard. Then solvent was removed under vacuum, and the residue was purified by column chromatography on silica gel with petroleum ether/ethyl acetate (20:1) to afford the product $\mathbf{1 0}(9.9 \mathrm{mg}, 29 \%$ yield, $92 \%$ ee) as light yellow oil.

$[\alpha]_{\mathrm{D}}^{25.2}-9.70\left(c 0.3, \mathrm{CHCl}_{3}\right)$

${ }^{1} \mathrm{H}$ NMR (400 MHz, $\left.\mathrm{CDCl}_{3}\right) \delta 7.45-7.43(\mathrm{~m}, 2 \mathrm{H}), 7.35-7.32(\mathrm{~m}, 3 \mathrm{H}), 3.72(\mathrm{t}, J=$ $6.8 \mathrm{~Hz}, 1 \mathrm{H}), 2.03-1.96(\mathrm{~m}, 2 \mathrm{H}), 1.22(\mathrm{t}, J=7.2 \mathrm{~Hz}, 3 \mathrm{H})$

${ }^{13} \mathrm{C}$ NMR $\left(100 \mathrm{MHz} \mathrm{CDCl}_{3}\right) \delta 131.8,128.9,128.4,121.8,117.5,84.1,81.0,27.1$, $25.3,11.2$.

IR (neat): 2925, 2247, 1490, 1459, 1258, 1070, 1027, 799, 756, 690, $528 \mathrm{~cm}^{-1}$.

HRMS: $m / z$ (EI) calculated for $\mathrm{C}_{12} \mathrm{H}_{11} \mathrm{~N}^{+}[\mathrm{M}]^{+}$: 169.0886, found: 169.0885.

HPLC (AY-3, 0.46*15 cm, $3 \mu \mathrm{m}$, hexane/isopropanol = 99/1, flow $0.5 \mathrm{~mL} / \mathrm{min}$, detection at $214 \mathrm{~nm}$ ) retention time $=9.49 \mathrm{~min}$ (major) and $11.84 \mathrm{~min}$ (minor).

\section{Reaction with (8-Chlorooct-3-yn-2-yl)benzee (12)}

\subsection{Synthesis of (8-chlorooct-3-yn-2-yl)benzee (12)}




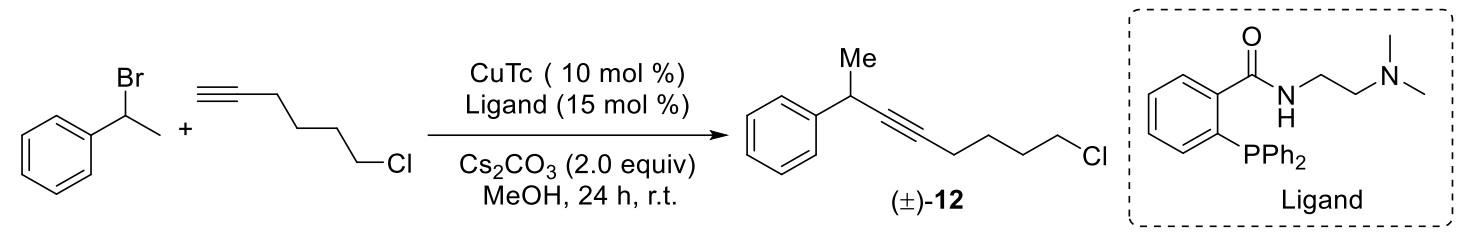

(8-Chlorooct-3-yn-2-yl)benzee (12) was synthesized according to the reported literature ${ }^{7}$. To a dried sealed $100 \mathrm{~mL}$ Schlenk tube with a magnetic stir bar was charged $\mathrm{CuTc} \quad(95 \mathrm{mg}, \quad 0.5 \mathrm{mmol}), \quad \mathrm{N}$-(2-(dimethylamino)ethyl)-2-(diphenyl phosphaneyl)benzamide (282 $\mathrm{mg}, 0.75 \mathrm{mmol})$, and $\mathrm{Cs}_{2} \mathrm{CO}_{3}(3.3 \mathrm{~g}, 10 \mathrm{mmol})$. The tube was evacuated and back-filled with argon for three times. Then, dry $\mathrm{MeOH}$ (50 $\mathrm{mL})$ and (1-bromoethyl) benzene (1.4 g, $7.5 \mathrm{mmol})$, 6-chlorohex-1-yne (580 mg, 5 mmol) were added sequentially and the reaction mixture was stirred at room temperature for 24 hours. Upon reaction completion, the precipitate was filtered off and washed by $\mathrm{Et}_{2} \mathrm{O}$. The filtrate was evaporated and the residue was purified by column chromatography on silica gel with a gradient eluent of petroleum ether to afford the corresponding product $\mathbf{1 2}$ as colorless oil ( $0.91 \mathrm{~g}, 83 \%$ yield).

${ }^{1} \mathrm{H}$ NMR $\left(400 \mathrm{MHz}, \mathrm{CDCl}_{3}\right) \delta 7.39-7.37(\mathrm{~m}, 2 \mathrm{H}), 7.35-7.31(\mathrm{~m}, 2 \mathrm{H}), 7.25-7.21$ $(\mathrm{m}, 1 \mathrm{H}), 3.74(\mathrm{q}, J=7.2 \mathrm{~Hz}, 1 \mathrm{H}), 3.58(\mathrm{t}, J=6.4 \mathrm{~Hz}, 2 \mathrm{H}), 2.29(\mathrm{t}, J=6.8 \mathrm{~Hz}, 2 \mathrm{H})$, $1.95-1.88(\mathrm{~m}, 2 \mathrm{H}), 1.73-1.65(\mathrm{~m}, 2 \mathrm{H}), 1.47(\mathrm{~d}, J=6.8 \mathrm{~Hz}, 3 \mathrm{H})$.

${ }^{13} \mathrm{C} \mathrm{NMR}\left(100 \mathrm{MHz}, \mathrm{CDCl}_{3}\right) \delta 143.9,128.4,126.7,128.5,83.6,81.3,44.6,31.9,31.6$, $26.1,24.8,18.1$.

IR (neat): 2928, 1709, 1493, 1449, 1299, 1029, 760, 698, $650 \mathrm{~cm}^{-1}$.

HRMS: $m / z$ (FI) calculated for $\mathrm{C}_{14} \mathrm{H}_{17} \mathrm{Cl}^{+}[\mathrm{M}]^{+}:$220.1013, found: 220.1016 .

\subsection{Reaction with (8-Chlorooct-3-yn-2-yl)benzee (12)}

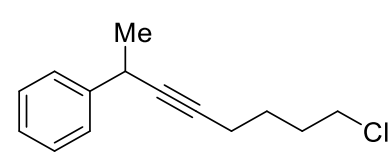

$( \pm)-12$

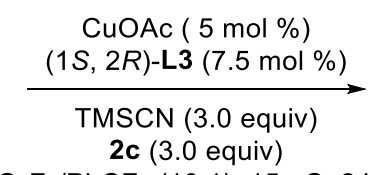

$\mathrm{C}_{6} \mathrm{~F}_{6} / \mathrm{PhCF}_{3}(10: 1), 15$ oC, $24 \mathrm{~h}$

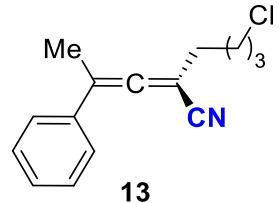

13

In a dried sealed $10 \mathrm{~mL}$ Schlenk tube, CuOAc (0.01 mmol, $5 \mathrm{~mol} \%)$, chiral bisoxazoline ligand (1S, 2R)-L3 (0.015 mmol, $7.5 \mathrm{~mol} \%$ ), and NFAS $2 \mathrm{c}$ (196 mg, 0.3 
mmol, 3.0 equiv) were dissolved in a mixed solvent of $\mathrm{C}_{6} \mathrm{~F}_{6}(1.0 \mathrm{~mL})$ and $\mathrm{PhCF}_{3}(100$ $\mu \mathrm{L}$ ) under a $N_{2}$ atmosphere, and the mixture was stirred for $30 \mathrm{~min}$. Then, (8-chlorooct-3-yn-2-yl)benzee (12) (0.2 mmol, 1.0 equiv), TMSCN (0.6 mmol, 3.0 equiv) were sequentially added. The reaction mixture was stirred at $15^{\circ} \mathrm{C}$ for 24 hours. After the reaction was completed, the mixture was analyzed by ${ }^{1} \mathrm{H}-\mathrm{NMR}$ with $\mathrm{CF}_{3}$-DMAc $(0.03 \mathrm{mmol})$ as an internal standard. Then solvent was removed under vacuum, and the residue was purified by column chromatography on silica gel with petroleum ether/ethyl acetate (20:1) to afford the product 13 (6.5 mg, 13\% yield, 53\% ee) as light yellow oil.

$[\alpha]_{\mathrm{D}}^{24.8}-19.35\left(c 0.21, \mathrm{CHCl}_{3}\right)$

${ }^{1} \mathrm{H}$ NMR (400 MHz, $\left.\mathrm{CDCl}_{3}\right) \delta 7.41-7.32(\mathrm{~m}, 5 \mathrm{H}), 3.53(\mathrm{t}, J=6.0 \mathrm{~Hz}), 2.35(\mathrm{t}, J=$ $7.2 \mathrm{~Hz}), 2.20(\mathrm{~s}, 3 \mathrm{H}), 1.87-1.82(\mathrm{~m}, 2 \mathrm{H}), 1.78-1.72(\mathrm{~m}, 2 \mathrm{H})$.

${ }^{13} \mathrm{C}$ NMR $\left(100 \mathrm{MHz}, \mathrm{CDCl}_{3}\right) \delta 212.4,133.6,128.8,128.5,126.3,115.3,107.3,83.2$, 44.2, 31.4, 31.0, 24.9, 16.6.

IR (neat): 2927, 2859, 2216, 1941, 1494, 1443, 1066, 1026, 760, 692, $649 \mathrm{~cm}^{-1}$.

HRMS: $m / z$ (FI) calculated for $\mathrm{C}_{15} \mathrm{H}_{16} \mathrm{NCl}^{+}[\mathrm{M}]^{+}: 245.0966$, found: 245.0968 .

HPLC (IG, 0.46*25 cm, $5 \mu \mathrm{m}$, hexane/isopropanol = 98/2, flow $0.7 \mathrm{~mL} / \mathrm{min}$, detection at $214 \mathrm{~nm}$ ) retention time $=12.72 \mathrm{~min}$ (minor) and $13.98 \mathrm{~min}$ (major).

The reaction with $(1 S, 2 R)-\mathbf{L 1}$ gave the desired product $\mathbf{1 3}(15.3 \mathrm{mg}, 31 \%$ yield, $-8 \%$ ee) as light yellow oil.

$[\alpha]_{\mathrm{D}}^{24.4}+6.47\left(c 0.70, \mathrm{CHCl}_{3}\right)$

HPLC (IG, 0.46*25 cm, $5 \mu \mathrm{m}$, hexane/isopropanol = 98/2, flow $0.7 \mathrm{~mL} / \mathrm{min}$, detection at $214 \mathrm{~nm}$ ) retention time $=13.45 \mathrm{~min}$ (major) and $14.56 \mathrm{~min}$ (minor).

\section{Product Characterizations}

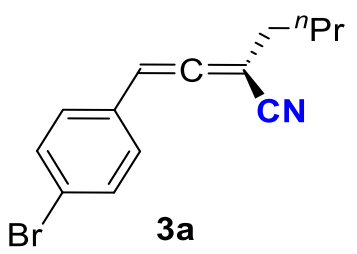

The reaction was conducted according to the general procedure on a $0.2 \mathrm{mmol}$ scale. 
The residue was purified by column chromatography on silica gel with petroleum ether/ethyl acetate (20:1) to afford the product 3a (42.7 $\mathrm{mg}, 78 \%$ yield, $90 \%$ ee) as colorless oil.

$[\alpha]_{\mathrm{D}}^{27.1}-84.70\left(c 0.83, \mathrm{CHCl}_{3}\right)$

${ }^{1} \mathrm{H}$ NMR (400 MHz, $\left.\mathrm{CDCl}_{3}\right) \delta 7.49(\mathrm{~d}, J=8.0 \mathrm{~Hz}, 2 \mathrm{H}), 7.14(\mathrm{~d}, J=8.4 \mathrm{~Hz}, 2 \mathrm{H}), 6.54$ $(\mathrm{t}, J=2.4 \mathrm{~Hz}, 1 \mathrm{H}), 2.35-2.31(\mathrm{~m}, 2 \mathrm{H}), 1.59-1.56(\mathrm{~m}, 2 \mathrm{H}), 1.43-1.37(\mathrm{~m}, 2 \mathrm{H})$, $0.92(\mathrm{t}, J=7.2 \mathrm{~Hz}, 3 \mathrm{H})$.

${ }^{13} \mathrm{C}$ NMR $\left(100 \mathrm{MHz}, \mathrm{CDCl}_{3}\right) \delta 213.1,132.2,129.7,129.1,122.6,114.6,98.8,86.8$, 31.3, 29.6, 21.8, 13.6.

IR (neat): 2961, 2927, 2859, 2219, 1943, 1486, 1259, 1070, 1010, 797, $501 \mathrm{~cm}^{-1}$. HRMS: $m / z$ (EI) calculated for $\mathrm{C}_{14} \mathrm{H}_{13} \mathrm{BrN}^{+}[\mathrm{M}-\mathrm{H}]^{+}:$274.0226, found: 274.0226 . HPLC (AD-H, 0.46*25 cm, $5 \mu \mathrm{m}$, hexane/isopropanol = 98/2, flow $0.7 \mathrm{~mL} / \mathrm{min}$, detection at $214 \mathrm{~nm}$ ) retention time $=6.95 \mathrm{~min}$ (major) and $7.31 \mathrm{~min}$ (minor).

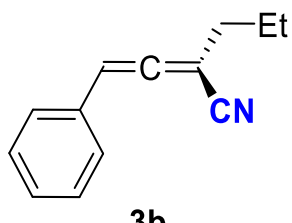

3b

The reaction was conducted according to the general procedure on a $0.2 \mathrm{mmol}$ scale. The residue was purified by column chromatography on silica gel with petroleum ether/ethyl acetate (20:1) to afford the product $\mathbf{3 b}(25.8 \mathrm{mg}, 71 \%$ yield, $93 \%$ ee) as colorless oil.

$[\alpha]_{\mathrm{D}}^{26.6}-143.30\left(c 1.05, \mathrm{CHCl}_{3}\right)$

${ }^{1} \mathrm{H}$ NMR $\left(400 \mathrm{MHz}, \mathrm{CDCl}_{3}\right) \delta 7.38-7.26(\mathrm{~m}, 5 \mathrm{H}), 6.60(\mathrm{t}, J=2.4 \mathrm{~Hz}, 1 \mathrm{H}), 2.34-$ $2.29(\mathrm{~m}, 2 \mathrm{H}), 1.67-1.58(\mathrm{~m}, 2 \mathrm{H}), 1.00(\mathrm{t}, J=7.2 \mathrm{~Hz}, 3 \mathrm{H})$.

${ }^{13} \mathrm{C} \mathrm{NMR}\left(100 \mathrm{MHz}, \mathrm{CDCl}_{3}\right) \delta 213.3,130.7,129.0,128.7,127.7,114.9,99.6,86.0$, $33.6,21.0,13.2$.

IR (neat): 2962, 2930, 2873, 2219, 1942, 1600, 1496, 827, 748, 691, $498 \mathrm{~cm}^{-1}$. HRMS: $m / z$ (EI) calculated for $\mathrm{C}_{13} \mathrm{H}_{12} \mathrm{~N}^{+}[\mathrm{M}-\mathrm{H}]^{+}$: 182.0964, found: 182.0962 . HPLC (IG, 0.46*25 cm, $5 \mu \mathrm{m}$, hexane/isopropanol = 98/2, flow $0.7 \mathrm{~mL} / \mathrm{min}$, detection at $214 \mathrm{~nm}$ ) retention time $=11.20 \mathrm{~min}$ (minor) and $12.64 \mathrm{~min}$ (major). 


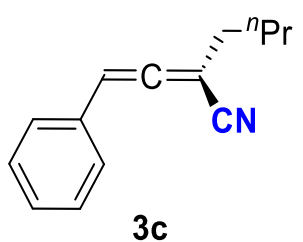

The reaction was conducted according to the general procedure on a $0.2 \mathrm{mmol}$ scale. The residue was purified by column chromatography on silica gel with petroleum ether/ethyl acetate (20:1) to afford the product 3c (26.3 $\mathrm{mg}, 67 \%$ yield, 92\% ee) as colorless oil.

$[\alpha]_{\mathrm{D}}^{26.8}-97.47\left(c\right.$ 1.01, $\left.\mathrm{CHCl}_{3}\right)$

${ }^{1} \mathrm{H}$ NMR $\left(400 \mathrm{MHz}, \mathrm{CDCl}_{3}\right) \delta 7.38-7.31(\mathrm{~m}, 2 \mathrm{H}), 7.30-7.26(\mathrm{~m}, 3 \mathrm{H}), 6.59(\mathrm{t}, J=$ $2.8 \mathrm{~Hz}, 1 \mathrm{H}), 2.35-2.31(\mathrm{~m}, 2 \mathrm{H}), 1.61-1.55(\mathrm{~m}, 2 \mathrm{H}), 1.44-1.39(\mathrm{~m}, 2 \mathrm{H}), 0.93(\mathrm{t}, J$ $=7.2 \mathrm{~Hz}, 3 \mathrm{H})$

${ }^{13} \mathrm{C}$ NMR $\left(100 \mathrm{MHz}, \mathrm{CDCl}_{3}\right) \delta 213.2,130.8,129.0,128.7,127.7,114.9,99.6,86.2$, 31.4, 29.7, 21.8, 13.6.

IR (neat): 2958, 2929, 2860, 2218, 1943, 1600, 1495, 829, 750, 692, $553 \mathrm{~cm}^{-1}$.

HRMS: $m / z$ (EI) calculated for $\mathrm{C}_{14} \mathrm{H}_{14} \mathrm{~N}^{+}[\mathrm{M}-\mathrm{H}]^{+}$: 196.1121, found: 196.1121.

HPLC (OJ-H, 0.46*25 cm, $5 \mu \mathrm{m}$, hexane/isopropanol $=98 / 2$, flow $0.7 \mathrm{~mL} / \mathrm{min}$, detection at $214 \mathrm{~nm}$ ) retention time $=10.71 \mathrm{~min}$ (minor) and 11.39 min (major)

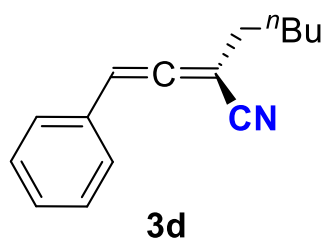

The reaction was conducted according to the general procedure on a $0.2 \mathrm{mmol}$ scale. The residue was purified by column chromatography on silica gel with petroleum ether/ethyl acetate (20:1) to afford the product 3d (33.3 $\mathrm{mg}$, 79\% yield, 93\% ee) as colorless oil.

$[\alpha]_{\mathrm{D}}^{26.0}-76.65\left(c 0.95, \mathrm{CHCl}_{3}\right)$

${ }^{1} \mathrm{H}$ NMR $\left(400 \mathrm{MHz}, \mathrm{CDCl}_{3}\right) \delta 7.38-7.35(\mathrm{~m}, 2 \mathrm{H}), 7.32-7.26(\mathrm{~m}, 3 \mathrm{H}), 6.60(\mathrm{t}, J=$ $2.4 \mathrm{~Hz}, 1 \mathrm{H}), 2.35-2.30(\mathrm{~m}, 2 \mathrm{H}), 1.63-1.58(\mathrm{~m}, 2 \mathrm{H}), 1.38-1.33(\mathrm{~m}, 4 \mathrm{H}), 0.89(\mathrm{t}, J$ $=6.8 \mathrm{~Hz}, 3 \mathrm{H})$.

${ }^{13} \mathrm{C}$ NMR $\left(100 \mathrm{MHz}, \mathrm{CDCl}_{3}\right) \delta 213.2,130.8,129.0,128.7,127.7,114.9,99.6(\mathrm{~d}, J=$ 
4.1 Hz), 86.2, 31.6, 30.8, 27.3, 22.2, 13.9.

IR (neat): 2927, 2857, 2218, 1943, 1495, 1457, 827, 748, 692, $492 \mathrm{~cm}^{-1}$.

HRMS: $m / z$ (EI) calculated for $\mathrm{C}_{15} \mathrm{H}_{17} \mathrm{~N}^{+}[\mathrm{M}]^{+}:$211.1356, found: 211.1353 .

HPLC (AS-RH, 0.46*15 cm, $3 \mu \mathrm{m}$, acetonitrile/water $=40 / 60$, flow $0.7 \mathrm{~mL} / \mathrm{min}$, detection at $214 \mathrm{~nm}$ ) retention time $=78.14 \mathrm{~min}$ (minor) and $83.49 \mathrm{~min}$ (major).

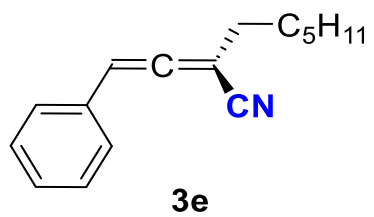

The reaction was conducted according to the general procedure on a $0.2 \mathrm{mmol}$ scale. The residue was purified by column chromatography on silica gel with petroleum ether/ethyl acetate (20:1) to afford the product 3e (31.5 mg, 70\% yield, 93\% ee) as colorless oil.

$[\alpha]_{\mathrm{D}}^{25.6}-62.60\left(c 1.71, \mathrm{CHCl}_{3}\right)$

${ }^{1} \mathrm{H}$ NMR (400 MHz, $\left.\mathrm{CDCl}_{3}\right) \delta 7.38-7.35(\mathrm{~m}, 2 \mathrm{H}), 7.32-7.26(\mathrm{~m}, 3 \mathrm{H}), 6.60-6.58$ $(\mathrm{m}, 1 \mathrm{H}), 2.35-2.31(\mathrm{~m}, 2 \mathrm{H}), 1.62-1.56(\mathrm{~m}, 2 \mathrm{H}), 1.39-1.29(\mathrm{~m}, 6 \mathrm{H}), 0.88(\mathrm{t}, J=$ $6.0 \mathrm{~Hz}, 3 \mathrm{H})$.

${ }^{13} \mathrm{C}$ NMR $\left(100 \mathrm{MHz}, \mathrm{CDCl}_{3}\right) \delta$ 213.2, 130.8, 129.0, 128.7, 127.7, 115.0, 99.6, 86.3, $31.7,31.4,28.4,27.6,22.5,14.0$.

HRMS: $m / z$ (EI) calculated for $\mathrm{C}_{16} \mathrm{H}_{19} \mathrm{~N}^{+}[\mathrm{M}]^{+}: 225.1512$, found: 225.1511 .

IR (neat): 2926, 2856, 2217, 1943, 1495, 1456, 827, 749, 692, $493 \mathrm{~cm}^{-1}$.

HPLC (IG, 0.46*25 cm, $5 \mu \mathrm{m}$, hexane/isopropanol =98/2, flow $0.7 \mathrm{~mL} / \mathrm{min}$, detection at $214 \mathrm{~nm}$ ) retention time $=10.28 \mathrm{~min}$ (minor) and $11.20 \mathrm{~min}$ (major).

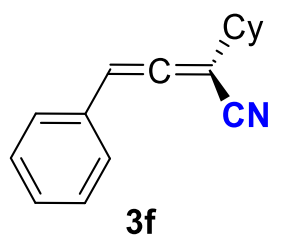

The reaction was conducted according to the general procedure on a $0.2 \mathrm{mmol}$ scale. The residue was purified by column chromatography on silica gel with petroleum ether/ethyl acetate (20:1) to afford the product $\mathbf{3 f}(28.9 \mathrm{mg}, 65 \%$ yield, $83 \%$ ee) as colorless oil. 
$[\alpha]_{\mathrm{D}}^{29.3}+3.18\left(c 1.06, \mathrm{CHCl}_{3}\right)$

${ }^{1} \mathrm{H}$ NMR (400 MHz, $\left.\mathrm{CDCl}_{3}\right) \delta 7.38-7.34(\mathrm{~m}, 2 \mathrm{H}), 7.32-7.27(\mathrm{~m}, 3 \mathrm{H}), 6.62(\mathrm{~d}, J=$ $2.4 \mathrm{~Hz}, 1 \mathrm{H}), 2.28-2.26(\mathrm{~m}, 1 \mathrm{H}), 2.00-1.96(\mathrm{~m}, 2 \mathrm{H}), 1.80-1.78(\mathrm{~m}, 2 \mathrm{H}), 1.70-$ $1.68(\mathrm{~m}, 1 \mathrm{H}), 1.34-1.17(\mathrm{~m}, 5 \mathrm{H})$.

${ }^{13} \mathrm{C} \mathrm{NMR}\left(100 \mathrm{MHz}, \mathrm{CDCl}_{3}\right) \delta 212.3,130.9,129.0,128.6,127.5,114.4,100.4,92.0$, $40.0,31.7,31.6,25.7,25.6$.

IR (neat): 2926, 2853, 2217, 1941, 1494, 1449, 827, 746, 691, 579, $506 \mathrm{~cm}^{-1}$.

HRMS: $m / z$ (EI) calculated for $\mathrm{C}_{16} \mathrm{H}_{16} \mathrm{~N}^{+}[\mathrm{M}-\mathrm{H}]^{+}:$: 222.1277, found: 222.1277 .

HPLC (IG, 0.46*25 cm, $5 \mu \mathrm{m}$, hexane/isopropanol = 98/2, flow $0.7 \mathrm{~mL} / \mathrm{min}$, detection at $214 \mathrm{~nm}$ ) retention time $=10.22 \min$ (minor) and $11.93 \min$ (major).

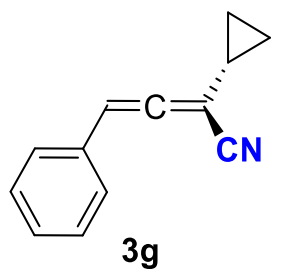

The reaction was conducted according to the general procedure on a $0.2 \mathrm{mmol}$ scale. The residue was purified by column chromatography on silica gel with petroleum ether/ethyl acetate (20:1) to afford the product $3 \mathrm{~g}(23.4 \mathrm{mg}, 65 \%$ yield, $89 \%$ ee) as colorless oil.

$[\alpha]_{\mathrm{D}}^{28.6}-69.62\left(c 1.07, \mathrm{CHCl}_{3}\right)$

${ }^{1} \mathrm{H}$ NMR (400 MHz, $\left.\mathrm{CDCl}_{3}\right) \delta 7.39-7.35(\mathrm{~m}, 2 \mathrm{H}), 7.33-7.27(\mathrm{~m}, 3 \mathrm{H}), 6.65(\mathrm{~d}, J=$ $0.8 \mathrm{~Hz}, 1 \mathrm{H}), 1.61-1.55(\mathrm{~m}, 1 \mathrm{H}), 0.91-0.88(\mathrm{~m}, 2 \mathrm{H}), 0.73-0.69(\mathrm{~m}, 2 \mathrm{H})$.

${ }^{13} \mathrm{C}$ NMR $\left(100 \mathrm{MHz}, \mathrm{CDCl}_{3}\right) \delta 212.6,130.6,129.0,128.8,127.7,113.9,101.1,89.9$, $11.5,6.6,6.5$.

IR (neat): 2924, 2853, 2213, 1940, 1494, 1455, 1028, 824, 749, 692, $507 \mathrm{~cm}^{-1}$. HRMS: $m / z$ (EI) calculated for $\mathrm{C}_{13} \mathrm{H}_{10} \mathrm{~N}^{+}[\mathrm{M}-\mathrm{H}]^{+}: 180.0808$, found: 180.0806 . HPLC (AD-RH, 0.46*15 cm, $3 \mu \mathrm{m}$, acetonitrile/water $=50 / 50$, flow $0.7 \mathrm{~mL} / \mathrm{min}$, detection at $214 \mathrm{~nm}$ ) retention time $=27.36 \mathrm{~min}$ (major) and $29.80 \mathrm{~min}$ (minor). 


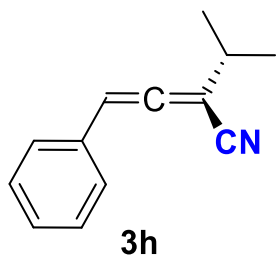

The reaction was conducted according to the general procedure on a $0.2 \mathrm{mmol}$ scale. The residue was purified by column chromatography on silica gel with petroleum ether/ethyl acetate (40:1) to afford the product $\mathbf{3 h}(25.7 \mathrm{mg}, 70 \%$ yield, $92 \%$ ee) as colorless oil.

$[\alpha]_{\mathrm{D}}^{23.6}-74.91\left(c 1.25, \mathrm{CHCl}_{3}\right)$

${ }^{1} \mathrm{H}$ NMR $\left(400 \mathrm{MHz}, \mathrm{CDCl}_{3}\right) \delta 7.39-7.35(\mathrm{~m}, 2 \mathrm{H}), 7.33-7.28(\mathrm{~m}, 3 \mathrm{H}), 6.64(\mathrm{~d}, J=$ $2.4 \mathrm{~Hz}, 1 \mathrm{H}), 2.64-2.60(\mathrm{~m}, 1 \mathrm{H}), 1.22(\mathrm{dd}, J=3.6,6.8 \mathrm{~Hz}, 2 \mathrm{H})$.

${ }^{13} \mathrm{C}$ NMR $\left(100 \mathrm{MHz}, \mathrm{CDCl}_{3}\right) \delta 211.7,130.8,129.0,128.7,127.5,114.4,100.6,93.0$, 31.4, 21.3.

IR (neat): 2967, 2217, 1940, 1494, 1458, 1217, 1053, 914, 829, 749, $691 \mathrm{~cm}^{-1}$.

HRMS: $m / z$ (EI) calculated for $\mathrm{C}_{13} \mathrm{H}_{13} \mathrm{~N}^{+}[\mathrm{M}]^{+}:$183.1043, found: 183.1046 .

HPLC (IG, 0.46*25 cm, $5 \mu \mathrm{m}$, hexane/isopropanol = 99/1, flow $0.7 \mathrm{~mL} / \mathrm{min}$, detection at $254 \mathrm{~nm}$ ) retention time $=10.30 \mathrm{~min}$ (minor) and $11.54 \mathrm{~min}$ (major).

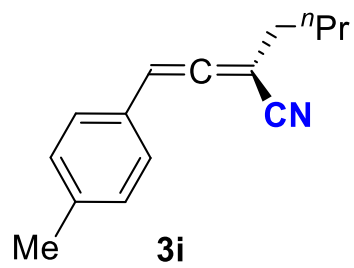

The reaction was conducted according to the general procedure on a $0.2 \mathrm{mmol}$ scale. The residue was purified by column chromatography on silica gel with petroleum ether/ethyl acetate (20:1) to afford the product $3 \mathbf{i}(28.6 \mathrm{mg}, 68 \%$ yield, $91 \%$ ee) as colorless oil.

$[\alpha]_{\mathrm{D}}^{26.0}-109.26\left(c 0.83, \mathrm{CHCl}_{3}\right)$.

${ }^{1} \mathrm{H}$ NMR (400 MHz, $\left.\mathrm{CDCl}_{3}\right) \delta 7.26-7.17(\mathrm{~m}, 4 \mathrm{H}), 6.57(\mathrm{t}, J=2.4 \mathrm{~Hz}, 1 \mathrm{H}), 2.36(\mathrm{~s}$, $3 \mathrm{H}), 2.34-2.30(\mathrm{~m}, 2 \mathrm{H}), 1.60-1.56(\mathrm{~m}, 2 \mathrm{H}), 1.43-1.40(\mathrm{~m}, 2 \mathrm{H}), 0.92(\mathrm{t}, J=7.2 \mathrm{~Hz}$, $3 \mathrm{H})$.

${ }^{13} \mathrm{C}$ NMR $\left(100 \mathrm{MHz}, \mathrm{CDCl}_{3}\right) \delta 213.2,138.8,129.7,127.7,127.6,115.1,99.5,86.0$, 
$31.4,29.6,21.8,21.3,13.7$.

IR (neat): 2958, 2925, 2858, 2219, 1942, 1611, 1513, 1459, 846, 800, 753, $506 \mathrm{~cm}^{-1}$. HRMS: $m / z$ (EI) calculated for $\mathrm{C}_{15} \mathrm{H}_{16} \mathrm{~N}^{+}[\mathrm{M}-\mathrm{H}]^{+}: 210.1277$, found: 210.1280 .

HPLC (OJ-H, 0.46*25 cm, $5 \mu \mathrm{m}$, hexane/isopropanol $=98 / 2$, flow $0.7 \mathrm{~mL} / \mathrm{min}$, detection at $214 \mathrm{~nm}$ ) retention time $=9.62 \mathrm{~min}$ (major) and $10.32 \mathrm{~min}$ (minor).

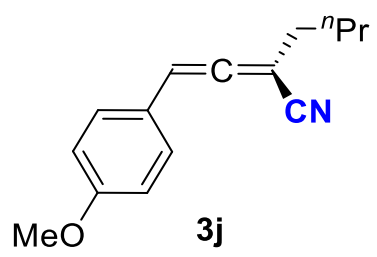

The reaction was conducted according to the general procedure on a $0.2 \mathrm{mmol}$ scale. The residue was purified by column chromatography on silica gel with petroleum ether/ethyl acetate (20:1) to afford the product $\mathbf{3 j}$ (37.8 $\mathrm{mg}, 83 \%$ yield, 91\% ee) as colorless oil.

$[\alpha]_{\mathrm{D}}^{28.4}-96.14\left(c 0.76, \mathrm{CHCl}_{3}\right)$

${ }^{1} \mathrm{H}$ NMR (400 MHz, $\left.\mathrm{CDCl}_{3}\right) \delta 7.21(\mathrm{~d}, J=8.8 \mathrm{~Hz}, 2 \mathrm{H}), 6.89(\mathrm{~d}, J=8.4 \mathrm{~Hz}, 2 \mathrm{H}), 6.55$ $(\mathrm{t}, J=2.8 \mathrm{~Hz}, 1 \mathrm{H}), 3.82(\mathrm{~s}, 3 \mathrm{H}), 2.33-2.29(\mathrm{~m}, 2 \mathrm{H}), 1.59-1.56(\mathrm{~m}, 2 \mathrm{H}), 1.43-1.38$ (m, 2H), $0.92(\mathrm{t}, J=7.6 \mathrm{~Hz}, 3 \mathrm{H})$.

${ }^{13} \mathrm{C}$ NMR $\left(100 \mathrm{MHz}, \mathrm{CDCl}_{3}\right) \delta 213.1,160.0,128.9,122.8,115.2,114.5,99.2,86.0$, $55.3,31.5,29.7,21.8,13.7$.

IR (neat): 2957, 2928, 2858, 2217, 1941, 1606, 1511, 1462, 1299, 1249, 1031, 847, $523 \mathrm{~cm}^{-1}$.

HRMS: $m / z$ (ESI) calculated for $\mathrm{C}_{15} \mathrm{H}_{18} \mathrm{ON}^{+}[\mathrm{M}+\mathrm{H}]^{+}: 228.1383$, found: 228.1381 .

HPLC (IG, $0.46^{*} 25 \mathrm{~cm}, 5 \mu \mathrm{m}$, hexane/isopropanol = 98/2, flow $0.7 \mathrm{~mL} / \mathrm{min}$, detection at $214 \mathrm{~nm}$ ) retention time $=14.91 \mathrm{~min}$ (major) and $15.74 \mathrm{~min}$ (minor).

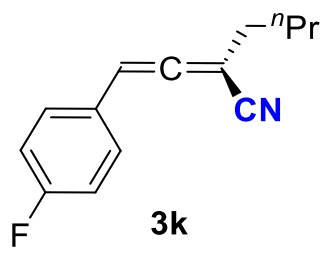

The reaction was conducted according to the general procedure on a $0.2 \mathrm{mmol}$ scale. The residue was purified by column chromatography on silica gel with petroleum ether/ethyl acetate (20:1) to afford the product $3 \mathbf{k}(35.1 \mathrm{mg}, 82 \%$ yield, $85 \%$ ee) as 
colorless oil.

$[\alpha]_{\mathrm{D}}^{28.1}-83.19\left(c 0.72, \mathrm{CHCl}_{3}\right)$

${ }^{1} \mathrm{H}$ NMR (400 MHz, $\left.\mathrm{CDCl}_{3}\right) \delta 7.25(\mathrm{q}, J=5.6 \mathrm{~Hz}, 2 \mathrm{H}), 7.06(\mathrm{t}, J=8.4 \mathrm{~Hz}, 2 \mathrm{H}), 6.57$

$(\mathrm{t}, J=2.8 \mathrm{~Hz}, 1 \mathrm{H}), 2.35-2.30(\mathrm{~m}, 2 \mathrm{H}), 1.60-1.54(\mathrm{~m}, 2 \mathrm{H}), 1.43-1.38(\mathrm{~m}, 2 \mathrm{H})$, $0.93(\mathrm{t}, J=7.2 \mathrm{~Hz}, 3 \mathrm{H})$.

${ }^{13} \mathrm{C} \mathrm{NMR}\left(100 \mathrm{MHz}, \mathrm{CDCl}_{3}\right) \delta 212.9,162.8(\mathrm{~d}, J=247.2 \mathrm{~Hz}), 129.3(\mathrm{~d}, J=8.5 \mathrm{~Hz})$, $126.7(\mathrm{~d}, J=3.7 \mathrm{~Hz}), 116.1(\mathrm{~d}, J=21.9 \mathrm{~Hz}), 114.8,98.7,86.5,31.4,29.6,21.8,13.6$.

${ }^{19} \mathrm{~F}$ NMR $\left(376 \mathrm{MHz}, \mathrm{CDCl}_{3}\right) \delta-112.2(\mathrm{~m})$.

IR (neat): 2959, 2929, 2860, 2219, 1945, 1601, 1508, 1227, 1157, 850, $516 \mathrm{~cm}^{-1}$.

HRMS: $m / z$ (EI) calculated for $\mathrm{C}_{14} \mathrm{H}_{13} \mathrm{FN}^{+}[\mathrm{M}-\mathrm{H}]^{+}: 214.1027$, found: 214.1029 .

HPLC (OJ-H, 0.46*25 cm, $5 \mu \mathrm{m}$, hexane/isopropanol $=98 / 2$, flow $0.7 \mathrm{~mL} / \mathrm{min}$, detection at $214 \mathrm{~nm}$ ) retention time $=9.24 \mathrm{~min}$ (minor) and $10.22 \mathrm{~min}$ (major).

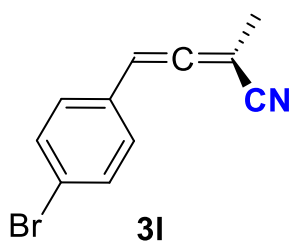

The reaction was conducted according to the general procedure on a $0.2 \mathrm{mmol}$ scale. The residue was purified by column chromatography on silica gel with petroleum ether/ethyl acetate (20:1) to afford the product 31 (32.9 $\mathrm{mg}, 70 \%$ yield, $86 \%$ ee) as colorless oil.

$[\alpha]_{\mathrm{D}}^{24.3}-163.31\left(c 0.50, \mathrm{CHCl}_{3}\right)$

${ }^{1} \mathrm{H}$ NMR (400 MHz, $\left.\mathrm{CDCl}_{3}\right) \delta 7.49(\mathrm{~d}, J=8.0 \mathrm{~Hz}, 2 \mathrm{H}), 7.15(\mathrm{~d}, J=8.0 \mathrm{~Hz}, 2 \mathrm{H}), 6.52$ $(\mathrm{q}, J=2.8 \mathrm{~Hz}, 1 \mathrm{H}), 2.06(\mathrm{~d}, J=2.8 \mathrm{~Hz}, 3 \mathrm{H})$

${ }^{13} \mathrm{C}$ NMR $\left(100 \mathrm{MHz}, \mathrm{CDCl}_{3}\right) \delta 213.9,132.2,129.6,129.2,122.7,115.0,98.4,81.6$, 17.5.

IR (neat): 2925, 2219, 1947, 1586, 1486, 1438, 1069, 1009, 847, 818, $499 \mathrm{~cm}^{-1}$. HRMS: $m / z$ (EI) calculated for $\mathrm{C}_{11} \mathrm{H}_{8} \mathrm{BrN}^{+}[\mathrm{M}-\mathrm{H}]^{+}:$232.9835, found: 232.9835 . HPLC (IG, 0.46*25 cm, $5 \mu \mathrm{m}$, hexane/isopropanol = 98/2, flow $0.7 \mathrm{~mL} / \mathrm{min}$, detection at $214 \mathrm{~nm}$ ) retention time $=13.17 \mathrm{~min}$ (minor) and $14.40 \mathrm{~min}$ (major). 


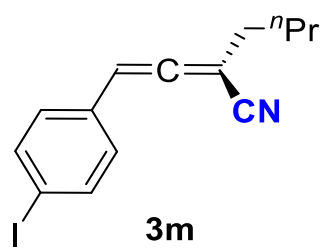

The reaction was conducted according to the general procedure on a $0.2 \mathrm{mmol}$ scale. The residue was purified by column chromatography on silica gel with petroleum ether/ethyl acetate (20:1) to afford the product $\mathbf{3 m}(47.0 \mathrm{mg}, 73 \%$ yield, $85 \%$ ee) as colorless oil.

$[\alpha]_{\mathrm{D}}^{27.9}-96.51\left(c 1.03, \mathrm{CHCl}_{3}\right)$

${ }^{1} \mathrm{H}$ NMR (400 MHz, $\left.\mathrm{CDCl}_{3}\right) \delta 7.69(\mathrm{~d}, J=7.6 \mathrm{~Hz}, 2 \mathrm{H}), 7.01(\mathrm{~d}, J=8.0 \mathrm{~Hz}, 2 \mathrm{H}), 6.53$ $(\mathrm{t}, J=2.6 \mathrm{~Hz}, 1 \mathrm{H}), 2.33(\mathrm{t}, J=6.0 \mathrm{~Hz}, 2 \mathrm{H}), 1.58-1.53(\mathrm{~m}, 2 \mathrm{H}), 1.42-1.37(\mathrm{~m}, 2 \mathrm{H})$, $0.92(\mathrm{t}, J=7.2 \mathrm{~Hz}, 3 \mathrm{H})$.

${ }^{13} \mathrm{C}$ NMR $\left(100 \mathrm{MHz}, \mathrm{CDCl}_{3}\right) \delta 213.2,138.1,130.3,129.2,114.6,99.0,94.2,86.8$ $31.3,29.6,21.8,13.6$.

IR (neat): 2957, 2926, 2858, 2219, 1943, 1484, 1461, 1059, 1005, 846, 817, 753, 498 $\mathrm{cm}^{-1}$.

HRMS: $m / z$ (EI) calculated for $\mathrm{C}_{14} \mathrm{H}_{13} \mathrm{IN}^{+}[\mathrm{M}-\mathrm{H}]^{+}: 322.0087$, found: 322.0083 .

HPLC (IG, 0.46*25 cm, $5 \mu \mathrm{m}$, hexane/isopropanol = 98/2, flow $0.7 \mathrm{~mL} / \mathrm{min}$, detection at $214 \mathrm{~nm}$ ) retention time $=10.80 \mathrm{~min}$ (minor) and $11.45 \mathrm{~min}$ (major).

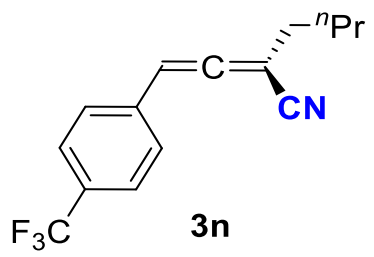

The reaction was conducted according to the general procedure on a $0.2 \mathrm{mmol}$ scale. The residue was purified by column chromatography on silica gel with petroleum ether/ethyl acetate (20:1) to afford the product $3 \mathrm{n}(33.5 \mathrm{mg}, 68 \%$ yield, $82 \%$ ee) as colorless oil.

$[\alpha]_{\mathrm{D}}^{23.9}-76.91\left(c 0.32, \mathrm{CHCl}_{3}\right)$

${ }^{1} \mathrm{H}$ NMR (400 MHz, $\left.\mathrm{CDCl}_{3}\right) \delta 7.62(\mathrm{~d}, J=8.0 \mathrm{~Hz}, 2 \mathrm{H}), 7.39(\mathrm{~d}, J=8.0 \mathrm{~Hz}, 2 \mathrm{H}), 6.63$ $(\mathrm{t}, J=2.4 \mathrm{~Hz}, 1 \mathrm{H}), 2.38-2.33(\mathrm{~m}, 2 \mathrm{H}), 1.63-1.55(\mathrm{~m}, 2 \mathrm{H}), 1.44-1.38(\mathrm{~m}, 2 \mathrm{H})$, $0.93(\mathrm{t}, J=7.2 \mathrm{~Hz}, 3 \mathrm{H})$. 
${ }^{13} \mathrm{C} \mathrm{NMR}\left(100 \mathrm{MHz}, \mathrm{CDCl}_{3}\right) \delta 213.5,134.7$ (q, $\left.J=1.6 \mathrm{~Hz}\right), 130.6(\mathrm{q}, J=33.1 \mathrm{~Hz})$,, 127.8, 126.0 (q, $J=3.7 \mathrm{~Hz}), 123.9$ (q, $J=270.4 \mathrm{~Hz}$ ), 114.4, 98.7, 87.1, 31.3, 29.6, 21.8, 13.6.

${ }^{19} \mathrm{~F}$ NMR $\left(376 \mathrm{MHz}, \mathrm{CDCl}_{3}\right) \delta-62.74(\mathrm{~s})$.

IR (neat): 2931, 2222, 1946, 1616, 1320, 1165, 1123, 1065, 1017, 857, 754, $597 \mathrm{~cm}^{-1}$. HRMS: $m / z$ (EI) calculated for $\mathrm{C}_{15} \mathrm{H}_{13} \mathrm{~F}_{3} \mathrm{~N}^{+}[\mathrm{M}-\mathrm{H}]^{+}: 264.0995$, found: 264.0994 .

HPLC (OJ-H, 0.46*25 cm, $5 \mu \mathrm{m}$, hexane/isopropanol $=99 / 1$, flow $0.7 \mathrm{~mL} / \mathrm{min}$, detection at $214 \mathrm{~nm}$ ) retention time $=13.16 \mathrm{~min}$ (minor) and $15.11 \mathrm{~min}$ (major).

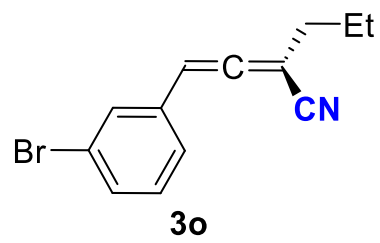

The reaction was conducted according to the general procedure on a $0.2 \mathrm{mmol}$ scale. The residue was purified by column chromatography on silica gel with petroleum ether/ethyl acetate (20:1) to afford the product 30 (40.7 $\mathrm{mg}, 78 \%$ yield, $89 \%$ ee) as colorless oil.

$[\alpha]_{\mathrm{D}}^{27.7}-88.41\left(c 0.96, \mathrm{CHCl}_{3}\right)$

${ }^{1} \mathrm{H}$ NMR (400 MHz, $\mathrm{CDCl}_{3}$ ) $\delta 7.44-7.42(\mathrm{~m}, 2 \mathrm{H}), 7.24-7.22(\mathrm{~m}, 2 \mathrm{H}), 6.55-6.52$ (m, 1H), $2.34-2.30(\mathrm{~m}, 2 \mathrm{H}), 1.64-1.59(\mathrm{~m}, 2 \mathrm{H}), 1.00(\mathrm{t}, J=7.6 \mathrm{~Hz}, 3 \mathrm{H})$.

${ }^{13} \mathrm{C}$ NMR $\left(100 \mathrm{MHz}, \mathrm{CDCl}_{3}\right) \delta 213.2,132.9,131.6,130.5,130.4,126.2,123.0,114.5$, $98.5,86.7,33.5,20.9,13.3$.

IR (neat): 2962, 2929, 2872, 2220, 1944, 1591, 1566, 1474, 1070, 755, 681, $434 \mathrm{~cm}^{-1}$. HRMS: $m / z(\mathrm{EI})$ calculated for $\mathrm{C}_{13} \mathrm{H}_{11} \mathrm{BrN}^{+}[\mathrm{M}-\mathrm{H}]^{+}: 260.0069$, found: 260.0067 .

HPLC (IG, 0.46*25 cm, $5 \mu \mathrm{m}$, hexane/isopropanol = 98/2, flow $0.7 \mathrm{~mL} / \mathrm{min}$, detection at $214 \mathrm{~nm}$ ) retention time $=13.02 \mathrm{~min}$ (minor) and $15.72 \mathrm{~min}$ (major).

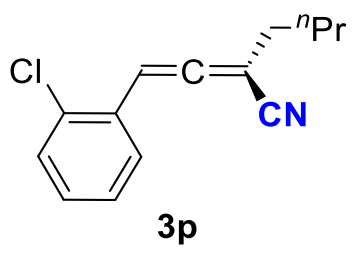

The reaction was conducted according to the general procedure on a $0.2 \mathrm{mmol}$ scale. The residue was purified by column chromatography on silica gel with petroleum 
ether/ethyl acetate (20:1) to afford the product $\mathbf{3 p}(26.1 \mathrm{mg}, 57 \%$ yield, $94 \%$ ee) as colorless oil.

$[\alpha]_{\mathrm{D}}^{27.3}-85.90\left(c 0.93, \mathrm{CHCl}_{3}\right)$

${ }^{1} \mathrm{H}$ NMR (400 MHz, $\left.\mathrm{CDCl}_{3}\right) \delta 7.41-7.35(\mathrm{~m}, 2 \mathrm{H}), 7.28-7.24(\mathrm{~m}, 2 \mathrm{H}), 7.04(\mathrm{t}, J=$ $2.8 \mathrm{~Hz}, 1 \mathrm{H}), 2.34(\mathrm{t}, J=6.0 \mathrm{~Hz}, 2 \mathrm{H}), 1.63-1.56(\mathrm{~m}, 2 \mathrm{H}), 1.44-1.38(\mathrm{~m}, 2 \mathrm{H}), 0.93(\mathrm{t}$, $J=7.2 \mathrm{~Hz}, 3 \mathrm{H})$.

${ }^{13} \mathrm{C}$ NMR $\left(100 \mathrm{MHz}, \mathrm{CDCl}_{3}\right) \delta 213.8,133.0,130.1,129.7,129.1,128.8,127.2,114.7$, 96.1, 86.4, 31.3, 29.6, 21.8, 13.6.

IR (neat): 2959, 2929, 2860, 2220, 1945, 1477, 1442, 1216, 1049, 749, $452 \mathrm{~cm}^{-1}$.

HRMS: $m / z(\mathrm{EI})$ calculated for $\mathrm{C}_{14} \mathrm{H}_{13} \mathrm{ClN}^{+}[\mathrm{M}-\mathrm{H}]^{+}: 230.0731$, found: 230.0732 .

HPLC (IG, 0.46*25 cm, $5 \mu \mathrm{m}$, hexane/isopropanol = 98/2, flow $0.7 \mathrm{~mL} / \mathrm{min}$, detection at $214 \mathrm{~nm}$ ) retention time $=8.64 \mathrm{~min}$ (minor) and $9.56 \min$ (major).

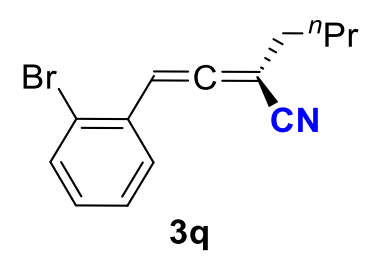

The reaction was conducted according to the general procedure on a $0.2 \mathrm{mmol}$ scale. The residue was purified by column chromatography on silica gel with petroleum ether/ethyl acetate (20:1) to afford the product 3q (29.4 mg, 50\% yield, 94\% ee) as colorless oil.

$[\alpha]_{\mathrm{D}}^{23.2}-44.47\left(c 1.34, \mathrm{CHCl}_{3}\right)$

${ }^{1} \mathrm{H}$ NMR (400 MHz, $\left.\mathrm{CDCl}_{3}\right) \delta 7.59-7.57(\mathrm{~m}, 1 \mathrm{H}), 7.37$ - $7.29(\mathrm{~m}, 2 \mathrm{H}), 7.18-7.14$ (m, 1H), $7.06(\mathrm{t}, J=2.8 \mathrm{~Hz}, 1 \mathrm{H}), 2.34(\mathrm{td}, J=7.6,2.8 \mathrm{~Hz}, 2 \mathrm{H}), 1.61-1.55(\mathrm{~m}, 2 \mathrm{H})$, $1.44-1.38(\mathrm{~m}, 2 \mathrm{H}), 0.93(\mathrm{t}, J=7.2 \mathrm{~Hz}, 3 \mathrm{H})$.

${ }^{13} \mathrm{C}$ NMR $\left(100 \mathrm{MHz}, \mathrm{CDCl}_{3}\right) \delta 213.7,133.4,130.6,129.9,129.3,127.8,123.2,114.7$, 98.7, 86.5, 31.3, 29.6, 21.8, 13.6.

IR (neat): 2958, 2928, 2860, 2220, 1944, 1472, 1438, 1266, 1023, 827, $749 \mathrm{~cm}^{-1}$. HRMS: $m / z$ (FI) calculated for $\mathrm{C}_{14} \mathrm{H}_{14} \mathrm{BrN}^{+}[\mathrm{M}]^{+}:$275.0304, found: 275.0308 . HPLC (IG, $0.46^{*} 25 \mathrm{~cm}, 5 \mu \mathrm{m}$, hexane/isopropanol = 99/1, flow $0.7 \mathrm{~mL} / \mathrm{min}$, detection at $214 \mathrm{~nm}$ ) retention time $=10.98 \mathrm{~min}$ (minor) and $11.87 \mathrm{~min}$ (major). 


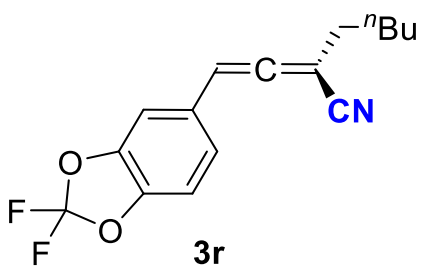

The reaction was conducted according to the general procedure on a $0.2 \mathrm{mmol}$ scale. The residue was purified by column chromatography on silica gel with petroleum ether/ethyl acetate (20:1) to afford the product $3 \mathbf{r}(36.7 \mathrm{mg}, 63 \%$ yield, $81 \%$ ee) as colorless oil.

$[\alpha]_{\mathrm{D}}^{29.2}:-64.16\left(c 0.95, \mathrm{CHCl}_{3}\right)$

${ }^{1} \mathrm{H}$ NMR (400 MHz, $\left.\mathrm{CDCl}_{3}\right) \delta 7.06-7.04(\mathrm{~m}, 1 \mathrm{H}), 7.00-6.99(\mathrm{~m}, 2 \mathrm{H}), 6.57(\mathrm{t}, J=$ $2.9 \mathrm{~Hz}, 1 \mathrm{H}), 2.35-2.31(\mathrm{~m}, 2 \mathrm{H}), 1.61-1.56(\mathrm{~m}, 2 \mathrm{H}), 1.35-1.31(\mathrm{~m}, 4 \mathrm{H}), 0.89(\mathrm{t}, J$ $=6.8 \mathrm{~Hz}, 3 \mathrm{H})$.

${ }^{13} \mathrm{C} \mathrm{NMR}\left(100 \mathrm{MHz}, \mathrm{CDCl}_{3}\right) \delta 212.8,144.4,143.9,131.6(\mathrm{t}, J=254.6 \mathrm{~Hz}), 127.1$, $123.4,114.5,109.8,108.3,99.0,87.2,31.6,30.8,27.2,22.2,13.9$.

${ }^{19} \mathrm{~F} \mathrm{NMR}\left(376 \mathrm{MHz}, \mathrm{CDCl}_{3}\right) \delta-50.1$ (s).

IR (neat): 2929, 2860, 2220, 1947, 1499, 1452, 1235, 1145, 1033, 868, 756, $703 \mathrm{~cm}^{-1}$. HRMS: $m / z$ (EI) calculated for $\mathrm{C}_{16} \mathrm{H}_{15} \mathrm{~F}_{2} \mathrm{NO}_{2}{ }^{+}[\mathrm{M}]^{+}: 291.1065$, found: 291.1064 .

HPLC (IG, $0.46^{*} 25 \mathrm{~cm}, 5 \mu \mathrm{m}$, hexane/isopropanol = 98/2, flow $0.7 \mathrm{~mL} / \mathrm{min}$, detection at $214 \mathrm{~nm}$ ) retention time $=8.78 \mathrm{~min}$ (minor) and $9.22 \mathrm{~min}$ (major).

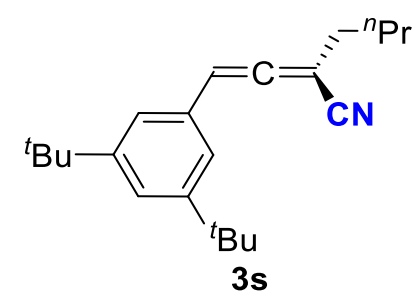

The reaction was conducted according to the general procedure on a $0.2 \mathrm{mmol}$ scale. The residue was purified by column chromatography on silica gel with petroleum ether/ethyl acetate (20:1) to afford the product 3s (48.9 mg, 79\% yield, 91\% ee) as colorless oil.

$[\alpha]_{\mathrm{D}}^{29.1}-99.30\left(c 1.50, \mathrm{CHCl}_{3}\right)$

${ }^{1} \mathrm{H}$ NMR (400 MHz, $\left.\mathrm{CDCl}_{3}\right) \delta 7.39(\mathrm{~s}, 1 \mathrm{H}), 7.13(\mathrm{~d}, J=1.6 \mathrm{~Hz}, 2 \mathrm{H}), 6.61(\mathrm{t}, J=2.4$ 
$\mathrm{Hz}, 1 \mathrm{H}), 2.38-2.32(\mathrm{~m}, 2 \mathrm{H}), 1.64-1.60(\mathrm{~m}, 2 \mathrm{H}), 1.49-1.42(\mathrm{~m}, 2 \mathrm{H}), 1.34(\mathrm{~s}, 18 \mathrm{H})$, $0.94(\mathrm{t}, J=7.2 \mathrm{~Hz}, 3 \mathrm{H})$.

${ }^{13} \mathrm{C} \mathrm{NMR}\left(100 \mathrm{MHz}, \mathrm{CDCl}_{3}\right) \delta 213.3,151.6,129.7,123.1,122.0,115.1,100.2,85.7$, $34.8,31.5,31.3,29.7,21.8,13.7$.

IR (neat): 2959, 2865, 2219, 1943, 1595, 1462, 1363, 1248, 879, 755, $706 \mathrm{~cm}^{-1}$.

HRMS: $m / z$ (EI) calculated for $\mathrm{C}_{22} \mathrm{H}_{31} \mathrm{~N}^{+}[\mathrm{M}]^{+}: 309.2451$, found: 309.2442 .

HPLC (IG, $0.46 * 25 \mathrm{~cm}, 5 \mu \mathrm{m}$, hexane/isopropanol =99/1, flow $0.7 \mathrm{~mL} / \mathrm{min}$, detection at $214 \mathrm{~nm}$ ) retention time $=5.49 \min$ (major) and $5.95 \min$ (minor).

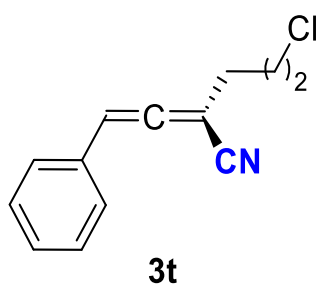

The reaction was conducted according to the general procedure on a $0.2 \mathrm{mmol}$ scale. The residue was purified by column chromatography on silica gel with petroleum ether/ethyl acetate (20:1) to afford the product $\mathbf{3 t}(32.3 \mathrm{mg}, 74 \%$ yield, $91 \%$ ee) as colorless oil.

$[\alpha]_{\mathrm{D}}^{28.7}-67.69\left(c 1.02, \mathrm{CHCl}_{3}\right)$

${ }^{1} \mathrm{H}$ NMR (400 MHz, $\left.\mathrm{CDCl}_{3}\right) \delta 7.40-7.32(\mathrm{~m}, 3 \mathrm{H}), 7.30-7.26(\mathrm{~m}, 2 \mathrm{H}), 6.66(\mathrm{t}, J=$ $2.9 \mathrm{~Hz}, 1 \mathrm{H}), 3.61(\mathrm{t}, J=6.4 \mathrm{~Hz}, 2 \mathrm{H}), 2.55-2.51(\mathrm{~m}, 2 \mathrm{H}), 2.09-2.06(\mathrm{~m}, 2 \mathrm{H})$.

${ }^{13} \mathrm{C}$ NMR $\left(100 \mathrm{MHz}, \mathrm{CDCl}_{3}\right) \delta 213.4,130.2,129.1,128.9,127.8,114.5,100.3,84.9$, $43.3,30.1,28.8$.

IR (neat): 2925, 2218, 1943, 1443, 1217, 829, 749, 691, $498 \mathrm{~cm}^{-1}$.

HRMS: $m / z$ (FI) calculated for $\mathrm{C}_{13} \mathrm{H}_{12} \mathrm{ClN}^{+}[\mathrm{M}]^{+}: 217.0653$, found: 217.0649.

HPLC (IG, $0.46^{*} 25 \mathrm{~cm}, 5 \mu \mathrm{m}$, hexane/isopropanol = 98/2, flow $0.7 \mathrm{~mL} / \mathrm{min}$, detection at $214 \mathrm{~nm}$ ) retention time $=16.49 \mathrm{~min}$ (minor) and $20.73 \mathrm{~min}$ (major).

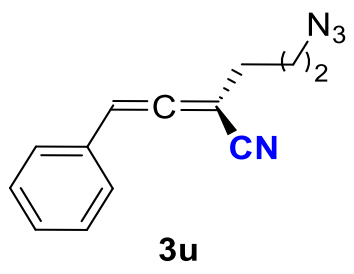


The reaction was conducted according to the general procedure on a $0.2 \mathrm{mmol}$ scale. The residue was purified by column chromatography on silica gel with petroleum ether/ethyl acetate (10:1) to afford the product $3 \mathbf{u}(28.2 \mathrm{mg}, 63 \%$ yield, $93 \%$ ee) as colorless oil.

$[\alpha]_{\mathrm{D}}^{28.9}:+13.83\left(c 1.06, \mathrm{CHCl}_{3}\right)$

${ }^{1} \mathrm{H}$ NMR (400 MHz, $\left.\mathrm{CDCl}_{3}\right) \delta 7.39-7.32(\mathrm{~m}, 3 \mathrm{H}), 7.29-7.26(\mathrm{~m}, 2 \mathrm{H}), 6.66(\mathrm{t}, J=$ $2.4 \mathrm{~Hz}, 1 \mathrm{H}), 3.39$ (t, $J=6.4 \mathrm{~Hz}, 2 \mathrm{H}), 2.46-2.42(\mathrm{~m}, 2 \mathrm{H}), 1.91-1.84(\mathrm{~m}, 2 \mathrm{H})$.

${ }^{13} \mathrm{C}$ NMR (100 MHz, $\left.\mathrm{CDCl}_{3}\right) \delta 213.3,130.2$ 129.1, 129.0, 127.8, 114.5, 100.4, 85.1, $50.1,28.7,26.8$.

IR (neat): 2928, 2097, 1497, 1450, 1355, 1217, 748, 693, $666 \mathrm{~cm}^{-1}$.

HRMS: $m / z$ (EI) calculated for $\mathrm{C}_{13} \mathrm{H}_{12} \mathrm{~N}_{4}{ }^{+}[\mathrm{M}]^{+}:$224.1056, found: 224.1052 .

HPLC (OJ-H, 0.46*25 cm, $5 \mu \mathrm{m}$, hexane/isopropanol $=95 / 5$, flow $0.7 \mathrm{~mL} / \mathrm{min}$, detection at $214 \mathrm{~nm}$ ) retention time $=28.89 \mathrm{~min}$ (minor) and $30.18 \mathrm{~min}$ (major).

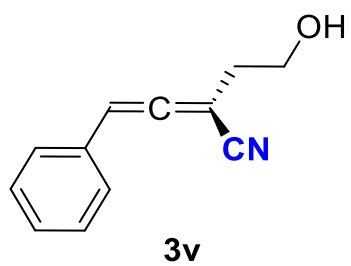

The reaction was conducted according to the general procedure on a $5 \mathrm{mmol}$ scale. The resulting suspension was added $1 \mathrm{M} \mathrm{HCl}(100 \mathrm{~mL})$ and stirred overnight, then the reaction mixture was extracted with ethyl acetate, the organic layer was dried over anhydrous $\mathrm{MgSO}_{4}$, filtered and evaporated. The residue was purified by column chromatography on silica gel with petroleum ether/ethyl acetate $(2: 1)$ to afford the product 3v (677.7 mg, 73\% yield, 92\% ee) as colorless oil.

$[\alpha]_{\mathrm{D}}^{29.4}-151.61\left(c 1.01, \mathrm{CHCl}_{3}\right)$

${ }^{1} \mathrm{H}$ NMR (400 MHz, $\left.\mathrm{CDCl}_{3}\right) \delta 7.38-7.35(\mathrm{~m}, 2 \mathrm{H}), 7.33-7.27(\mathrm{~m}, 3 \mathrm{H}), 6.65(\mathrm{t}, J=$ $2.4 \mathrm{~Hz}, 1 \mathrm{H}), 3.90-3.88(\mathrm{~m}, 2 \mathrm{H}), 2.59-2.56(\mathrm{~m}, 2 \mathrm{H}), 1.70(\mathrm{br}, 1 \mathrm{H})$.

${ }^{13} \mathrm{C} \mathrm{NMR}\left(100 \mathrm{MHz}, \mathrm{CDCl}_{3}\right) \delta 214.2,130.3,129.0,128.9,127.8,114.6,99.9,83.2$, 59.9, 34.7.

IR (neat): 3416, 2927, 2218, 1942, 1705, 1494, 1456, 1050, 828, 749, 691, 584, 494 
$\mathrm{cm}^{-1}$.

HRMS: $m / z$ (EI) calculated for $\mathrm{C}_{12} \mathrm{H}_{11} \mathrm{NO}^{+}[\mathrm{M}]^{+}$: 185.0835 , found: 185.0833 .

HPLC (AD-H, 0.46*25 cm, $5 \mu \mathrm{m}$, hexane/isopropanol $=95 / 5$, flow $0.7 \mathrm{~mL} / \mathrm{min}$, detection at $214 \mathrm{~nm}$ ) retention time $=24.12 \mathrm{~min}$ (major) and $29.72 \mathrm{~min}$ (minor).

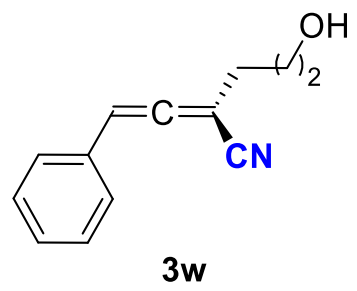

The reaction was conducted according to the general procedure on a $5 \mathrm{mmol}$ scale. The resulting suspension was added $1 \mathrm{M} \mathrm{HCl}(100 \mathrm{~mL})$ and stirred overnight, then the reaction mixture was extracted with ethyl acetate, the organic layer was dried over anhydrous $\mathrm{MgSO}_{4}$, filtered and evaporated. The residue was purified by column chromatography on silica gel with petroleum ether/ethyl acetate $(2: 1)$ to afford the product 3w (759.4 mg, 76\% yield, 92\% ee) as colorless oil.

$[\alpha]_{\mathrm{D}}^{29.4}:-114.40\left(c 1.02, \mathrm{CHCl}_{3}\right)$

${ }^{1} \mathrm{H}$ NMR $\left(400 \mathrm{MHz}, \mathrm{CDCl}_{3}\right) \delta 7.39-7.37(\mathrm{~m}, 2 \mathrm{H}), 7.35-7.31(\mathrm{~m}, 3 \mathrm{H}), 6.62(\mathrm{t}, J=$ $2.9 \mathrm{~Hz}, 1 \mathrm{H}), 3.72(\mathrm{t}, J=6.4 \mathrm{~Hz}, 2 \mathrm{H}), 2.48-2.42(\mathrm{~m}, 2 \mathrm{H}), 1.87-1.83(\mathrm{~m}, 2 \mathrm{H}), 1.58$ (br, 1H).

${ }^{13} \mathrm{C} \mathrm{NMR}\left(100 \mathrm{MHz}, \mathrm{CDCl}_{3}\right) \delta 213.2,130.5,129.0,128.8,127.7,114.8,100.1,85.7$, $61.2,30.4,28.0$.

IR (neat): 3382, 2930, 2218, 1942, 1494, 1455, 1055, 829, 749, 691, $492 \mathrm{~cm}^{-1}$.

HRMS: $m / z$ (EI) calculated for $\mathrm{C}_{13} \mathrm{H}_{13} \mathrm{NO}^{+}[\mathrm{M}]^{+}:$199.0992, found: 199.0992.

HPLC (OD-H, 0.46*25 cm, $5 \mu \mathrm{m}$, hexane/isopropanol = 95/5, flow $0.7 \mathrm{~mL} / \mathrm{min}$, detection at $214 \mathrm{~nm}$ ) retention time $=19.54 \mathrm{~min}$ (major) and $24.90 \mathrm{~min}$ (minor).

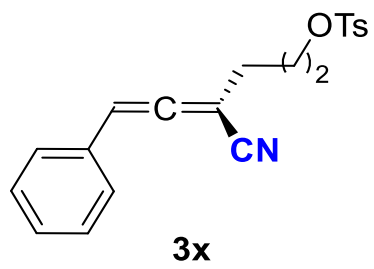

The reaction was conducted according to the general procedure on a $0.2 \mathrm{mmol}$ scale. 
The residue was purified by column chromatography on silica gel with petroleum ether/ethyl acetate (5:1) to afford the product $\mathbf{3 x}(47.5 \mathrm{mg}, 67 \%$ yield, $88 \%$ ee) as colorless oil.

$[\alpha]_{\mathrm{D}}^{28.8}-34.80\left(c 1.15, \mathrm{CHCl}_{3}\right)$

${ }^{1} \mathrm{H}$ NMR (400 MHz, $\left.\mathrm{CDCl}_{3}\right) \delta 7.77(\mathrm{~d}, J=8.0 \mathrm{~Hz}, 2 \mathrm{H}), 7.39-7.33(\mathrm{~m}, 5 \mathrm{H}), 7.25(\mathrm{~d}$, $J=8.0 \mathrm{~Hz}, 2 \mathrm{H}), 6.62(\mathrm{t}, J=2.8 \mathrm{~Hz}, 1 \mathrm{H}), 4.10(\mathrm{t}, J=5.6 \mathrm{~Hz}, 2 \mathrm{H}), 2.45(\mathrm{~s}, 3 \mathrm{H}), 2.42-$ $2.39(\mathrm{~m}, 2 \mathrm{H}), 1.95-1.93(\mathrm{~m}, 2 \mathrm{H})$.

${ }^{13} \mathrm{C}$ NMR $\left(100 \mathrm{MHz}, \mathrm{CDCl}_{3}\right) \delta$ 213.2, 145.0, 132.7, 130.1, 129.9, 129.1, 129.0, 127.8, $127.7,114.4,100.6,84.8,68.5,27.6,26.8,21.6$

IR (neat): 3028, 2925, 2219, 1942, 1598, 1356, 1174, 1096, 972, 924, 814, 662, 552 $\mathrm{cm}^{-1}$.

HRMS: $m / z$ (ESI) calculated for $\mathrm{C}_{20} \mathrm{H}_{19} \mathrm{NO}_{3} \mathrm{SNa}^{+}[\mathrm{M}+\mathrm{Na}]^{+}: 376.0978$, found: 379.0983 .

HPLC (IG, 0.46*25 $\mathrm{cm}, 5 \mu \mathrm{m}$, hexane/isopropanol $=80 / 20$, flow $0.7 \mathrm{~mL} / \mathrm{min}$, detection at $214 \mathrm{~nm}$ ) retention time $=34.18 \mathrm{~min}$ (minor) and $37.60 \mathrm{~min}$ (major).

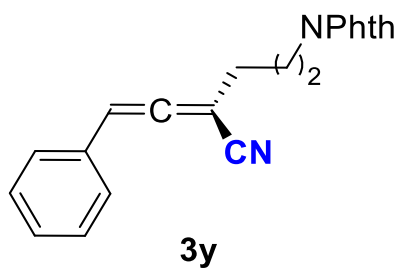

The reaction was conducted according to the general procedure on a $0.2 \mathrm{mmol}$ scale at $0{ }^{\circ} \mathrm{C}$ for 72 hours. The residue was purified by column chromatography on silica gel with petroleum ether/ethyl acetate (5:1) to afford the product $\mathbf{3 y}(51.1 \mathrm{mg}, 78 \%$ yield, $88 \%$ ee) as light yellow solid.

$[\alpha]_{\mathrm{D}}^{25.6}-13.35\left(c 1.43, \mathrm{CHCl}_{3}\right)$

${ }^{1} \mathrm{H}$ NMR (400 MHz, $\left.\mathrm{CDCl}_{3}\right) \delta 7.85-7.83(\mathrm{~m}, 2 \mathrm{H}), 7.73-7.71(\mathrm{~m}, 2 \mathrm{H}), 7.38-7.34$ (m, 2H), $7.32-7.28(\mathrm{~m}, 3 \mathrm{H}), 6.66(\mathrm{t}, J=2.8 \mathrm{~Hz}, 1 \mathrm{H}), 3.76(\mathrm{t}, J=7.2 \mathrm{~Hz}, 2 \mathrm{H}), 2.42-$ $2.38(\mathrm{~m}, 2 \mathrm{H}), 1.99-1.95(\mathrm{~m}, 2 \mathrm{H})$.

${ }^{13} \mathrm{C} \mathrm{NMR}\left(100 \mathrm{MHz}, \mathrm{CDCl}_{3}\right) \delta 213.2,168.2,134.0,131.9,130.3,129.0,128.9,127.8$, $123.3,114.6,100.7,85.3,36.8,28.9,26.6$. 
IR (neat): 3021, 2929, 2216, 1943, 1771, 1706, 1395, 1360, 1018, 748, 717, 693, 529 $\mathrm{cm}^{-1}$.

HRMS: $m / z$ (ESI) calculated for $\mathrm{C}_{21} \mathrm{H}_{16} \mathrm{~N}_{2} \mathrm{O}_{2} \mathrm{Na}^{+}[\mathrm{M}+\mathrm{Na}]^{+}$: 351.1104, found: 351.1107 .

HPLC $\left(\mathrm{IG}, 0.46^{*} 25 \mathrm{~cm}, 5 \mu \mathrm{m}\right.$, hexane/isopropanol $=80 / 20$, flow $0.7 \mathrm{~mL} / \mathrm{min}$, detection at $214 \mathrm{~nm}$ ) retention time $=27.80 \mathrm{~min}$ (major) and $32.08 \mathrm{~min}$ (minor).

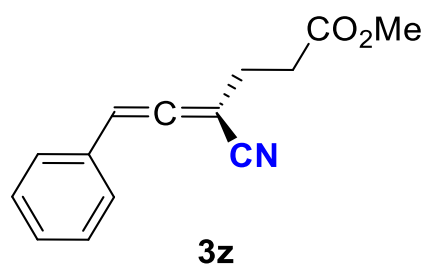

The reaction was conducted according to the general procedure on a $0.2 \mathrm{mmol}$ scale. The residue was purified by column chromatography on silica gel with petroleum ether/ethyl acetate (10:1) to afford the product $\mathbf{3 z}(28.8 \mathrm{mg}, 63 \%$ yield, $83 \%$ ee) as colorless oil.

$[\alpha]_{\mathrm{D}}^{28.5}-36.75\left(c 0.48, \mathrm{CHCl}_{3}\right)$

${ }^{1} \mathrm{H}$ NMR (400 MHz, $\left.\mathrm{CDCl}_{3}\right) \delta 7.38-7.31(\mathrm{~m}, 3 \mathrm{H}), 7.28-7.26(\mathrm{~m}, 2 \mathrm{H}), 6.67(\mathrm{t}, J=$ $2.8 \mathrm{~Hz}, 1 \mathrm{H}), 3.58(\mathrm{~s}, 3 \mathrm{H}), 2.75-2.64(\mathrm{~m}, 2 \mathrm{H}), 2.60-2.57(\mathrm{~m}, 2 \mathrm{H})$.

${ }^{13} \mathrm{C}$ NMR $\left(100 \mathrm{MHz}, \mathrm{CDCl}_{3}\right) \delta 213.0,171.8,130.1,129.0,128.9,127.9,114.4,101.2$, $85.1,51.9,31.5,26.6$

HRMS: $m / z$ (EI) calculated for $\mathrm{C}_{14} \mathrm{H}_{13} \mathrm{NO}_{2}{ }^{+}[\mathrm{M}]^{+}: 227.0941$, found: 227.0942 .

IR (neat): 2925, 2217, 1944, 1735, 1437, 1366, 1170, 1042, 832, 751, 692, $588 \mathrm{~cm}^{-1}$. HPLC (IG, 0.46*25 cm, $5 \mu \mathrm{m}$, hexane/isopropanol = 95/5, flow $0.7 \mathrm{~mL} / \mathrm{min}$, detection at $214 \mathrm{~nm}$ ) retention time $=21.30 \mathrm{~min}$ (minor) and $23.65 \mathrm{~min}$ (major).

HPLC (IG, $0.46^{*} 25 \mathrm{~cm}, 5 \mu \mathrm{m}$, hexane/isopropanol =98/2, flow $0.7 \mathrm{~mL} / \mathrm{min}$, detection at $214 \mathrm{~nm}$ ) retention time $=21.40 \mathrm{~min}$ (minor) and $23.25 \mathrm{~min}$ (major).

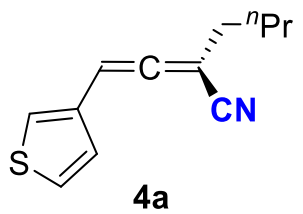

The reaction was conducted according to the general procedure on a $0.2 \mathrm{mmol}$ scale. 
The residue was purified by column chromatography on silica gel with petroleum ether/ethyl acetate (20:1) to afford the product $4 \mathbf{a}(24.8 \mathrm{mg}, 61 \%$ yield, $93 \%$ ee) as orange oil.

$[\alpha]_{\mathrm{D}}^{29.0}-108.09\left(c 1.10, \mathrm{CHCl}_{3}\right)$

${ }^{1} \mathrm{H}$ NMR (400 MHz, $\left.\mathrm{CDCl}_{3}\right) \delta 7.34-7.33(\mathrm{~m}, 1 \mathrm{H}), 7.24-7.23(\mathrm{~m}, 1 \mathrm{H}), 7.03$ (d, $J=$ $4.8 \mathrm{~Hz}, 1 \mathrm{H}), 6.66(\mathrm{t}, J=2.8 \mathrm{~Hz}, 1 \mathrm{H}), 2.33-2.29(\mathrm{~m}, 2 \mathrm{H}), 1.61-1.54(\mathrm{~m}, 2 \mathrm{H}), 1.45-$ $1.38(\mathrm{~m}, 2 \mathrm{H}), 0.93(\mathrm{t}, J=7.2 \mathrm{~Hz}, 3 \mathrm{H})$.

${ }^{13} \mathrm{C}$ NMR $\left(100 \mathrm{MHz}, \mathrm{CDCl}_{3}\right) \delta 213.7,131.4,126.9,126.1,124.0,114.9,94.0,85.3$, 31.4, 29.6, 21.8, 13.6.

IR (neat): 2958, 2928, 2860, 2218, 1944, 1462, 1379, 1238, 1146, 1081, 910, 864, 831, $772,731,620 \mathrm{~cm}^{-1}$.

HRMS: $m / z$ (EI) calculated for $\mathrm{C}_{12} \mathrm{H}_{12} \mathrm{NS}^{+}[\mathrm{M}-\mathrm{H}]^{+}:$202.0685, found: 202.0682 .

HPLC (IG, 0.46*25 cm, $5 \mu \mathrm{m}$, hexane/isopropanol = 98/2, flow $0.7 \mathrm{~mL} / \mathrm{min}$, detection at $214 \mathrm{~nm}$ ) retention time $=19.15 \mathrm{~min}$ (minor) and $20.98 \mathrm{~min}$ (major).

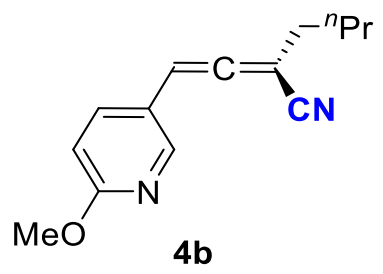

The reaction was conducted according to the general procedure on a $0.2 \mathrm{mmol}$ scale. The residue was purified by column chromatography on silica gel with petroleum ether/ethyl acetate (10:1) to afford the product $\mathbf{4 b}(30.5 \mathrm{mg}, 67 \%$ yield, $86 \%$ ee) as colorless oil.

$[\alpha]_{\mathrm{D}}^{29.2}-83.03\left(c 1.47, \mathrm{CHCl}_{3}\right)$

${ }^{1} \mathrm{H}$ NMR (400 MHz, $\left.\mathrm{CDCl}_{3}\right) \delta 8.05(\mathrm{~s}, 1 \mathrm{H}), 7.49-7.47(\mathrm{~m}, 1 \mathrm{H}), 6.75(\mathrm{~d}, J=8.8 \mathrm{~Hz}$, $1 \mathrm{H}), 6.56(\mathrm{t}, J=3.0 \mathrm{~Hz}, 1 \mathrm{H}), 3.94(\mathrm{~s}, 3 \mathrm{H}), 2.32(\mathrm{t}, J=6.8 \mathrm{~Hz}, 2 \mathrm{H}), 1.58-1.53(\mathrm{~m}$, $2 \mathrm{H}), 1.42-1.37(\mathrm{~m}, 2 \mathrm{H}), 0.92(\mathrm{t}, J=6.8 \mathrm{~Hz}, 3 \mathrm{H})$.

${ }^{13} \mathrm{C} \mathrm{NMR}\left(100 \mathrm{MHz}, \mathrm{CDCl}_{3}\right) \delta 212.6,164.3,146.0,137.0,119.8,114.8,111.6,96.3$, 86.7, 53.7, 31.4, 29.6, 21.8, 13.6.

IR (neat): 2929, 2860, 2218, 1943, 1602, 1493, 1355, 1308, 1127, 1022, 838, 753, 628 
$\mathrm{cm}^{-1}$.

HRMS: $m / z$ (EI) calculated for $\mathrm{C}_{14} \mathrm{H}_{15} \mathrm{~N}_{2} \mathrm{O}^{+}[\mathrm{M}-\mathrm{H}]^{+}: 227.1179$, found: 227.1175 .

HPLC (OD-H, 0.46*25 cm, $5 \mu \mathrm{m}$, hexane/isopropanol $=98 / 2$, flow $0.7 \mathrm{~mL} / \mathrm{min}$, detection at $214 \mathrm{~nm}$ ) retention time $=11.32 \mathrm{~min}$ (minor) and $13.18 \mathrm{~min}$ (major).

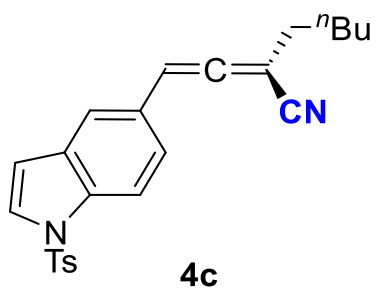

The reaction was conducted according to the general procedure on a $0.2 \mathrm{mmol}$ scale. The residue was purified by column chromatography on silica gel with petroleum ether/ethyl acetate (5:1) to afford the product $4 \mathrm{c}(39.1 \mathrm{mg}, 48 \%$ yield, $93 \%$ ee) as colorless oil.

$[\alpha]_{\mathrm{D}}^{29.4}+21.74\left(c\right.$ 1.07, $\left.\mathrm{CHCl}_{3}\right)$

${ }^{1} \mathrm{H}$ NMR (400 MHz, $\mathrm{CDCl}_{3}$ ) $\delta 7.87(\mathrm{~s}, 1 \mathrm{H}), 7.75$ (d, $\left.J=8.4 \mathrm{~Hz}, 2 \mathrm{H}\right), 7.58$ (d, $J=3.6$ $\mathrm{Hz}, 1 \mathrm{H}), 7.49$ (d, $J=8.4 \mathrm{~Hz}, 1 \mathrm{H}), 7.25$ (d, $J=8.0 \mathrm{~Hz}, 2 \mathrm{H}), 7.16$ (d, $J=8.8 \mathrm{~Hz}, 1 \mathrm{H})$, $6.71(\mathrm{t}, J=2.8 \mathrm{~Hz}, 1 \mathrm{H}), 6.64(\mathrm{~d}, J=3.2 \mathrm{~Hz}, 1 \mathrm{H}), 2.38-2.34(\mathrm{~m}, 5 \mathrm{H}), 1.66-1.62(\mathrm{~m}$, 2H), $1.41-1.34(\mathrm{~m}, 4 \mathrm{H}), 0.89(\mathrm{t}, J=7.2 \mathrm{~Hz}, 3 \mathrm{H})$.

${ }^{13} \mathrm{C}$ NMR $\left(100 \mathrm{MHz}, \mathrm{CDCl}_{3}\right) \delta 213.5,145.2,135.1,135.0,131.1,130.0,127.3,127.2$, $126.8,122.8,121.8,114.9,112.8,108.9,100.2,86.5,31.7,30.9,27.4,22.2,21.6$, 13.9.

IR (neat): 2927, 2858, 2217, 1941, 1735, 1372, 1307, 1269, 1171, 1119, 703, 673, 577, $539 \mathrm{~cm}^{-1}$.

HRMS: $m / z$ (ESI) calculated for $\mathrm{C}_{24} \mathrm{H}_{24} \mathrm{~N}_{2} \mathrm{O}_{2} \mathrm{SNa}^{+}[\mathrm{M}+\mathrm{Na}]^{+}:$427.1451, found: 427.1455 .

HPLC (IG, $0.46^{*} 25 \mathrm{~cm}, 5 \mu \mathrm{m}$, hexane/isopropanol =98/2, flow $0.7 \mathrm{~mL} / \mathrm{min}$, detection at $214 \mathrm{~nm}$ ) retention time $=28.70 \mathrm{~min}$ (major) and $33.95 \mathrm{~min}$ (minor).

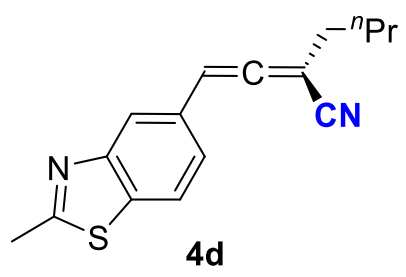


The reaction was conducted according to the general procedure on a $0.2 \mathrm{mmol}$ scale. The residue was purified by column chromatography on silica gel with petroleum ether/ethyl acetate (5:1) to afford the product $\mathbf{4 d}(23.2 \mathrm{mg}, 43 \%$ yield, $90 \%$ ee) as light yellow oil.

$[\alpha]_{\mathrm{D}}^{29.0}-101.51\left(c\right.$ 1.01, $\left.\mathrm{CHCl}_{3}\right)$

${ }^{1} \mathrm{H}$ NMR (400 MHz, $\left.\mathrm{CDCl}_{3}\right) \delta 7.83(\mathrm{~s}, 1 \mathrm{H}), 7.79(\mathrm{~d}, J=8.0 \mathrm{~Hz}, 1 \mathrm{H}), 7.27$ (d, $J=8.8$ $\mathrm{Hz}, 1 \mathrm{H}), 6.72(\mathrm{t}, J=2.8 \mathrm{~Hz}, 1 \mathrm{H}), 2.84(\mathrm{~s}, 3 \mathrm{H}), 2.37-2.33(\mathrm{~m}, 2 \mathrm{H}), 1.63-1.58(\mathrm{~m}$, 2H), $1.44-1.39(\mathrm{~m}, 2 \mathrm{H}), 0.92(\mathrm{t}, J=7.6 \mathrm{~Hz}, 3 \mathrm{H})$.

${ }^{13} \mathrm{C} \mathrm{NMR}\left(100 \mathrm{MHz}, \mathrm{CDCl}_{3}\right) \delta 213.3,168.3,153.9,135.9,128.9,124.0,121.9,121.4$, $114.8,99.6,86.6,31.4,29.7,21.9,20.2,13.7$.

IR (neat): 2959, 2926, 2857, 2218, 1944, 1523, 1376, 1257, 1064, 1006, 884, 789, 756, $634 \mathrm{~cm}^{-1}$.

HRMS: $m / z$ (EI) calculated for $\mathrm{C}_{16} \mathrm{H}_{15} \mathrm{~N}_{2} \mathrm{~S}^{+}[\mathrm{M}-\mathrm{H}]^{+}: 267.0950$, found: 267.0951 .

HPLC (IG, 0.46*25 cm, $5 \mu \mathrm{m}$, hexane/isopropanol =95/5, flow $0.7 \mathrm{~mL} / \mathrm{min}$, detection at $214 \mathrm{~nm}$ ) retention time $=28.37$ min (major) and $32.37 \mathrm{~min}$ (minor).

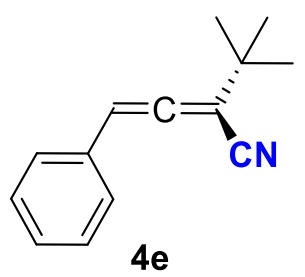

The reaction was conducted according to the general procedure on a $0.2 \mathrm{mmol}$ scale. The residue was purified by column chromatography on silica gel with petroleum ether/ethyl acetate (20:1) to afford the product 4 e (5.3 $\mathrm{mg}, 13 \%$ yield, $70 \%$ ee) as light yellow oil.

$[\alpha]_{\mathrm{D}}^{26.4}:-41.16\left(c 0.4, \mathrm{CHCl}_{3}\right)$

${ }^{1} \mathrm{H}$ NMR (400 MHz, $\left.\mathrm{CDCl}_{3}\right) \delta 7.36-7.35(\mathrm{~m}, 2 \mathrm{H}), 7.32-7.26(\mathrm{~m}, 3 \mathrm{H}), 6.64(\mathrm{~s}, 1 \mathrm{H})$, $1.26(\mathrm{~s}, 9 \mathrm{H})$.

${ }^{13} \mathrm{C}$ NMR $\left(100 \mathrm{MHz}, \mathrm{CDCl}_{3}\right) \delta 211.0,131.0,129.0,128.6,127.5,114.0,100.8,97.3$, 35.2, 28.9 .

IR (neat): 2962, 2927, 2211, 1940, 1732, 1459, 1367, 1246, 1062, 1022, 977, 828, 749, 
$692 \mathrm{~cm}^{-1}$.

HRMS: $m / z$ (EI) calculated for $\mathrm{C}_{14} \mathrm{H}_{15} \mathrm{~N}^{+}[\mathrm{M}]^{+}$: 197.1199, found: 197.1198.

HPLC (IG, 0.46*25 cm, $5 \mu \mathrm{m}$, hexane/isopropanol = 99/1, flow $0.7 \mathrm{~mL} / \mathrm{min}$, detection at $214 \mathrm{~nm}$ ) retention time $=9.40 \mathrm{~min}$ (minor) and 10.47 min (major).

\section{Single Crystal X-Ray Diffraction Data $\left(7, \mathrm{~L} \mathrm{CuCl}_{2}\right.$ and $\left.\mathrm{L}_{4} \mathrm{CuCl}_{2}\right)$}

\subsection{X-ray of 7}
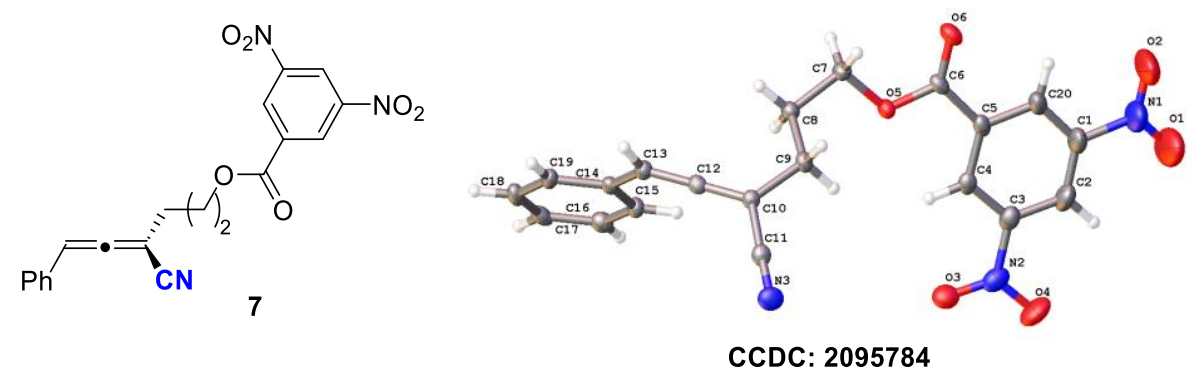

Table S8. Crystal data and structure refinement for mj21271_0m.

Identification code

Empirical formula

Formula weight

Temperature

Wavelength

Crystal system

Space group

Unit cell dimensions

Volume

Z

Density (calculated)

Absorption coefficient

$\mathrm{F}(000)$

Crystal size mj21271_0m

C20 H15 N3 O6

393.35

$173.01 \mathrm{~K}$

$1.34139 \AA$

Monoclinic

C 121

$a=108.194(4) \AA$

$\alpha=90^{\circ}$.

$\mathrm{b}=7.5307(3) \AA$

$\beta=90.272(2)^{\circ}$.

$\mathrm{c}=7.6351$

(3) $\AA$

$\gamma=90^{\circ}$.
12

$1.260 \mathrm{Mg} / \mathrm{m}^{3}$

$0.513 \mathrm{~mm}^{-1}$

2448

$0.05 \times 0.05 \times 0.01 \mathrm{~mm}^{3}$ 
Theta range for data collection

Index ranges

Reflections collected

Independent reflections

Completeness to theta $=53.594^{\circ}$

Absorption correction

Max. and min. transmission

Refinement method

Data / restraints / parameters

Goodness-of-fit on $\mathrm{F}^{2}$

Final $\mathrm{R}$ indices $[\mathrm{I}>2 \operatorname{sigma}(\mathrm{I})]$

$\mathrm{R}$ indices (all data)

Absolute structure parameter

Extinction coefficient

Largest diff. peak and hole
3.554 to $54.969^{\circ}$.

$-131<=\mathrm{h}<=132,-9<=\mathrm{k}<=9,-9<=\mathrm{l}<=9$

30647

$11565[\mathrm{R}(\mathrm{int})=0.0820]$

$98.8 \%$

Semi-empirical from equivalents

0.7508 and 0.5357

Full-matrix least-squares on $\mathrm{F}^{2}$

$11565 / 79 / 784$

1.002

$\mathrm{R} 1=0.0616, w \mathrm{R} 2=0.1402$

$\mathrm{R} 1=0.0724, \mathrm{wR} 2=0.1455$

$0.09(13)$

$\mathrm{n} / \mathrm{a}$

0.446 and -0.330 e. $\AA^{-3}$

\subsection{X-ray of $\mathrm{L}^{1 C u C l}{ }_{2}$}
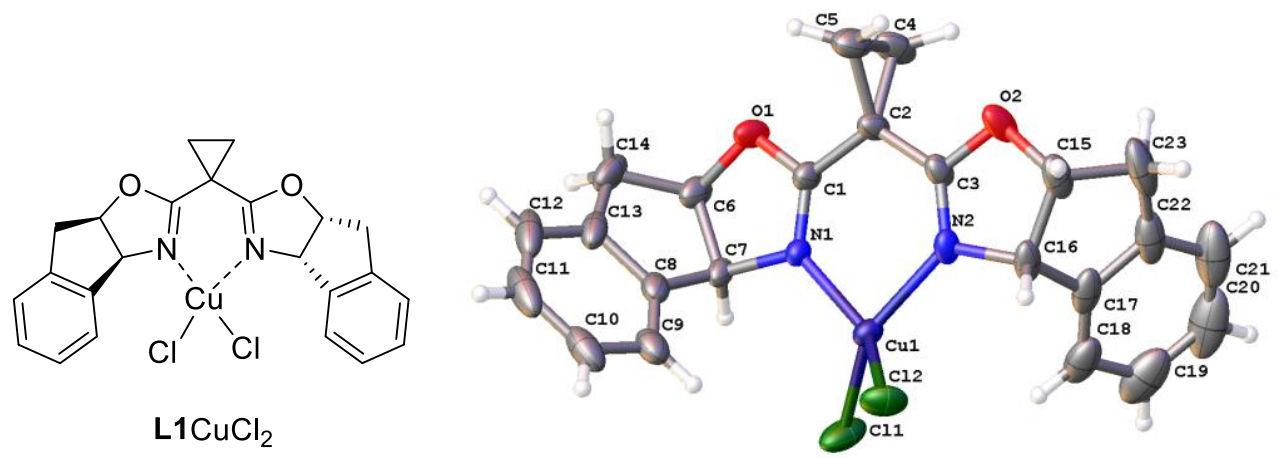

CCDC: 2104644

Table S9. Crystal data and structure refinement for mo_dm21093_0m_4.

Identification code

Empirical formula

Formula weight

Temperature

Wavelength

Crystal system mo_dm21093_0m_4

$\mathrm{C} 23 \mathrm{H} 20 \mathrm{Cl} 2 \mathrm{Cu}$ N2 O2

490.85

$296.15 \mathrm{~K}$

$0.71073 \AA$

Orthorhombic 
Space group

Unit cell dimensions

Volume

Z

Density (calculated)

Absorption coefficient

$\mathrm{F}(000)$

Crystal size

Theta range for data collection

Index ranges

Reflections collected

Independent reflections

Completeness to theta $=25.242^{\circ}$

Absorption correction

Refinement method

Data / restraints / parameters

Goodness-of-fit on $\mathrm{F}^{2}$

Final $\mathrm{R}$ indices [I $>2 \operatorname{sigma}(\mathrm{I})]$

$\mathrm{R}$ indices (all data)

Absolute structure parameter

Extinction coefficient

Largest diff. peak and hole
$\mathrm{P} 212121$

$$
\begin{array}{ll}
\mathrm{a}=9.4902(7) \AA & \alpha=90^{\circ} . \\
\mathrm{b}=10.2776(7) \AA & \beta=90^{\circ} . \\
\mathrm{c}=22.6113(17) \AA & \gamma=90^{\circ} .
\end{array}
$$$$
\text { 2205.4(3) } \AA^{3}
$$

4

\section{$1.478 \mathrm{Mg} / \mathrm{m}^{3}$}

$1.255 \mathrm{~mm}^{-1}$

1004

$0.18 \times 0.16 \times 0.15 \mathrm{~mm}^{3}$

1.801 to $27.512^{\circ}$.

$-12<=\mathrm{h}<=12,0<=\mathrm{k}<=13,0<=\mathrm{l}<=29$

5342

$5077[\mathrm{R}($ int $)=0.0156]$

$100.0 \%$

None

Full-matrix least-squares on $\mathrm{F}^{2}$

5077 / 0 / 272

1.054

$\mathrm{R} 1=0.0325, \mathrm{wR} 2=0.0750$

$\mathrm{R} 1=0.0444, \mathrm{wR} 2=0.0787$

$0.297(15)$

$\mathrm{n} / \mathrm{a}$

0.244 and -0.419 e. $\AA^{-3}$

\subsection{X-ray of $\mathrm{L}^{4} \mathrm{CuCl}_{2}$}
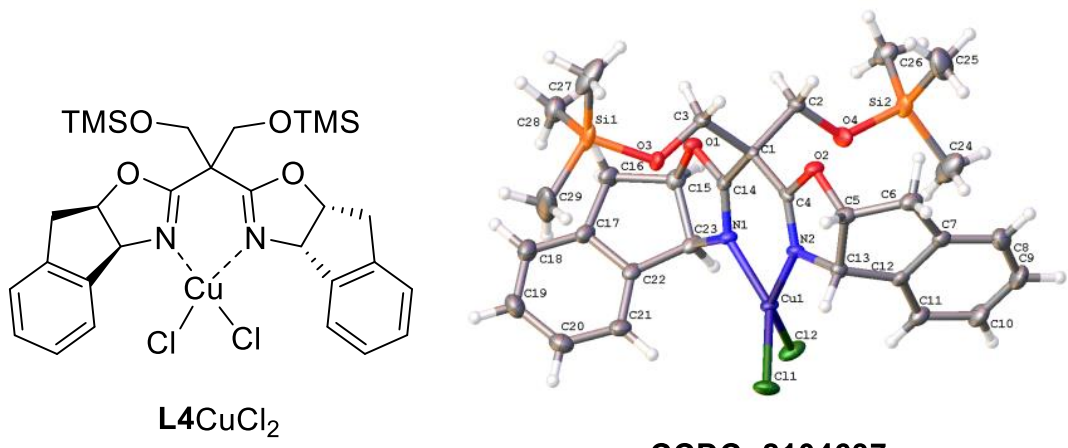

CCDC: 2104637

Table S10. Crystal data and structure refinement for mj21340_0m.

Identification code

Empirical formula mj21340_0m

$\mathrm{C} 31 \mathrm{H} 41 \mathrm{Cl} 2 \mathrm{Cu}$ N3 O4 Si2 
Formula weight

Temperature

Wavelength

Crystal system

Space group

Unit cell dimensions

Volume

Z

Density (calculated)

Absorption coefficient

$\mathrm{F}(000)$

Crystal size

Theta range for data collection

Index ranges

Reflections collected

Independent reflections

Completeness to theta $=53.594^{\circ}$

Absorption correction

Max. and min. transmission

Refinement method

Data / restraints / parameters

Goodness-of-fit on $\mathrm{F}^{2}$

Final R indices [I>2sigma(I)]

$\mathrm{R}$ indices (all data)

Absolute structure parameter

Extinction coefficient

Largest diff. peak and hole
710.29

$193.0 \mathrm{~K}$

$1.34139 \AA$

Monoclinic

P 1211

$\mathrm{a}=9.2845(2) \AA$

$\alpha=90^{\circ}$.

$\mathrm{b}=15.0354(4) \AA$

$\beta=97.4090(10)^{\circ}$.

$\mathrm{c}=13.0562(3) \AA$

$\gamma=90^{\circ}$.

1807.38(7) $\AA^{3}$

2

$1.305 \mathrm{Mg} / \mathrm{m}^{3}$

$4.754 \mathrm{~mm}^{-1}$

742

$0.18 \times 0.16 \times 0.15 \mathrm{~mm}^{3}$

3.920 to $54.877^{\circ}$.

$-10<=\mathrm{h}<=11,-17<=\mathrm{k}<=18,-15<=\mathrm{l}<=15$

17719

$6268[\mathrm{R}(\mathrm{int})=0.0855]$

$99.6 \%$

Semi-empirical from equivalents

0.7508 and 0.4352

Full-matrix least-squares on $\mathrm{F}^{2}$

$6268 / 37 / 396$

1.030

$\mathrm{R} 1=0.0659, \mathrm{wR} 2=0.1691$

$\mathrm{R} 1=0.0695, \mathrm{wR} 2=0.1717$

$0.063(7)$

$\mathrm{n} / \mathrm{a}$

0.701 and -0.699 e. $\AA^{-3}$

\section{DFT Calculations}

\subsection{DFT calculations for ring-opening of the cyclopropyl group in radical}

intermediated for $\mathbf{3 g}$

These calculations were performed using the Gaussian 16 package $^{10}$. All of the structures are optimized using the B3LYP functional ${ }^{11}$ with the basis set def2-TZVP ${ }^{12}$. 
Grimme's D3 correction with Becke-Johnson damping was used ${ }^{13,14}$. Vibrational analyses were performed to ensure intermediates to have no imaginary frequencies and the transition state structures to have only one imaginary frequency. Free energies were estimated at $298 \mathrm{~K}$. Transition state structures were confirmed to connect appropriate reactants or products by intrinsic reaction coordinate (IRC) calculations ${ }^{17}$. Rate constants were calculated by Erying equation under TST theory. We assumed that tunneling effect should play little role in these reactions due to the high atom weight of $\mathrm{C}$ atom.

we calculated the energetics and the rate constant for ring-opening of the cyclopropyl group in radical intermediated for $\mathbf{3 g}$ in Table $\mathbf{2}$. In order to facilitate understanding, we also calculated other molecular structures which were frequently used in three-membered ring opening reactions (see Figure S5). The results indicate that the energy barrier for the ring opening of the cyclopropyl group in the allenyl radical intermediate is as high as $17.4 \mathrm{kcal} / \mathrm{mol}$, thus the rate is very slow. Compared with alkyl radical and benzyl radical intermediates, the ring opening of the cyclopropyl group in the allenyl radical intermediate is almost impossible. This is also consistent with the results in our reaction.

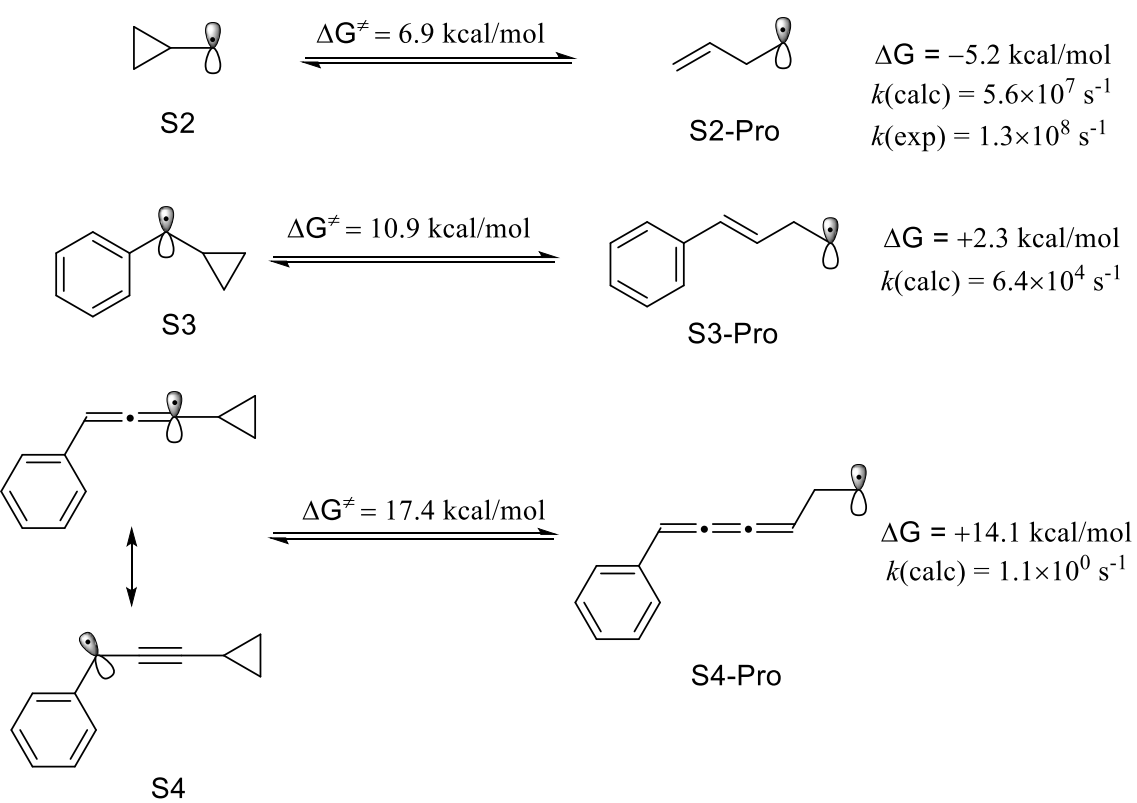

Figure S5: energetics and the rate constant for ring-opening of the cyclopropyl group. 
Table S11. Computational Details: ring-opening of the cyclopropyl group

\begin{tabular}{|c|c|c|c|c|c|c|c|}
\hline $\mathrm{S} 2$ & & & & $\mathrm{C}$ & 0.96085600 & -0.63564000 & -0.19168300 \\
\hline 02 & & & & $\mathrm{H}$ & 1.18866700 & -1.68680800 & -0.04050800 \\
\hline $\mathrm{C}$ & -0.90772100 & 0.74465400 & -0.13462000 & $\mathrm{C}$ & 2.01784700 & 0.21457400 & -0.44390800 \\
\hline $\mathrm{C}$ & -0.90764700 & -0.74463000 & -0.13459000 & $\mathrm{C}$ & 3.43575300 & 0.34530600 & 0.92090100 \\
\hline $\mathrm{C}$ & 0.27438700 & -0.00008900 & 0.48586300 & $\mathrm{C}$ & 3.44641200 & -0.21044800 & -0.44930200 \\
\hline $\mathrm{H}$ & -0.72009200 & 1.25802600 & -1.06792900 & $\mathrm{H}$ & 1.83040300 & 1.24663100 & -0.70630000 \\
\hline $\mathrm{H}$ & -1.57460000 & 1.26388400 & 0.54075700 & $\mathrm{H}$ & 3.17951600 & -0.28182600 & 1.75944100 \\
\hline $\mathrm{H}$ & -0.72013400 & -1.25795700 & -1.06796400 & $\mathrm{H}$ & 3.66992700 & 1.38277100 & 1.10293000 \\
\hline $\mathrm{H}$ & -1.57456500 & -1.26381700 & 0.54078100 & $\mathrm{H}$ & 4.07993400 & 0.30706100 & -1.16515000 \\
\hline $\mathrm{H}$ & 0.29425500 & -0.00005800 & 1.56845000 & $\mathrm{H}$ & 3.58810700 & -1.28690500 & -0.51152300 \\
\hline $\mathrm{C}$ & 1.56917000 & 0.00005500 & -0.16364800 & S3-Pro & & & \\
\hline $\mathrm{H}$ & 1.64298800 & -0.00035800 & -1.24313600 & 02 & & & \\
\hline \multirow[t]{2}{*}{$\mathrm{H}$} & 2.48301200 & 0.00033900 & 0.41100900 & $\mathrm{C}$ & -2.84195900 & -0.98279300 & 0.16156100 \\
\hline & & & & $\mathrm{C}$ & -1.48551100 & -1.26602600 & 0.07322000 \\
\hline S2-TS & & & & $\mathrm{C}$ & -0.53708700 & -0.24642500 & -0.06377900 \\
\hline 02 & & & & $\mathrm{C}$ & -0.99888800 & 1.07597700 & -0.10552500 \\
\hline $\mathrm{C}$ & -0.80424100 & 0.72800700 & -0.14895300 & $\mathrm{C}$ & -2.35151800 & 1.36058400 & -0.01736500 \\
\hline $\mathrm{C}$ & -1.17072300 & -0.70284500 & -0.11015300 & $\mathrm{C}$ & -3.28217000 & 0.33323200 & 0.11614300 \\
\hline $\mathrm{C}$ & 0.41556100 & 0.14942100 & 0.48381500 & $\mathrm{H}$ & -3.55442100 & -1.79107900 & 0.26639400 \\
\hline $\mathrm{H}$ & -0.64224800 & 1.14900900 & -1.13752100 & $\mathrm{H}$ & -1.14845700 & -2.29512400 & 0.10931200 \\
\hline $\mathrm{H}$ & -1.38988900 & 1.39422200 & 0.47807400 & $\mathrm{H}$ & -0.29375500 & 1.89032500 & -0.20446400 \\
\hline $\mathrm{H}$ & -0.96898000 & -1.33728800 & -0.95783500 & $\mathrm{H}$ & -2.68501000 & 2.39006500 & -0.05115500 \\
\hline $\mathrm{H}$ & -1.72747400 & -1.11195200 & 0.71871600 & $\mathrm{H}$ & -4.33814300 & 0.55962800 & 0.18530100 \\
\hline $\mathrm{H}$ & 0.38638100 & 0.03809100 & 1.55989400 & $\mathrm{C}$ & 0.88047200 & -0.60653800 & -0.15488200 \\
\hline $\mathrm{C}$ & 1.59550500 & -0.11316900 & -0.18187100 & $\mathrm{H}$ & 1.08533500 & -1.67056400 & -0.06476400 \\
\hline $\mathrm{H}$ & 1.69280500 & 0.06905900 & -1.24461000 & $\mathrm{C}$ & 1.91650700 & 0.21299100 & -0.33801700 \\
\hline $\mathrm{H}$ & 2.43279000 & -0.56963000 & 0.32625600 & $\mathrm{C}$ & 4.18665600 & 0.33861700 & 0.68641800 \\
\hline S2-Pro & & & & $\mathrm{C}$ & 3.34798500 & -0.23306200 & -0.40419200 \\
\hline 02 & & & & $\mathrm{H}$ & 1.75760700 & 1.28274500 & -0.43033100 \\
\hline $\mathrm{C}$ & 0.61779100 & 0.55119700 & -0.12653800 & $\mathrm{H}$ & 3.77949900 & 0.45345100 & 1.68169700 \\
\hline $\mathrm{C}$ & 1.74568700 & -0.35759800 & 0.21937700 & $\mathrm{H}$ & 5.23590100 & 0.54955500 & 0.53320500 \\
\hline $\mathrm{C}$ & -0.66110700 & -0.18008500 & -0.42199400 & $\mathrm{H}$ & 3.77753900 & 0.04514800 & -1.37382200 \\
\hline $\mathrm{H}$ & 0.43443200 & 1.26395700 & 0.69300600 & $\mathrm{H}$ & 3.37697900 & -1.33349500 & -0.37286400 \\
\hline $\mathrm{H}$ & 0.89802400 & 1.16413000 & -0.99130800 & $\mathrm{~S} 4$ & & & \\
\hline $\mathrm{H}$ & 1.56392000 & -1.26245900 & 0.78296000 & 02 & & & \\
\hline $\mathrm{H}$ & 2.76982700 & -0.07603400 & 0.01756800 & $\mathrm{C}$ & -2.80887200 & 1.53447600 & -0.00011300 \\
\hline $\mathrm{H}$ & -0.62409700 & -0.87170300 & -1.25931400 & $\mathrm{C}$ & -1.57428500 & 0.91381000 & -0.00006200 \\
\hline $\mathrm{C}$ & -1.78682000 & -0.05162600 & 0.26763900 & $\mathrm{C}$ & -1.47421300 & -0.49430200 & 0.00002800 \\
\hline $\mathrm{H}$ & -1.85851500 & 0.62302600 & 1.11371000 & $\mathrm{C}$ & -2.67212500 & -1.24052000 & 0.00006700 \\
\hline $\mathrm{H}$ & -2.67689800 & -0.61224500 & 0.01247000 & $\mathrm{C}$ & -3.90323000 & -0.61151500 & 0.00001700 \\
\hline $\mathrm{S} 3$ & & & & $\mathrm{C}$ & -3.98210300 & 0.78008700 & -0.00007400 \\
\hline 02 & & & & $\mathrm{H}$ & -2.86449600 & 2.61582300 & -0.00018300 \\
\hline $\mathrm{C}$ & -2.72013800 & -1.01731700 & 0.00039800 & $\mathrm{H}$ & -0.66605900 & 1.50188000 & -0.00009200 \\
\hline $\mathrm{C}$ & -1.36865900 & -1.29010400 & 0.00020200 & $\mathrm{H}$ & -2.61567100 & -2.32236900 & 0.00013800 \\
\hline $\mathrm{C}$ & -0.40023600 & -0.24966800 & -0.00023800 & $\mathrm{H}$ & -4.80924700 & -1.20417200 & 0.00004800 \\
\hline $\mathrm{C}$ & -0.89142900 & 1.08303700 & -0.00042200 & $\mathrm{H}$ & -4.94611400 & 1.27143100 & -0.00011400 \\
\hline $\mathrm{C}$ & -2.24750900 & 1.34377900 & -0.00022200 & $\mathrm{C}$ & -0.21463500 & -1.16755200 & 0.00008300 \\
\hline $\mathrm{C}$ & -3.17622700 & 0.30268300 & 0.00017700 & $\mathrm{H}$ & -0.22450500 & -2.25204600 & 0.00016000 \\
\hline $\mathrm{H}$ & -3.43164500 & -1.83360600 & 0.00072500 & $\mathrm{C}$ & 1.01394900 & -0.53852500 & 0.00006100 \\
\hline $\mathrm{H}$ & -1.02432300 & -2.31731700 & 0.00036400 & $\mathrm{C}$ & 2.09950800 & 0.01475000 & 0.00000600 \\
\hline $\mathrm{H}$ & -0.19589200 & 1.91063600 & -0.00065200 & $\mathrm{C}$ & 3.37790400 & 0.64613200 & -0.00012700 \\
\hline $\mathrm{H}$ & -2.59203700 & 2.37048000 & -0.00035900 & $\mathrm{C}$ & 4.54137300 & 0.01017100 & 0.74613400 \\
\hline $\mathrm{H}$ & -4.23684000 & 0.51556500 & 0.0003310 & $\mathrm{C}$ & 4.54140800 & 0.00979900 & -0.74601100 \\
\hline $\mathrm{C}$ & 0.97041600 & -0.57400500 & -0.00046200 & $\mathrm{H}$ & 3.35497800 & 1.72932200 & -0.00039500 \\
\hline $\mathrm{H}$ & 1.22800500 & -1.62861600 & 0.00001400 & $\mathrm{H}$ & 4.33822300 & -0.92001200 & 1.25689400 \\
\hline $\mathrm{C}$ & 2.08589900 & 0.36182900 & -0.00105500 & $\mathrm{H}$ & 5.21323600 & 0.68027400 & 1.26464500 \\
\hline $\mathrm{C}$ & 3.36503200 & 0.00816000 & 0.74617100 & $\mathrm{H}$ & 5.21330100 & 0.67964100 & -1.26482200 \\
\hline $\mathrm{C}$ & 3.36626200 & 0.00572400 & -0.74481500 & $\mathrm{H}$ & 4.33828500 & -0.92063900 & -1.25631700 \\
\hline $\mathrm{H}$ & 1.83495400 & 1.41317400 & -0.00294600 & S4-TS & & & \\
\hline $\mathrm{H}$ & 3.38294400 & -0.94322400 & 1.26038500 & 02 & & & \\
\hline $\mathrm{H}$ & 3.87355100 & 0.80986300 & 1.26438200 & $\mathrm{C}$ & -2.83456700 & 1.53236600 & -0.00069300 \\
\hline $\mathrm{H}$ & 3.87572300 & 0.80569200 & -1.26477700 & $\mathrm{C}$ & -1.59743300 & 0.91722700 & -0.06967000 \\
\hline $\mathrm{H}$ & 3.38509000 & -0.94734900 & -1.25587000 & $\mathrm{C}$ & -1.48955200 & -0.48303900 & -0.02857800 \\
\hline S3-TS & & & & $\mathrm{C}$ & -2.66895200 & -1.23586200 & 0.08379700 \\
\hline 02 & & & & $\mathrm{C}$ & -3.90653300 & -0.61578100 & 0.15276300 \\
\hline $\mathrm{C}$ & -2.74631100 & -0.98383400 & 0.14661800 & $\mathrm{C}$ & -3.99703000 & 0.77147700 & 0.11086000 \\
\hline $\mathrm{C}$ & -1.39772400 & -1.28350500 & 0.05520700 & $\mathrm{H}$ & -2.89847600 & 2.61267800 & -0.03342400 \\
\hline $\mathrm{C}$ & -0.42886500 & -0.27189900 & -0.08938600 & $\mathrm{H}$ & -0.69654200 & 1.51072400 & -0.15544100 \\
\hline $\mathrm{C}$ & -0.88483200 & 1.06073100 & -0.12651900 & $\mathrm{H}$ & -2.60288400 & -2.31672100 & 0.11633700 \\
\hline $\mathrm{C}$ & -2.23344300 & 1.35629900 & -0.03502100 & $\mathrm{H}$ & -4.80386700 & -1.21513200 & 0.23944900 \\
\hline $\mathrm{C}$ & -3.17608000 & 0.33910100 & 0.09996800 & $\mathrm{H}$ & -4.96270800 & 1.25681200 & 0.16458200 \\
\hline $\mathrm{H}$ & -3.46746100 & -1.78407500 & 0.25571700 & $\mathrm{C}$ & -0.21131900 & -1.16072400 & -0.09952300 \\
\hline $\mathrm{H}$ & -1.07238400 & -2.31641800 & 0.09050000 & $\mathrm{H}$ & -0.23215500 & -2.24556400 & -0.05490800 \\
\hline $\mathrm{H}$ & -0.17266900 & 1.87004900 & -0.21491400 & $\mathrm{C}$ & 0.98087200 & -0.55873400 & -0.21771600 \\
\hline $\mathrm{H}$ & -2.55636600 & 2.38951100 & -0.06371200 & $\mathrm{C}$ & 2.08252100 & 0.00870600 & -0.33289500 \\
\hline $\mathrm{H}$ & -4.22935200 & 0.57590600 & 0.17226500 & $\mathrm{C}$ & 3.28737600 & 0.60075200 & -0.44647900 \\
\hline
\end{tabular}




$\begin{array}{cccccccr}\mathrm{C} & 4.70573600 & 0.18244100 & 1.04186600 & \mathrm{H} & -0.76968300 & 1.52873500 & -0.11163800 \\ \mathrm{C} & 4.59297400 & -0.13774000 & -0.39813900 & \mathrm{H} & -2.63675200 & -2.31897400 & 0.08658300 \\ \mathrm{H} & 3.34183000 & 1.67821500 & -0.54540300 & \mathrm{H} & -4.85158900 & -1.24247800 & 0.21767700 \\ \mathrm{H} & 4.20763000 & -0.43730300 & 1.77102300 & \mathrm{H} & -5.03428900 & 1.22782600 & 0.18436500 \\ \mathrm{H} & 5.13180200 & 1.11993900 & 1.36689400 & \mathrm{C} & -0.25361800 & -1.14061100 & -0.09777300 \\ \mathrm{H} & 5.36347500 & 0.30690200 & -1.02472200 & \mathrm{H} & -0.27462400 & -2.22699700 & -0.07419000 \\ \mathrm{H} & 4.48734600 & -1.19708900 & -0.61795600 & \mathrm{C} & 0.92259800 & -0.54054900 & -0.19208700 \\ \mathrm{~S} 4-\mathrm{Pro} & & & & \mathrm{C} & 2.03313500 & 0.04410400 & -0.28163200 \\ 0 \mathrm{C} & & & & \mathrm{C} & 3.21020000 & 0.62887100 & -0.38143000 \\ \mathrm{C} & -2.90913700 & 1.52794700 & 0.03392500 & \mathrm{C} & 5.24397200 & 0.11324200 & 0.88662200 \\ \mathrm{C} & -1.66538900 & 0.92553700 & -0.03965400 & \mathrm{C} & 4.53074200 & -0.11638000 & -0.40161800 \\ \mathrm{C} & -1.54666300 & -0.47131200 & -0.02183000 & \mathrm{H} & 3.26651700 & 1.71318900 & -0.39656200 \\ \mathrm{C} & -2.71426800 & -1.23849600 & 0.07193100 & \mathrm{H} & 4.91239700 & -0.39890000 & 1.77939400 \\ \mathrm{C} & -3.96022100 & -0.63257400 & 0.14559900 & \mathrm{H} & 5.94081800 & 0.93228000 & 1.00008200 \\ \mathrm{C} & -4.06353300 & 0.75304000 & 0.12693100 & \mathrm{H} & 5.13134700 & 0.23945700 & -1.24328200 \\ \mathrm{H} & -2.98389800 & 2.60784800 & 0.01913200 & \mathrm{H} & 4.33285500 & -1.17890000 & -0.55546000\end{array}$

\subsection{Bond Dissociation Energy (BDE) Calculation of substrate}

These calculations were performed by the ORCA 5.0.0 program package ${ }^{16}$. All geometry was optimized at B3LYP ${ }^{11} /$ Def2-SVP ${ }^{12}$ level with Grimme's D4 dispersion ${ }^{13}$ correction in vacuum. Frequency calculation was performed to confirm all structures as local minimum (no imaginary frequencies) and to obtain enthalpy correction at $298.15 \mathrm{~K}$. Single point energy was calculated at M06-2X $\mathrm{X}^{17} / \mathrm{def}^{2}-\mathrm{TZVPP}^{12}$ level in vacuum. RIJCOSX approximation was engaged to accelerate all these calculations, utilizing the def $2 / \mathrm{J}$ auxiliary basis $\operatorname{set}^{18}$. Tight SCF convergence and tight geometry optimization criterions were chosen to ensure accuracy. Bond dissociation energy was calculation by formula below, where $\Delta_{f} \mathrm{H}^{0}=\mathrm{E}^{\mathrm{M} 062 \mathrm{X}}+\mathrm{H}_{\text {Corr }}{ }^{\text {B3LYP }}$.

$$
\mathrm{BDE}=\Delta_{f} \mathrm{H}^{0}{ }_{\text {Radical }}+\Delta_{f} \mathrm{H}^{0}{ }_{H}-\Delta_{f} \mathrm{H}^{0} \text { Molecule }
$$

Table S12. The energies of calculated structures

\begin{tabular}{|l|r|r|r|r|r|}
\hline & $\begin{array}{r}\mathrm{E}(\mathrm{B} 3 \mathrm{LYP}- \\
\text { D4/Def2-SVP) }\end{array}$ & $\begin{array}{r}\mathrm{H}(\mathrm{B} 3 \mathrm{LYP}- \\
\text { D4/Def2-SVP) }\end{array}$ & $\begin{array}{r}\mathrm{H} \\
\text { Corr(B3LYP- }\end{array}$ & $\begin{array}{r}\text { E(M06- } \\
\text { 2X/Def2- }\end{array}$ & $\begin{array}{r}\text { H(M06- } \\
\text { 2X/Def2- }\end{array}$ \\
\hline $\mathrm{H}$ & -0.49785992 & -0.49549944 & 0.00236048 & -0.498140796 & -0.495780316 \\
\hline Sub1 $(3 \boldsymbol{c})$ & & & & & \\
\hline 1 & -465.0471394 & -464.81125 & 0.23588935 & -465.6114385 & -465.3755492 \\
\hline $1-1 \mathrm{H}$ & -464.4219301 & -464.1993008 & 0.22262933 & -464.9766601 & -464.7540308 \\
\hline $1-2 \mathrm{H}$ & -464.4048789 & -464.1828573 & 0.22202154 & -464.9609901 & -464.7389686 \\
\hline $1 \mathrm{H}-\mathrm{BDE}$ & & & & & 78.90187521 \\
\hline $2 \mathrm{H}-\mathrm{BDE}$ & & & & & 88.35357643 \\
\hline Sub2 (9) & & & & & \\
\hline 1 & -425.7922122 & -425.5857288 & 0.2064834 & -426.3072396 & -426.1007562 \\
\hline $1-1 \mathrm{H}$ & -425.1553877 & -424.9626547 & 0.19273301 & -425.6611048 & -425.4683718 \\
\hline $1 \mathrm{H}-\mathrm{BDE}$ & & & & & 85.72043755 \\
\hline Sub3 (12) & & & & & \\
\hline 1 & -504.309021 & -504.0436386 & 0.26538238 & -504.9200461 & -504.6546637 \\
\hline $1-1 \mathrm{H}$ & -503.6861369 & -503.4339304 & 0.25220655 & -504.2880223 & -504.0358157 \\
\hline $1 \mathrm{H}-\mathrm{BDE}$ & & & & & 77.22619329 \\
\hline
\end{tabular}

Table S13. Computational Details 
$\mathrm{H}$

$\mathrm{E}(\mathrm{B} 3 \mathrm{LYP})=-0.49785992 \mathrm{H}(\mathrm{B} 3 \mathrm{LYP})=-0.49549944$

$\mathrm{E}(\mathrm{M} 062 \mathrm{X})=-0.498140796088$

xyz 02

$\mathrm{H}$

0.00 .00 .0

Sub1

$E(B 3 L Y P)=-465.04713936$

$\mathrm{H}(\mathrm{B} 3 \mathrm{LYP})=-464.8112500$

$\mathrm{E}(\mathrm{M} 062 \mathrm{X})=-465.611438514298$ xyz 01

$\begin{array}{lrrr}\mathrm{C} & -2.88868 & 1.61713 & -0.05053 \\ \mathrm{C} & -1.71027 & 0.86444 & -0.00211 \\ \mathrm{C} & -1.75987 & -0.53323 & 0.02456 \\ \mathrm{C} & -3.01359 & -1.16408 & 0.00183 \\ \mathrm{C} & -4.19024 & -0.41591 & -0.04655 \\ \mathrm{C} & -4.13162 & 0.98185 & -0.07304 \\ \mathrm{H} & -2.83161 & 2.70885 & -0.07084 \\ \mathrm{H} & -0.73929 & 1.36357 & 0.01550 \\ \mathrm{H} & -3.06688 & -2.25724 & 0.02228 \\ \mathrm{H} & -5.15771 & -0.92490 & -0.06375 \\ \mathrm{H} & -5.05181 & 1.57056 & -0.11113 \\ \mathrm{C} & -0.50529 & -1.39696 & 0.07766 \\ \mathrm{H} & -0.56261 & -2.04680 & 0.97063 \\ \mathrm{H} & -0.51716 & -2.08828 & -0.78536 \\ \mathrm{C} & 0.76655 & -0.67704 & 0.09289 \\ \mathrm{C} & 1.82098 & -0.07783 & 0.10523 \\ \mathrm{C} & 3.11236 & 0.60923 & 0.11560 \\ \mathrm{H} & 3.23131 & 1.15328 & 1.07098 \\ \mathrm{H} & 3.12149 & 1.38399 & -0.67344 \\ \mathrm{C} & 4.31314 & -0.33434 & -0.08526 \\ \mathrm{H} & 4.29680 & -1.10373 & 0.70555 \\ \mathrm{H} & 4.18603 & -0.87365 & -1.03949 \\ \mathrm{C} & 5.64800 & 0.40802 & -0.07179 \\ \mathrm{H} & 5.80608 & 0.93177 & 0.88627 \\ \mathrm{H} & 6.49358 & -0.28269 & -0.21740 \\ \mathrm{H} & 5.69404 & 1.16513 & -0.87290\end{array}$

Sub1-1H

$\mathrm{E}(\mathrm{B} 3 \mathrm{LYP})=-464.42193009$

$\mathrm{H}(\mathrm{B} 3 \mathrm{LYP})=-464.19930076$

$\mathrm{E}(\mathrm{M} 062 \mathrm{X})=-464.976660141831$ xyz 02

$\begin{array}{lrrr}\mathrm{C} & 3.03278 & 1.46418 & -0.78426 \\ \mathrm{C} & 1.80733 & 0.89683 & -0.45532 \\ \mathrm{C} & 1.73824 & -0.39872 & 0.12101 \\ \mathrm{C} & 2.95661 & -1.08969 & 0.34788 \\ \mathrm{C} & 4.17866 & -0.51488 & 0.01609 \\ \mathrm{C} & 4.22667 & 0.76556 & -0.55205 \\ \mathrm{H} & 3.06474 & 2.46319 & -1.22753 \\ \mathrm{H} & 0.87850 & 1.44219 & -0.63691 \\ \mathrm{H} & 2.92393 & -2.08908 & 0.79086 \\ \mathrm{H} & 5.10513 & -1.06540 & 0.20002 \\ \mathrm{H} & 5.18757 & 1.21639 & -0.81224 \\ \mathrm{C} & 0.48948 & -1.01341 & 0.47265 \\ \mathrm{H} & 0.52655 & -2.01688 & 0.91108 \\ \mathrm{C} & -0.76066 & -0.42952 & 0.29630 \\ \mathrm{C} & -1.86264 & 0.08974 & 0.14375 \\ \mathrm{C} & -3.18823 & 0.67405 & -0.02649 \\ \mathrm{H} & -3.26530 & 1.11886 & -1.03699 \\ \mathrm{H} & -3.30863 & 1.52095 & 0.67670 \\ \mathrm{C} & -4.34013 & -0.32795 & 0.18097 \\ \mathrm{H} & -4.21245 & -1.16708 & -0.52382 \\ \mathrm{H} & -4.25628 & -0.76170 & 1.19196 \\ \mathrm{C} & -5.71243 & 0.31582 & -0.00620 \\ \mathrm{H} & -5.82482 & 0.73346 & -1.02106 \\ \mathrm{H} & -6.52215 & -0.41540 & 0.14475 \\ \mathrm{H} & -5.87014 & 1.14061 & 0.70926 \\ & & & \end{array}$

Sub1-2H

$\mathrm{E}(\mathrm{B} 3 \mathrm{LYP})=-464.40487888$

$\mathrm{H}(\mathrm{B} 3 \mathrm{LYP})=-464.18285734$

$\mathrm{E}(\mathrm{M} 062 \mathrm{X})=-464.960990119795$

xyz 02

$\begin{array}{lrrr}\mathrm{C} & -2.63369 & 1.67186 & 0.22749 \\ \mathrm{C} & -1.51422 & 0.83552 & 0.16188 \\ \mathrm{C} & -1.66308 & -0.53177 & -0.09345 \\ \mathrm{C} & -2.95456 & -1.04811 & -0.27941\end{array}$

$\begin{array}{lrrr}\mathrm{C} & -4.07258 & -0.21587 & -0.21446 \\ \mathrm{C} & -3.91534 & 1.15089 & 0.03994 \\ \mathrm{H} & -2.50008 & 2.73834 & 0.42770 \\ \mathrm{H} & -0.51328 & 1.24584 & 0.31173 \\ \mathrm{H} & -3.08461 & -2.11683 & -0.47695 \\ \mathrm{H} & -5.07148 & -0.63528 & -0.36134 \\ \mathrm{H} & -4.78975 & 1.80462 & 0.09272 \\ \mathrm{C} & -0.47209 & -1.47902 & -0.19062 \\ \mathrm{H} & -0.66421 & -2.35298 & 0.46201 \\ \mathrm{H} & -0.43037 & -1.89216 & -1.21645 \\ \mathrm{C} & 0.82667 & -0.90901 & 0.14364 \\ \mathrm{C} & 1.92583 & -0.43562 & 0.44190 \\ \mathrm{C} & 3.16012 & 0.08425 & 0.76078 \\ \mathrm{H} & 3.21938 & 0.82356 & 1.56999 \\ \mathrm{C} & 4.43114 & -0.25930 & 0.03597 \\ \mathrm{H} & 5.17249 & -0.63782 & 0.76631 \\ \mathrm{H} & 4.24071 & -1.08192 & -0.67205 \\ \mathrm{C} & 5.04069 & 0.94198 & -0.70505 \\ \mathrm{H} & 5.24660 & 1.77468 & -0.01213 \\ \mathrm{H} & 5.99085 & 0.66600 & -1.19096 \\ \mathrm{H} & 4.35496 & 1.31708 & -1.48159\end{array}$

Sub2

$\mathrm{E}(\mathrm{B} 3 \mathrm{LYP})=-425.79221221$

$\mathrm{H}(\mathrm{B} 3 \mathrm{LYP})=-425.58572881$

$\mathrm{E}(\mathrm{M} 062 \mathrm{X})=-426.307239587394$ xyz 01

$\begin{array}{lrrr}\mathrm{C} & -3.11415 & 1.38479 & -0.01152 \\ \mathrm{C} & -1.73562 & 1.17507 & -0.01204 \\ \mathrm{C} & -1.21053 & -0.13377 & -0.00185 \\ \mathrm{C} & -2.10686 & -1.22256 & 0.00875 \\ \mathrm{C} & -3.48423 & -1.00499 & 0.00921 \\ \mathrm{C} & -3.99375 & 0.29747 & -0.00090 \\ \mathrm{H} & -3.50601 & 2.40526 & -0.01947 \\ \mathrm{H} & -1.04730 & 2.02282 & -0.02033 \\ \mathrm{H} & -1.70714 & -2.23875 & 0.01663 \\ \mathrm{H} & -4.16627 & -1.85927 & 0.01755 \\ \mathrm{H} & -5.07383 & 0.46470 & -0.00049 \\ \mathrm{C} & 0.20394 & -0.35198 & -0.00222 \\ \mathrm{C} & 1.40452 & -0.54463 & -0.00160 \\ \mathrm{C} & 2.85189 & -0.74188 & -0.00106 \\ \mathrm{H} & 3.13726 & -1.34645 & 0.88035 \\ \mathrm{H} & 3.13783 & -1.34762 & -0.88148 \\ \mathrm{C} & 3.65453 & 0.57248 & -0.00163 \\ \mathrm{H} & 3.36258 & 1.16743 & -0.88385 \\ \mathrm{H} & 3.36180 & 1.16862 & 0.87951 \\ \mathrm{C} & 5.16328 & 0.33606 & -0.00080 \\ \mathrm{H} & 5.71788 & 1.28777 & -0.00165 \\ \mathrm{H} & 5.48045 & -0.23364 & 0.88909 \\ \mathrm{H} & 5.48113 & -0.23563 & -0.88918\end{array}$

Sub2-1H

$\mathrm{E}(\mathrm{B} 3 \mathrm{LYP})=-425.15538766$

$\mathrm{H}(\mathrm{B} 3 \mathrm{LYP})=-424.96265465$

$\mathrm{E}(\mathrm{M} 062 \mathrm{X})=-425.661104783290$ xyz 02

$\begin{array}{lrrr}\mathrm{C} & 3.01853 & 1.38475 & -0.04189 \\ \mathrm{C} & 1.64705 & 1.17857 & 0.06909 \\ \mathrm{C} & 1.11258 & -0.13563 & 0.06037 \\ \mathrm{C} & 2.01017 & -1.22743 & -0.06340 \\ \mathrm{C} & 3.37951 & -1.00719 & -0.17365 \\ \mathrm{C} & 3.89214 & 0.29647 & -0.16386 \\ \mathrm{H} & 3.41414 & 2.40377 & -0.03358 \\ \mathrm{H} & 0.96454 & 2.02561 & 0.16424 \\ \mathrm{H} & 1.60871 & -2.24281 & -0.07083 \\ \mathrm{H} & 4.05749 & -1.85944 & -0.26865 \\ \mathrm{H} & 4.96856 & 0.46374 & -0.25081 \\ \mathrm{C} & -0.27639 & -0.35146 & 0.17093 \\ \mathrm{C} & -1.49893 & -0.54792 & 0.26696 \\ \mathrm{C} & -2.84341 & -0.74455 & 0.37563 \\ \mathrm{H} & -3.22214 & -1.77478 & 0.35088 \\ \mathrm{C} & -3.85386 & 0.36344 & 0.48394 \\ \mathrm{H} & -4.45089 & 0.21631 & 1.40467 \\ \mathrm{H} & -3.33236 & 1.32734 & 0.59819 \\ \mathrm{C} & -4.80763 & 0.41945 & -0.71988 \\ \mathrm{H} & -5.55789 & 1.21681 & -0.59233 \\ \mathrm{H} & -4.25448 & 0.61460 & -1.65247 \\ \mathrm{H} & -5.34790 & -0.53357 & -0.84569\end{array}$




\begin{tabular}{lrrr} 
Sub3 & \multicolumn{5}{l}{} \\
$\mathrm{E}(\mathrm{B} 3 \mathrm{LYP})=$ & -504.30902100 & \\
$\mathrm{H}(\mathrm{B} 3 \mathrm{LYP})=-504.04363862$ & & \\
$\mathrm{E}(\mathrm{M} 062 \mathrm{X})=$ & -504.920046088704 & \\
xyz 0 1 & \multicolumn{3}{l}{} \\
$\mathrm{C}$ & 2.34090 & -1.80746 & 0.45306 \\
$\mathrm{C}$ & 1.37411 & -0.79966 & 0.50347 \\
$\mathrm{C}$ & 1.56168 & 0.40380 & -0.18825 \\
$\mathrm{C}$ & 2.73613 & 0.57508 & -0.93444 \\
$\mathrm{C}$ & 3.70372 & -0.43068 & -0.98824 \\
$\mathrm{C}$ & 3.50911 & -1.62714 & -0.29211 \\
$\mathrm{H}$ & 2.17766 & -2.74196 & 0.99681 \\
$\mathrm{H}$ & 0.45610 & -0.94800 & 1.07685 \\
$\mathrm{H}$ & 2.89318 & 1.50839 & -1.48409 \\
$\mathrm{H}$ & 4.61064 & -0.28129 & -1.58020 \\
$\mathrm{H}$ & 4.26321 & -2.41745 & -0.33454 \\
$\mathrm{C}$ & 0.55753 & 1.55034 & -0.09559 \\
$\mathrm{H}$ & 0.59584 & 2.09492 & -1.05664 \\
$\mathrm{C}$ & -0.82314 & 1.09424 & 0.10219 \\
$\mathrm{C}$ & -1.96395 & 0.73438 & 0.30160 \\
$\mathrm{C}$ & -3.33766 & 0.27165 & 0.49861 \\
$\mathrm{H}$ & -4.02443 & 1.13789 & 0.46531 \\
$\mathrm{H}$ & -3.44031 & -0.15044 & 1.51587 \\
$\mathrm{C}$ & -3.78849 & -0.77653 & -0.53575 \\
$\mathrm{H}$ & -3.68764 & -0.34494 & -1.54629 \\
$\mathrm{H}$ & -3.09399 & -1.63347 & -0.49973 \\
$\mathrm{C}$ & -5.22252 & -1.24786 & -0.30337 \\
$\mathrm{H}$ & -5.93566 & -0.40831 & -0.36340 \\
$\mathrm{H}$ & -5.52553 & -1.99645 & -1.05261 \\
$\mathrm{H}$ & -5.33764 & -1.70765 & 0.69292 \\
$\mathrm{C}$ & 0.97155 & 2.54295 & 1.01672 \\
$\mathrm{H}$ & 0.95765 & 2.04353 & 1.99772 \\
$\mathrm{H}$ & 1.99117 & 2.91655 & 0.83583 \\
$\mathrm{H}$ & 0.28050 & 3.39921 & 1.05284
\end{tabular}

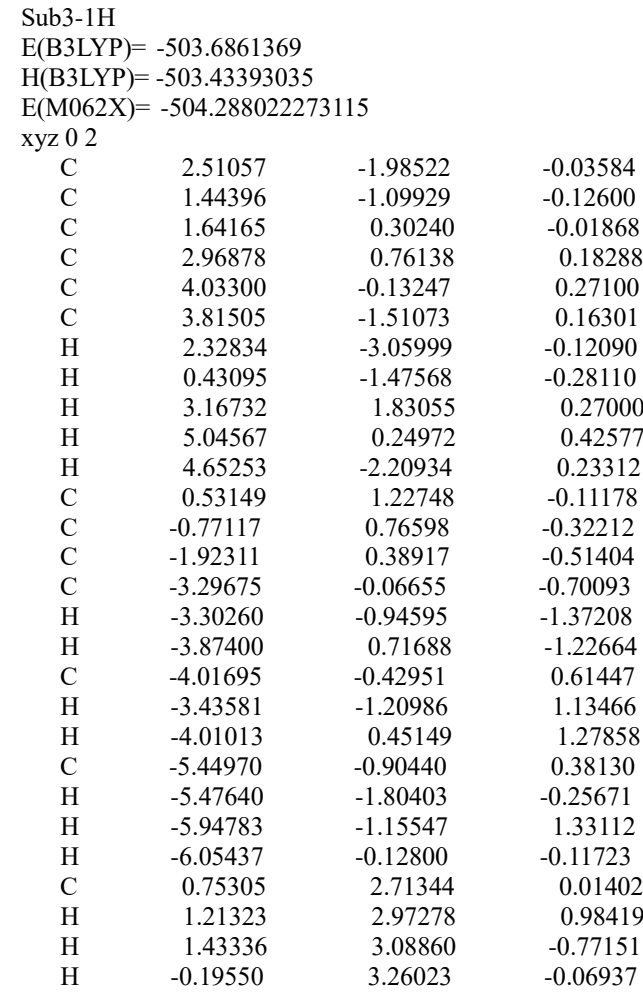

\subsection{DFT calculation on the enantioselective cyanation of allenyl radical.}

DFT calculations were performed using the Gaussian 16 package $^{10}$. All of the structures are optimized using the M06 functional ${ }^{15}$ with the basis set $6-311 \mathrm{G}^{*}$ for $\mathrm{Cu}$ 19 and 6-31G* for other elements ${ }^{20}$. The van der Waals complex of $\mathbf{L} 4 \mathbf{C u}(\mathbf{I I})(\mathbf{C N})_{2}$ with the allenyl radical and the transition states for allenyl radical addition are singlet biradicals which were calculated using unrestricted broken symmetry method. Vibrational analyses were also performed to ensure intermediates to have no imaginary frequencies and the transition state structures to have only one imaginary frequency. Free energies were estimated at $298 \mathrm{~K}$. Transition state structures were confirmed to connect appropriate reactants or products by intrinsic reaction coordinate $\left(\right.$ IRC) calculations ${ }^{20}$. Solvent effect (solvent $=$ perfluorobenzene) was considered by carrying out single-point energy calculations using the SMD model ${ }^{21}$ at the M06-D3/6-311++G** level ${ }^{14}$ with $6-311 \mathrm{G}^{*}$ for $\mathrm{Cu}^{19}$

Figure S6 shows the energy profiles calculated. The results indicate that the reaction follows a stepwise mechanism passing through a $\mathrm{Cu}(\mathrm{III})$ intermediate. The allenyl radical adds to the $\mathrm{Cu}$ (II) center gives a diasteromeric pair of the allenyl- $\mathrm{Cu}(\mathrm{III})$ 
intermediates $\mathbf{B}(\boldsymbol{R})$ and $\mathbf{B}(\boldsymbol{S})$, followed by reductive elimination to produce a pair of the enantiomeric allenyl nitrile products. The allenyl radical addition step was found to be enantio-determining. The energy difference between the enantio-determining transition states $\mathbf{T} \mathbf{S}_{\mathbf{A} \_} \mathbf{B}(\boldsymbol{R})$ and $\mathbf{T} \mathbf{S}_{\mathbf{A}_{-}} \mathbf{B}(\boldsymbol{S})$ was calculated to be $1.8 \mathrm{kcal} / \mathrm{mol}$.

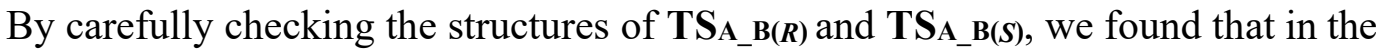

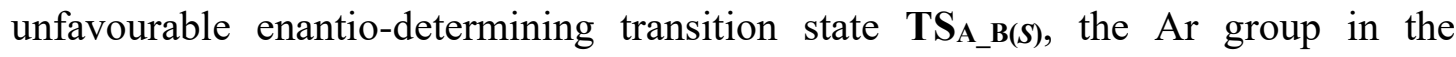
substrate experiences much steric repulsion from the -OTMS of ligand, while the Ar group is pointing away from the ligand in $\mathbf{T S}_{\mathbf{A} \_} \mathbf{B}(\boldsymbol{R})$. This finding is consistent with the experimental observations shown in Table 1 that steric bulkier Box ligands improve the enantioselectivity.

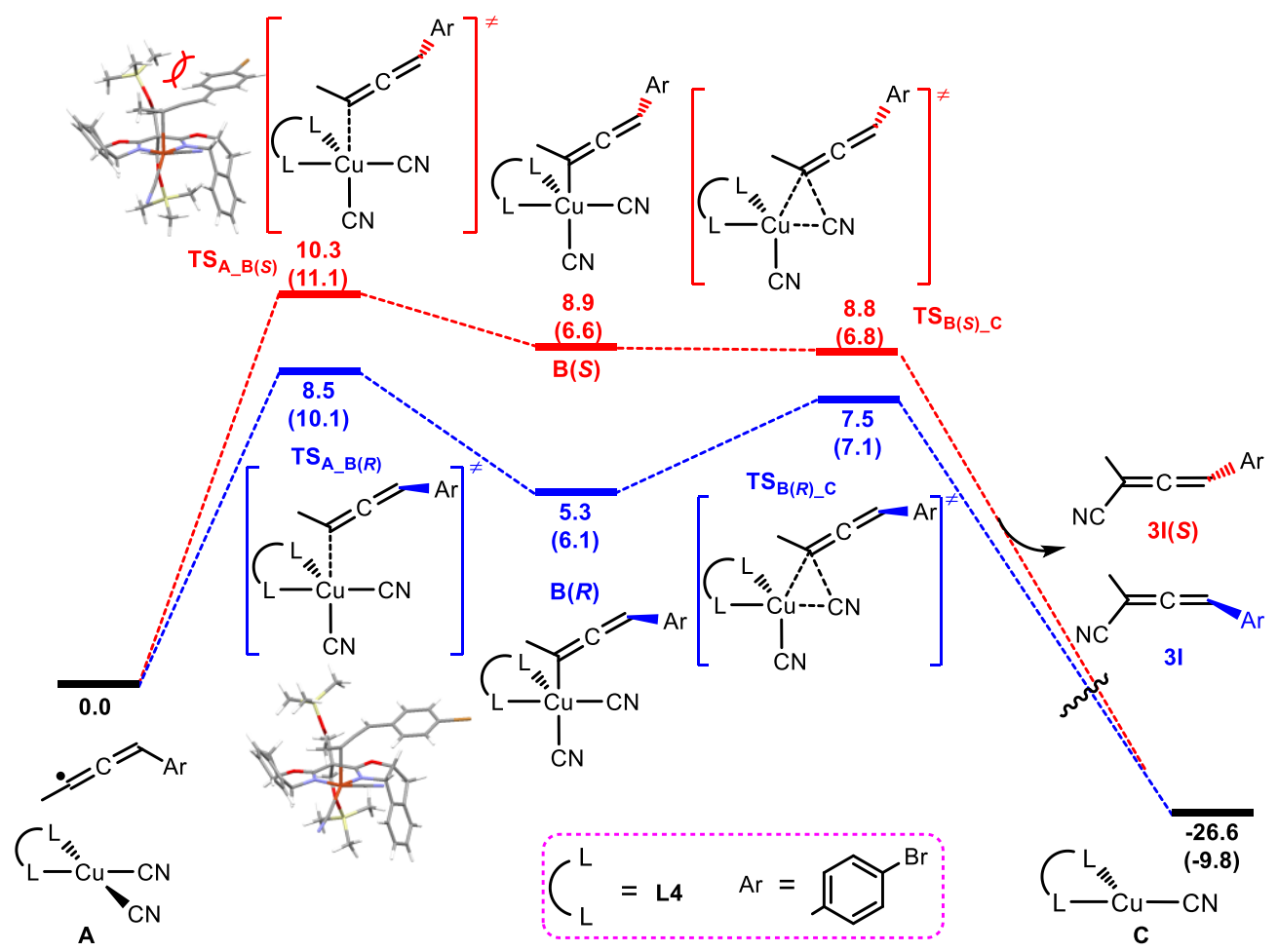

Figure S6. Free energy profiles calculated for the reaction of $\mathrm{L} 4 \mathrm{Cu}(\mathrm{II})(\mathrm{CN})_{2}$ with the allenyl radical $\mathrm{MeC}=\mathrm{C}=\mathrm{CH}\left(p-\mathrm{C}_{6} \mathrm{H}_{4} \mathrm{Br}\right.$ ) (considering both the $\mathrm{Re}$ and $\mathrm{Si}$ face additions). Relative free energies and electronic energies (in parenthesis) are given in $\mathrm{kcal} / \mathrm{mol}$.

We also considered the mechanistic possibility of a direct $\mathrm{C}-\mathrm{C}$ coupling of an allenyl radical with an isocyanoCu(II) complex, proposed by Zhang, Bao and co-coworker. ${ }^{8}$ We have also calculated/optimized the transition state of this type mode 
(TSA'_C) for our system and found that it is lying very significantly higher in energy (by $13.2 \mathrm{kcal} / \mathrm{mol}$ in triplet and $22.6 \mathrm{kcal} / \mathrm{mol}$ in singlet) than the favorable enantio-determining transition state $\mathrm{TS}_{\mathrm{A}_{-} \mathrm{B}(\mathrm{R})}$ reported above, suggesting that the unusual $\mathrm{C}-\mathrm{C}$ coupling mode is unlikely in our case (Figure $\mathbf{S} 7$ ).

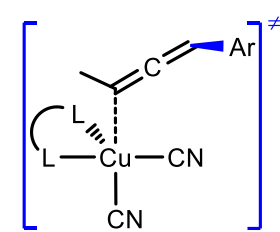

$\frac{(10.5}{\mathrm{TS}_{\mathrm{A}_{-} \mathrm{B}(R)}}$<smiles>CC(C=N[Ge](C)(C)C)=CC[Te]</smiles>

$\frac{21.7}{(21.5)}$

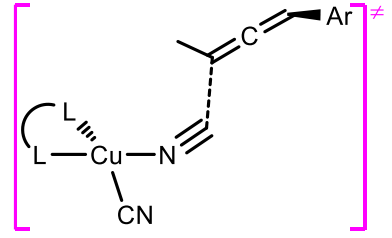

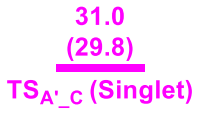

Figure S7. Comparison of the transition states for $\mathrm{C}-\mathrm{C}$ coupling of an allenyl radical with an isocyanoCu(II) complex ( $\left.\mathbf{T S}_{\mathbf{A}_{-}} \mathbf{C}\right)$ and the favourable enantio-determining

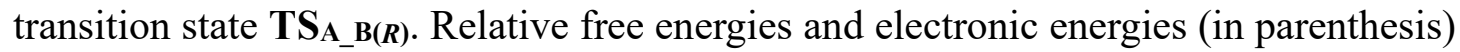
are given in $\mathrm{kcal} / \mathrm{mol}$.

Table S14. The energies of calculated structures

\begin{tabular}{lcccc}
\hline & E(gas) & G(gas) & E(Sol) & G(Sol) \\
\hline $\mathbf{A}$ & -6899.0769 & -6898.3869 & -6902.5724 & -6901.8823 \\
$\mathbf{T S}_{\mathbf{A}_{-} \mathbf{B}(\boldsymbol{R})}$ & -6899.0619 & -6898.3744 & -6902.5563 & -6901.8689 \\
$\mathbf{T S}_{\mathbf{A}_{-} \mathbf{C}}($ triplet) & -68990427 & -6898.3524 & -6902.5380 & -6901.8478 \\
$\mathbf{T S}_{\mathbf{A}_{-} \mathbf{C}}($ (singlet) & -6899.0312 & -6898.3393 & -6902.5248 & -6901.8329 \\
$\mathbf{B}(\boldsymbol{R})$ & -6899.0756 & -6898.3867 & -6902.5627 & -6901.8738 \\
$\mathbf{T S}$ & -6899.0734 & -6898.3828 & -6902.5610 & -6901.8704 \\
$\mathbf{C}$ & -3849.5503 & -3849.0005 & -3850.1318 & -3849.5820 \\
$\mathbf{T} \mathbf{S}_{\mathbf{A}_{-} \mathbf{B}(\boldsymbol{S})}$ & -6899.0569 & -6898.3682 & -6902.5547 & -6901.8660 \\
$\mathbf{B}(\boldsymbol{S})$ & -6899.0733 & -6898.3795 & -6902.5619 & -6901.8681 \\
$\mathbf{T S}$ & -6899.0701 & -6898.3770 & -6902.5615 & -6901.8684 \\
\hline
\end{tabular}

Table S15. Computational Details

$\begin{array}{llll}99 & & & \\ \mathrm{~A} & & & \\ \mathrm{Cu} & -0.99779 & 0.952201 & -1.673522 \\ \mathrm{O} & 0.313414 & 0.08693 & 2.239024 \\ \mathrm{O} & -4.091117 & 1.321486 & 1.134013 \\ \mathrm{~N} & -0.041939 & 0.493523 & 0.068692 \\ \mathrm{~N} & -2.700311 & 1.014651 & -0.589117 \\ \mathrm{C} & 1.334113 & -0.049183 & 0.145277 \\ \mathrm{C} & 2.448055 & 0.962214 & 0.033349 \\ \mathrm{C} & 2.77046 & 1.798684 & -1.031718 \\ \mathrm{C} & 3.884522 & 2.626448 & -0.920636 \\ \mathrm{C} & 4.676191 & 2.605609 & 0.227749 \\ \mathrm{C} & 4.366467 & 1.751795 & 1.281844 \\ \mathrm{C} & 3.247768 & 0.933835 & 1.177926\end{array}$

$\begin{array}{llll}\mathrm{C} & 2.766838 & -0.092957 & 2.164584 \\ \mathrm{C} & 1.441388 & -0.565448 & 1.587548 \\ \mathrm{C} & -0.49233 & 0.528184 & 1.267359 \\ \mathrm{C} & -2.852794 & 1.121801 & 0.676072 \\ \mathrm{C} & -4.974227 & 1.043447 & 0.025672 \\ \mathrm{C} & -5.515132 & -0.393421 & 0.120482 \\ \mathrm{C} & -5.209666 & -0.986964 & -1.225808 \\ \mathrm{C} & -5.634051 & -2.205296 & -1.74325 \\ \mathrm{C} & -5.235145 & -2.56573 & -3.028758 \\ \mathrm{C} & -4.434799 & -1.713408 & -3.789613 \\ \mathrm{C} & -4.010428 & -0.489946 & -3.275513 \\ \mathrm{C} & -4.39731 & -0.143664 & -1.985177\end{array}$




\begin{tabular}{|c|c|c|c|}
\hline $\mathrm{C}$ & -4.04883 & 1.095313 & -1.206382 \\
\hline $\mathrm{H}$ & 1.416027 & -0.84362 & -0.601794 \\
\hline $\mathrm{H}$ & 2.180865 & 1.784576 & -1.946828 \\
\hline $\mathrm{H}$ & 4.147759 & 3.283779 & -1.747526 \\
\hline $\mathrm{H}$ & 5.552481 & 3.24877 & 0.292325 \\
\hline $\mathrm{H}$ & 4.995074 & 1.719319 & 2.171628 \\
\hline $\mathrm{H}$ & 2.640705 & 0.294416 & 3.18568 \\
\hline $\mathrm{H}$ & 3.466952 & -0.940632 & 2.223495 \\
\hline $\mathrm{H}$ & 1.269366 & -1.645145 & 1.687613 \\
\hline $\mathrm{H}$ & -5.754697 & 1.808512 & 0.038224 \\
\hline $\mathrm{H}$ & -6.583847 & -0.423564 & 0.370457 \\
\hline $\mathrm{H}$ & -4.978538 & -0.922375 & 0.926605 \\
\hline $\mathrm{H}$ & -6.276464 & -2.863732 & -1.157711 \\
\hline $\mathrm{H}$ & -5.562754 & -3.515745 & -3.448344 \\
\hline $\mathrm{H}$ & -4.144795 & -2.001573 & -4.79838 \\
\hline $\mathrm{H}$ & -3.400113 & 0.191186 & -3.86847 \\
\hline $\mathrm{H}$ & -4.097025 & 2.014714 & -1.802426 \\
\hline $\mathrm{C}$ & -1.830491 & 0.991798 & 1.765899 \\
\hline $\mathrm{C}$ & -1.697095 & 2.355861 & 2.485376 \\
\hline $\mathrm{C}$ & -2.36025 & -0.091261 & 2.72163 \\
\hline $\mathrm{H}$ & -2.602988 & 2.500188 & 3.094919 \\
\hline $\mathrm{H}$ & -0.834652 & 2.306224 & 3.172179 \\
\hline $\mathrm{H}$ & -1.639647 & -0.227994 & 3.544662 \\
\hline $\mathrm{C}$ & -1.827654 & 2.015219 & -3.067618 \\
\hline $\mathrm{N}$ & -2.430079 & 2.69 & -3.805913 \\
\hline $\mathrm{C}$ & 0.429887 & 0.17 & -2.734065 \\
\hline $\mathrm{N}$ & 1.270355 & -0.447929 & -3.263413 \\
\hline $\mathrm{C}$ & 0.570435 & -3.408125 & -0.385454 \\
\hline $\mathrm{C}$ & -0.456305 & -2.938665 & -0.863366 \\
\hline $\mathrm{C}$ & -1.647364 & -2.371681 & -1.464564 \\
\hline $\mathrm{H}$ & -2.151303 & -1.670285 & -0.777609 \\
\hline $\mathrm{H}$ & -2.383015 & -3.14186 & -1.73642 \\
\hline $\mathrm{H}$ & -1.382951 & -1.823399 & -2.380371 \\
\hline $\mathrm{C}$ & 1.778653 & -3.809122 & 0.171264 \\
\hline $\mathrm{H}$ & 1.774228 & -4.558136 & 0.967097 \\
\hline $\mathrm{C}$ & 3.025759 & -3.188078 & -0.159378 \\
\hline $\mathrm{C}$ & 3.15548 & -2.328633 & -1.274806 \\
\hline $\mathrm{C}$ & 4.146709 & -3.353578 & 0.684341 \\
\hline $\mathrm{C}$ & 4.311541 & -1.597255 & -1.481264 \\
\hline $\mathrm{H}$ & 2.32 & -2.208203 & -1.9 \\
\hline $\mathrm{C}$ & 5.309032 & -2.628702 & 0.478244 \\
\hline $\mathrm{H}$ & 4.07759 & -4.036782 & 1.531852 \\
\hline $\mathrm{C}$ & 5.374967 & -1.733491 & -0.590124 \\
\hline $\mathrm{H}$ & 4.373769 & -0.909814 & -2.32295 \\
\hline $\mathrm{H}$ & 6.161067 & -2.739068 & 1.145771 \\
\hline $\mathrm{Br}$ & 6.92802 & -0.678566 & -0.822346 \\
\hline $\mathrm{H}$ & -3.315507 & 0.247252 & 3.152027 \\
\hline $\mathrm{O}$ & -1.602848 & 3.382585 & 1.561812 \\
\hline $\mathrm{O}$ & -2.538607 & -1.254432 & 1.963024 \\
\hline $\mathrm{Si}$ & -0.255654 & 4.284788 & 1.094846 \\
\hline $\mathrm{Si}$ & -2.553094 & -2.787057 & 2.666149 \\
\hline $\mathrm{C}$ & -0.009664 & 4.027634 & -0.736431 \\
\hline $\mathrm{H}$ & 0.784916 & 4.683305 & -1.11957 \\
\hline $\mathrm{H}$ & 0.293841 & & -0.963788 \\
\hline $\mathrm{H}$ & -0.924039 & 4.239575 & -1.306903 \\
\hline $\mathrm{C}$ & -0.65602 & 6.078162 & 1.443931 \\
\hline $\mathrm{H}$ & -1.561958 & 6.389358 & 0.908747 \\
\hline $\mathrm{H}$ & -0.819194 & 6.256576 & 2.51439 \\
\hline $\mathrm{H}$ & 0.16376 .731 & 1.115 & 458 \\
\hline $\mathrm{C}$ & 1.232149 & 3.737716 & \\
\hline $\mathrm{H}$ & 1.166855 & 4.057492 & 3.144794 \\
\hline $\mathrm{H}$ & 1.385481 & 2.649824 & 2.077073 \\
\hline $\mathrm{H}$ & 2.142808 & 4.181088 & 1.668956 \\
\hline $\mathrm{C}$ & -3.74588 & -2.739187 & 4.113318 \\
\hline $\mathrm{H}$ & -3.872635 & -3.734404 & 4.559056 \\
\hline $\mathrm{H}$ & -3.403384 & -2.06549 & 4.910505 \\
\hline $\mathrm{H}$ & -4.736717 & -2.393441 & 3.786844 \\
\hline $\mathrm{C}$ & -3.146122 & -3.922712 & 1.309772 \\
\hline $\mathrm{H}$ & -3.525146 & -4.871461 & 1.710686 \\
\hline $\mathrm{H}$ & -3.954839 & -3.449762 & 0.73374 \\
\hline $\mathrm{H}$ & -2.327092 & -4.15164 & 0.61438 \\
\hline $\mathrm{C}$ & -0.80995 & -3.194661 & 3.213948 \\
\hline $\mathrm{H}$ & -0.174992 & -3.309934 & 2.32321 \\
\hline $\mathrm{H}$ & -0.377332 & -2.401447 & 3.839499 \\
\hline $\mathrm{H}$ & -0.766117 & -4.131146 & 3.784927 \\
\hline \multicolumn{4}{|l|}{99} \\
\hline \multicolumn{4}{|c|}{ TSA_B(R) } \\
\hline $\mathrm{Cu}$ & -0.894637 & -0.110604 & -1.856686 \\
\hline $\mathrm{O}$ & 1.022057 & 1.318371 & 1.734552 \\
\hline $\mathrm{O}$ & -3.615146 & 0.486191 & 1.263128 \\
\hline
\end{tabular}

\begin{tabular}{|c|c|c|c|}
\hline $\mathrm{N}$ & 0.30966 & 0.77518 & -0.322287 \\
\hline $\mathrm{N}$ & -2.412982 & 0.029769 & -0.568194 \\
\hline $\mathrm{C}$ & 1.708343 & 1.187655 & -0.500715 \\
\hline $\mathrm{C}$ & 1.792031 & 2.556996 & -1.117407 \\
\hline $\mathrm{C}$ & 1.328519 & 2.955556 & -2.365767 \\
\hline $\mathrm{C}$ & 1.485487 & 4.288867 & -2.735179 \\
\hline $\mathrm{C}$ & \multicolumn{3}{|c|}{$2.097 \quad 5.198218$} \\
\hline $\mathrm{C}$ & 2.564539 & 4.789382 & -0.623478 \\
\hline $\mathrm{C}$ & 2.40591 & 3.458395 & -0.249093 \\
\hline $\mathrm{C}$ & 2.862878 & 2.776303 & 1.012783 \\
\hline $\mathrm{C}$ & 2.235644 & 1.386086 & 0.935828 \\
\hline $\mathrm{C}$ & 0.035339 & 0.924366 & 0.909407 \\
\hline $\mathrm{C}$ & -2.419805 & 0.406163 & 0.657716 \\
\hline $\mathrm{C}$ & -4.557542 & -0.105124 & 0.351757 \\
\hline $\mathrm{C}$ & -4.778757 & -1.594725 & 0.65195 \\
\hline $\mathrm{C}$ & -4.78659 & -2.216733 & -0.716939 \\
\hline $\mathrm{C}$ & -5.252188 & -3.466638 & -1.103863 \\
\hline $\mathrm{C}$ & -5.190146 & -3.816242 & -2.452698 \\
\hline $\mathrm{C}$ & -4.685412 & -2.923861 & -3.397785 \\
\hline $\mathrm{C}$ & -4.21108 & -1.670958 & -3.012394 \\
\hline $\mathrm{C}$ & -4.249234 & -1.33921 & -1.663379 \\
\hline $\mathrm{C}$ & -3.839727 & -0.057359 & -1.000097 \\
\hline $\mathrm{H}$ & 2.241338 & 0.422893 & -1.082116 \\
\hline $\mathrm{H}$ & 0.862517 & 2.235905 & -3.039225 \\
\hline $\mathrm{H}$ & 1.127908 & 4.623061 & -3.707312 \\
\hline $\mathrm{H}$ & 2.212069 & 6.237532 & -2.174521 \\
\hline $\mathrm{H}$ & 3.05146 & 5.500417 & 0.045374 \\
\hline $\mathrm{H}$ & 2.557196 & 3.292905 & 1.933796 \\
\hline $\mathrm{H}$ & \multicolumn{3}{|c|}{$\begin{array}{ll}3.95922 .693044 & 1.047037\end{array}$} \\
\hline $\mathrm{H}$ & 2.892037 & 0.583181 & 1.294159 \\
\hline $\mathrm{H}$ & -5.475296 & 0.487621 & 0.39828 \\
\hline $\mathrm{H}$ & -5.689578 & -1.789453 & 1.232521 \\
\hline $\mathrm{H}$ & -3.924009 & -1.955505 & 1.248193 \\
\hline $\mathrm{H}$ & -5.674588 & -4.155072 & -0.371591 \\
\hline $\mathrm{H}$ & -5.557502 & -4.790034 & -2.77311 \\
\hline $\mathrm{H}$ & -4.667362 & -3.204949 & -4.449241 \\
\hline $\mathrm{H}$ & -3.816786 & -0.96018 & -3.738922 \\
\hline $\mathrm{H}$ & -4.038127 & 0.827415 & -1.618711 \\
\hline $\mathrm{C}$ & -1.298248 & 0.790383 & 1.586335 \\
\hline $\mathrm{C}$ & -1.617596 & 2.180511 & 2.176226 \\
\hline $\mathrm{C}$ & -1.263738 & -0.24761 & 2.723143 \\
\hline $\mathrm{H}$ & -2.612542 & 2.149551 & 2.648632 \\
\hline $\mathrm{H}$ & -0.872002 & 2.391578 & 2.963681 \\
\hline $\mathrm{H}$ & -0.332431 & -0.109602 & 3.300335 \\
\hline $\mathrm{C}$ & -1.929455 & 0.808463 & -3.224931 \\
\hline $\mathrm{N}$ & -2.691189 & 1.272609 & -3.981761 \\
\hline $\mathrm{C}$ & 0.609533 & -0.564044 & -2.943859 \\
\hline $\mathrm{N}$ & 1.635928 & -0.728832 & -3.480989 \\
\hline $\mathrm{C}$ & 0.455876 & -2.282931 & -0.202026 \\
\hline $\mathrm{C}$ & -0.406497 & -2.362573 & -1.094353 \\
\hline $\mathrm{C}$ & -1.293129 & -3.141857 & -1.961936 \\
\hline $\mathrm{H}$ & -2.328186 & -3.109952 & -1.597436 \\
\hline $\mathrm{H}$ & -0.966375 & -4.191001 & -1.98399 \\
\hline $\mathrm{H}$ & -1.290263 & -2.765143 & -2.993019 \\
\hline $\mathrm{C}$ & 1.419453 & -2.09313 & 0.743986 \\
\hline $\mathrm{H}$ & 1.098541 & -1.937149 & 1.778957 \\
\hline $\mathrm{C}$ & 2.833504 & -2.08804 & 0.4792 \\
\hline $\mathrm{C}$ & 3.343269 & -2.081118 & -0.836016 \\
\hline $\mathrm{C}$ & 3.742965 & -2.043098 & 1.554178 \\
\hline $\mathrm{C}$ & 4.708366 & -2.022757 & -1.061041 \\
\hline $\mathrm{H}$ & 2.656757 & -2.077387 & -1.684965 \\
\hline $\mathrm{C}$ & 5.109846 & -1.994843 & 1.333671 \\
\hline $\mathrm{H}$ & 3.357046 & -2.048656 & 2.575297 \\
\hline $\mathrm{C}$ & 5.583275 & -1.984738 & 0.023437 \\
\hline $\mathrm{H}$ & 5.095716 & -1.997089 & -2.076943 \\
\hline $\mathrm{H}$ & 5.810648 & -1.964478 & 2.165053 \\
\hline $\mathrm{Br}$ & 7.448631 & -1.906249 & -0.285011 \\
\hline $\mathrm{H}$ & -2.115662 & -0.046284 & 3.397274 \\
\hline $\mathrm{O}$ & -1.570824 & 3.103181 & 1.137489 \\
\hline $\mathrm{O}$ & -1.346131 & -1.523031 & 2.165707 \\
\hline $\mathrm{Si}$ & -1.393842 & 4.757648 & 1.39363 \\
\hline $\mathrm{Si}$ & -1.601668 & -2.924569 & 3.057195 \\
\hline $\mathrm{C}$ & -1.342154 & 5.489217 & -0.31506 \\
\hline $\mathrm{H}$ & -1.062165 & 6.550586 & -0.29373 \\
\hline $\mathrm{H}$ & -0.593395 & 4.956984 & -0.918849 \\
\hline $\mathrm{H}$ & -2.307919 & 5.400338 & -0.827064 \\
\hline $\mathrm{C}$ & -2.850788 & 5.351857 & 2.416369 \\
\hline $\mathrm{H}$ & -3.803725 & 5.114146 & 1.926323 \\
\hline $\mathrm{H}$ & -2.8612 & 4.88963 & 3.41345 \\
\hline $\mathrm{H}$ & -2.813433 & 6.439418 & 2.563378 \\
\hline
\end{tabular}




\begin{tabular}{|c|c|c|c|}
\hline $\mathrm{C}$ & 0.205873 & 5.073283 & 2.322051 \\
\hline $\mathrm{H}$ & 0.365041 & 4.372351 & 3.153511 \\
\hline $\mathrm{H}$ & 1.056431 & 4.986184 & 1.633264 \\
\hline $\mathrm{H}$ & 0.218185 & 6.087448 & 2.743767 \\
\hline $\mathrm{C}$ & -3.050551 & -2.63457 & 4.215922 \\
\hline $\mathrm{H}$ & -3.400958 & -3.581118 & 4.648173 \\
\hline $\mathrm{H}$ & -2.78034 & -1.976535 & 5.05289 \\
\hline $\mathrm{H}$ & -3.899893 & -2.174179 & 3.691599 \\
\hline $\mathrm{C}$ & -1.952838 & -4.227277 & 1.768541 \\
\hline $\mathrm{H}$ & -2.17246 & -5.202625 & 2.221546 \\
\hline $\mathrm{H}$ & -2.80562 & -3.947891 & 1.132974 \\
\hline $\mathrm{H}$ & -1.081031 & -4.343876 & 1.110699 \\
\hline $\mathrm{C}$ & -0.060109 & -3.320563 & 4.050452 \\
\hline $\mathrm{H}$ & 0.767862 & -3.631602 & 3.398964 \\
\hline $\mathrm{H}$ & 0.276967 & -2.452155 & 4.634439 \\
\hline $\mathrm{H}$ & -0.24652 & -4.136172 & 4.761771 \\
\hline 99 & & & \\
\hline \multicolumn{4}{|c|}{ TSA'C(triplet) } \\
\hline $\mathrm{Cu}$ & \multicolumn{3}{|c|}{$1.56830 .789033 \quad 1.567565$} \\
\hline $\mathrm{O}$ & 2.82048 & -1.346571 & -1.784984 \\
\hline $\mathrm{O}$ & -1.192005 & 1.169955 & -1.512799 \\
\hline $\mathrm{N}$ & 2.376365 & -0.350087 & 0.15896 \\
\hline $\mathrm{N}$ & 0.003548 & 1.020579 & 0.3652 \\
\hline $\mathrm{C}$ & 3.465492 & -1.291262 & 0.45439 \\
\hline $\mathrm{C}$ & 2.898335 & -2.543173 & 1.069644 \\
\hline $\mathrm{C}$ & 2.09351 & -2.641012 & 2.197104 \\
\hline $\mathrm{C}$ & 1.610387 & -3.896407 & 2.557555 \\
\hline $\mathrm{C}$ & 1.939312 & -5.024011 & 1.803613 \\
\hline $\mathrm{C}$ & 2.735144 & -4.91441 & 0.663931 \\
\hline $\mathrm{C}$ & \multicolumn{3}{|c|}{$3.2061-3.659882 \quad 0.292789$} \\
\hline $\mathrm{C}$ & 4.056005 & -3.271799 & -0.888435 \\
\hline $\mathrm{C}$ & 3.935504 & -1.753919 & -0.93279 \\
\hline $\mathrm{C}$ & 2.044377 & -0.534667 & -1.060198 \\
\hline $\mathrm{C}$ & -0.106357 & 0.737944 & -0.877036 \\
\hline $\mathrm{C}$ & -1.993702 & 1.919731 & -0.557642 \\
\hline $\mathrm{C}$ & -2.124864 & 3.372915 & -1.013787 \\
\hline $\mathrm{C}$ & -1.208755 & 4.137953 & -0.100516 \\
\hline $\mathrm{C}$ & -0.905572 & 5.49468 & -0.13489 \\
\hline $\mathrm{C}$ & -0.07187 & 6.024954 & 0.846146 \\
\hline $\mathrm{C}$ & 0.449223 & 5.208814 & 1.850353 \\
\hline $\mathrm{C}$ & 0.150055 & 3.849994 & 1.88653 \\
\hline $\mathrm{C}$ & -0.681509 & 3.324306 & 0.901742 \\
\hline $\mathrm{C}$ & -1.12958 & 1.894815 & 0.724274 \\
\hline $\mathrm{H}$ & 4.230004 & -0.789754 & 1.061363 \\
\hline $\mathrm{H}$ & 1.823026 & -1.753895 & 2.775173 \\
\hline $\mathrm{H}$ & 0.960164 & -3.990808 & 3.425808 \\
\hline $\mathrm{H}$ & 1.555471 & -5.999155 & 2.099445 \\
\hline $\mathrm{H}$ & 2.974651 & -5.797577 & 0.070533 \\
\hline $\mathrm{H}$ & 3.740072 & -3.723734 & -1.838189 \\
\hline $\mathrm{H}$ & 5.109928 & -3.55071 & -0.739115 \\
\hline $\mathrm{H}$ & 4.823585 & -1.239407 & -1.317592 \\
\hline $\mathrm{H}$ & -2.946311 & 1.387613 & -0.474409 \\
\hline $\mathrm{H}$ & -3.1657 & 3.714805 & -0.921645 \\
\hline $\mathrm{H}$ & -1.855555 & 3.463898 & -2.07509 \\
\hline $\mathrm{H}$ & -1.320922 & 6.134908 & -0.913256 \\
\hline $\mathrm{H}$ & 0.171605 & 7.086351 & 0.831899 \\
\hline $\mathrm{H}$ & 1.099083 & 5.635614 & 2.612183 \\
\hline $\mathrm{H}$ & 0.545266 & 3.213172 & 2.677955 \\
\hline $\mathrm{H}$ & -1.63146 & 1.472715 & 1.604882 \\
\hline $\mathrm{C}$ & 0.887964 & 0.071014 & -1.791224 \\
\hline $\mathrm{C}$ & 0.208004 & -1.046418 & -2.590725 \\
\hline $\mathrm{C}$ & 1.417943 & 1.19728 & -2.71774 \\
\hline $\mathrm{H}$ & -0.713641 & -0.650523 & -3.048684 \\
\hline $\mathrm{H}$ & \multicolumn{3}{|c|}{$0.8864-1.360496 \quad-3.404502$} \\
\hline $\mathrm{H}$ & 2.277128 & 0.802762 & -3.29056 \\
\hline $\mathrm{C}$ & 3.317144 & 1.293843 & 2.204 \\
\hline $\mathrm{N}$ & 4.434103 & 1.616156 & 2.335066 \\
\hline $\mathrm{H}$ & 0.623043 & 1.449809 & -3.437847 \\
\hline $\mathrm{O}$ & -0.072264 & -2.090744 & -1.705211 \\
\hline $\mathrm{O}$ & 1.730498 & 2.302698 & -1.939384 \\
\hline $\mathrm{Si}$ & -0.39164 & -3.661419 & -2.23388 \\
\hline $\mathrm{Si}$ & 3.247323 & 3.039775 & -1.833354 \\
\hline $\mathrm{C}$ & -0.901242 & -4.582448 & -0.698558 \\
\hline $\mathrm{H}$ & -0.96016 & -5.662638 & -0.887513 \\
\hline $\mathrm{H}$ & -0.157896 & -4.422281 & 0.09666 \\
\hline $\mathrm{H}$ & -1.875486 & -4.250119 & -0.317501 \\
\hline $\mathrm{C}$ & -1.745901 & -3.573614 & -3.528841 \\
\hline $\mathrm{H}$ & -2.654135 & -3.103262 & -3.127239 \\
\hline $\mathrm{H}$ & -1.431906 & -3.001042 & -4.41258 \\
\hline $\mathrm{H}$ & -2.022318 & -4.578379 & -3.874929 \\
\hline
\end{tabular}

\begin{tabular}{|c|c|c|c|}
\hline $\mathrm{C}$ & 1.174138 & -4.386681 & -2.970551 \\
\hline $\mathrm{H}$ & 1.749256 & -3.645433 & -3.542603 \\
\hline $\mathrm{H}$ & 1.816686 & -4.762147 & -2.162896 \\
\hline $\mathrm{H}$ & 0.952524 & -5.227092 & -3.641409 \\
\hline $\mathrm{C}$ & 3.570569 & 3.90029 & -3.468739 \\
\hline $\mathrm{H}$ & 4.552142 & 4.392623 & -3.470831 \\
\hline $\mathrm{H}$ & 3.561444 & 3.187104 & -4.305217 \\
\hline $\mathrm{H}$ & 2.81089 & 4.66483 & -3.67427 \\
\hline $\mathrm{C}$ & 3.104212 & 4.205555 & -0.394606 \\
\hline $\mathrm{H}$ & 3.924887 & 4.934726 & -0.388587 \\
\hline $\mathrm{H}$ & 2.155211 & 4.757625 & -0.420454 \\
\hline $\mathrm{H}$ & 3.154202 & 3.649351 & 0.552827 \\
\hline $\mathrm{C}$ & 4.573668 & 1.751383 & -1.521174 \\
\hline $\mathrm{H}$ & 4.528156 & 1.40823 & -0.477268 \\
\hline $\mathrm{H}$ & 4.505139 & 0.886909 & -2.19772 \\
\hline $\mathrm{H}$ & 5.565447 & 2.199194 & -1.671418 \\
\hline $\mathrm{C}$ & -0.046118 & 0.378341 & 4.067546 \\
\hline $\mathrm{N}$ & 0.554506 & 0.888472 & 3.181815 \\
\hline $\mathrm{C}$ & -1.215969 & -1.687228 & 2.848568 \\
\hline $\mathrm{C}$ & -0.871774 & -1.40803 & 4.029981 \\
\hline $\mathrm{C}$ & -0.793801 & -1.894608 & 5.416857 \\
\hline $\mathrm{H}$ & -1.290723 & -1.191452 & 6.097192 \\
\hline $\mathrm{H}$ & 0.252935 & -1.959904 & 5.74627 \\
\hline $\mathrm{H}$ & -1.258564 & -2.883367 & 5.520757 \\
\hline $\mathrm{C}$ & -1.514415 & -1.690088 & 1.525141 \\
\hline $\mathrm{H}$ & -0.780833 & -2.090605 & 0.817185 \\
\hline $\mathrm{C}$ & -2.769704 & -1.225766 & 0.984255 \\
\hline $\mathrm{C}$ & -3.76625 & -0.647428 & 1.800354 \\
\hline $\mathrm{C}$ & -3.039098 & -1.321984 & -0.396763 \\
\hline $\mathrm{C}$ & -4.960911 & -0.192469 & 1.268225 \\
\hline $\mathrm{H}$ & -3.57875 & -0.557155 & 2.870399 \\
\hline $\mathrm{C}$ & -4.235457 & -0.874533 & -0.937933 \\
\hline $\mathrm{H}$ & -2.272161 & -1.736692 & -1.050494 \\
\hline $\mathrm{C}$ & -5.194631 & -0.309923 & -0.101799 \\
\hline $\mathrm{H}$ & -5.718512 & 0.253589 & 1.909619 \\
\hline $\mathrm{H}$ & -4.426084 & -0.954021 & -2.007189 \\
\hline $\mathrm{Br}$ & -6.827956 & 0.318458 & -0.83428 \\
\hline \multicolumn{4}{|l|}{99} \\
\hline \multicolumn{4}{|c|}{ TSA'_C(singlet) } \\
\hline $\mathrm{Cu}$ & 1.592532 & 0.545869 & 1.542756 \\
\hline $\mathrm{O}$ & -1.093723 & 0.983326 & -1.632537 \\
\hline $\mathrm{O}$ & 3.516915 & 0.029171 & -2.196396 \\
\hline $\mathrm{N}$ & 0.080774 & 0.669472 & 0.241926 \\
\hline $\mathrm{N}$ & 2.822166 & 0.256294 & -0.080241 \\
\hline $\mathrm{C}$ & -1.297318 & 0.935012 & 0.691701 \\
\hline $\mathrm{C}$ & -1.473257 & 2.328439 & 1.231835 \\
\hline $\mathrm{C}$ & -0.791873 & 2.929046 & 2.284818 \\
\hline $\mathrm{C}$ & -1.093184 & 4.25106 & 2.598529 \\
\hline $\mathrm{C}$ & -2.063836 & 4.948544 & 1.877417 \\
\hline $\mathrm{C}$ & -2.741498 & 4.340927 & 0.823652 \\
\hline $\mathrm{C}$ & -2.432986 & 3.024987 & 0.496914 \\
\hline $\mathrm{C}$ & -2.999533 & 2.177377 & -0.61066 \\
\hline $\mathrm{C}$ & -2.126317 & 0.926299 & -0.602948 \\
\hline $\mathrm{C}$ & 0.08309 & 0.755279 & -1.036387 \\
\hline $\mathrm{C}$ & 2.537602 & 0.296392 & -1.327018 \\
\hline $\mathrm{C}$ & 4.592684 & -0.521501 & -1.409818 \\
\hline $\mathrm{C}$ & 4.535919 & -2.056318 & -1.424042 \\
\hline $\mathrm{C}$ & 4.725158 & -2.427468 & 0.018125 \\
\hline $\mathrm{C}$ & 5.02742 & -3.673956 & 0.551635 \\
\hline $\mathrm{C}$ & 5.167253 & -3.797729 & 1.931845 \\
\hline $\mathrm{C}$ & 5.02234 & -2.686233 & 2.761203 \\
\hline $\mathrm{C}$ & 4.723659 & -1.433612 & 2.228739 \\
\hline $\mathrm{C}$ & 4.560451 & -1.31912 & 0.851723 \\
\hline $\mathrm{C}$ & 4.26265 & -0.089394 & 0.032983 \\
\hline $\mathrm{H}$ & -1.574057 & 0.155989 & 1.408782 \\
\hline $\mathrm{H}$ & -0.03744 & 2.375833 & 2.845802 \\
\hline $\mathrm{H}$ & -0.568391 & 4.742136 & 3.416021 \\
\hline $\mathrm{H}$ & -2.291767 & 5.980293 & 2.140933 \\
\hline $\mathrm{H}$ & -3.490407 & 4.892633 & 0.255518 \\
\hline $\mathrm{H}$ & -2.974167 & 2.669402 & -1.593504 \\
\hline $\mathrm{H}$ & -4.049945 & 1.899928 & -0.428826 \\
\hline $\mathrm{H}$ & -2.687261 & 0.000569 & -0.777462 \\
\hline $\mathrm{H}$ & 5.526586 & -0.112702 & -1.80456 \\
\hline $\mathrm{H}$ & 5.280196 & -2.505049 & -2.094288 \\
\hline $\mathrm{H}$ & 3.540664 & -2.366025 & -1.786596 \\
\hline $\mathrm{H}$ & 5.161443 & -4.538154 & -0.099159 \\
\hline $\mathrm{H}$ & 5.405614 & -4.767824 & 2.365394 \\
\hline $\mathrm{H}$ & 5.152504 & -2.793145 & 3.836508 \\
\hline $\mathrm{H}$ & 4.620528 & -0.560748 & 2.87129 \\
\hline $\mathrm{H}$ & 4.786682 & 0.805461 & 0.396436 \\
\hline
\end{tabular}




\begin{tabular}{|c|c|c|c|}
\hline $\mathrm{C}$ & 1.227837 & 0.596951 & -1.99486 \\
\hline $\mathrm{C}$ & 1.416713 & 1.873005 & -2.867529 \\
\hline $\mathrm{C}$ & 0.889281 & -0.591135 & -2.921782 \\
\hline $\mathrm{H}$ & 1.904756 & 1.555927 & -3.80156 \\
\hline $\mathrm{H}$ & 0.420819 & 2.267289 & -3.128378 \\
\hline $\mathrm{H}$ & -0.062833 & -0.361497 & -3.431517 \\
\hline $\mathrm{C}$ & 2.982658 & 1.270661 & 2.653505 \\
\hline $\mathrm{N}$ & 3.886552 & 1.806485 & 3.166694 \\
\hline $\mathrm{C}$ & -1.079016 & -3.461528 & 1.707013 \\
\hline $\mathrm{C}$ & 0.027047 & -2.86705 & 1.832473 \\
\hline $\mathrm{C}$ & 1.374682 & -2.677786 & 1.269766 \\
\hline $\mathrm{H}$ & 1.415042 & -1.773716 & 0.644809 \\
\hline $\mathrm{H}$ & 1.658063 & -3.530875 & 0.638833 \\
\hline $\mathrm{H}$ & 2.133961 & -2.570571 & 2.056766 \\
\hline $\mathrm{C}$ & -2.346264 & -3.919361 & 1.617424 \\
\hline $\mathrm{H}$ & -2.567839 & -4.972643 & 1.80318 \\
\hline $\mathrm{C}$ & -3.437134 & -3.029291 & 1.255385 \\
\hline $\mathrm{C}$ & -3.391139 & -1.684054 & 1.660867 \\
\hline $\mathrm{C}$ & -4.539789 & -3.456469 & 0.499758 \\
\hline $\mathrm{C}$ & -4.366442 & -0.780836 & 1.267547 \\
\hline $\mathrm{H}$ & -2.583666 & -1.37292 & 2.326107 \\
\hline $\mathrm{C}$ & -5.511774 & -2.556849 & 0.082096 \\
\hline $\mathrm{H}$ & -4.609906 & -4.503184 & 0.20253 \\
\hline $\mathrm{C}$ & -5.405386 & -1.219029 & 0.451267 \\
\hline $\mathrm{H}$ & -4.333363 & 0.255617 & 1.604525 \\
\hline $\mathrm{H}$ & -6.345927 & -2.884309 & -0.53482 \\
\hline $\mathrm{Br}$ & -6.696251 & 0.032409 & -0.155643 \\
\hline $\mathrm{H}$ & 1.675306 & -0.6629 & -3.688969 \\
\hline $\mathrm{O}$ & 2.227167 & 2.811539 & -2.252338 \\
\hline $\mathrm{O}$ & 0.834904 & -1.767302 & -2.170188 \\
\hline $\mathrm{Si}$ & 1.802756 & 4.169356 & -1.34873 \\
\hline $\mathrm{Si}$ & -0.30468 & -2.969485 & -2.510906 \\
\hline $\mathrm{C}$ & 2.206602 & 3.814187 & 0.43567 \\
\hline $\mathrm{H}$ & 1.909581 & 4.638173 & 1.098303 \\
\hline $\mathrm{H}$ & 1.685723 & 2.913599 & 0.786932 \\
\hline $\mathrm{H}$ & 3.278775 & 3.630557 & 0.58878 \\
\hline $\mathrm{C}$ & 2.846537 & 5.575635 & -2.001449 \\
\hline $\mathrm{H}$ & 3.914084 & 5.328905 & -1.942913 \\
\hline $\mathrm{H}$ & 2.610522 & 5.802746 & -3.048668 \\
\hline $\mathrm{H}$ & 2.684895 & 6.488403 & -1.412795 \\
\hline $\mathrm{C}$ & -0.022049 & 4.516754 & -1.592116 \\
\hline $\mathrm{H}$ & -0.252459 & 4.829792 & -2.619352 \\
\hline $\mathrm{H}$ & -0.644683 & 3.644635 & -1.350723 \\
\hline $\mathrm{H}$ & -0.339006 & 5.321818 & -0.914901 \\
\hline $\mathrm{C}$ & -0.309109 & -3.268824 & -4.364312 \\
\hline $\mathrm{H}$ & -0.999759 & -4.083995 & -4.618733 \\
\hline $\mathrm{H}$ & -0.626983 & -2.385894 & -4.934786 \\
\hline $\mathrm{H}$ & 0.688065 & -3.558172 & -4.721755 \\
\hline $\mathrm{C}$ & 0.245387 & -4.496115 & -1.592028 \\
\hline $\mathrm{H}$ & -0.211546 & -5.392259 & -2.032816 \\
\hline $\mathrm{H}$ & 1.335709 & -4.618016 & -1.642227 \\
\hline $\mathrm{H}$ & -0.053634 & -4.454695 & -0.535863 \\
\hline $\mathrm{C}$ & -1.97136 & -2.362791 & -1.919847 \\
\hline $\mathrm{H}$ & -1.915013 & -2.127777 & -0.845175 \\
\hline $\mathrm{H}$ & -2.2965 & -1.463175 & -2.461494 \\
\hline $\mathrm{H}$ & -2.75177 & -3.12603 & -2.041326 \\
\hline $\mathrm{N}$ & 0.428976 & -0.209575 & 2.93666 \\
\hline $\mathrm{C}$ & -0.009089 & -1.282231 & 3.201672 \\
\hline \multicolumn{4}{|c|}{-1.202201} \\
\hline \multicolumn{4}{|c|}{$\mathrm{B}(\mathrm{R})$} \\
\hline $\mathrm{Cu}$ & -0.881719 & -0.36205 & -1.810808 \\
\hline $\mathrm{O}$ & 0.708058 & 1.505449 & 1.813815 \\
\hline $\mathrm{O}$ & -3.709707 & -0.144086 & 1.202181 \\
\hline $\mathrm{N}$ & 0.096464 & 0.982025 & -0.284771 \\
\hline $\mathrm{N}$ & -2.393957 & -0.337652 & -0.583578 \\
\hline $\mathrm{C}$ & 1.426396 & 1.58057 & -0.415342 \\
\hline $\mathrm{C}$ & 1.349688 & 2.970451 & -0.983857 \\
\hline $\mathrm{C}$ & 0.862523 & 3.352498 & -2.228863 \\
\hline $\mathrm{C}$ & 0.863876 & 4.706217 & -2.553219 \\
\hline $\mathrm{C}$ & 1.342755 & 5.653314 & -1.645982 \\
\hline $\mathrm{C}$ & 1.829066 & 5.262557 & -0.401261 \\
\hline $\mathrm{C}$ & 1.828585 & 3.909783 & -0.072714 \\
\hline $\mathrm{C}$ & 2.32589 & 3.250855 & 1.186696 \\
\hline $\mathrm{C}$ & 1.904555 & 1.789007 & 1.040399 \\
\hline $\mathrm{C}$ & -0.199965 & 1.007953 & 0.947641 \\
\hline $\mathrm{C}$ & -2.499086 & 0.015027 & 0.644336 \\
\hline $\mathrm{C}$ & -4.498085 & -0.870628 & 0.240029 \\
\hline $\mathrm{C}$ & -4.439339 & -2.390646 & 0.46589 \\
\hline $\mathrm{C}$ & -4.291011 & -2.936682 & -0.929734 \\
\hline $\mathrm{C}$ & -4.471689 & -4.232564 & -1.394413 \\
\hline
\end{tabular}

\begin{tabular}{|c|c|c|c|}
\hline $\mathrm{C}$ & -4.250756 & -4.49618 & -2.74684 \\
\hline $\mathrm{C}$ & -3.861227 & -3.482518 & -3.621858 \\
\hline $\mathrm{C}$ & -3.678192 & -2.180496 & -3.158813 \\
\hline $\mathrm{C}$ & -3.890728 & -1.929075 & -1.810625 \\
\hline $\mathrm{C}$ & -3.762475 & -0.632752 & -1.078539 \\
\hline $\mathrm{H}$ & 2.068572 & 0.911259 & -1.007095 \\
\hline $\mathrm{H}$ & 0.476144 & 2.609868 & -2.92692 \\
\hline $\mathrm{H}$ & 0.480724 & 5.02727 & -3.52002 \\
\hline $\mathrm{H}$ & 1.335318 & 6.708715 & -1.91459 \\
\hline $\mathrm{H}$ & 2.203478 & 6.004672 & 0.305009 \\
\hline $\mathrm{H}$ & 1.908406 & 3.686925 & 2.10597 \\
\hline $\mathrm{H}$ & 3.420002 & 3.323728 & 1.272604 \\
\hline $\mathrm{H}$ & 2.668626 & 1.07438 & 1.371731 \\
\hline $\mathrm{H}$ & -5.512241 & -0.464115 & 0.276948 \\
\hline $\mathrm{H}$ & -5.307883 & -2.782011 & 1.010341 \\
\hline $\mathrm{H}$ & -3.541852 & -2.614615 & 1.068792 \\
\hline $\mathrm{H}$ & -4.787354 & -5.029077 & -0.720638 \\
\hline $\mathrm{H}$ & -4.393276 & -5.506795 & -3.126139 \\
\hline $\mathrm{H}$ & -3.704811 & -3.708877 & -4.674809 \\
\hline $\mathrm{H}$ & -3.381437 & -1.373582 & -3.830343 \\
\hline $\mathrm{H}$ & -4.083169 & 0.240793 & -1.663471 \\
\hline $\mathrm{C}$ & -1.498281 & 0.611763 & 1.597746 \\
\hline $\mathrm{C}$ & -2.109999 & 1.916632 & 2.155658 \\
\hline $\mathrm{C}$ & -1.295342 & -0.393465 & 2.743728 \\
\hline $\mathrm{H}$ & -3.066781 & 1.683627 & 2.650877 \\
\hline $\mathrm{H}$ & -1.416858 & 2.311151 & 2.920099 \\
\hline $\mathrm{H}$ & -0.416844 & -0.086018 & 3.33637 \\
\hline $\mathrm{C}$ & -1.739553 & 0.984429 & -2.889267 \\
\hline $\mathrm{N}$ & -2.420283 & 1.710645 & -3.502364 \\
\hline $\mathrm{C}$ & 0.535043 & -0.614755 & -2.965057 \\
\hline $\mathrm{N}$ & 1.507352 & -0.642871 & -3.612234 \\
\hline $\mathrm{C}$ & 0.797677 & -1.901114 & -0.166348 \\
\hline $\mathrm{C}$ & -0.094272 & -2.015584 & -1.086496 \\
\hline $\mathrm{C}$ & -0.644372 & -3.266226 & -1.689941 \\
\hline $\mathrm{H}$ & -1.709513 & -3.389536 & -1.448428 \\
\hline $\mathrm{H}$ & -0.101976 & -4.145248 & -1.315883 \\
\hline $\mathrm{H}$ & -0.556582 & -3.246575 & -2.784323 \\
\hline $\mathrm{C}$ & 1.732528 & -1.72306 & 0.755331 \\
\hline $\mathrm{H}$ & 1.420395 & -1.620948 & 1.802161 \\
\hline $\mathrm{C}$ & 3.163078 & -1.624614 & 0.48103 \\
\hline $\mathrm{C}$ & 3.658789 & -1.557516 & -0.83198 \\
\hline $\mathrm{C}$ & 4.07175 & -1.563087 & 1.546017 \\
\hline $\mathrm{C}$ & 5.019714 & -1.439015 & -1.066945 \\
\hline $\mathrm{H}$ & 2.965323 & -1.581267 & -1.675504 \\
\hline $\mathrm{C}$ & 5.43728 & -1.447878 & 1.319844 \\
\hline $\mathrm{H}$ & 3.69585 & -1.610443 & 2.569709 \\
\hline $\mathrm{C}$ & 5.900025 & -1.389017 & 0.011003 \\
\hline $\mathrm{H}$ & 5.399671 & -1.379931 & -2.084293 \\
\hline $\mathrm{H}$ & 6.140023 & -1.40446 & 2.149031 \\
\hline $\mathrm{Br}$ & 7.760741 & -1.230579 & -0.310693 \\
\hline $\mathrm{H}$ & -2.18337 & -0.349775 & 3.400025 \\
\hline $\mathrm{O}$ & -2.284233 & 2.784654 & 1.087753 \\
\hline $\mathrm{O}$ & -1.13269 & -1.66646 & 2.19507 \\
\hline $\mathrm{Si}$ & -2.351758 & 4.460301 & 1.222193 \\
\hline $\mathrm{Si}$ & -1.13241 & -3.077278 & 3.105911 \\
\hline $\mathrm{C}$ & -2.305135 & 5.056474 & -0.537262 \\
\hline $\mathrm{H}$ & -2.189135 & 6.146268 & -0.601526 \\
\hline $\mathrm{H}$ & -1.44785 & 4.595509 & -1.048944 \\
\hline $\mathrm{H}$ & -3.206767 & 4.770783 & -1.092358 \\
\hline $\mathrm{C}$ & -3.927106 & 4.911062 & 2.137367 \\
\hline $\mathrm{H}$ & -4.814953 & 4.547106 & 1.604933 \\
\hline $\mathrm{H}$ & -3.939674 & 4.47622 & 3.146928 \\
\hline $\mathrm{H}$ & -4.026616 & 5.998794 & 2.249984 \\
\hline $\mathrm{C}$ & -0.863013 & 5.063909 & 2.190449 \\
\hline $\mathrm{H}$ & -0.695551 & 4.510044 & 3.124463 \\
\hline $\mathrm{H}$ & 0.039476 & 4.980024 & 1.570748 \\
\hline $\mathrm{H}$ & -0.984456 & 6.122397 & 2.458322 \\
\hline $\mathrm{C}$ & -2.68653 & -3.096107 & 4.159786 \\
\hline $\mathrm{H}$ & -2.868093 & -4.094017 & 4.579933 \\
\hline $\mathrm{H}$ & -2.621318 & -2.395254 & 5.002877 \\
\hline $\mathrm{H}$ & -3.568863 & -2.818945 & 3.565208 \\
\hline $\mathrm{C}$ & -1.1076 & -4.437928 & 1.830215 \\
\hline $\mathrm{H}$ & -1.083263 & -5.433416 & 2.291782 \\
\hline $\mathrm{H}$ & -1.98386 & -4.392446 & 1.167869 \\
\hline $\mathrm{H}$ & -0.213558 & -4.331145 & 1.200461 \\
\hline $\mathrm{C}$ & 0.393774 & -3.119683 & 4.194463 \\
\hline $\mathrm{H}$ & 1.30816 & -3.208943 & 3.592047 \\
\hline $\mathrm{H}$ & 0.480279 & -2.212347 & 4.808468 \\
\hline $\mathrm{H}$ & 0.365869 & -3.975311 & 4.882255 \\
\hline
\end{tabular}


TSB(R) C

\begin{tabular}{|c|c|c|c|}
\hline $\mathrm{Cu}$ & -0.903476 & -0.505037 & -1.854256 \\
\hline $\mathrm{O}$ & 0.51275 & 1.717711 & 1.629907 \\
\hline $\mathrm{O}$ & -3.709123 & -0.437132 & 1.237429 \\
\hline $\mathrm{N}$ & -0.101356 & 1.033753 & -0.421728 \\
\hline $\mathrm{N}$ & -2.407433 & -0.586926 & -0.568931 \\
\hline $\mathrm{C}$ & 1.126893 & 1.803847 & -0.625203 \\
\hline $\mathrm{C}$ & 0.820331 & 3.159215 & -1.199636 \\
\hline $\mathrm{C}$ & 0.186362 & 3.444467 & -2.403221 \\
\hline $\mathrm{C}$ & -0.023107 & 4.779366 & -2.739093 \\
\hline $\mathrm{C}$ & 0.38851 & 5.800934 & -1.880476 \\
\hline $\mathrm{C}$ & 1.013364 & 5.505537 & -0.67161 \\
\hline $\mathrm{C}$ & 1.227992 & 4.172455 & -0.33394 \\
\hline $\mathrm{C}$ & 1.90112 & 3.60815 & 0.889548 \\
\hline $\mathrm{C}$ & 1.640288 & 2.105939 & 0.800549 \\
\hline $\mathrm{C}$ & -0.35728 & 1.076288 & 0.81991 \\
\hline $\mathrm{C}$ & -2.533884 & -0.178888 & 0.637416 \\
\hline $\mathrm{C}$ & -4.421682 & -1.314104 & 0.343363 \\
\hline $\mathrm{C}$ & -4.186043 & -2.796741 & 0.680818 \\
\hline $\mathrm{C}$ & -3.948814 & -3.425529 & -0.667141 \\
\hline $\mathrm{C}$ & -3.948246 & -4.767582 & -1.025374 \\
\hline $\mathrm{C}$ & -3.693578 & -5.106217 & -2.354816 \\
\hline $\mathrm{C}$ & -3.449674 & -4.121677 & -3.312189 \\
\hline $\mathrm{C}$ & -3.445781 & -2.773782 & -2.956425 \\
\hline $\mathrm{C}$ & -3.69016 & -2.446981 & -1.629668 \\
\hline $\mathrm{C}$ & -3.732692 & -1.089333 & -1.004548 \\
\hline $\mathrm{H}$ & 1.826631 & 1.220036 & -1.241645 \\
\hline $\mathrm{H}$ & -0.153247 & 2.640049 & -3.05709 \\
\hline $\mathrm{H}$ & -0.519873 & 5.026538 & -3.675514 \\
\hline $\mathrm{H}$ & 0.213509 & 6.839721 & -2.156902 \\
\hline $\mathrm{H}$ & 1.328585 & 6.305807 & -0.000928 \\
\hline $\mathrm{H}$ & 1.518092 & 4.017068 & 1.835662 \\
\hline $\mathrm{H}$ & 2.983669 & 3.803499 & 0.877739 \\
\hline $\mathrm{H}$ & 2.491834 & 1.491051 & 1.118596 \\
\hline $\mathrm{H}$ & -5.475067 & -1.023204 & 0.373565 \\
\hline $\mathrm{H}$ & -5.01446 & -3.248297 & 1.241227 \\
\hline $\mathrm{H}$ & -3.284354 & -2.866954 & 1.313684 \\
\hline $\mathrm{H}$ & -4.153026 & -5.54313 & -0.287144 \\
\hline $\mathrm{H}$ & -3.695798 & -6.154072 & -2.650897 \\
\hline $\mathrm{H}$ & -3.26571 & -4.40809 & -4.346007 \\
\hline $\mathrm{H}$ & -3.260275 & -1.987425 & -3.69001 \\
\hline $\mathrm{H}$ & -4.171506 & -0.318735 & -1.653694 \\
\hline $\mathrm{C}$ & -1.571484 & 0.550484 & 1.536529 \\
\hline $\mathrm{C}$ & -2.29187 & \multicolumn{2}{|c|}{1.78212 .125538} \\
\hline $\mathrm{C}$ & -1.199232 & -0.405461 & 2.682691 \\
\hline $\mathrm{H}$ & -3.212592 & 1.450786 & 2.632714 \\
\hline $\mathrm{H}$ & -1.62474 & 2.226655 & 2.886097 \\
\hline $\mathrm{H}$ & -0.357588 & 0.030581 & 3.248579 \\
\hline $\mathrm{C}$ & -1.934904 & 0.493438 & -3.134736 \\
\hline $\mathrm{N}$ & -2.731977 & 0.95038 & -3.858103 \\
\hline $\mathrm{C}$ & 0.626148 & -0.848035 & -2.811268 \\
\hline $\mathrm{N}$ & 1.570131 & -0.789611 & -3.502312 \\
\hline $\mathrm{C}$ & 1.083198 & -1.694553 & -0.224079 \\
\hline $\mathrm{C}$ & 0.231953 & -1.964154 & -1.162316 \\
\hline $\mathrm{C}$ & -0.22902 & -3.321507 & -1.595701 \\
\hline $\mathrm{H}$ & -1.25123 & -3.516582 & -1.240437 \\
\hline $\mathrm{H}$ & 0.431349 & -4.100679 & -1.193287 \\
\hline $\mathrm{H}$ & -0.236294 & -3.405741 & -2.689399 \\
\hline $\mathrm{C}$ & 1.971325 & -1.358054 & 0.692852 \\
\hline $\mathrm{H}$ & 1.613255 & -1.159985 & 1.710946 \\
\hline $\mathrm{C}$ & 3.405579 & -1.204486 & 0.45502 \\
\hline $\mathrm{C}$ & 3.941758 & -1.229816 & -0.842531 \\
\hline $\mathrm{C}$ & 4.268407 & -0.995272 & 1.538156 \\
\hline $\mathrm{C}$ & 5.302942 & -1.061648 & -1.044148 \\
\hline $\mathrm{H}$ & 3.279587 & -1.361257 & -1.701177 \\
\hline $\mathrm{C}$ & 5.633706 & -0.828233 & 1.345185 \\
\hline $\mathrm{H}$ & 3.857776 & -0.965648 & 2.549001 \\
\hline $\mathrm{C}$ & 6.139625 & -0.866344 & 0.051862 \\
\hline $\mathrm{H}$ & 5.717264 & -1.074947 & -2.049691 \\
\hline $\mathrm{H}$ & 6.302722 & -0.669677 & 2.188043 \\
\hline $\mathrm{Br}$ & 8.000219 & -0.638615 & -0.224922 \\
\hline $\mathrm{H}$ & -2.067201 & -0.476296 & 3.362736 \\
\hline $\mathrm{O}$ & -2.568357 & 2.657783 & 1.082872 \\
\hline $\mathrm{O}$ & -0.875194 & -1.652445 & 2.14471 \\
\hline $\mathrm{Si}$ & -2.853106 & 4.299005 & 1.319826 \\
\hline $\mathrm{Si}$ & -0.619073 & -3.018576 & 3.090211 \\
\hline $\mathrm{C}$ & -3.014637 & 4.996966 & -0.395714 \\
\hline $\mathrm{H}$ & -3.032158 & \multicolumn{2}{|c|}{$6.0947-0.388588$} \\
\hline $\mathrm{H}$ & -2.155566 & 4.67771 & -1.002447 \\
\hline $\mathrm{H}$ & -3.923829 & 4.642742 & -0.896356 \\
\hline
\end{tabular}

$\begin{array}{llll}\text { C } & -4.409043 & 4.48903 & 2.351824\end{array}$

$\begin{array}{llll}\mathrm{H} & -5.269896 & 4.014025 & 1.864103\end{array}$

$\begin{array}{llll}\mathrm{H} & -4.292129 & 4.033242 & 3.345085\end{array}$

$\begin{array}{llll}\mathrm{H} & -4.655318 & 5.547805 & 2.507089\end{array}$

$\begin{array}{llll}\text { C } & -1.390819 & 5.044324 & 2.225444\end{array}$

$\begin{array}{llll}\mathrm{H} & -1.164357 & 4.540452 & 3.174831\end{array}$

$\begin{array}{llll}\mathrm{H} & -0.501056 & 4.991452 & 1.58472\end{array}$

$\begin{array}{llll}\mathrm{H} & -1.575077 & 6.102982 & 2.453131\end{array}$

$\begin{array}{llll}\mathrm{C} & -2.04408 & -3.178343 & 4.302095\end{array}$

$\begin{array}{llll}\mathrm{H} & -2.028674 & -4.160926 & 4.792025\end{array}$

$\begin{array}{llll}\mathrm{H} & -1.999897 & -2.419949 & 5.095268\end{array}$

$\begin{array}{llll}\mathrm{H} & -3.014459 & -3.075381 & 3.796112\end{array}$

$\begin{array}{llll}\text { C } & -0.562685 & -4.426516 & 1.86852\end{array}$

$\begin{array}{llll}\mathrm{H} & -0.439126 & -5.395092 & 2.370006\end{array}$

$\begin{array}{llll}\mathrm{H} & -1.475261 & -4.474166 & 1.257141\end{array}$

$\begin{array}{llll}\mathrm{H} & 0.28578 & -4.288446 & 1.184807\end{array}$

$\begin{array}{llll}\text { C } & 1.002109 & -2.847374 & 4.018268\end{array}$

$\begin{array}{lll}\mathrm{H} & 1.8585-2.935348 & 3.335949\end{array}$

$\begin{array}{llll}\mathrm{H} & 1.076887 & -1.878392 & 4.532173\end{array}$

$\begin{array}{llll}\mathrm{H} & 1.10445 & -3.628861 & 4.782978\end{array}$

78

C

$\begin{array}{llll}\mathrm{Cu} & -0.432928 & -1.987776 & -0.609558\end{array}$

$\begin{array}{llll}\text { O } & -1.416688 & 1.943983 & -1.22112\end{array}$

$\begin{array}{llll}\mathrm{O} & 1.765886 & 0.59785 & 2.056002\end{array}$

$\begin{array}{llll}\mathrm{N} & -0.88119 & -0.23014 & -1.237266\end{array}$

$\begin{array}{llll}\mathrm{N} & 1.059554 & -0.987815 & 0.621639\end{array}$

$\begin{array}{llll}\text { C } & -1.872886 & -0.064486 & -2.307872\end{array}$

$\begin{array}{llll}\text { C } & -3.208281 & -0.612845 & -1.8989\end{array}$

$\begin{array}{llll}\text { C } & -3.489542 & -1.919539 & -1.523035\end{array}$

$\begin{array}{llll}\text { C } & -4.796492 & -2.233762 & -1.16512\end{array}$

$\begin{array}{llll}\text { C } & -5.786373 & -1.248013 & -1.171787\end{array}$

$\begin{array}{llll}\text { C } & -5.486858 & 0.065543 & -1.526802\end{array}$

$\begin{array}{llll}\text { C } & -4.181487 & 0.381931 & -1.893318\end{array}$

$\begin{array}{llll}\text { C } & -3.607266 & 1.70711 & -2.323042\end{array}$

$\begin{array}{llll}\text { C } & -2.099879 & 1.456183 & -2.40069\end{array}$

$\begin{array}{llll}\text { C } & -0.721617 & 0.918522 & -0.696507\end{array}$

$\begin{array}{llll}\text { C } & 0.980178 & 0.211901 & 1.023155\end{array}$

$\begin{array}{llll}\text { C } & 2.64205 & -0.519062 & 2.323623\end{array}$

$\begin{array}{llll}\text { C } & 4.066873 & -0.2478 & 1.813763\end{array}$

$\begin{array}{llll}\mathrm{C} & 4.344267 & -1.366874 & 0.847803\end{array}$

$\begin{array}{llll}\text { C } & 5.529834 & -1.651134 & 0.178613\end{array}$

$\begin{array}{llll}\text { C } & 5.568781 & -2.747312 & -0.679899\end{array}$

$\begin{array}{llll}\text { C } & 4.442602 & -3.551376 & -0.860039\end{array}$

$\begin{array}{llll}\text { C } & 3.253827 & -3.272475 & -0.190098\end{array}$

$\begin{array}{llll}\text { C } & 3.223279 & -2.171899 & 0.658484\end{array}$

$\begin{array}{llll}\mathrm{C} & 2.058282 & -1.663326 & 1.456729\end{array}$

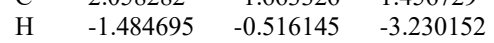

$\begin{array}{llll}\mathrm{H} & -2.673887 & -2.64359 & -1.467638\end{array}$

$\begin{array}{llll}\mathrm{H} & -5.044748 & -3.250005 & -0.864813\end{array}$

$\begin{array}{llll}\mathrm{H} & -6.804377 & -1.507379 & -0.884865\end{array}$

$\begin{array}{llll}\mathrm{H} & -6.262193 & 0.832081 & -1.512339\end{array}$

$\begin{array}{llll}\mathrm{H} & -3.828951 & 2.528958 & -1.626887\end{array}$

$\begin{array}{llll}\mathrm{H} & -3.99118 & 2.01629 & -3.306305\end{array}$

$\begin{array}{llll}\mathrm{H} & -1.62113 & 1.940055 & -3.259477\end{array}$

$\begin{array}{llll}\mathrm{H} & 2.601243 & -0.700407 & 3.401944\end{array}$

$\begin{array}{llll}\mathrm{H} & 4.798272 & -0.204884 & 2.632749\end{array}$

$\begin{array}{llll}\mathrm{H} & 4.092169 & 0.735574 & 1.316958\end{array}$

$\begin{array}{llll}\mathrm{H} & 6.414893 & -1.030884 & 0.326508\end{array}$

$\begin{array}{llll}\mathrm{H} & 6.490793 & -2.98388 & -1.209441\end{array}$

$\mathrm{H} \quad 4.494448 \quad-4.410261 \quad-1.527103$

$\begin{array}{llll}\mathrm{H} & 2.367335 & -3.898398 & -0.312091\end{array}$

H $\quad 1.5485-2.461589 \quad 2.014982$

$\begin{array}{llll}\text { C } & 0.090344 & 1.311251 & 0.509277\end{array}$

$\begin{array}{llll}\text { C } & -0.911027 & 1.700994 & 1.61301\end{array}$

$\begin{array}{llll}\text { C } & 0.980868 & 2.513821 & 0.165369\end{array}$

$\begin{array}{llll}\mathrm{H} & -0.349851 & 1.926295 & 2.535343\end{array}$

$\begin{array}{llll}\mathrm{H} & -1.432638 & 2.62268 & 1.296707\end{array}$

$\begin{array}{llll}\mathrm{H} & 0.359874 & 3.312019 & -0.273739\end{array}$

$\begin{array}{llll}\text { C } & -0.277009 & -3.787185 & -0.292967\end{array}$

$\begin{array}{llll}\mathrm{N} & 0.042424 & -4.872195 & 0.011891\end{array}$

$\begin{array}{llll}\mathrm{H} & 1.415041 & 2.895632 & 1.105084\end{array}$

$\begin{array}{llll}\mathrm{O} & -1.787587 & 0.63482 & 1.793835\end{array}$

$\begin{array}{llll}\text { O } & 1.966534 & 2.082147 & -0.723603\end{array}$

$\begin{array}{llll}\mathrm{Si} & -3.326098 & 0.809666 & 2.445823\end{array}$

$\begin{array}{llll}\mathrm{Si} & 3.379438 & 2.941975 & -1.012482\end{array}$

$\begin{array}{llll}\text { C } & -4.085143 & -0.879367 & 2.270263\end{array}$

$\begin{array}{llll}\mathrm{H} & -5.161308 & -0.873273 & 2.48691\end{array}$

$\begin{array}{llll}\mathrm{H} & -3.952952 & -1.225374 & 1.234884\end{array}$

$\mathrm{H} \quad-3.605808 \quad-1.613744 \quad 2.928835$ 


\begin{tabular}{|c|c|c|c|}
\hline $\mathrm{C}$ & -3.167369 & 1.38674 & 4.223093 \\
\hline $\mathrm{H}$ & -2.585748 & 0.674586 & 4.822066 \\
\hline $\mathrm{H}$ & -2.666089 & 2.363015 & 4.283471 \\
\hline $\mathrm{H}$ & -4.152139 & 1.49656 & 4.696185 \\
\hline $\mathrm{C}$ & -4.271475 & 2.080143 & 1.440686 \\
\hline $\mathrm{H}$ & -3.756475 & 3.047549 & 1.366773 \\
\hline $\mathrm{H}$ & -4.432843 & 1.694405 & 0.425334 \\
\hline $\mathrm{H}$ & -5.258467 & 2.268839 & 1.884354 \\
\hline $\mathrm{C}$ & 4.009463 & 3.655287 & 0.607604 \\
\hline $\mathrm{H}$ & 5.050854 & 3.986411 & 0.49797 \\
\hline $\mathrm{H}$ & 3.423333 & 4.527826 & 0.926736 \\
\hline $\mathrm{H}$ & 3.979776 & 2.920173 & 1.423852 \\
\hline $\mathrm{C}$ & 4.560804 & 1.699392 & -1.744889 \\
\hline $\mathrm{H}$ & 5.546595 & 2.145113 & -1.932234 \\
\hline $\mathrm{H}$ & 4.698063 & 0.834291 & -1.080903 \\
\hline $\mathrm{H}$ & 4.180635 & 1.314446 & -2.699424 \\
\hline $\mathrm{C}$ & 3.015448 & 4.345033 & -2.201465 \\
\hline $\mathrm{H}$ & 2.662147 & 3.96795 & -3.169421 \\
\hline $\mathrm{H}$ & 2.242329 & 5.014986 & -1.800114 \\
\hline $\mathrm{H}$ & 3.911476 & 4.952894 & -2.385318 \\
\hline 99 & & & \\
\hline \multicolumn{4}{|c|}{ TSA-B(S) } \\
\hline $\mathrm{Cu}$ & -1.784558 & 0.440729 & -1.591142 \\
\hline $\mathrm{O}$ & 1.771166 & 1.042633 & 0.793799 \\
\hline $\mathrm{O}$ & -2.268072 & -0.887156 & 2.336205 \\
\hline $\mathrm{N}$ & 0.107013 & 0.946554 & -0.706283 \\
\hline $\mathrm{N}$ & -2.302879 & -0.27408 & 0.185989 \\
\hline $\mathrm{C}$ & 1.141123 & 1.773556 & -1.344477 \\
\hline $\mathrm{C}$ & 0.761323 & 3.231022 & -1.358897 \\
\hline $\mathrm{C}$ & -0.294276 & 3.831253 & -2.037226 \\
\hline $\mathrm{C}$ & -0.464764 & 5.206818 & -1.911901 \\
\hline $\mathrm{C}$ & 0.402162 & 5.96262 & -1.120355 \\
\hline $\mathrm{C}$ & 1.460846 & 5.356424 & -0.449261 \\
\hline $\mathrm{C}$ & 1.639773 & 3.98254 & -0.578734 \\
\hline $\mathrm{C}$ & 2.752659 & 3.131998 & -0.028305 \\
\hline $\mathrm{C}$ & 2.338965 & 1.701639 & -0.367899 \\
\hline $\mathrm{C}$ & 0.532041 & 0.64437 & 0.451847 \\
\hline $\mathrm{C}$ & -1.619382 & -0.415794 & 1.259578 \\
\hline $\mathrm{C}$ & -3.599116 & -1.21446 & 1.89465 \\
\hline $\mathrm{C}$ & -3.764154 & -2.705137 & 1.563697 \\
\hline $\mathrm{C}$ & -4.537097 & -2.684336 & 0.271285 \\
\hline $\mathrm{C}$ & -5.195397 & -3.72356 & -0.3732 \\
\hline $\mathrm{C}$ & -5.817469 & -3.467283 & -1.59469 \\
\hline $\mathrm{C}$ & -5.792853 & -2.190903 & -2.157579 \\
\hline $\mathrm{C}$ & -5.1348 & -1.145048 & -1.512582 \\
\hline $\mathrm{C}$ & -4.502721 & -1.41287 & -0.305616 \\
\hline $\mathrm{C}$ & -3.730068 & -0.472235 & 0.56269 \\
\hline $\mathrm{H}$ & 1.341269 & 1.37887 & -2.350756 \\
\hline $\mathrm{H}$ & -0.958898 & 3.239274 & -2.665558 \\
\hline $\mathrm{H}$ & -1.287554 & 5.693954 & -2.431619 \\
\hline $\mathrm{H}$ & 0.250581 & 7.037323 & -1.029604 \\
\hline $\mathrm{H}$ & 2.142826 & 5.948812 & 0.161949 \\
\hline $\mathrm{H}$ & 2.911607 & 3.243714 & 1.054107 \\
\hline $\mathrm{H}$ & 3.711943 & 3.374379 & -0.508978 \\
\hline $\mathrm{H}$ & 3.168904 & 1.072135 & -0.708073 \\
\hline $\mathrm{H}$ & -4.289116 & -0.869896 & 2.669999 \\
\hline $\mathrm{H}$ & -4.250716 & -3.274142 & 2.366305 \\
\hline $\mathrm{H}$ & -2.761555 & -3.145126 & 1.419699 \\
\hline $\mathrm{H}$ & \multicolumn{3}{|c|}{$-5.232-4.720158 \quad 0.066646$} \\
\hline $\mathrm{H}$ & -6.340529 & -4.271522 & -2.10983 \\
\hline $\mathrm{H}$ & -6.298142 & -2.00863 & -3.104193 \\
\hline $\mathrm{H}$ & -5.114857 & -0.137538 & -1.929278 \\
\hline $\mathrm{H}$ & -4.172876 & 0.531519 & 0.619009 \\
\hline $\mathrm{C}$ & -0.201481 & -0.029579 & 1.578809 \\
\hline $\mathrm{C}$ & -0.323536 & 1.050671 & 2.684858 \\
\hline $\mathrm{C}$ & 0.538118 & -1.276861 & 2.075867 \\
\hline $\mathrm{H}$ & -0.781976 & 0.598674 & 3.580223 \\
\hline $\mathrm{H}$ & 0.688976 & \multicolumn{2}{|c|}{1.39972 .951386} \\
\hline $\mathrm{H}$ & 1.619603 & -1.064239 & 2.12376 \\
\hline $\mathrm{C}$ & -3.192625 & 1.779765 & -1.438237 \\
\hline $\mathrm{N}$ & -4.065861 & 2.510934 & -1.168085 \\
\hline $\mathrm{C}$ & -1.273335 & 0.733352 & -3.39941 \\
\hline $\mathrm{N}$ & -0.743251 & 1.029984 & -4.399148 \\
\hline $\mathrm{C}$ & 0.422211 & -1.634641 & -2.413781 \\
\hline $\mathrm{C}$ & -0.76626 & -1.851075 & -2.126557 \\
\hline $\mathrm{C}$ & -1.955015 & -2.698426 & -2.080679 \\
\hline $\mathrm{H}$ & -2.339497 & -2.819864 & -1.060763 \\
\hline $\mathrm{H}$ & -1.717056 & -3.694608 & -2.481199 \\
\hline $\mathrm{H}$ & -2.771148 & -2.278308 & -2.685121 \\
\hline $\mathrm{C}$ & \multicolumn{3}{|c|}{$1.6581-1.247528 \quad-2.84635$} \\
\hline
\end{tabular}

\begin{tabular}{|c|c|c|c|}
\hline $\mathrm{H}$ & 1.71617 & -0.873451 & -3.873485 \\
\hline $\mathrm{C}$ & 2.870774 & -1.274698 & -2.078467 \\
\hline $\mathrm{C}$ & 2.896279 & -1.70496 & -0.735824 \\
\hline $\mathrm{C}$ & 4.075258 & -0.82867 & -2.657976 \\
\hline $\mathrm{C}$ & 4.071544 & -1.677846 & -0.003769 \\
\hline $\mathrm{H}$ & 1.96553 & -2.040913 & -0.27347 \\
\hline $\mathrm{C}$ & 5.25595 & -0.805621 & -1.932502 \\
\hline $\mathrm{H}$ & 4.070665 & -0.492779 & -3.694952 \\
\hline $\mathrm{C}$ & 5.246994 & -1.22945 & -0.605512 \\
\hline $\mathrm{H}$ & 4.086722 & -1.99769 & 1.037448 \\
\hline $\mathrm{H}$ & 6.182398 & -0.460428 & -2.386053 \\
\hline $\mathrm{Br}$ & 6.851681 & -1.195081 & 0.396307 \\
\hline $\mathrm{H}$ & 0.192125 & -1.506932 & 3.099339 \\
\hline $\mathrm{O}$ & -1.117826 & 2.054089 & 2.147429 \\
\hline $\mathrm{O}$ & 0.262726 & -2.327819 & 1.19064 \\
\hline $\mathrm{Si}$ & -1.235807 & 3.636308 & 2.710901 \\
\hline $\mathrm{Si}$ & 0.490172 & -3.942726 & 1.612414 \\
\hline $\mathrm{C}$ & 0.064271 & -4.874551 & 0.053735 \\
\hline $\mathrm{H}$ & -1.008545 & -4.795696 & -0.170694 \\
\hline $\mathrm{H}$ & 0.614212 & -4.459052 & -0.80224 \\
\hline $\mathrm{H}$ & 0.312705 & -5.940489 & 0.134061 \\
\hline $\mathrm{C}$ & 2.277974 & -4.195458 & 2.115448 \\
\hline $\mathrm{H}$ & 2.955273 & -4.025464 & 1.267313 \\
\hline $\mathrm{H}$ & 2.577096 & -3.520662 & 2.930036 \\
\hline $\mathrm{H}$ & 2.443894 & -5.22187 & 2.468883 \\
\hline $\mathrm{C}$ & -0.65869 & -4.378981 & 3.036573 \\
\hline $\mathrm{H}$ & -0.244562 & -5.192394 & 3.646743 \\
\hline $\mathrm{H}$ & -0.833609 & -3.524488 & 3.705175 \\
\hline $\mathrm{H}$ & -1.639075 & -4.709237 & 2.668678 \\
\hline $\mathrm{C}$ & -2.117556 & 4.544389 & 1.352435 \\
\hline $\mathrm{H}$ & -2.893177 & 3.916416 & 0.891359 \\
\hline $\mathrm{H}$ & -2.590418 & 5.467151 & 1.713143 \\
\hline $\mathrm{H}$ & -1.411499 & 4.81484 & 0.555352 \\
\hline $\mathrm{C}$ & -2.184497 & 3.616296 & 4.330017 \\
\hline $\mathrm{H}$ & -3.209788 & 3.252858 & 4.18583 \\
\hline $\mathrm{H}$ & -1.697636 & 2.96763 & 5.071962 \\
\hline $\mathrm{H}$ & -2.245933 & 4.62233 & 4.766059 \\
\hline $\mathrm{C}$ & 0.508787 & 4.268544 & 2.996344 \\
\hline $\mathrm{H}$ & 1.140323 & 3.988049 & 2.140981 \\
\hline $\mathrm{H}$ & 0.522944 & 5.364539 & 3.06463 \\
\hline $\mathrm{H}$ & 0.967136 & 3.87052 & 3.911573 \\
\hline 99 & & & \\
\hline \multicolumn{4}{|c|}{$\mathrm{B}(\mathrm{S})$} \\
\hline $\mathrm{Cu}$ & -1.807911 & 0.295304 & -1.621677 \\
\hline $\mathrm{O}$ & 1.811131 & 1.02761 & 0.788545 \\
\hline $\mathrm{O}$ & -2.326973 & -0.650807 & 2.379228 \\
\hline $\mathrm{N}$ & 0.132539 & 0.993967 & -0.704707 \\
\hline $\mathrm{N}$ & -2.304677 & -0.237762 & 0.189962 \\
\hline $\mathrm{C}$ & 1.216375 & 1.726361 & -1.369368 \\
\hline $\mathrm{C}$ & 0.929396 & 3.202192 & -1.437142 \\
\hline $\mathrm{C}$ & -0.09438 & 3.838749 & -2.129715 \\
\hline $\mathrm{C}$ & -0.184098 & 5.225084 & -2.052758 \\
\hline $\mathrm{C}$ & 0.733183 & 5.95531 & -1.29447 \\
\hline $\mathrm{C}$ & 1.757729 & 5.311755 & -0.6048 \\
\hline $\mathrm{C}$ & 1.854386 & 3.925869 & -0.68528 \\
\hline $\mathrm{C}$ & 2.914232 & 3.028087 & -0.105004 \\
\hline $\mathrm{C}$ & 2.413498 & 1.61378 & -0.392971 \\
\hline $\mathrm{C}$ & 0.546874 & 0.693162 & 0.455533 \\
\hline $\mathrm{C}$ & -1.643168 & -0.277725 & 1.285602 \\
\hline $\mathrm{C}$ & -3.635956 & -1.03892 & 1.923573 \\
\hline $\mathrm{C}$ & -3.752249 & -2.550468 & 1.672708 \\
\hline $\mathrm{C}$ & -4.51842 & -2.621383 & 0.378013 \\
\hline $\mathrm{C}$ & -5.158735 & -3.704005 & -0.209622 \\
\hline $\mathrm{C}$ & -5.776435 & -3.526264 & -1.447776 \\
\hline $\mathrm{C}$ & -5.763049 & -2.285892 & -2.08538 \\
\hline $\mathrm{C}$ & -5.120795 & -1.197232 & -1.497858 \\
\hline $\mathrm{C}$ & -4.497506 & -1.385533 & -0.272601 \\
\hline $\mathrm{C}$ & -3.741667 & -0.38924 & 0.544382 \\
\hline $\mathrm{H}$ & 1.397585 & 1.276043 & -2.356548 \\
\hline $\mathrm{H}$ & -0.806392 & 3.265354 & -2.72295 \\
\hline $\mathrm{H}$ & -0.982986 & 5.741148 & -2.581742 \\
\hline $\mathrm{H}$ & 0.64606 & 7.039727 & -1.241604 \\
\hline $\mathrm{H}$ & 2.476123 & 5.884483 & -0.017053 \\
\hline $\mathrm{H}$ & 3.082498 & 3.168963 & 0.972517 \\
\hline $\mathrm{H}$ & 3.88556 & 3.193916 & -0.59405 \\
\hline $\mathrm{H}$ & 3.201916 & 0.923518 & -0.715438 \\
\hline $\mathrm{H}$ & -4.357654 & -0.666076 & 2.655434 \\
\hline $\mathrm{H}$ & -4.221032 & -3.092969 & 2.503551 \\
\hline $\mathrm{H}$ & -2.734413 & -2.960456 & 1.549369 \\
\hline $\mathrm{H}$ & -5.18556 & -4.674244 & 0.286029 \\
\hline
\end{tabular}




\begin{tabular}{|c|c|c|c|}
\hline $\mathrm{H}$ & -6.286004 & -4.365631 & -1.918216 \\
\hline $\mathrm{H}$ & -6.262055 & -2.16647 & -3.045156 \\
\hline $\mathrm{H}$ & -5.109746 & -0.219382 & -1.98099 \\
\hline $\mathrm{H}$ & -4.171526 & 0.622573 & 0.523882 \\
\hline $\mathrm{C}$ & -0.220845 & 0.09159 & 1.602744 \\
\hline $\mathrm{C}$ & -0.306688 & 1.216069 & 2.663192 \\
\hline $\mathrm{C}$ & 0.462657 & -1.16249 & 2.162372 \\
\hline $\mathrm{H}$ & -0.813308 & 0.826997 & 3.56218 \\
\hline $\mathrm{H}$ & 0.720892 & 1.505912 & 2.944163 \\
\hline $\mathrm{H}$ & 1.551696 & -0.995889 & 2.207429 \\
\hline $\mathrm{C}$ & -2.78747 & 1.927371 & -1.320956 \\
\hline $\mathrm{N}$ & -3.455436 & 2.830552 & -0.997645 \\
\hline $\mathrm{C}$ & -1.615195 & 0.616389 & -3.425726 \\
\hline $\mathrm{N}$ & -1.38155 & 0.95452 & -4.518714 \\
\hline C & 0.229538 & -1.468798 & -2.465346 \\
\hline $\mathrm{C}$ & -1.017691 & -1.431465 & -2.139609 \\
\hline $\mathrm{C}$ & -1.970629 & -2.5813 & -2.138774 \\
\hline $\mathrm{H}$ & -2.362445 & -2.779476 & -1.131686 \\
\hline $\mathrm{H}$ & -1.47431 & -3.489678 & -2.506779 \\
\hline $\mathrm{H}$ & -2.835834 & -2.376476 & -2.784487 \\
\hline $\mathrm{C}$ & 1.474473 & -1.431373 & -2.914472 \\
\hline $\mathrm{H}$ & 1.610304 & -1.301642 & -3.9939 \\
\hline $\mathrm{C}$ & 2.695831 & -1.504344 & -2.119591 \\
\hline $\mathrm{C}$ & 2.676791 & -1.755829 & -0.739107 \\
\hline $\mathrm{C}$ & 3.933876 & -1.276664 & -2.736066 \\
\hline $\mathrm{C}$ & 3.850825 & -1.7561 & 0.000272 \\
\hline $\mathrm{H}$ & 1.719641 & -1.939871 & -0.245499 \\
\hline $\mathrm{C}$ & 5.116097 & -1.281592 & -2.006528 \\
\hline $\mathrm{H}$ & 3.965443 & -1.083009 & -3.808447 \\
\hline $\mathrm{C}$ & 5.065142 & -1.517234 & -0.637916 \\
\hline $\mathrm{H}$ & 3.832091 & -1.933475 & 1.07486 \\
\hline $\mathrm{H}$ & 6.071996 & -1.100881 & -2.493258 \\
\hline $\mathrm{Br}$ & 6.667164 & -1.516005 & 0.374087 \\
\hline $\mathrm{H}$ & 0.101424 & -1.330725 & 3.192804 \\
\hline $\mathrm{O}$ & -1.018799 & 2.253214 & 2.074288 \\
\hline $\mathrm{O}$ & 0.139604 & -2.23694 & 1.323607 \\
\hline $\mathrm{Si}$ & -0.996154 & 3.842913 & 2.637177 \\
\hline $\mathrm{Si}$ & 0.357812 & -3.847971 & 1.763857 \\
\hline $\mathrm{C}$ & -0.083643 & -4.778077 & 0.208928 \\
\hline $\mathrm{H}$ & -1.149961 & -4.664107 & -0.031848 \\
\hline $\mathrm{H}$ & 0.492318 & -4.383777 & -0.640523 \\
\hline $\mathrm{H}$ & 0.129292 & -5.851177 & 0.295033 \\
\hline $\mathrm{C}$ & 2.140866 & -4.123806 & 2.266778 \\
\hline $\mathrm{H}$ & 2.819588 & -3.98341 & 1.414904 \\
\hline $\mathrm{H}$ & 2.456294 & -3.442251 & 3.069418 \\
\hline $\mathrm{H}$ & 2.283825 & -5.147476 & 2.63809 \\
\hline $\mathrm{C}$ & -0.794057 & -4.249577 & 3.198284 \\
\hline $\mathrm{H}$ & -0.34699 & -4.984671 & 3.880014 \\
\hline $\mathrm{H}$ & -1.033839 & -3.35506 & 3.790751 \\
\hline $\mathrm{H}$ & -1.744944 & -4.66622 & 2.840655 \\
\hline $\mathrm{C}$ & -1.772159 & 4.855511 & 1.28993 \\
\hline $\mathrm{H}$ & -2.532582 & 4.274644 & 0.748795 \\
\hline $\mathrm{H}$ & -2.24183 & 5.766403 & 1.68392 \\
\hline $\mathrm{H}$ & -1.014588 & 5.154545 & 0.552917 \\
\hline $\mathrm{C}$ & -1.953168 & 3.904301 & 4.251405 \\
\hline $\mathrm{H}$ & -3.005297 & 3.632196 & 4.09929 \\
\hline $\mathrm{H}$ & -1.531185 & 3.217356 & 4.998535 \\
\hline $\mathrm{H}$ & -1.929458 & 4.912924 & 4.685494 \\
\hline $\mathrm{C}$ & 0.79684 & 4.315094 & 2.940707 \\
\hline $\mathrm{H}$ & 1.409426 & 3.994041 & 2.085603 \\
\hline $\mathrm{H}$ & 0.898405 & 5.405866 & 3.020704 \\
\hline $\mathrm{H}$ & 1.217587 & 3.873345 & 3.853809 \\
\hline 99 & & & \\
\hline \multicolumn{4}{|c|}{$\mathrm{TSB}(\mathrm{S}) \_\mathrm{C}$} \\
\hline $\mathrm{Cu}$ & -1.821565 & 0.2506 & -1.603 \\
\hline $\mathrm{O}$ & 1.801206 & 1.096498 & 0.7556 \\
\hline $\mathrm{O}$ & -2.281482 & -0.605595 & 2.479118 \\
\hline $\mathrm{N}$ & 0.100032 & 0.971139 & -0.70654 \\
\hline $\mathrm{N}$ & -2.287506 & -0.21591 & 0.279839 \\
\hline $\mathrm{C}$ & 1.159056 & 1.700627 & -1.413504 \\
\hline $\mathrm{C}$ & 0.842505 & 3.167354 & -1.527104 \\
\hline $\mathrm{C}$ & -0.21571 & 3.758435 & -2.207106 \\
\hline $\mathrm{C}$ & -0.333347 & 5.144318 & -2.17646 \\
\hline $\mathrm{C}$ & 0.592313 & 5.919179 & -1.474789 \\
\hline $\mathrm{C}$ & 1.649512 & 5.320897 & -0.793547 \\
\hline $\mathrm{C}$ & 1.772252 & 3.935185 & -0.826964 \\
\hline $\mathrm{C}$ & 2.858208 & 3.07803 & -0.234283 \\
\hline $\mathrm{C}$ & 2.374503 & 1.645975 & -0.456444 \\
\hline $\mathrm{C}$ & 0.537259 & 0.724856 & 0.45987 \\
\hline $\mathrm{C}$ & -1.617833 & -0.240274 & 1.366561 \\
\hline
\end{tabular}

\begin{tabular}{|c|c|c|c|}
\hline $\mathrm{C}$ & -3.587235 & -1.022299 & 2.03667 \\
\hline $\mathrm{C}$ & -3.675395 & -2.538603 & 1.796079 \\
\hline $\mathrm{C}$ & -4.443749 & -2.636392 & 0.504181 \\
\hline $\mathrm{C}$ & -5.060593 & -3.737743 & -0.074147 \\
\hline $\mathrm{C}$ & -5.685301 & -3.58281 & -1.311943 \\
\hline $\mathrm{C}$ & -5.699048 & -2.347477 & -1.959518 \\
\hline $\mathrm{C}$ & -5.078838 & -1.240271 & -1.382692 \\
\hline $\mathrm{C}$ & -4.452186 & -1.405374 & -0.155572 \\
\hline $\mathrm{C}$ & -3.714147 & -0.387149 & 0.651172 \\
\hline $\mathrm{H}$ & 1.334153 & 1.21864 & -2.387019 \\
\hline $\mathrm{H}$ & -0.944871 & 3.150419 & -2.741502 \\
\hline $\mathrm{H}$ & -1.162339 & 5.623789 & -2.69333 \\
\hline $\mathrm{H}$ & 0.484096 & 7.002799 & -1.457031 \\
\hline $\mathrm{H}$ & 2.370938 & 5.928233 & -0.245697 \\
\hline $\mathrm{H}$ & 3.043777 & 3.264387 & 0.833419 \\
\hline $\mathrm{H}$ & 3.817533 & 3.238629 & -0.748111 \\
\hline $\mathrm{H}$ & 3.169314 & 0.957566 & -0.766528 \\
\hline $\mathrm{H}$ & -4.310174 & -0.659556 & 2.77243 \\
\hline $\mathrm{H}$ & -4.129531 & -3.085133 & 2.632587 \\
\hline $\mathrm{H}$ & -2.650886 & -2.930716 & 1.670095 \\
\hline $\mathrm{H}$ & -5.065069 & -4.704625 & 0.428967 \\
\hline $\mathrm{H}$ & -6.177873 & -4.436583 & -1.774683 \\
\hline $\mathrm{H}$ & -6.201322 & -2.246481 & -2.919777 \\
\hline $\mathrm{H}$ & -5.086082 & -0.265989 & -1.873288 \\
\hline $\mathrm{H}$ & -4.16543 & 0.615104 & 0.620719 \\
\hline $\mathrm{C}$ & -0.191774 & 0.143514 & 1.643787 \\
\hline $\mathrm{C}$ & -0.256107 & 1.284246 & 2.687744 \\
\hline $\mathrm{C}$ & 0.518613 & -1.096507 & 2.198032 \\
\hline $\mathrm{H}$ & -0.72629 & 0.903505 & 3.609852 \\
\hline $\mathrm{H}$ & 0.774541 & 1.597994 & 2.928646 \\
\hline $\mathrm{H}$ & 1.604901 & -0.91071 & 2.23916 \\
\hline $\mathrm{C}$ & -2.896268 & 1.845904 & -1.475402 \\
\hline $\mathrm{N}$ & -3.610428 & 2.736055 & -1.219377 \\
\hline $\mathrm{C}$ & -1.708453 & 0.036986 & -3.420814 \\
\hline $\mathrm{N}$ & -1.762976 & 0.169211 & -4.582675 \\
\hline $\mathrm{C}$ & 0.261775 & -1.515386 & -2.484249 \\
\hline $\mathrm{C}$ & -1.011871 & -1.430563 & -2.236475 \\
\hline $\mathrm{C}$ & -1.938362 & -2.604929 & -2.13895 \\
\hline $\mathrm{H}$ & -2.315743 & -2.730574 & -1.114603 \\
\hline $\mathrm{H}$ & -1.418148 & -3.524977 & -2.43586 \\
\hline $\mathrm{H}$ & -2.807982 & -2.469441 & -2.794849 \\
\hline $\mathrm{C}$ & 1.508178 & -1.556289 & -2.915059 \\
\hline $\mathrm{H}$ & 1.652493 & -1.569622 & -4.001868 \\
\hline $\mathrm{C}$ & 2.728111 & -1.553223 & -2.110052 \\
\hline $\mathrm{C}$ & 2.701575 & -1.706747 & -0.716882 \\
\hline $\mathrm{C}$ & 3.965748 & -1.36047 & -2.736801 \\
\hline $\mathrm{C}$ & 3.87171 & -1.645787 & 0.026821 \\
\hline $\mathrm{H}$ & 1.743205 & -1.866334 & -0.21748 \\
\hline $\mathrm{C}$ & 5.143859 & -1.302599 & -2.002667 \\
\hline $\mathrm{H}$ & 4.001902 & -1.244929 & -3.820224 \\
\hline $\mathrm{C}$ & 5.08694 & -1.44156 & -0.621053 \\
\hline $\mathrm{H}$ & 3.848327 & -1.748391 & 1.11093 \\
\hline $\mathrm{H}$ & 6.100979 & -1.14876 & -2.496053 \\
\hline $\mathrm{Br}$ & 6.683374 & -1.356323 & 0.39543 \\
\hline $\mathrm{H}$ & 0.165273 & -1.27337 & 3.229581 \\
\hline $\mathrm{O}$ & -1.006518 & 2.295911 & 2.104866 \\
\hline $\mathrm{O}$ & 0.211767 & -2.175317 & 1.357867 \\
\hline $\mathrm{Si}$ & -1.015781 & 3.910633 & 2.579758 \\
\hline $\mathrm{Si}$ & 0.448115 & -3.782622 & 1.804479 \\
\hline $\mathrm{C}$ & -0.003275 & -4.724022 & 0.259066 \\
\hline $\mathrm{H}$ & -1.071436 & -4.609003 & 0.027015 \\
\hline $\mathrm{H}$ & 0.567174 & -4.340295 & -0.59907 \\
\hline $\mathrm{H}$ & 0.207123 & -5.796948 & 0.353456 \\
\hline $\mathrm{C}$ & 2.238202 & -4.041521 & 2.292132 \\
\hline $\mathrm{H}$ & 2.911366 & -3.888813 & 1.43805 \\
\hline $\mathrm{H}$ & 2.550329 & -3.361418 & 3.097377 \\
\hline $\mathrm{H}$ & 2.393546 & -5.065699 & 2.657174 \\
\hline $\mathrm{C}$ & -0.682964 & -4.192524 & 3.253126 \\
\hline $\mathrm{H}$ & -0.206657 & -4.89719 & 3.947162 \\
\hline $\mathrm{H}$ & -0.952055 & -3.295533 & 3.829003 \\
\hline $\mathrm{H}$ & -1.620189 & -4.6497 & 2.909374 \\
\hline $\mathrm{C}$ & -1.857629 & 4.816189 & 1.193188 \\
\hline $\mathrm{H}$ & -2.57794 & 4.170043 & 0.670423 \\
\hline $\mathrm{H}$ & -2.388004 & 5.709333 & 1.548637 \\
\hline $\mathrm{H}$ & -1.117516 & 5.136277 & 0.447382 \\
\hline $\mathrm{C}$ & -1.9271 & 4.03622 & 4.216026 \\
\hline $\mathrm{H}$ & -2.975961 & 3.731172 & 4.111663 \\
\hline $\mathrm{H}$ & -1.46493 & 3.397487 & 4.982021 \\
\hline $\mathrm{H}$ & -1.915541 & 5.065747 & 4.598306 \\
\hline $\mathrm{C}$ & 0.769455 & 4.450795 & 2.800681 \\
\hline
\end{tabular}




\section{References}

(1) Li, J.; Zhang, Z.; Wu, L.; Zhang, W.; Chen, P.; Lin, Z.; Liu, G. Nature 2019, 574, $516-521$

(2) Kuno, A.; Saino, N.; Kamachi, T.; Okamoto, S. Tetrahedron Lett. 2006, 47, 2591-2594.

(3) Larsen, C. H.; Anderson, K. W.; Tundel, R. E.; Buchwald, S. L. Synlett 2006, 18, 2941-2946.

(4) Zhang, W.-W.; Zhang, X.-G.; Li, J.-H. J. Org. Chem. 2010, 75, 5259-5264.

(5) Egi, M.; Kawai, T.; Umemura, M.; Akai, S. J. Org. Chem. 2012, 77, 7092-7097.

(6) Xu, S.; Zhang, Z.; Han, C.; Hu, W.; Xiao, T.; Yuan, Y.; Zhao, J. J. Org. Chem. 2019, $84,12192-12197$.

(7) Dong, X.-Y.; Zhang, Y.-F.; Ma, C.-L.; Gu, Q.-S.; Wang, F.-L.; Li, Z.-L.; Jiang, S.-P.; Liu, X.-Y. Nat. Chem. 2019, 11, 1158-1166.

(8) Zeng, Y.; Chiou, M.-F.; Zhu, X.; Cao, J.; Lv, W.; Jian, W.; Li, Y.; Zhang, X.; Bao, H. J. Am. Chem. Soc. 2020, 142, 18014-18021.

(9) Falivene, L.; Cao, Z.; Petta, A.; Serra, L.; Poater, A.; Oliva, R.; Scarano V.; Cavallo L. Nat. Chem. 2019, 11, 872-879.

(10) Frisch, M. J.; Trucks, G. W.; Schlegel, H. B.; Scuseria, G. E.; Robb, M. A.; Cheeseman, J. R.; Scalmani, G.; Barone, V.; Petersson, G. A.; Nakatsuji, H.; Li, X.; Caricato, M.; Marenich, A. V.; Bloino, J.; Janesko, B. G.; Gomperts, R.; Mennucci, B.; Hratchian, H. P.; Ortiz, J. V.; Izmaylov, A. F.; Sonnenberg, J. L.; Williams-Young, D.; Ding, F.; Lipparini, F.; Egidi, F.; Goings, J.; Peng, B.; Petrone, A.; Henderson, T.; Ranasinghe, D.; Zakrzewski, V. G.; Gao, J.; Rega, N.; Zheng, G.; Liang, W.; Hada, M.; Ehara, M.; Toyota, K.; Fukuda, R.; Hasegawa, J.; Ishida, M.; Nakajima, T.; Honda, Y.; Kitao, O.; Nakai, H.; Vreven, T.; Throssell, K.; Montgomery, J. A.; Jr., Peralta, J. E.; Ogliaro, F.; Bearpark, M. J.; Heyd, J. J.; Brothers, E. N.; Kudin, K. N.; Staroverov, V. N.; Keith, T. A.; Kobayashi, R.; Normand, J.; Raghavachari, K.; Rendell, A. P.; Burant, 
J. C.; Iyengar, S. S.; Tomasi, J.; Cossi, M.; Millam, J. M.; Klene, M.; Adamo, C.;

Cammi, R.; Ochterski, J. W.; Martin, R. L.; Morokuma, K.; Farkas, O.; Foresman, J.

B.; Fox, D. J. Gaussian 16, Revision C.01, Gaussian, Inc., Wallingford CT, 2019.

(11) Stephens, P. J.; Devlin, F. J.; Chabalowski, C. F.; Frisch, M. J. The Journal of Physical Chemistry, 1994 98, 11623-11627.

(12) Weigend, F.; Ahlrichs, R. Phys. Chem. Chem. Phys., 2005, 7, 3297-3305.

(13) (a) Grimme, S. Journal of Computational Chemistry, 2004, 25, 1463-1473. (b)

Caldeweyher, E.; Bannwarth, C.; Grimme, S. J. Chem. Phys. 2017, 147, 034112.

(14) Grimme, S.; Antony, J.; Ehrlich, S.; Krieg, H. J. Chem. Phys. 2010, 132, 154104-154119.

(15) Fukui, K. Acc. Chem. Res. 1981, 14, 363-368.

(16) (a) Neese, F. "The ORCA program system" Wiley Interdisciplinary Reviews: Computational Molecular Science, 2012, 2, 73-78. (b) Neese, F. "Software update: the ORCA program system, version 4.0" Wiley Interdisciplinary Reviews: Computational Molecular Science, 2017, 8, 1327.

(17) Zhao, Y.; Truhlar, D. G. Theor. Chem. Account, 2008, 120, 215-241.

(18) Weigend, F. Phys. Chem. Chem. Phys. 2006, 8, 1057-1065.

(19) Raghavachari, K.; Trucks, G.W. J. Chem. Phys. 1989, 91, 1062-1065.

(20) (a) Petersson, G.A.; Bennett, A.; Tensfeldt, T.G.; Al-Laham, M.A.; Shirley, W.A.; Mantzaris, J. J. Chem. Phys. 1988. 89, 2193-2218. (b) Petersson, G.A.; Al-Laham, M.A. J. Chem. Phys. 1991, 94, 6081-6090.

(21) Marenich, A. V.; Cramer, C. J.; Truhlar, D. G. J. Phys. Chem. B. 2009, 113, 6378-6396.

\section{NMR Spectra of Starting Materials and Products}




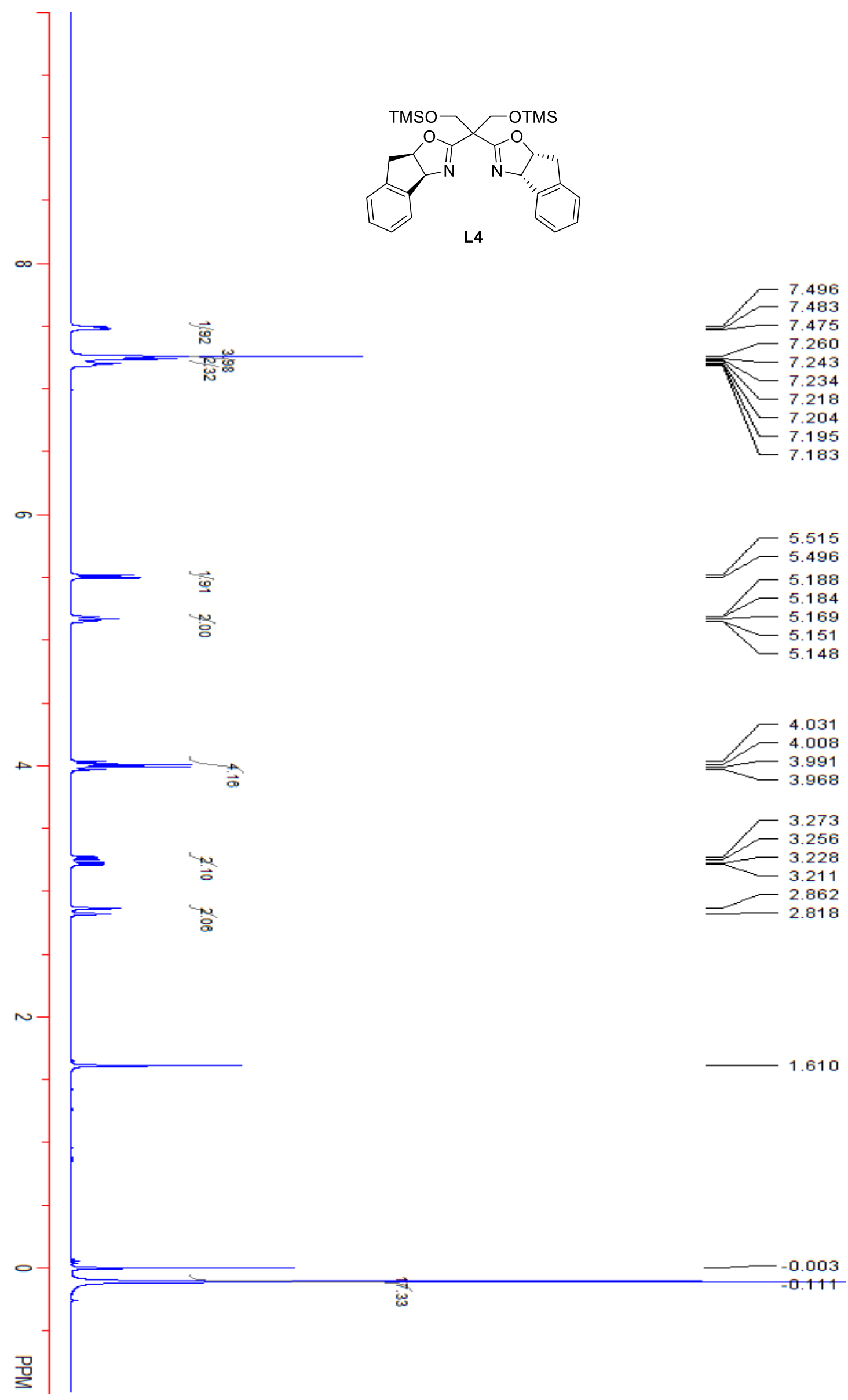




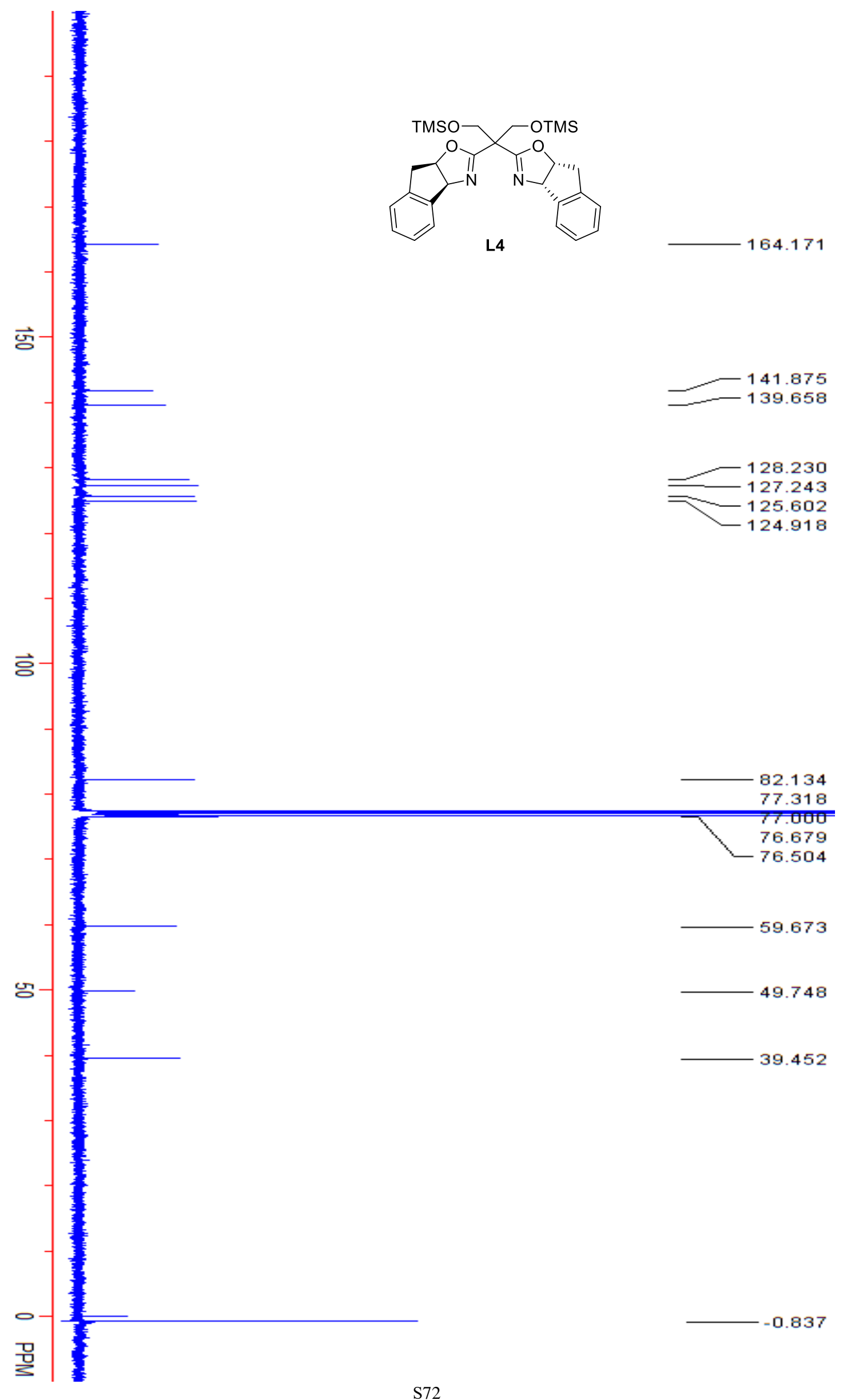



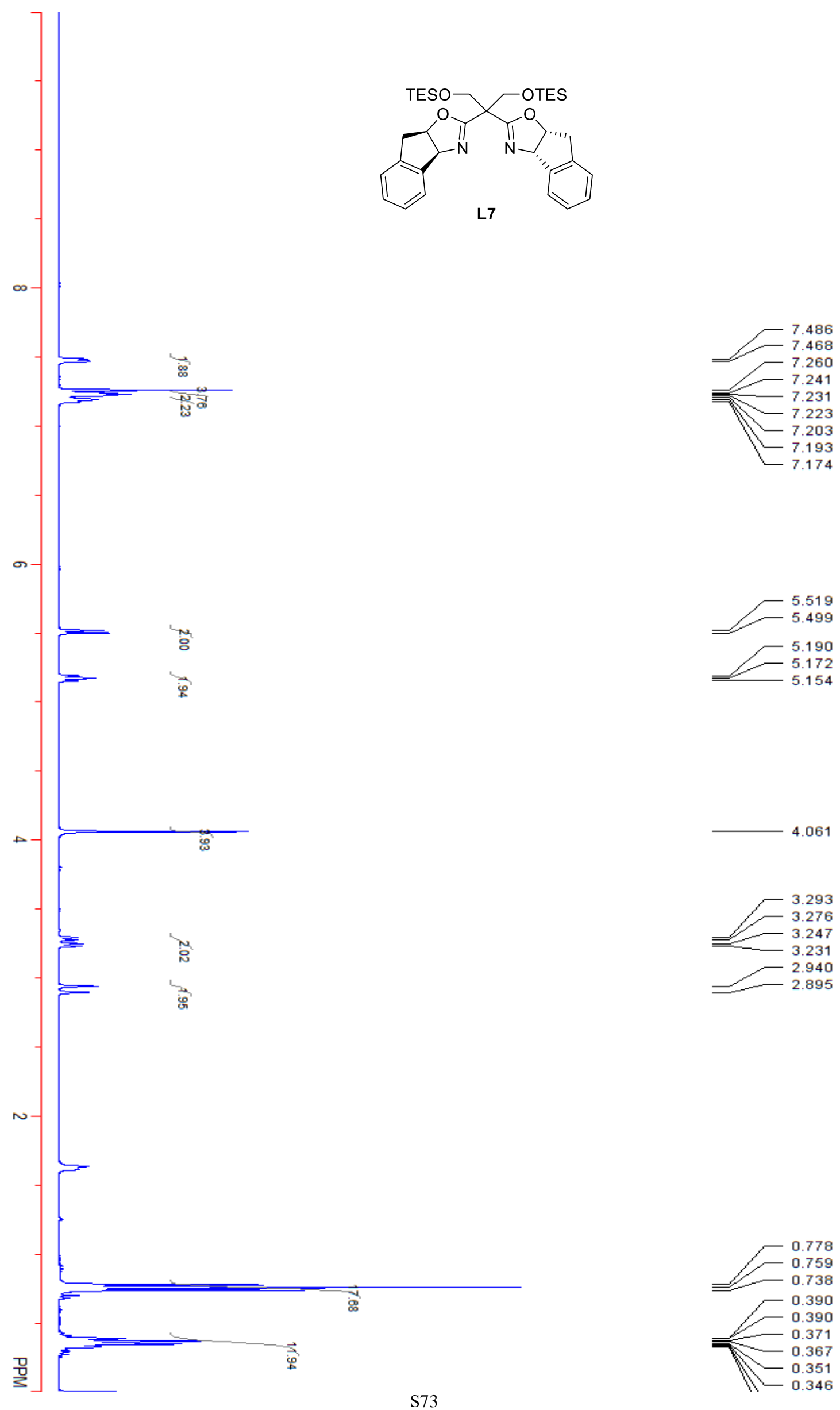

4.061
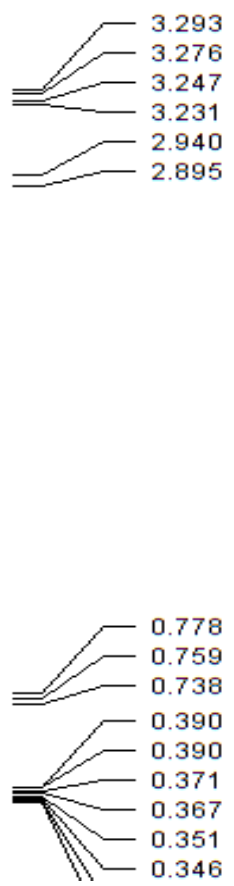

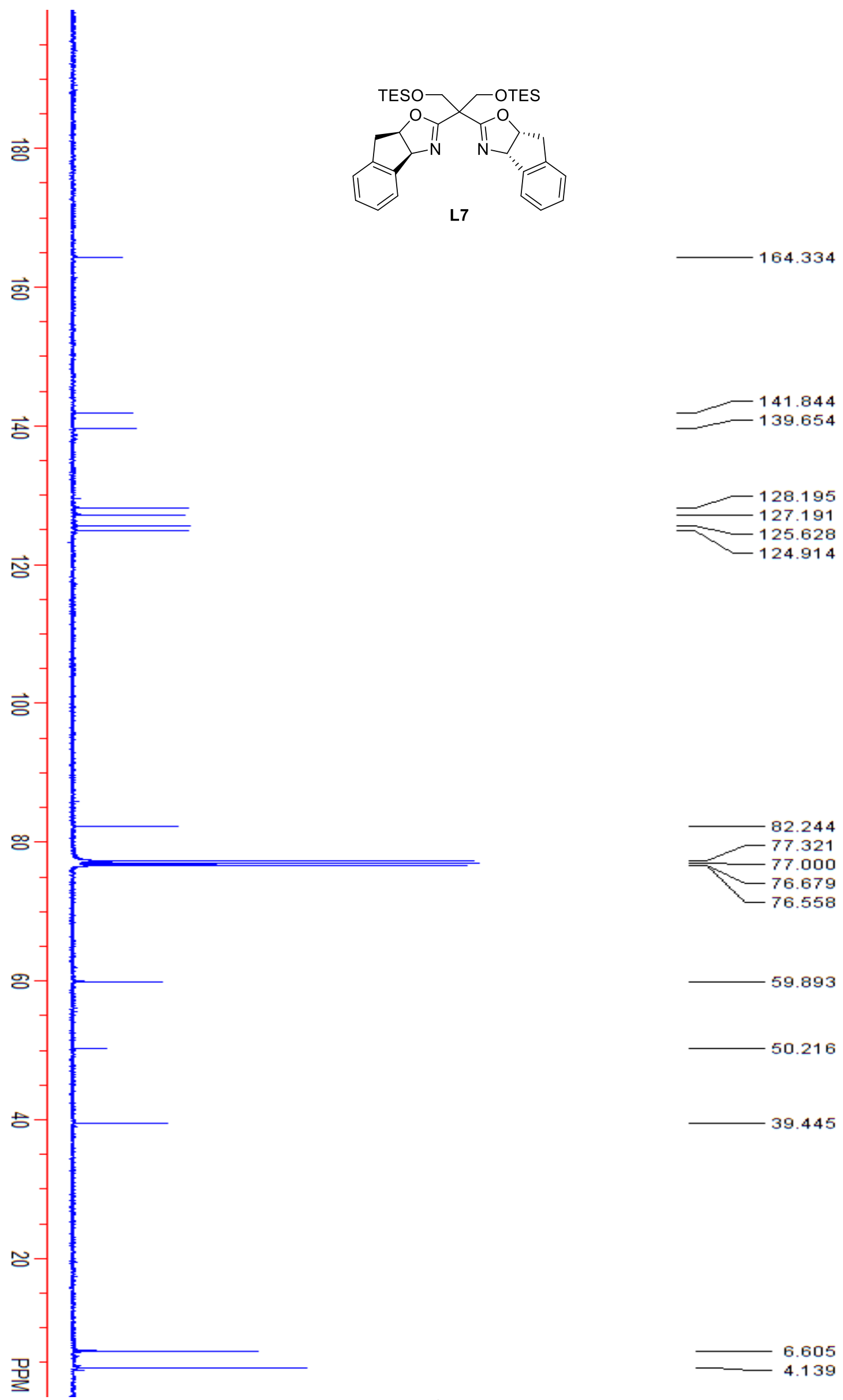

59.893

50. 216

39.445 


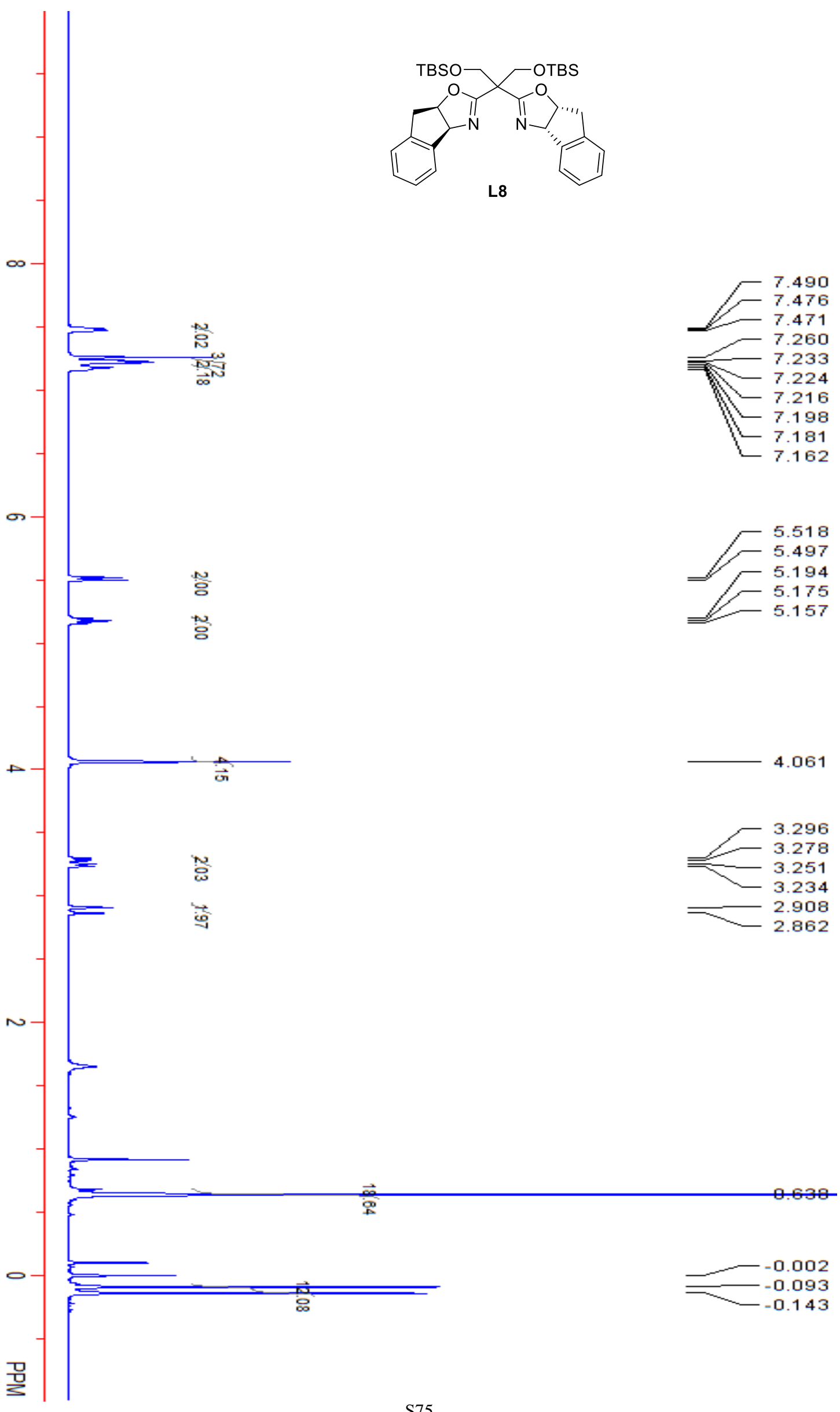




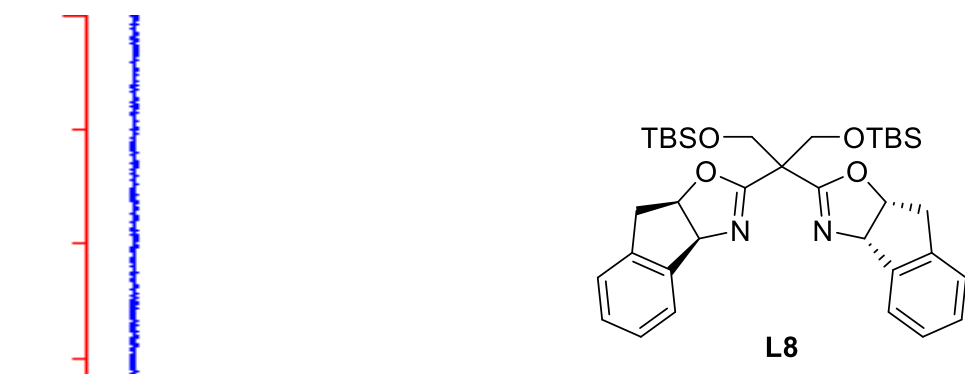

164.214

ज्ञ

ㅎㅇㅇ

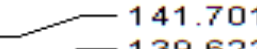

139.623
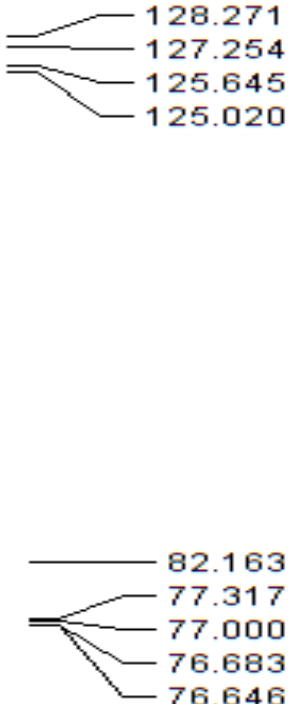

60.062

50.222

39.535

25.509

17.809

몰

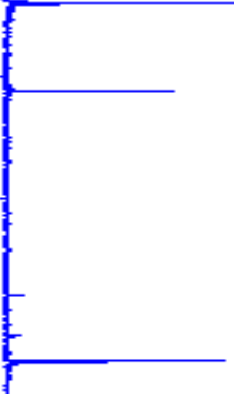

5.810 


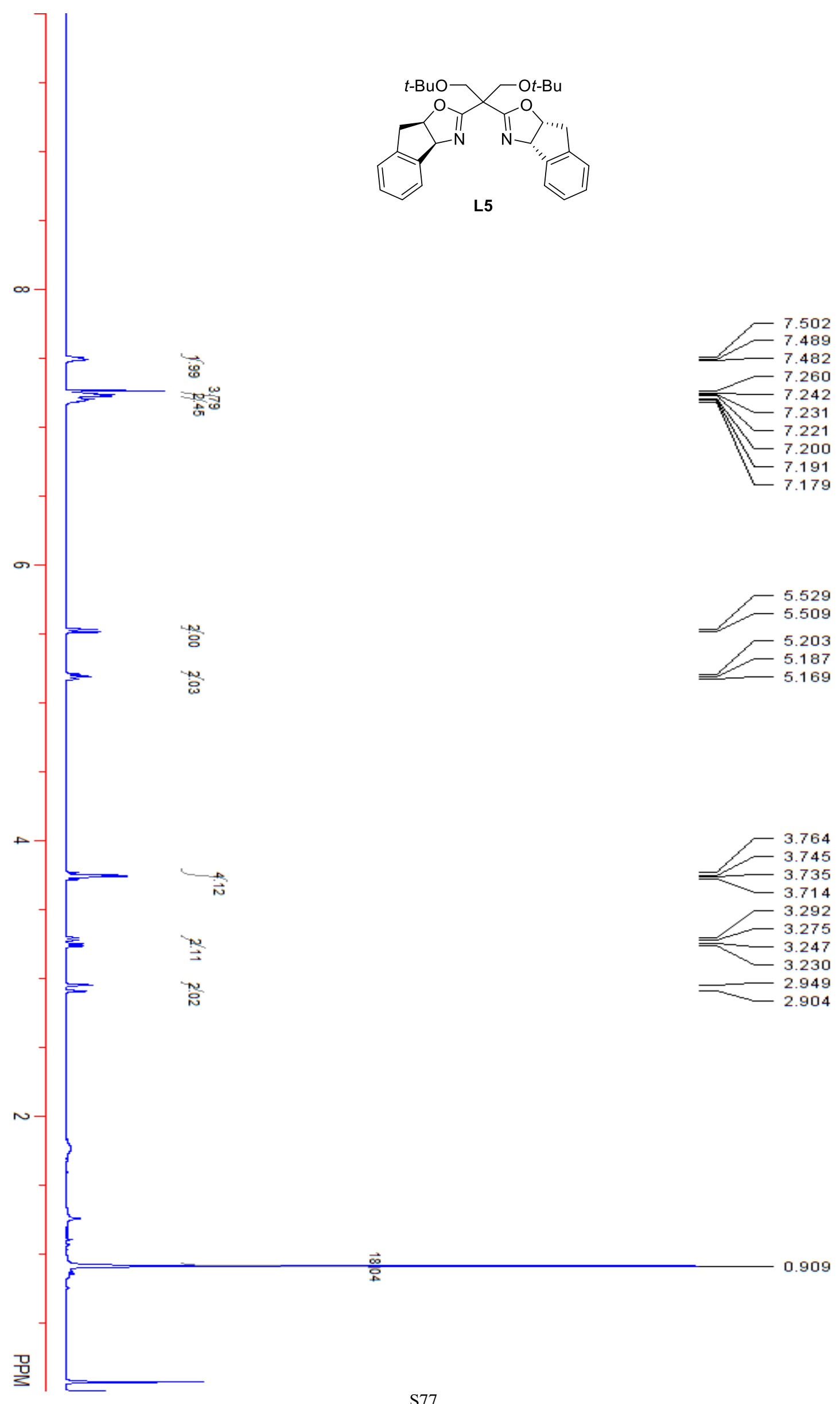




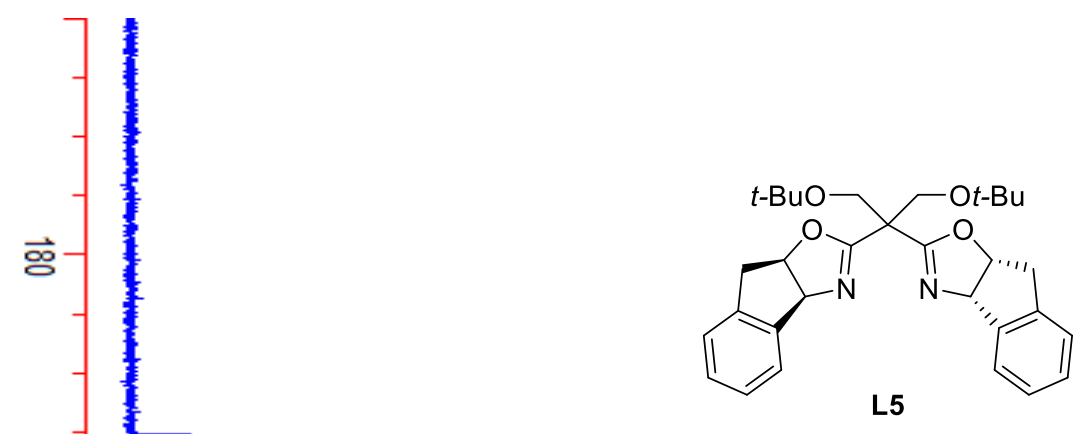
164.825

홍

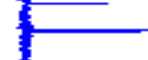
139.733
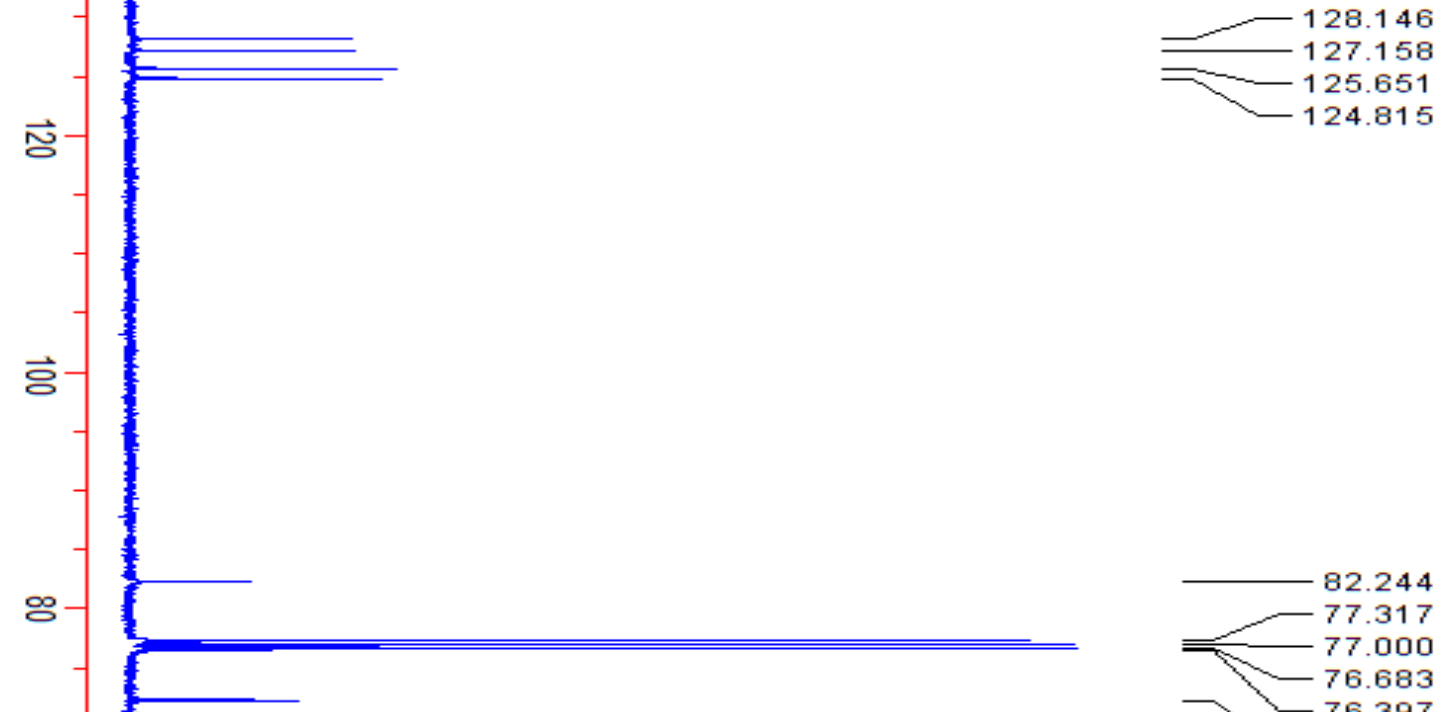

$8-$
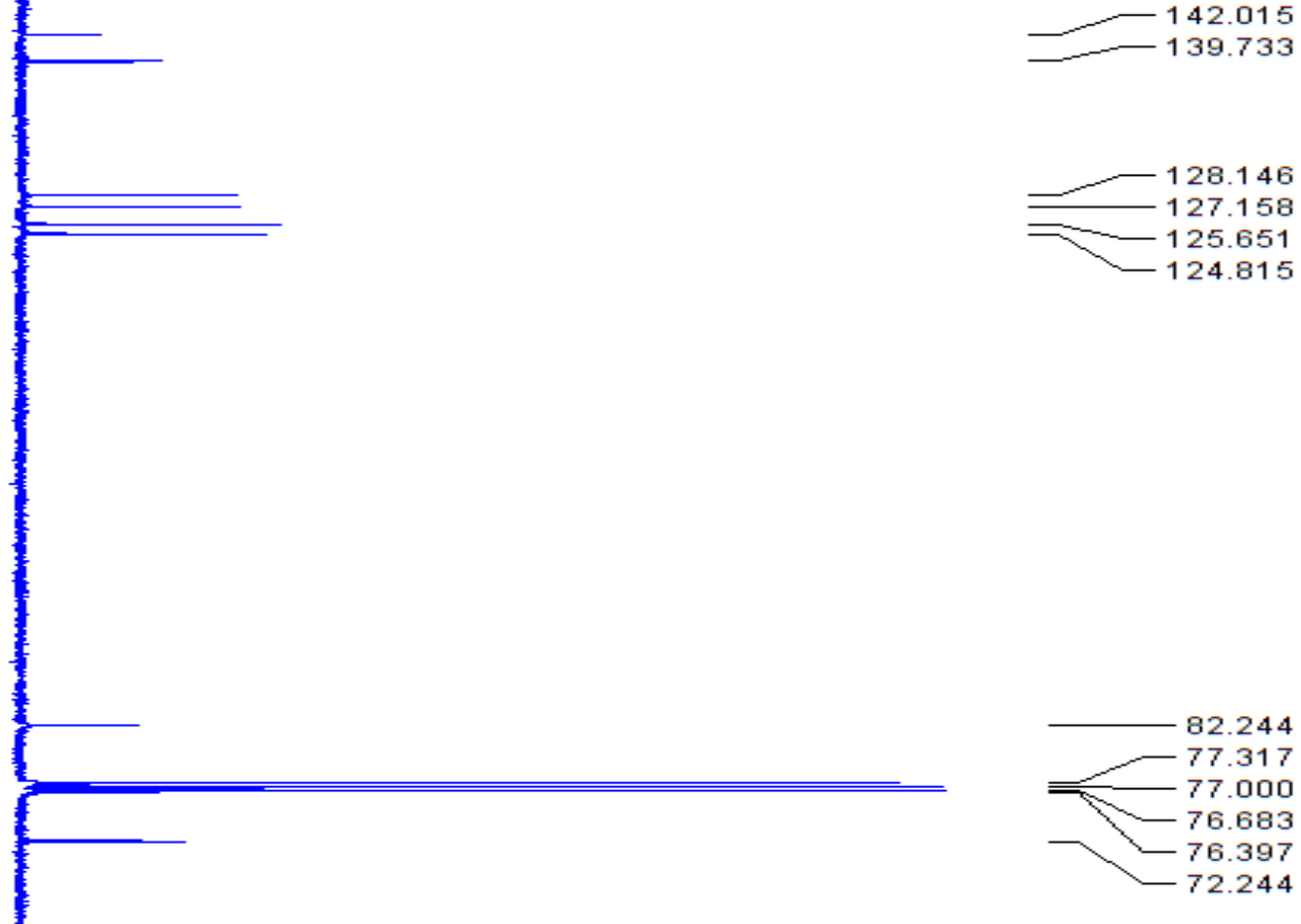

59.164

47.801

D

27.165

긍

몰

㔔 


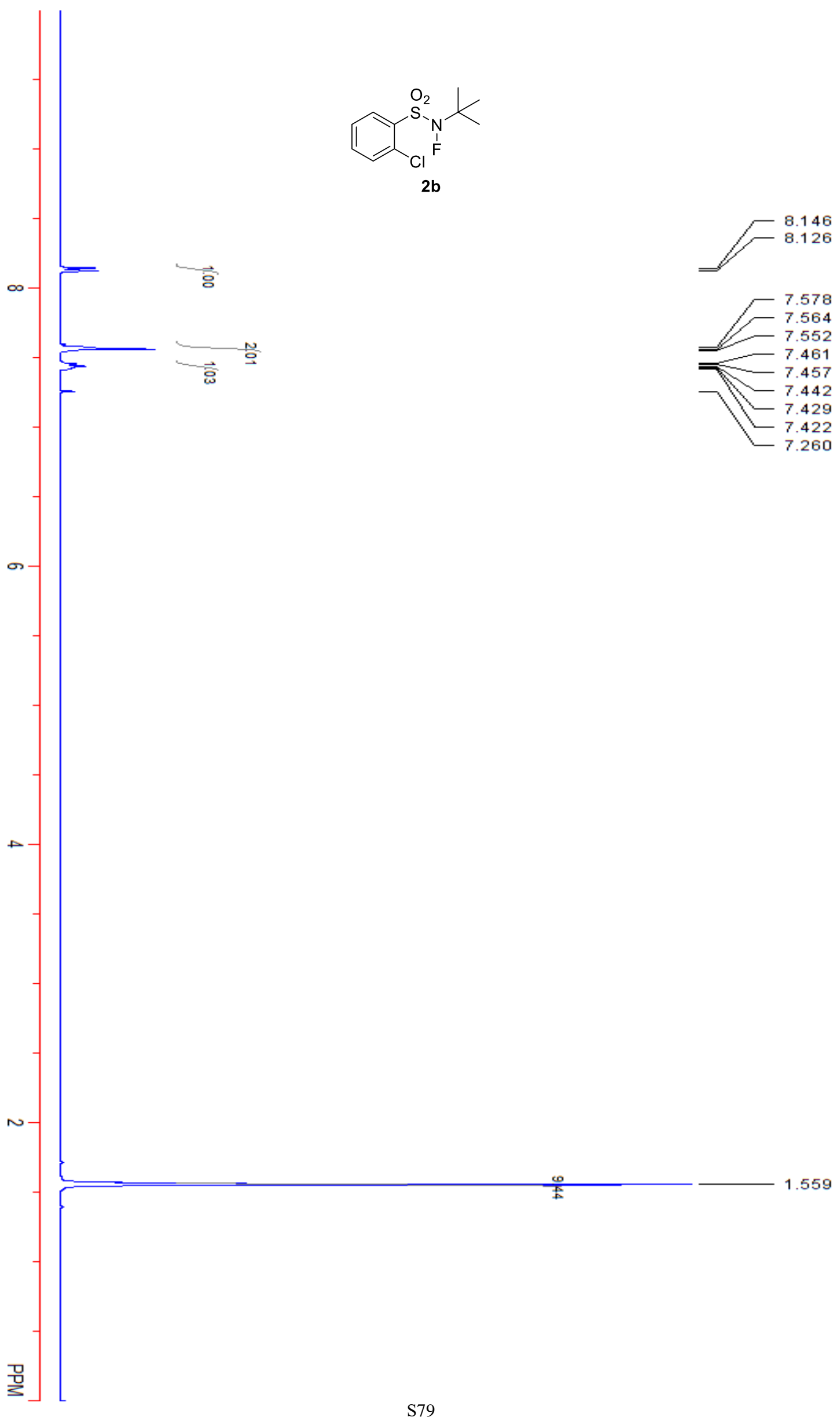




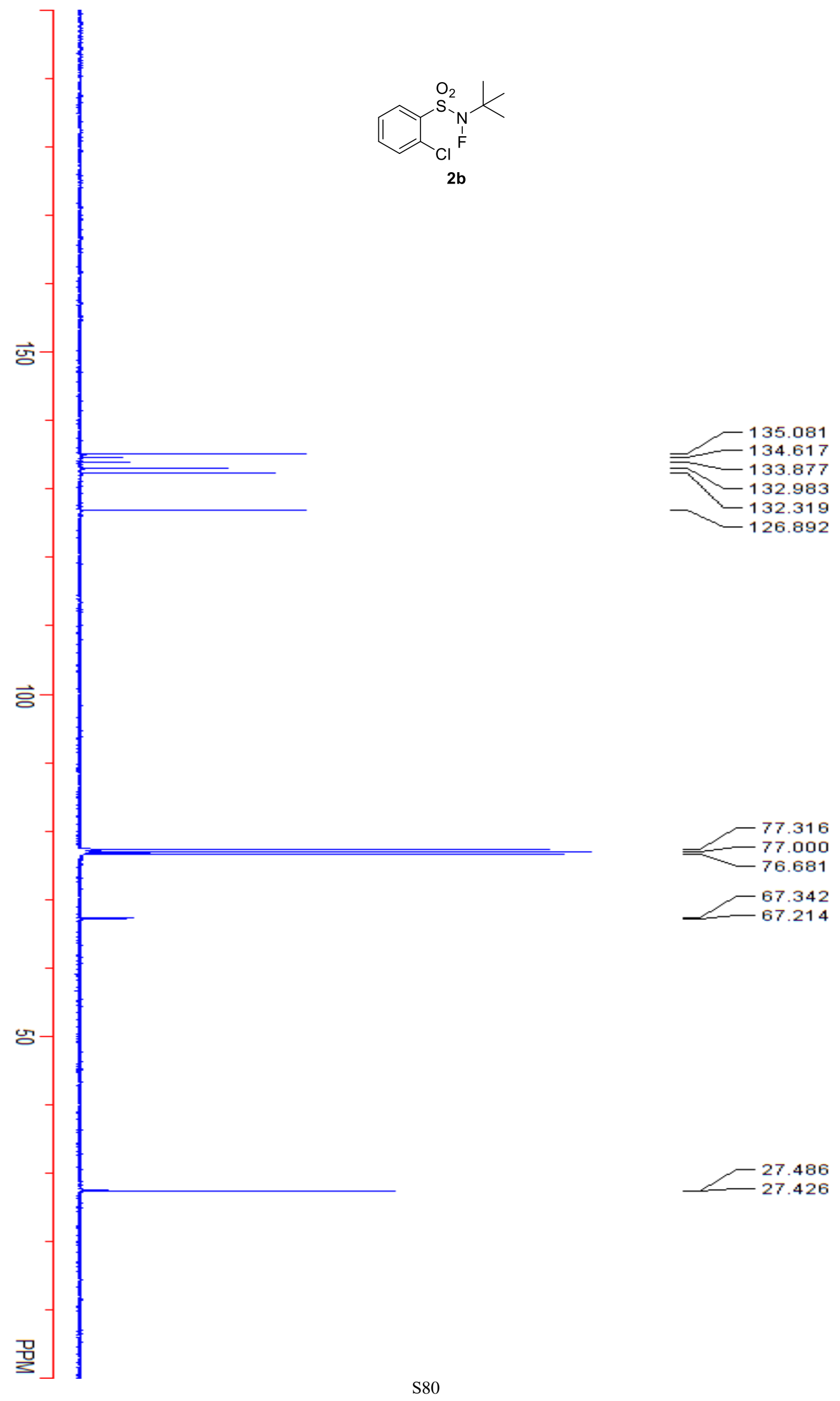




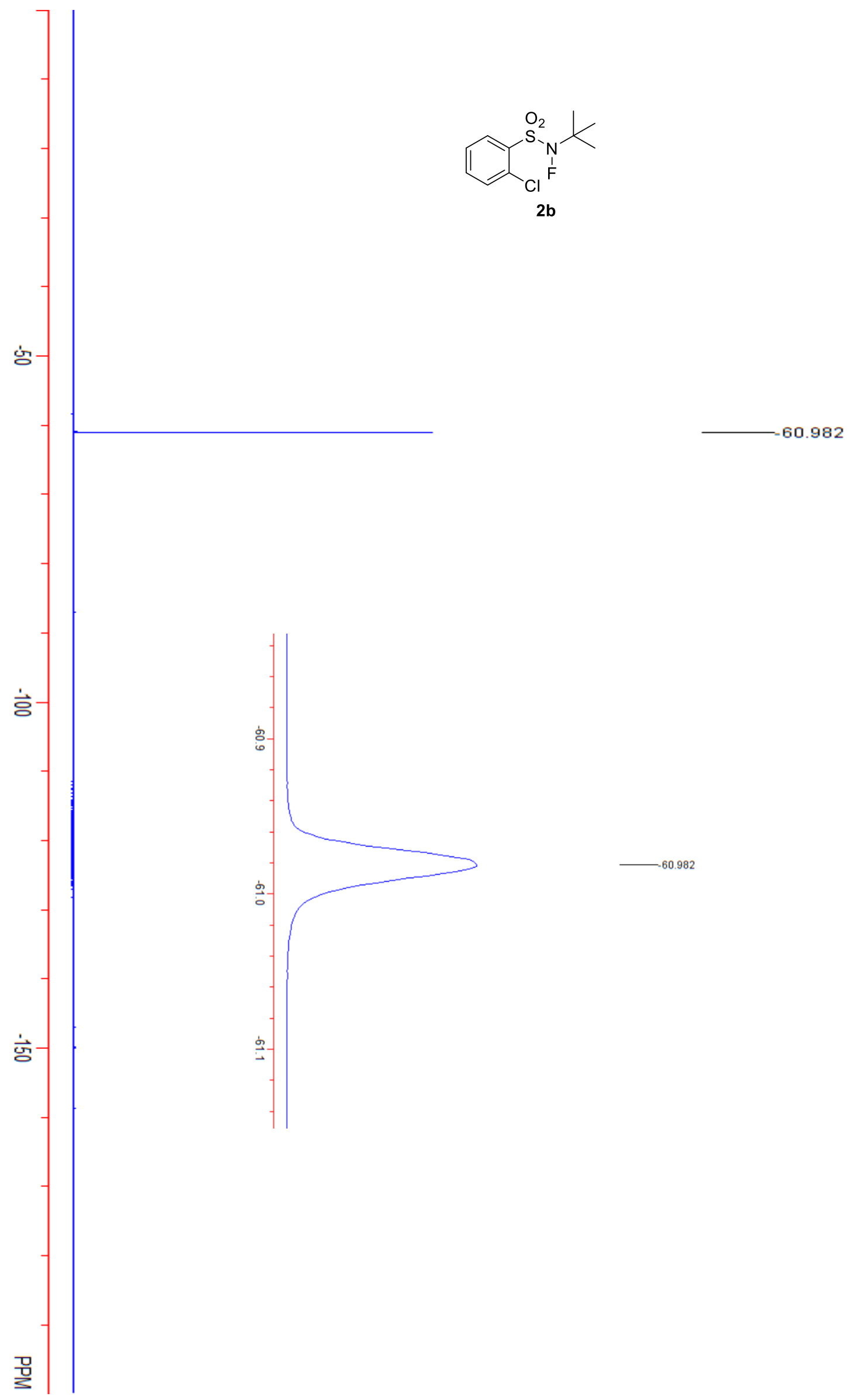




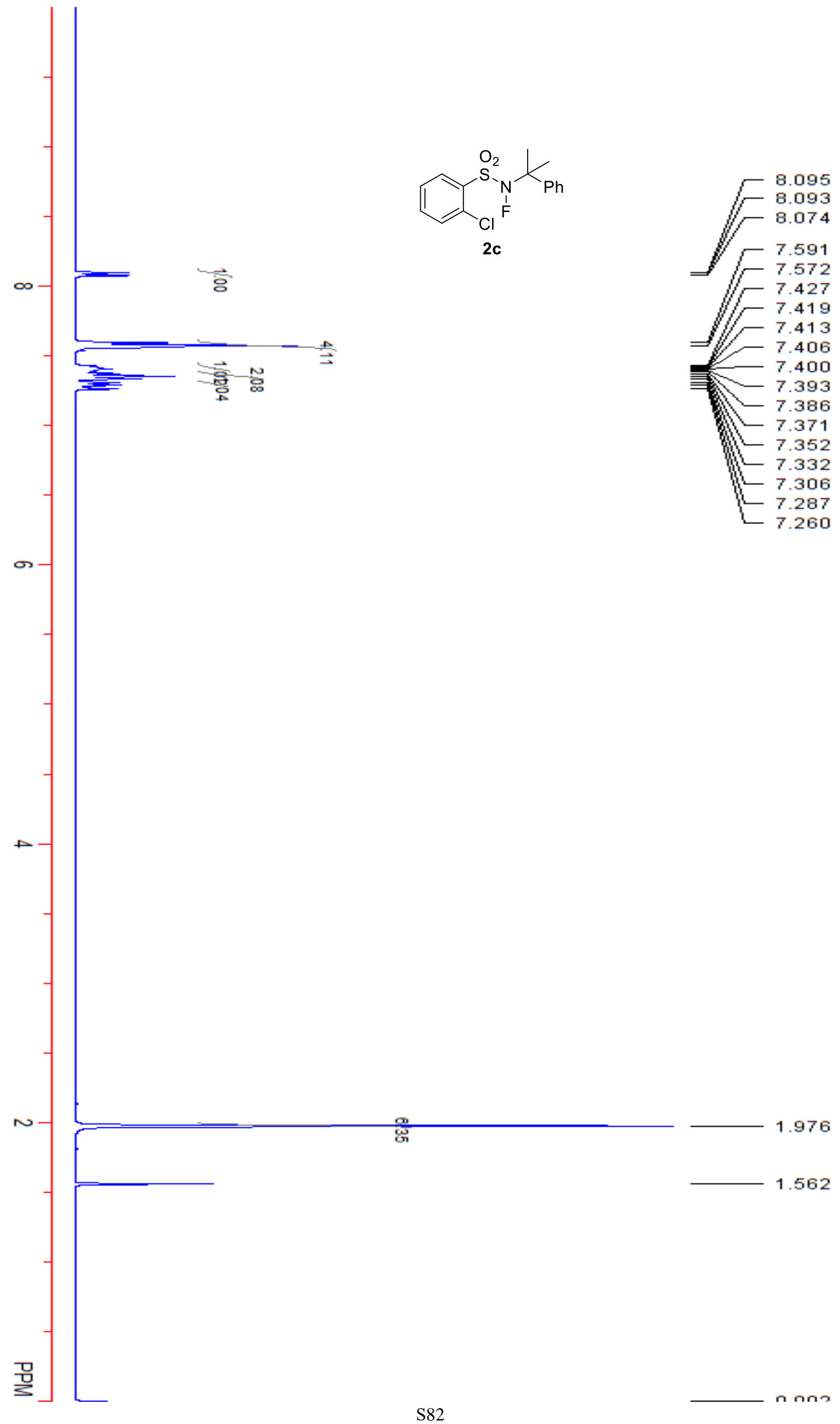



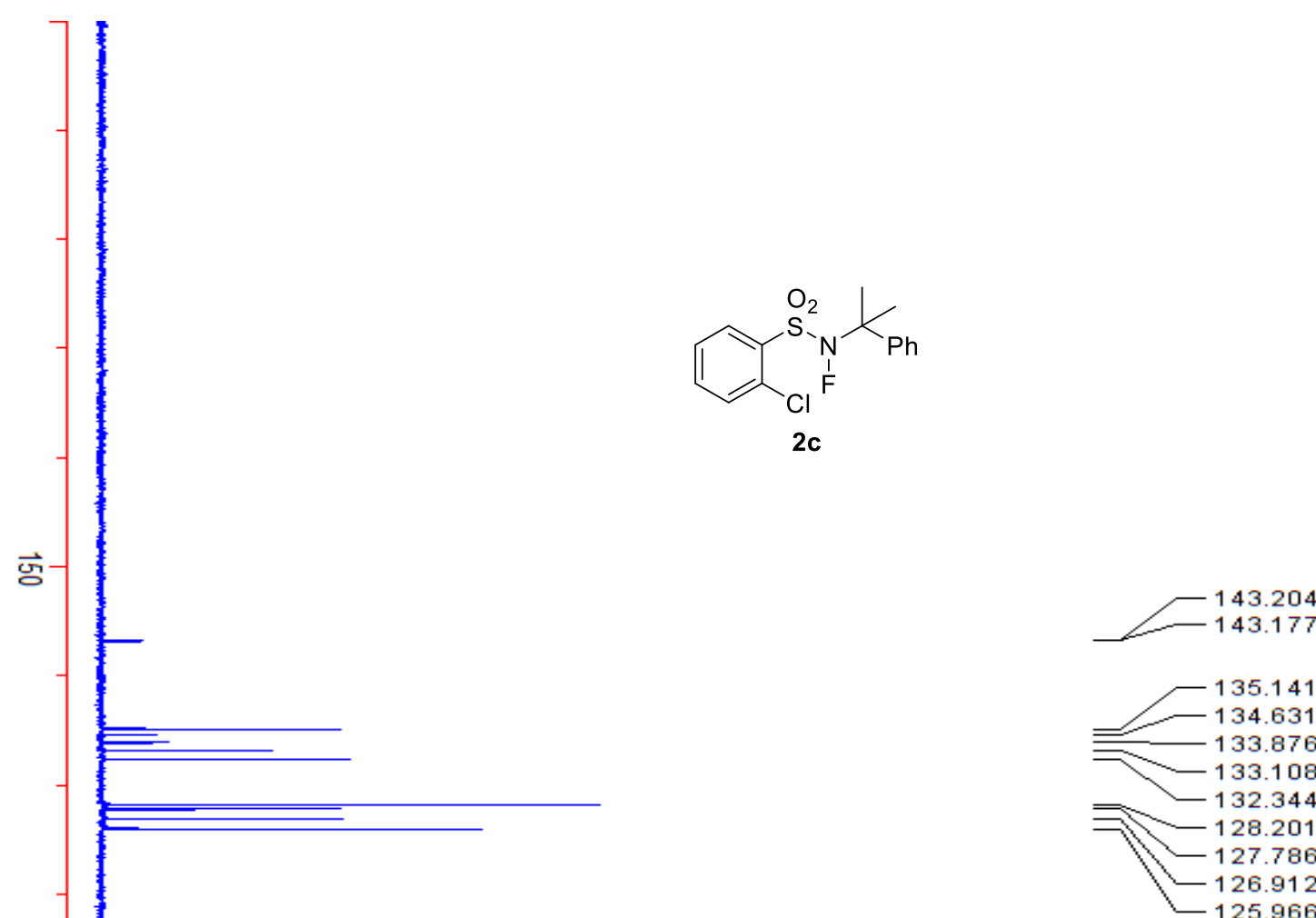

홍
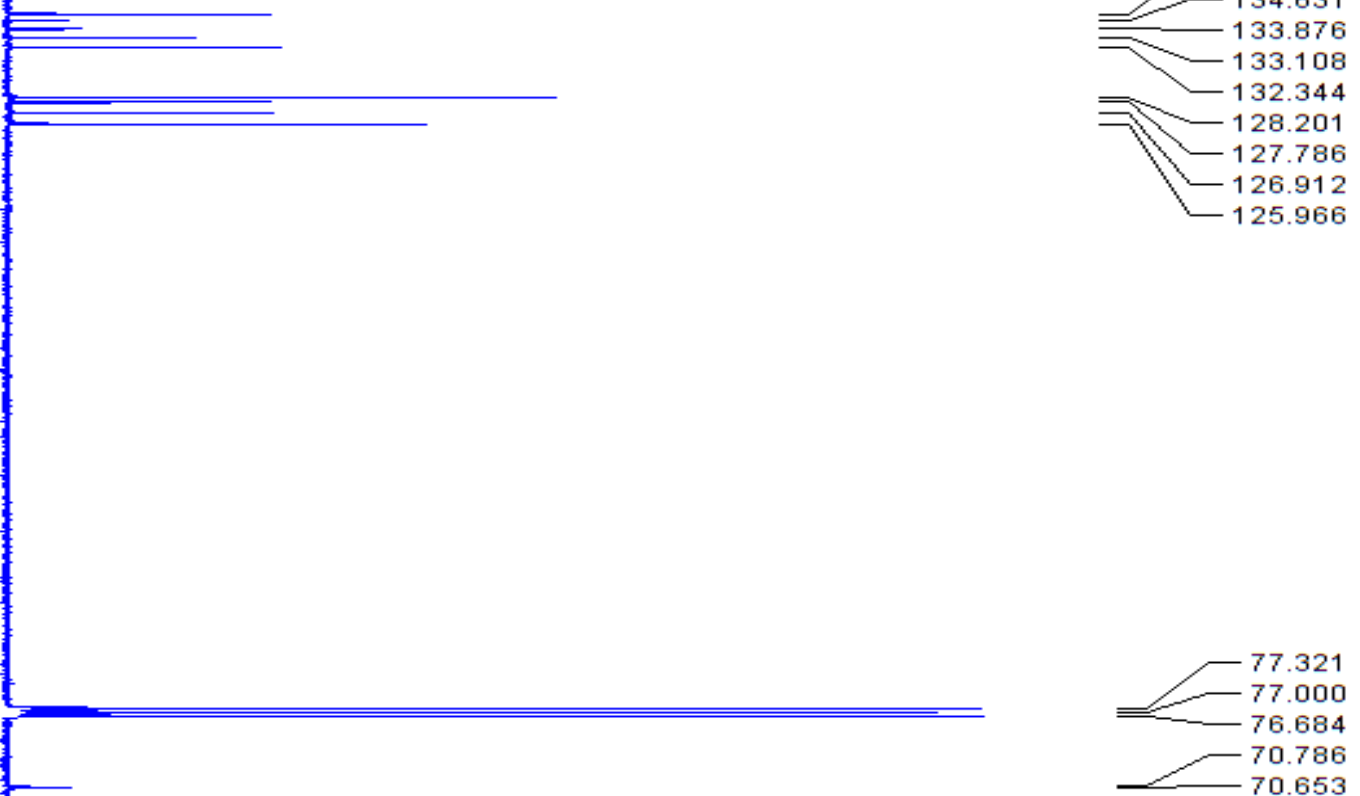

s

믄 


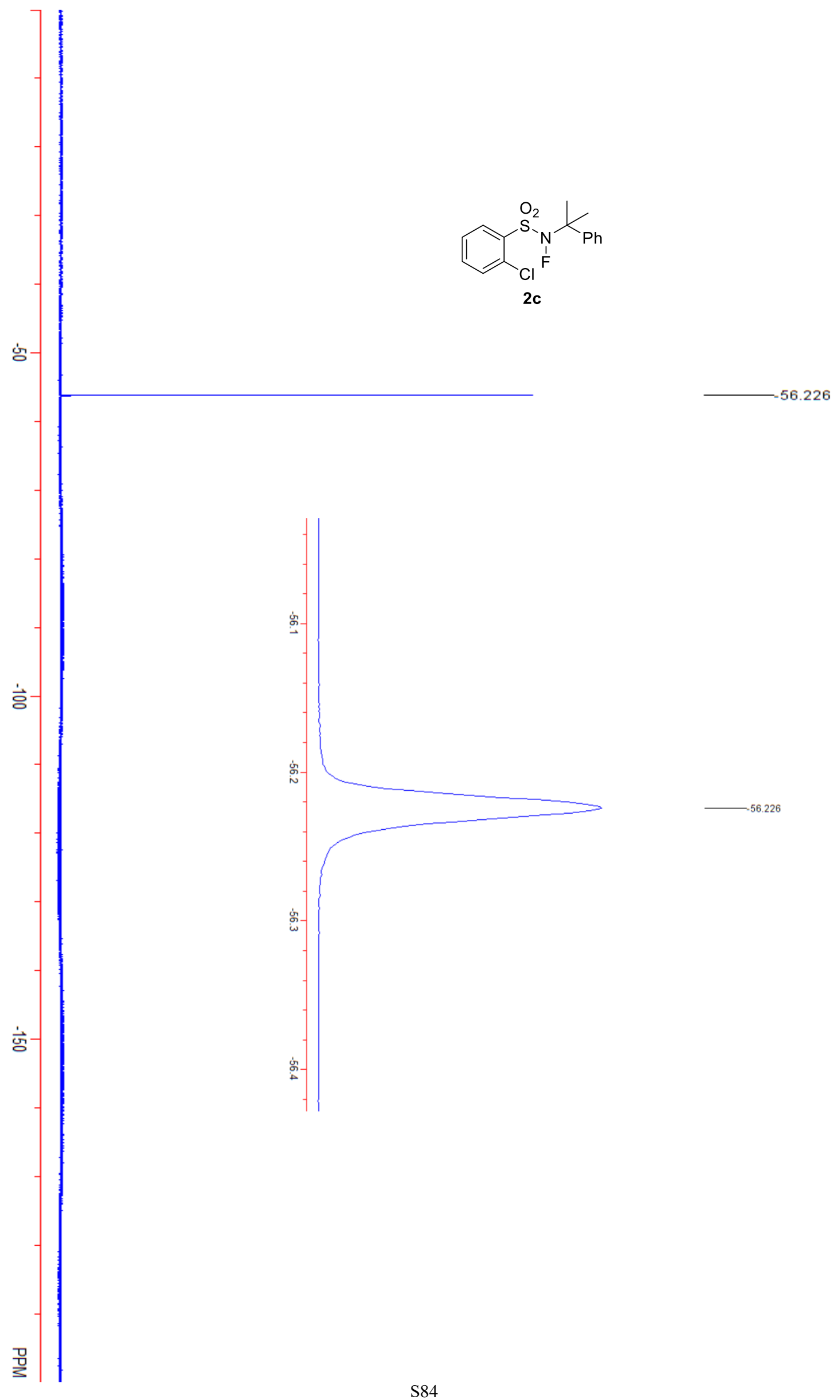




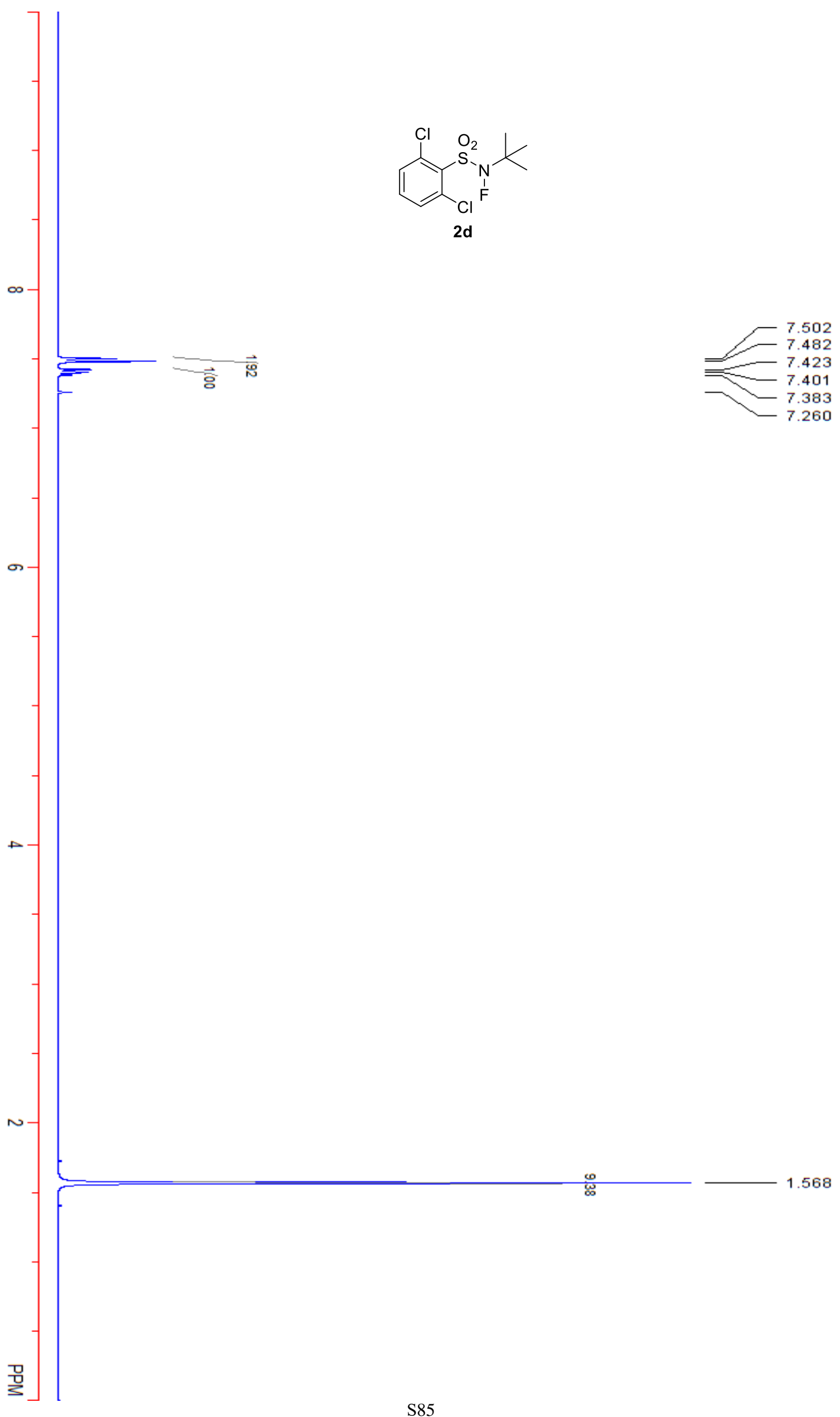




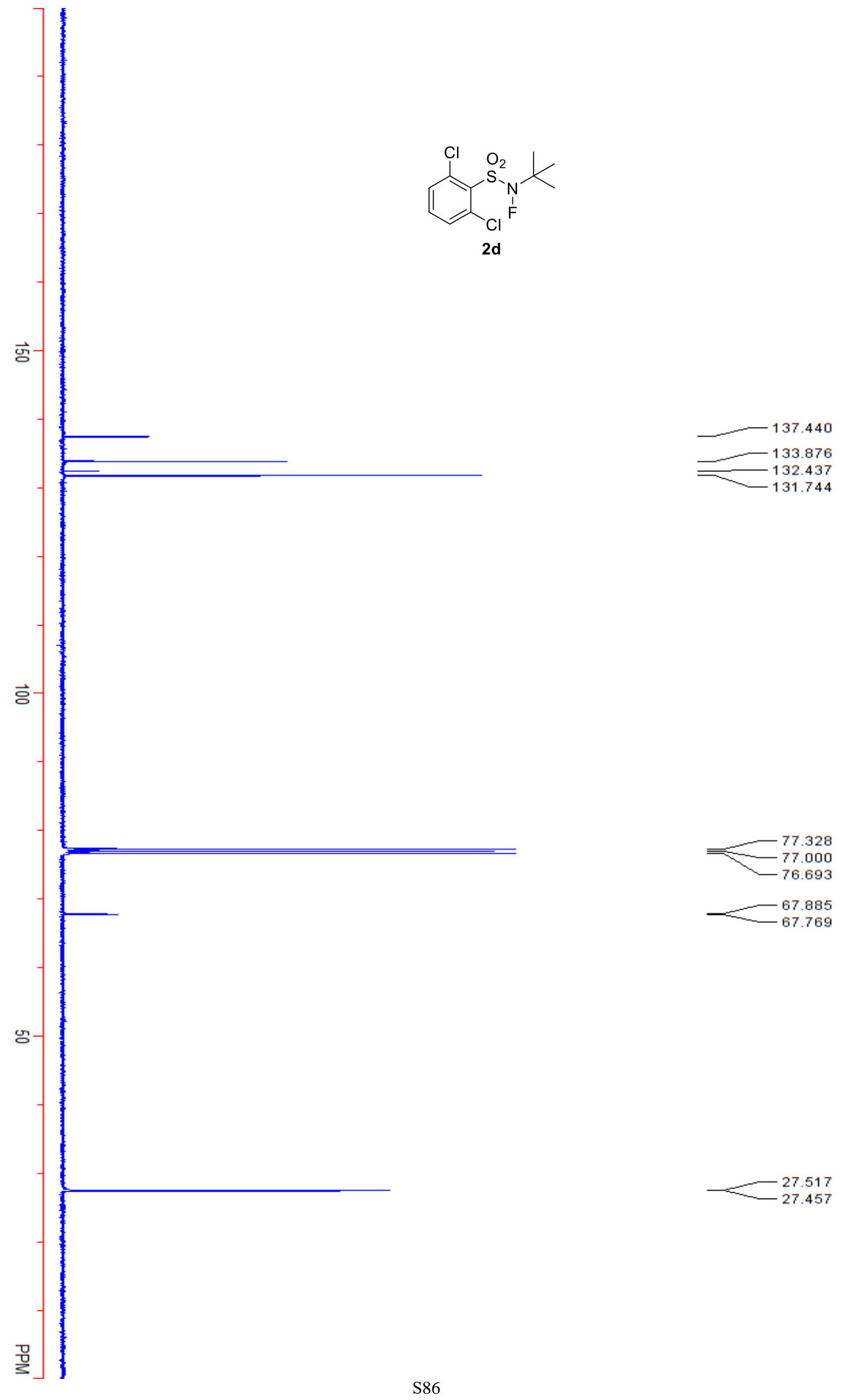




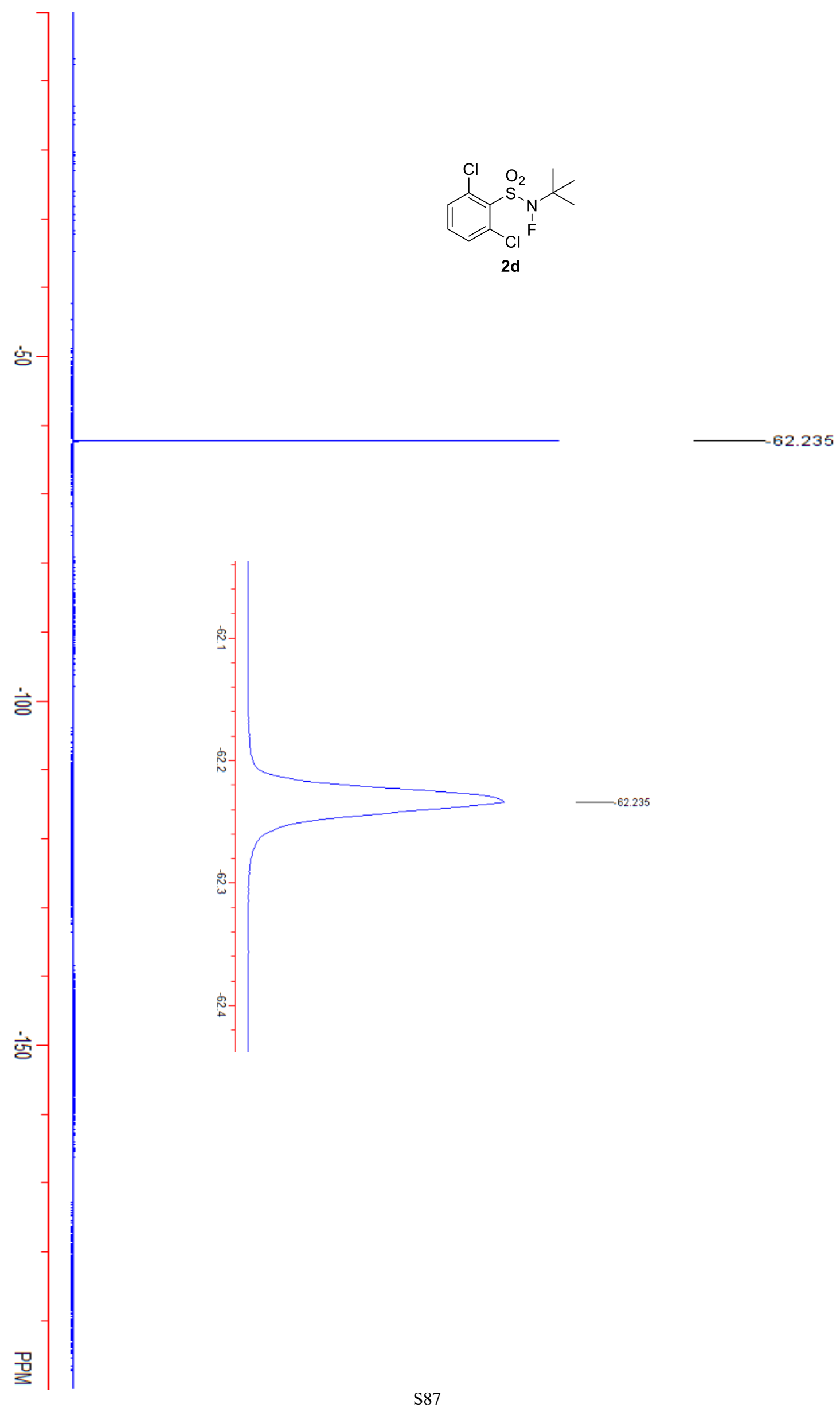



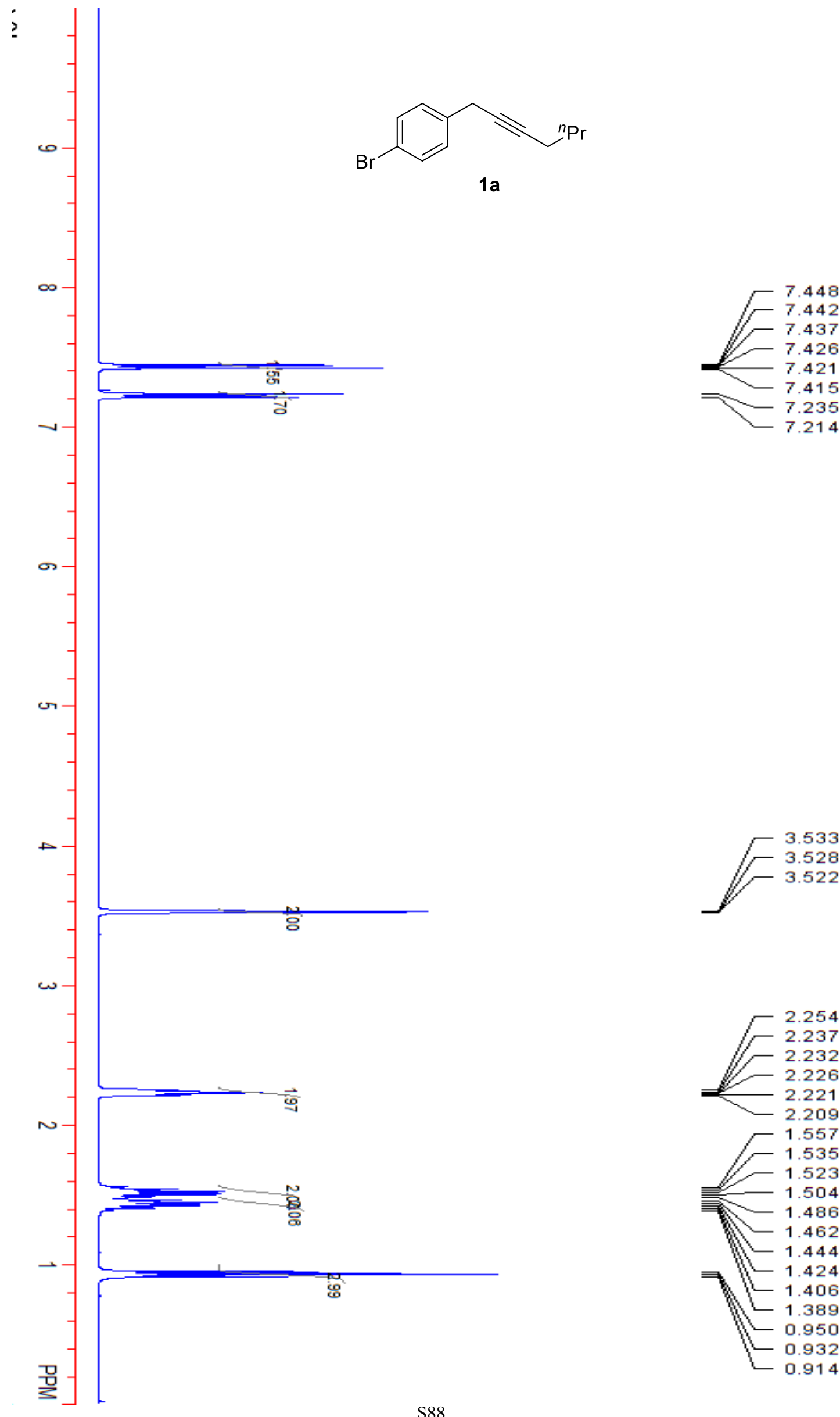

- 1.557

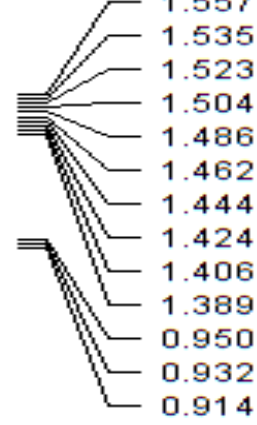




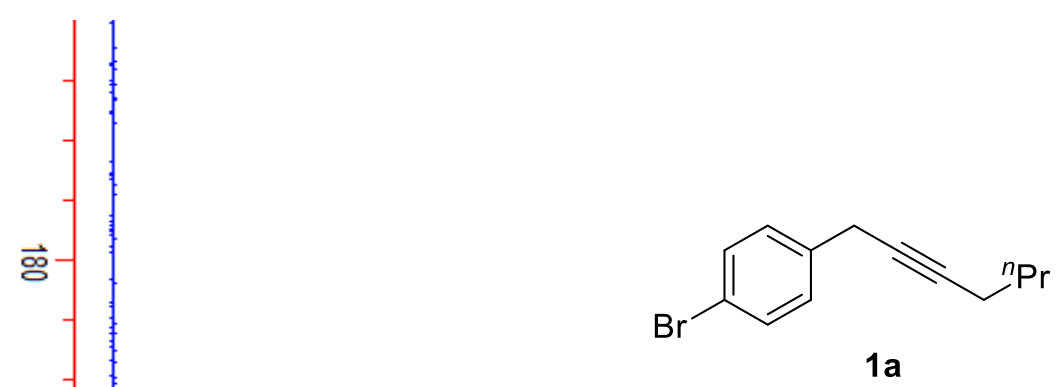

$\overrightarrow{8}$

客

홍

$\vec{\sigma}$

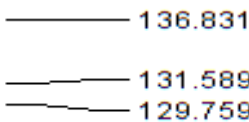

$-120.334$

8

E
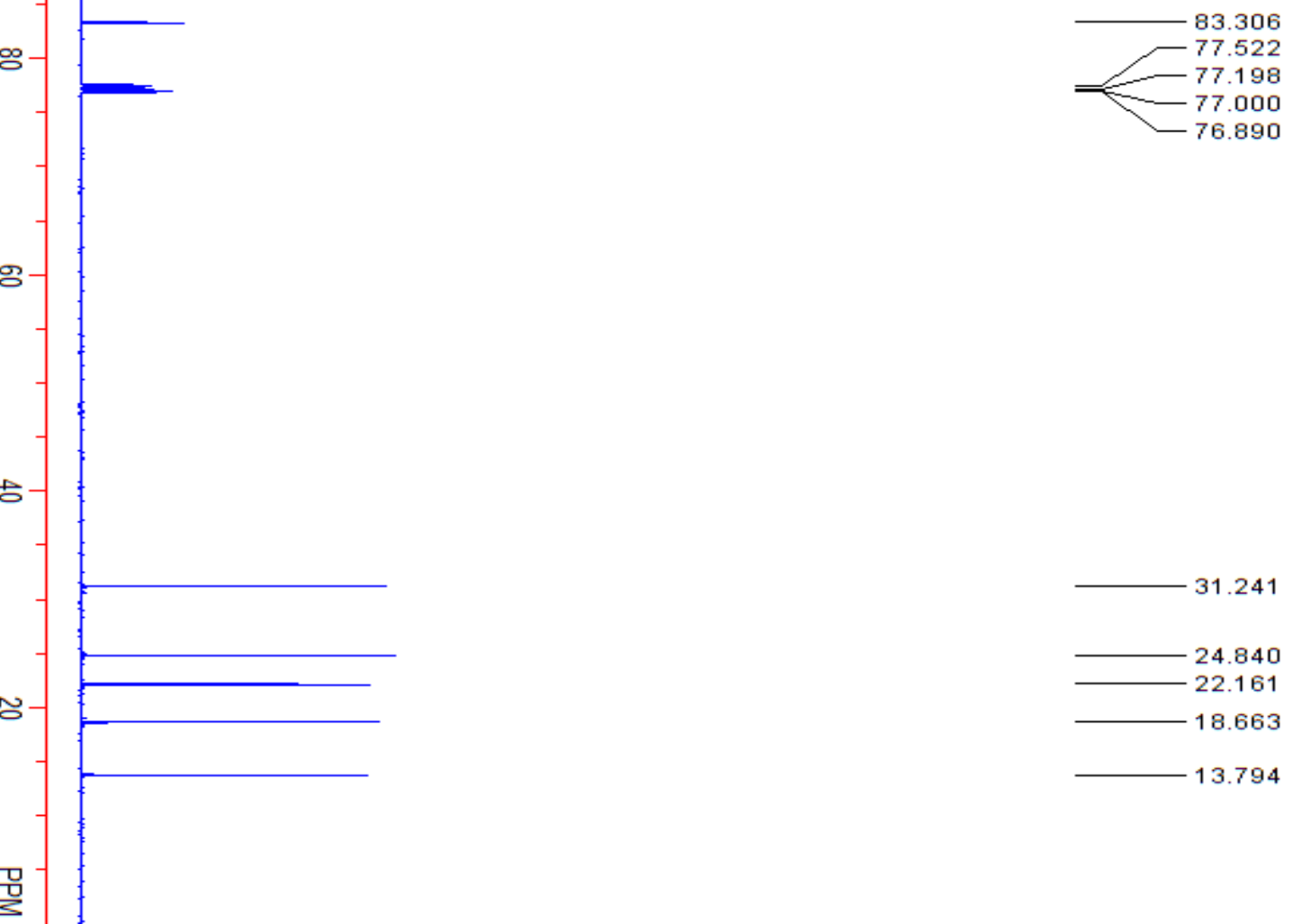

31.241

24.840

22.161

18.663

$-13.794$ 


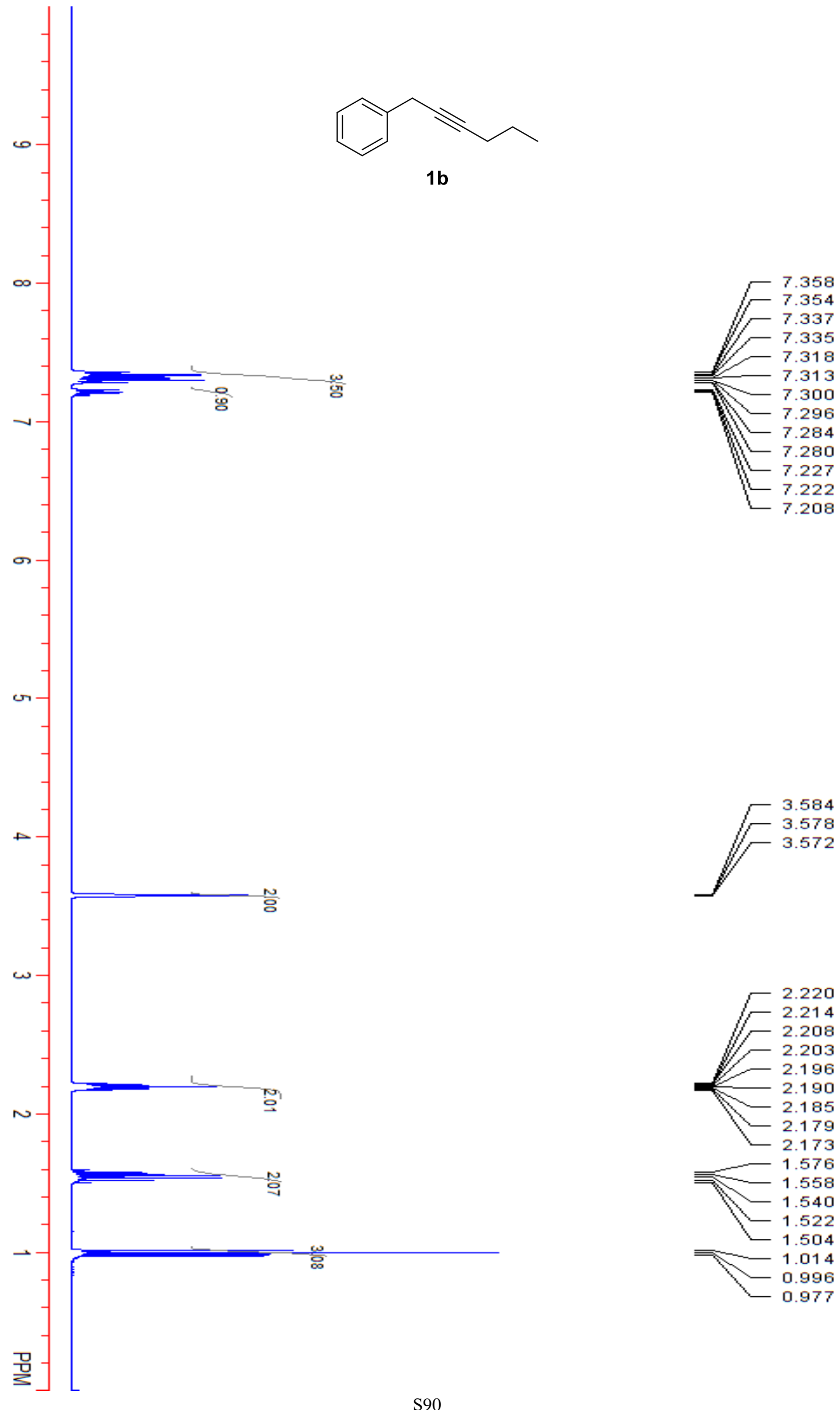



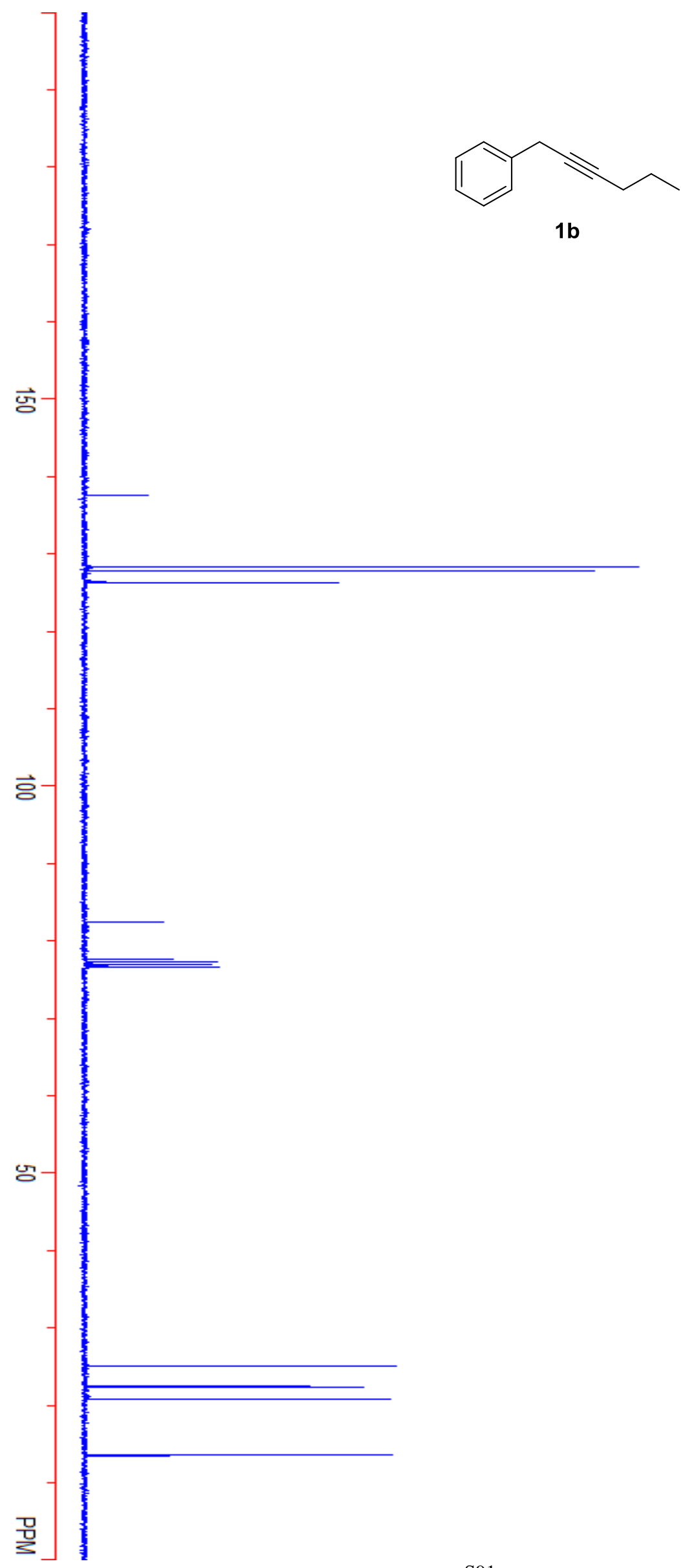

1b

137.617

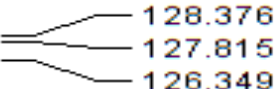

82.497

77.634

77.325

77.000

76.691

25.120

22.431

20.840

13.532 

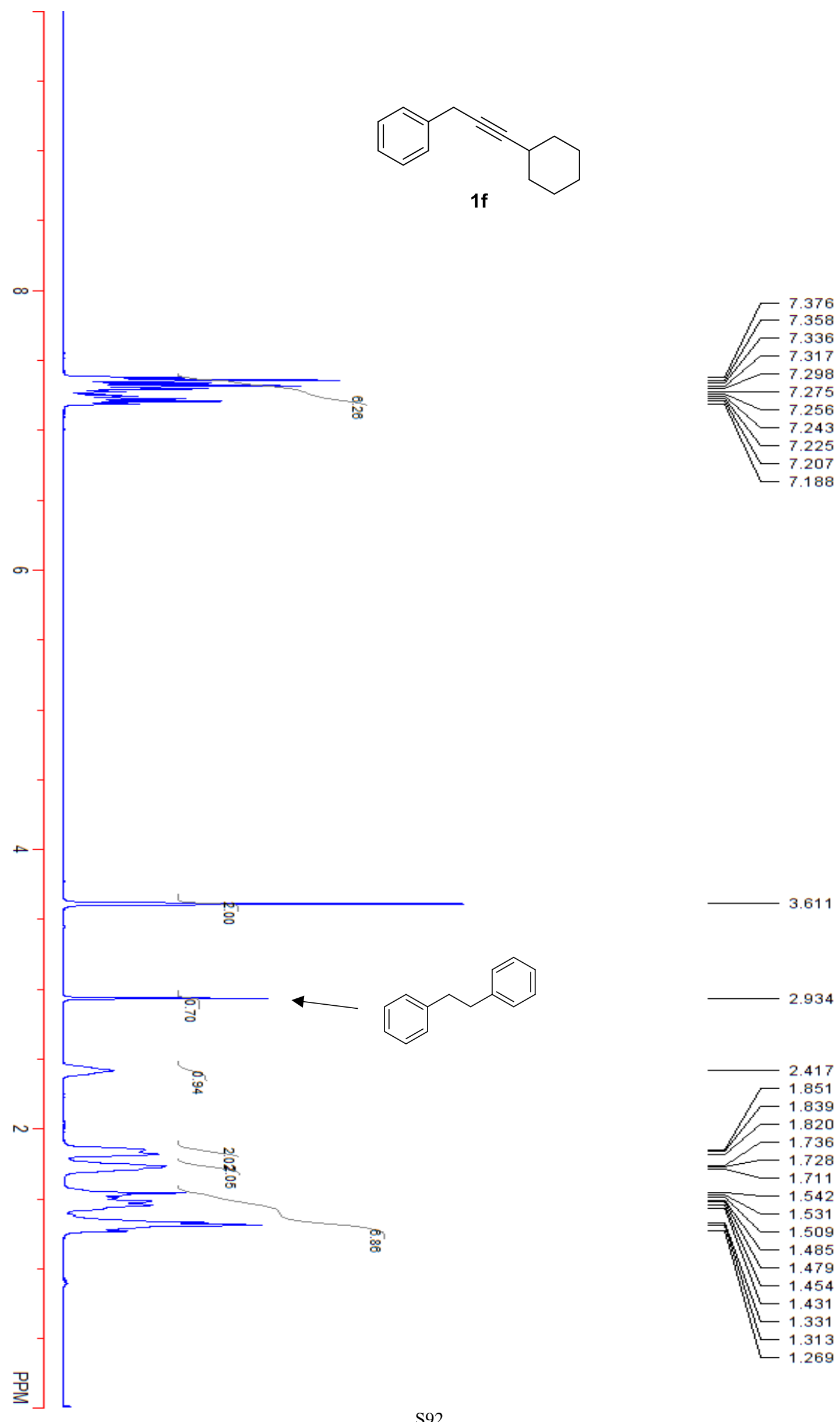

1.851

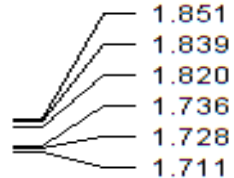

$\bar{\equiv}-1.542$

$\Rightarrow-1.531$

$\Rightarrow \mathrm{v}-1.509$

$\mathrm{M}-1.485$

$\mathrm{M}-1.479$

留 -1.454

留 -1.431

- 1.331

1.313
$-\quad 1.269$ 


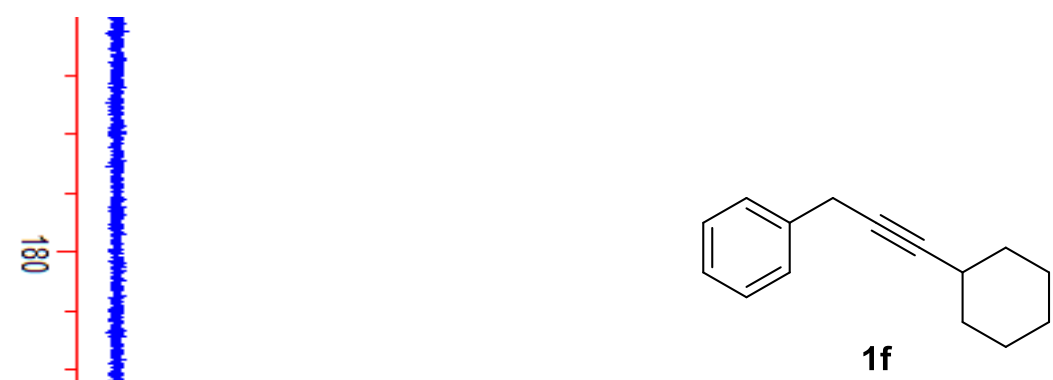

$\overrightarrow{8}$

点

点-

$-$

$-141.765$

$-137.682$

$-128.428$

128.337
-128.305

$=$

$\lceil-126.301$

125.890

굥

ㅎㅇㅇ

s-

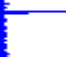

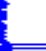
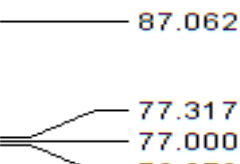

76.679

8

合
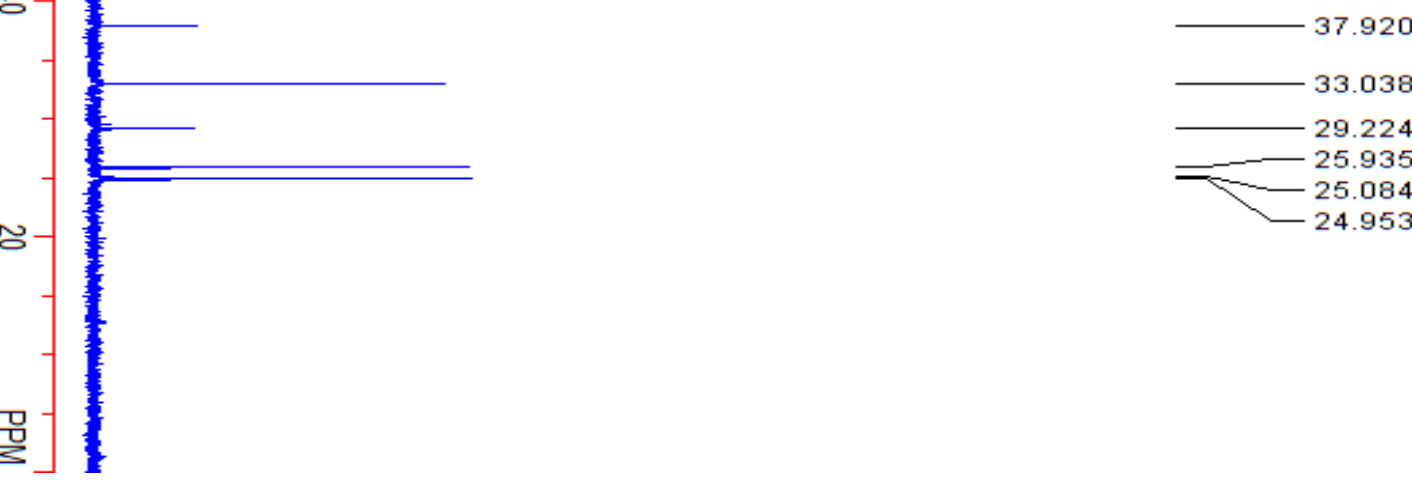

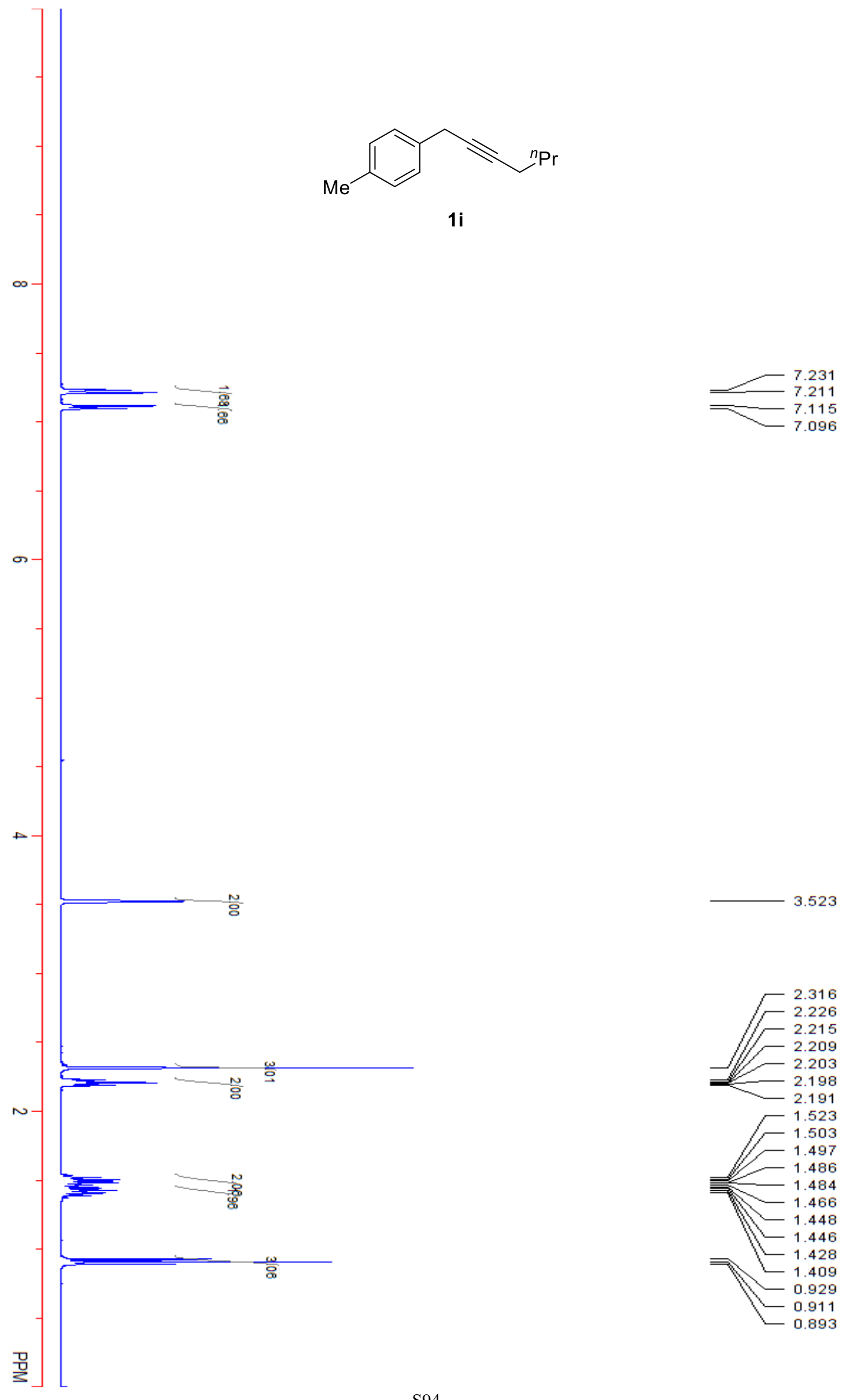

3.523

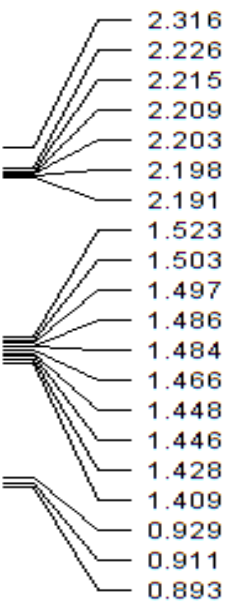




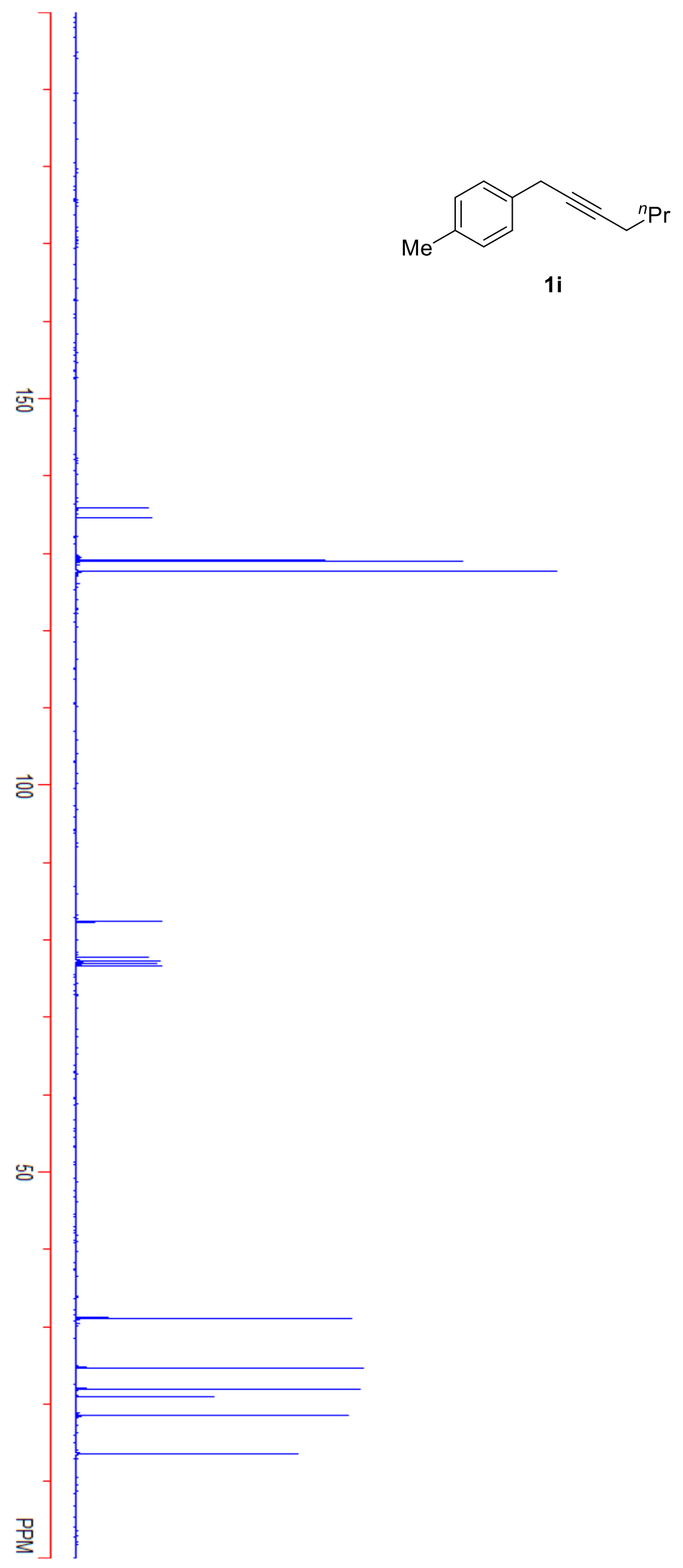

135.834

134.586

129.052

127.693

82.369

$-77.745$

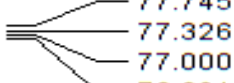

$-76.691$

31.135

24.701

21.974

$-20.965$

13.612 

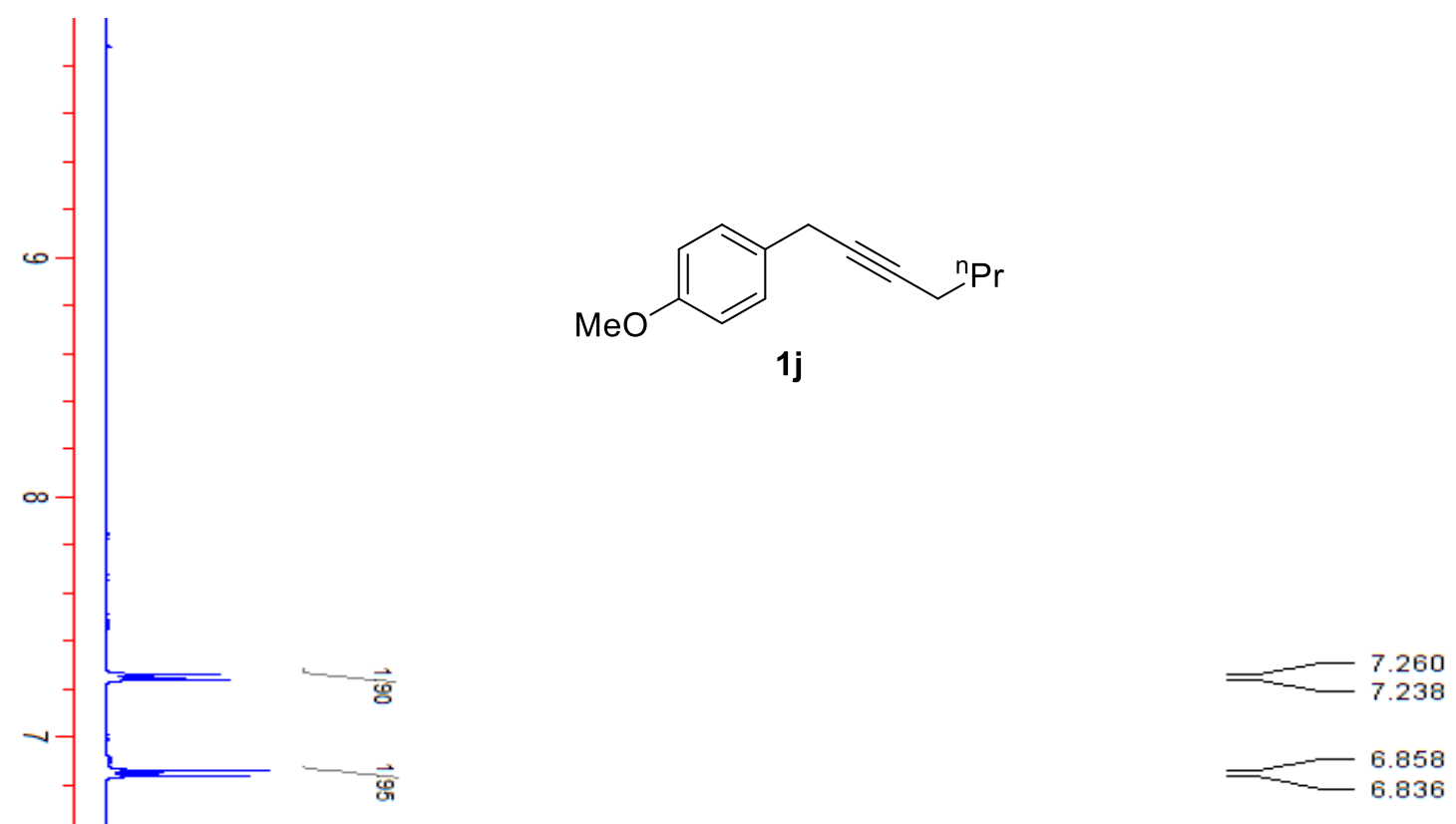

$\sigma$

$\checkmark$ 


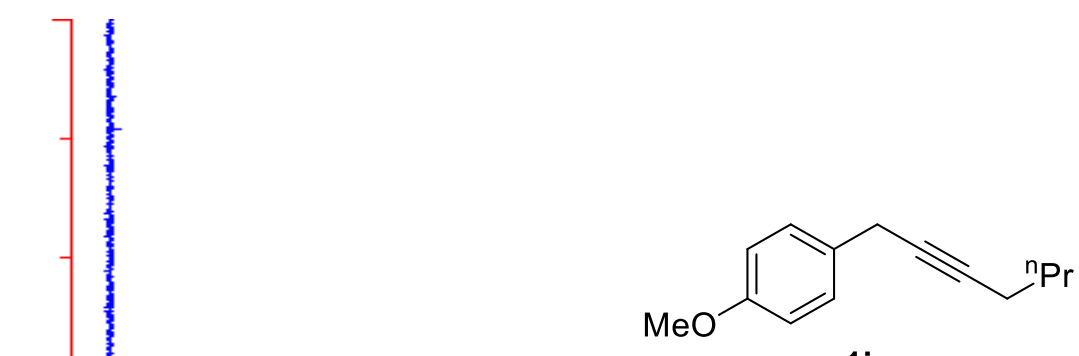

1j
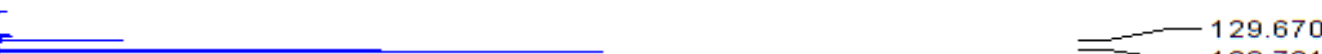


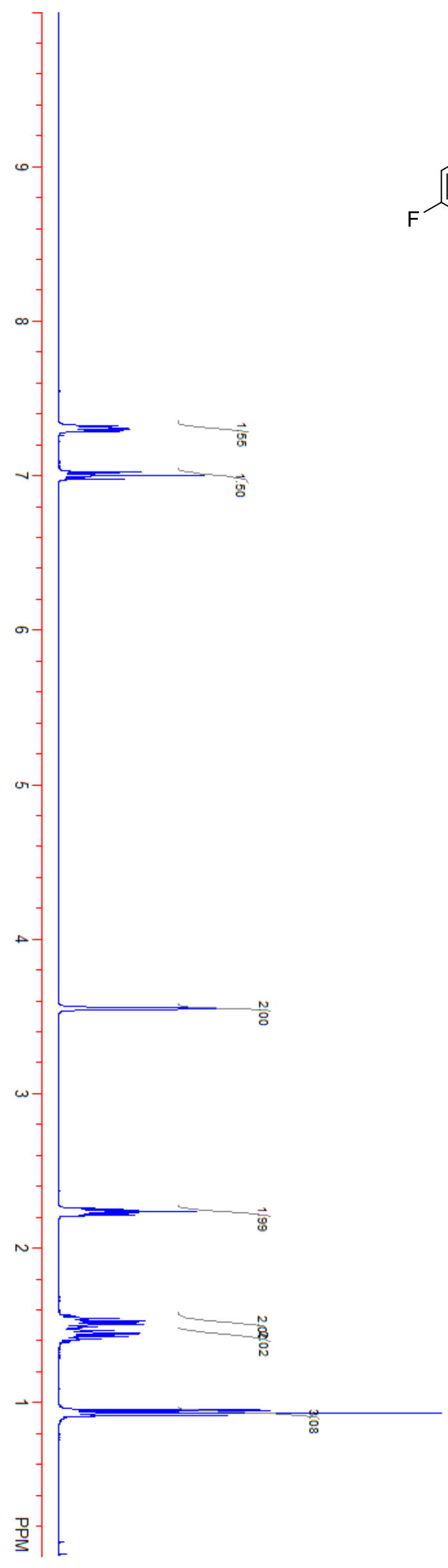<smiles>CC(C)CC#CCc1ccc(F)cc1</smiles>

$1 \mathrm{k}$
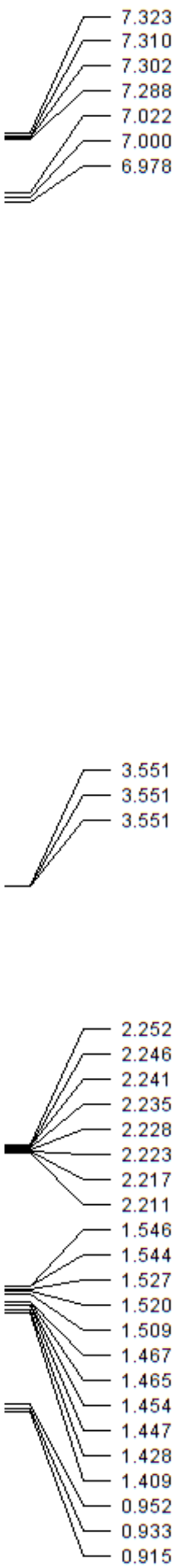


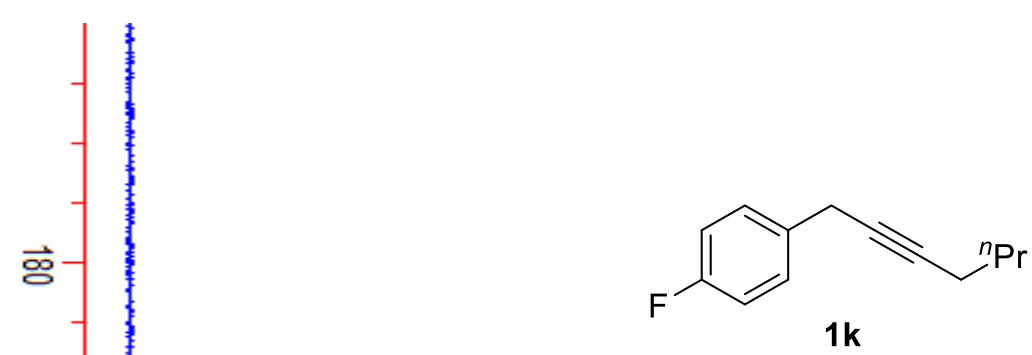

屋

$\vec{\Xi}$

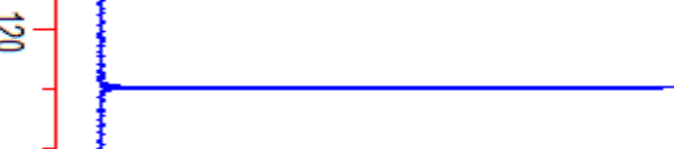

$\left[\begin{array}{r}133.238 \\ \hline\end{array}\right.$ 133.210

129.155

홍

용

8 


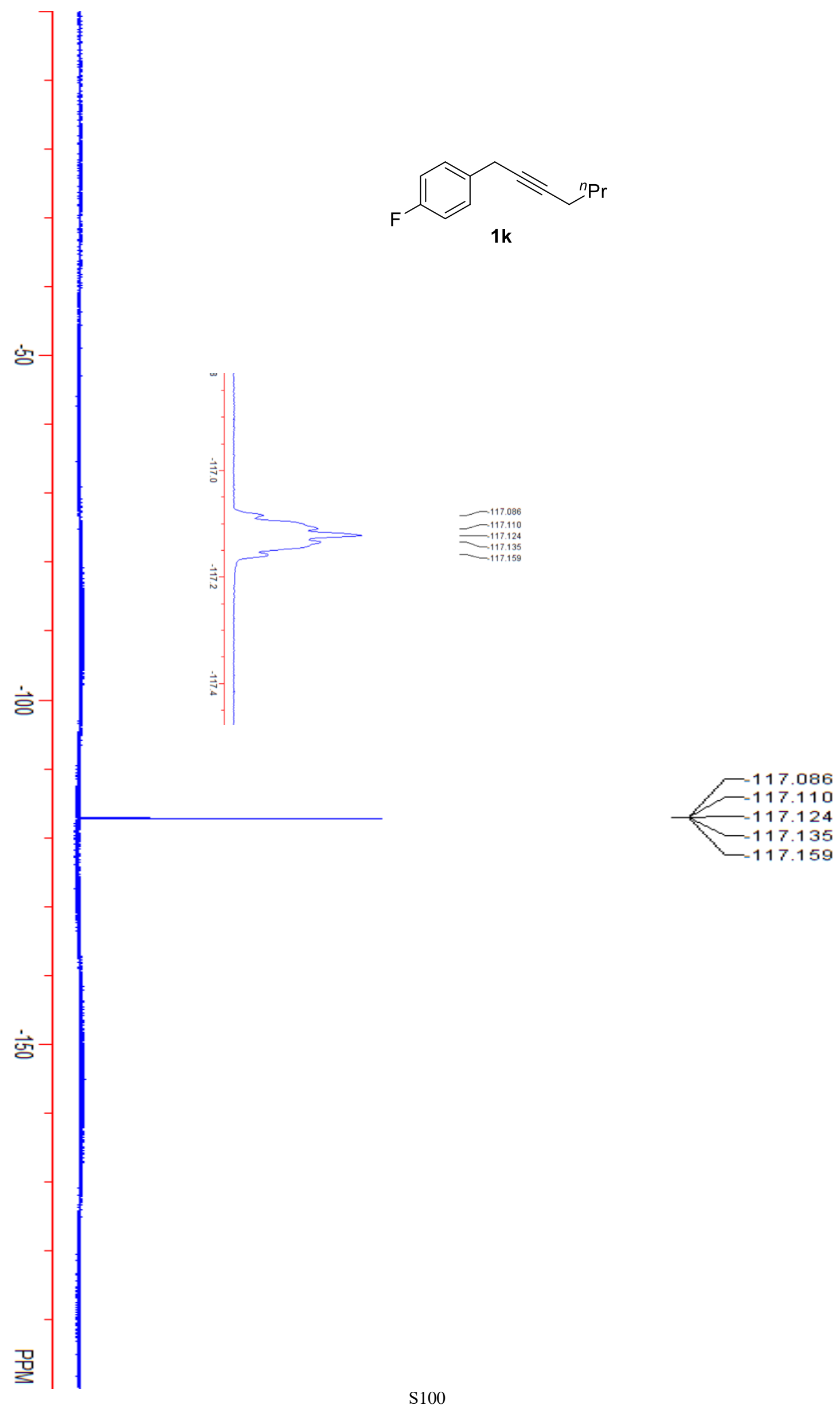



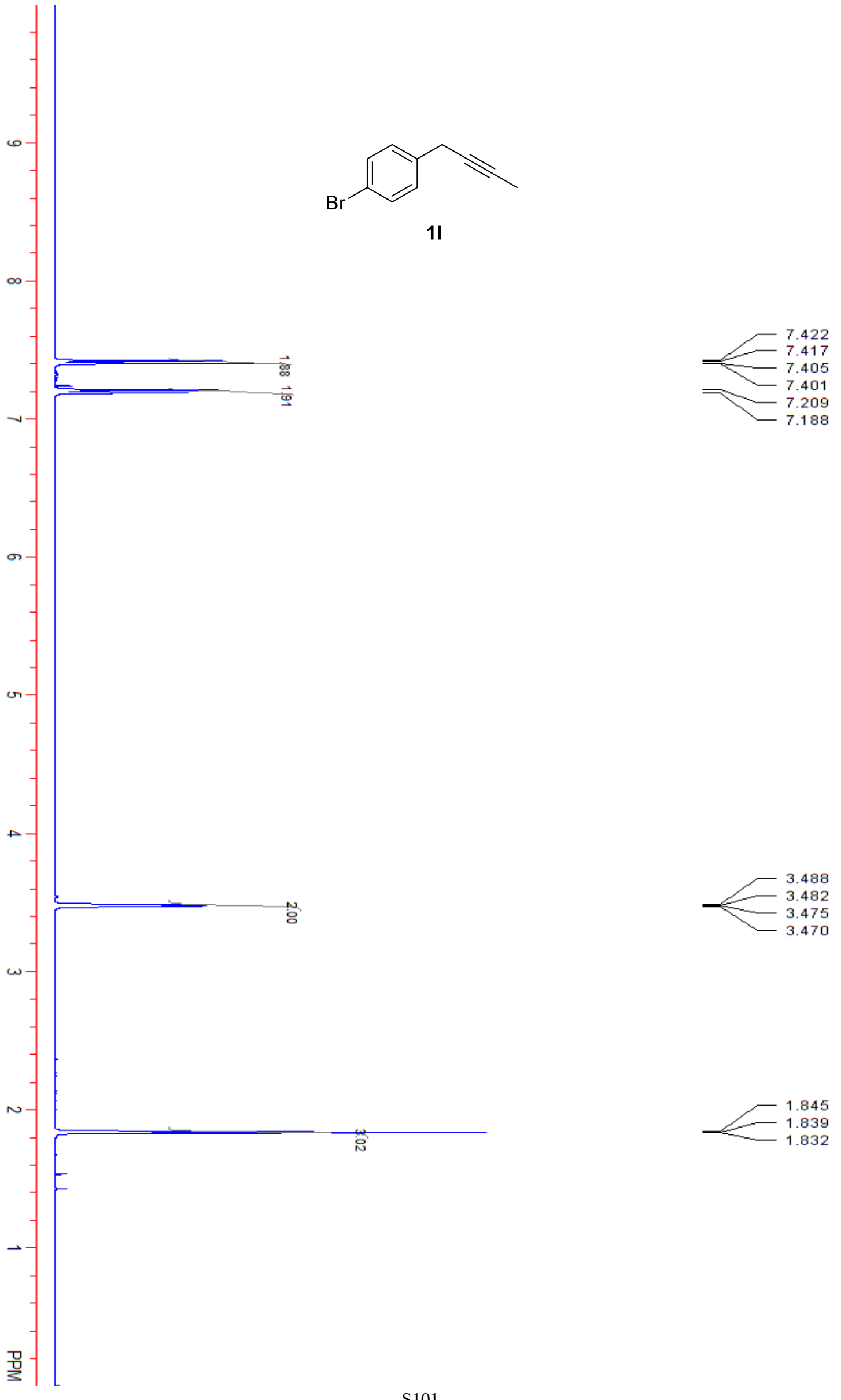


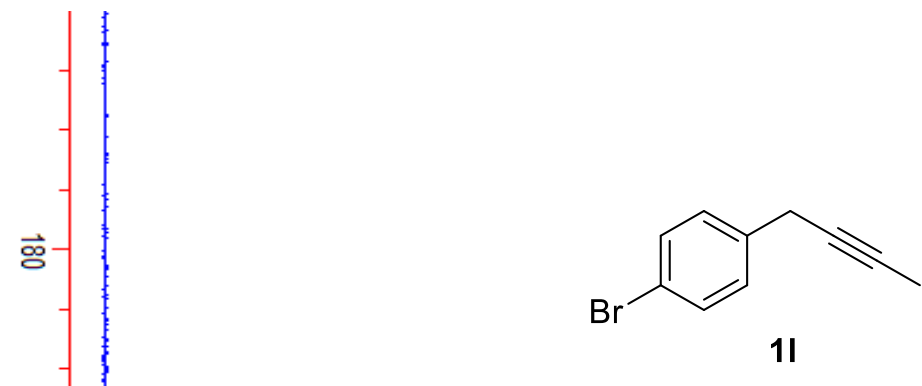

항
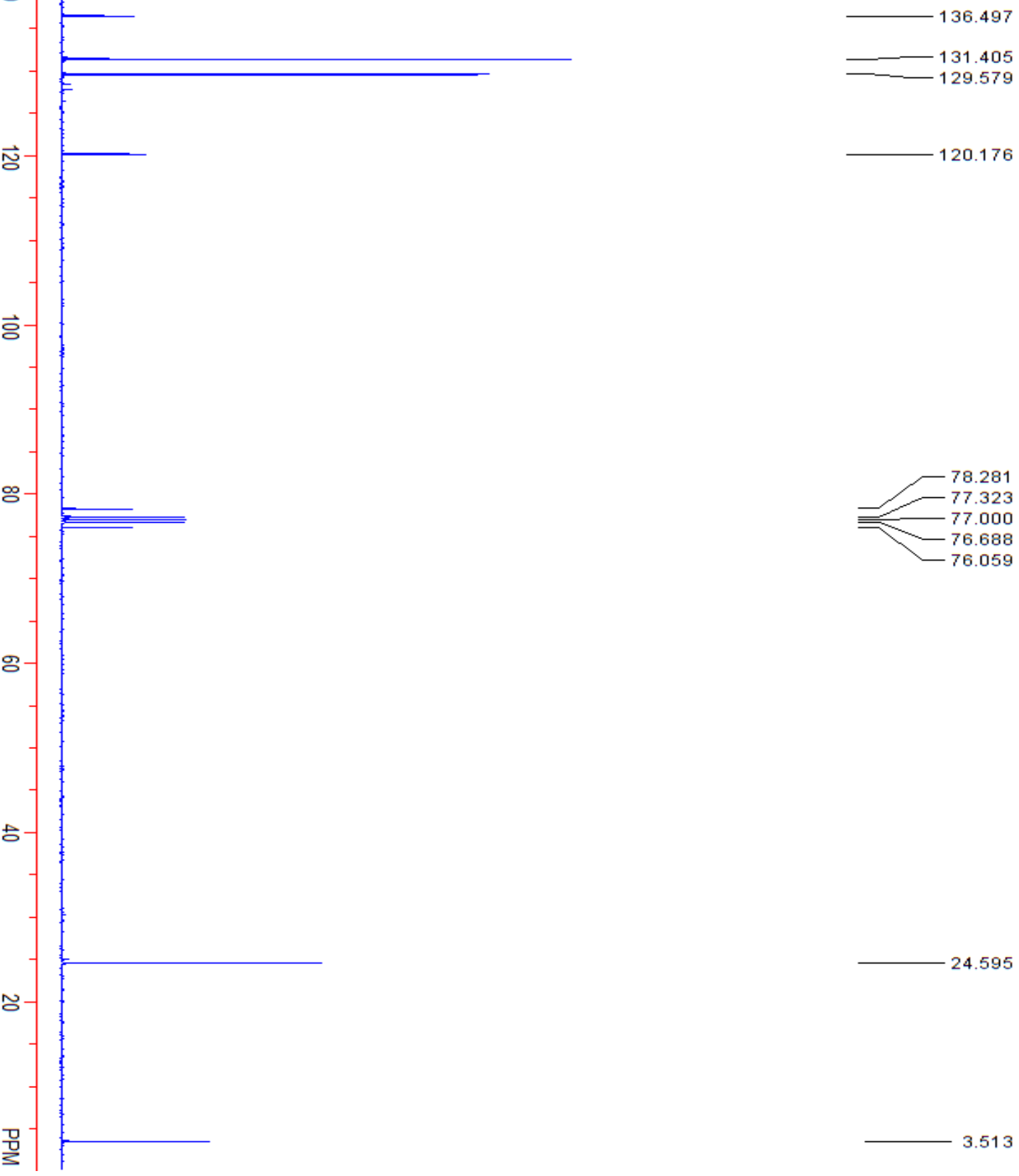

24.595 

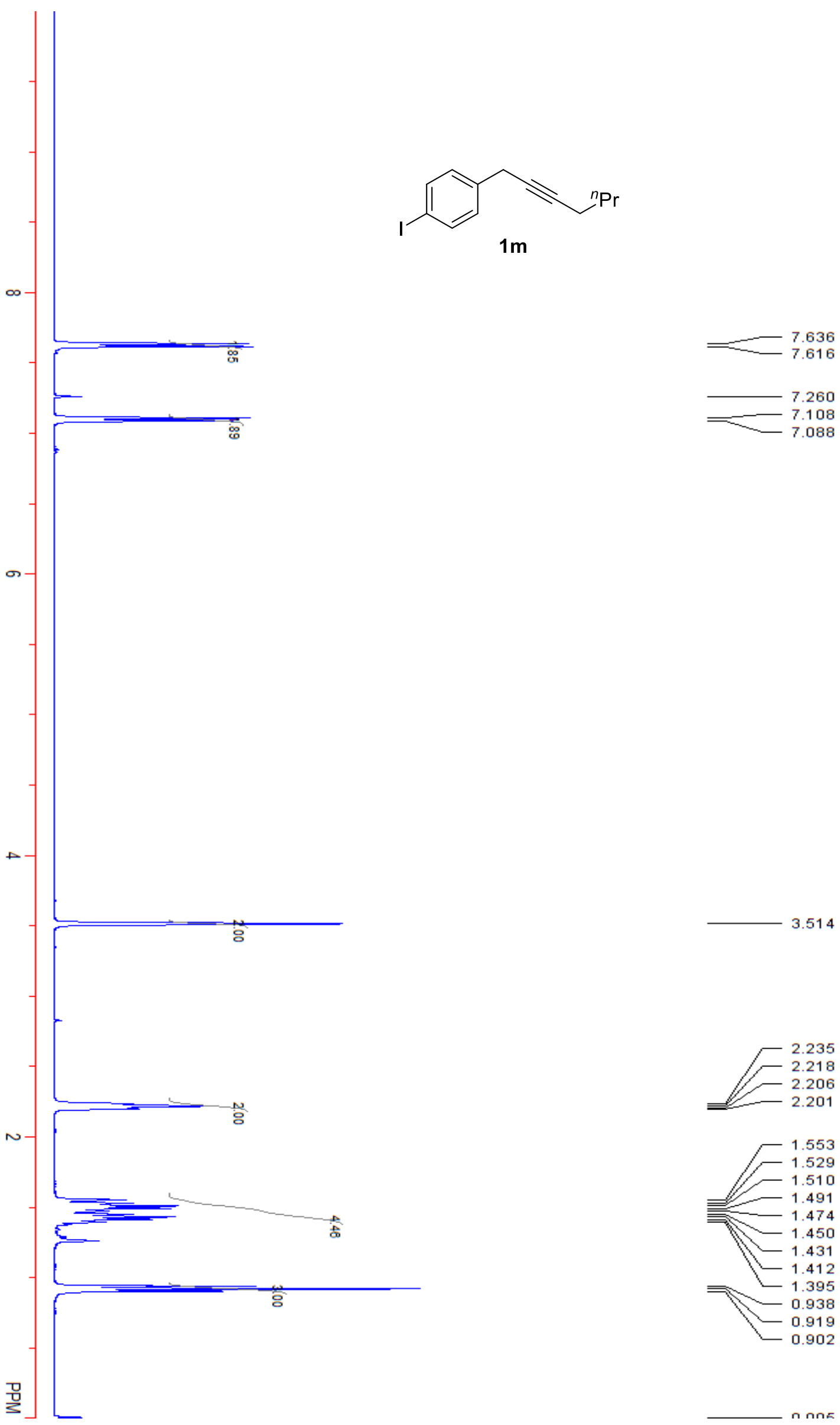

3.514
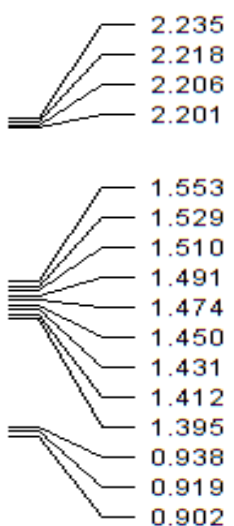


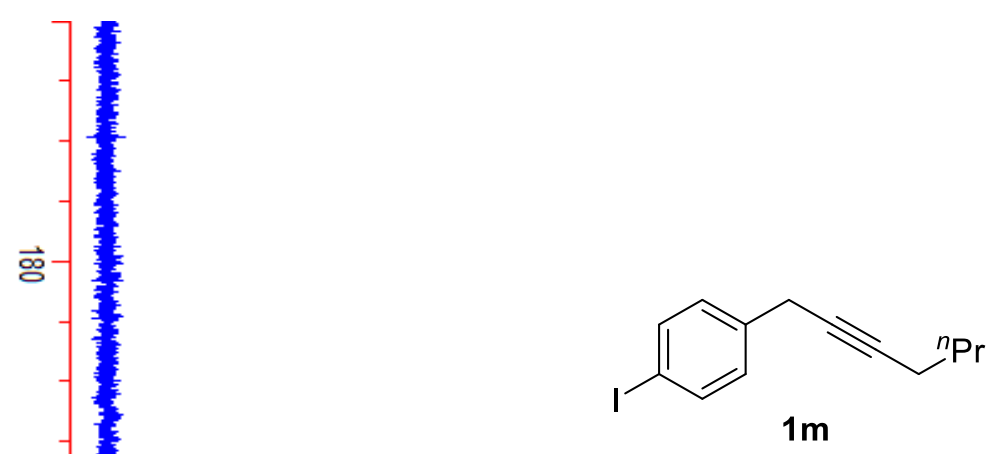

क्
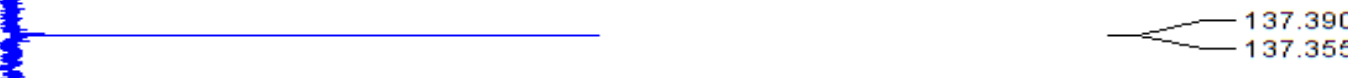

129.916

$\overrightarrow{\mathrm{N}}$

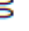
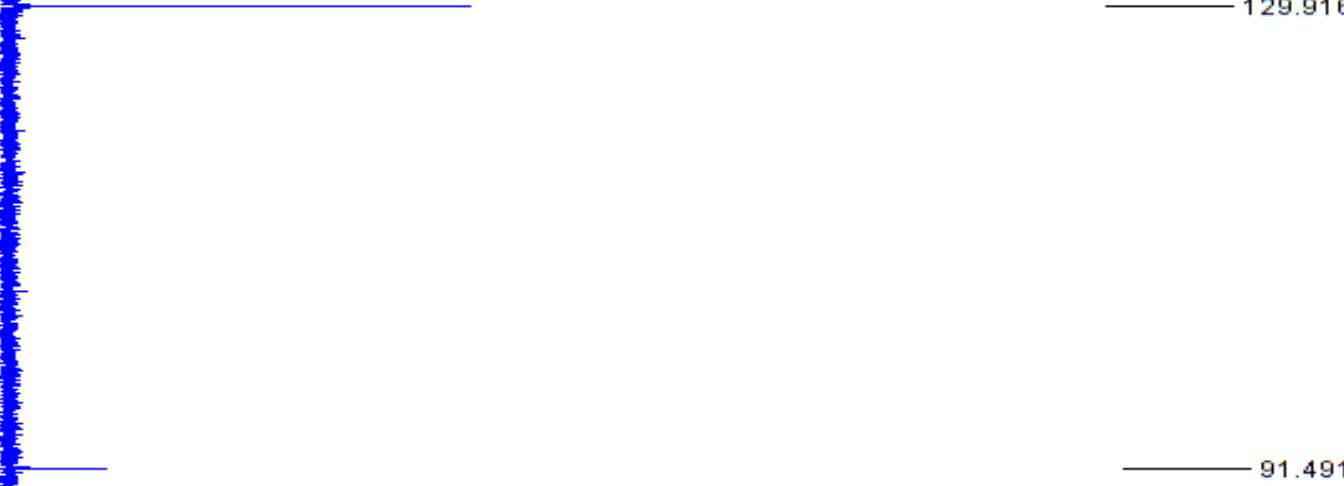

og-
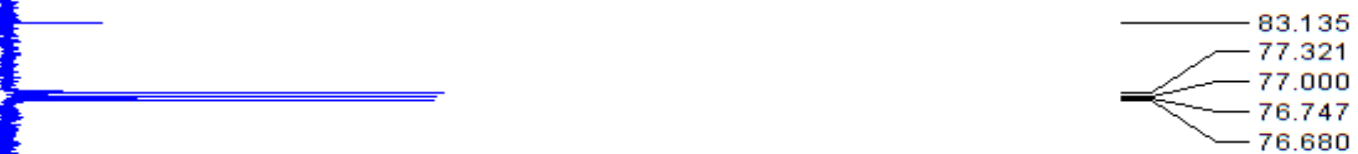

8

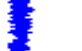

E

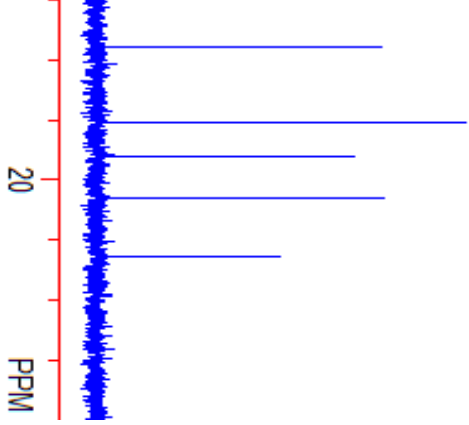

31.037

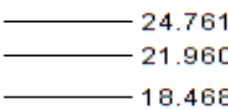

18.468

13.601 

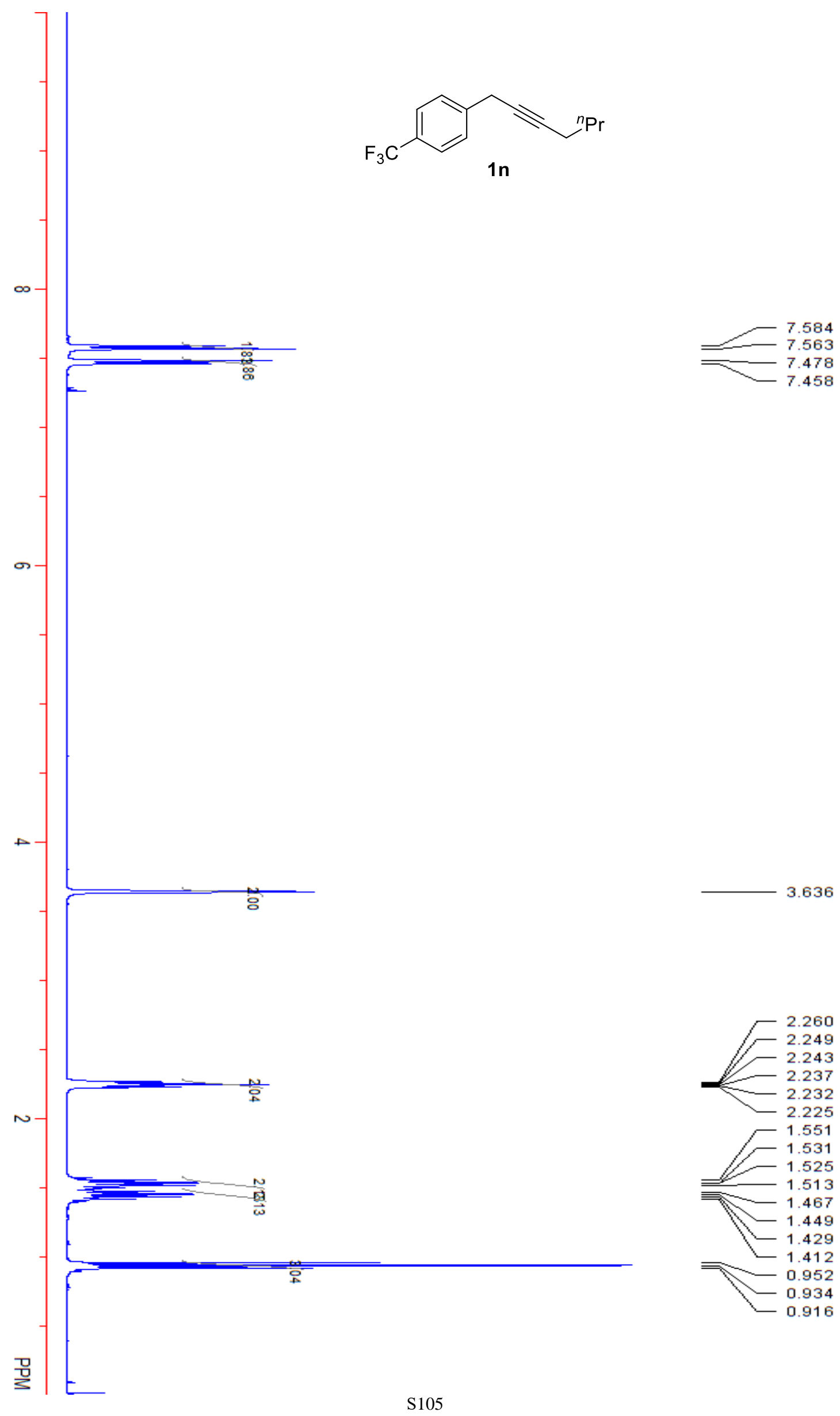


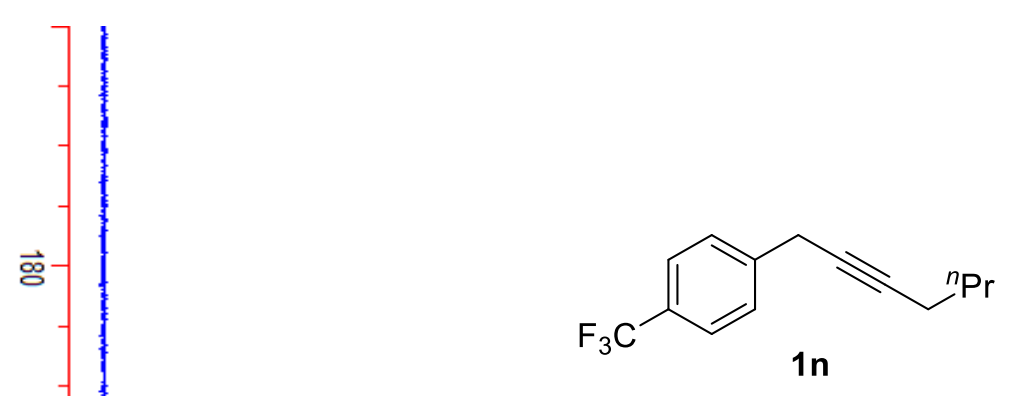

용

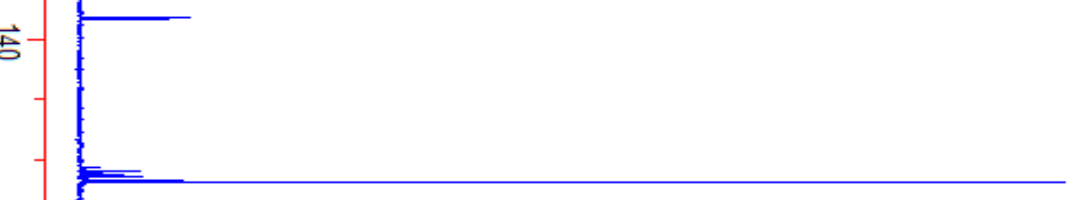

$-129.304$

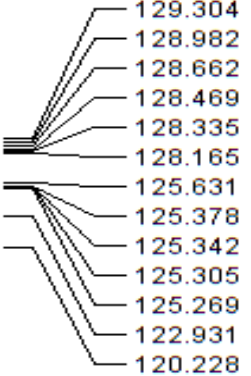

흥

$\overrightarrow{\tilde{O}}$

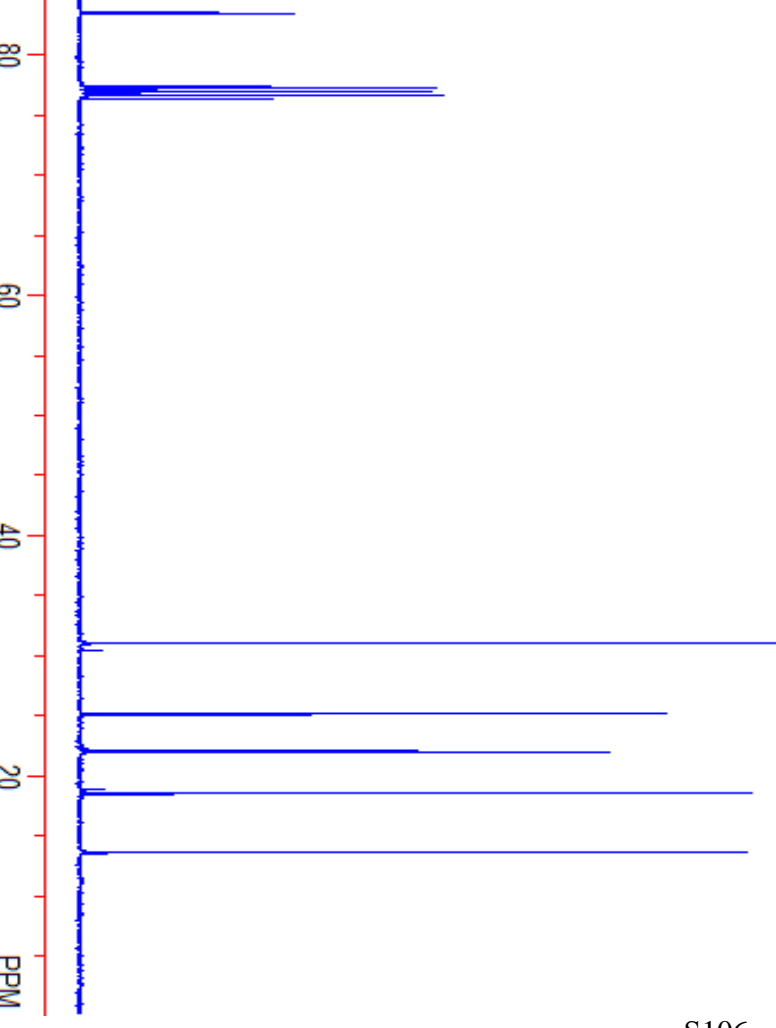

83.498

77.325

77.000

76.37
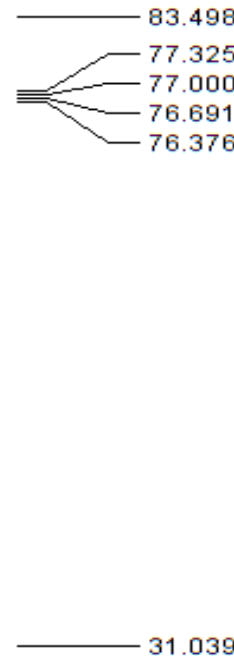

25.097

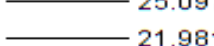

18.471

$-13.594$ 


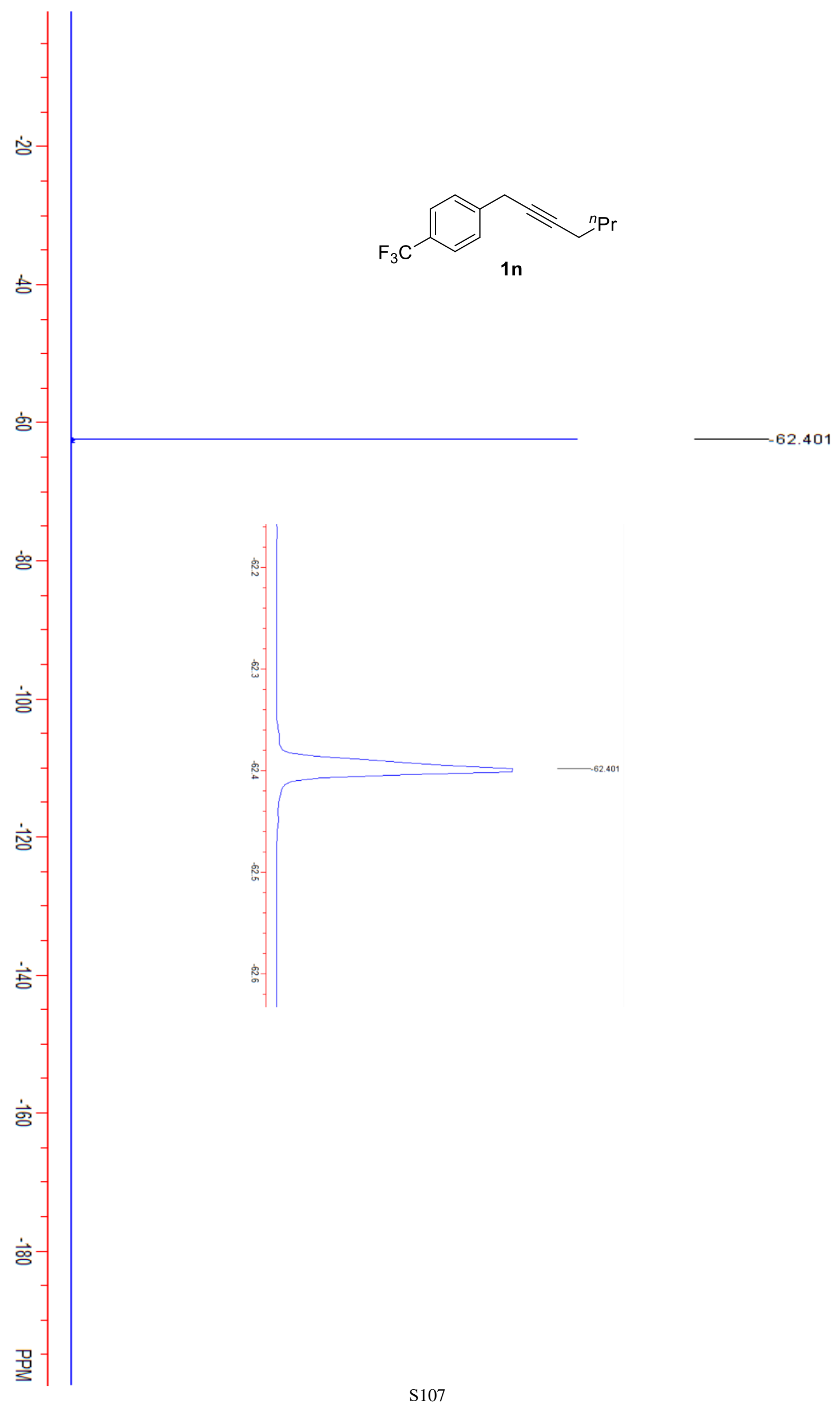



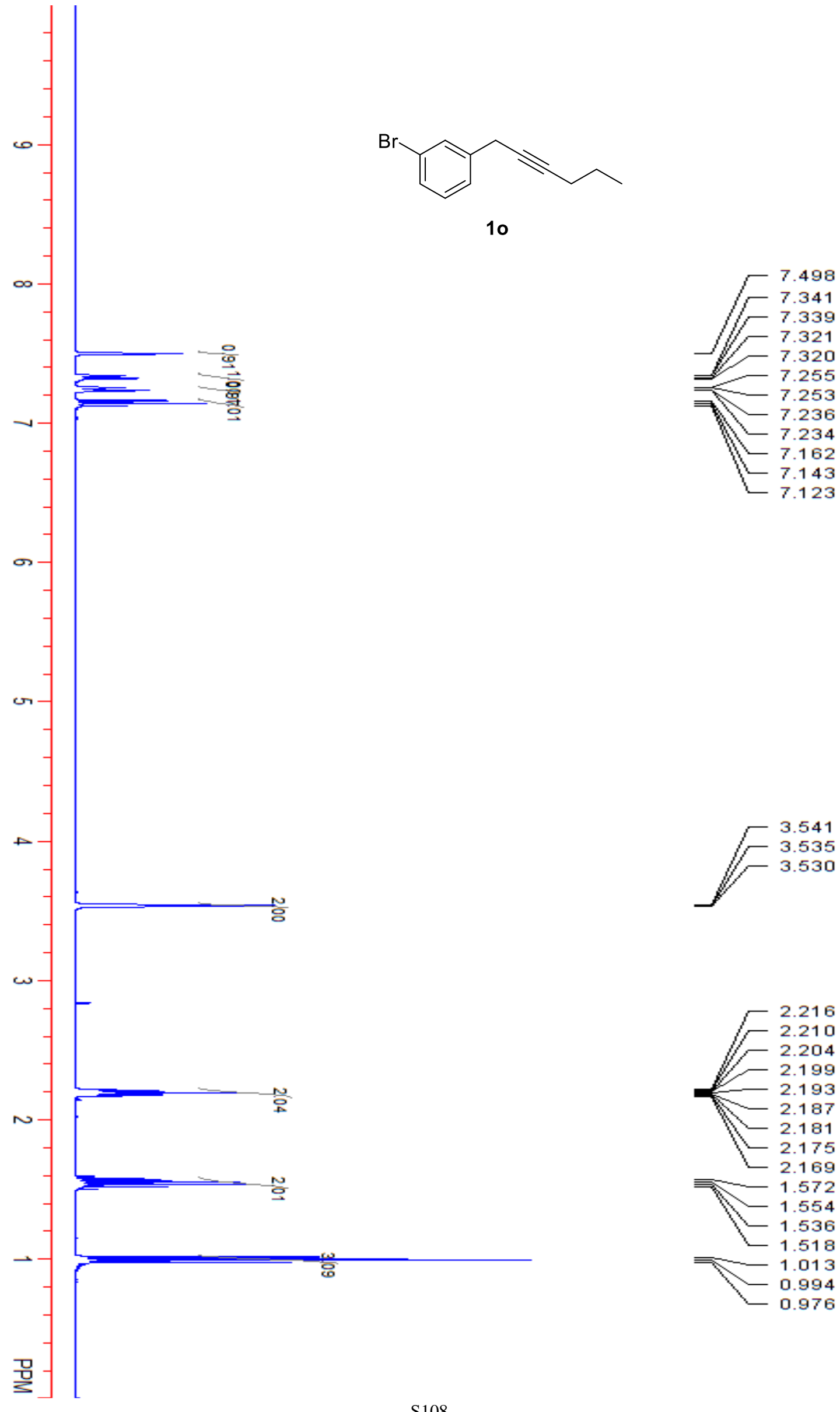

$-2.175$

$-2.169$

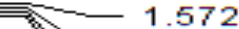

$=1.572$

$-1.518$

$\equiv-1.013$

0.994

0.976 


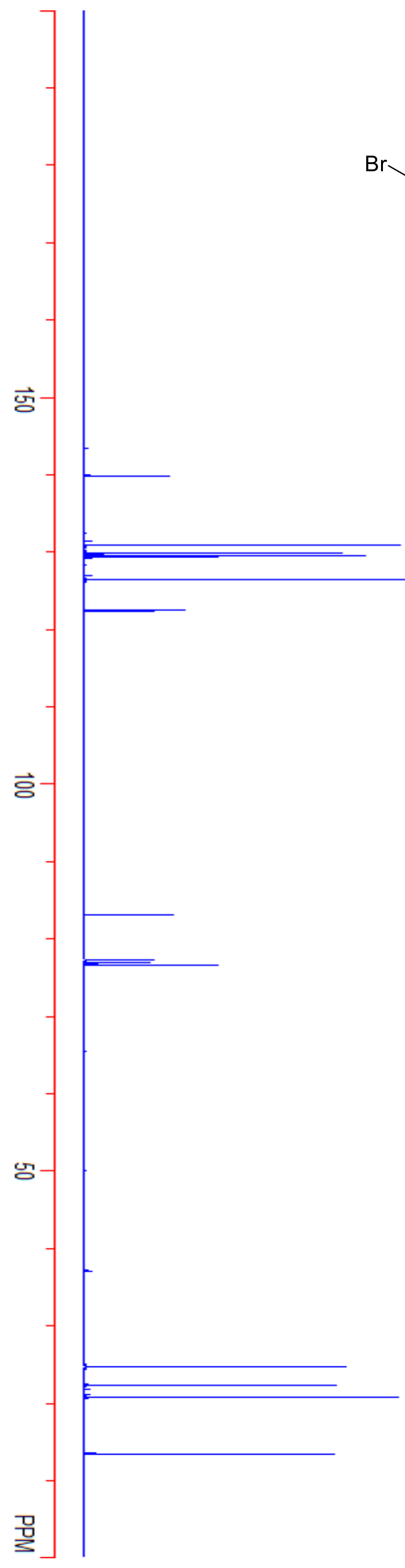

139.871
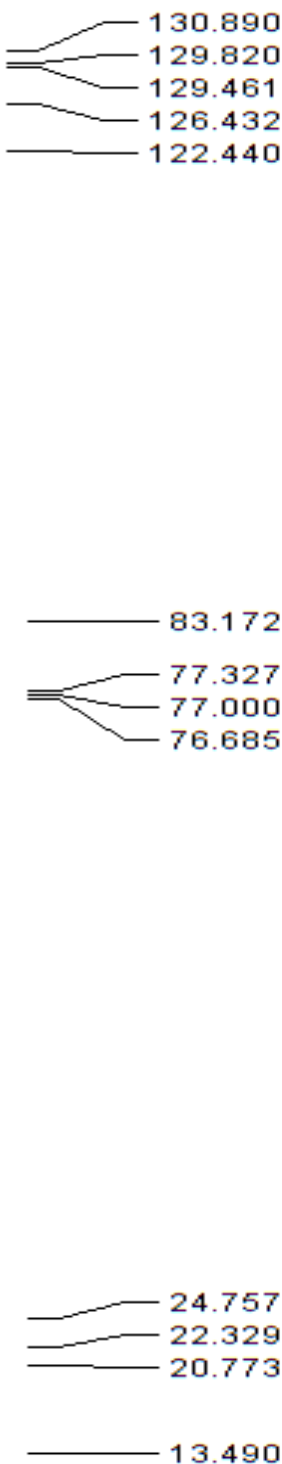


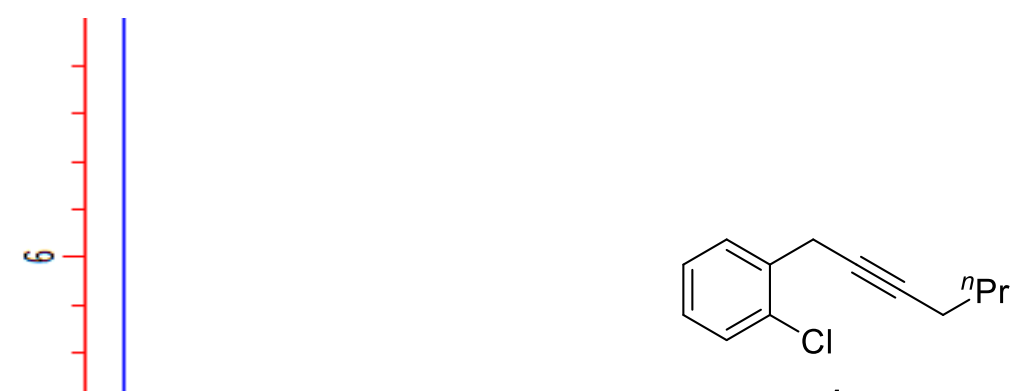

$1 p$

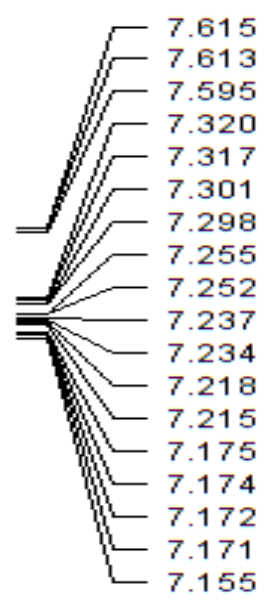

3.629

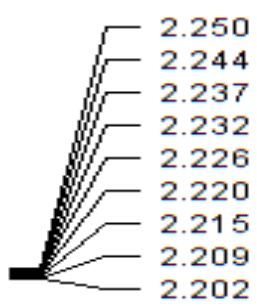

- 1530

1.521

1.511

1.509

1.504

$=1.492$

$\checkmark-1.475$

$\longrightarrow 1.445$

$\Rightarrow \quad 1.428$

$-1.415$

$\left\{\begin{array}{l}1.408 \\ -1.389\end{array}\right.$

$-0.922$

0.904
-0.886

믄 


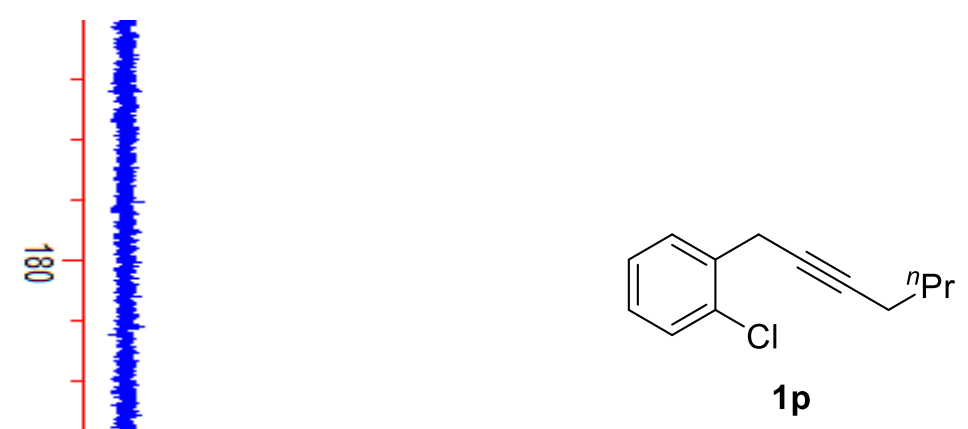

횽

횽

홍

$\overrightarrow{0}$

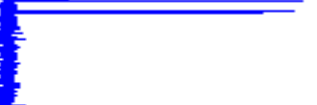

용
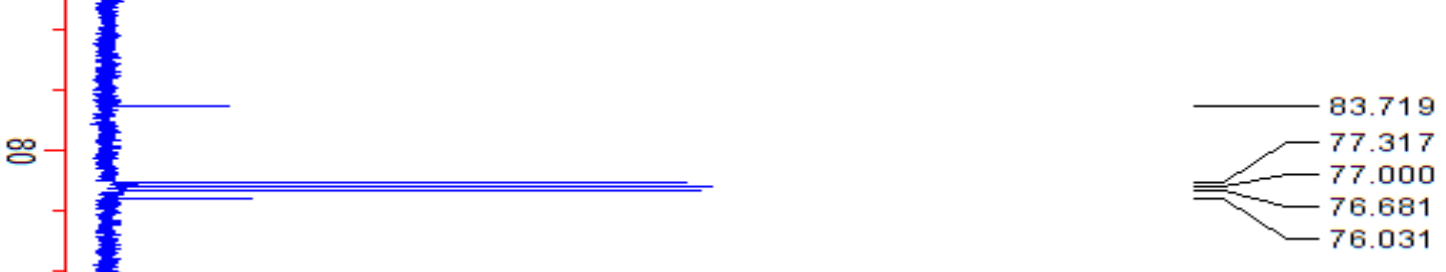

8

E-

㤎-

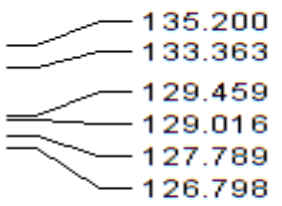

76.031

31.053

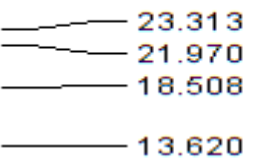



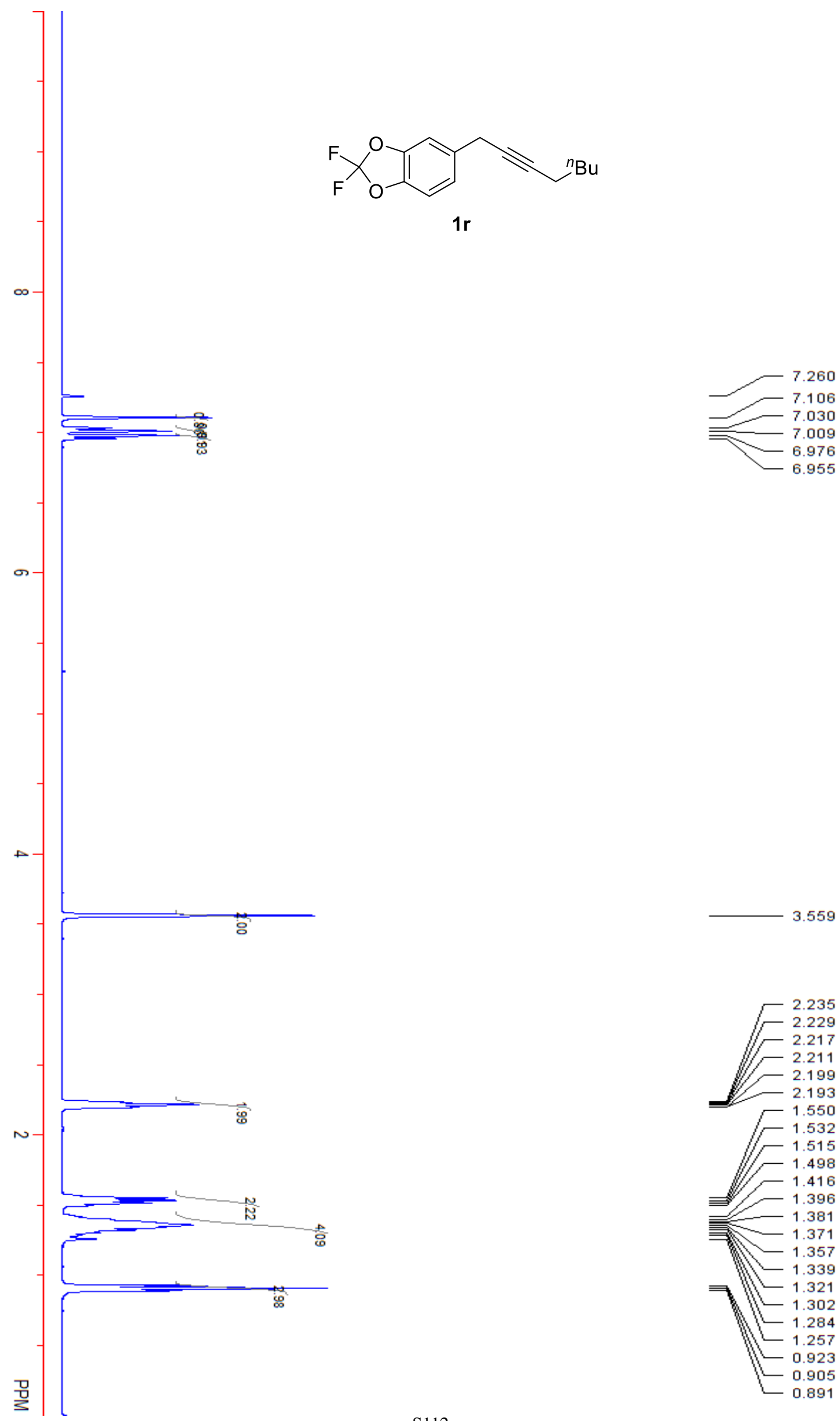

$\left[\begin{array}{r}1.550 \\ -1.532\end{array}\right.$

1.532

1.515 - 1.498 $-1.416$ $-1.396$ - 1.381 $-1.371$

$1-1.357$

$\widehat{L}-1.339$

$\Longrightarrow-1.321$

1.302

$\checkmark-1.284$

-

- 0.905

- 0.891 


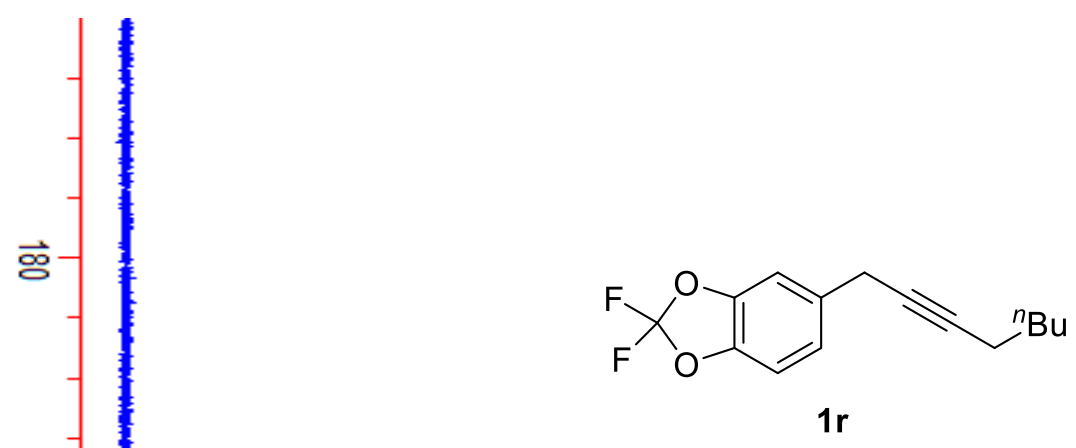

횽

$\vec{\Xi}$

点

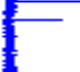

142.324

$-134.192$

133.830

131.663

$-131.663$

122.644
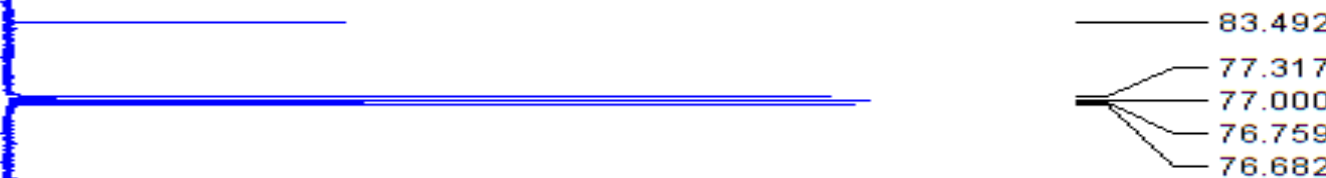

g

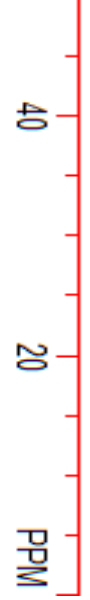
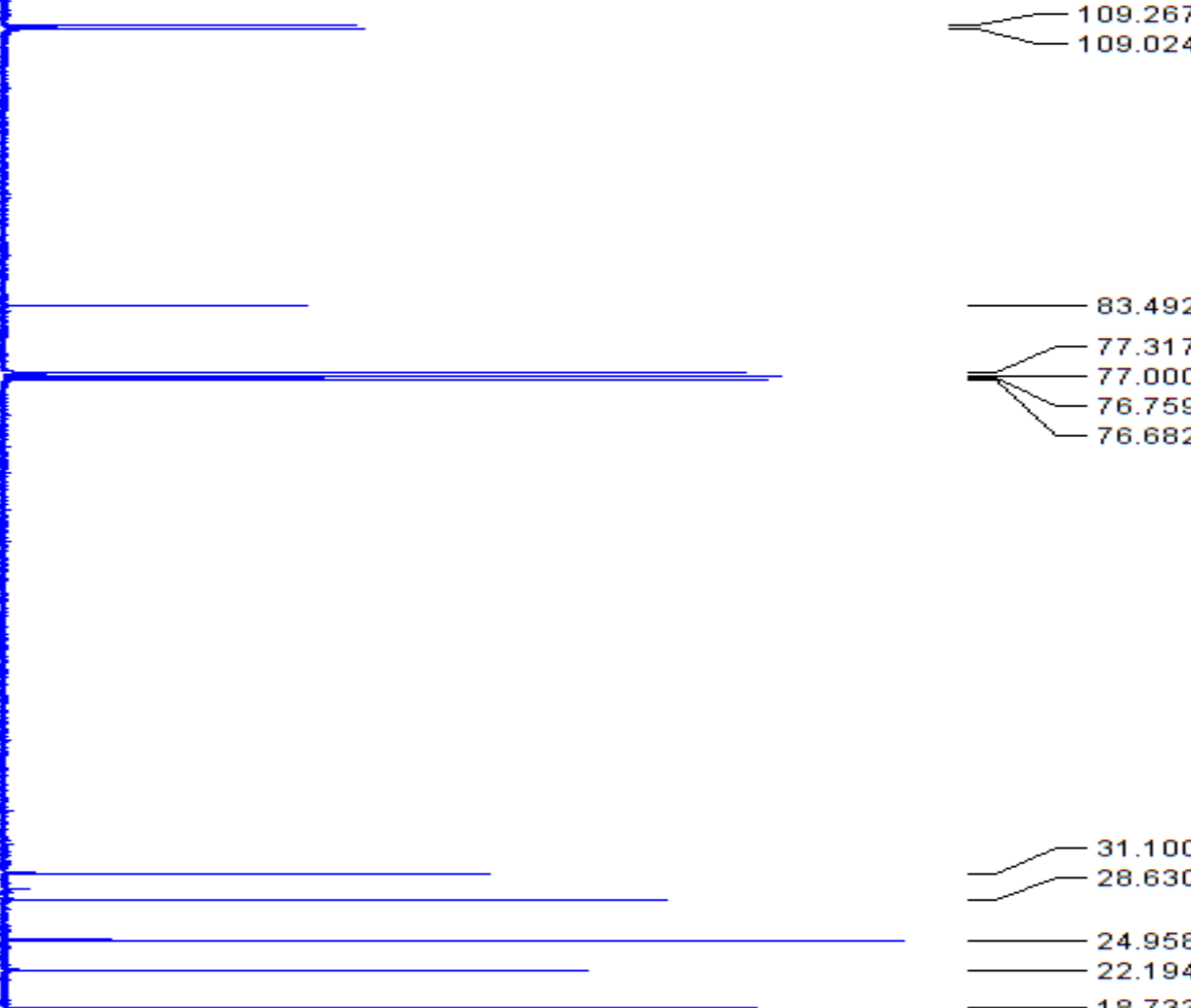

109.024

76.682

몬 


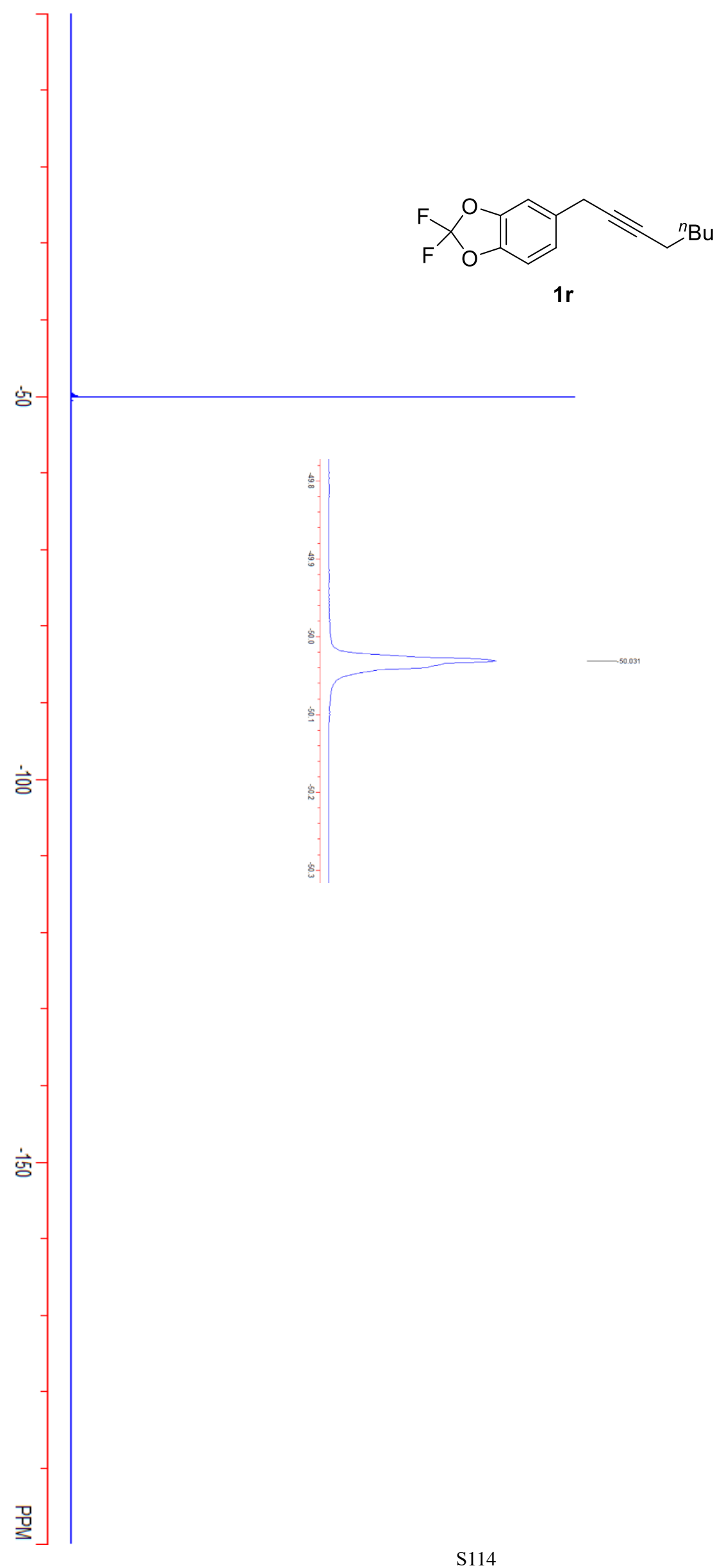

$-50.031$ 


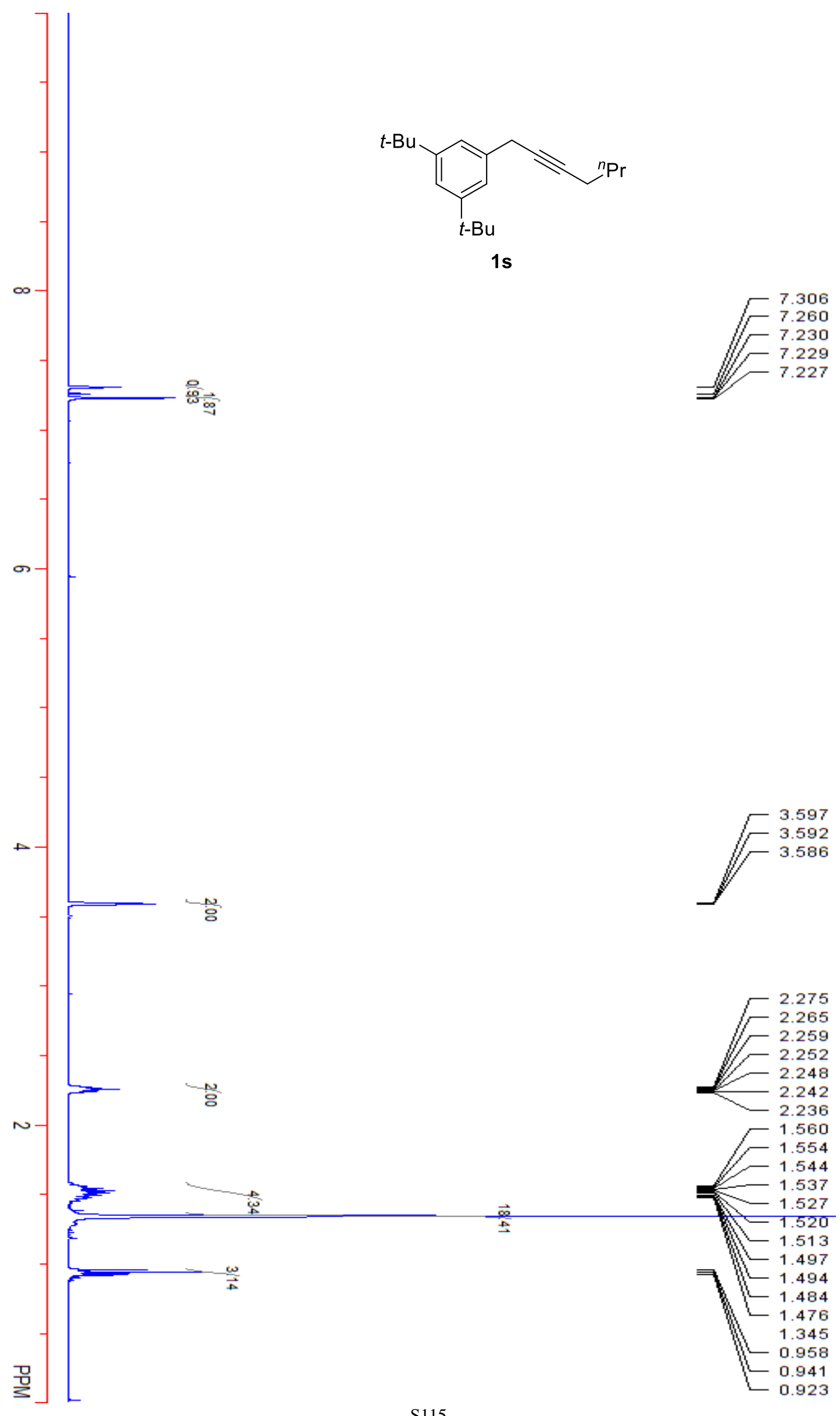




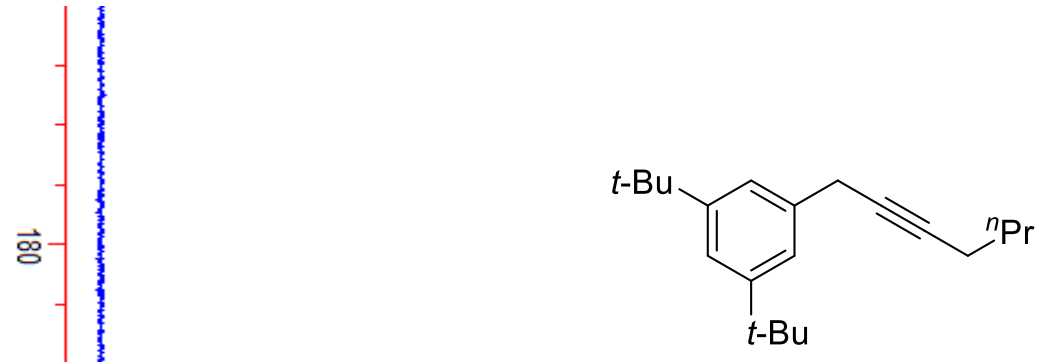

1s $\overrightarrow{8}$

点

$\vec{\Xi}$

흥

8

응

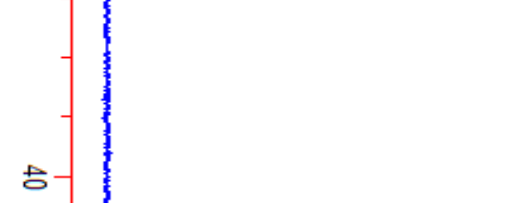

B

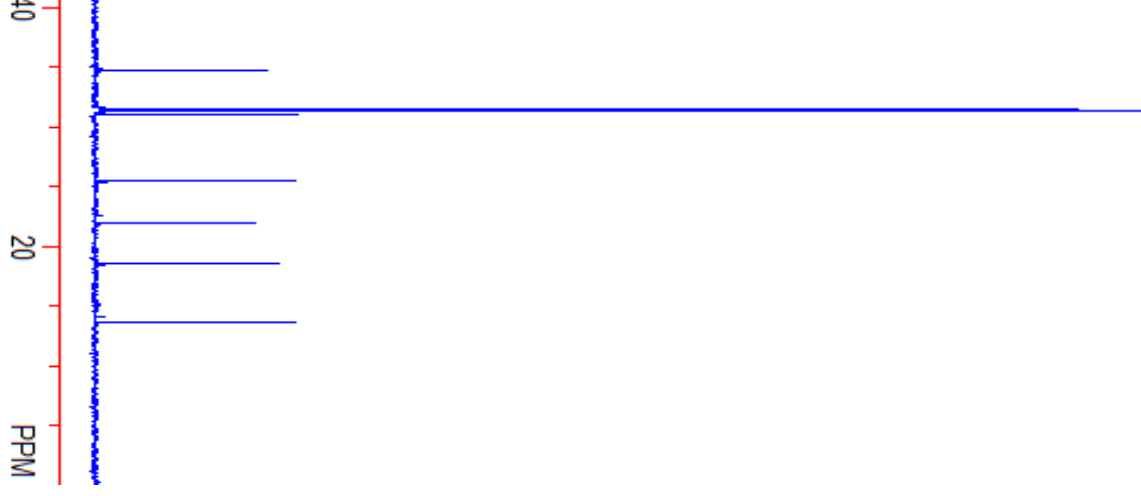

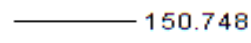
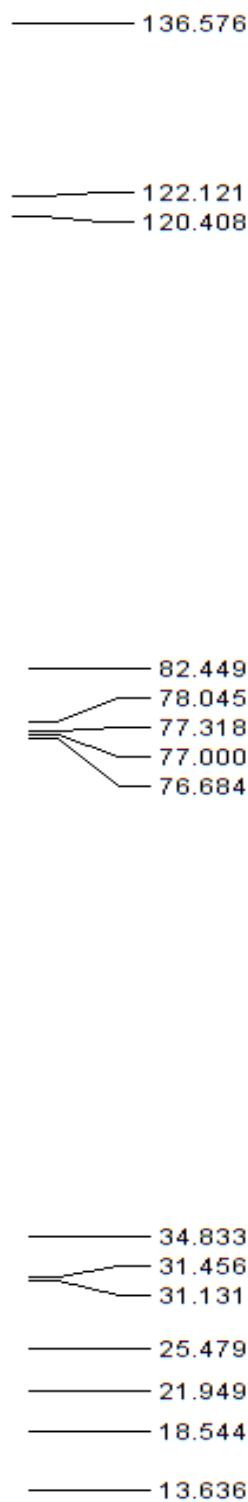


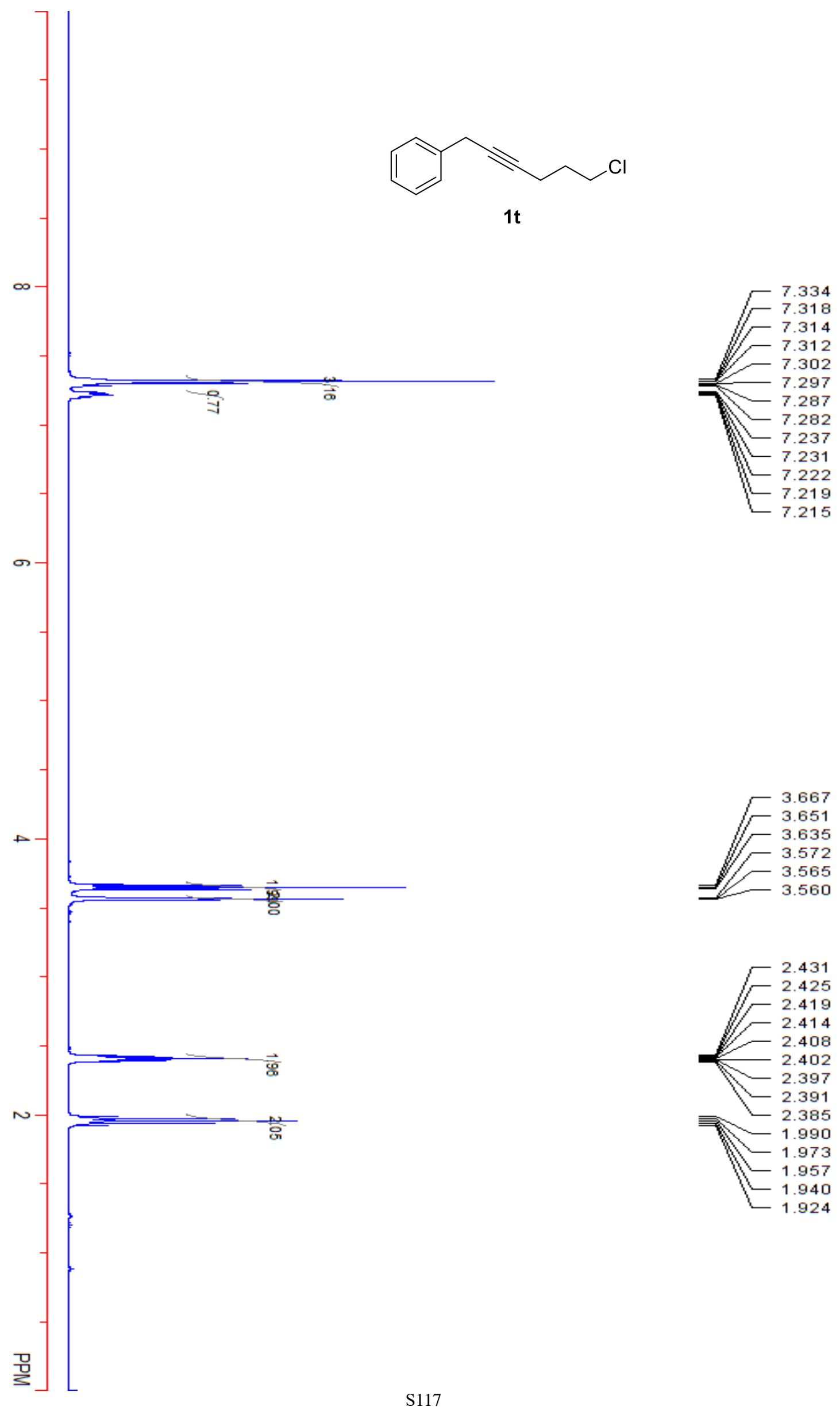




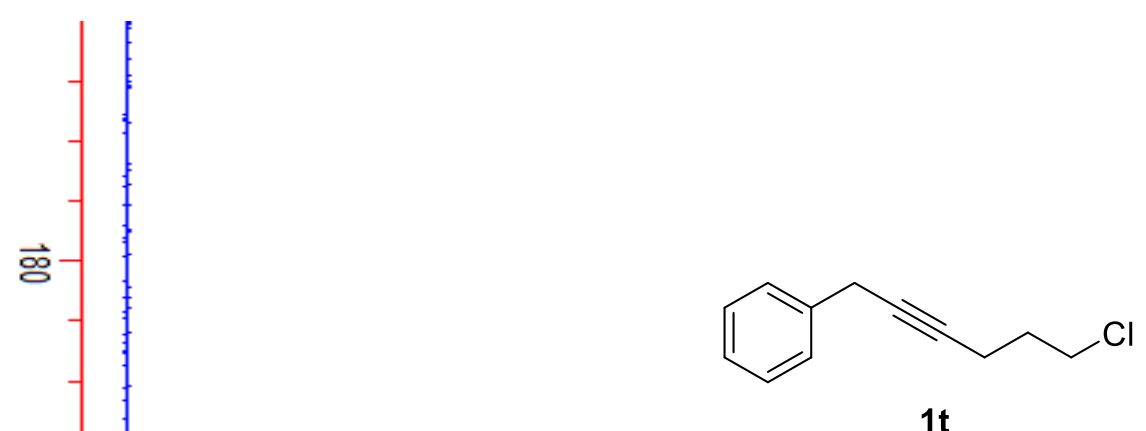

$\overrightarrow{8}$

횽

횽

응

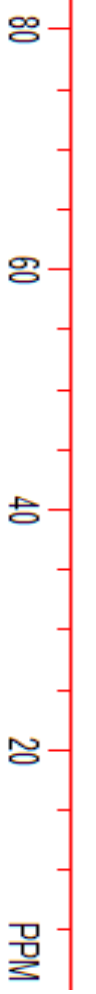

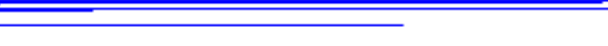

$-$

(

$-137.241$

$-128.420$

127.761

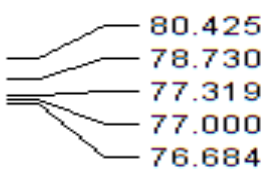

43.754

31.611

25.050 

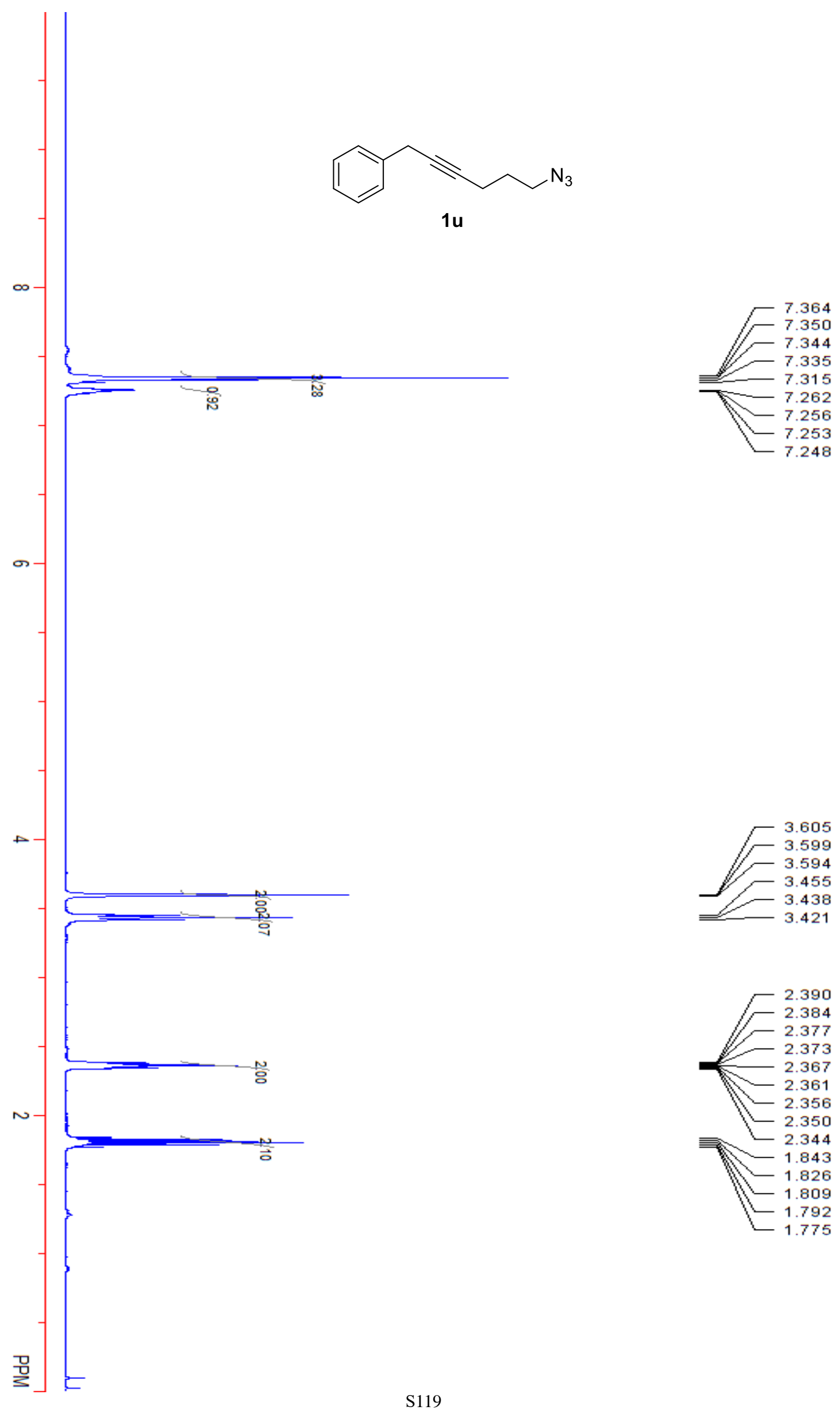


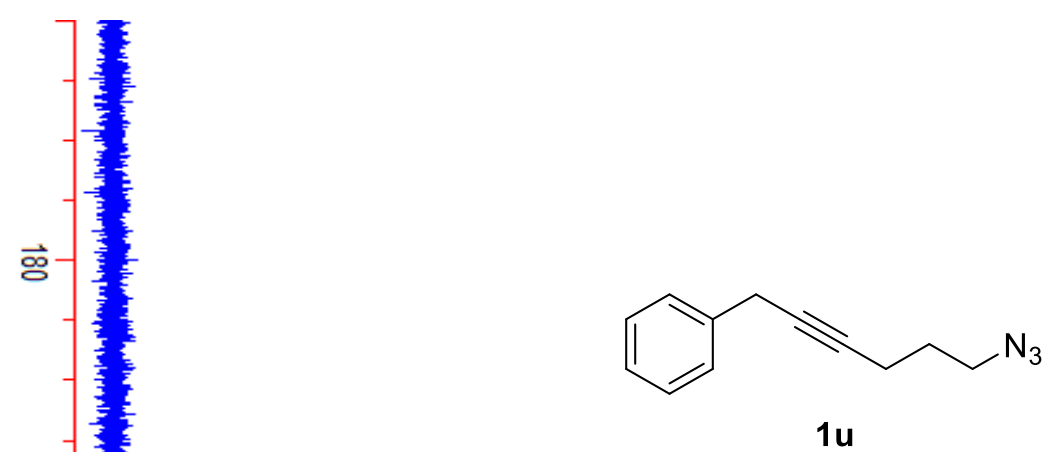

$\vec{\circ}$

E्

넝

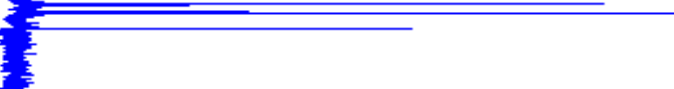

- 128.419

127.763

흥

。

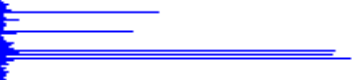

80.521

78.850

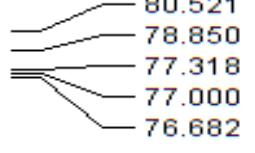

8 


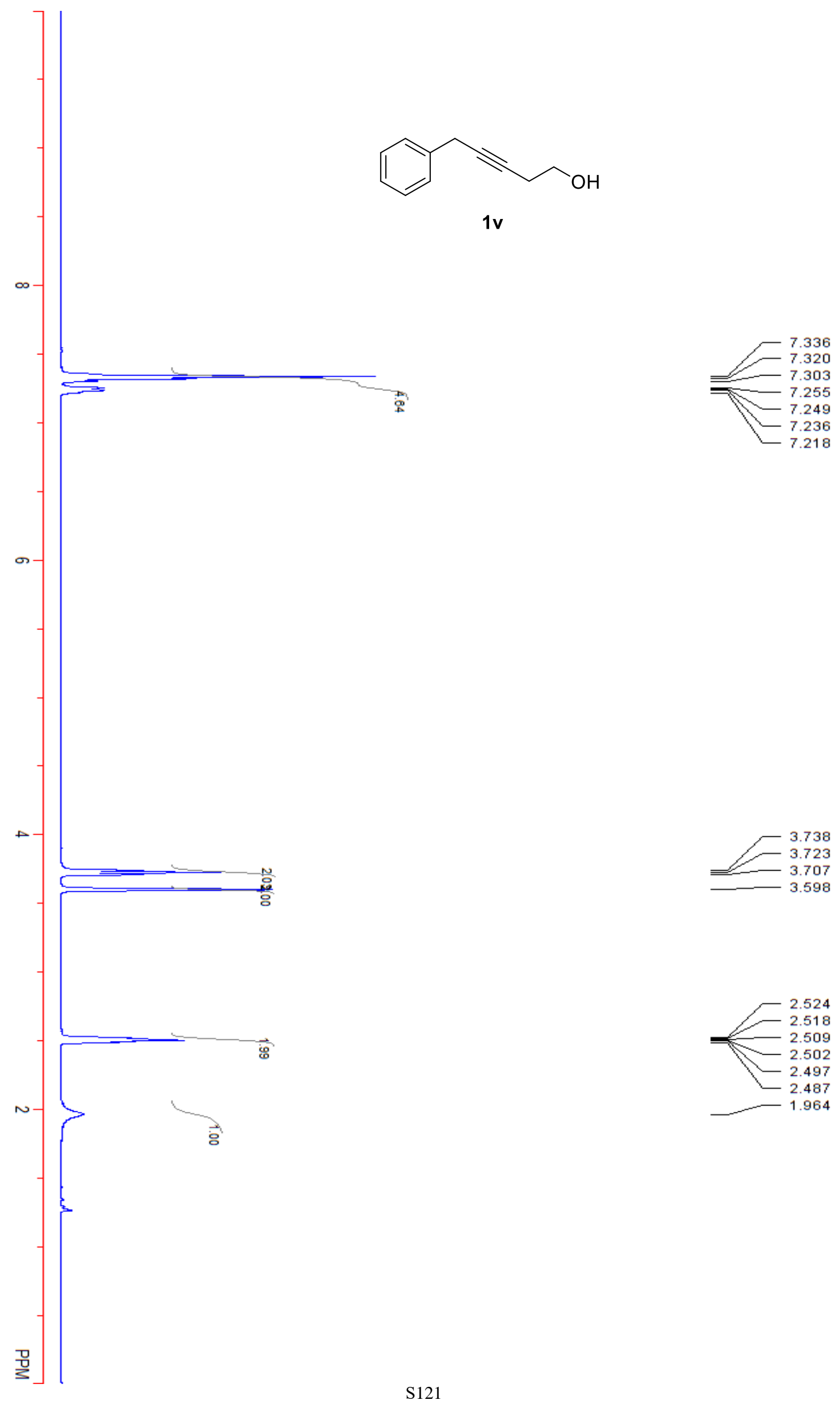




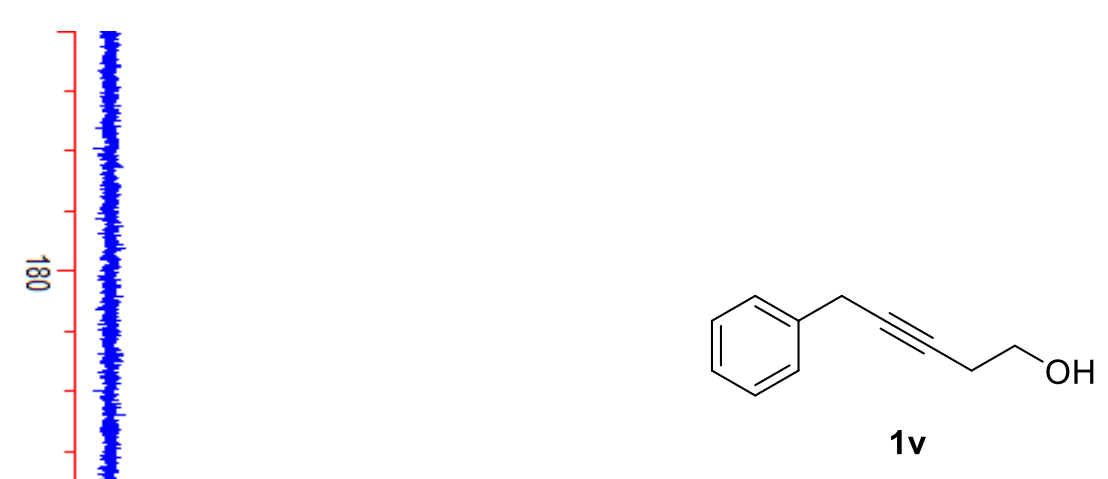

$\vec{\circ}$

횽

흥

\%
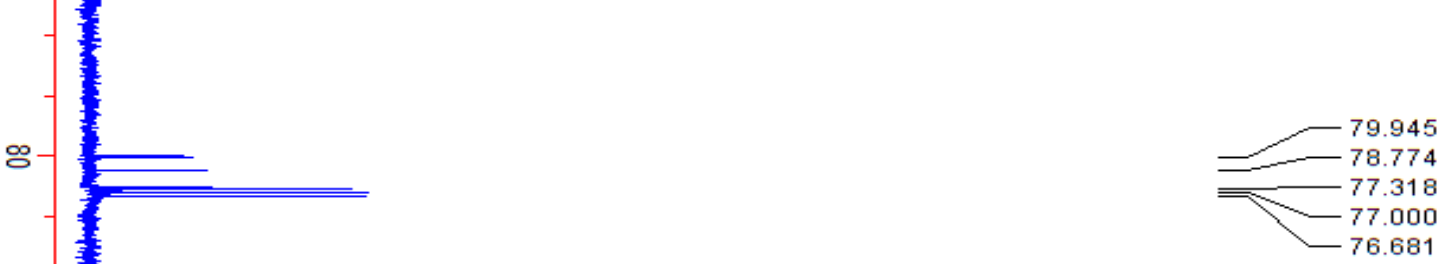

8

-
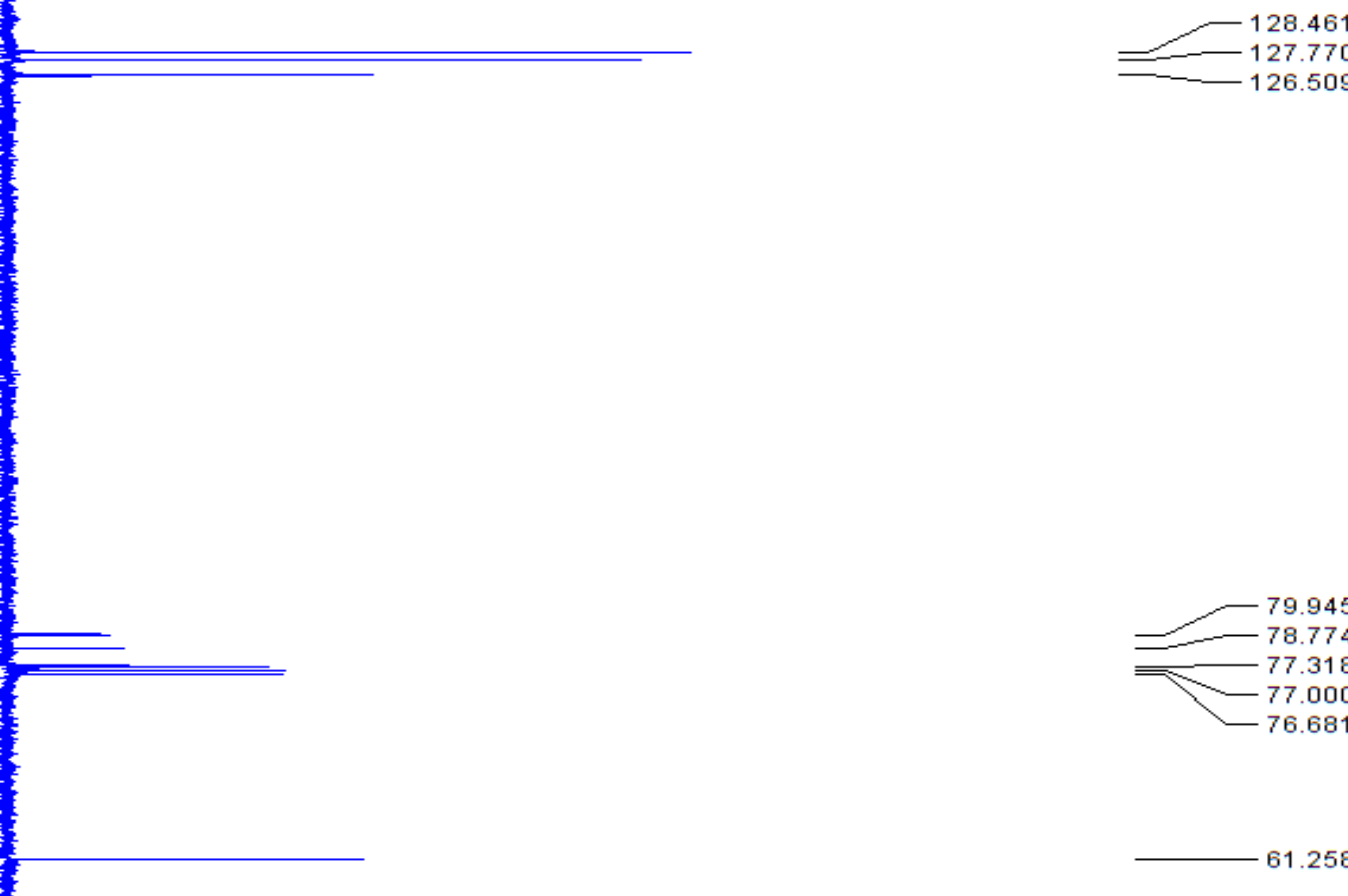

$\vec{\circ}-$

s-

$\pm$

$-$

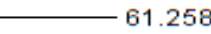

25.062

23.173 

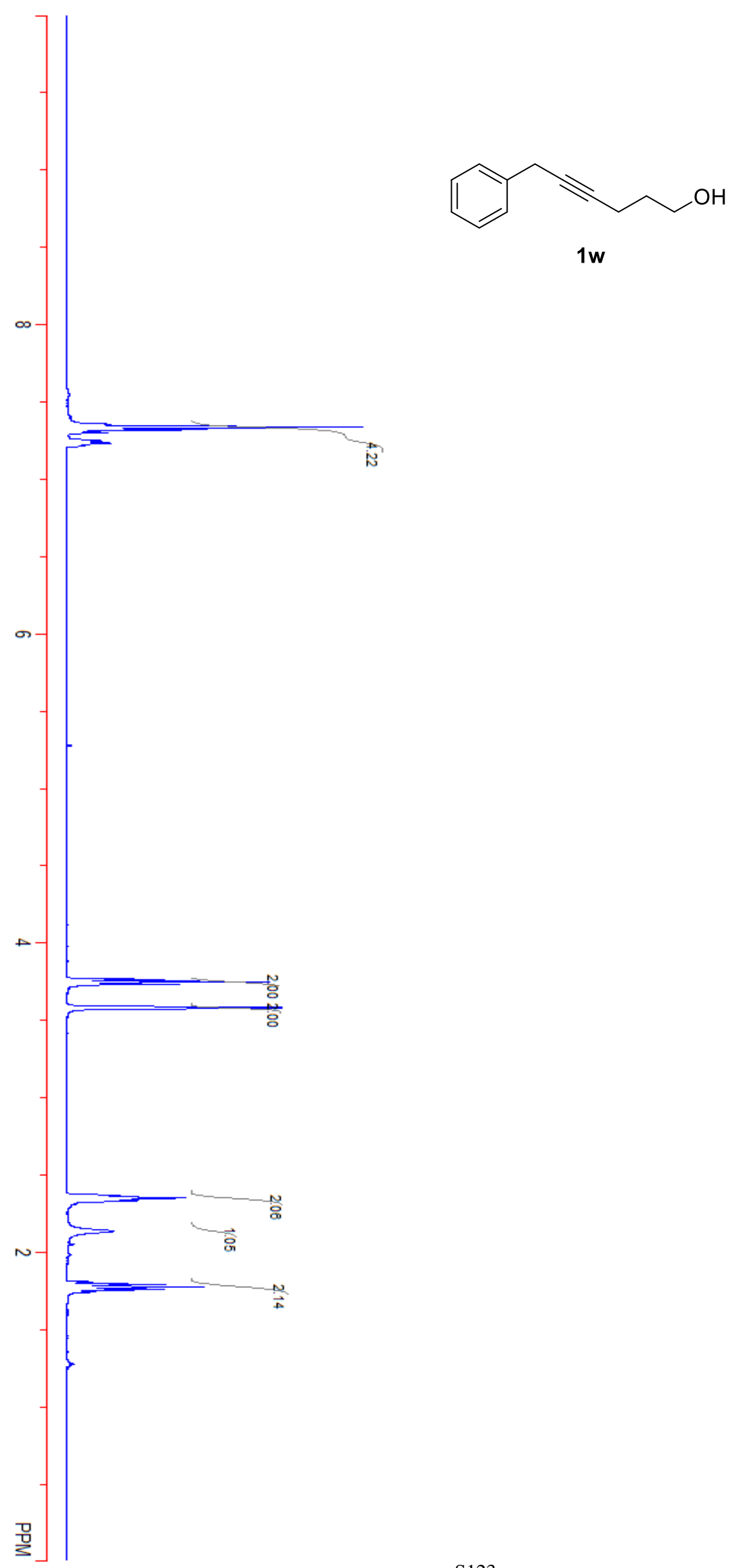

$1 w$
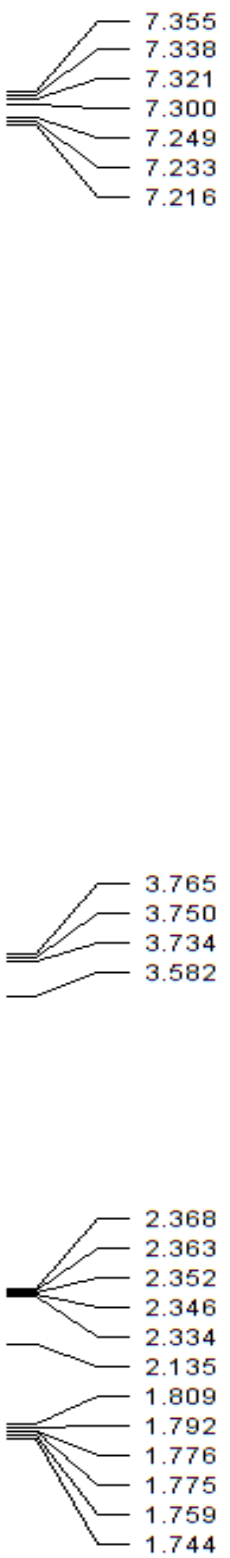


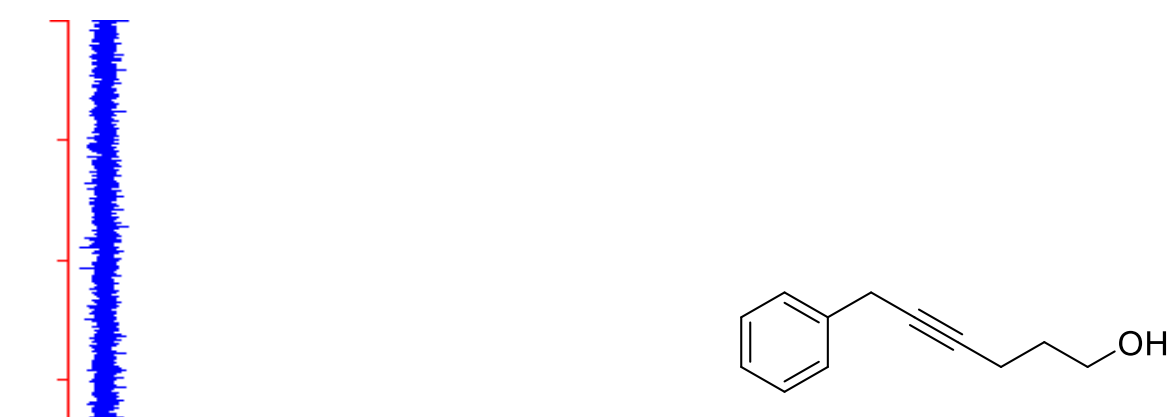

$1 w$
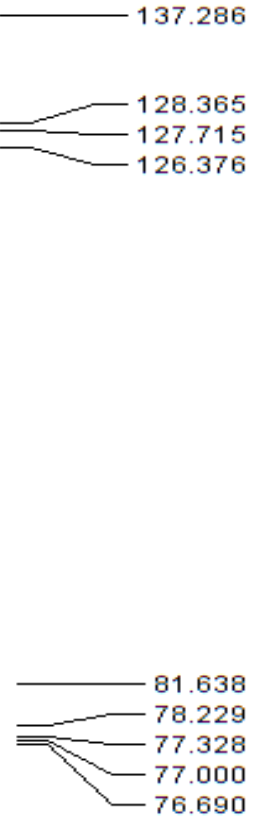

61.672

g

ㅎㅇ

$\overrightarrow{5}$
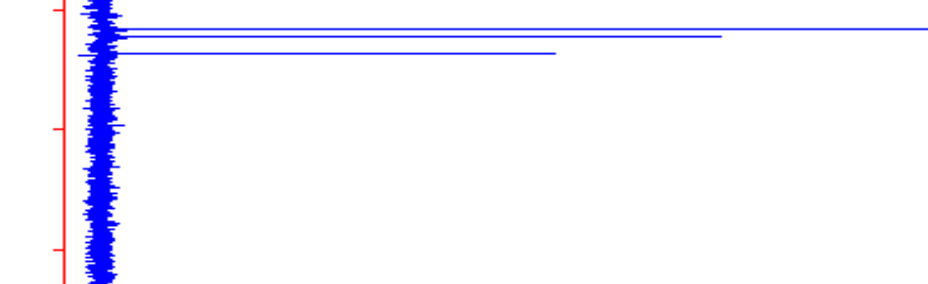

문

31.449

25.016 

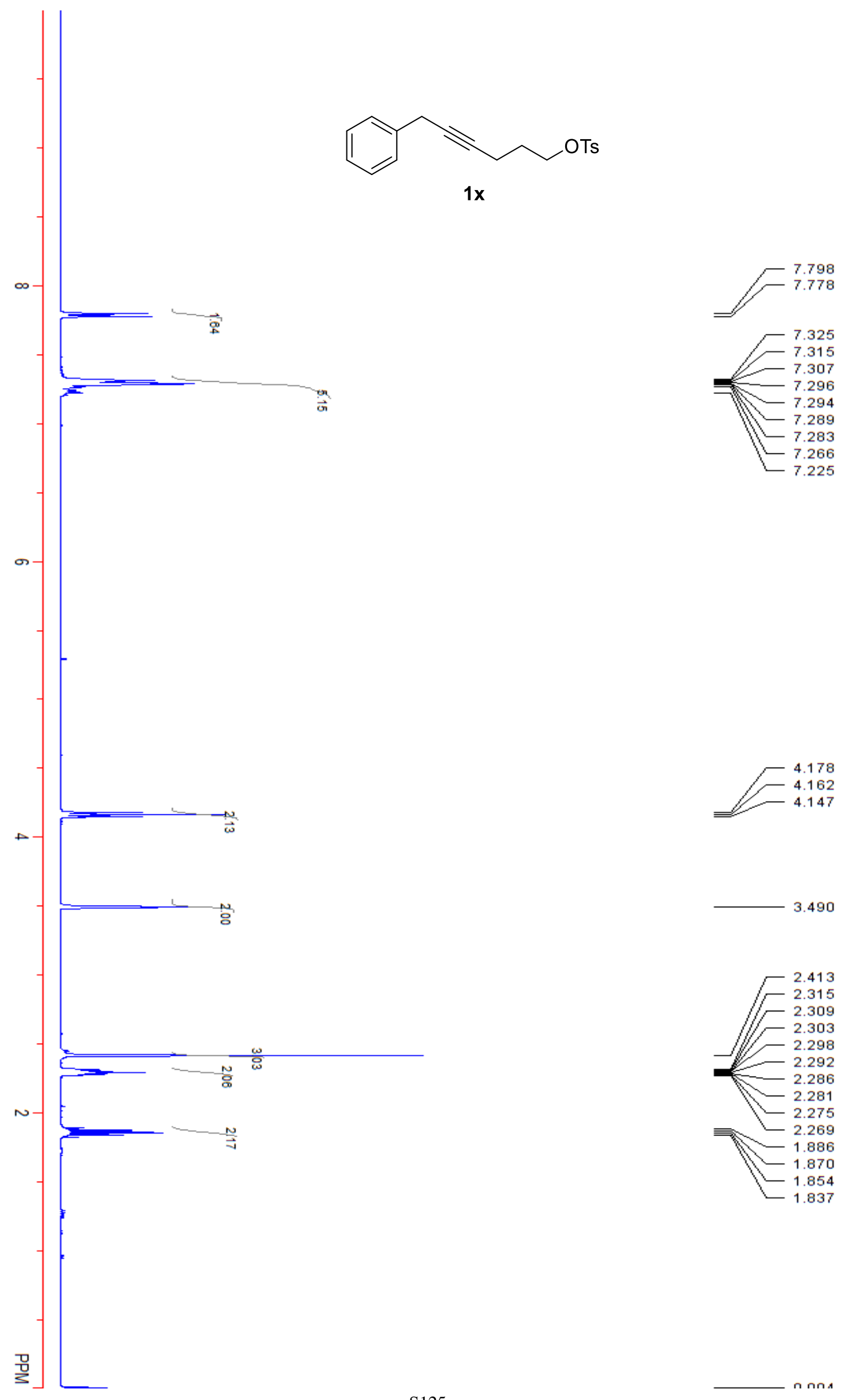

3.490

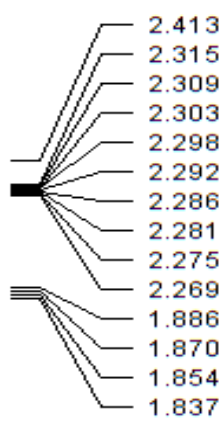



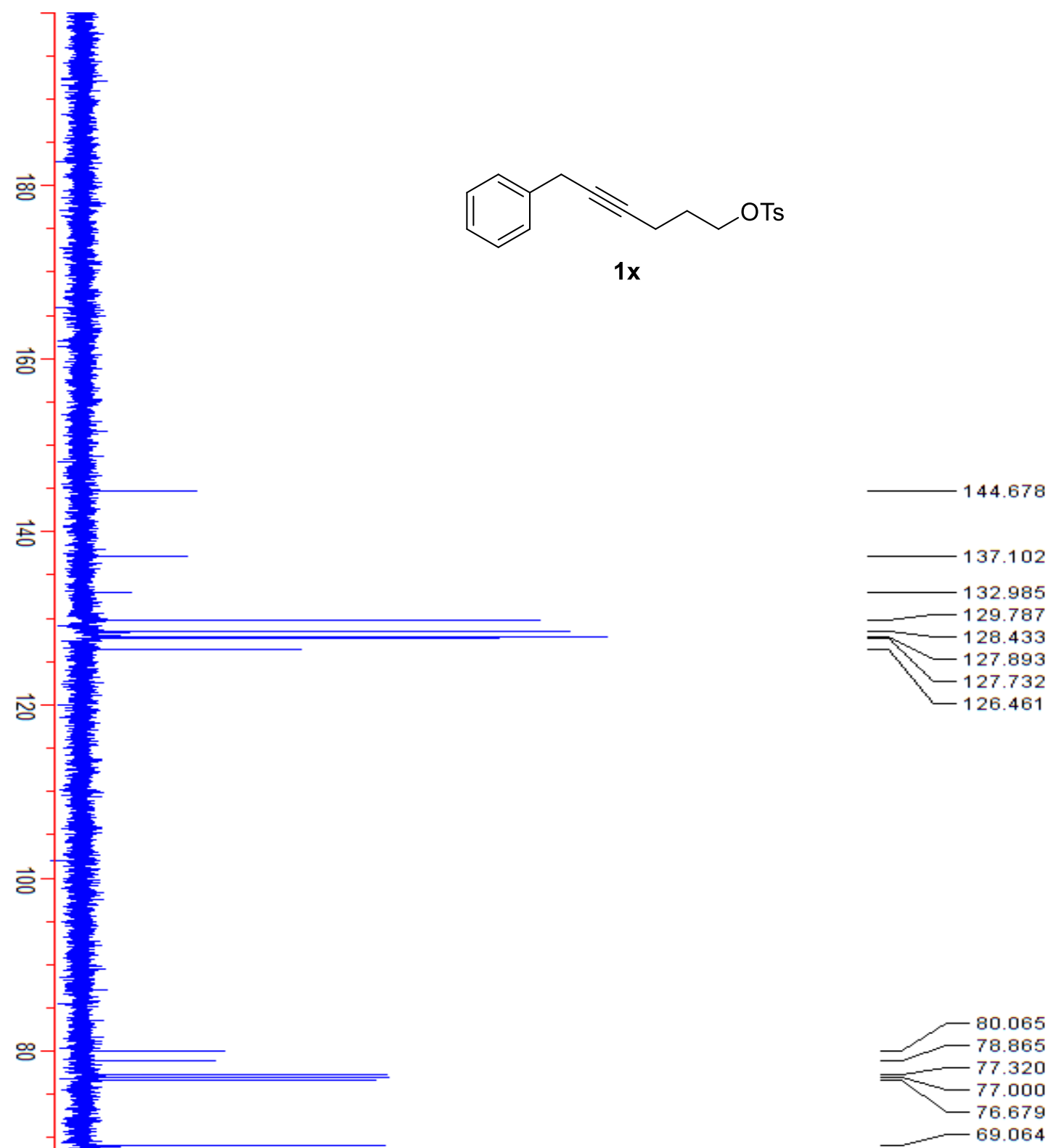

용

ㅇㅇㅇ

$\overrightarrow{8}$

릉

$\overrightarrow{\mathrm{O}}$
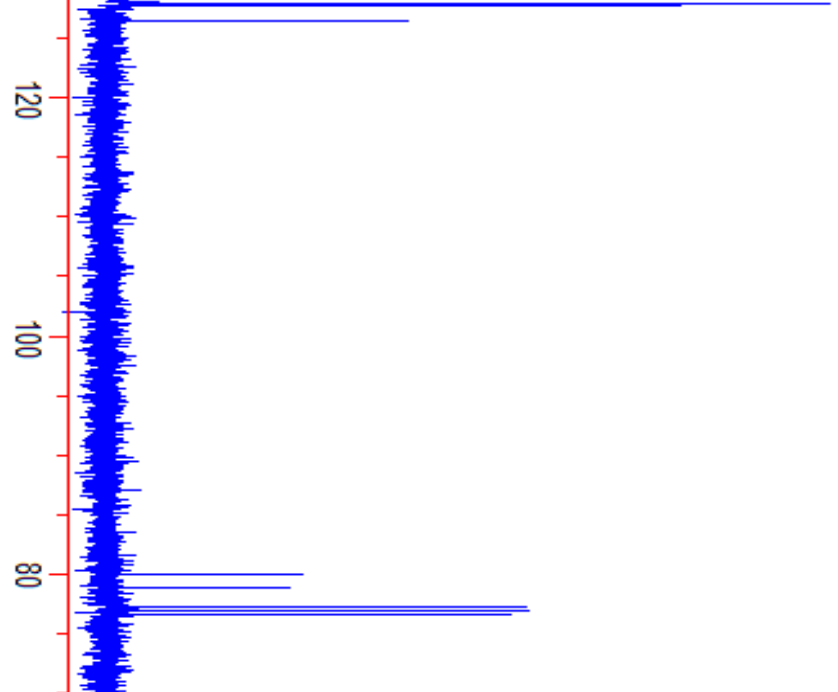

8

\&-

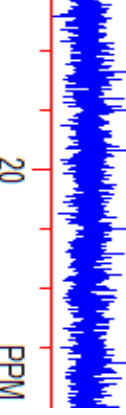

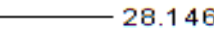

24.963

21.583

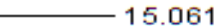




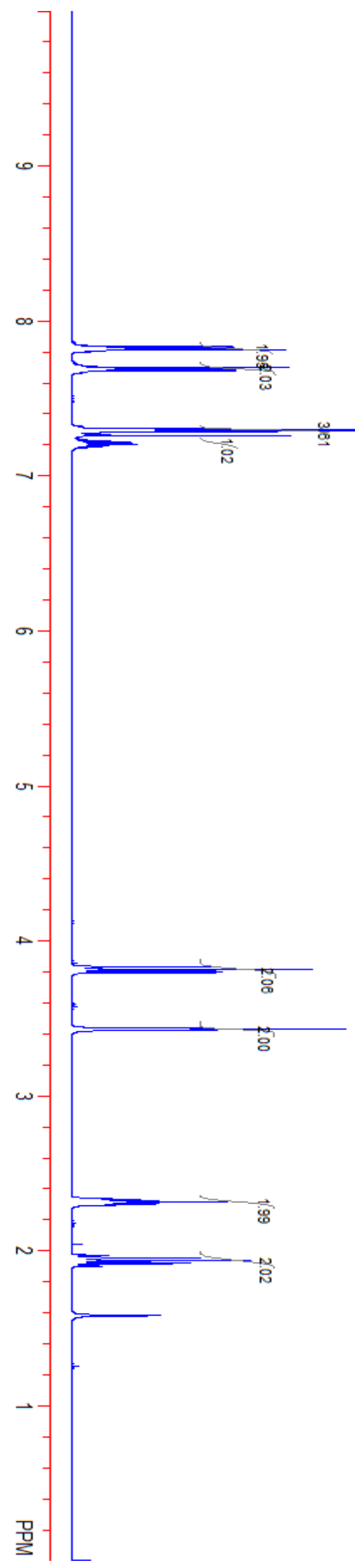<smiles>O=C1c2ccccc2C(=O)N1CCCC#CCc1ccccc1</smiles>
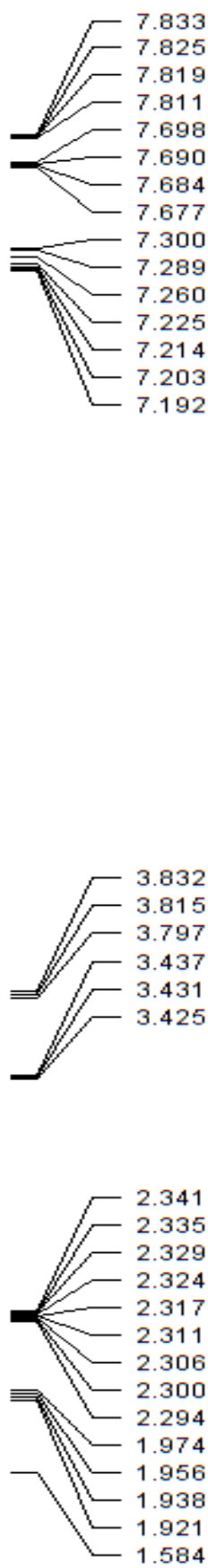


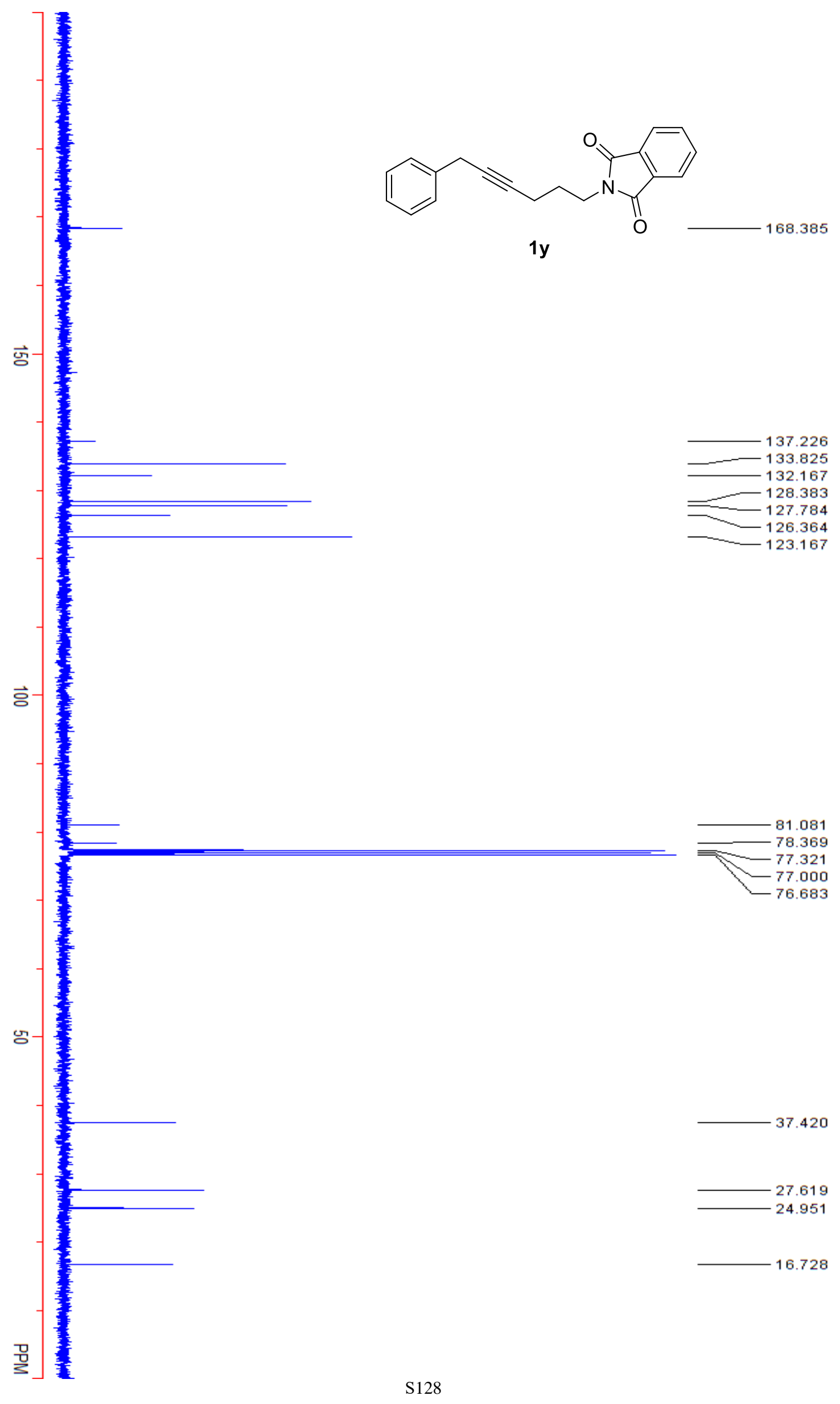




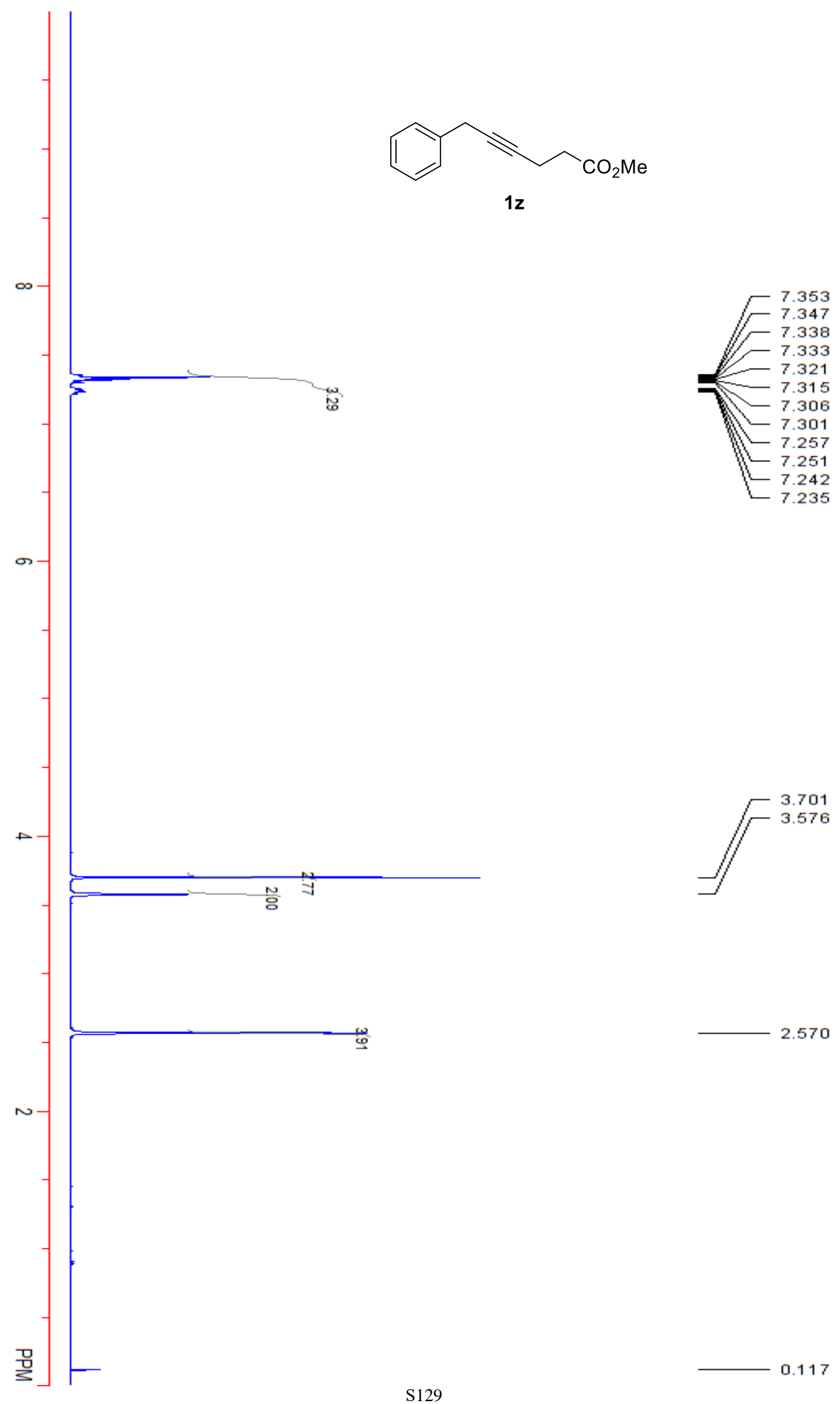




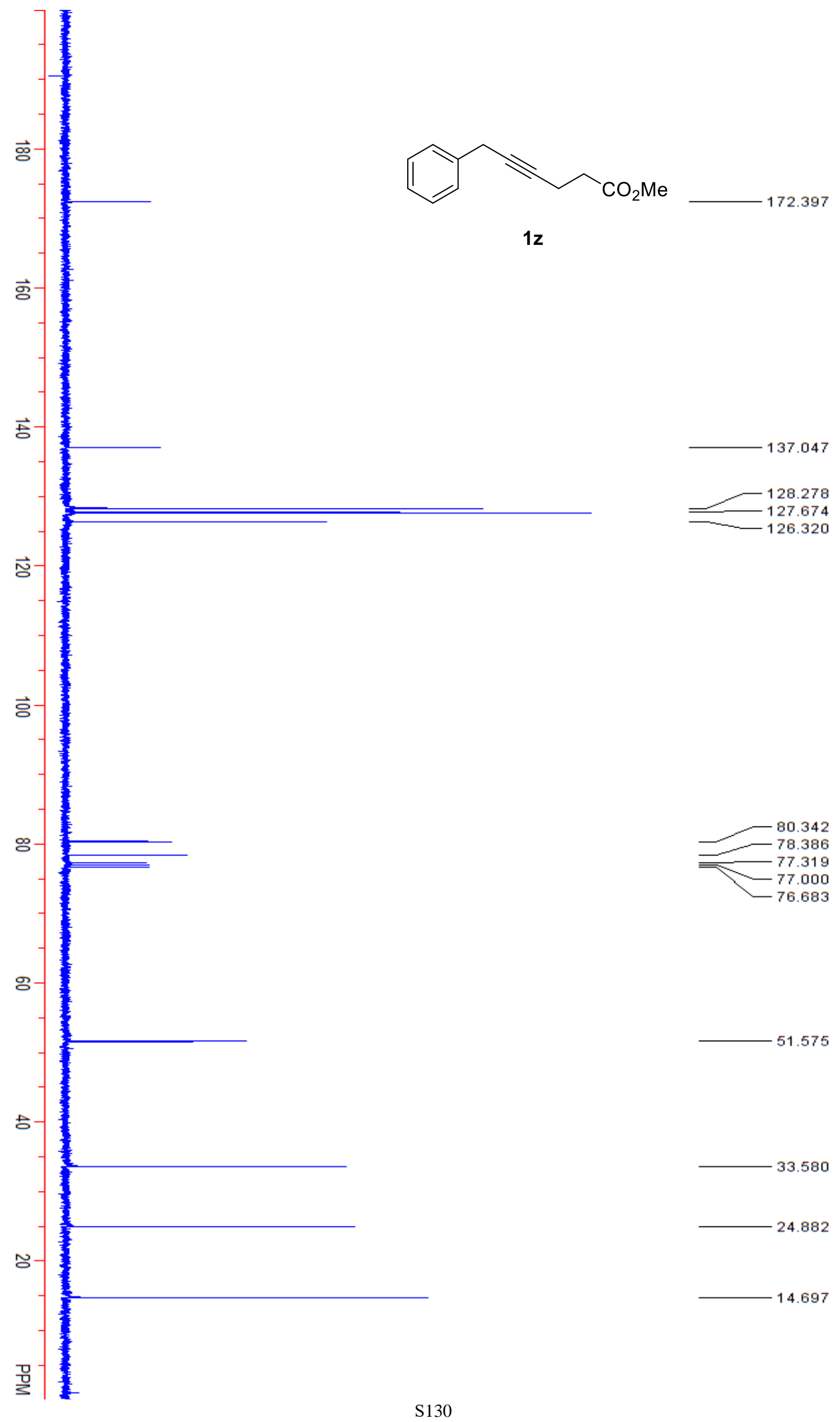



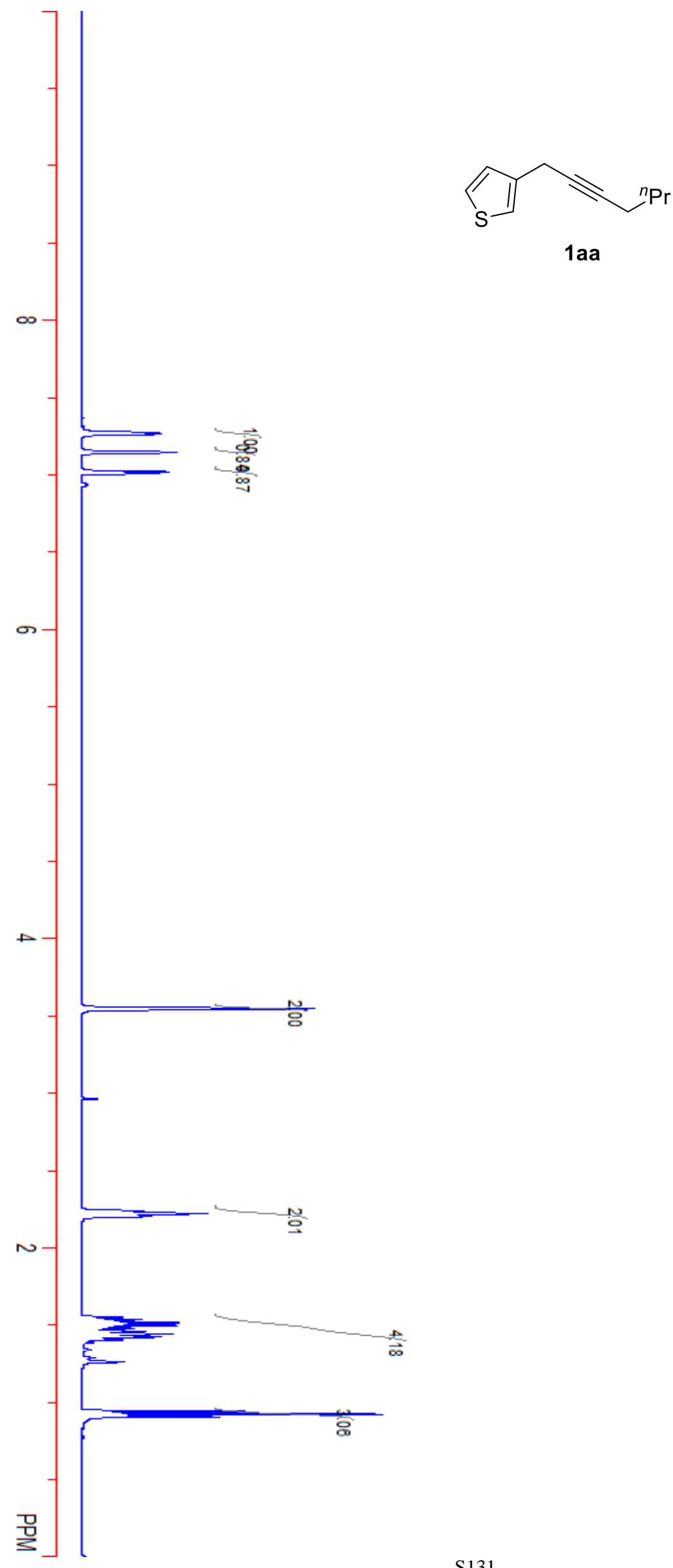

1 aa

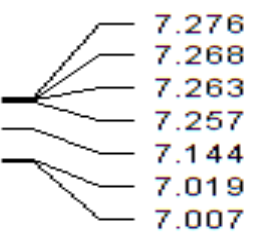

3.546

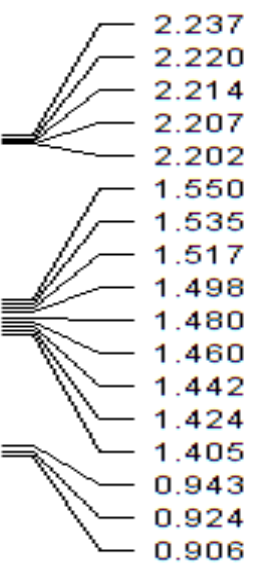



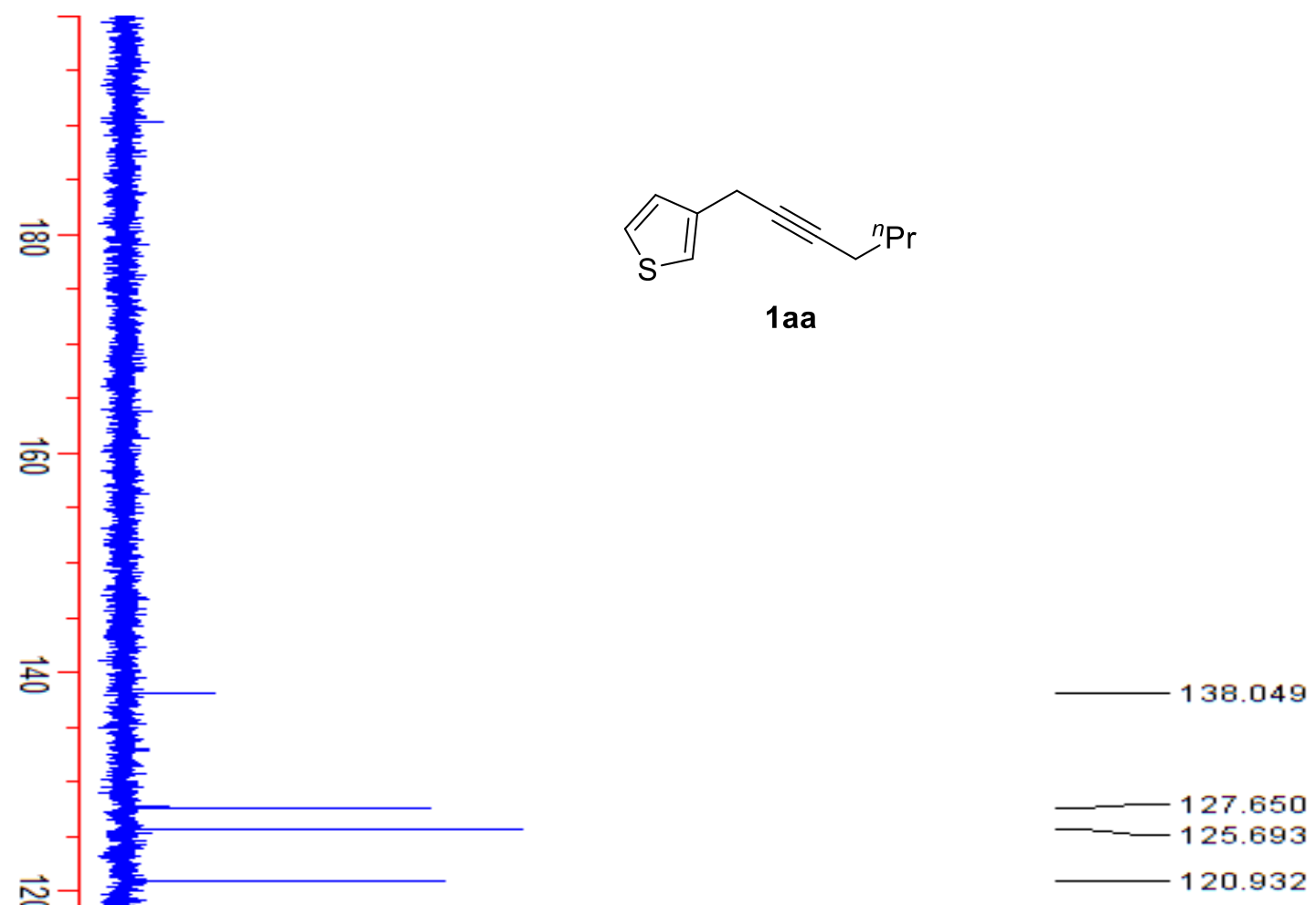

홍

용

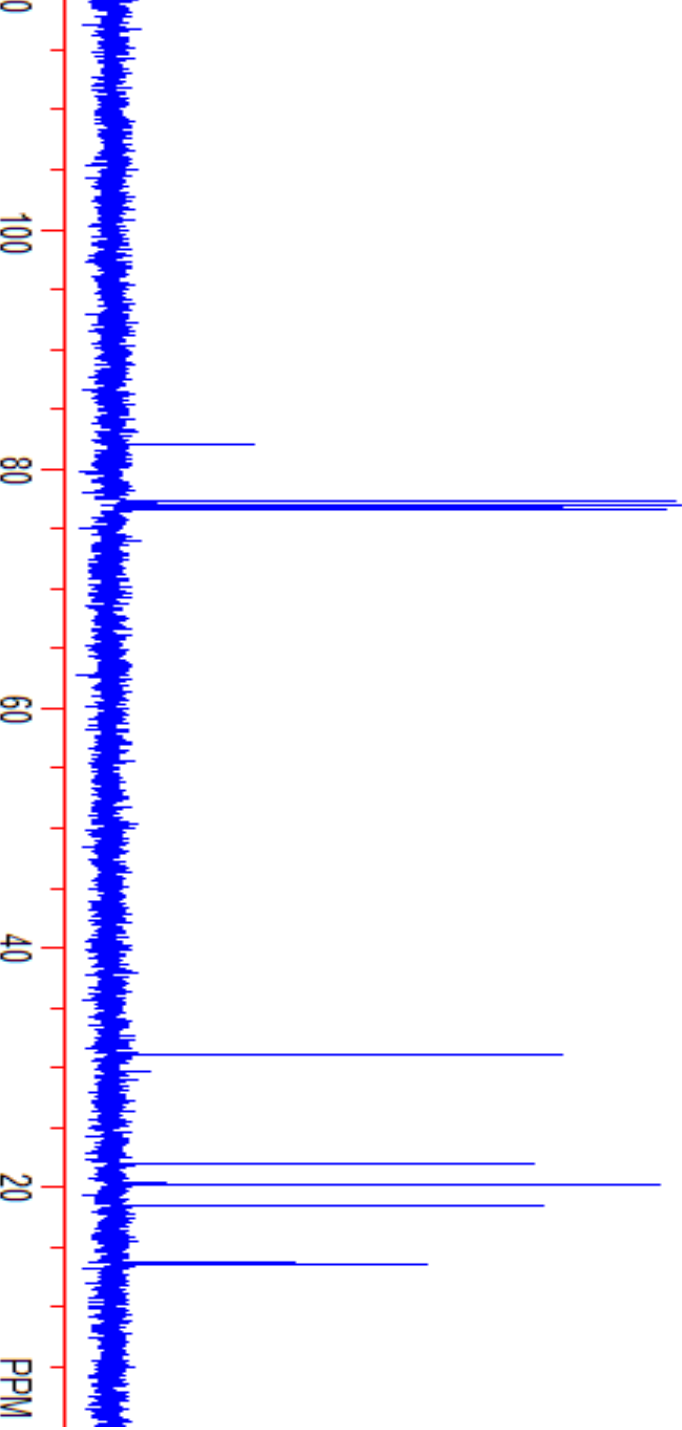

81.983 


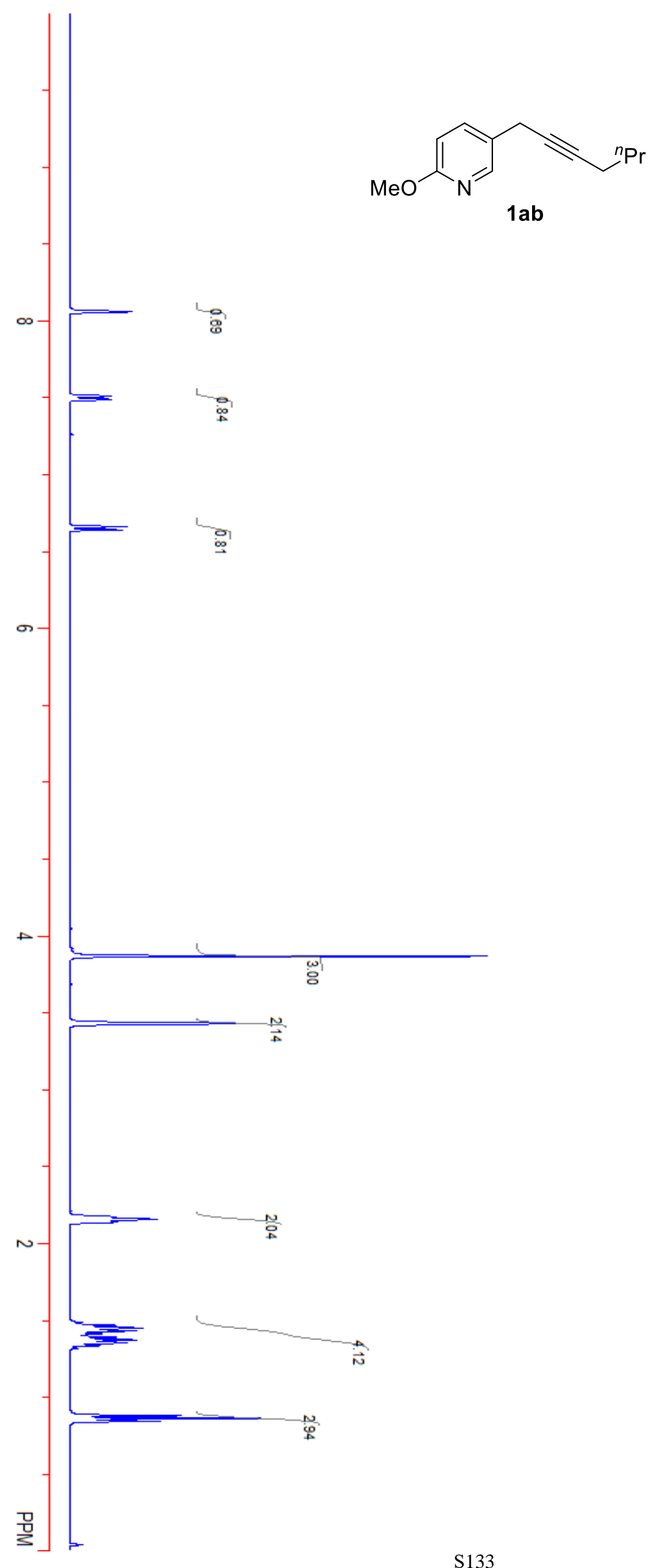

8.060
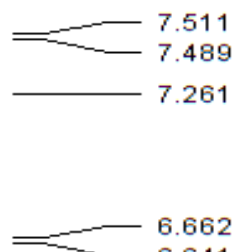

6.641

3.869

3.431

2.173

2.158

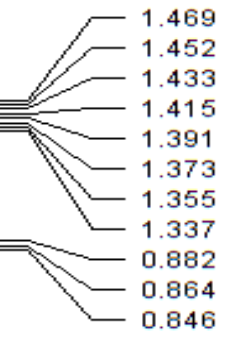




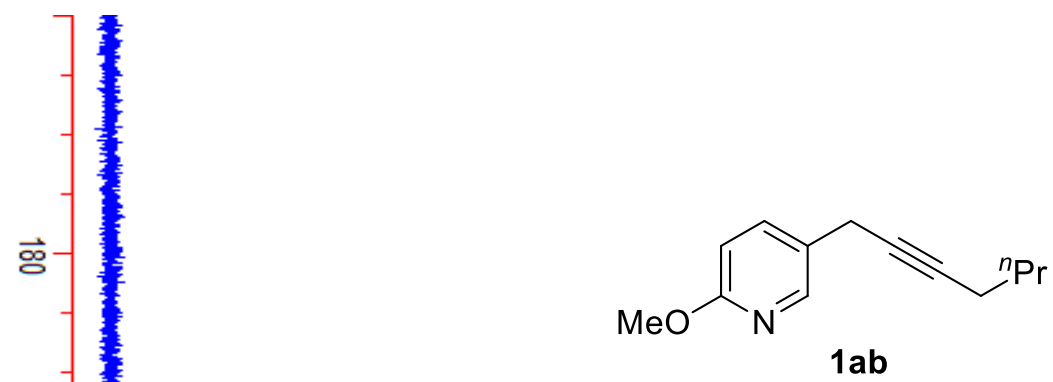

162.926

$\overrightarrow{8}$

E

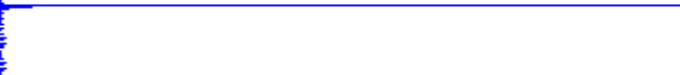

145.458

138.337

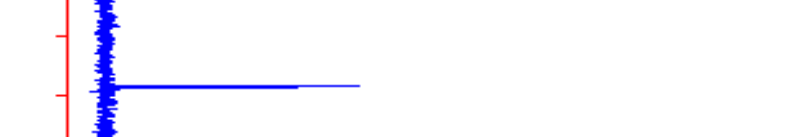

$\vec{\nabla}$
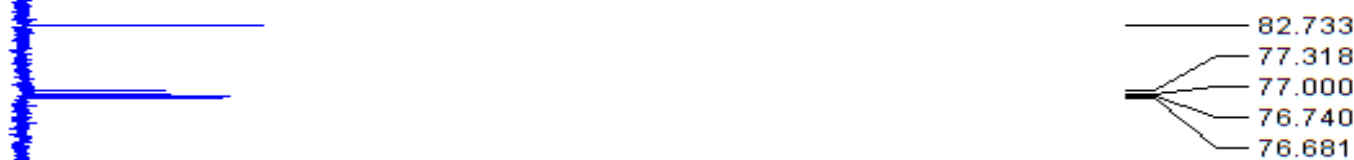

8

.

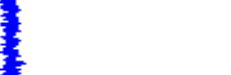

몰

용

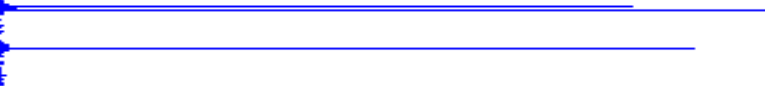

$-125.691$

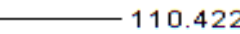

110.422

53.213

30.929

21.867

21.599

18.333

13.499 

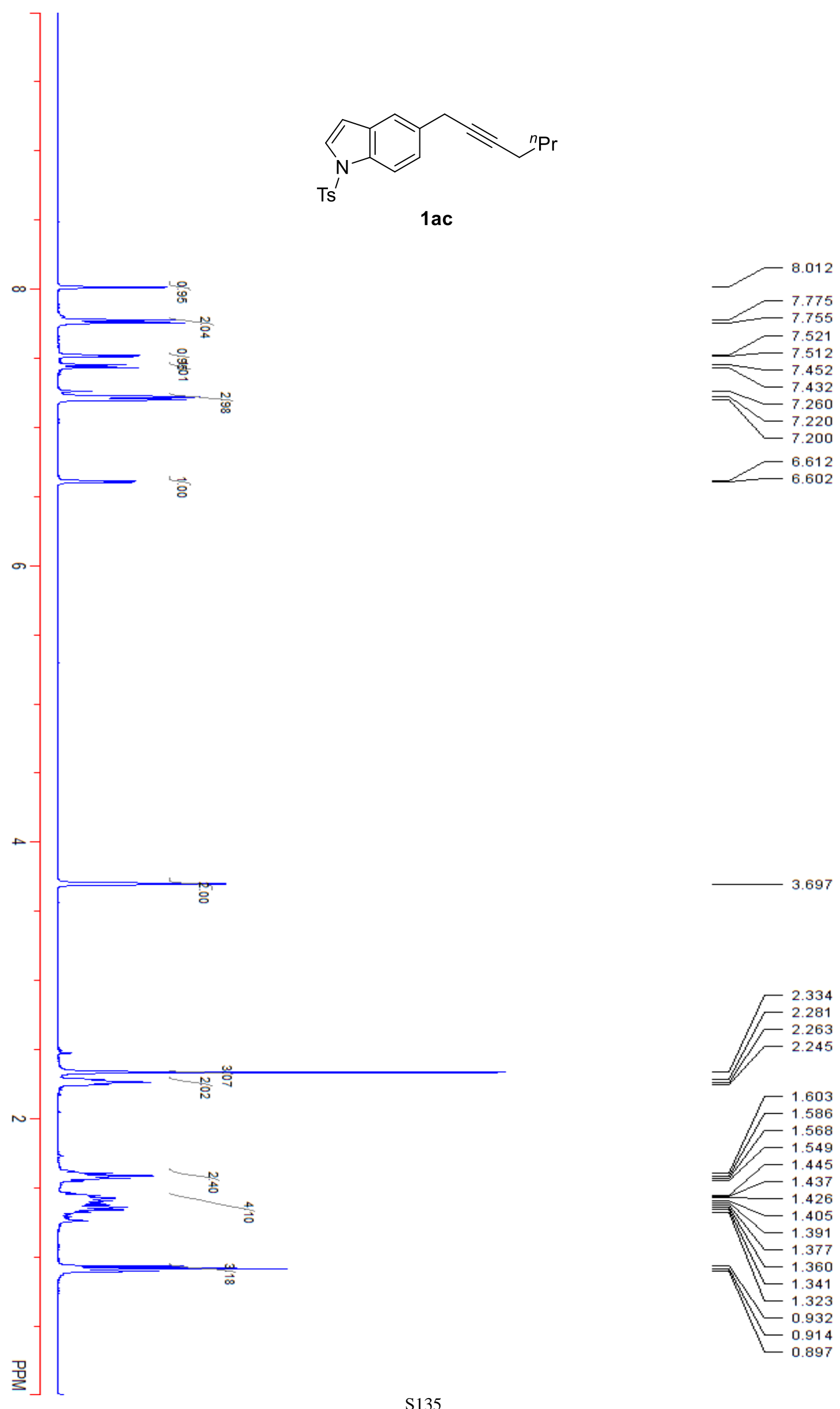

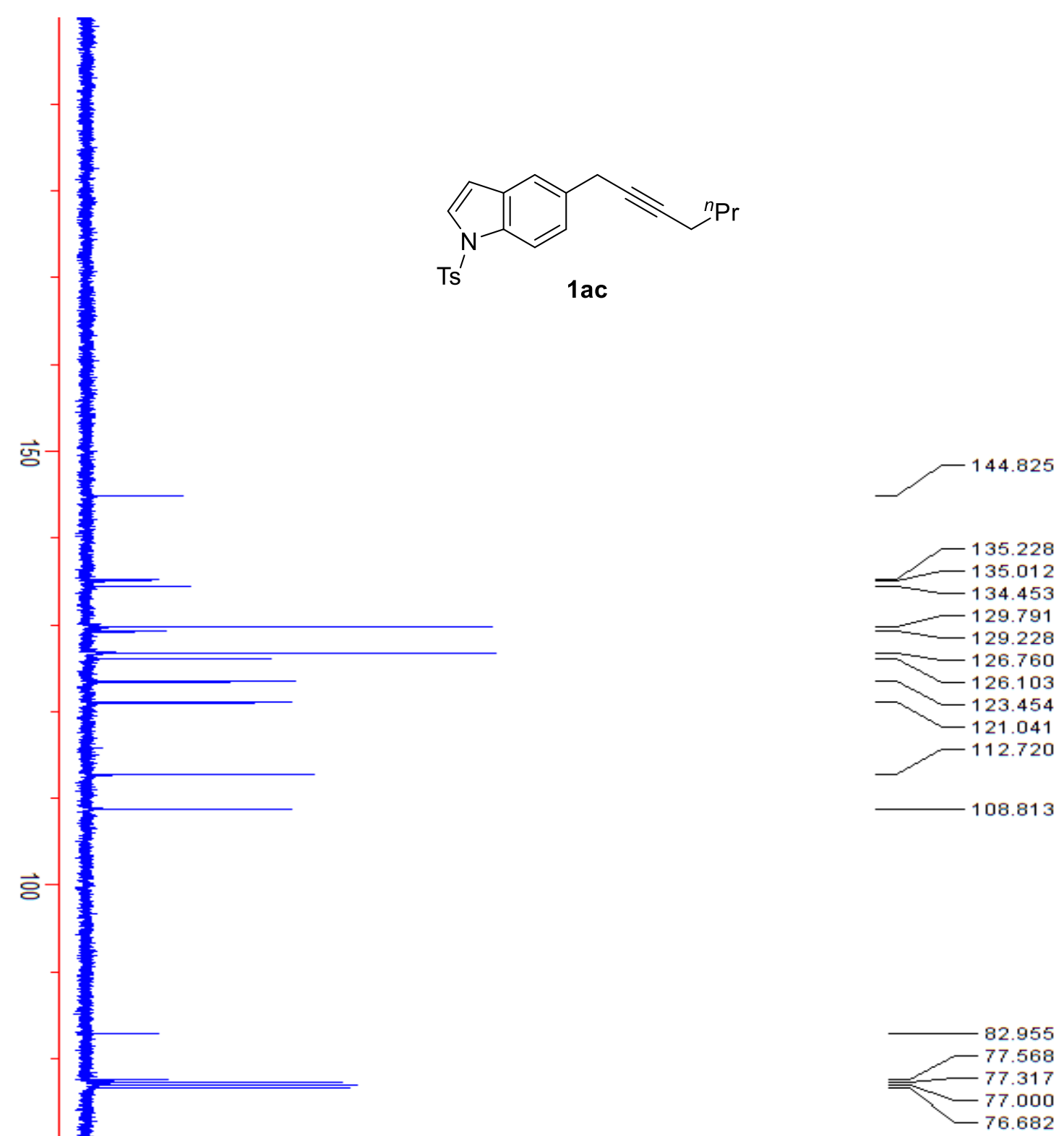

$\stackrel{\mathrm{g}}{\mathrm{y}}$

31.140

28.770

25.446

22.225

ב- 21.516

18.832 

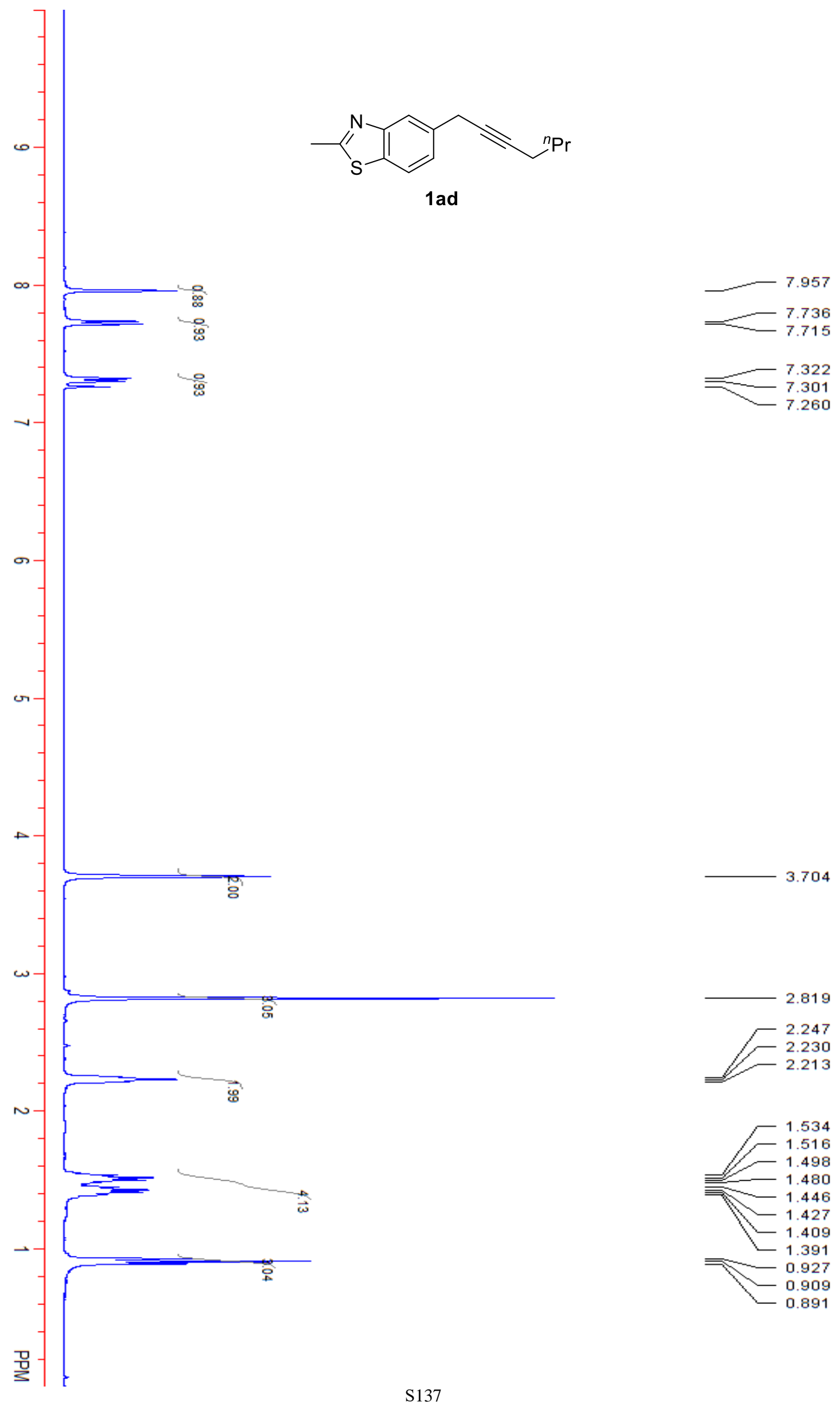

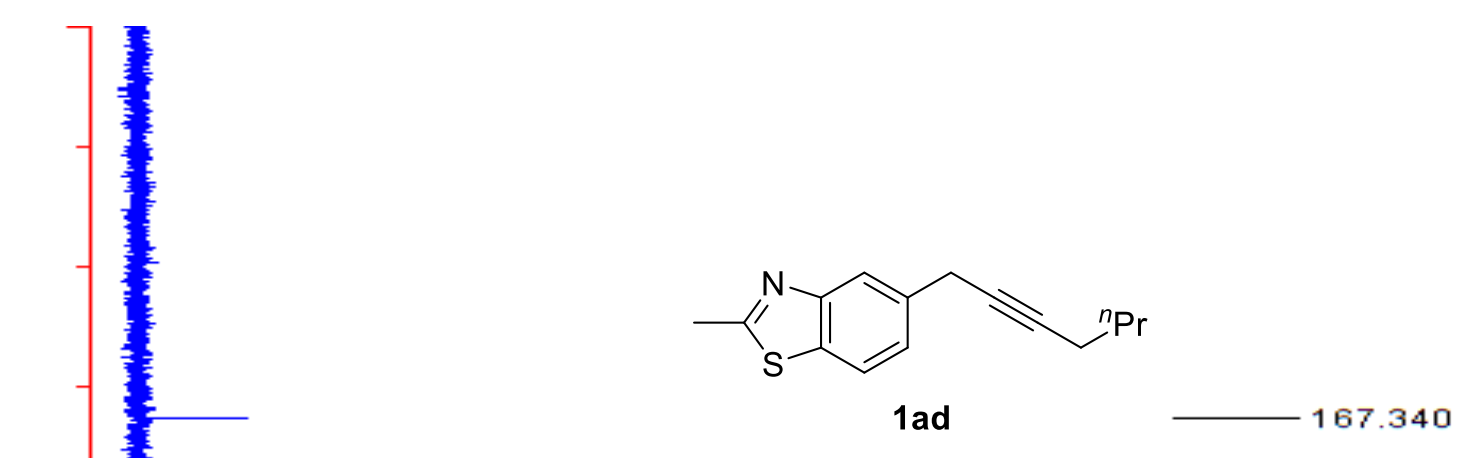

ज़ㅇㅇ

훙

낭

문

135.833

133.489

124.841

121.349
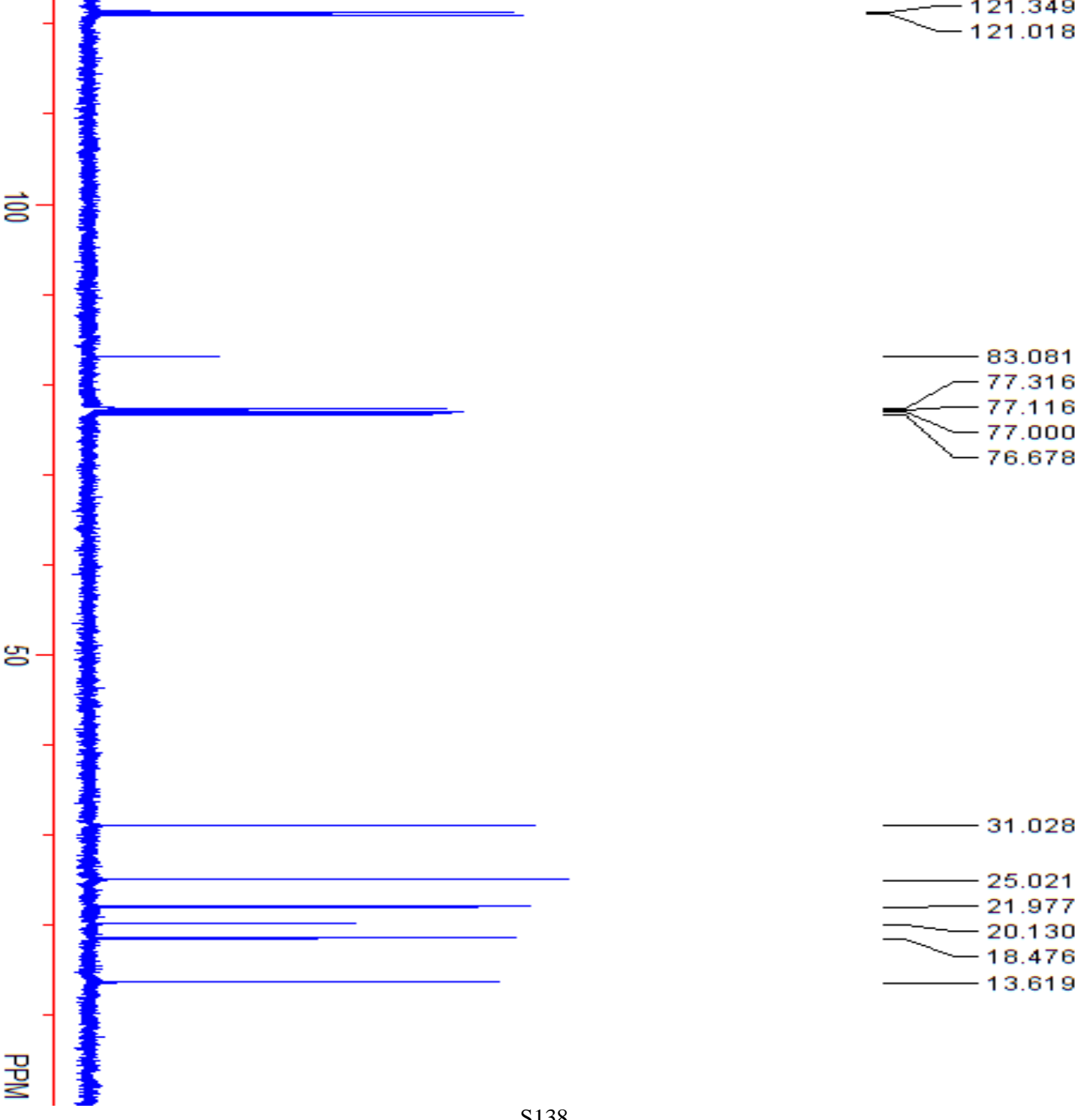

31.028 

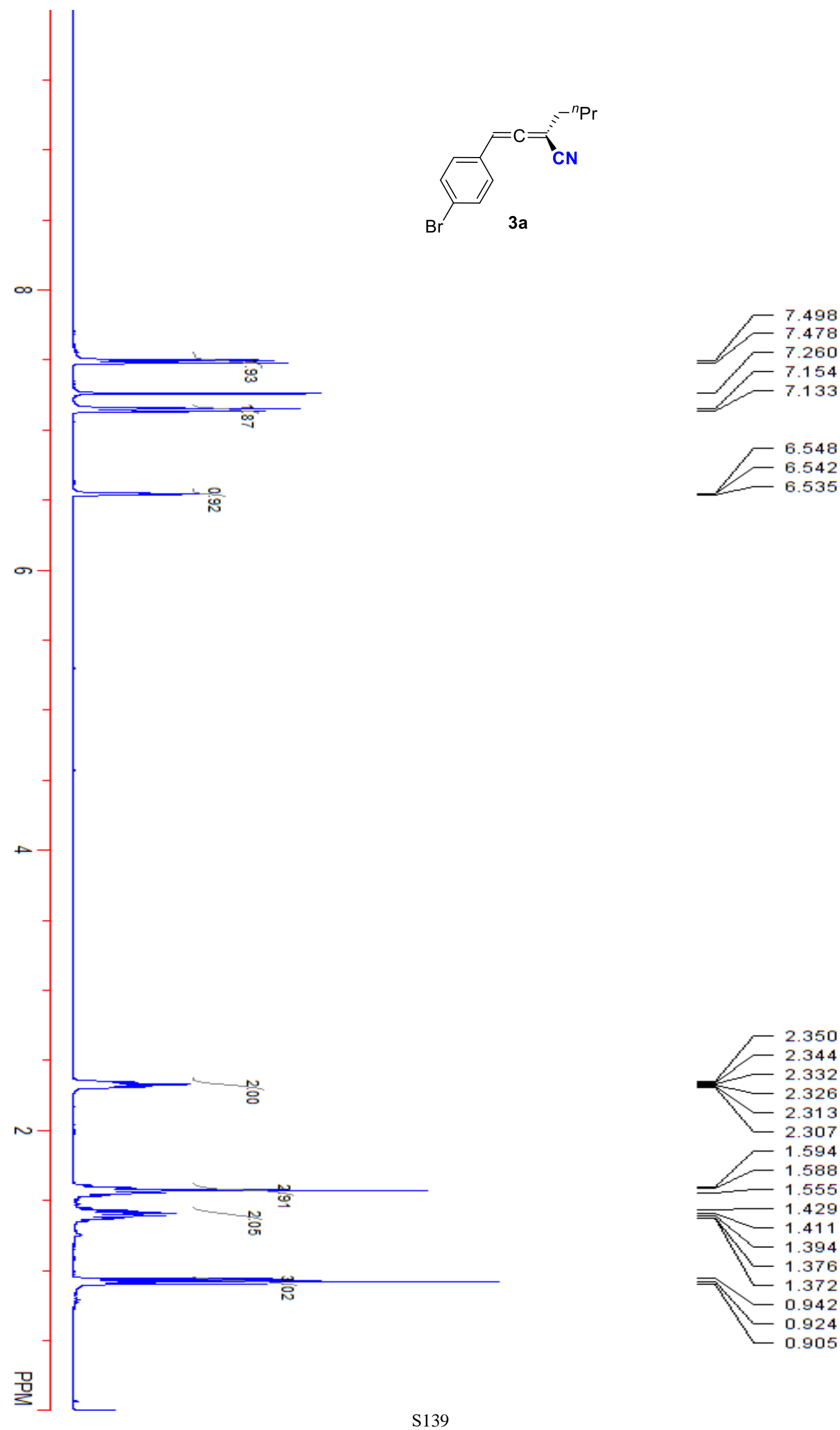

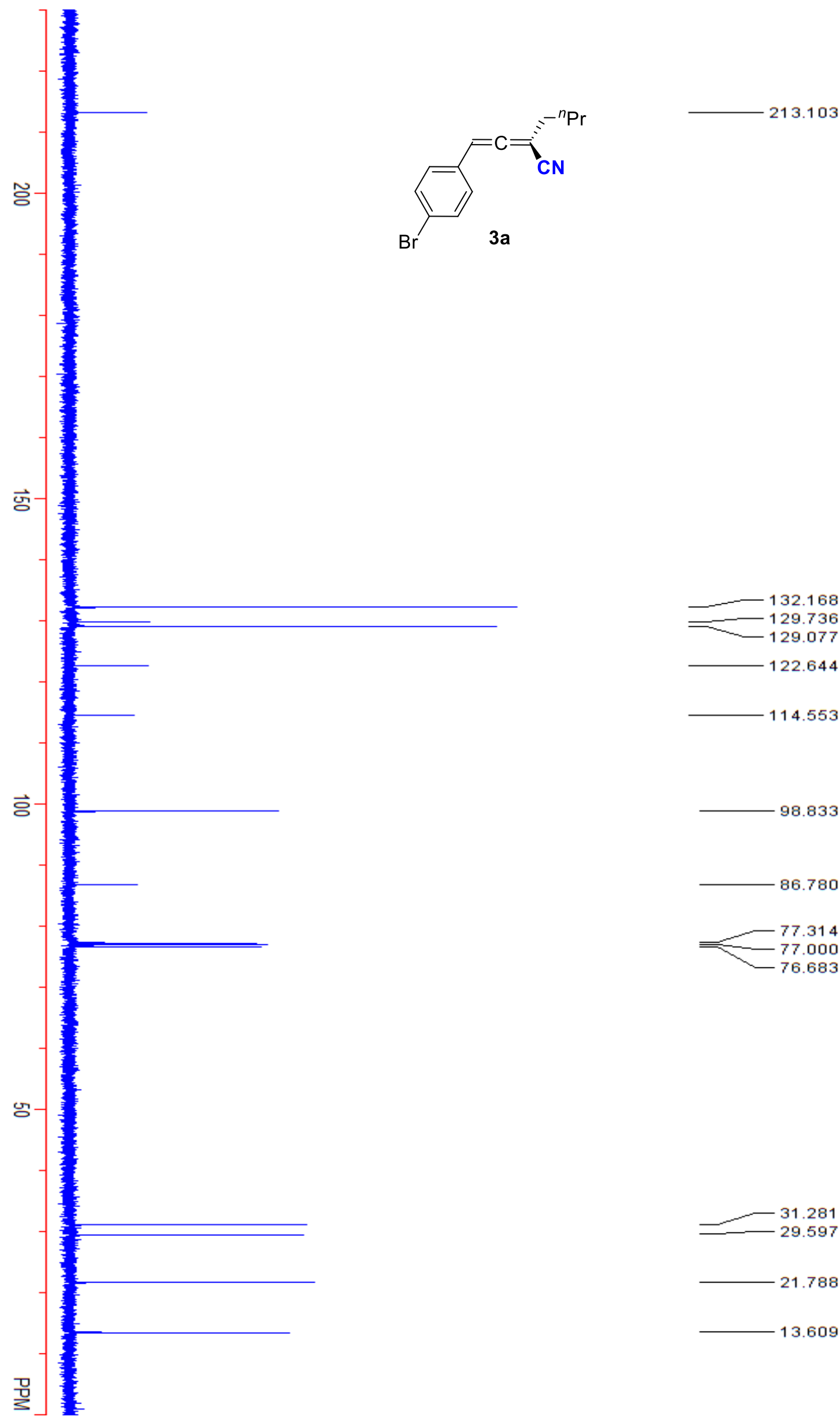

98.833

86.780

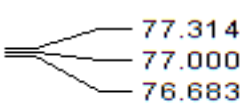



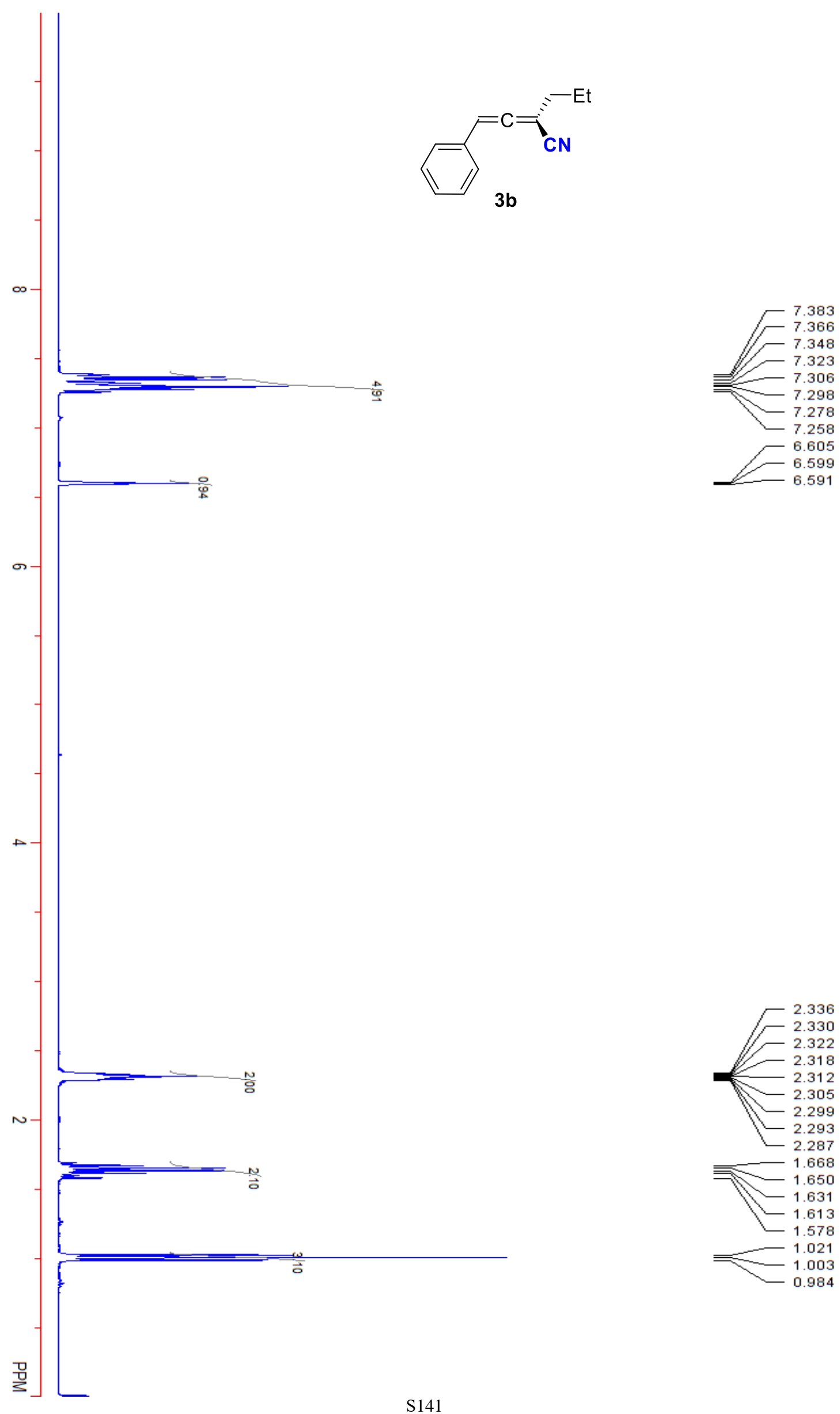


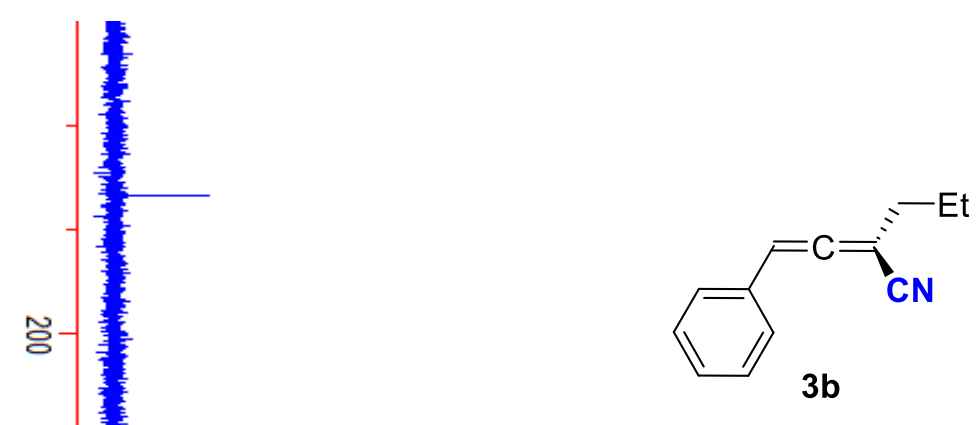

213.269

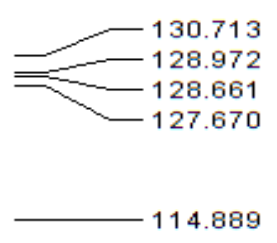

항

ज्ञ

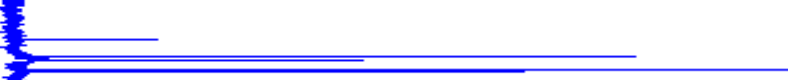

30.713

28.972

27.670

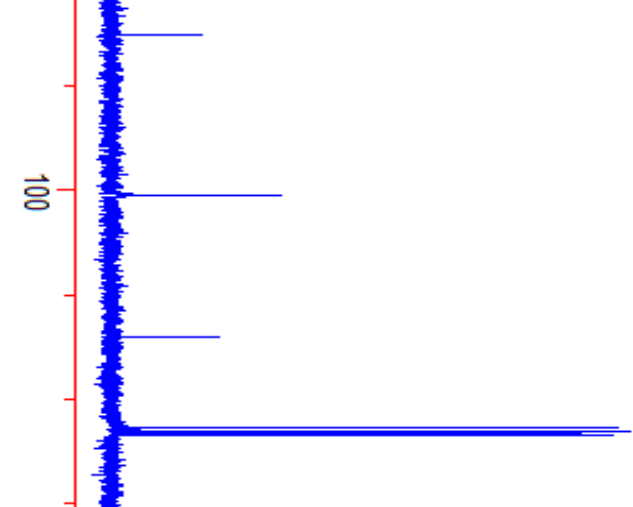

114.889

जs-

믄

99.586

86.028

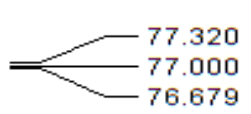

33.602

20.956

13.248 

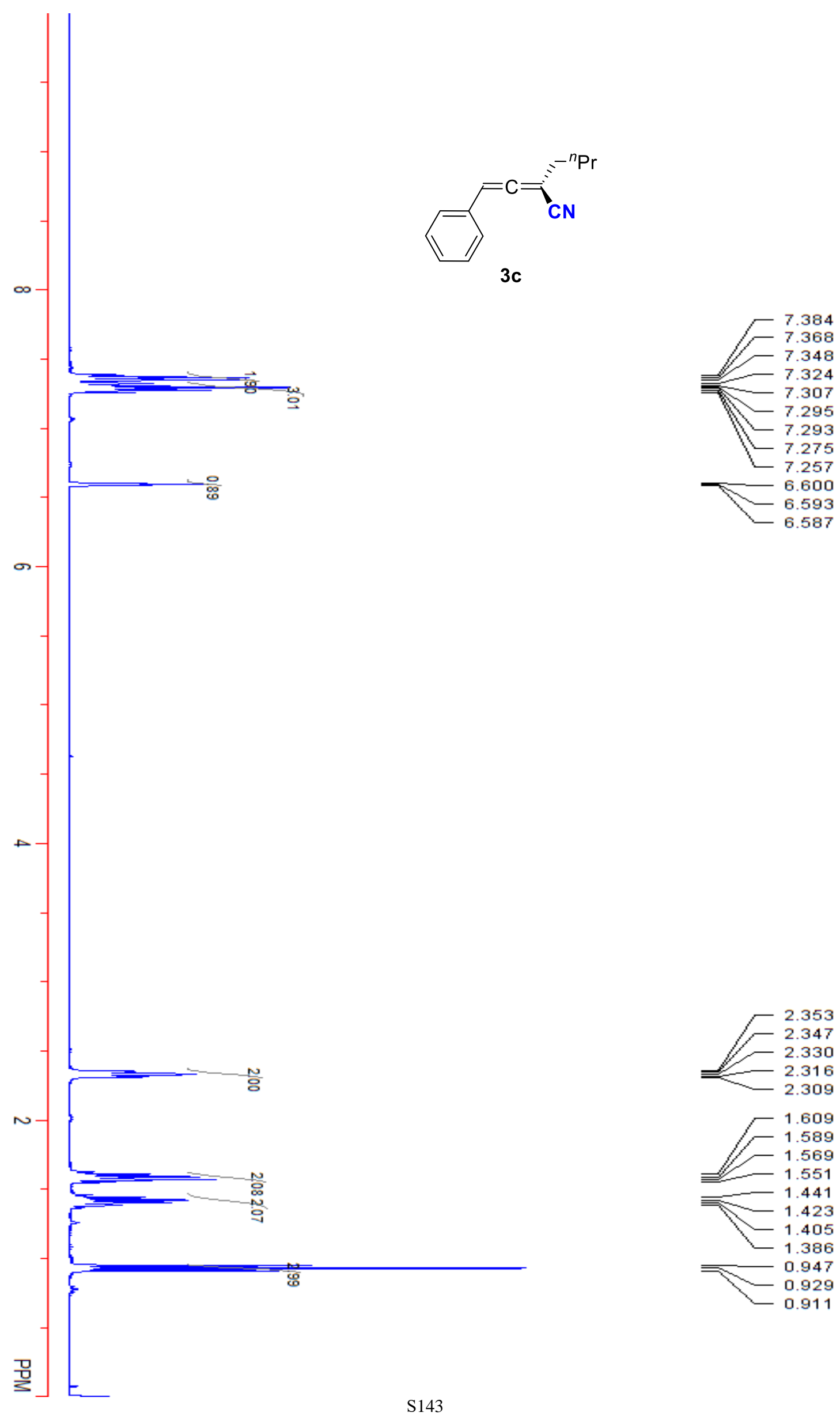

$-1609$

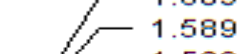

$-1.569$

1.551

$-1.441$

$-1423$

$-1.405$

$\overline{=0.947}$

0.929

- 0.911 

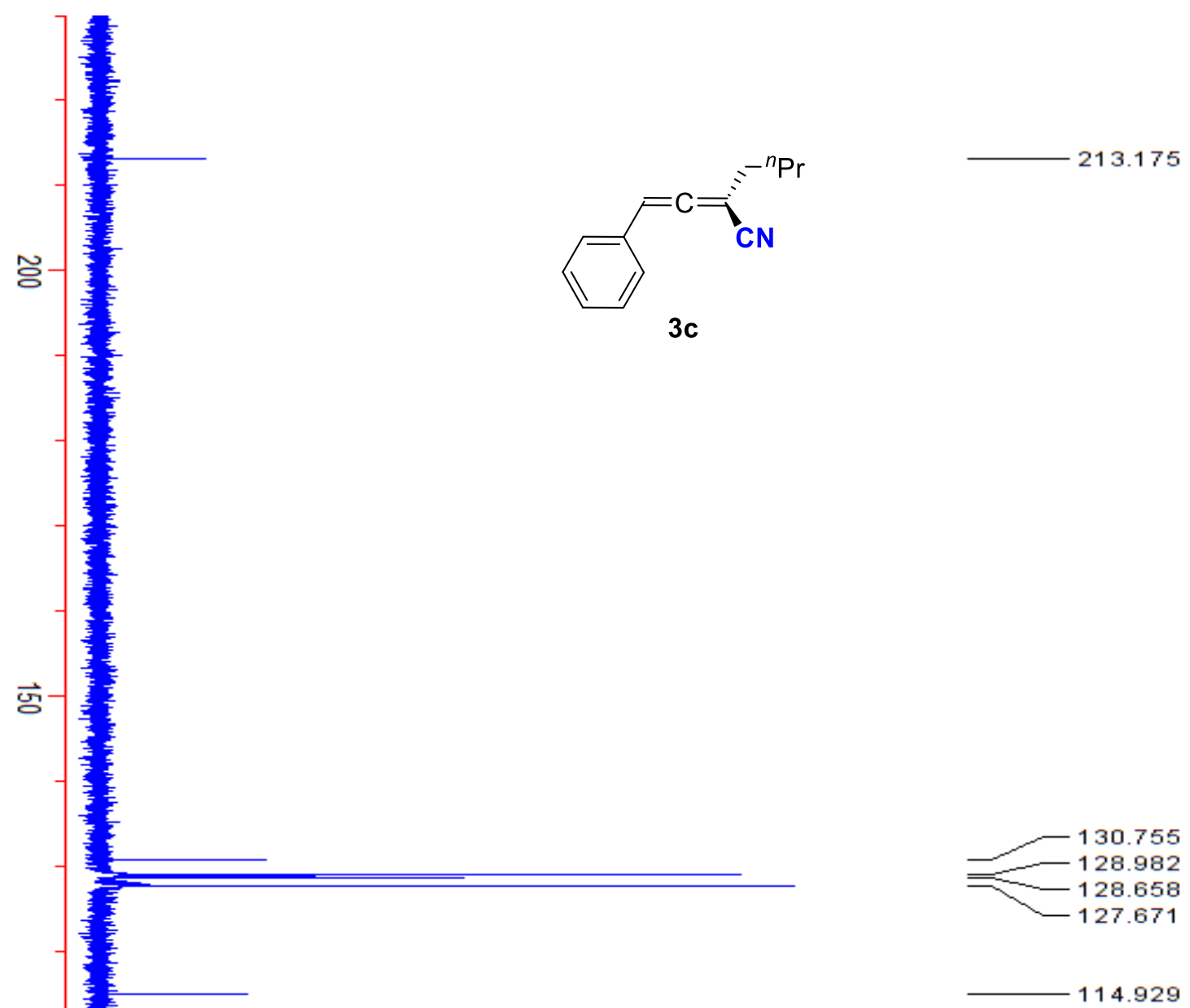

함

횽

86.226

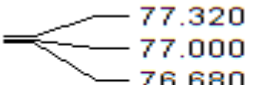

76.680

ㄷㅇㅇ

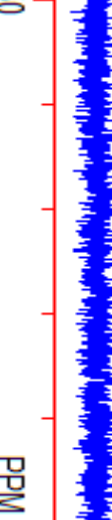

$-31.356$

29.666

21.807

13.626 

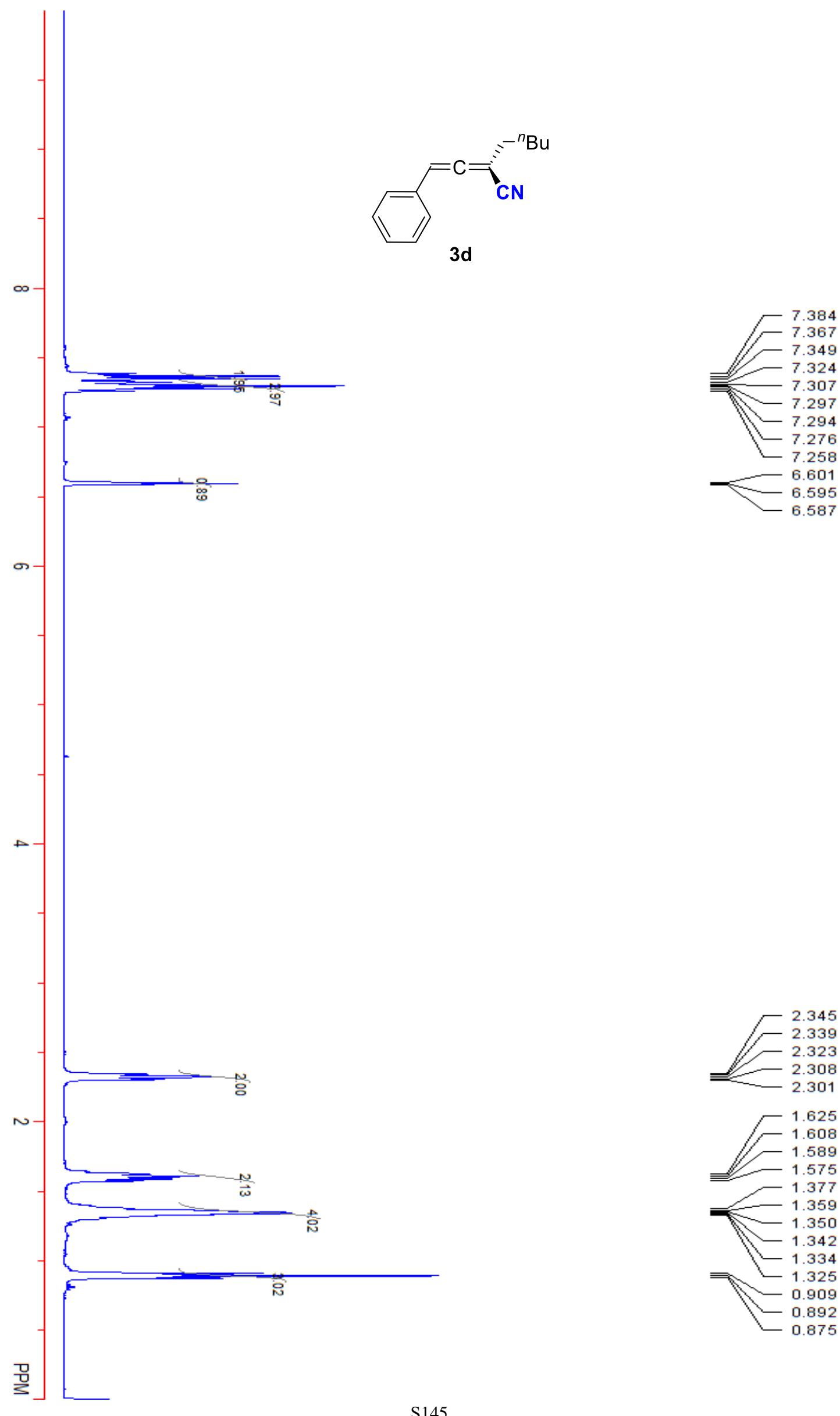

$-1.608$

$-1.589$

1.575

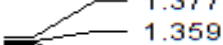

1.350



$\checkmark 1.334$

$\Longrightarrow-1.325$

0.875 

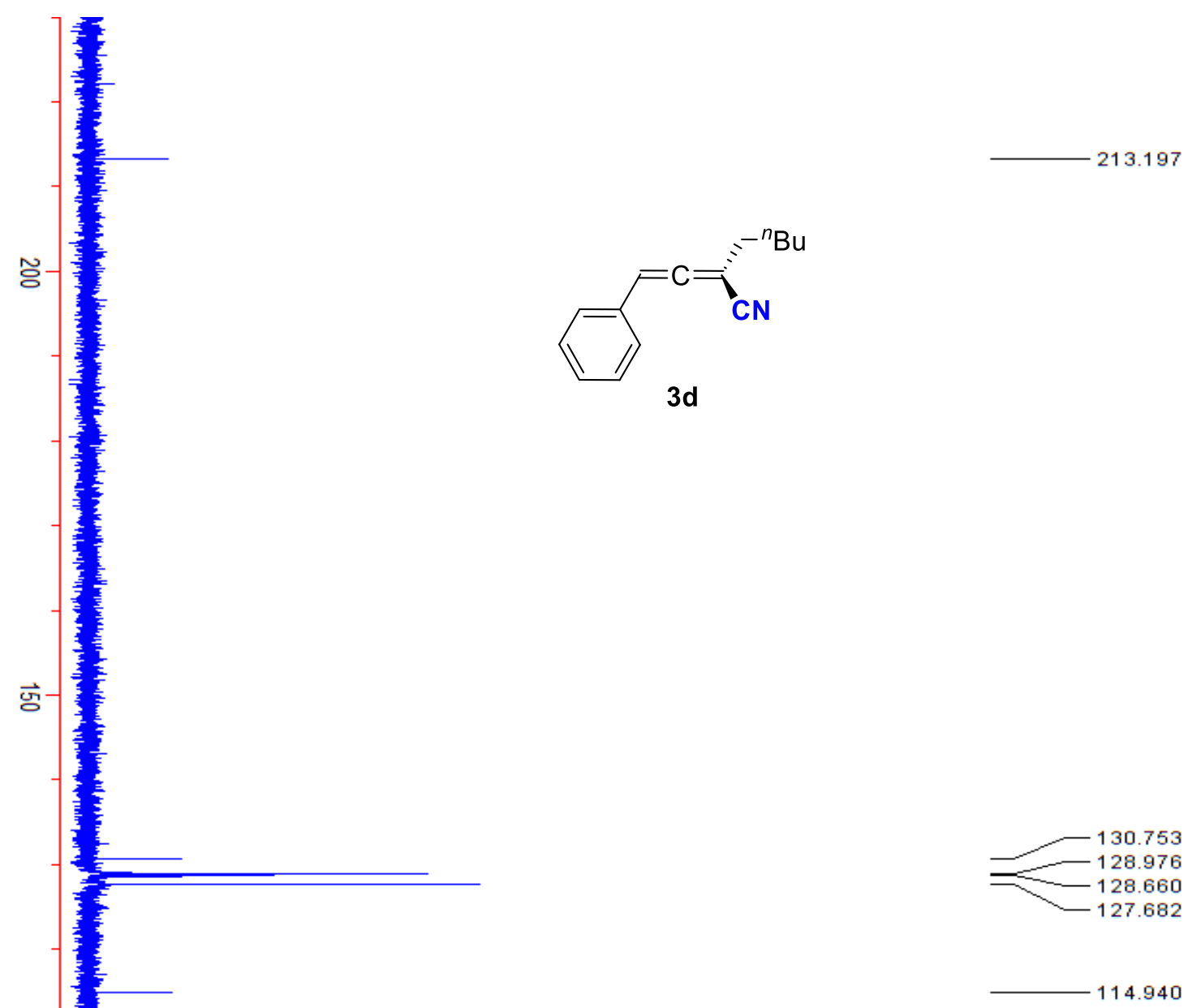

$\overrightarrow{8}$

겅-

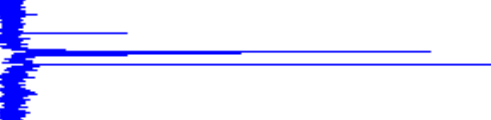

114.94

호-
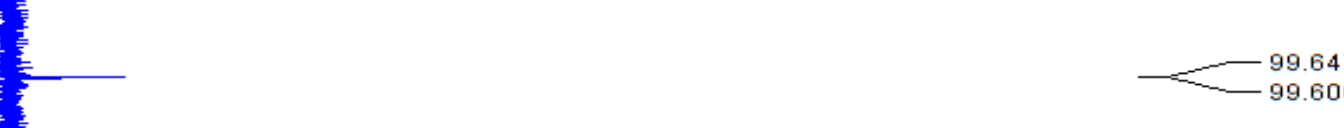

99.606

86.237

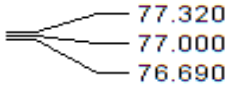

ch-

몰

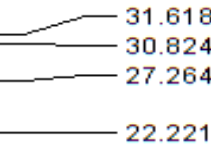

13.899 

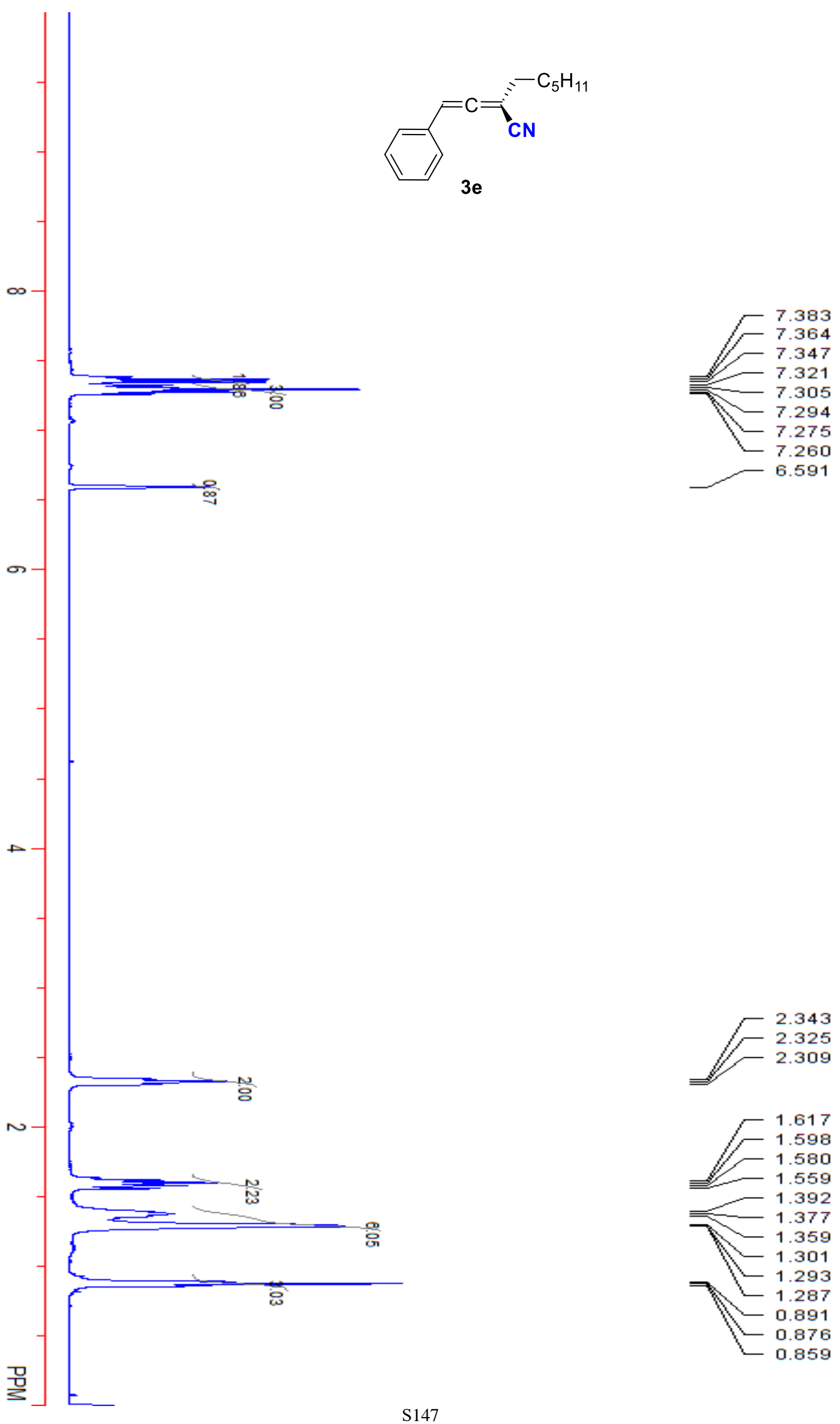

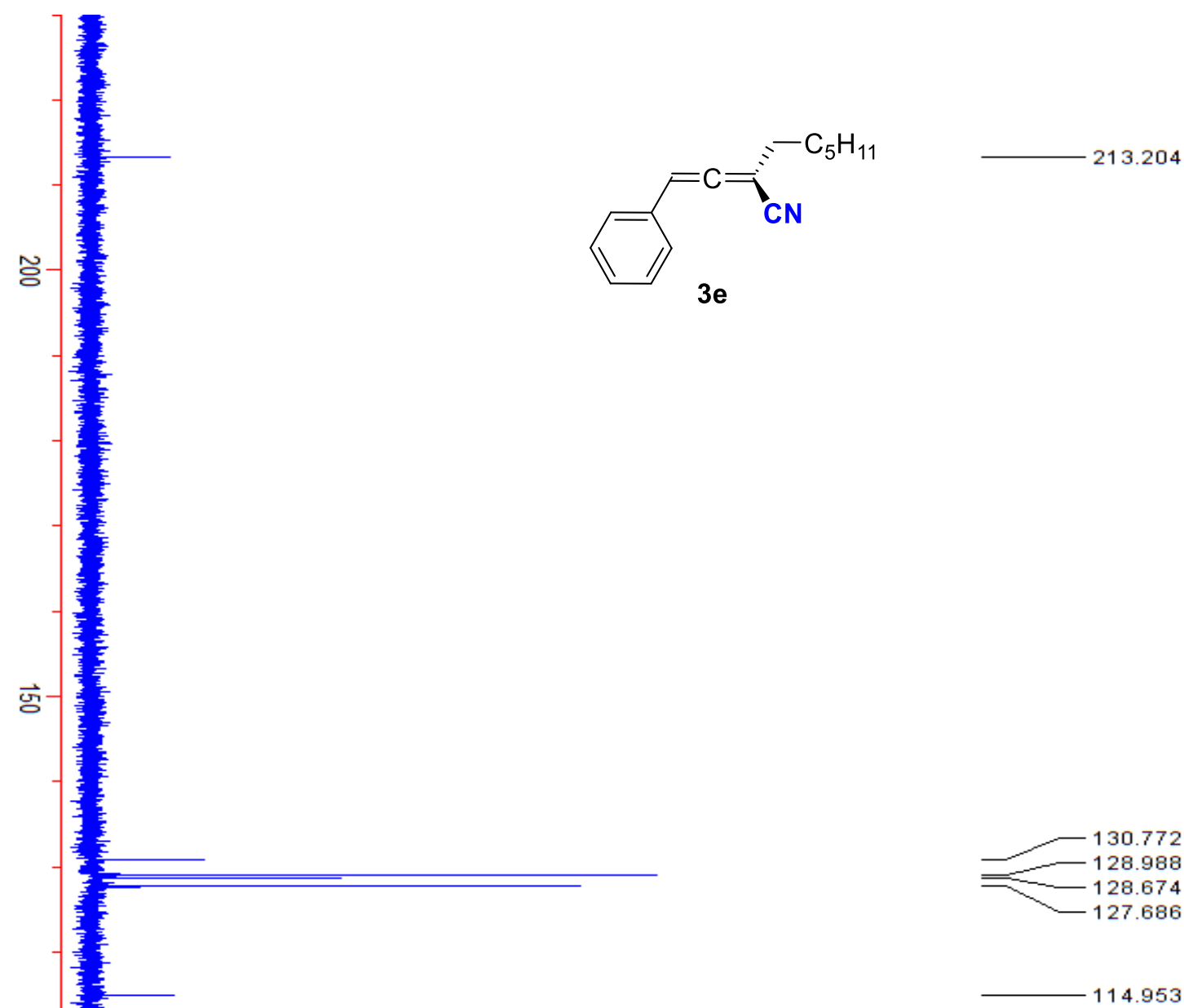

흥

$\overrightarrow{\mathrm{s}}$

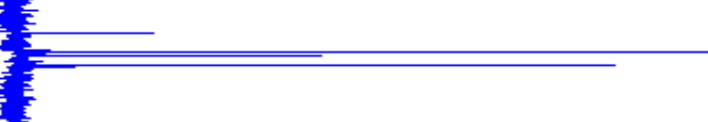

114.953

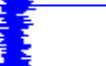



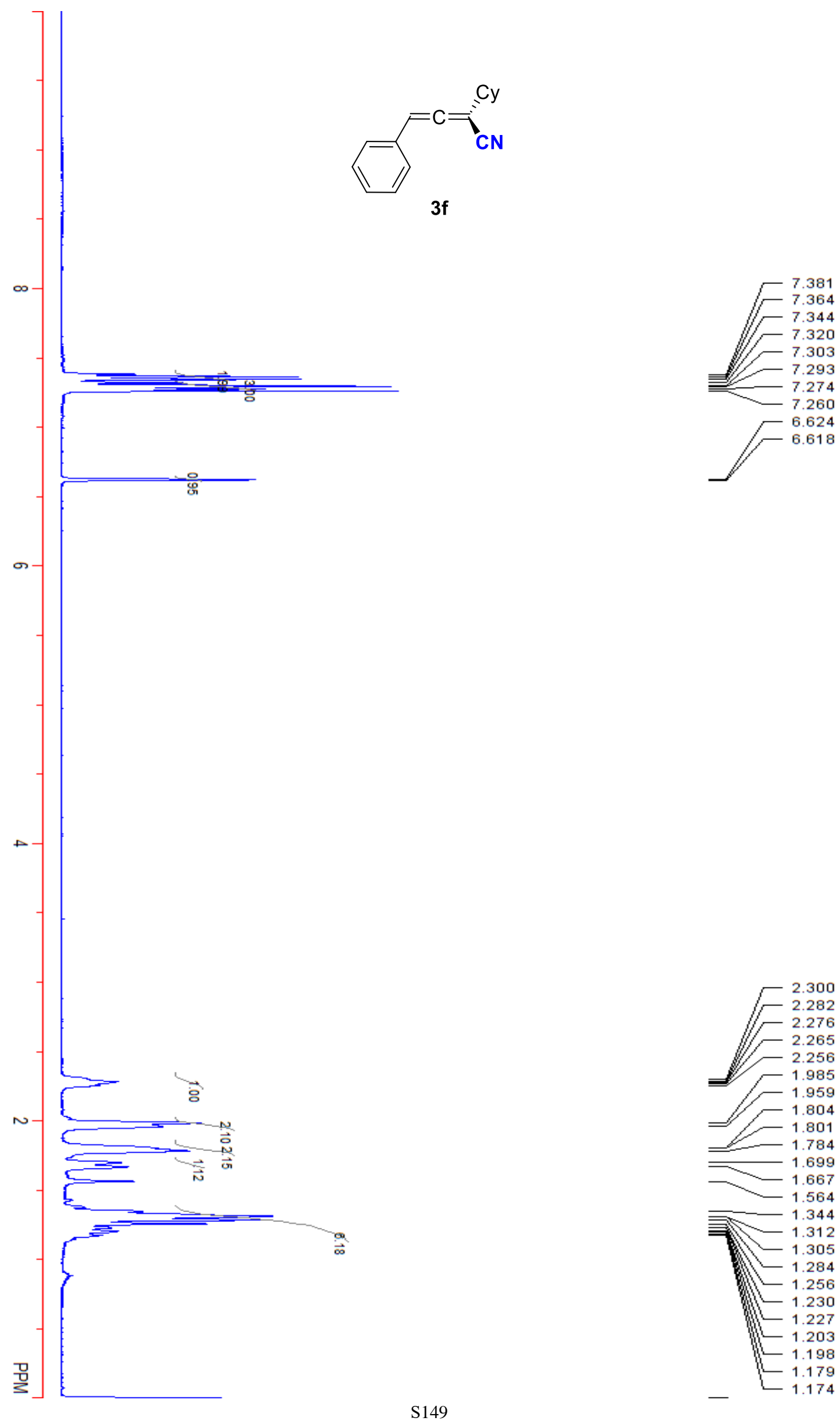


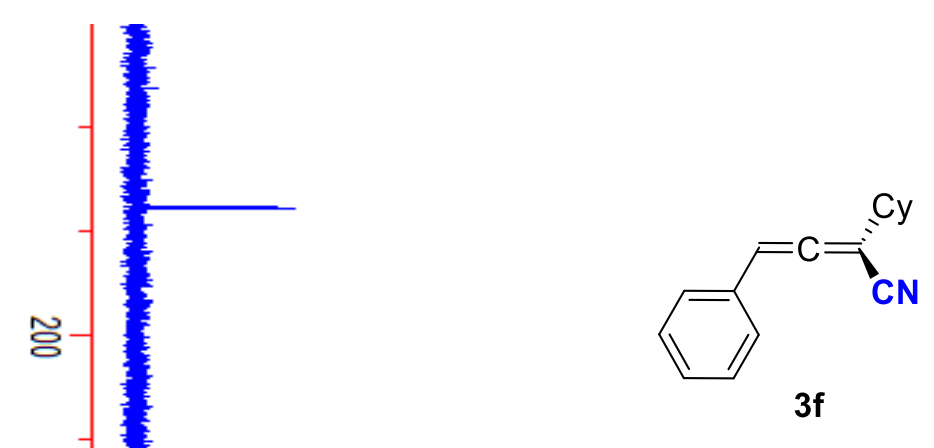

212.274

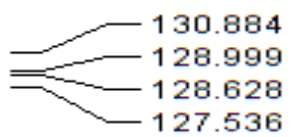

114.435

홍

굥

128.999

항-

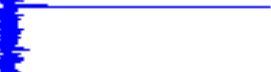

100.410

91.989

77.317

77.203

76.910

76.680

s.

몰

40.045

31.678

31.658

35.715
-25.550 

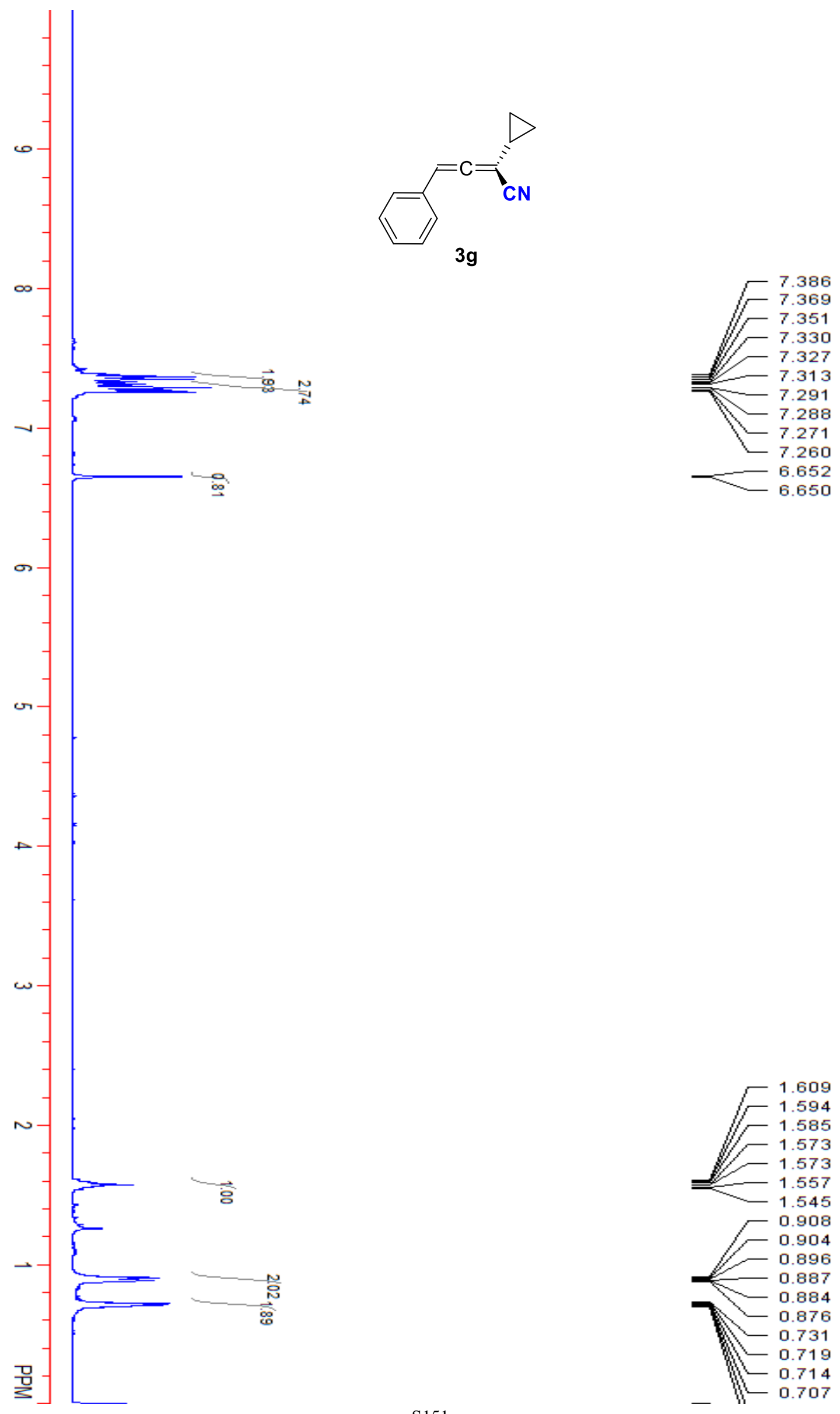

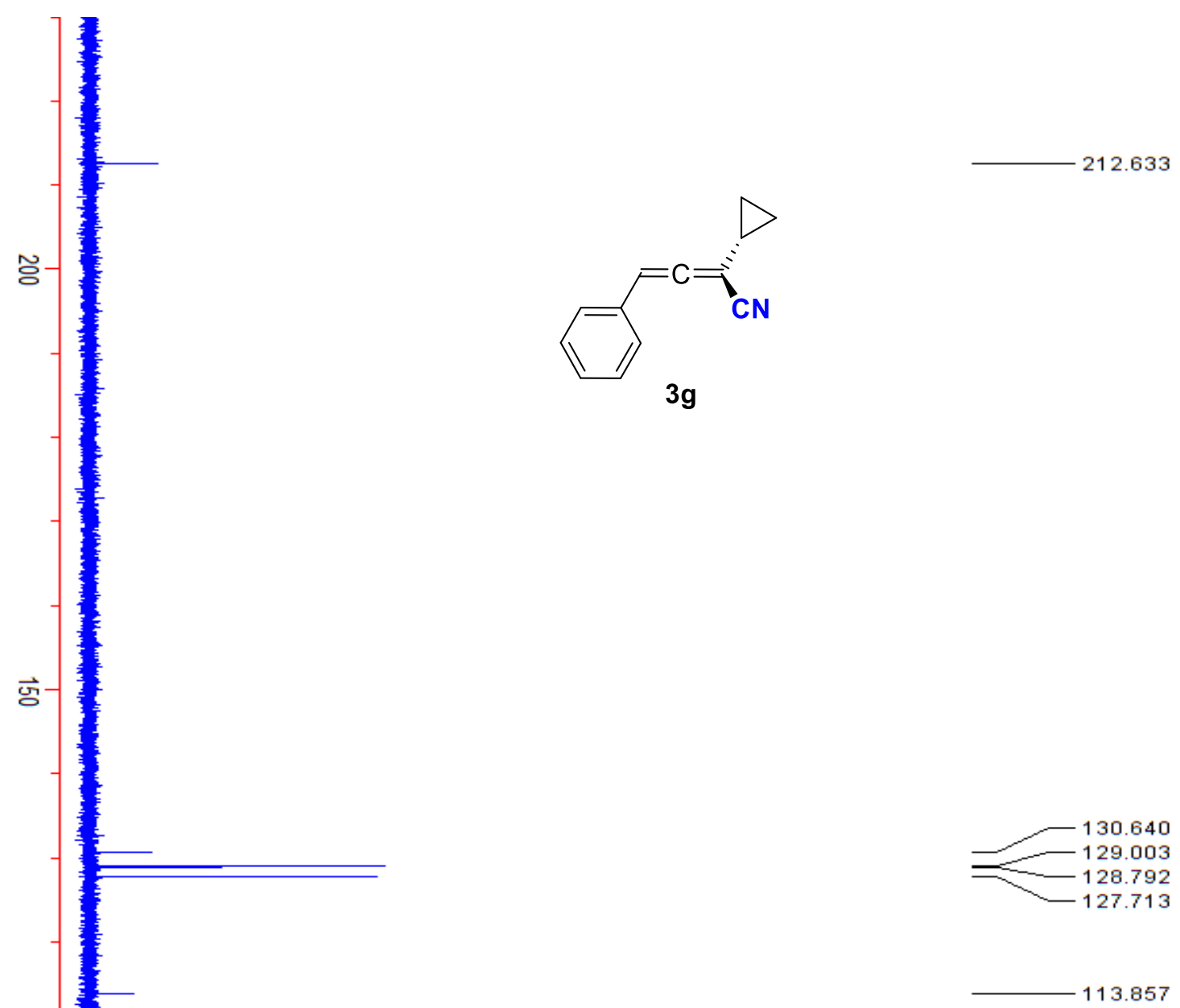

흥

형-

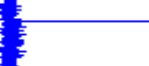

$-101.053$
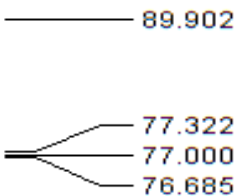

당

$\stackrel{\mathrm{g}}{\leq}$

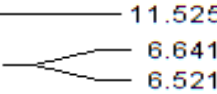



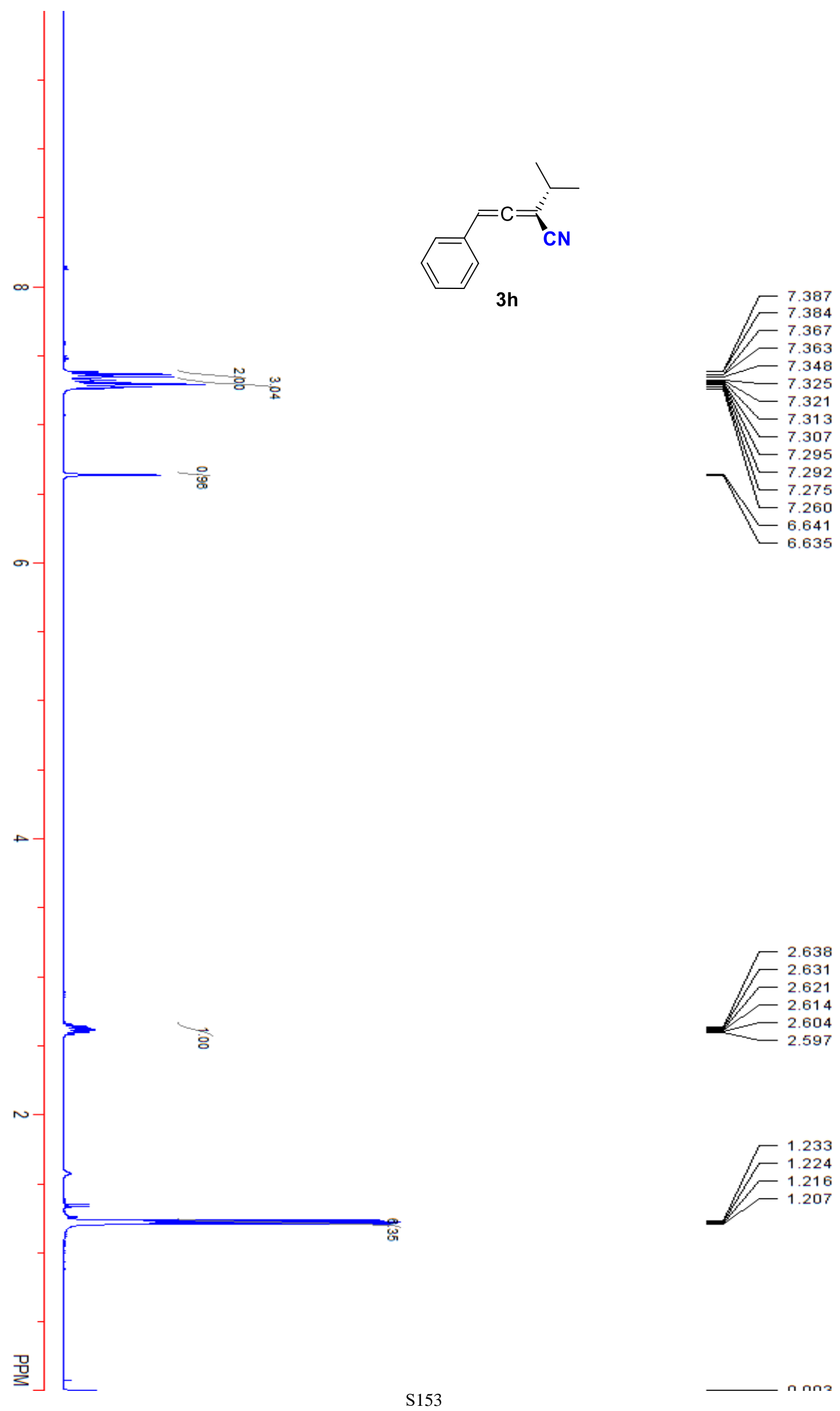


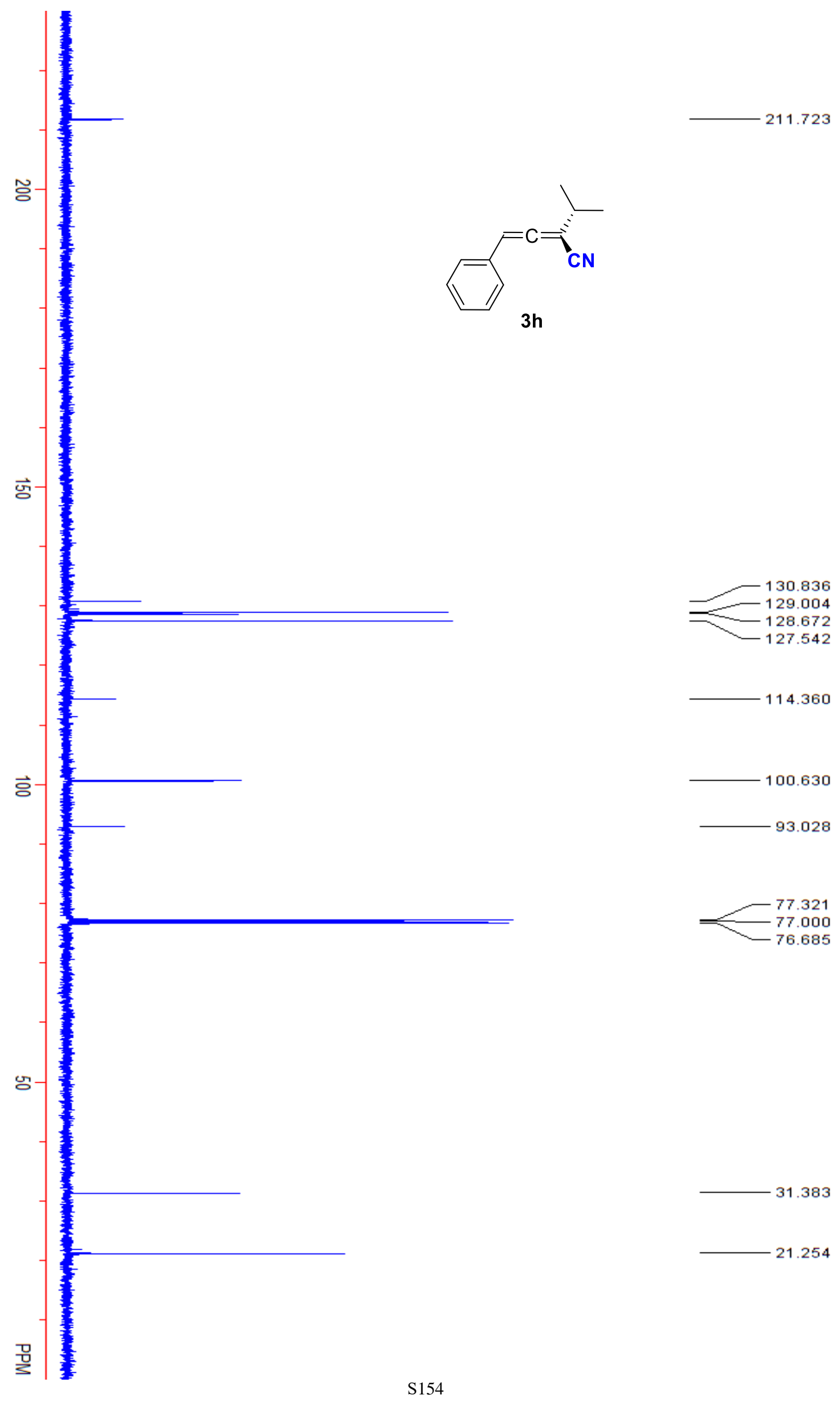



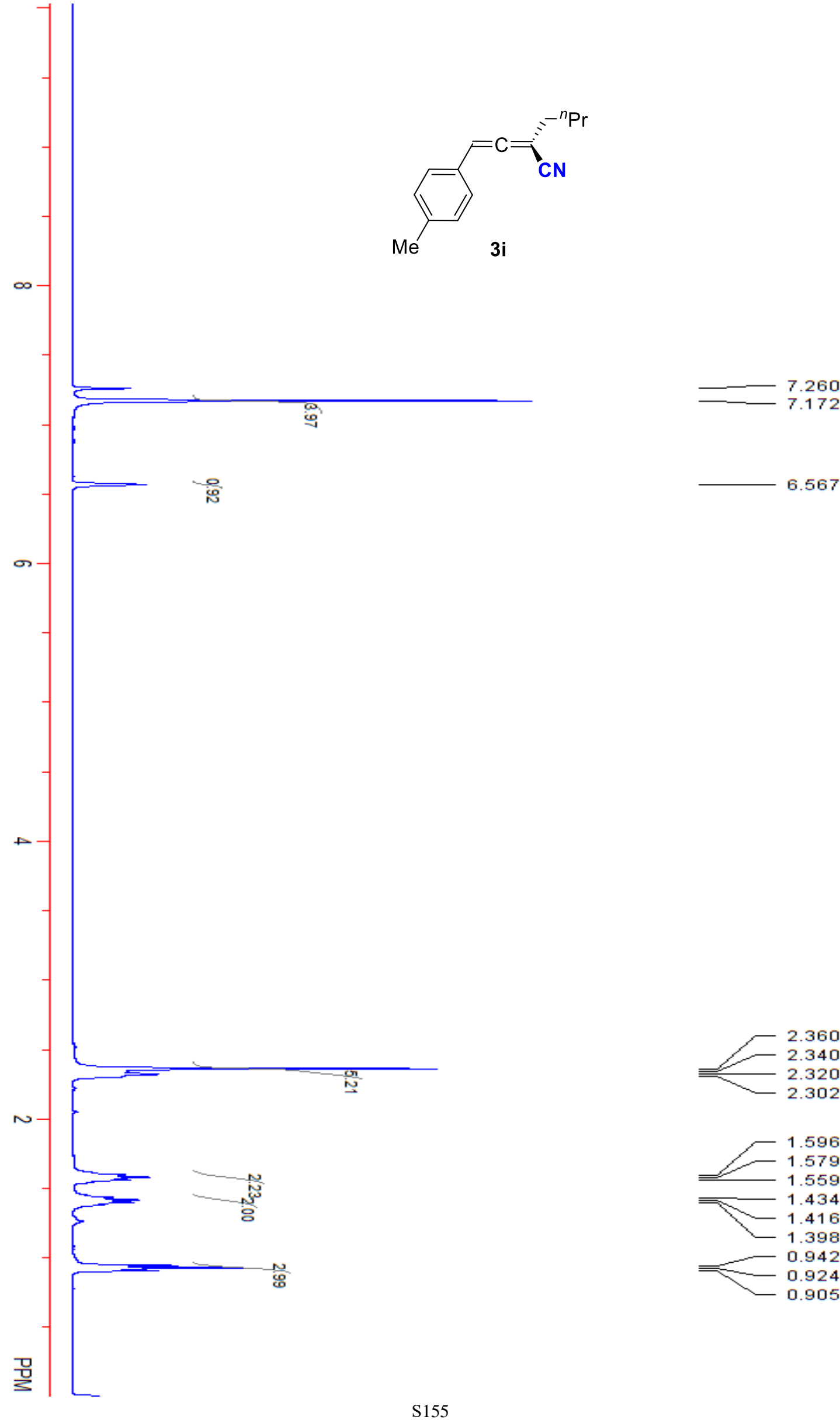

6.567

2.360 2.340 2.320
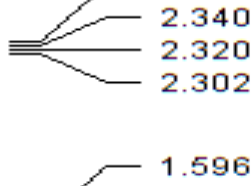
1.579 1.559 1.434 1.416 1.398 0.942 0.924

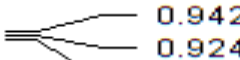




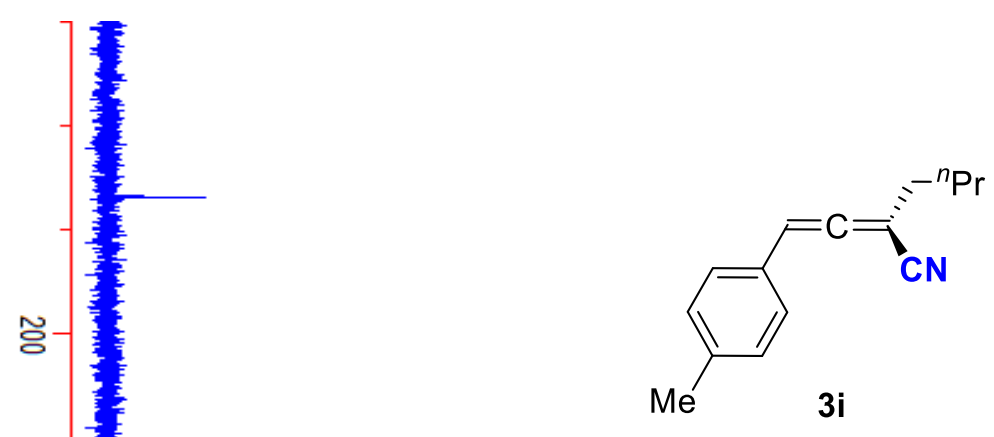

213.168

138.759

항

항
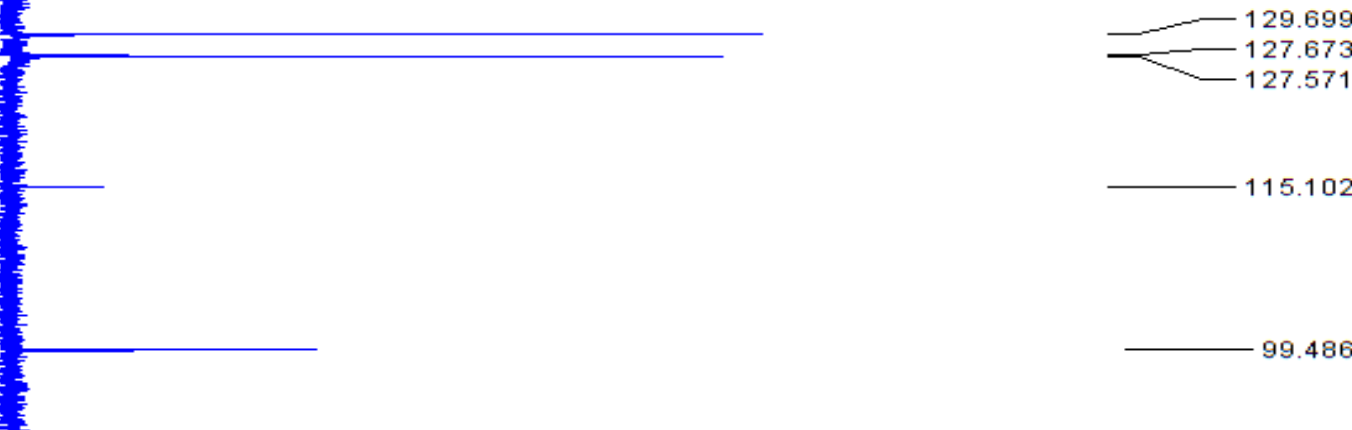

115.102

s
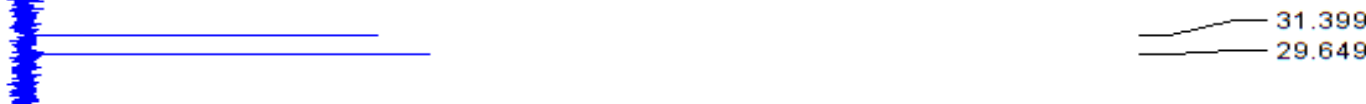

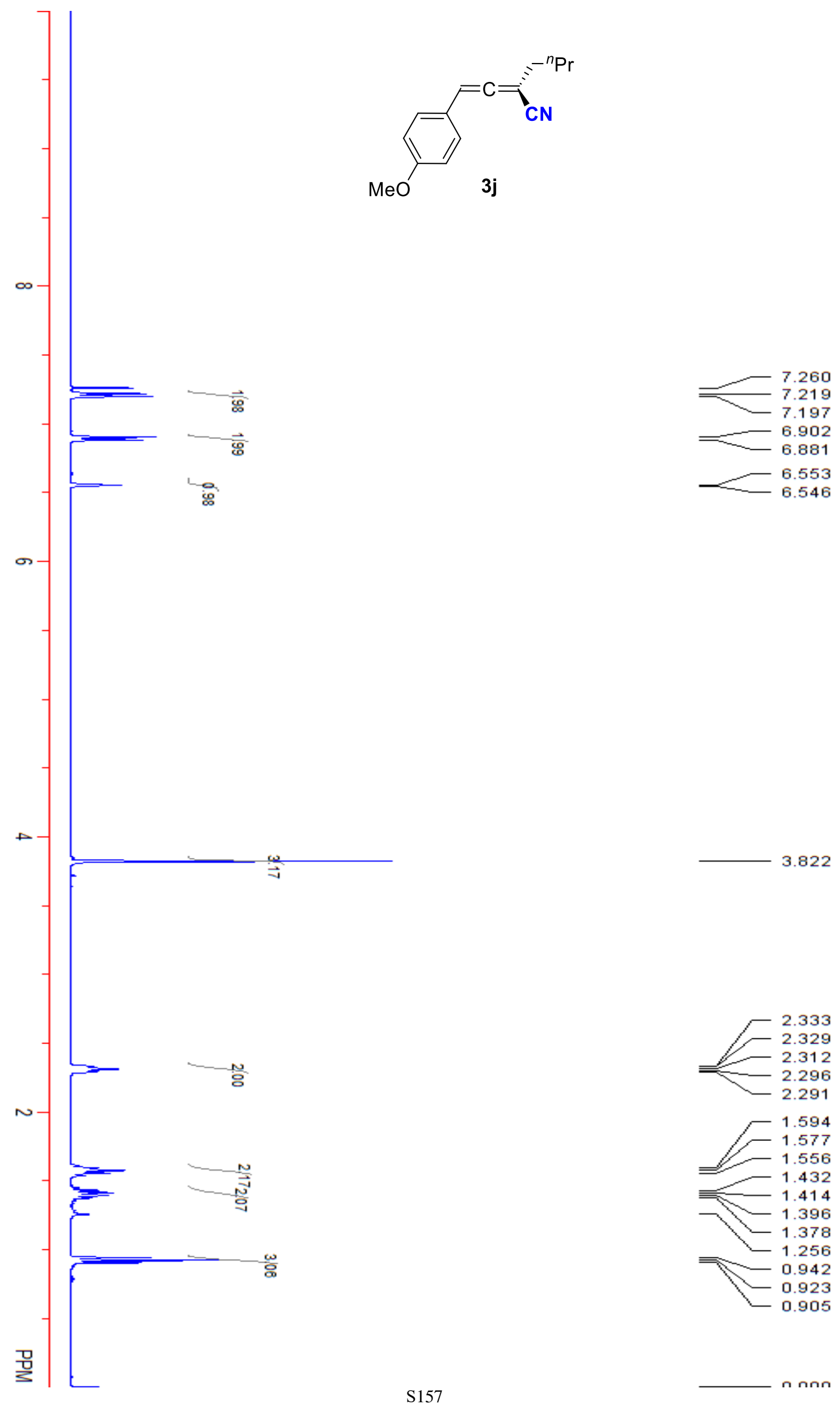


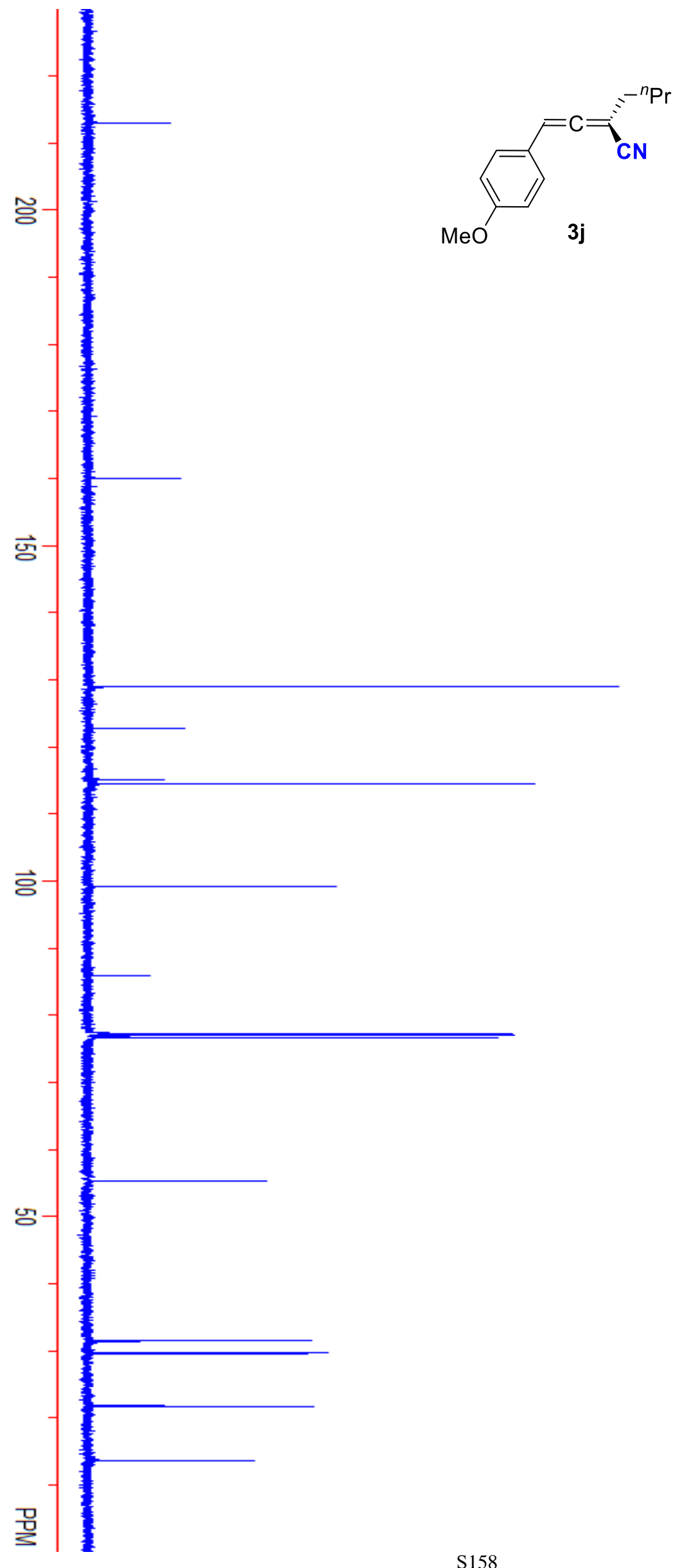

213.053

159.988

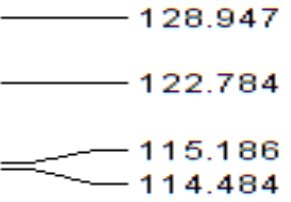

99.177

85.994

7.321

77.000

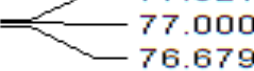

55.336 

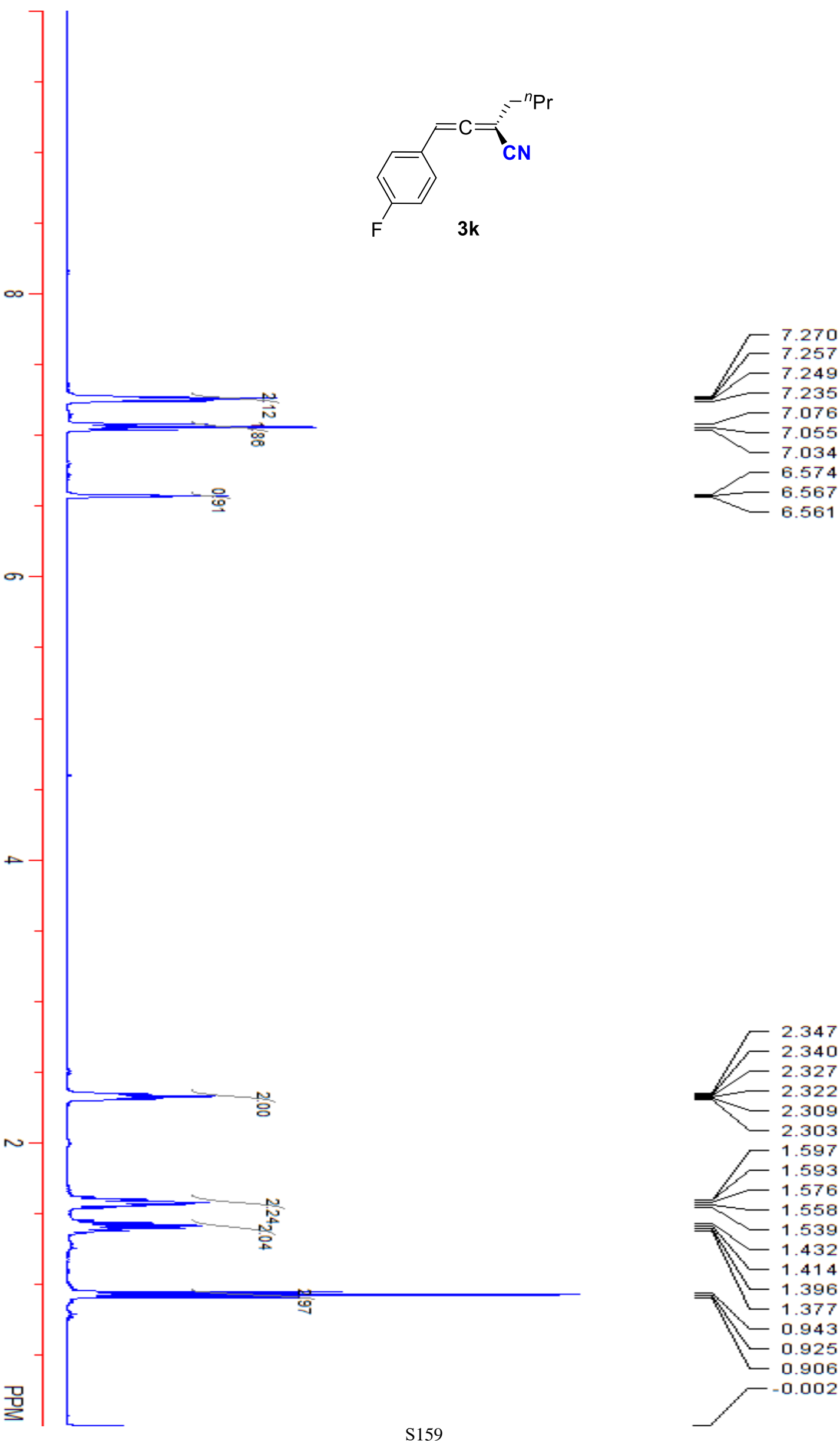

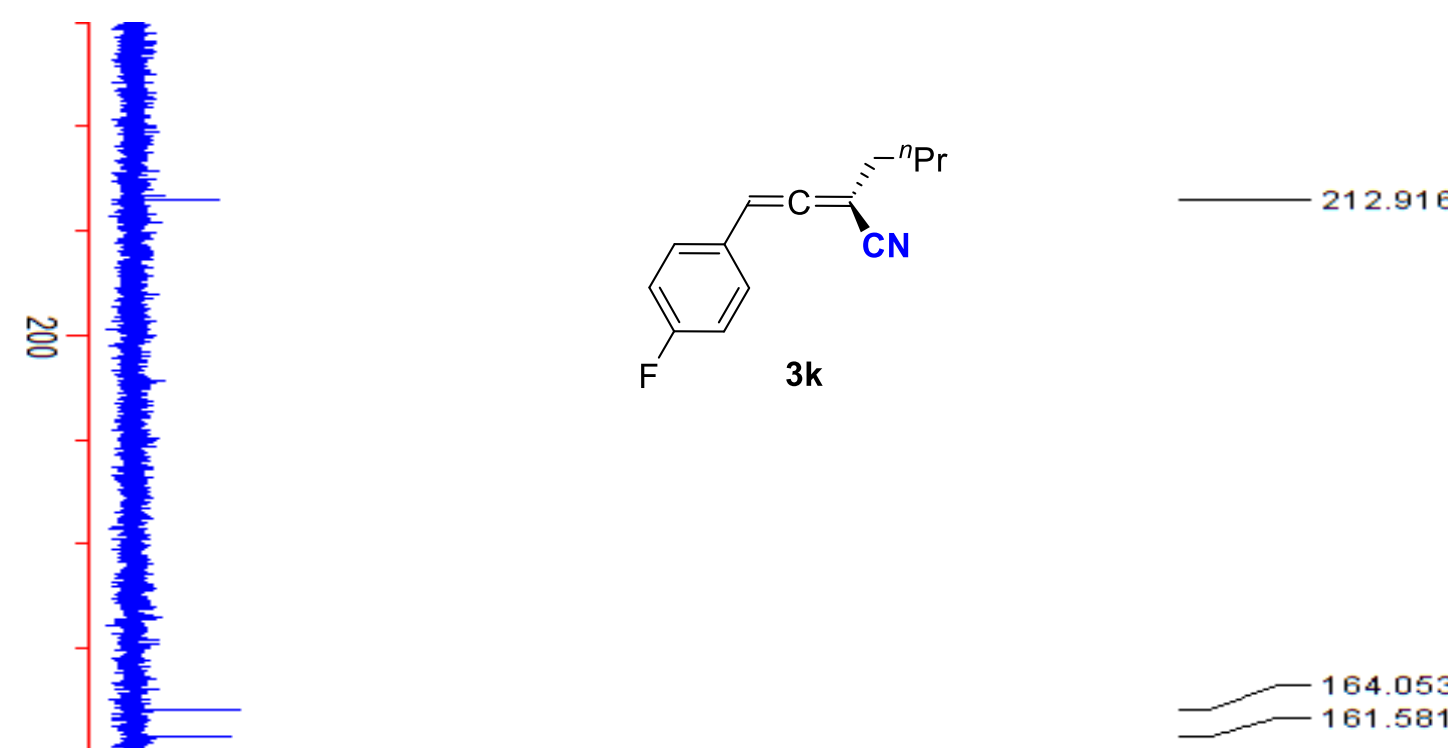


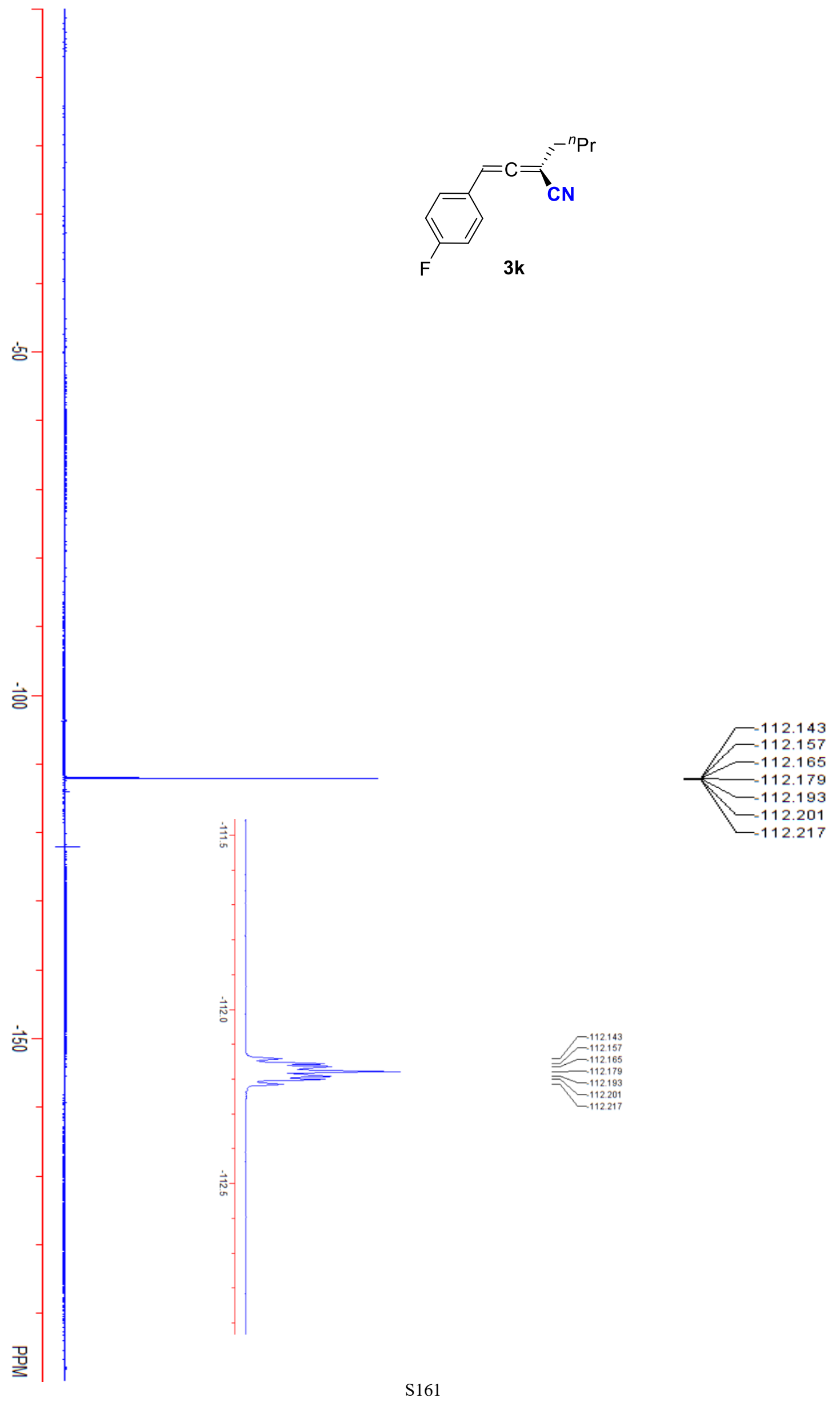



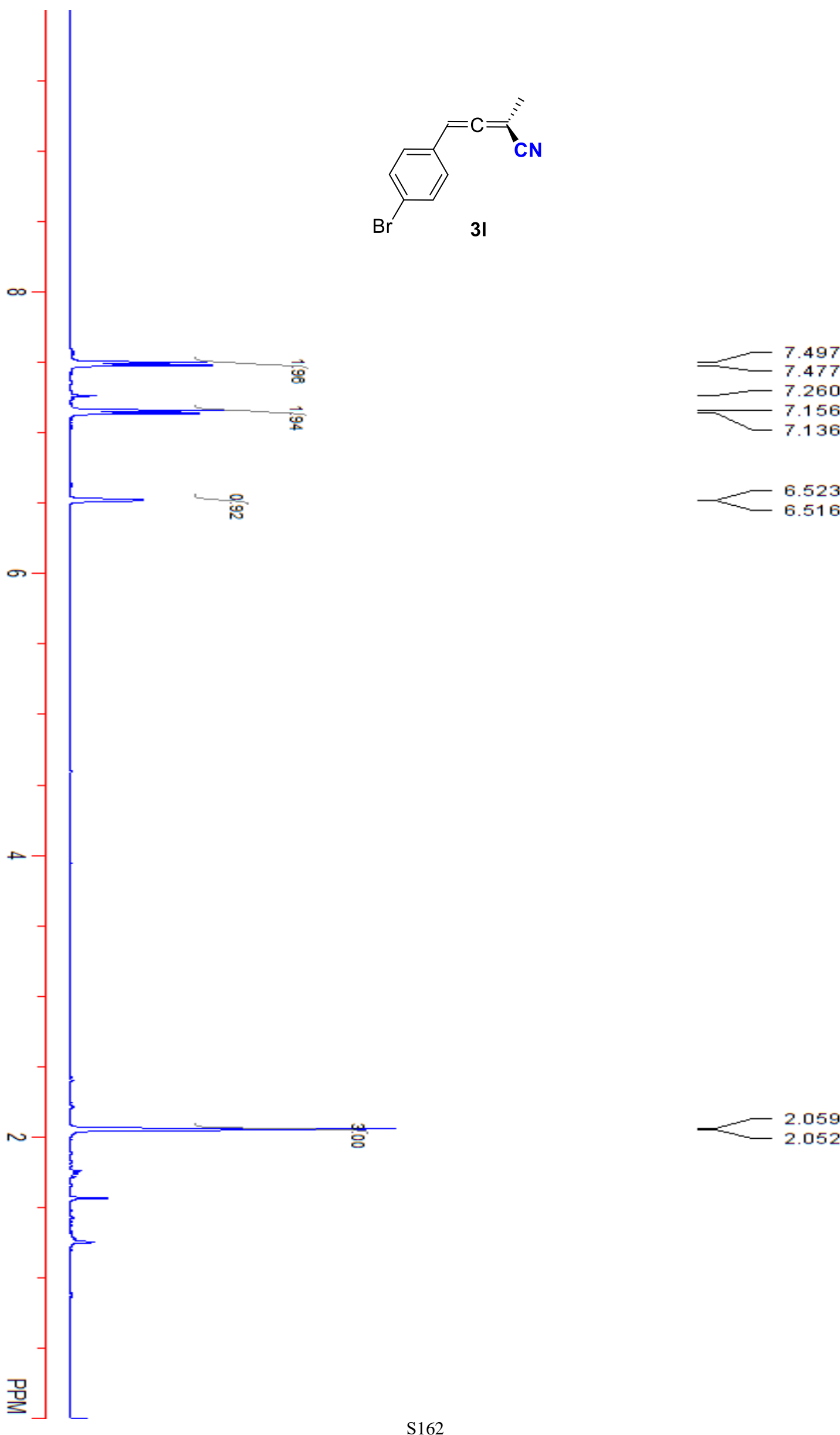

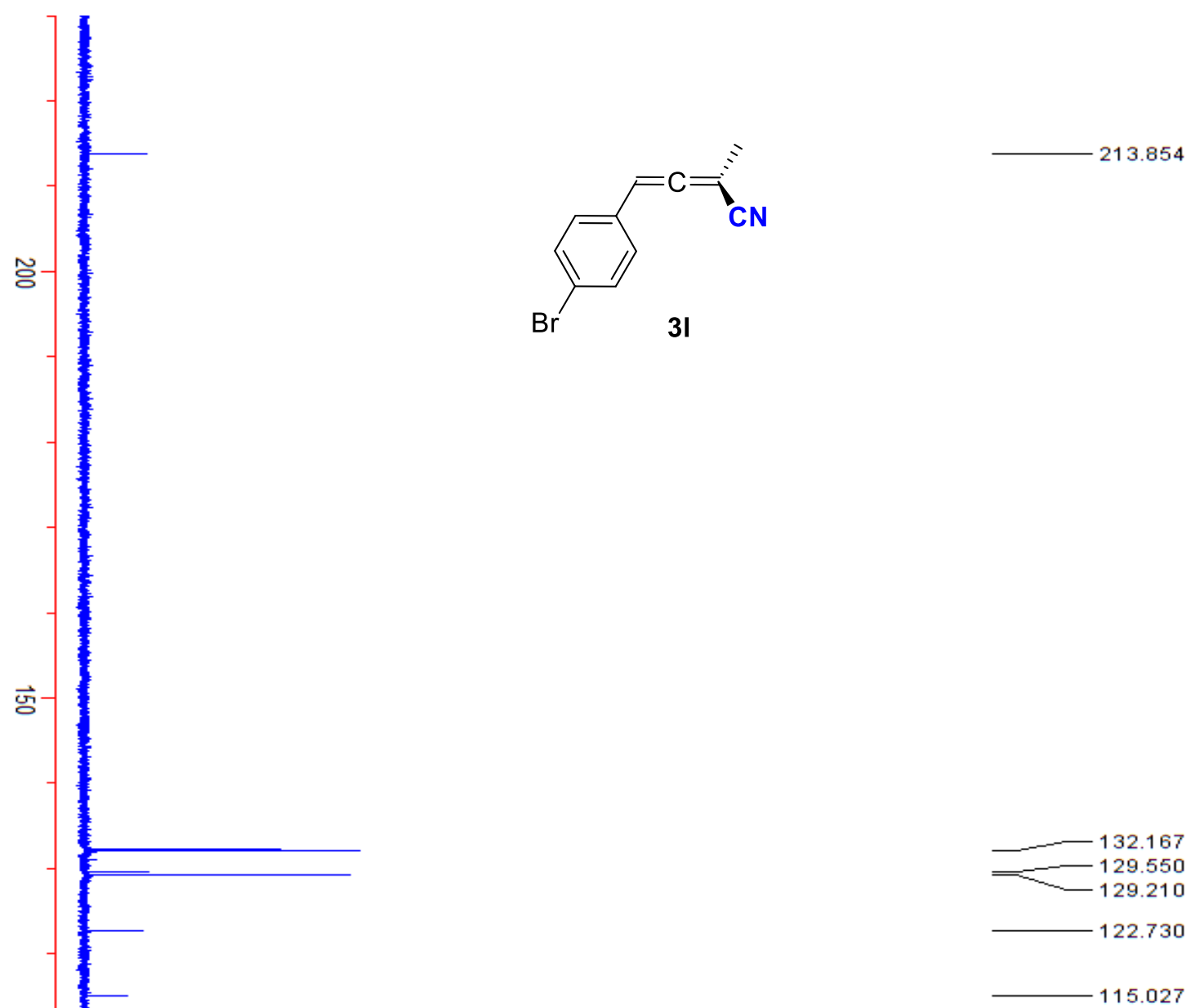

항
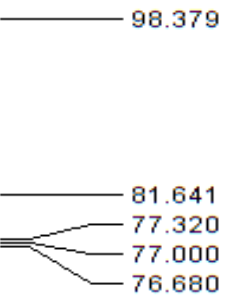

당

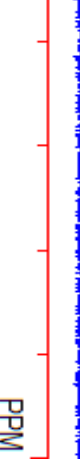

몬 

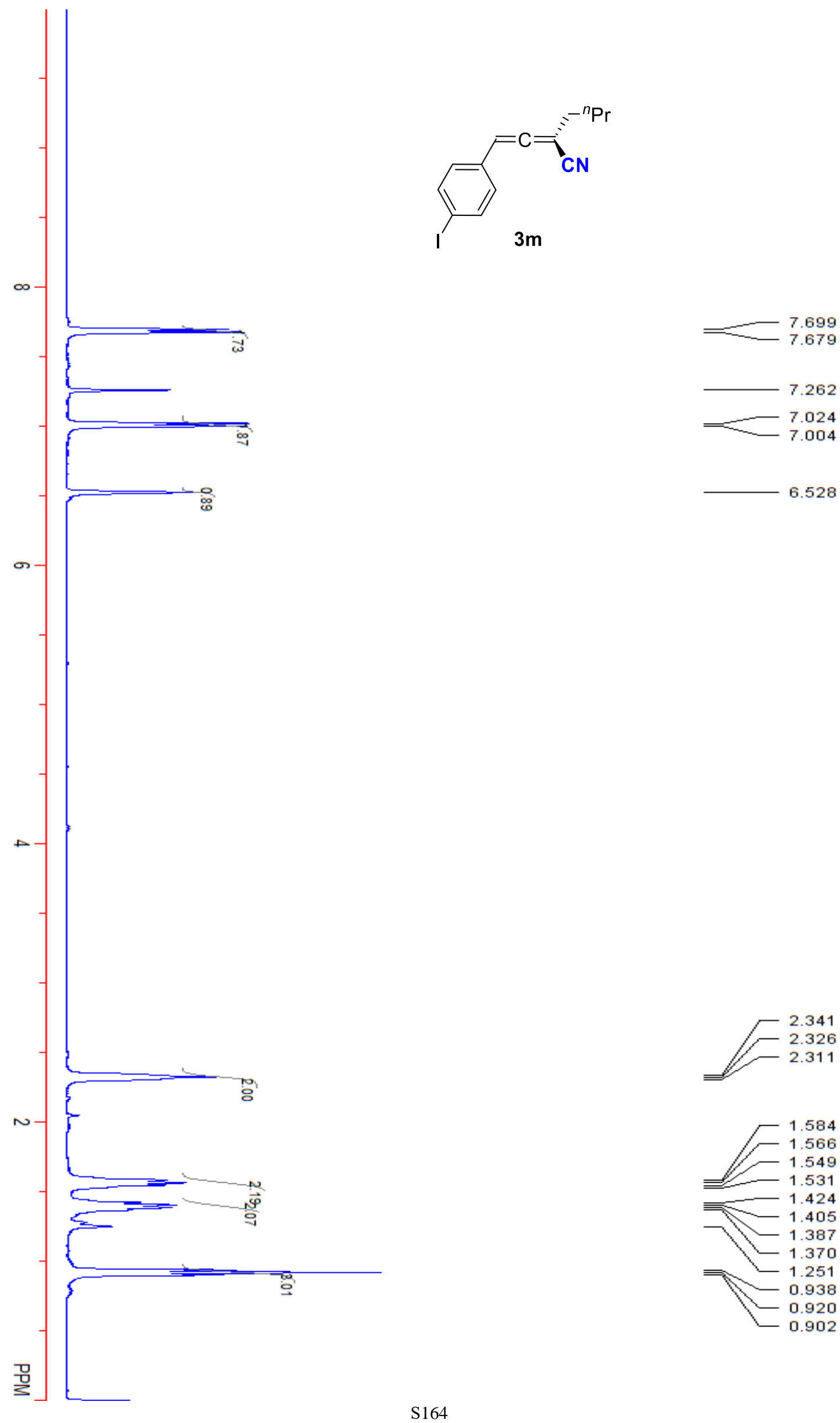
1.531 $=-1.424$

1.405

$-1.387$

$-1.370$

$\Longrightarrow-1.251$

a 0.938

$-0.920$

- 0.902 


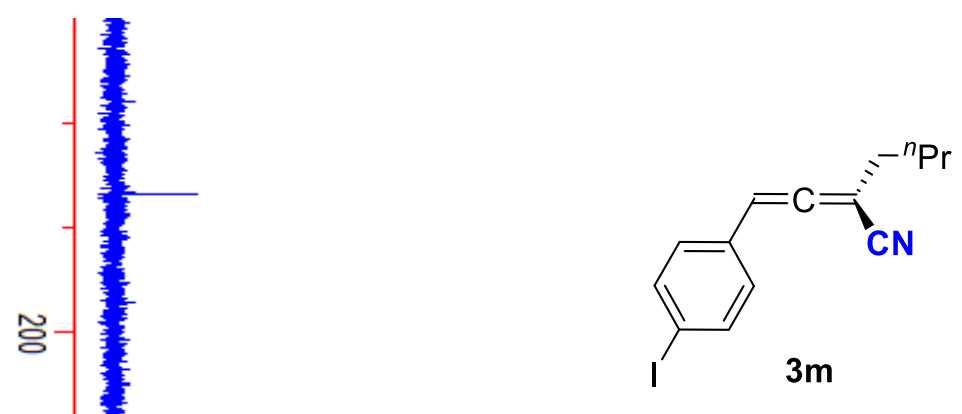

213.160

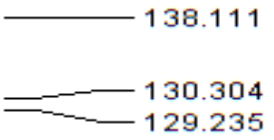

114.577

홍

뵹

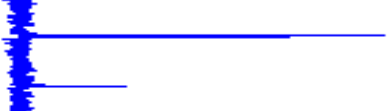

98.973

94.241

86.777

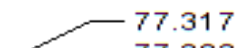

$-77.202$

76.680

s. 

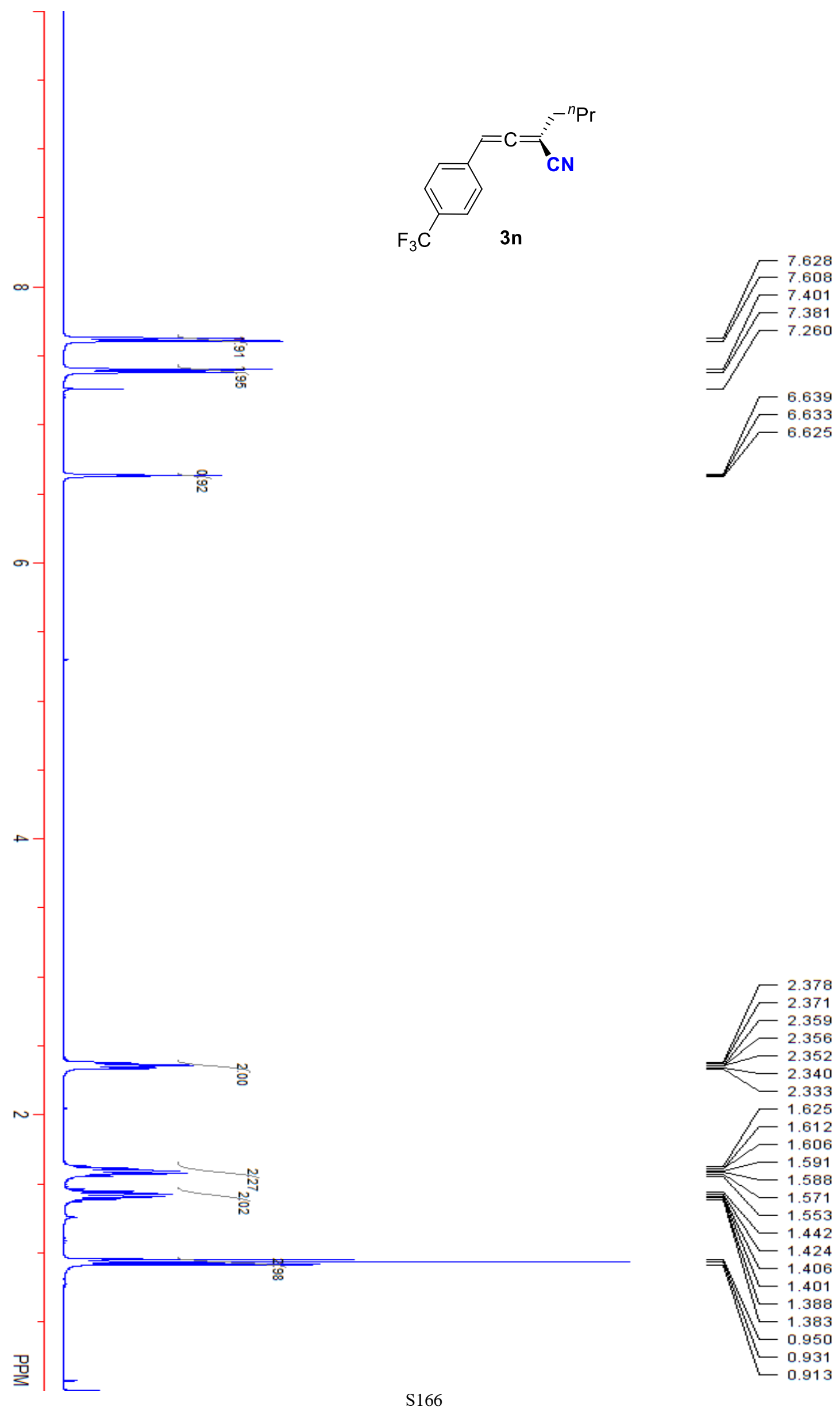

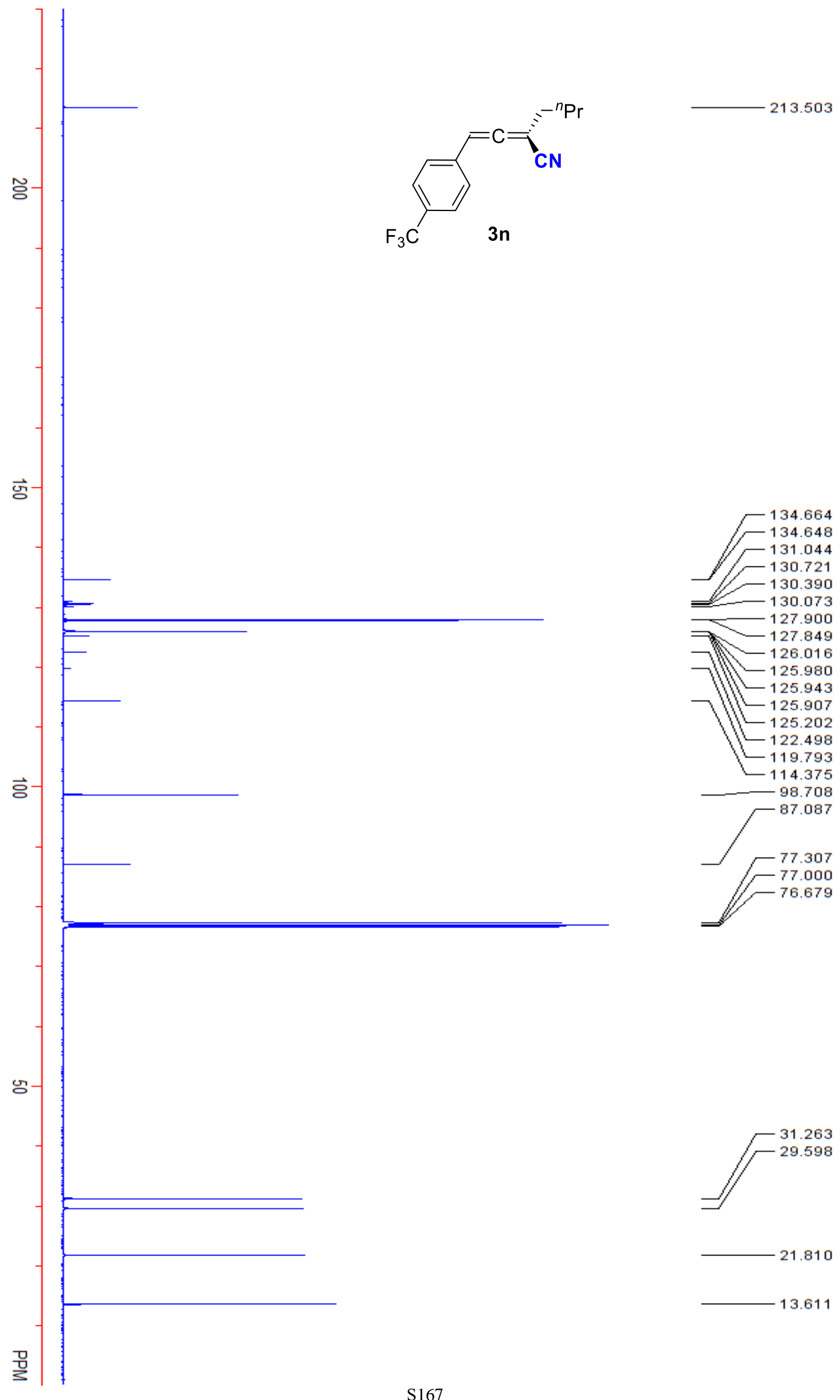

21.810

13.611 


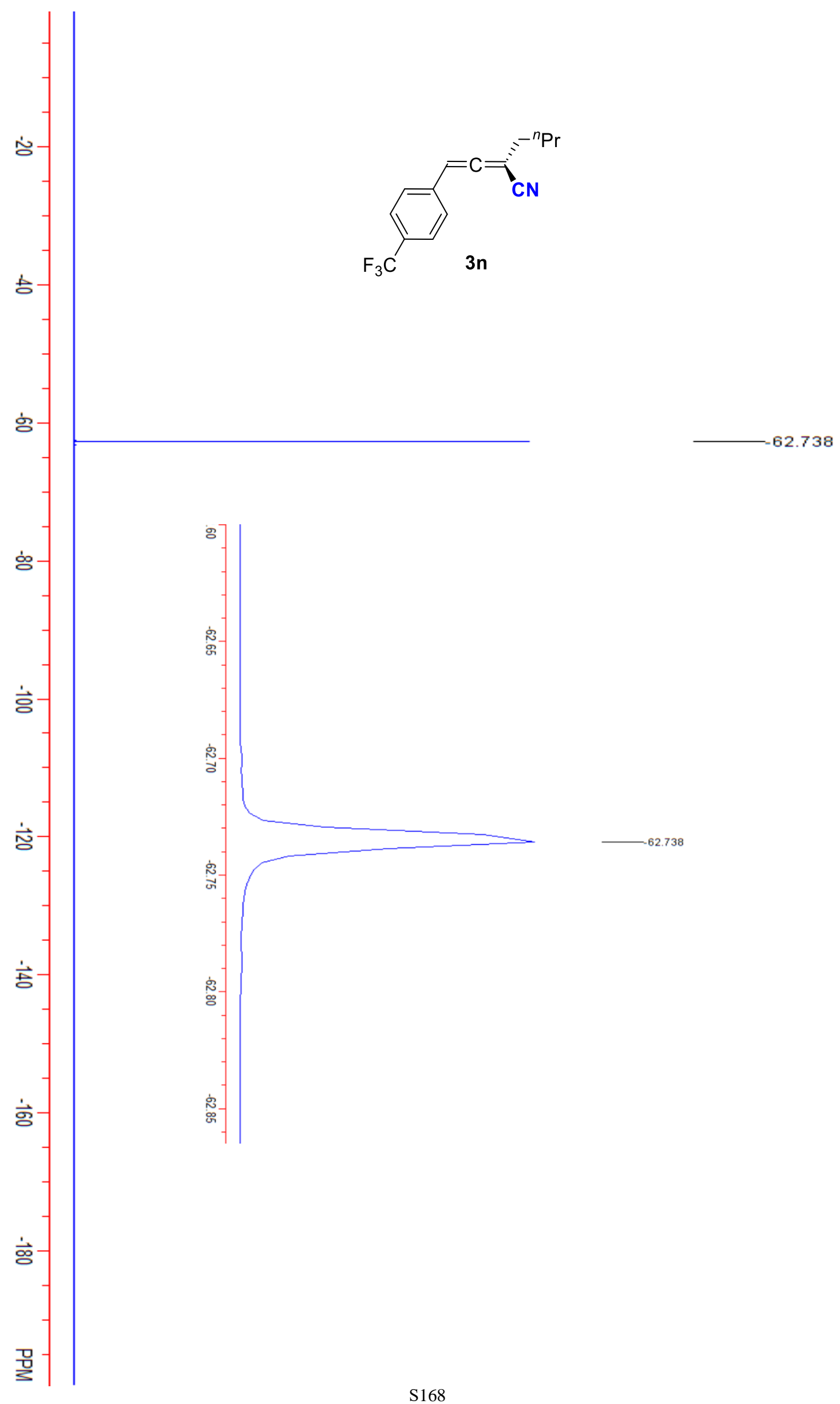



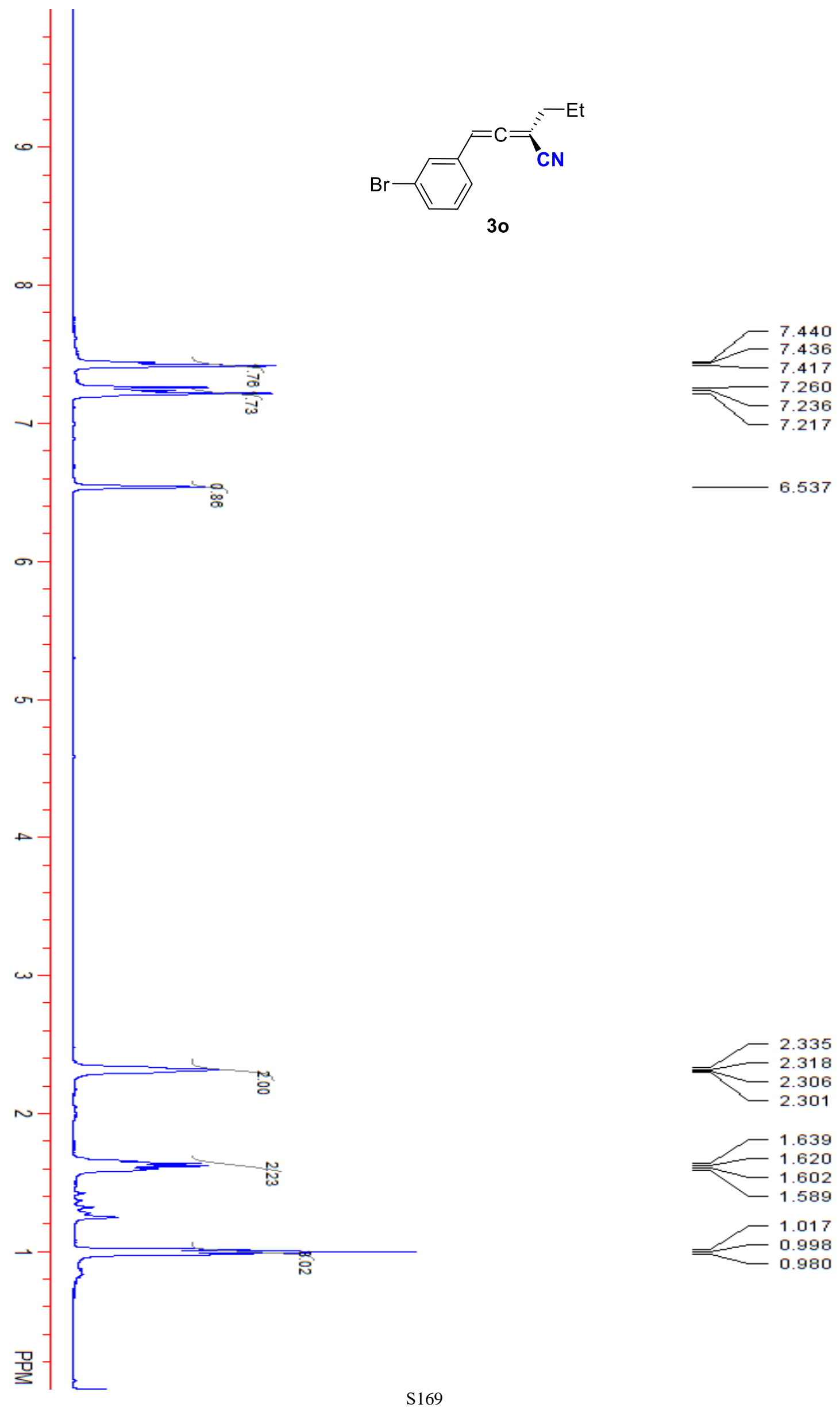

1.582

$-1.017$

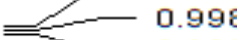

0.980 

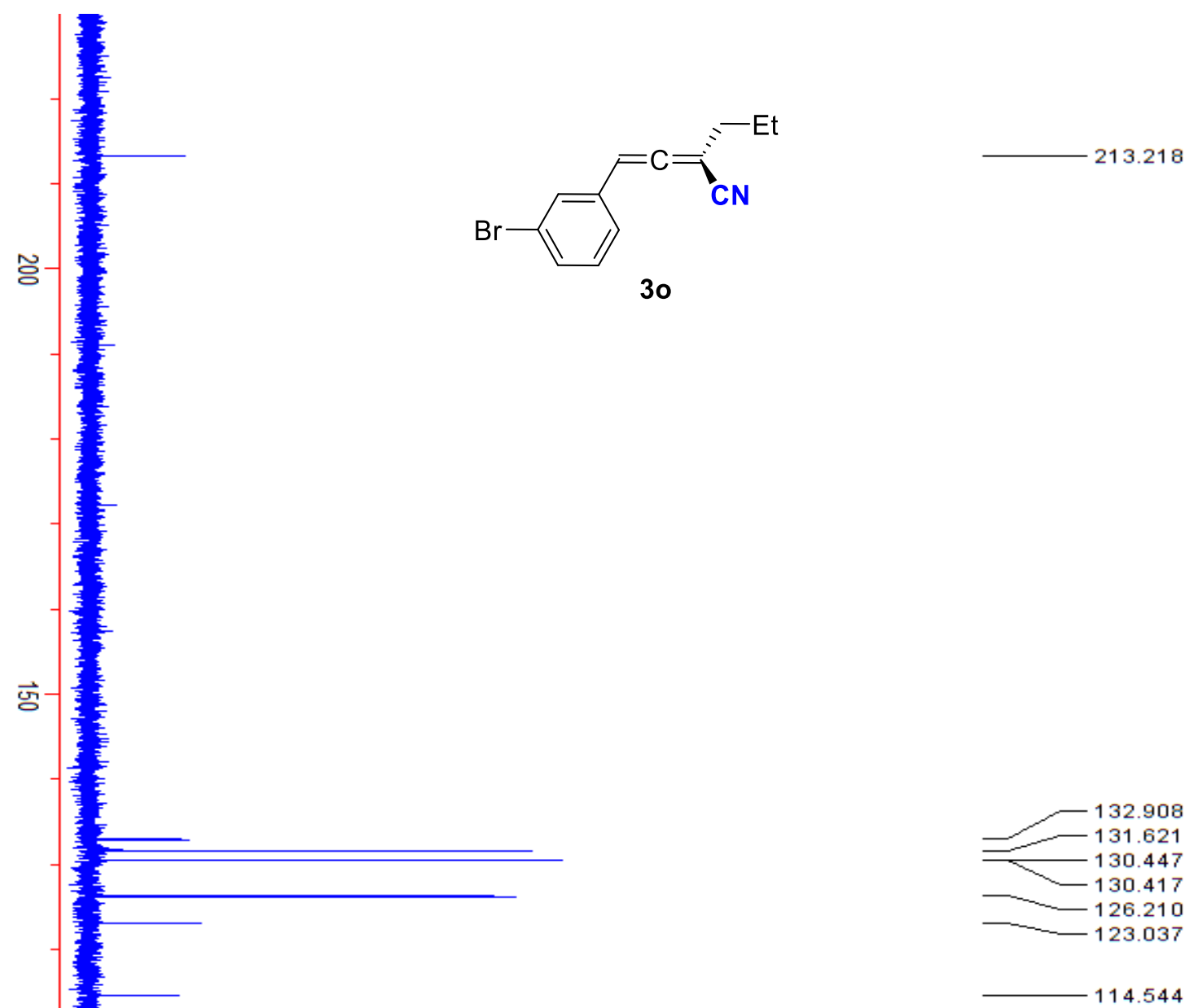

항

당

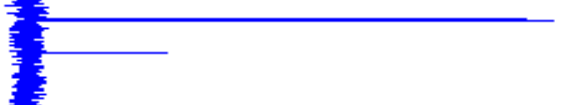

114.544

당 

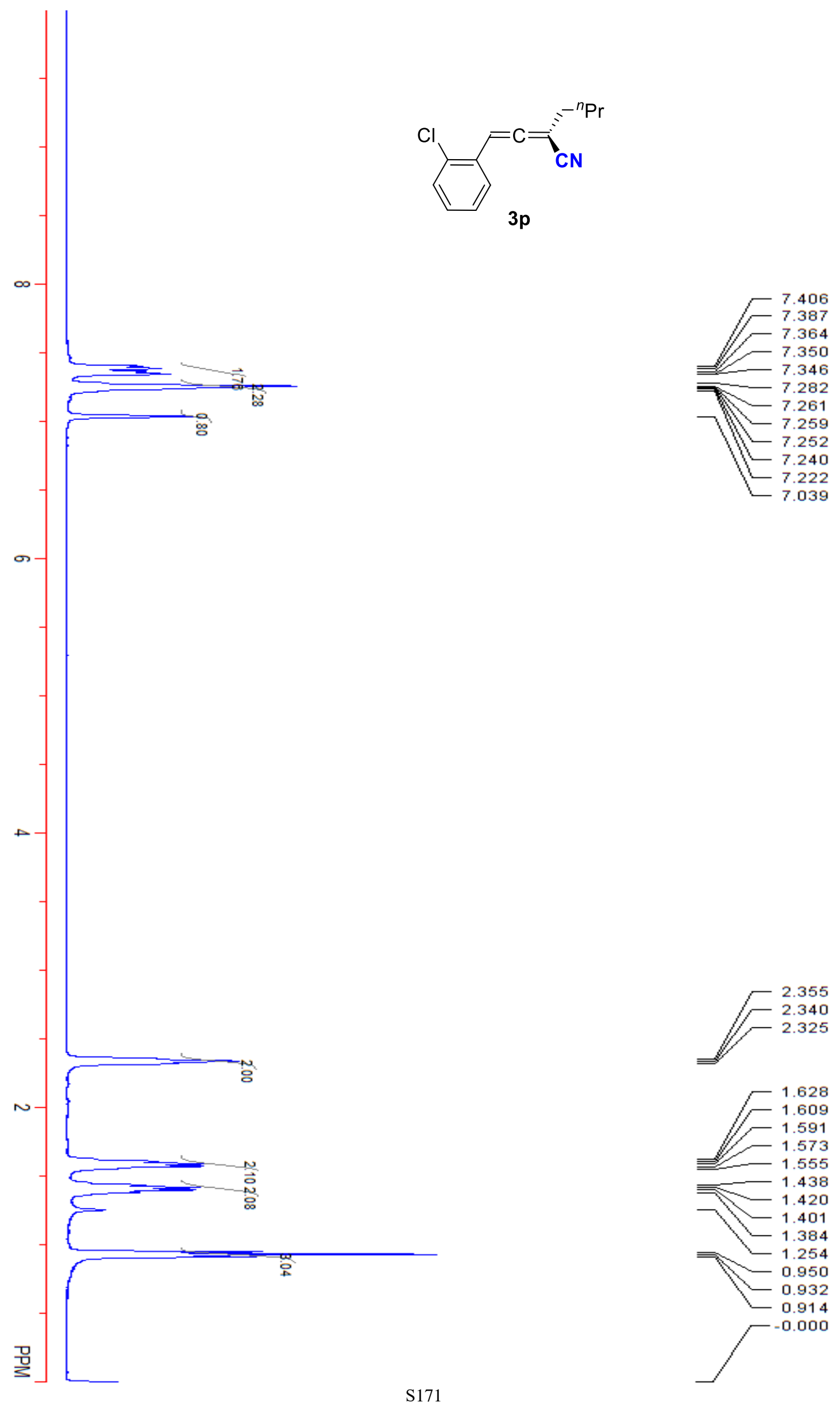

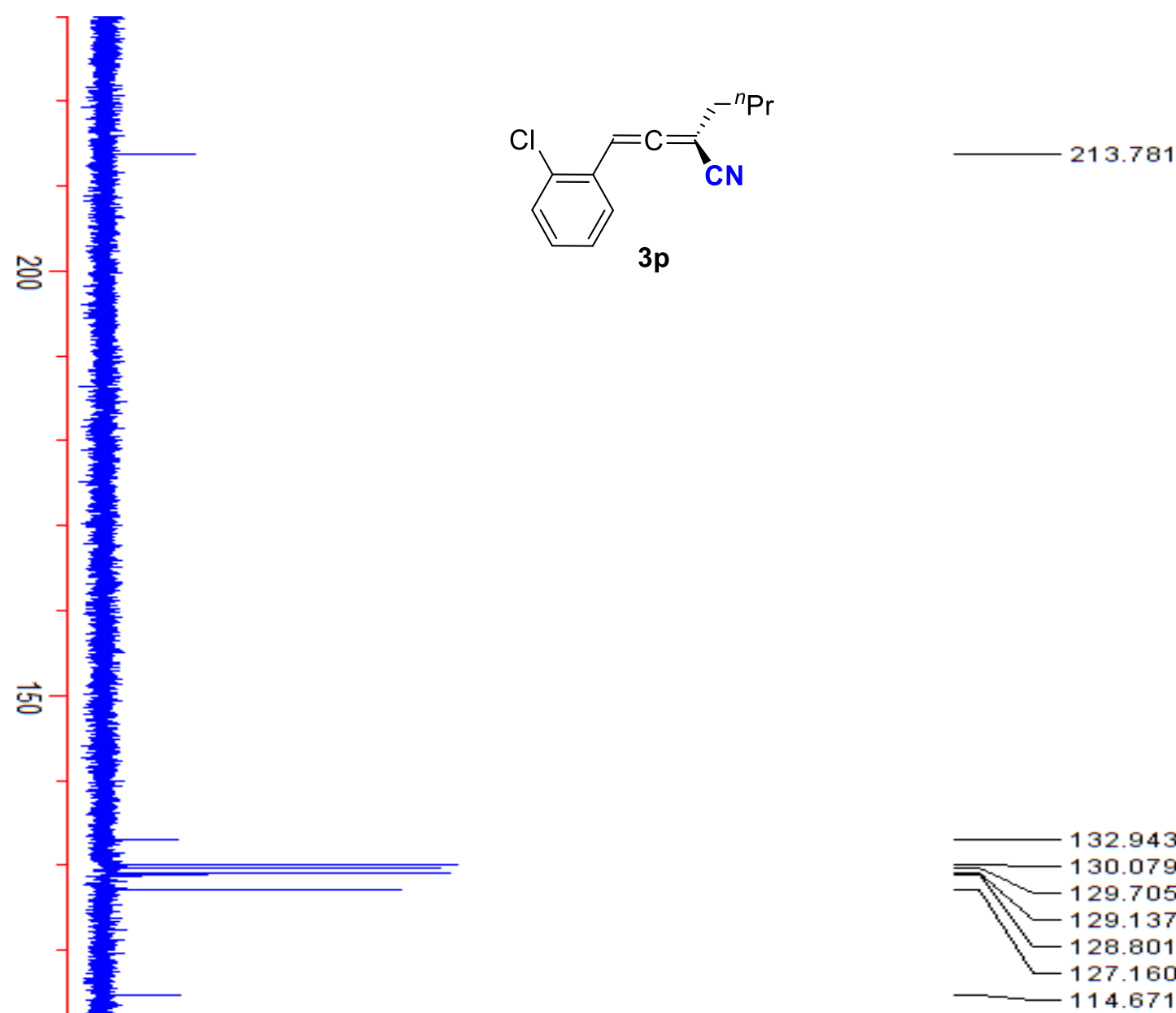

홍

형
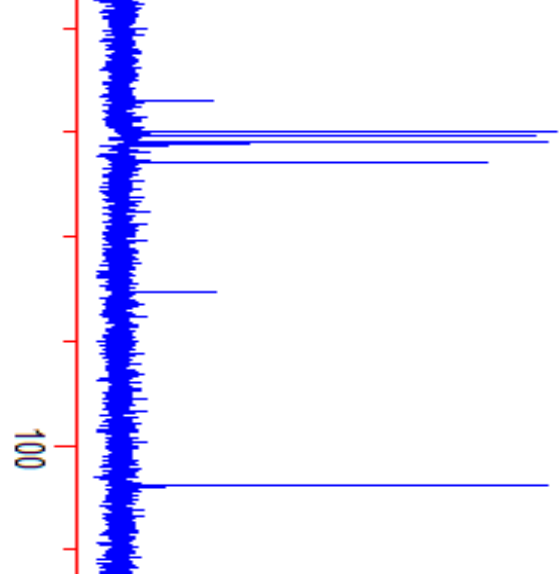

96.108

86.429

77.317

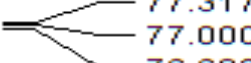

76.682

당

$\stackrel{\mathrm{g}}{3}$ 


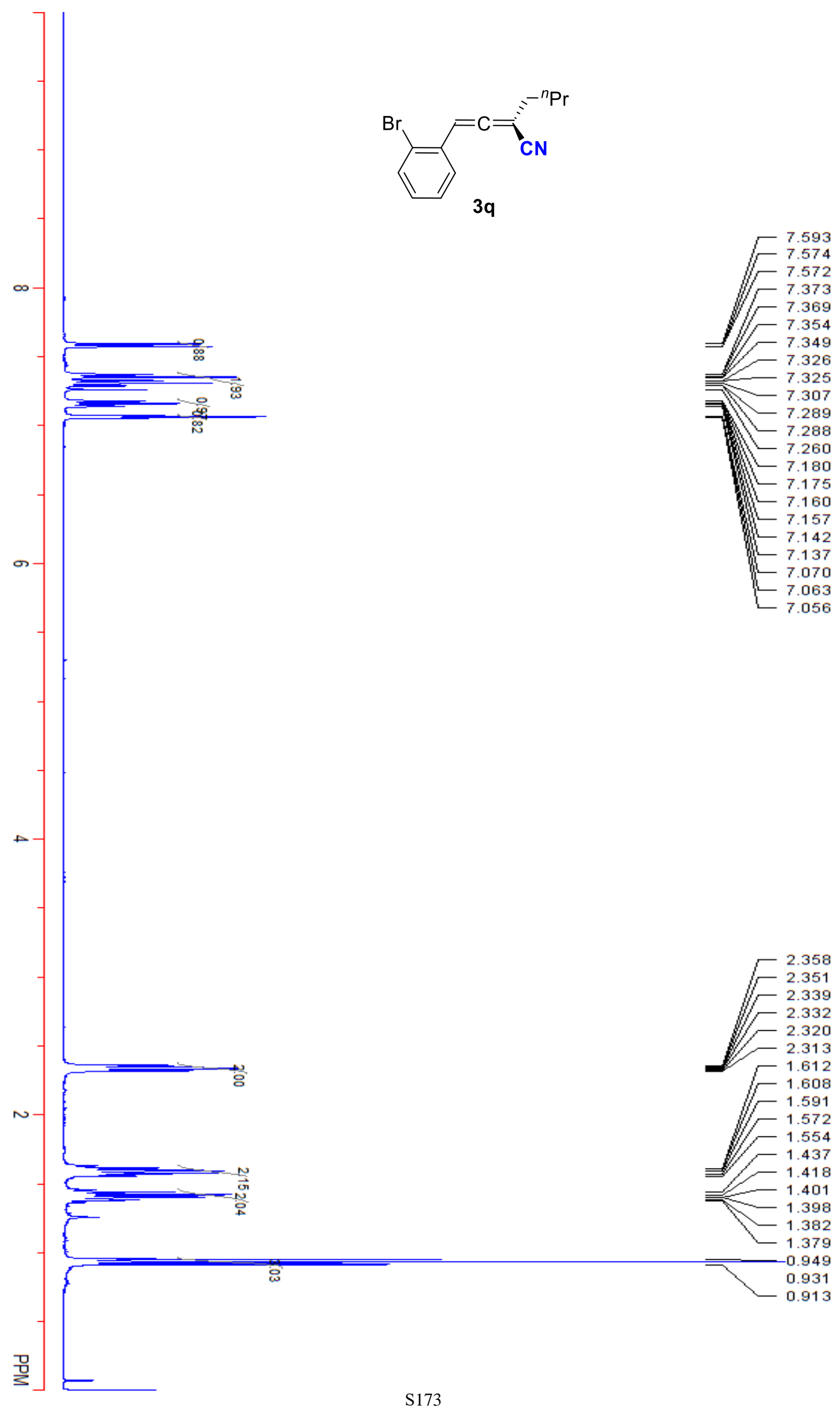



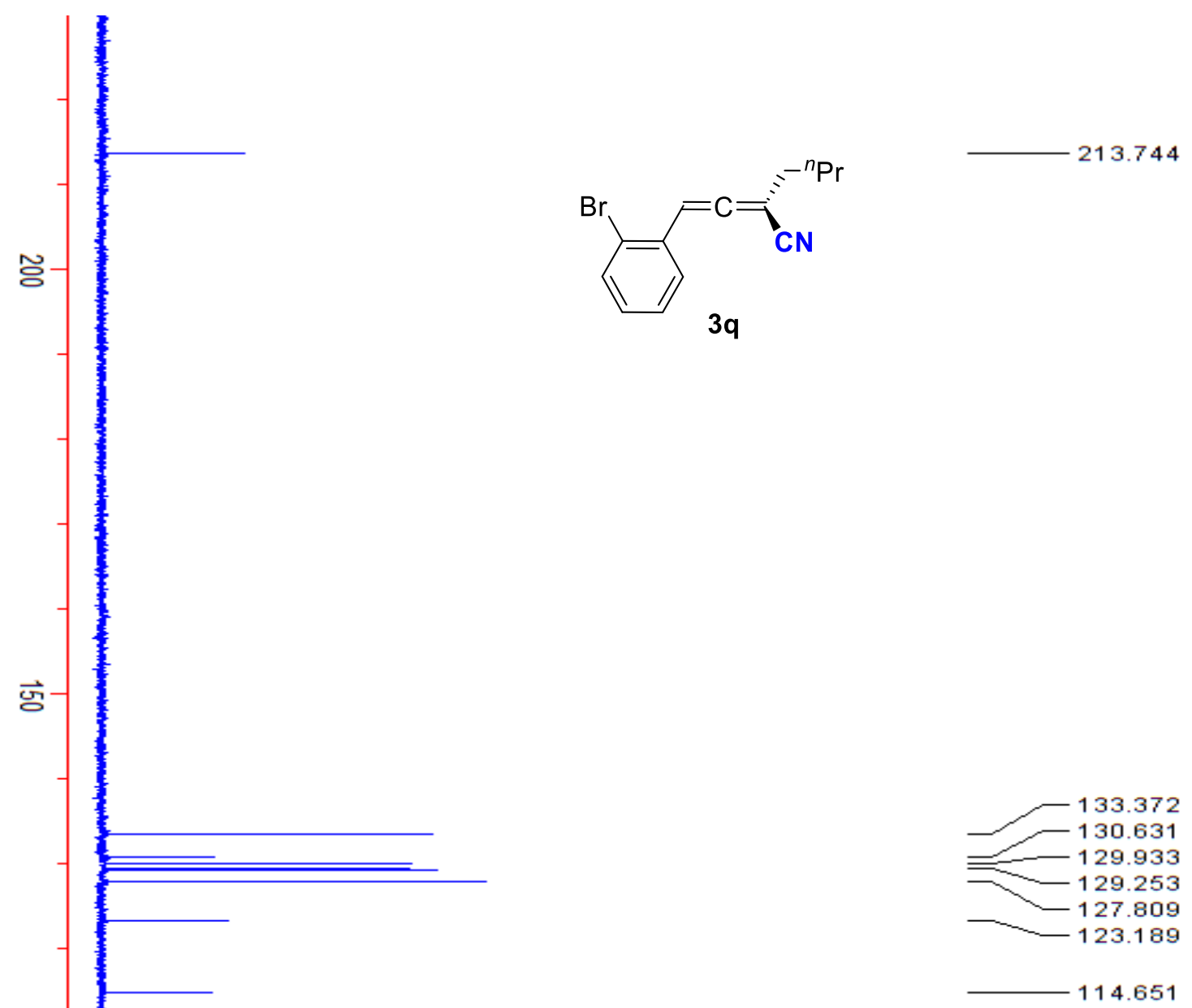

홍

항
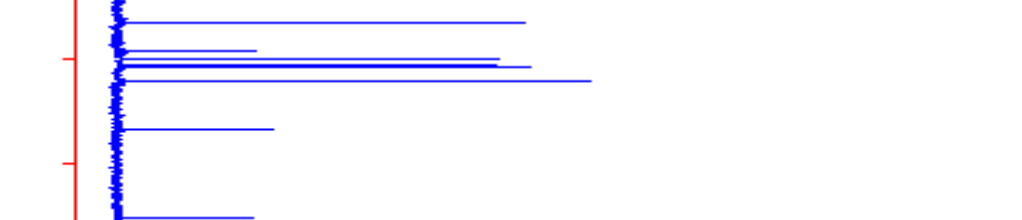

114.651
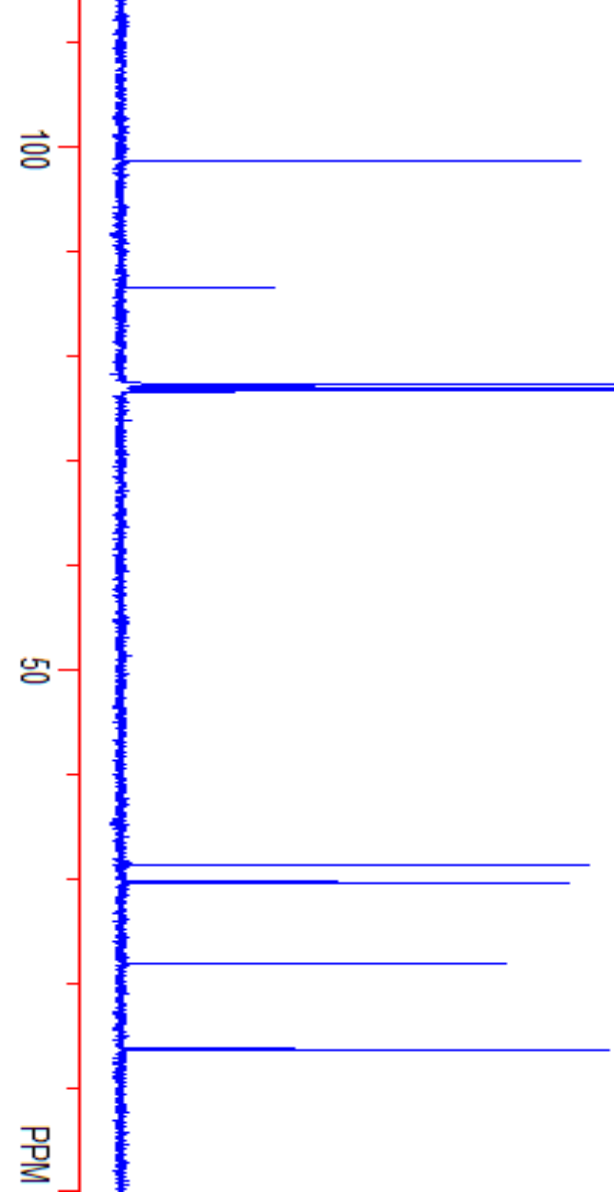

77.316

77.000

76.679

31.281 

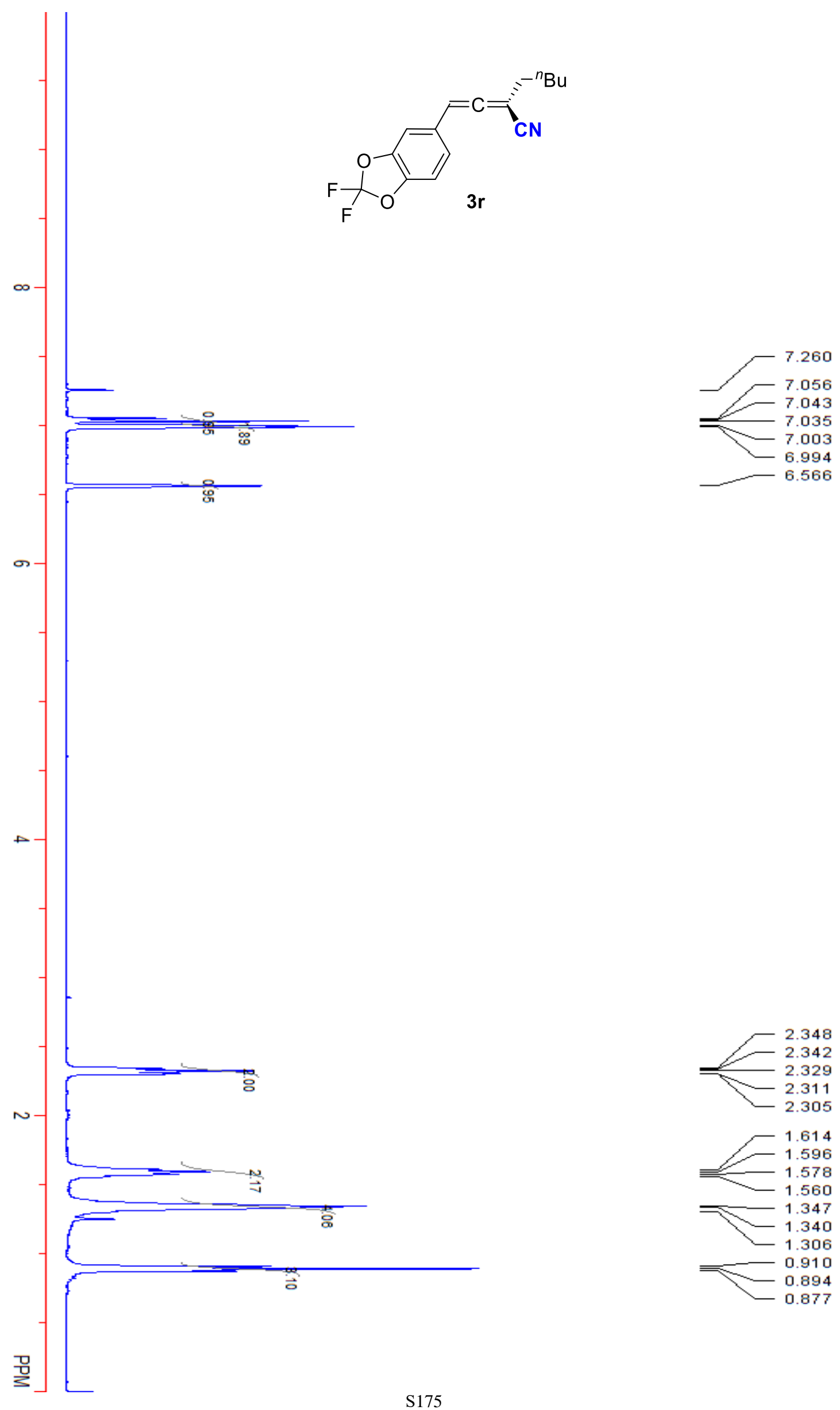


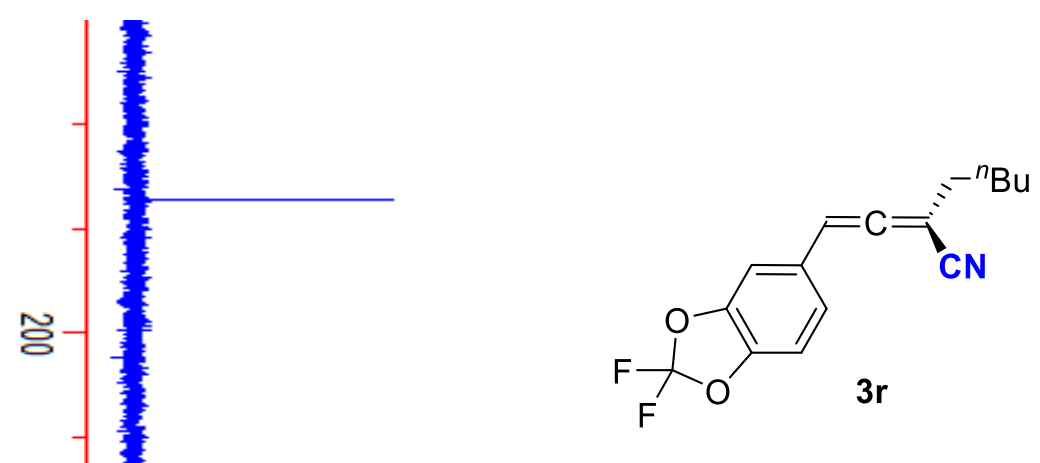

134.139

131.593

129.044

127.050

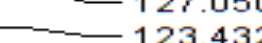

114.502

109.805

108.251

$\overrightarrow{8}$

98.964

87.169

77.316

77.000

76.683

s与

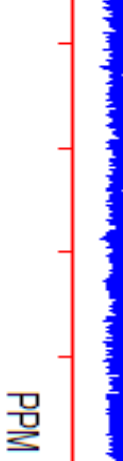

31.620

30.826

27.206

22.207

13.891 


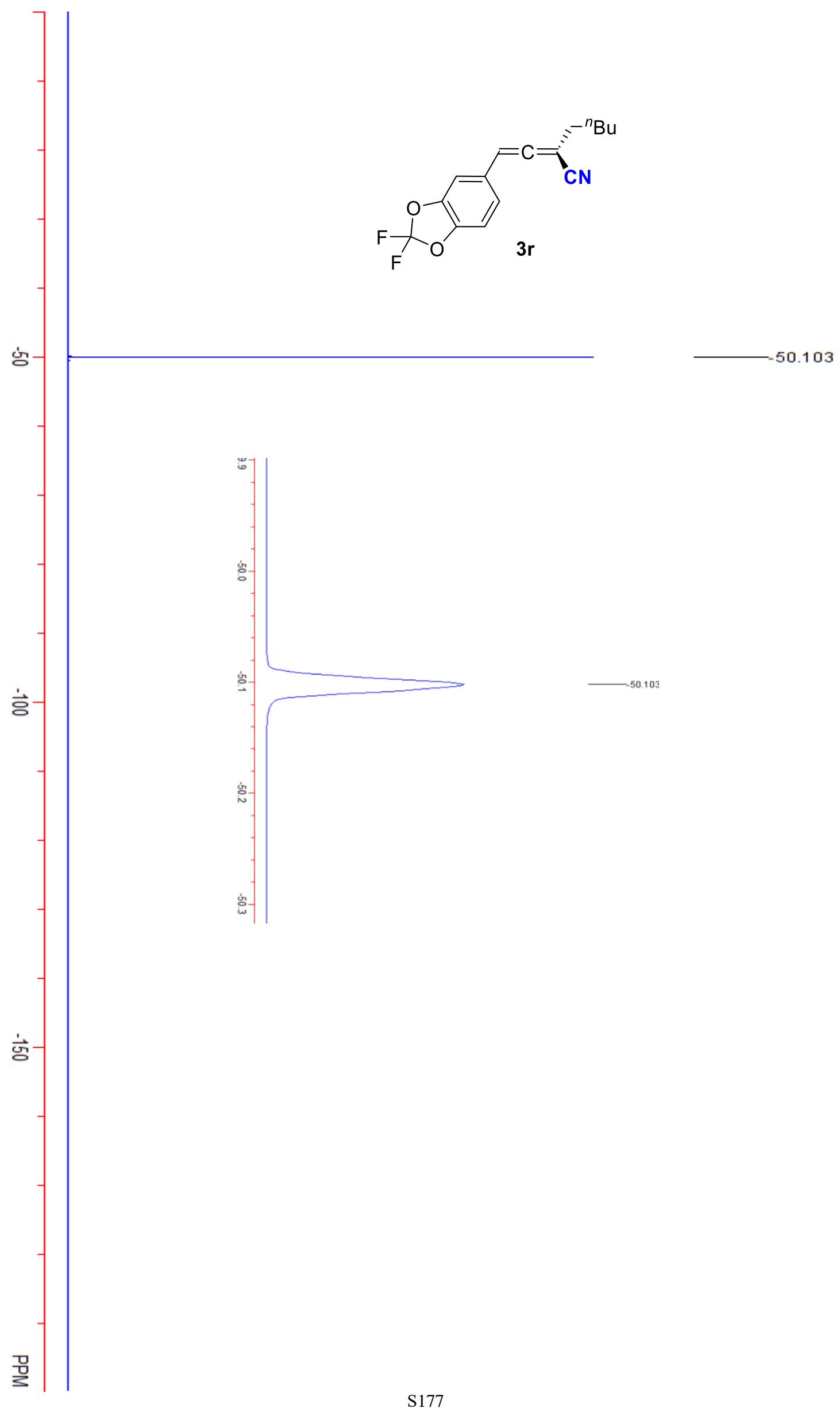




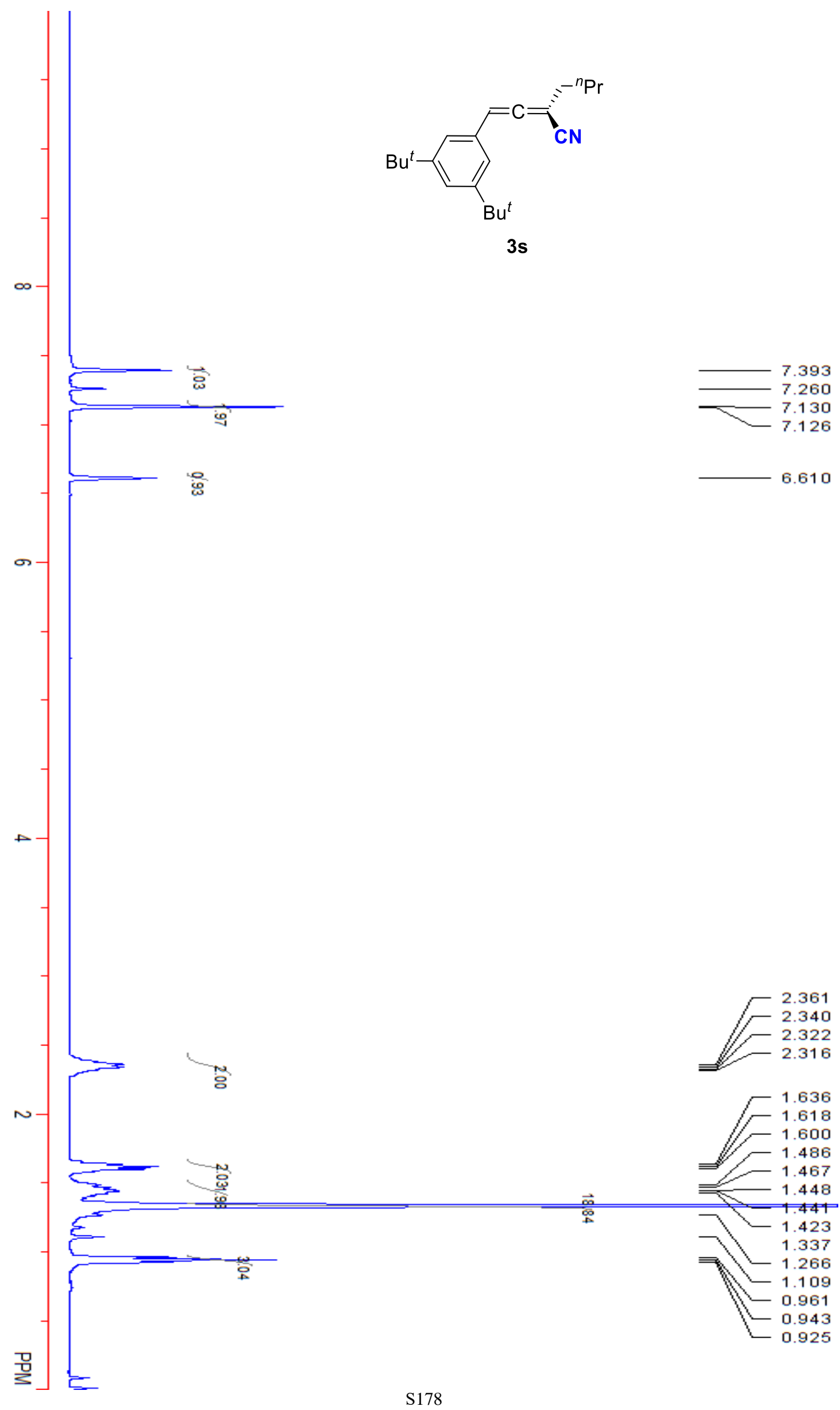




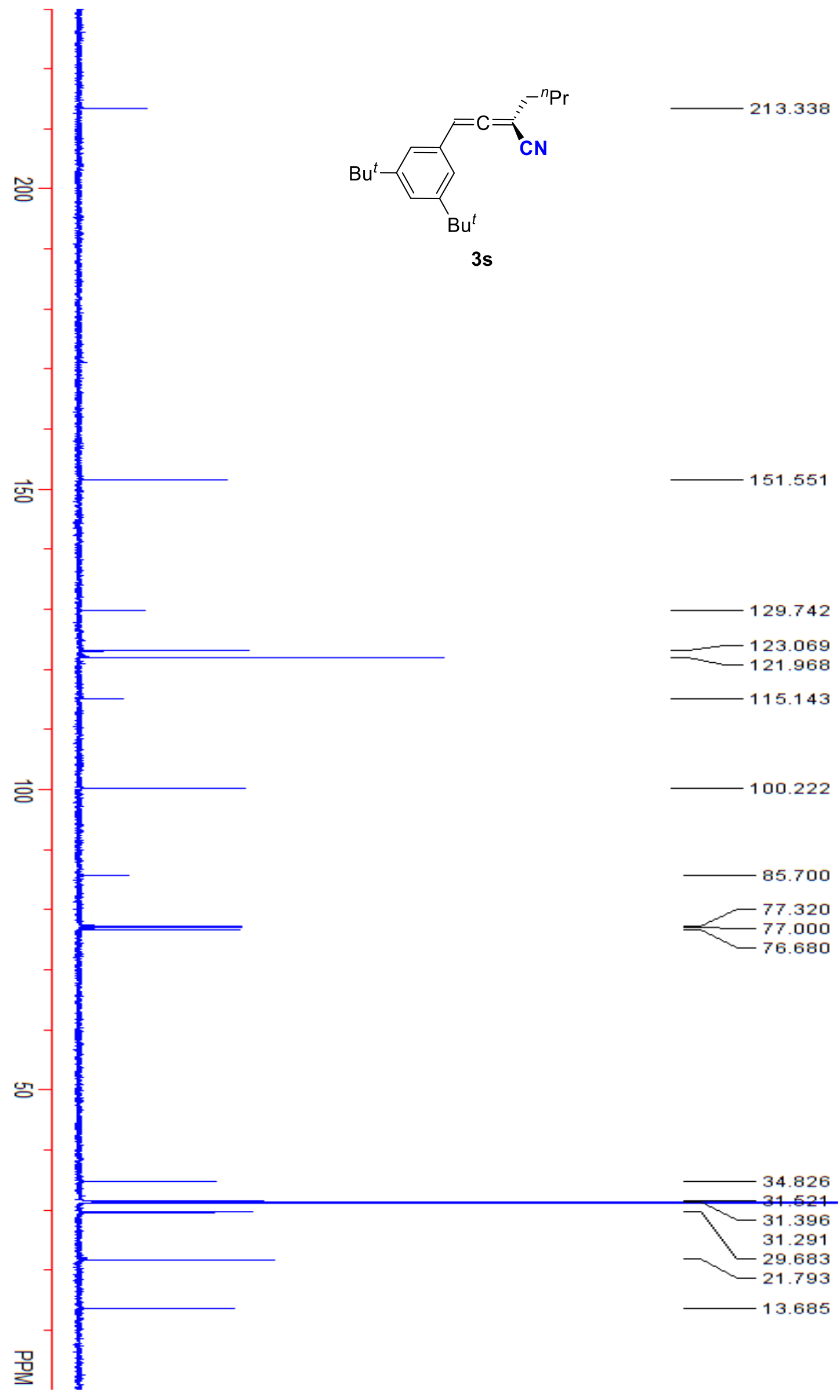



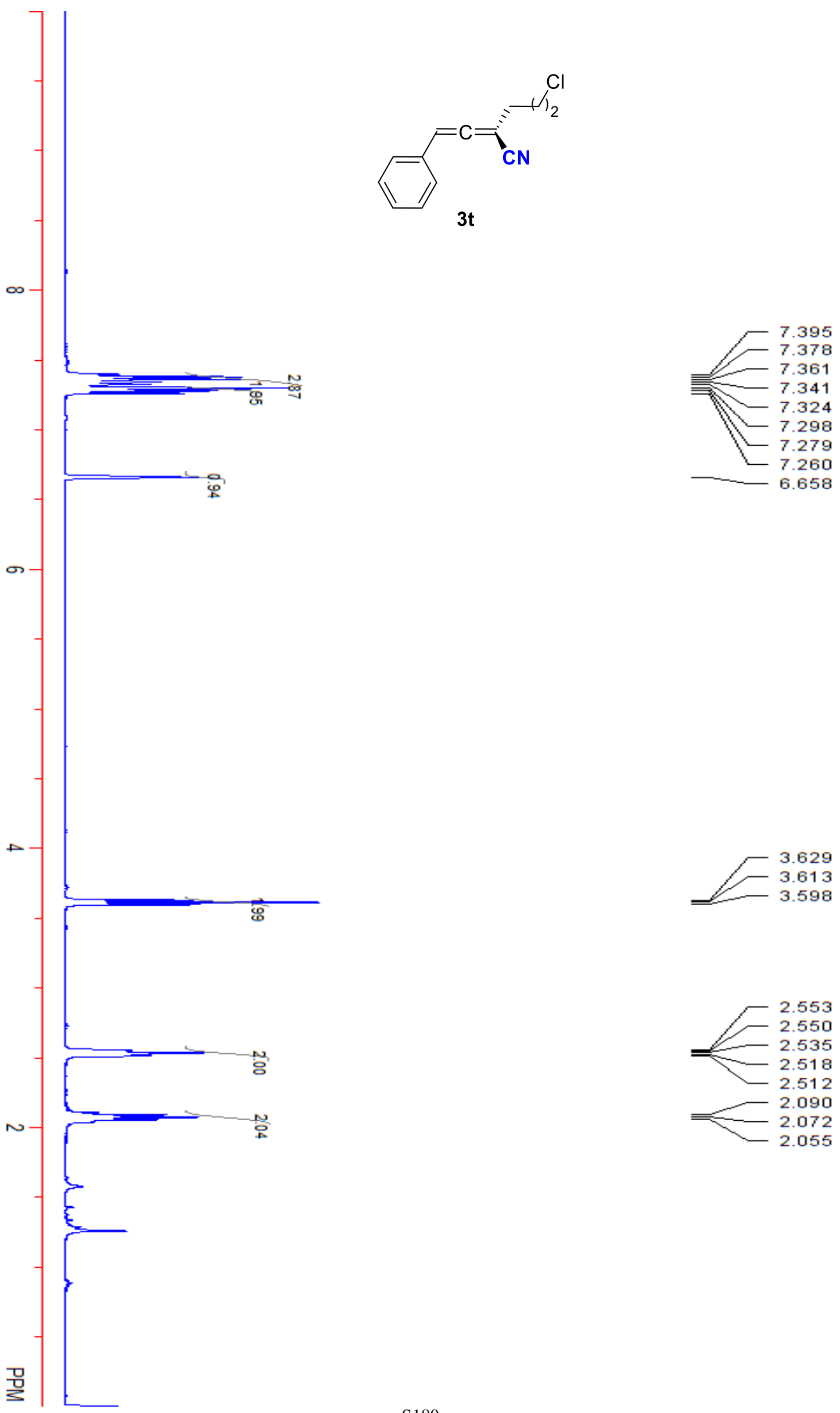


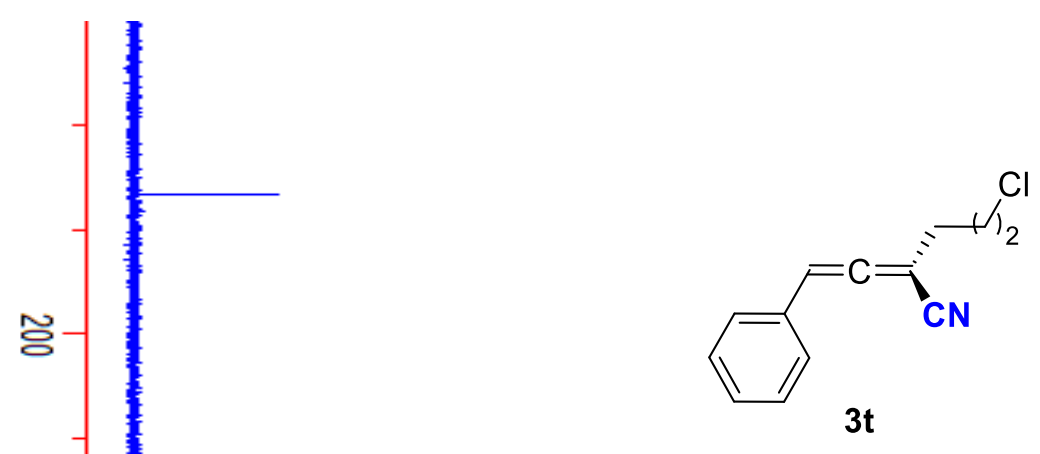

213.366

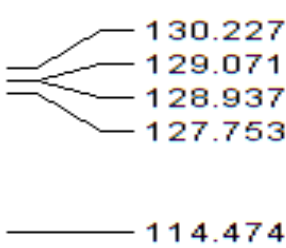

홍

ज्ञ

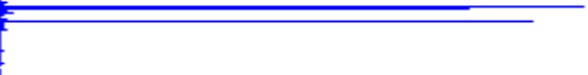

$-100.343$

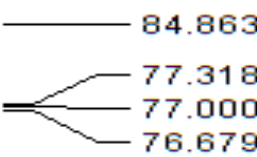

s与

몰 

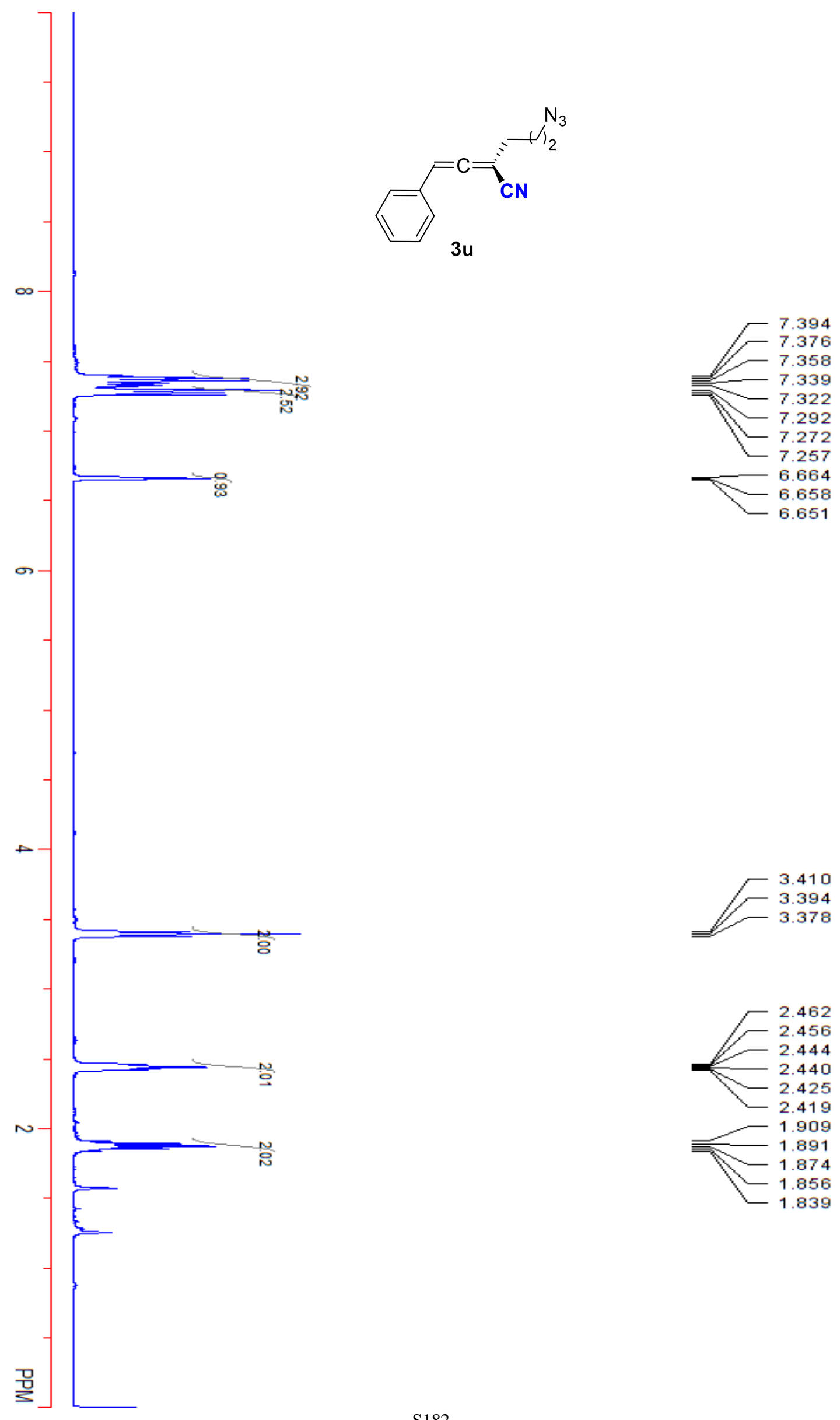

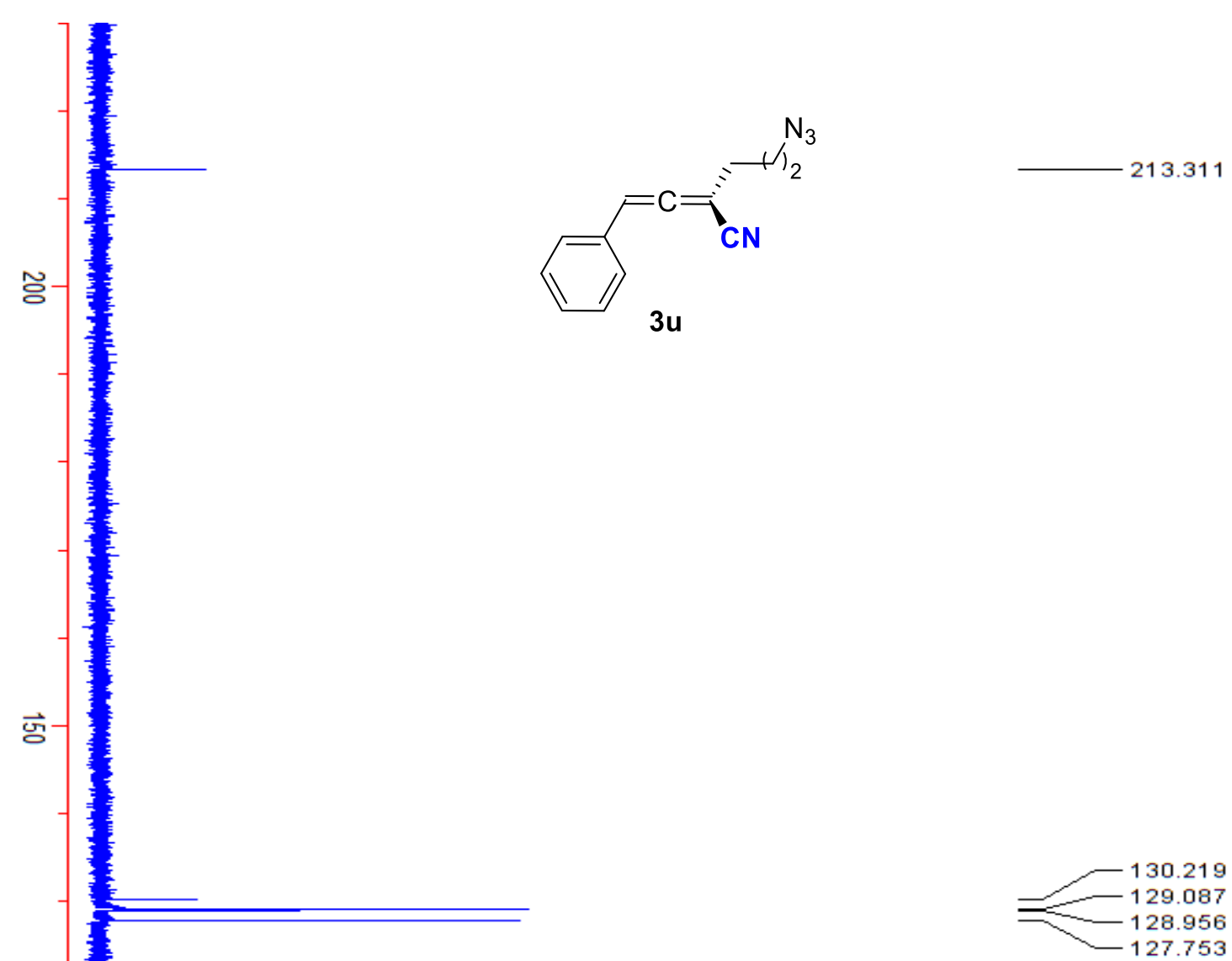

횽

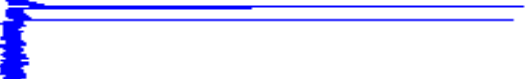

114.476
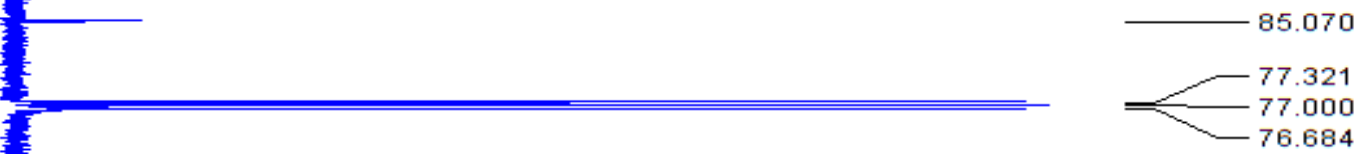

믄

100.386

7.000
6.684

50.083

8.738

26.823 

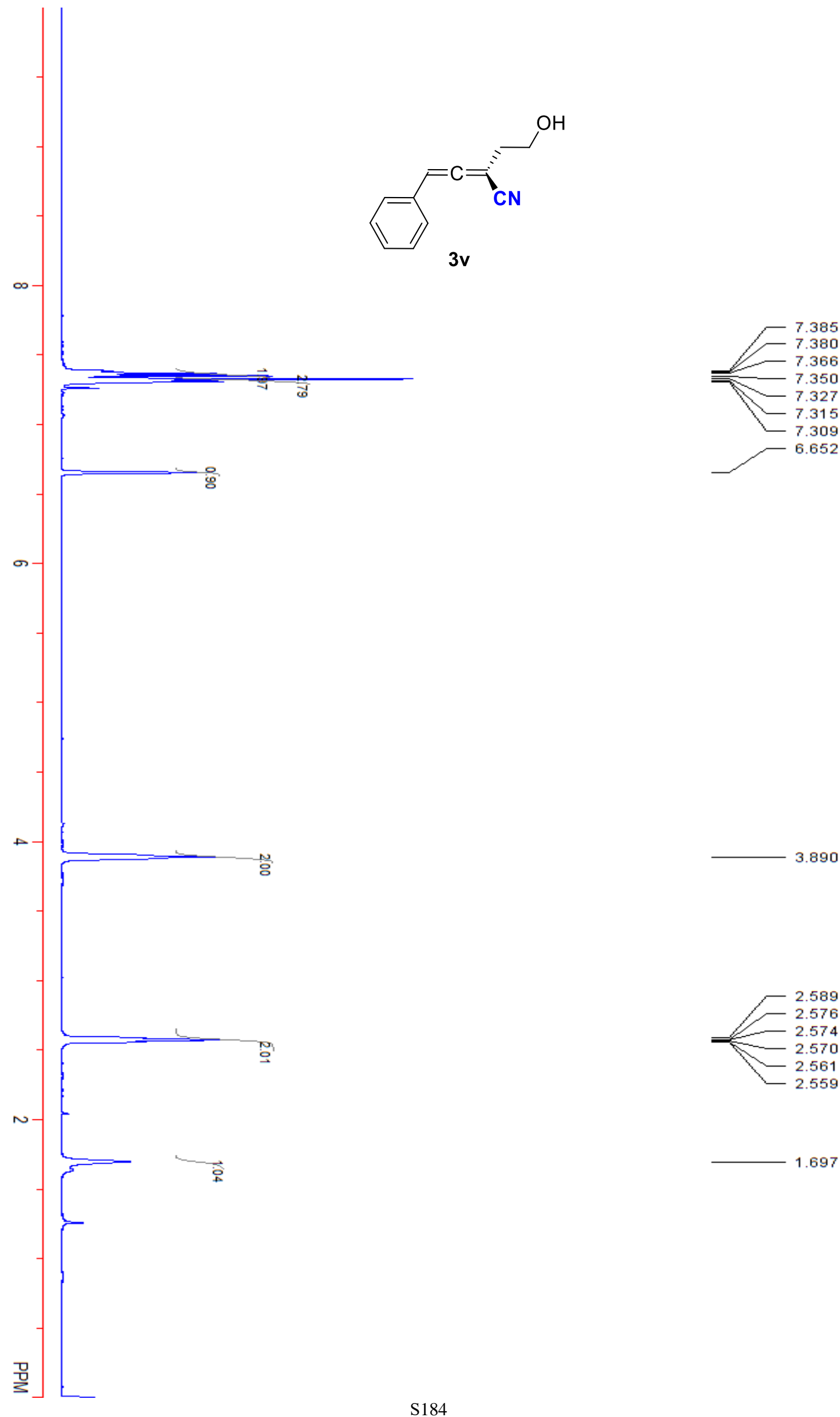

1.697 

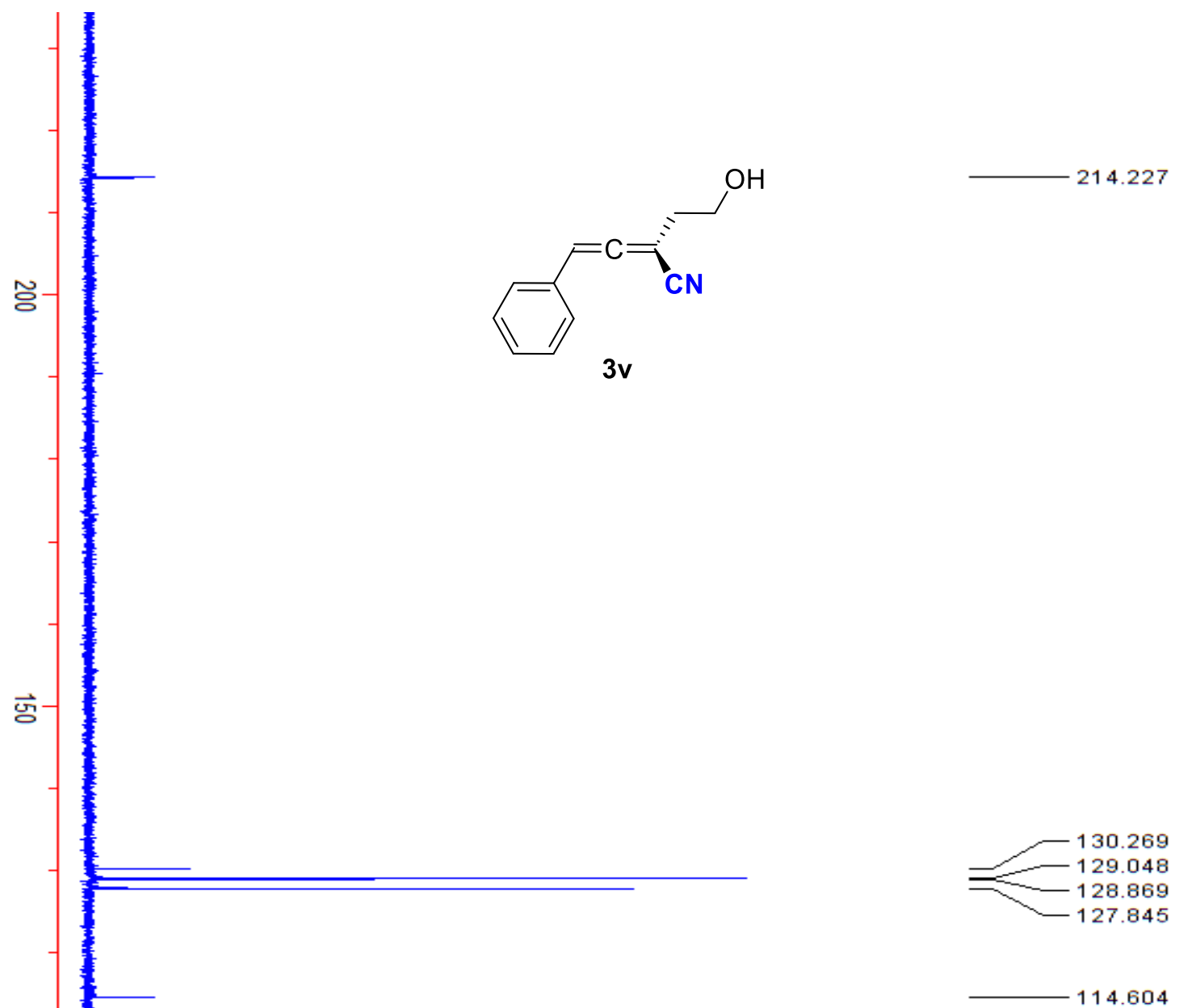

용

ज्

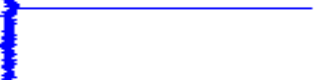

si

믄 

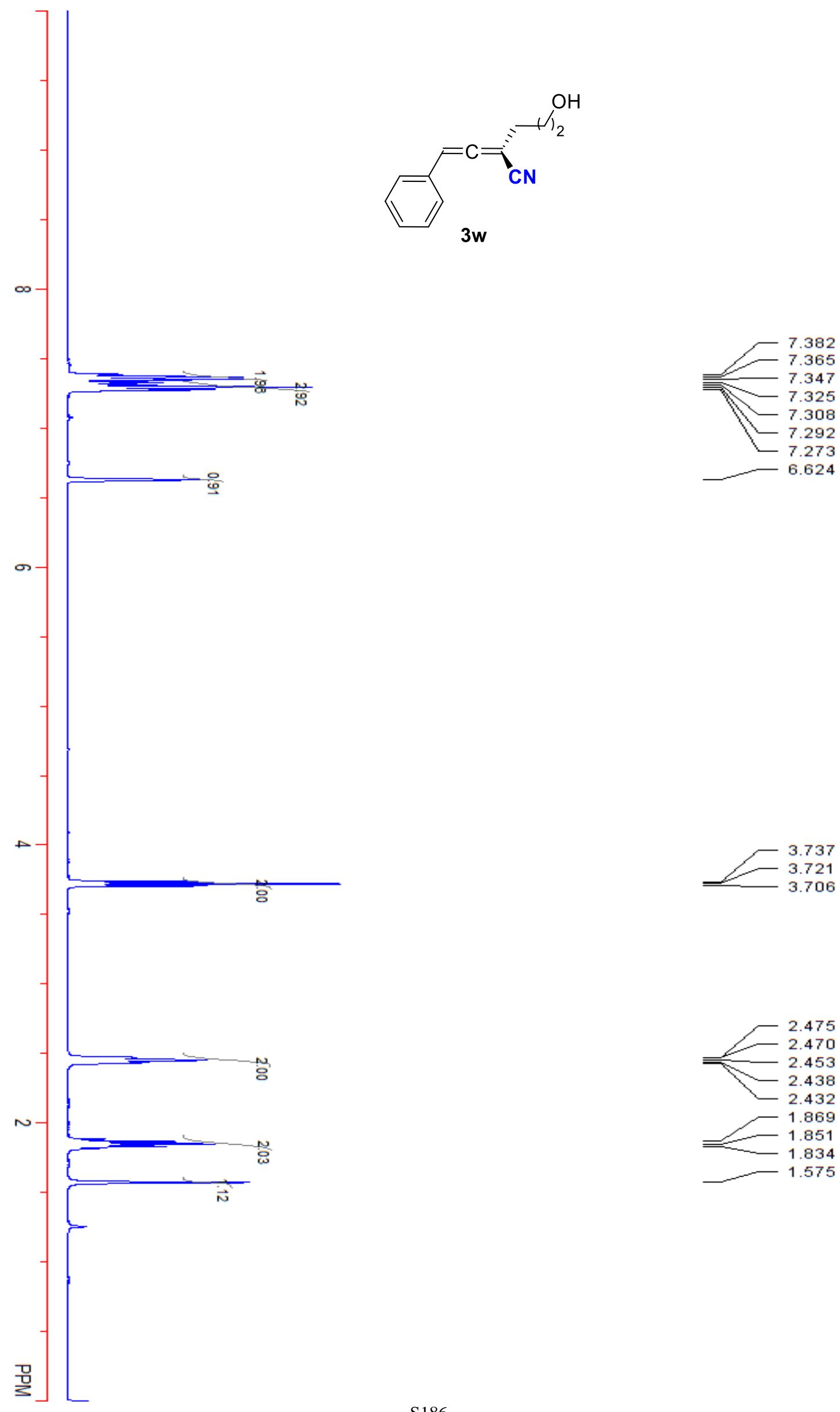


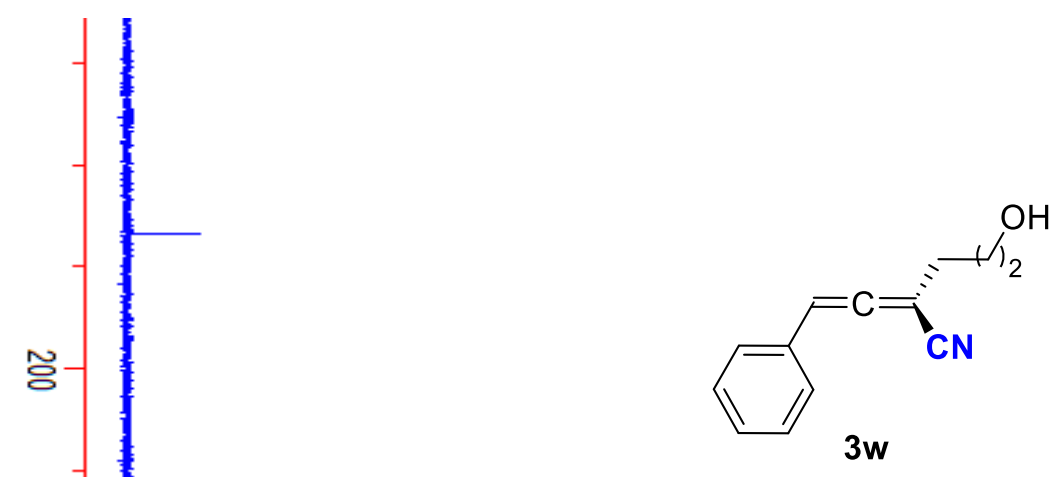

213.227

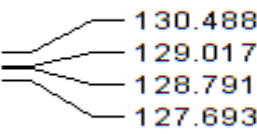

114.794

$\overrightarrow{\mathrm{s}}$

홍

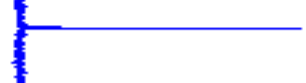

100.073

s

몬

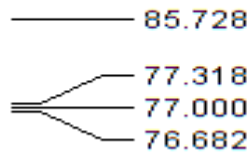

61.233 

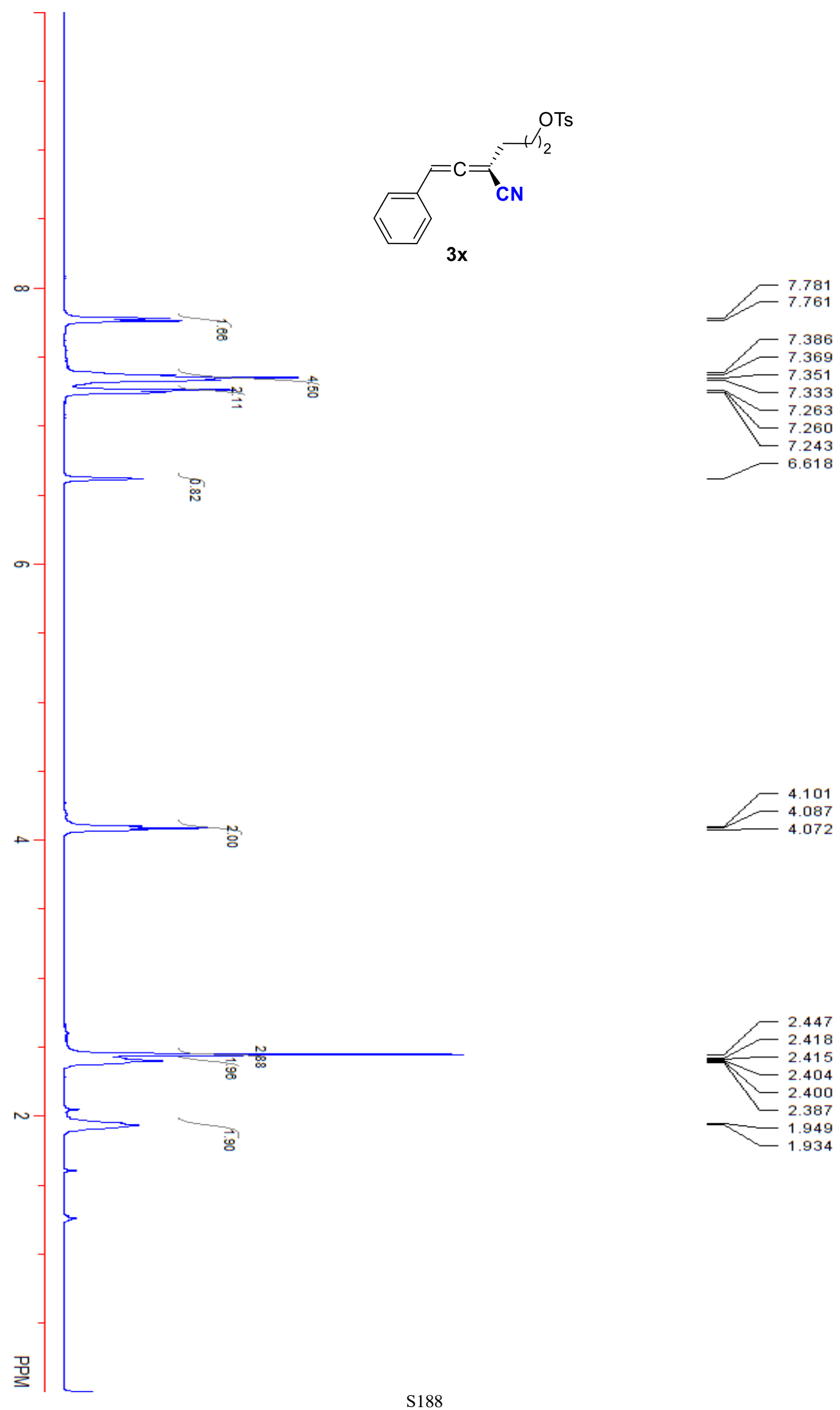


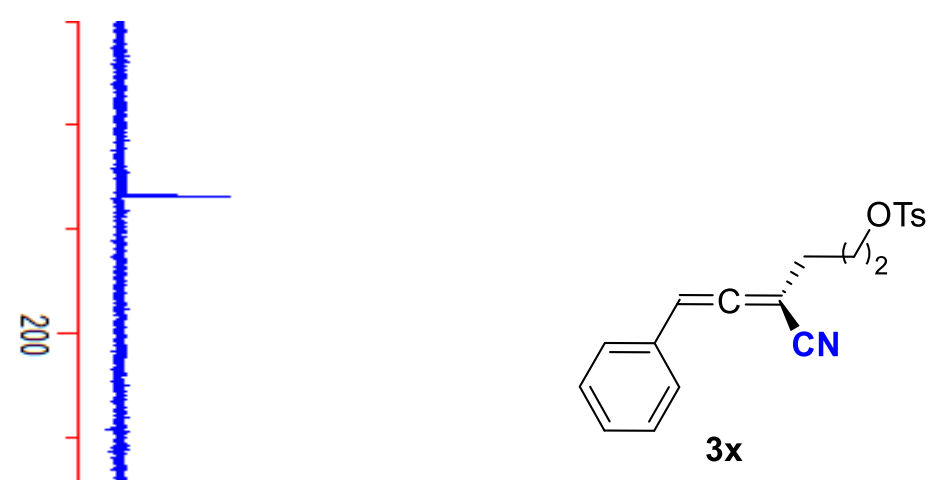

213.230

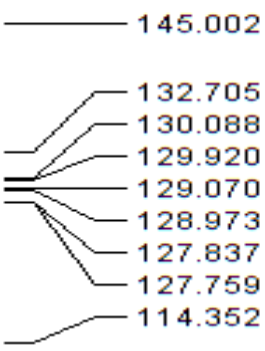

홍

ज्ञ
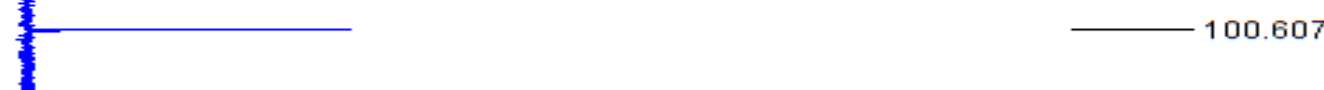

s.

D

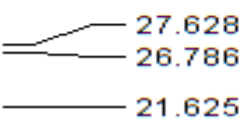



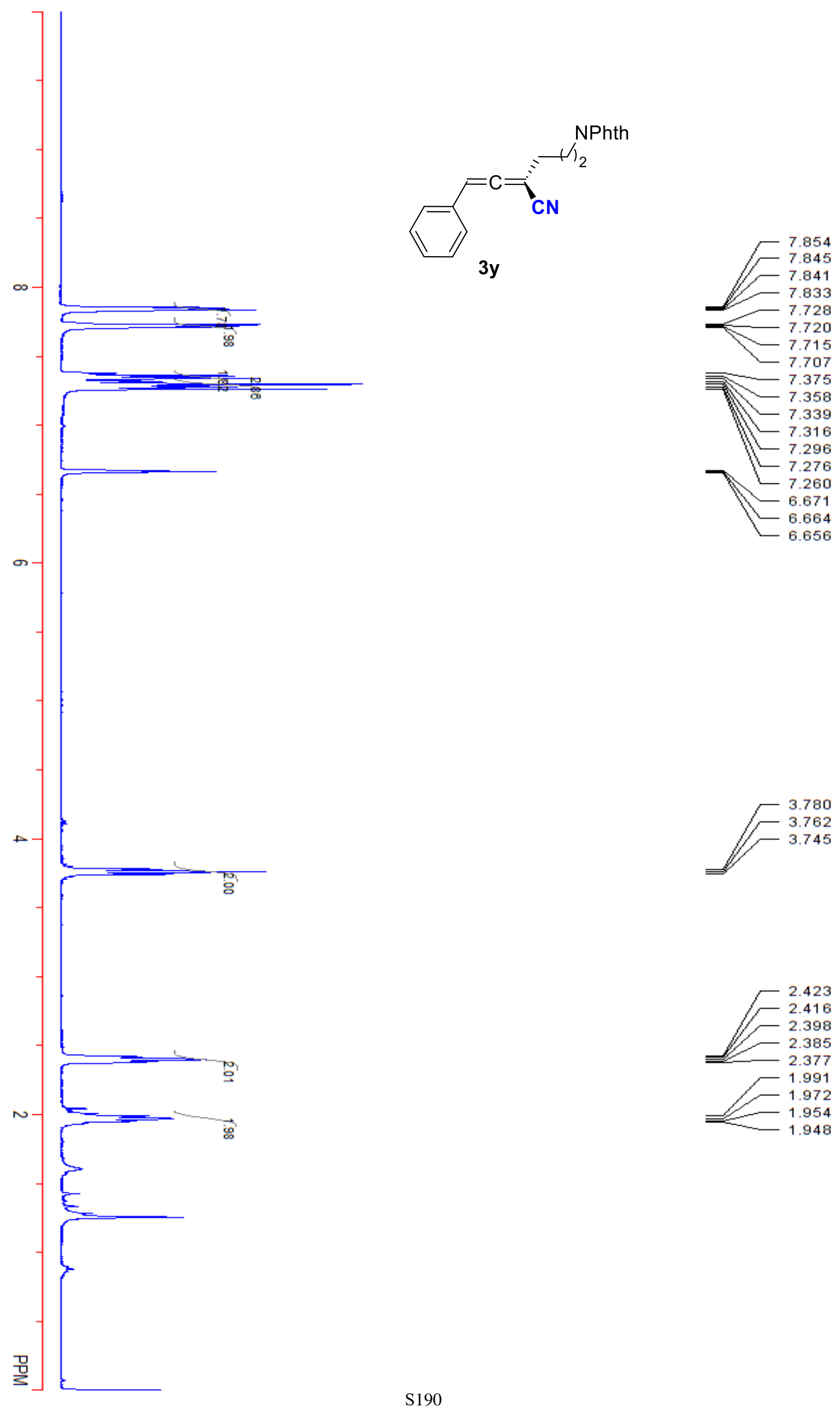

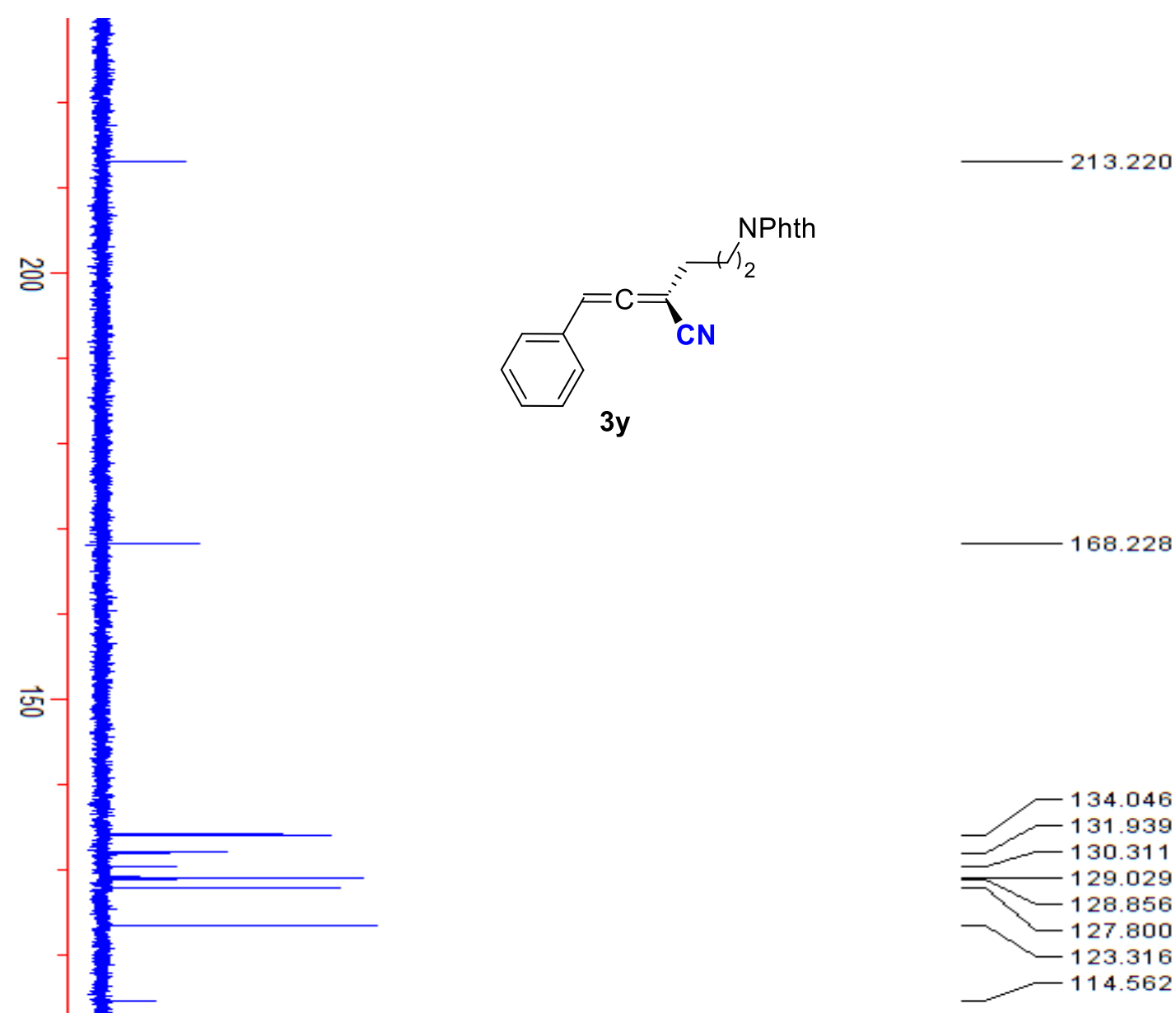

100.653

85.289

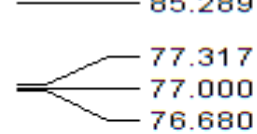

용

믄

36.828 

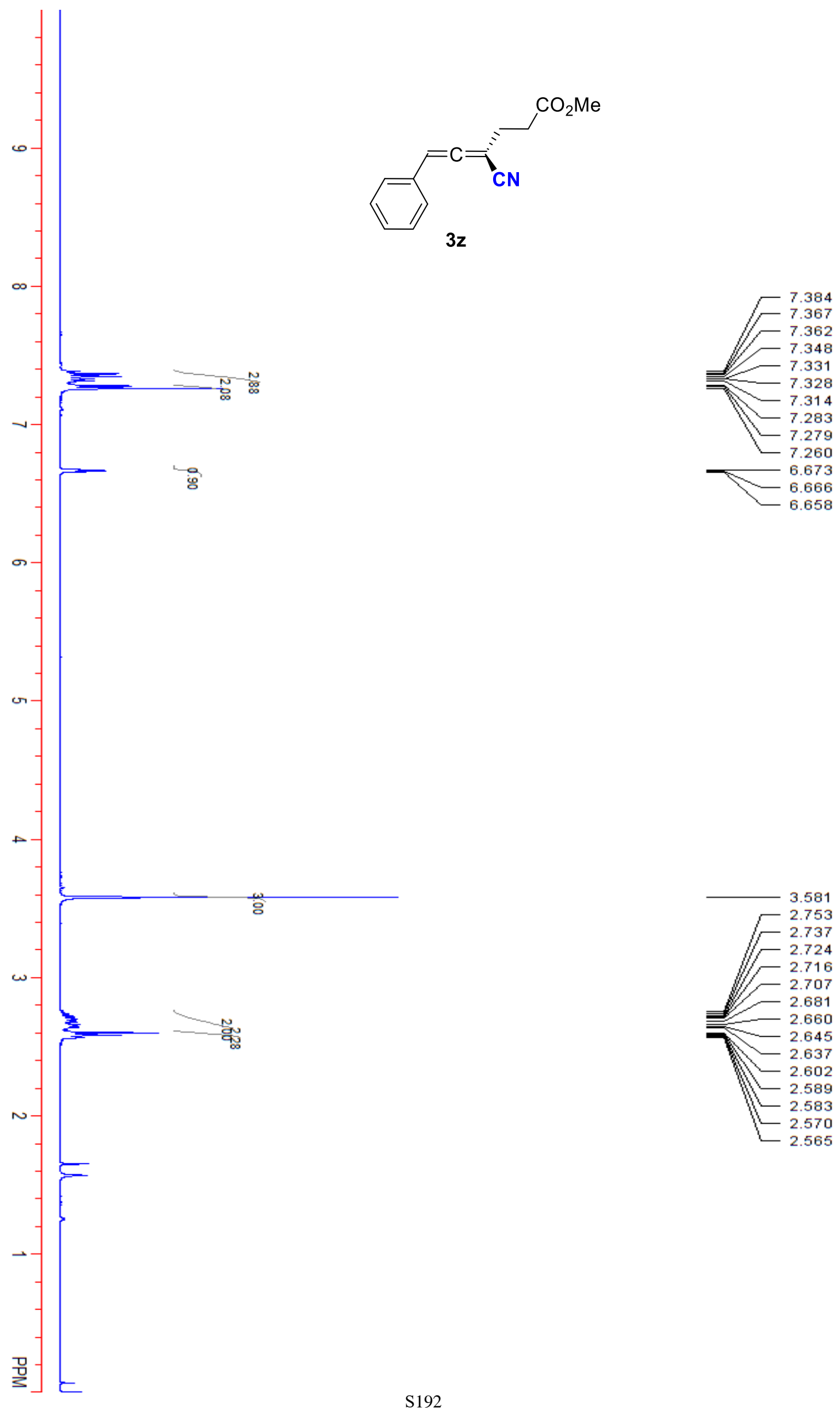


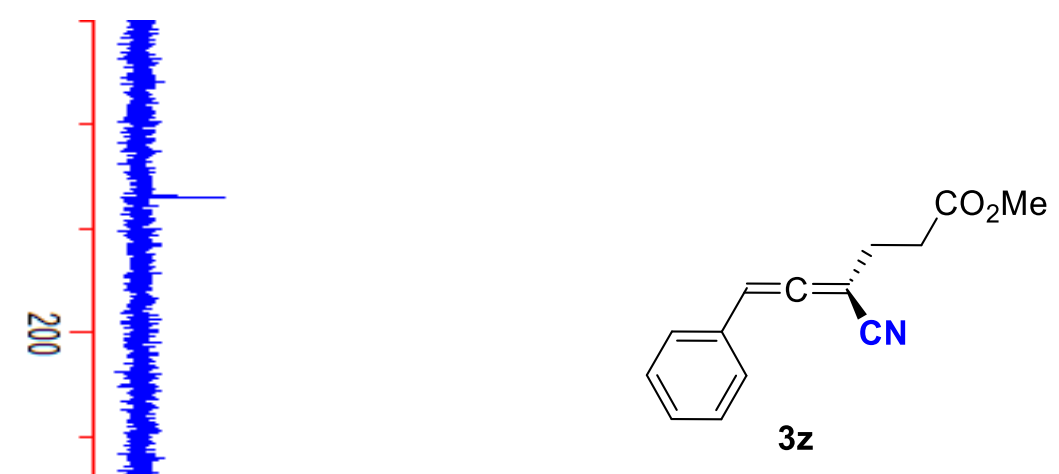

171.823

ज্ㅇㅇ

흥

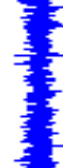

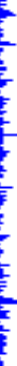

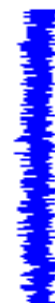

G

51.859

몬

31.482

26.568 

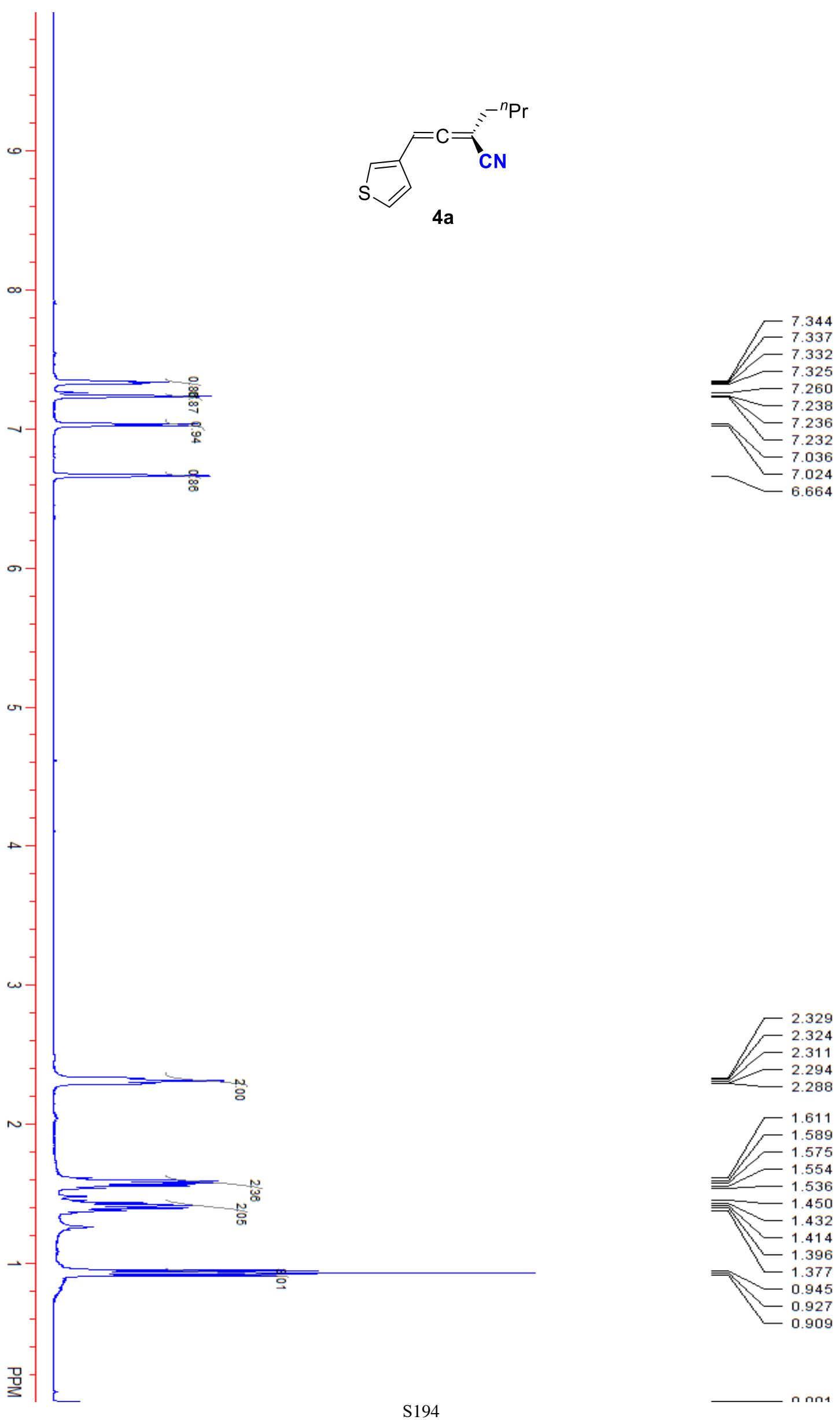


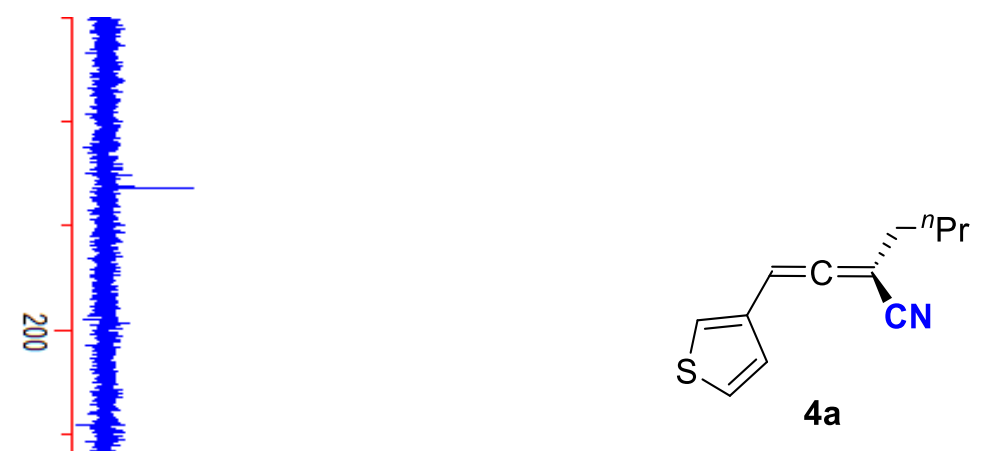

213.678

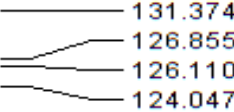

$-114.931$

$\overrightarrow{8}$

항

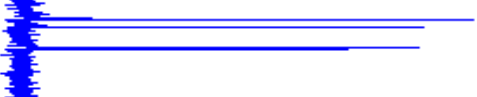

$-93.993$
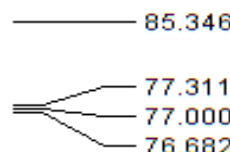

ㄷㅇㅇ

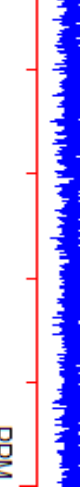

77.000

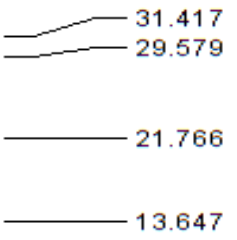



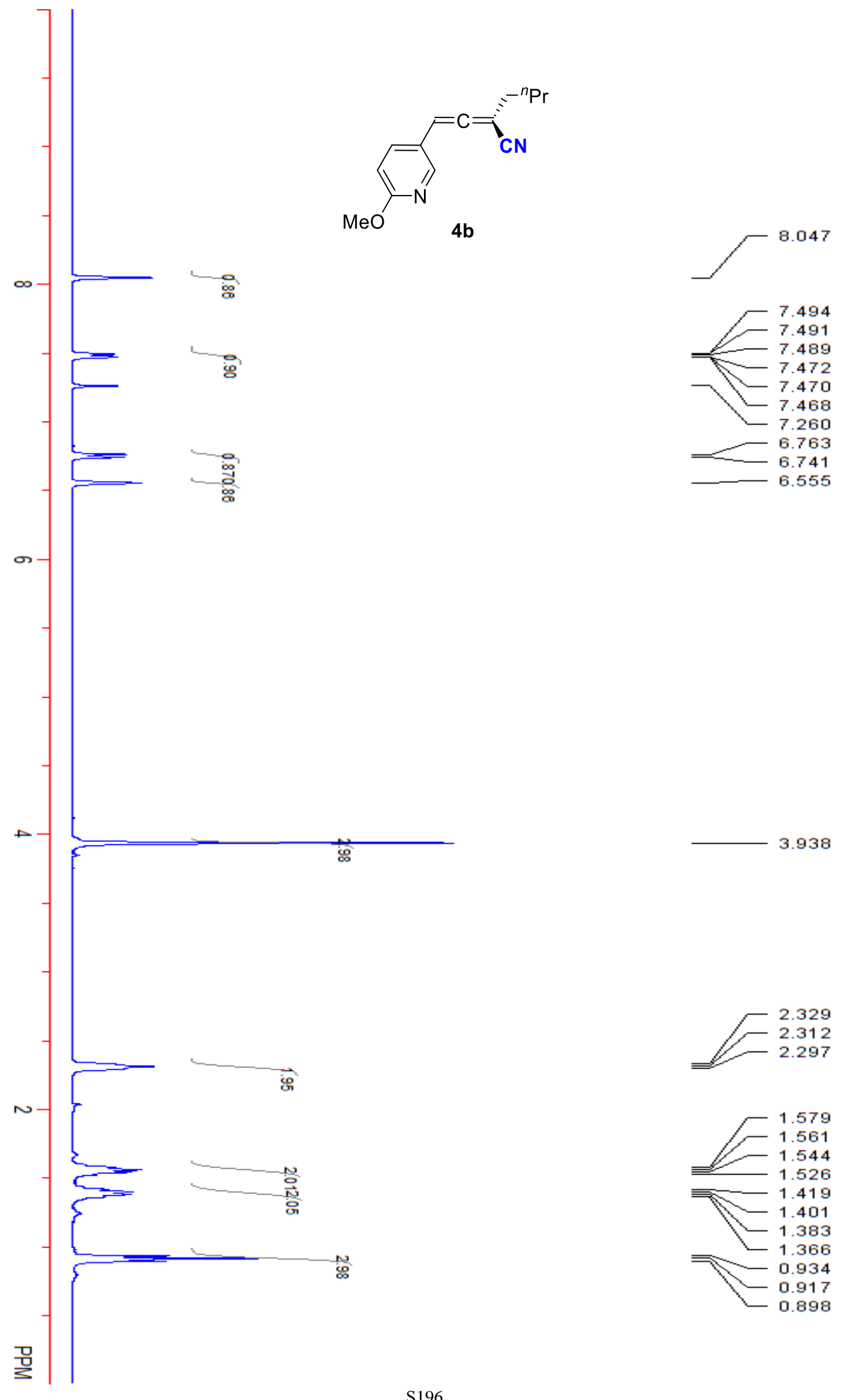


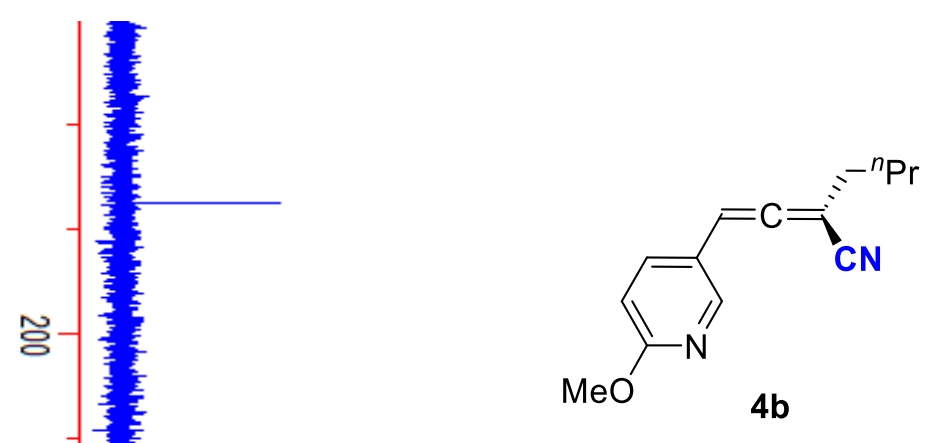

212.617

164.296

항

137.003

흥

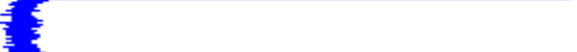

119.829

114.771

111.633

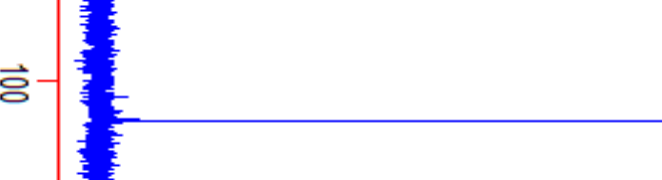

96.288

86.704

77.321

77.000

76.679

ज्ञ

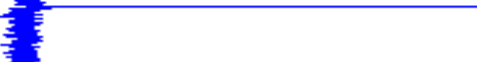

53.663

믈

g

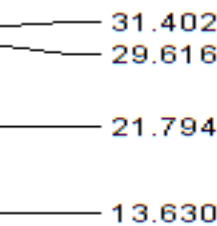



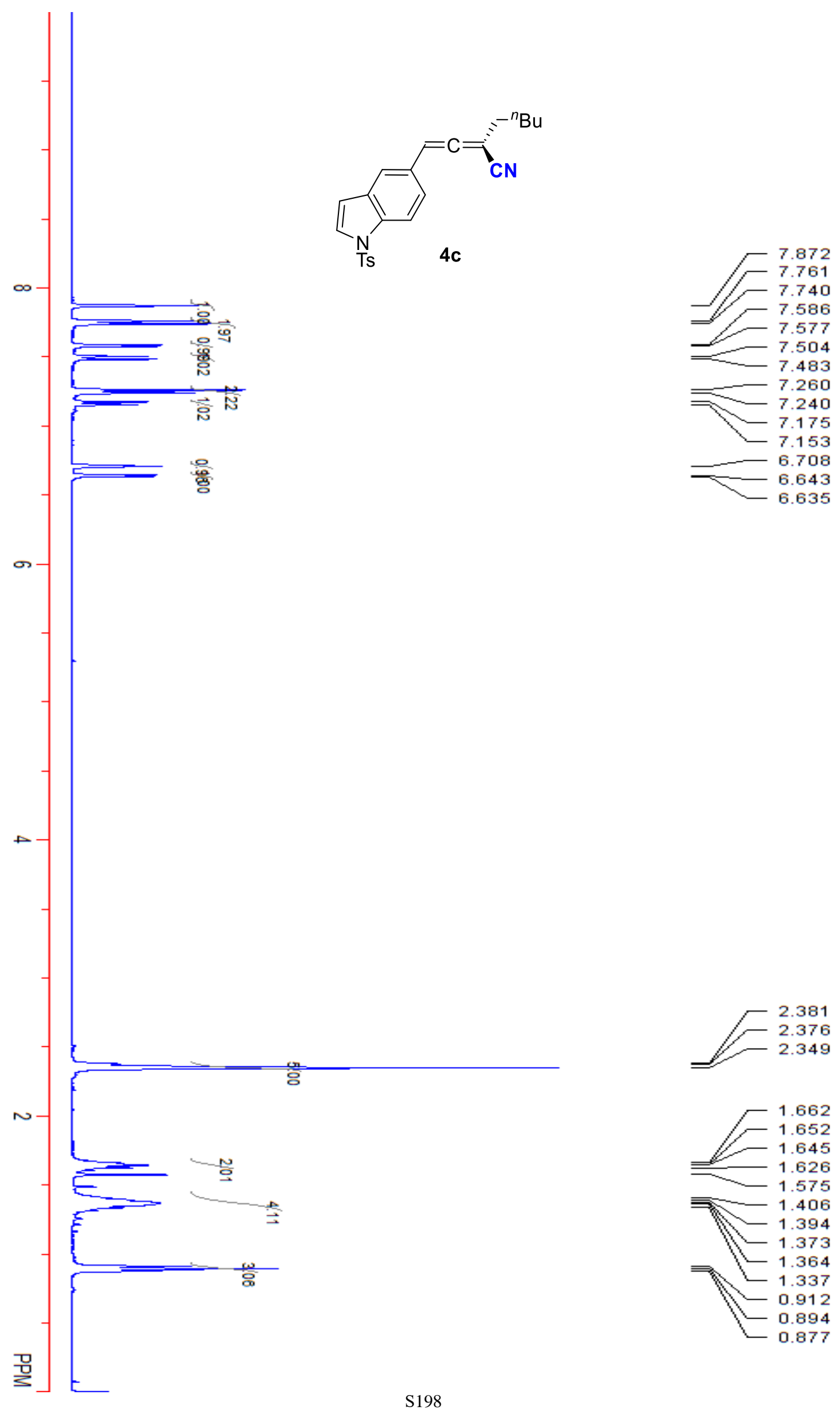

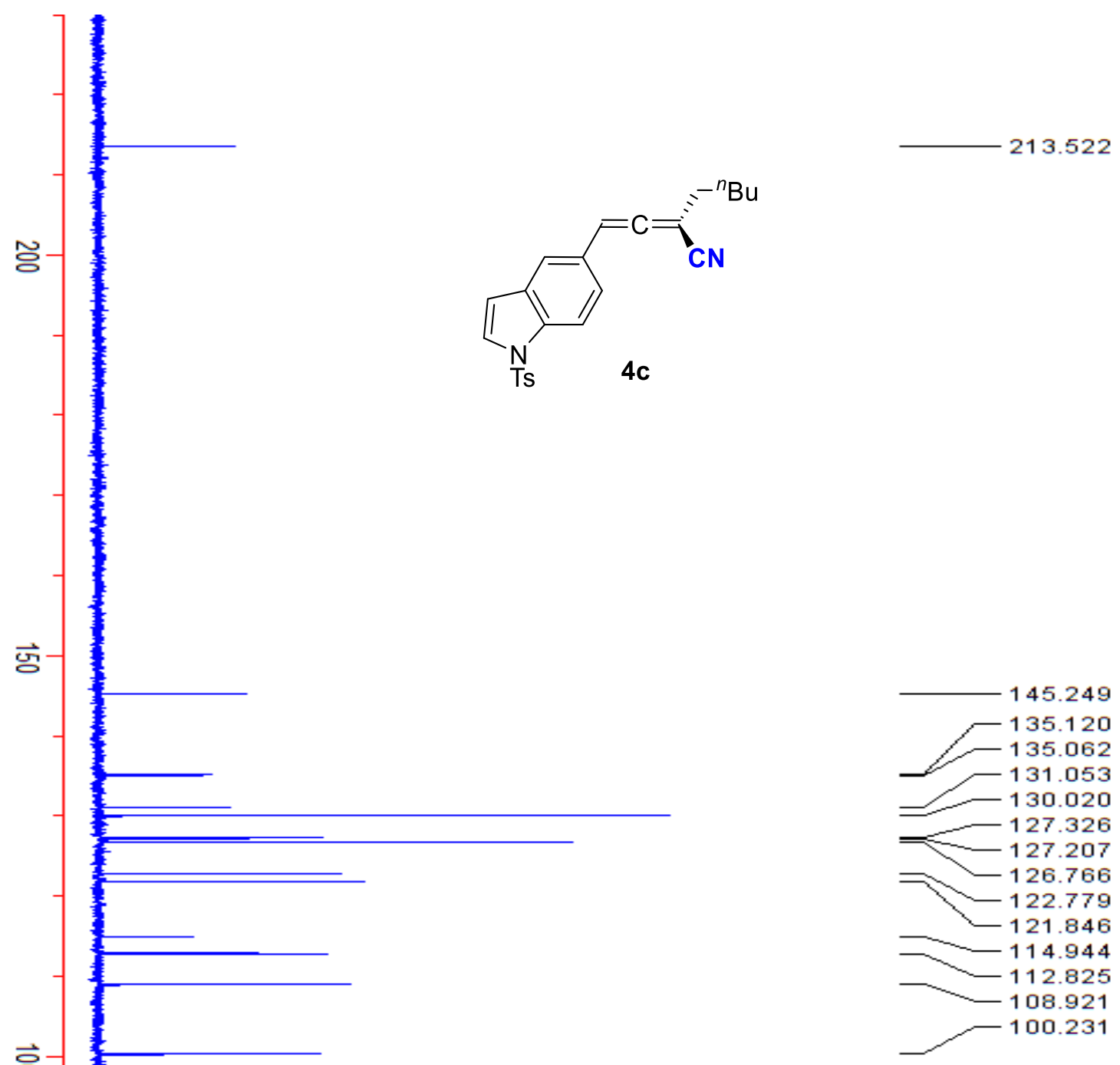

$\overrightarrow{8}$

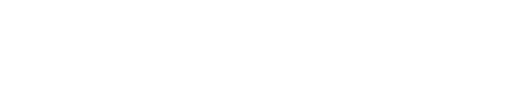

$-86.491$

77.320 77.000 76.685

댕

$-1$

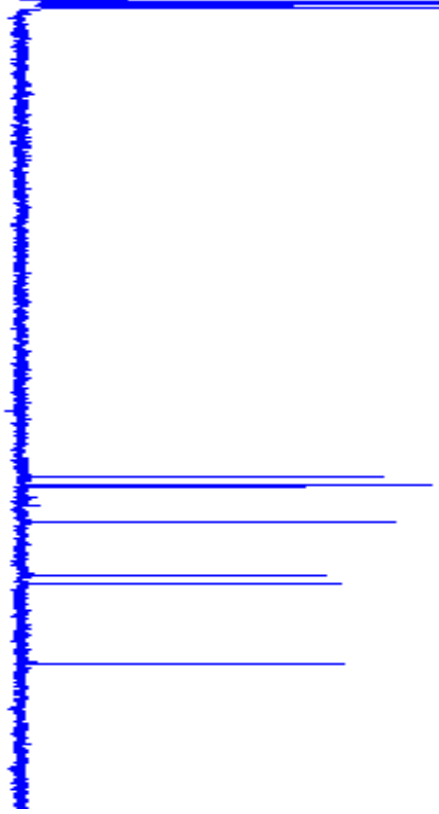

31.705

30.871

27.370

22.240

21.566

13.891 

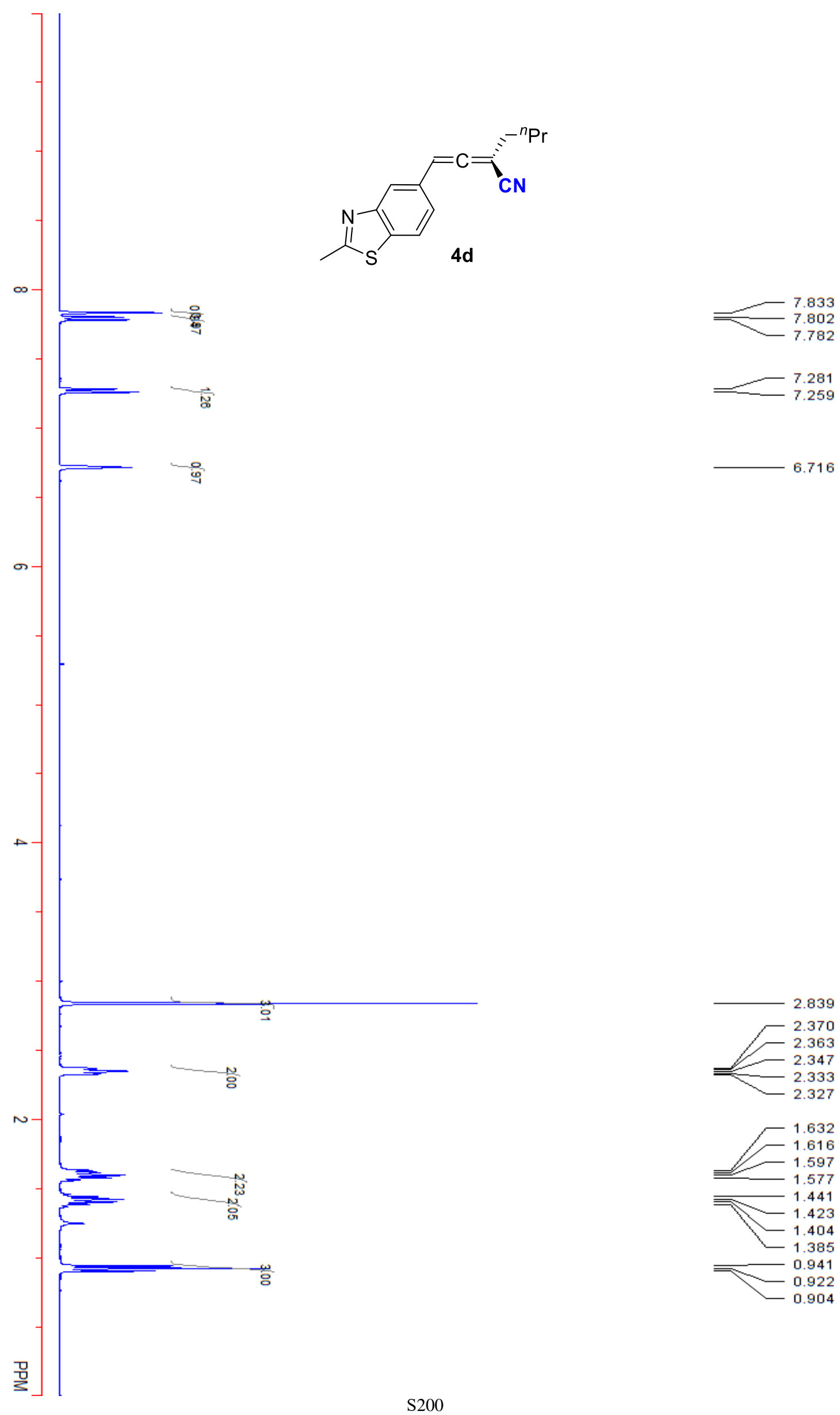

6.716

1.632 


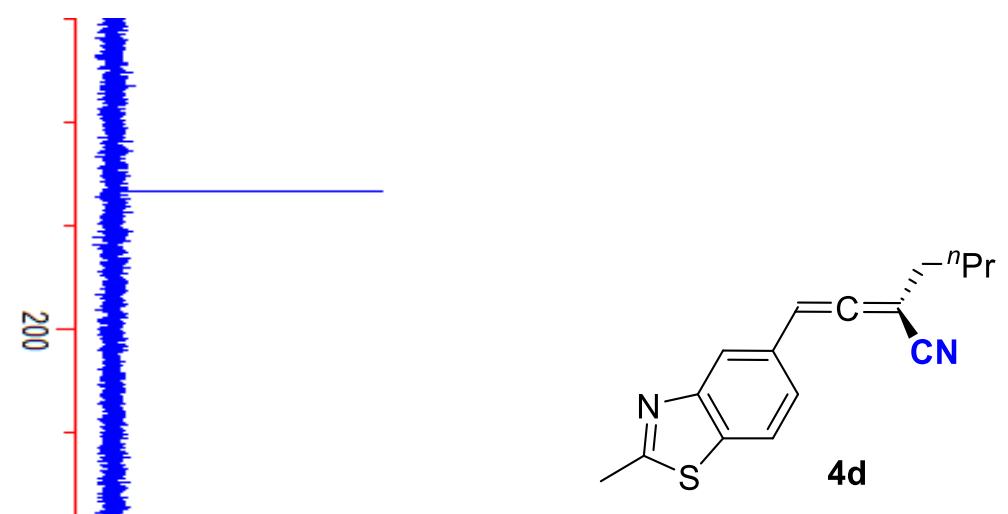



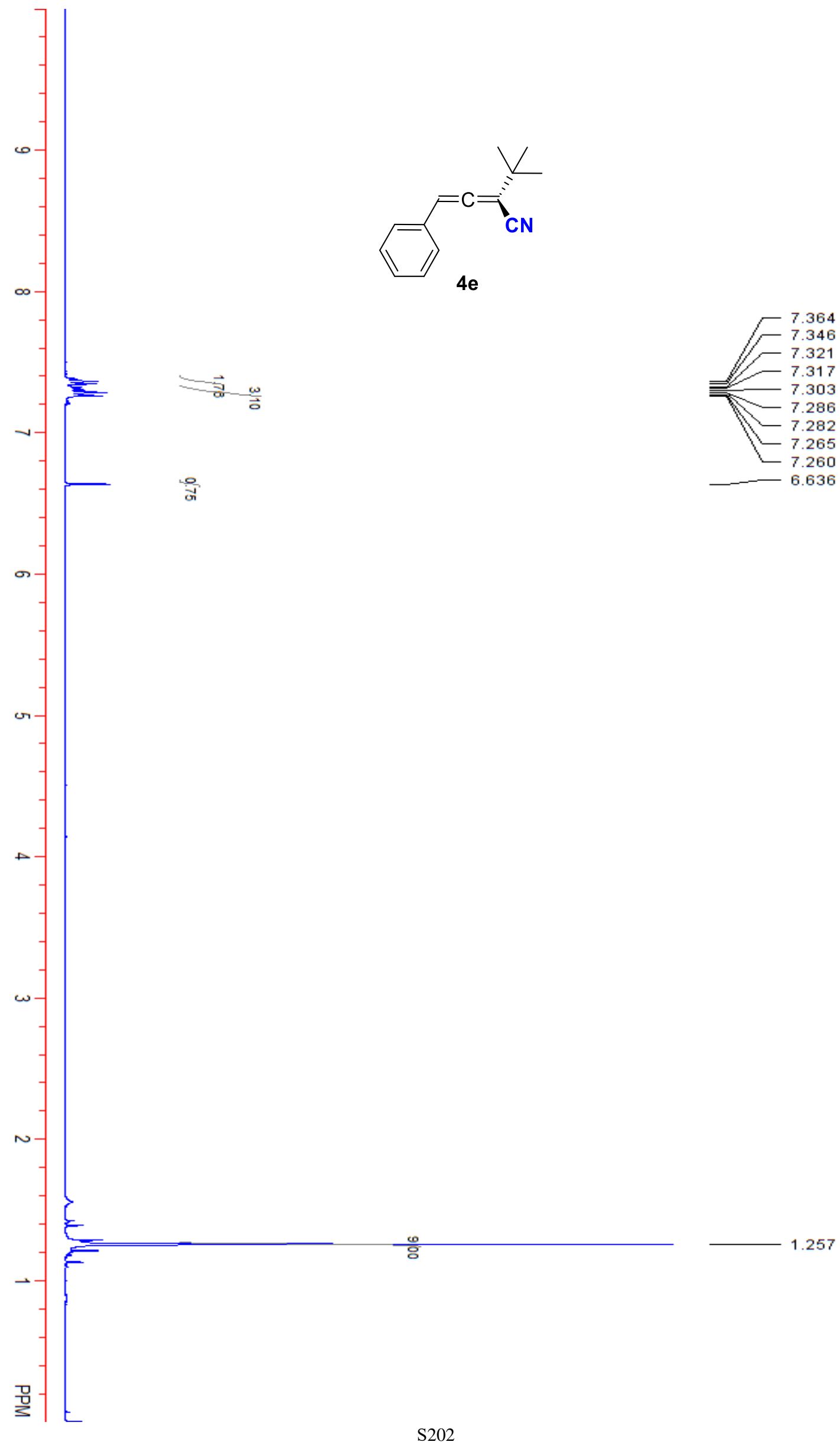


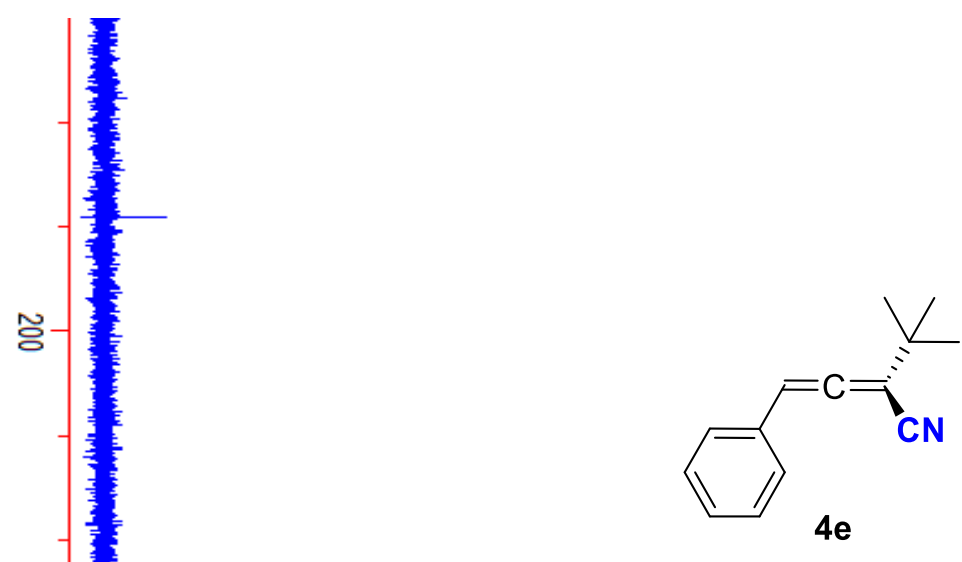

210.974
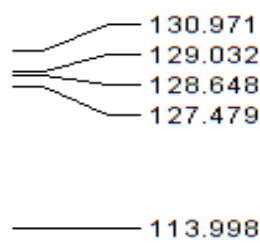

흥

항-

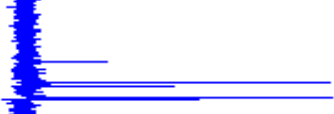

29.032

27.479

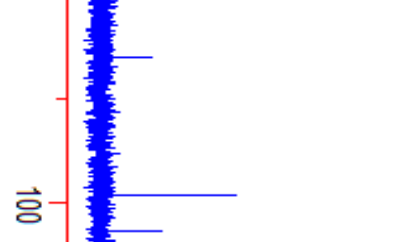

77.316

(1)

76.680

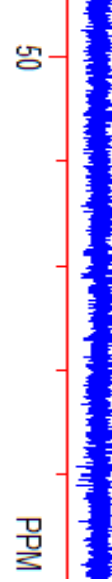

100.793

97.341

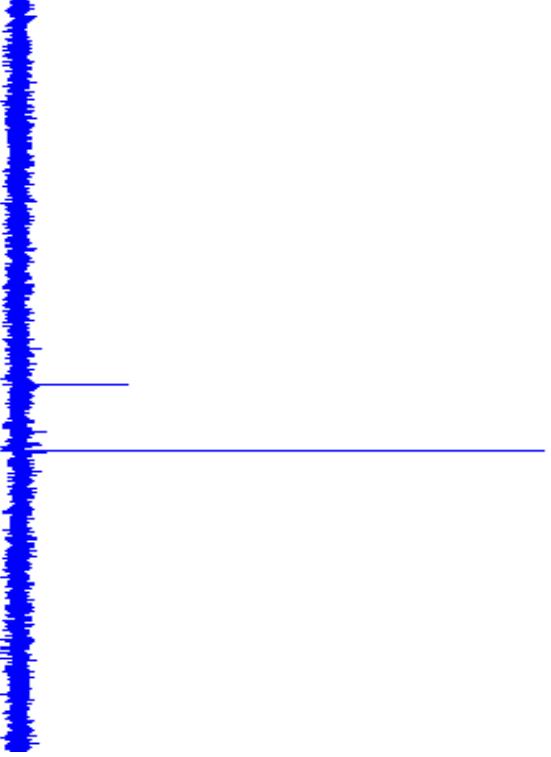

35.205

28.935 

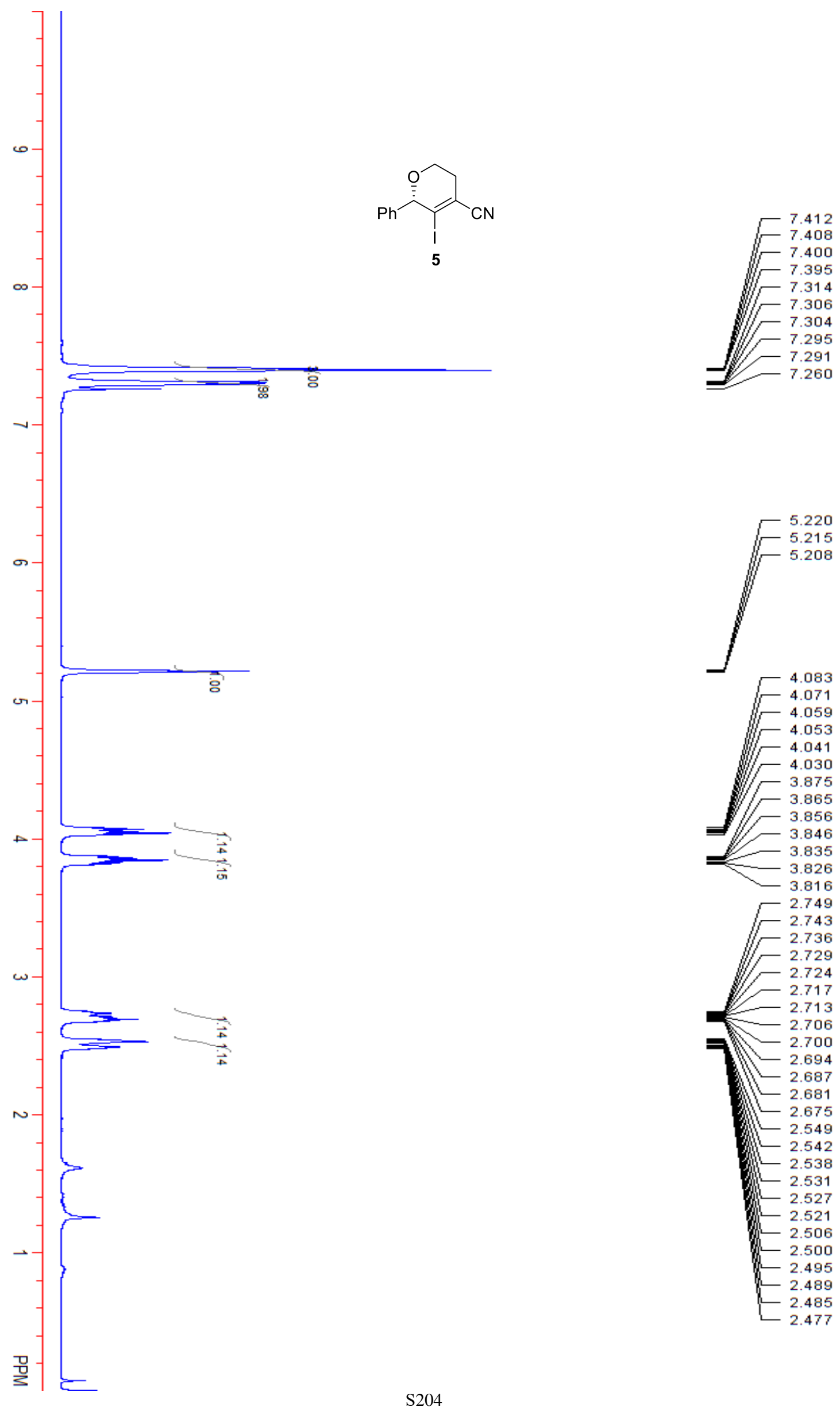

$-2.749$

$\left[\begin{array}{r}2.749 \\ -2.736\end{array}\right.$

$\sqrt{-2.729}$

2.729
-2.724 2.717

2.713

2.706

$=-2.700$

- 2694

2.687 2.681

$-2675$

$-2.549$

$-2.542$

- 2.538

- 2.531

2.527
-2521

2.521

2.506

M- 2.500

W 2495

$-2.489$

2.485 


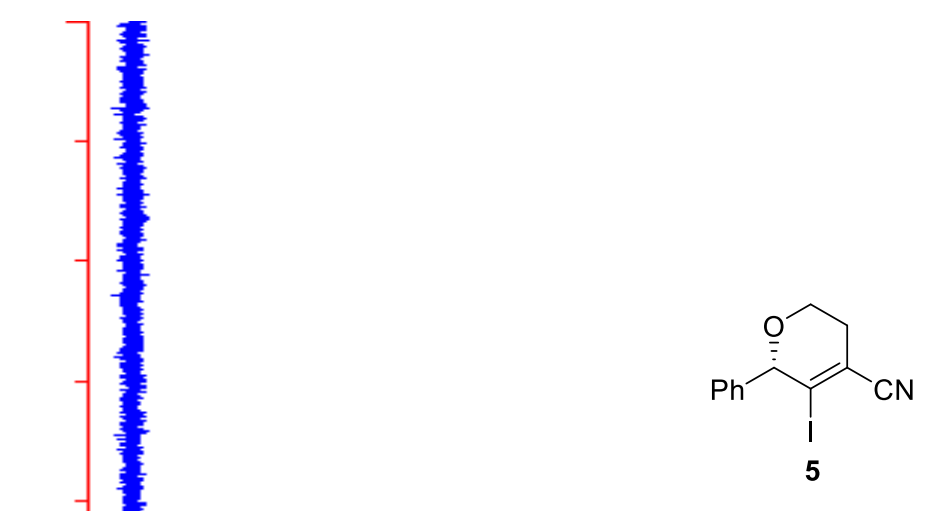

횽

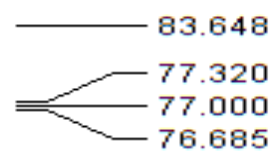

ss

章

118.939

117.887

61.010

30.853 

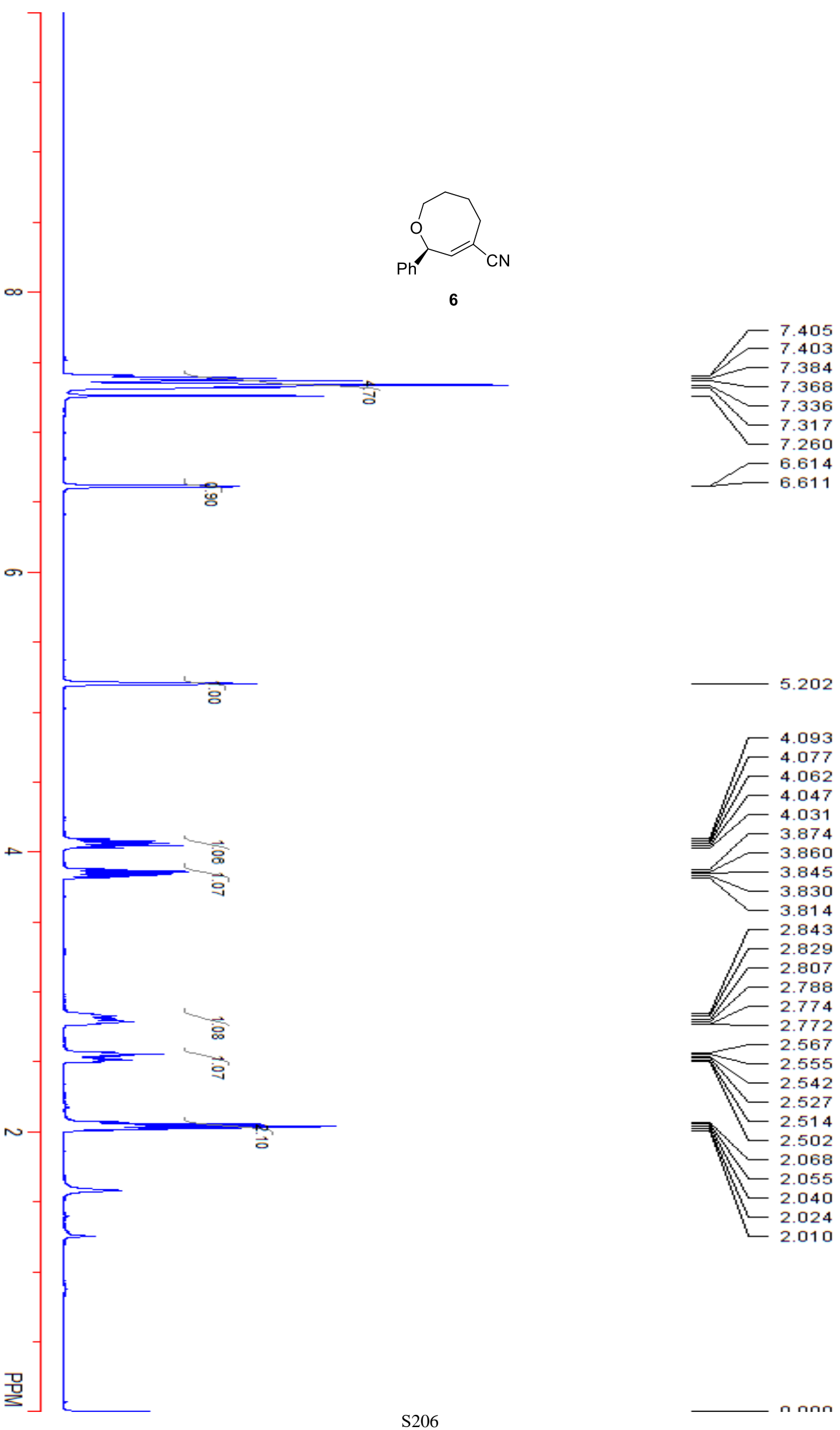

$\sqrt{-2.788}$

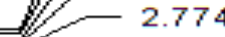

2.772

2.567

2.555

$k-2.542$

W 2.527

$\Longrightarrow 2.514$

諘 2.502

$\mathrm{x}-2.068$

$1-2.055$

$\checkmark 2.040$

2.024

$-2.010$ 

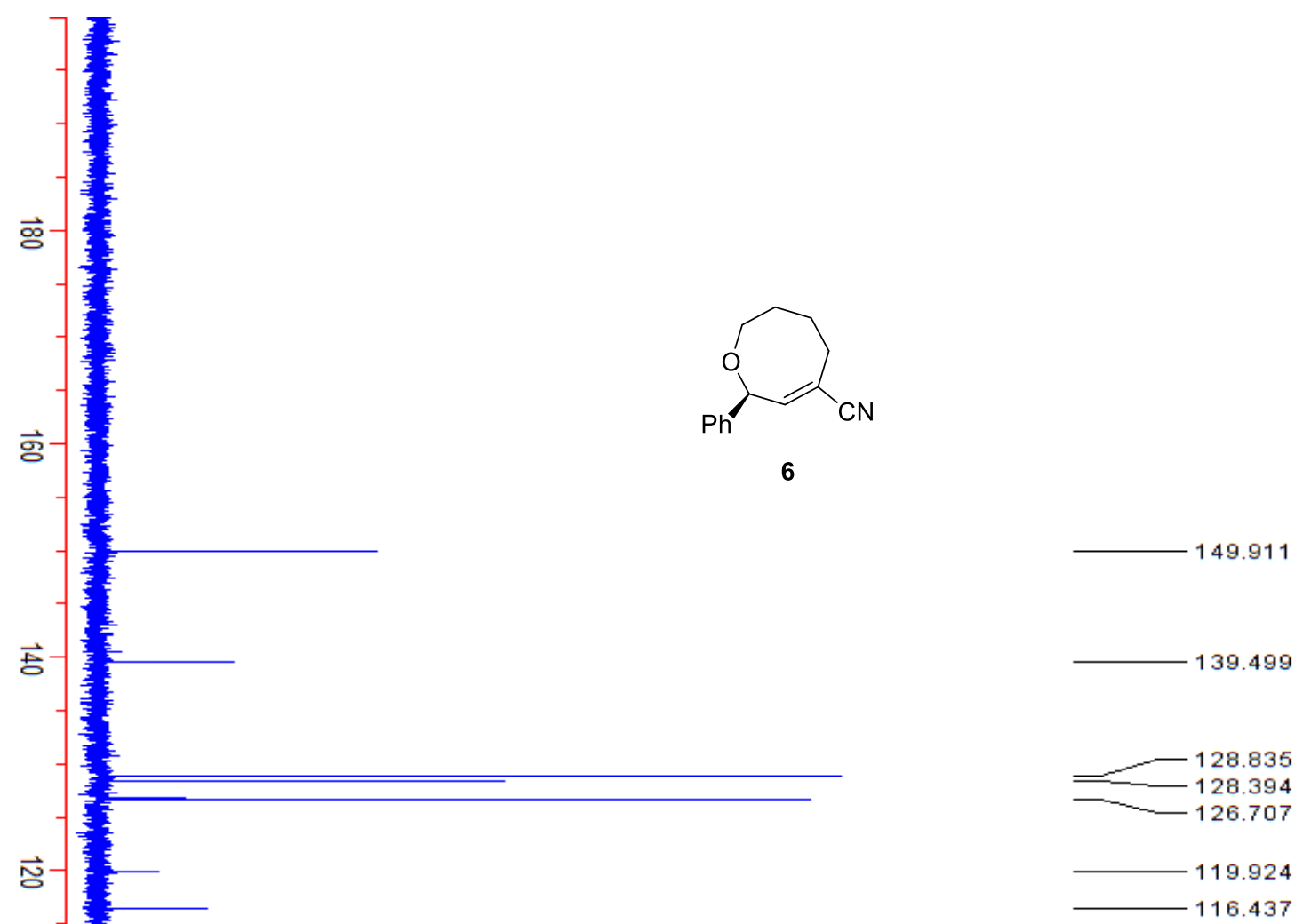

용

$\overrightarrow{8}$

$\vec{\circ}$

居

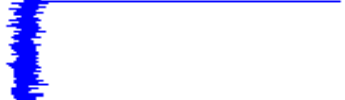

116.437

용

g

o-
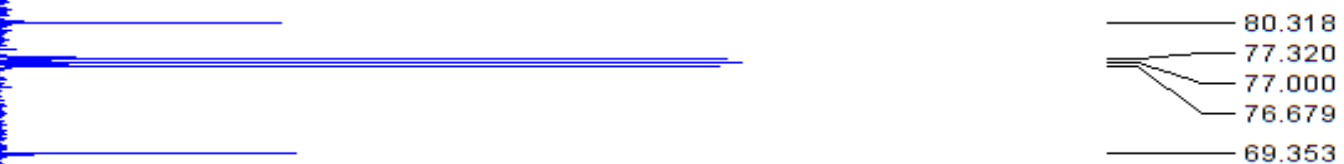

$\pm-$

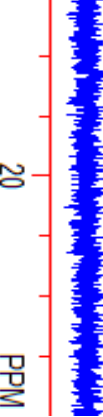

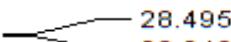

28.34 


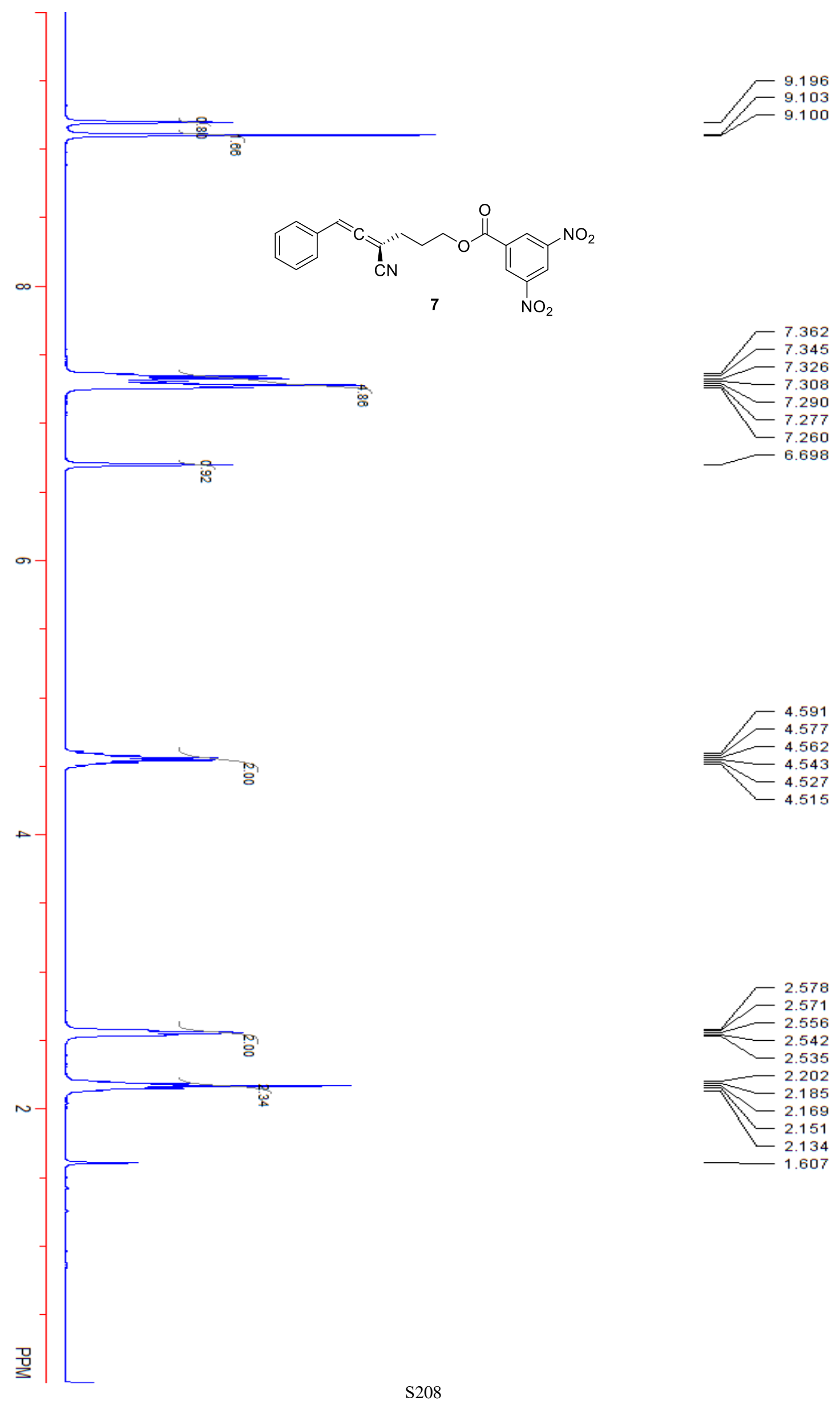



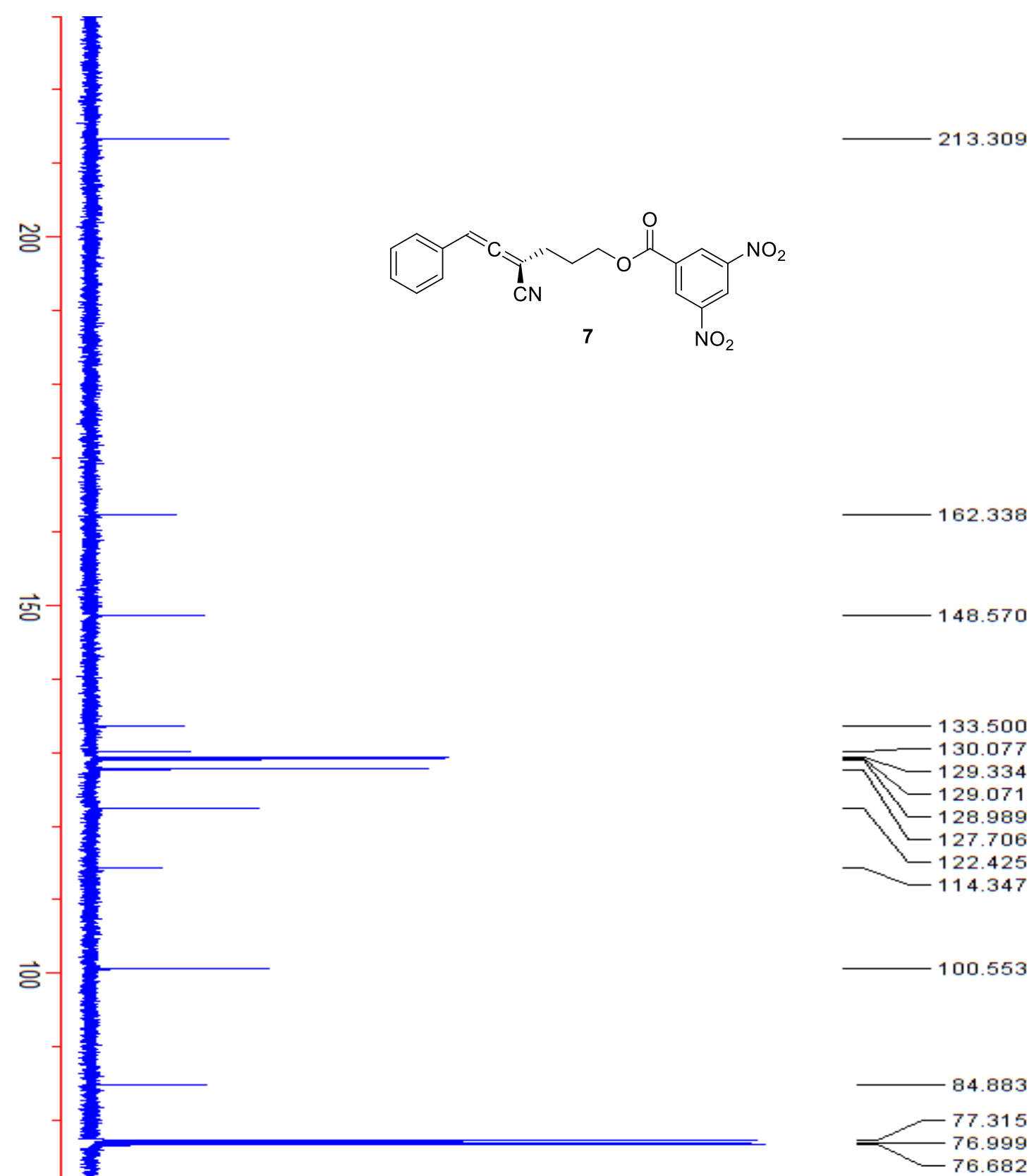

65.134

s.

믈 

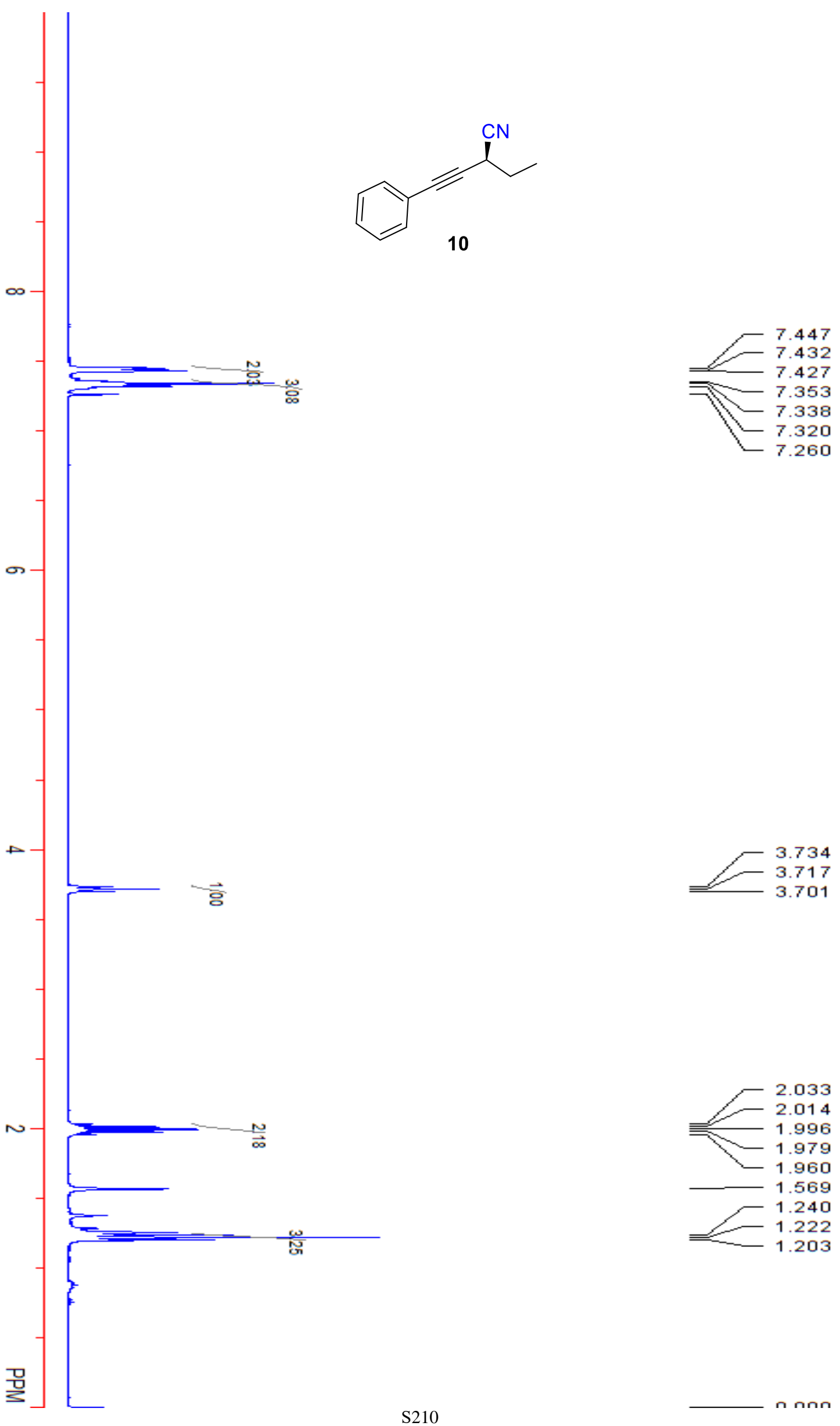


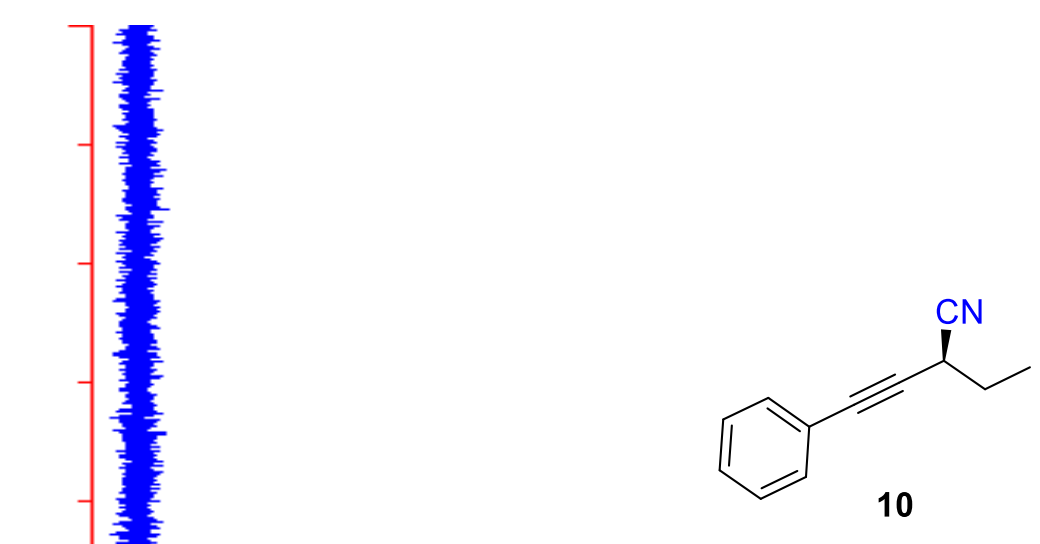




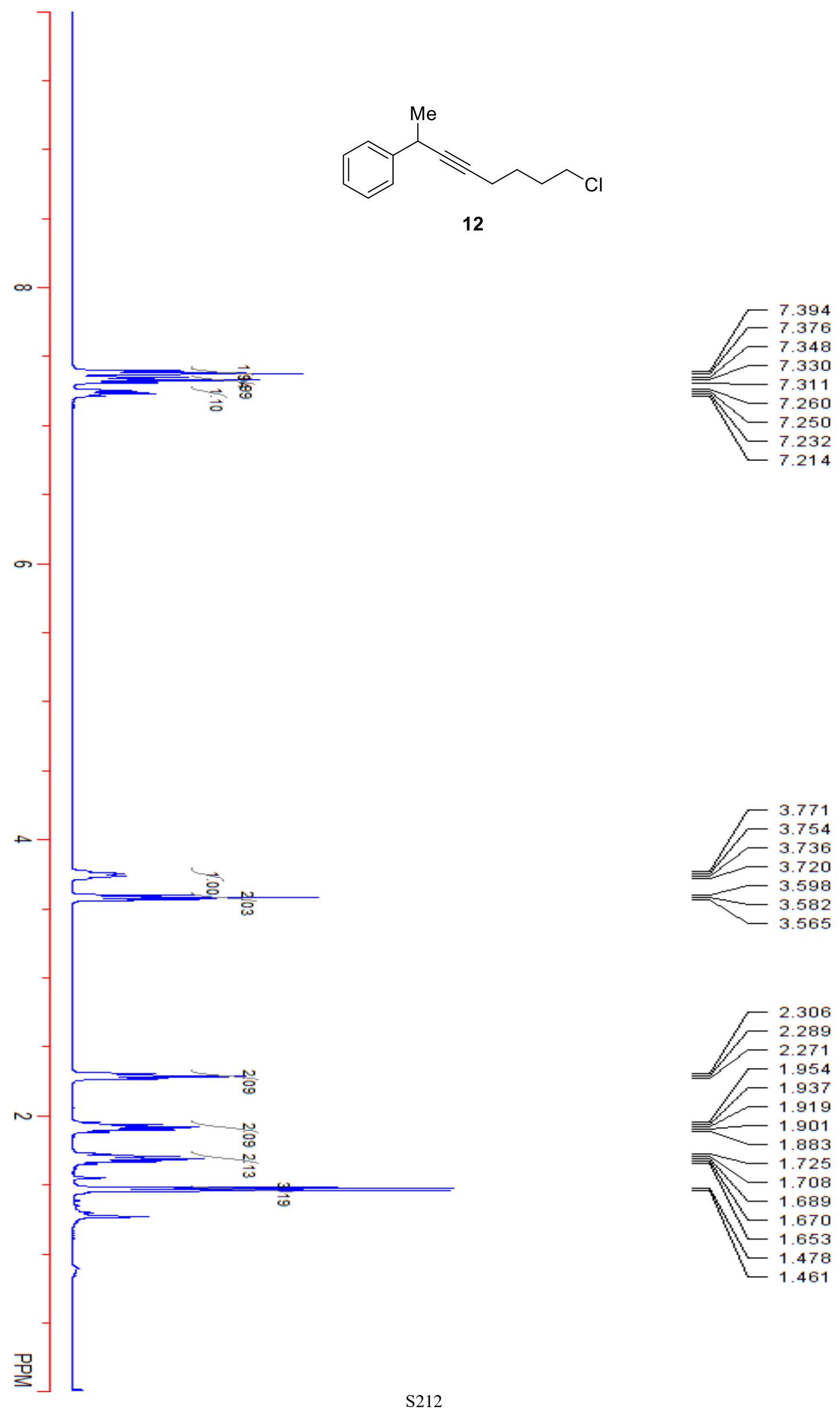




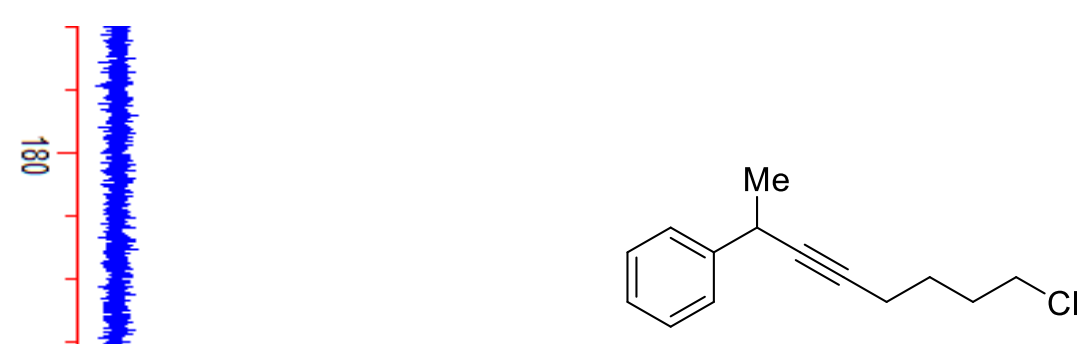

$\overrightarrow{8}$

12

143.870

吾

응

$\overrightarrow{8}$

s

81.320

$-77.318$

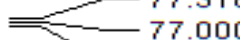

$-76.684$

8

44.623

용

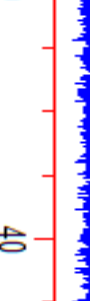
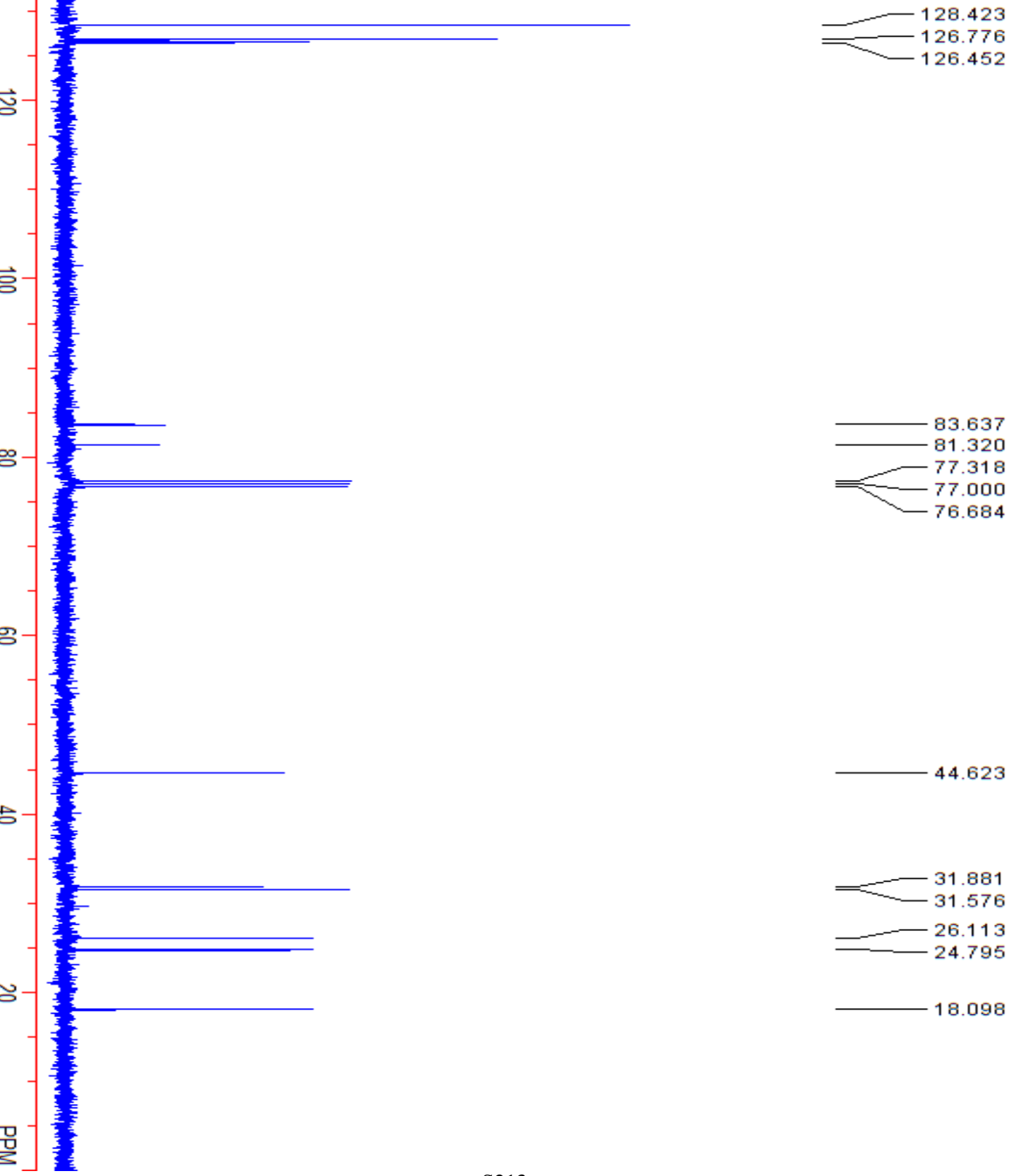

26.113

24.795

18.098 

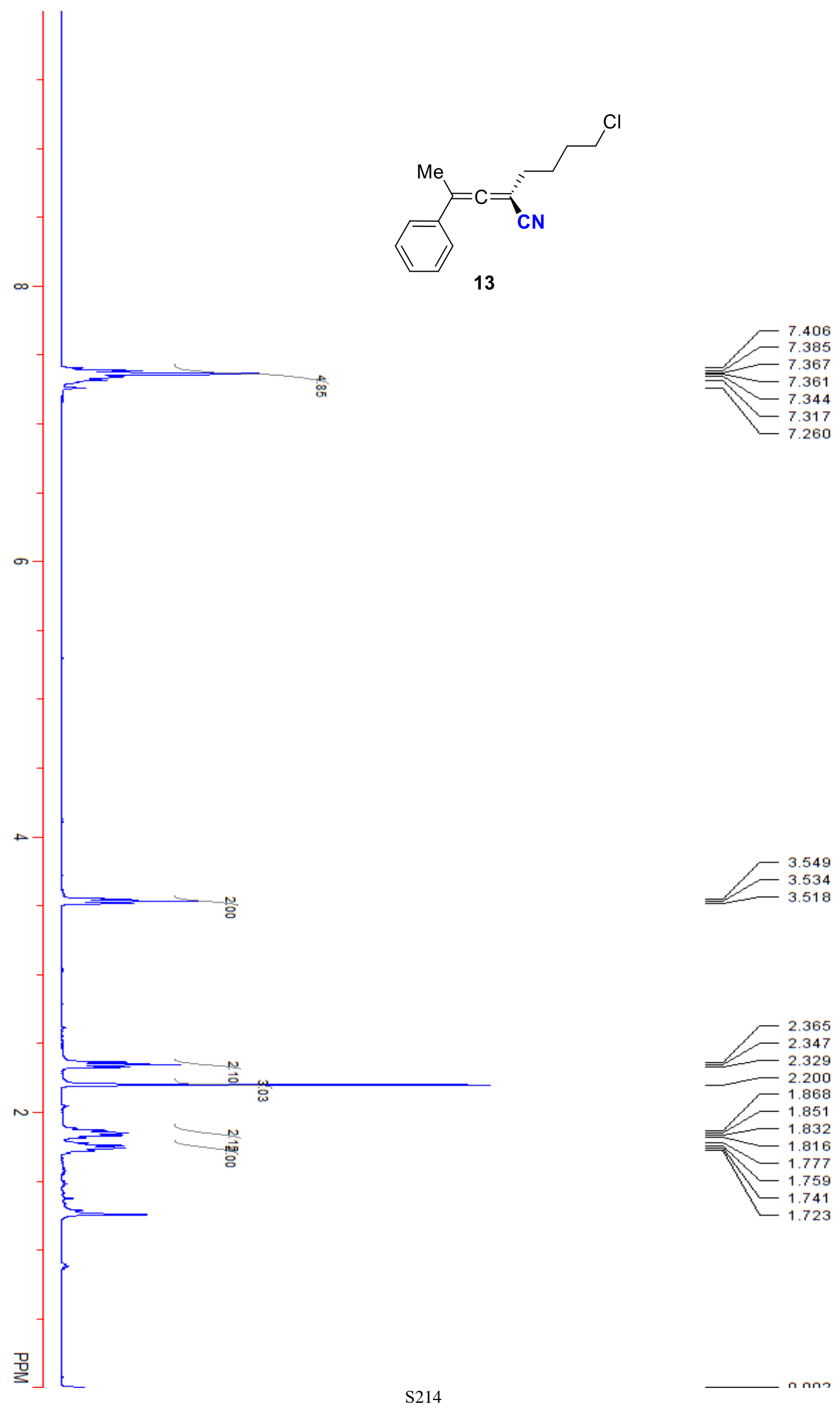

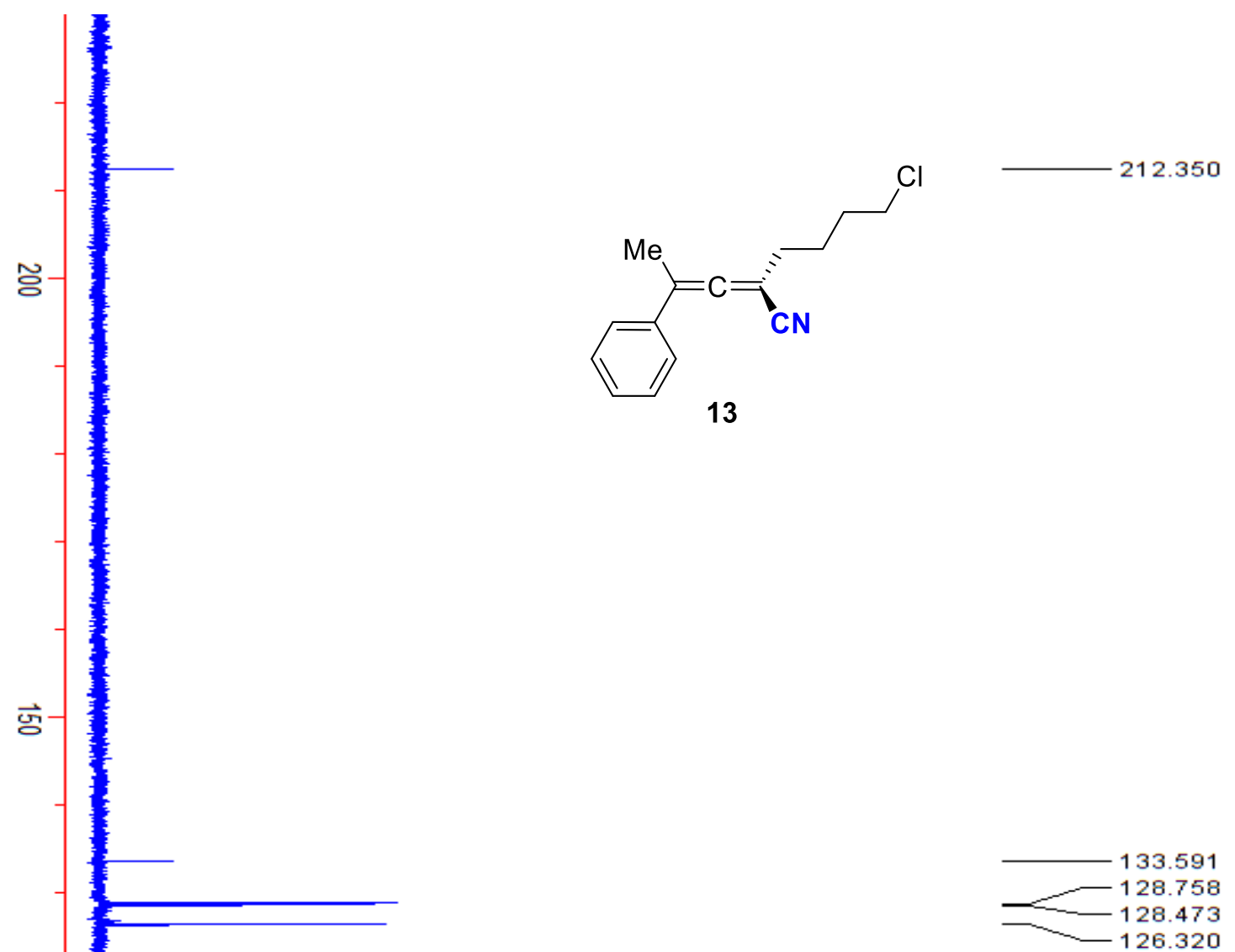

115.314

107.314

$\overrightarrow{8}$

s'

ㅋㅇㅇ

몬

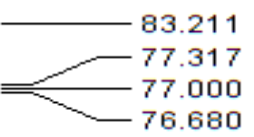

44.245

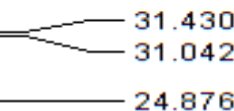

16.560 


\section{HPLC Analysis of Products}

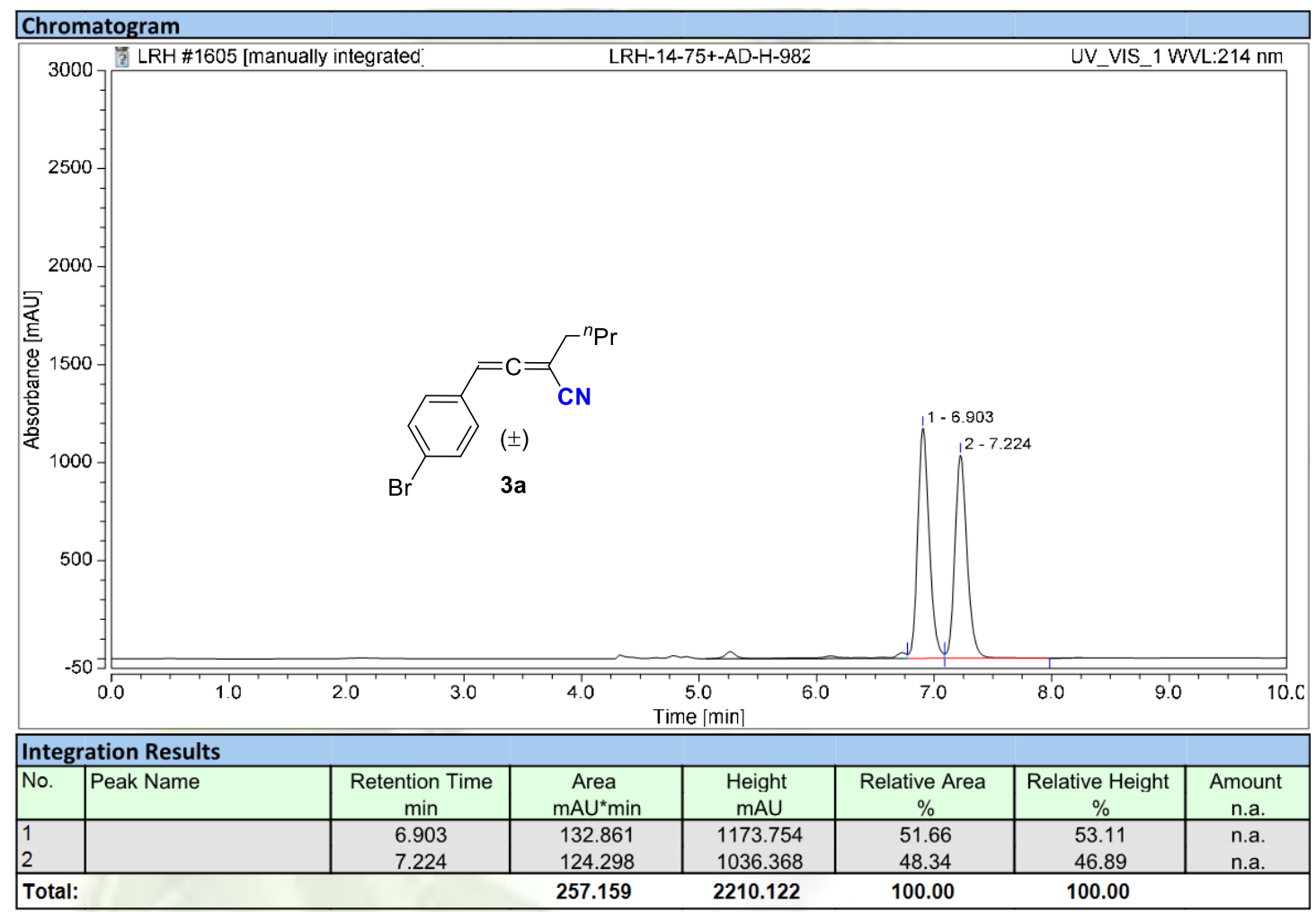

\section{Chromatogram}

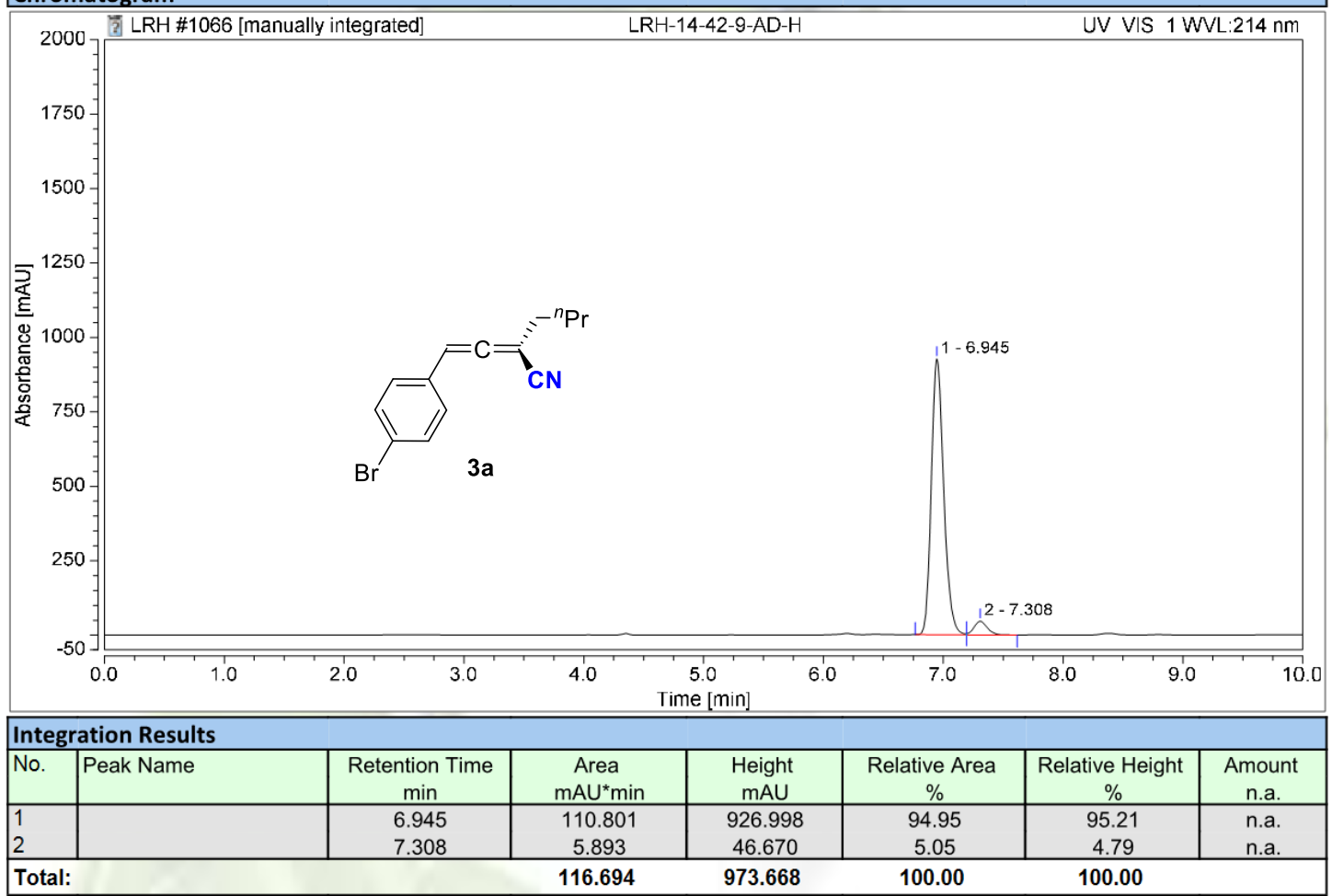



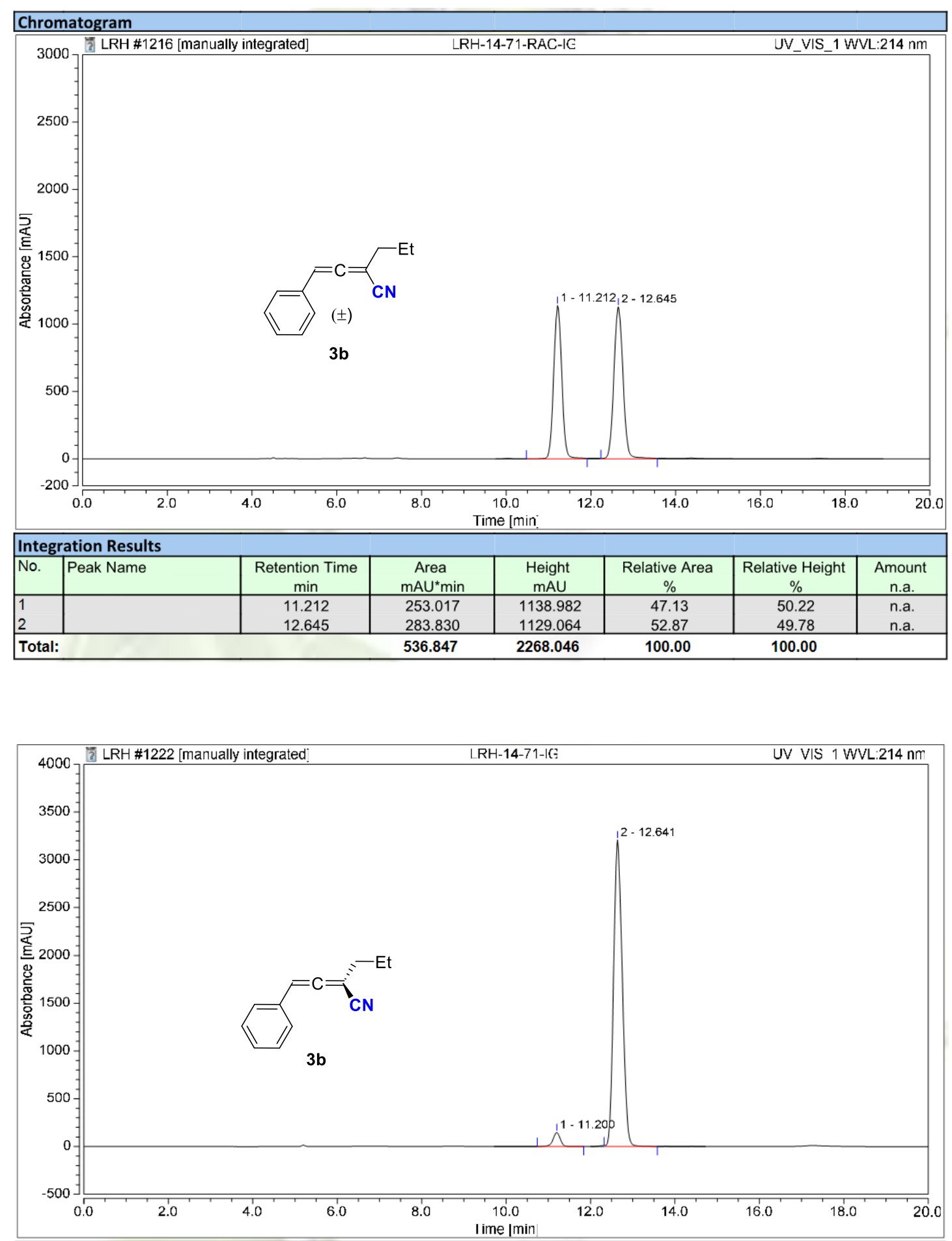

\begin{tabular}{|c|c|c|c|c|c|c|c|}
\hline \multicolumn{8}{|c|}{ Integration Results } \\
\hline No. & Peak Name & $\begin{array}{c}\text { Retention Time } \\
\text { min }\end{array}$ & $\begin{array}{c}\text { Area } \\
\mathrm{mAU}^{*} \min \end{array}$ & $\begin{array}{c}\text { Height } \\
\text { mAU }\end{array}$ & $\begin{array}{c}\text { Relative Area } \\
\%\end{array}$ & $\begin{array}{c}\text { Relative Height } \\
\%\end{array}$ & $\begin{array}{c}\text { Amount } \\
\text { n.a. }\end{array}$ \\
\hline $\begin{array}{l}1 \\
2\end{array}$ & & $\begin{array}{l}11.200 \\
12.641\end{array}$ & $\begin{array}{c}28.949 \\
759.266\end{array}$ & $\begin{array}{c}146.929 \\
3207.988 \\
\end{array}$ & $\begin{array}{c}3.67 \\
96.33 \\
\end{array}$ & $\begin{array}{c}4.38 \\
95.62 \\
\end{array}$ & $\begin{array}{l}\text { n.a. } \\
\text { n.a. }\end{array}$ \\
\hline \multicolumn{3}{|c|}{ Total: } & 788.215 & 3354.917 & 100.00 & 100.00 & \\
\hline
\end{tabular}



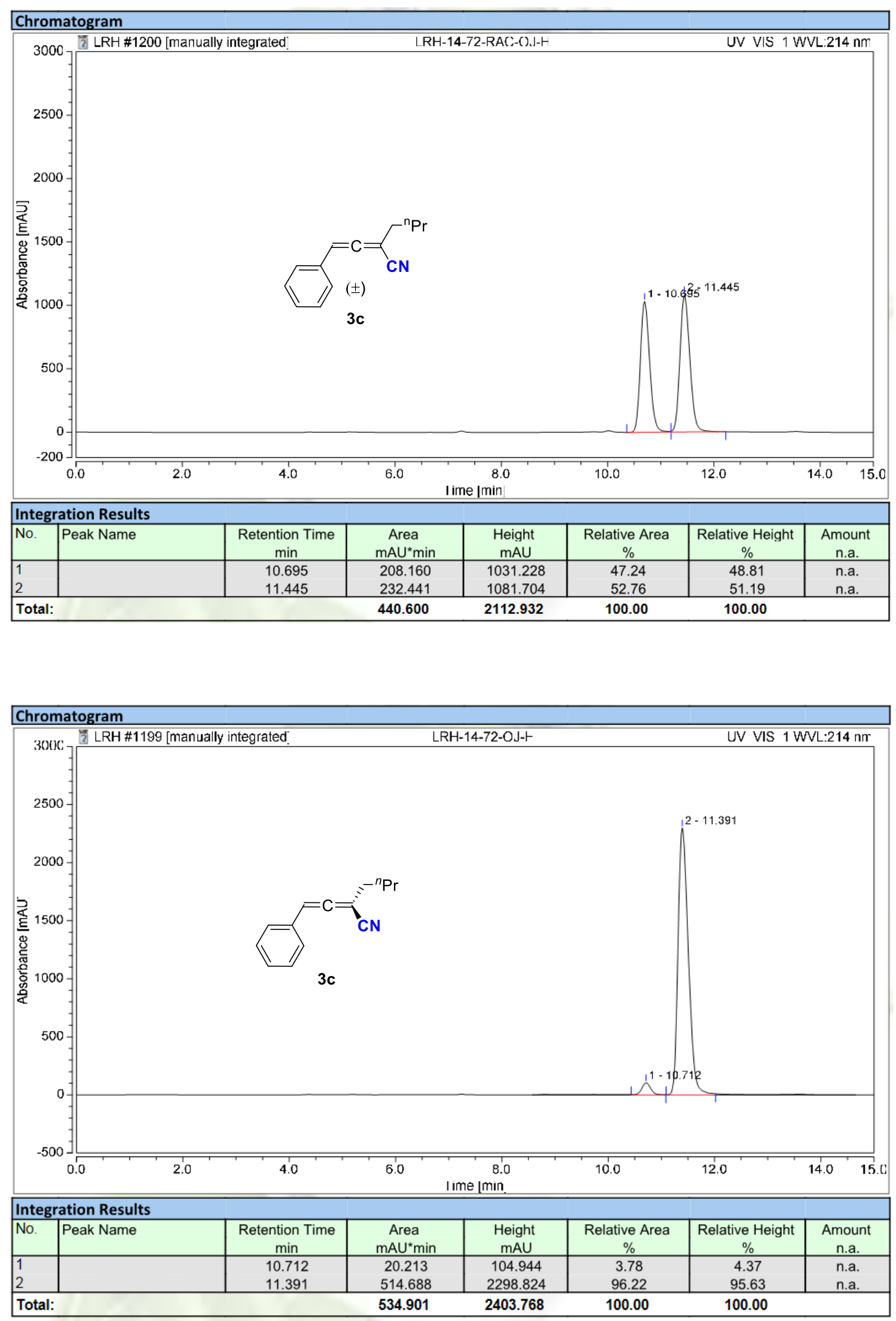


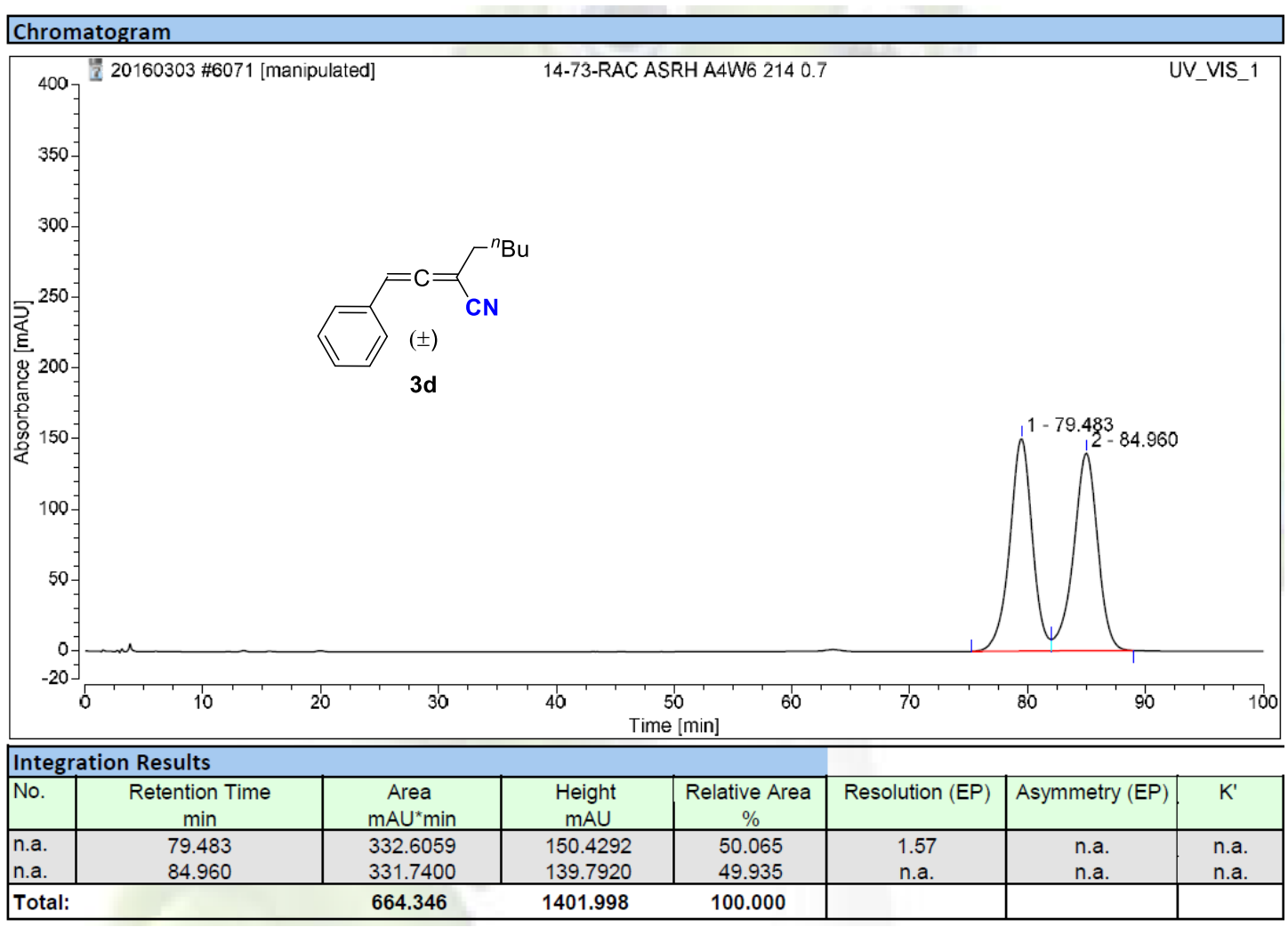

\section{Chromatogram}

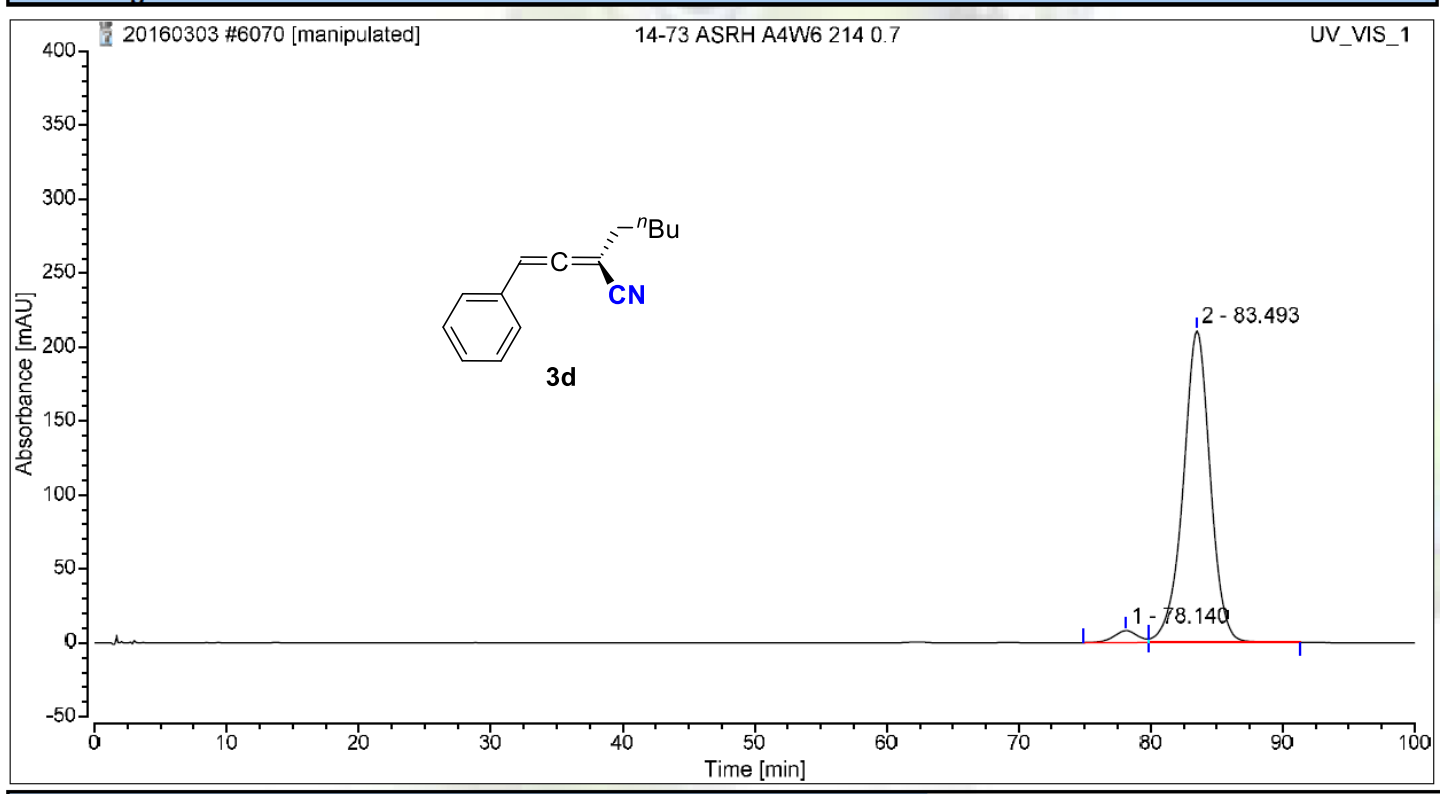

\begin{tabular}{|c|c|c|c|c|c|c|c|}
\hline \multicolumn{8}{|c|}{ Integration Results } \\
\hline No. & $\begin{array}{c}\text { Retention Time } \\
\text { min }\end{array}$ & $\begin{array}{c}\text { Area } \\
m A U^{*} \text { min }\end{array}$ & $\begin{array}{c}\text { Height } \\
\text { mAU }\end{array}$ & $\begin{array}{c}\text { Relative Area } \\
\%\end{array}$ & Resolution (EP) & Asymmetry (EP) & $K^{\prime}$ \\
\hline $\begin{array}{l}\text { n.a. } \\
\text { n.a. } \\
\end{array}$ & $\begin{array}{l}78.140 \\
83.493 \\
\end{array}$ & $\begin{array}{c}17.1849 \\
501.5144 \\
\end{array}$ & $\begin{array}{c}8.1395 \\
210.7876 \\
\end{array}$ & $\begin{array}{c}3.313 \\
96.687 \\
\end{array}$ & $\begin{array}{l}1.55 \\
\text { n.a. }\end{array}$ & $\begin{array}{l}\text { n.a. } \\
0.96 \\
\end{array}$ & $\begin{array}{l}\text { n.a. } \\
\text { n.a. }\end{array}$ \\
\hline \multicolumn{2}{|c|}{ Total: } & 518.699 & 1401.998 & 100.000 & & & \\
\hline
\end{tabular}



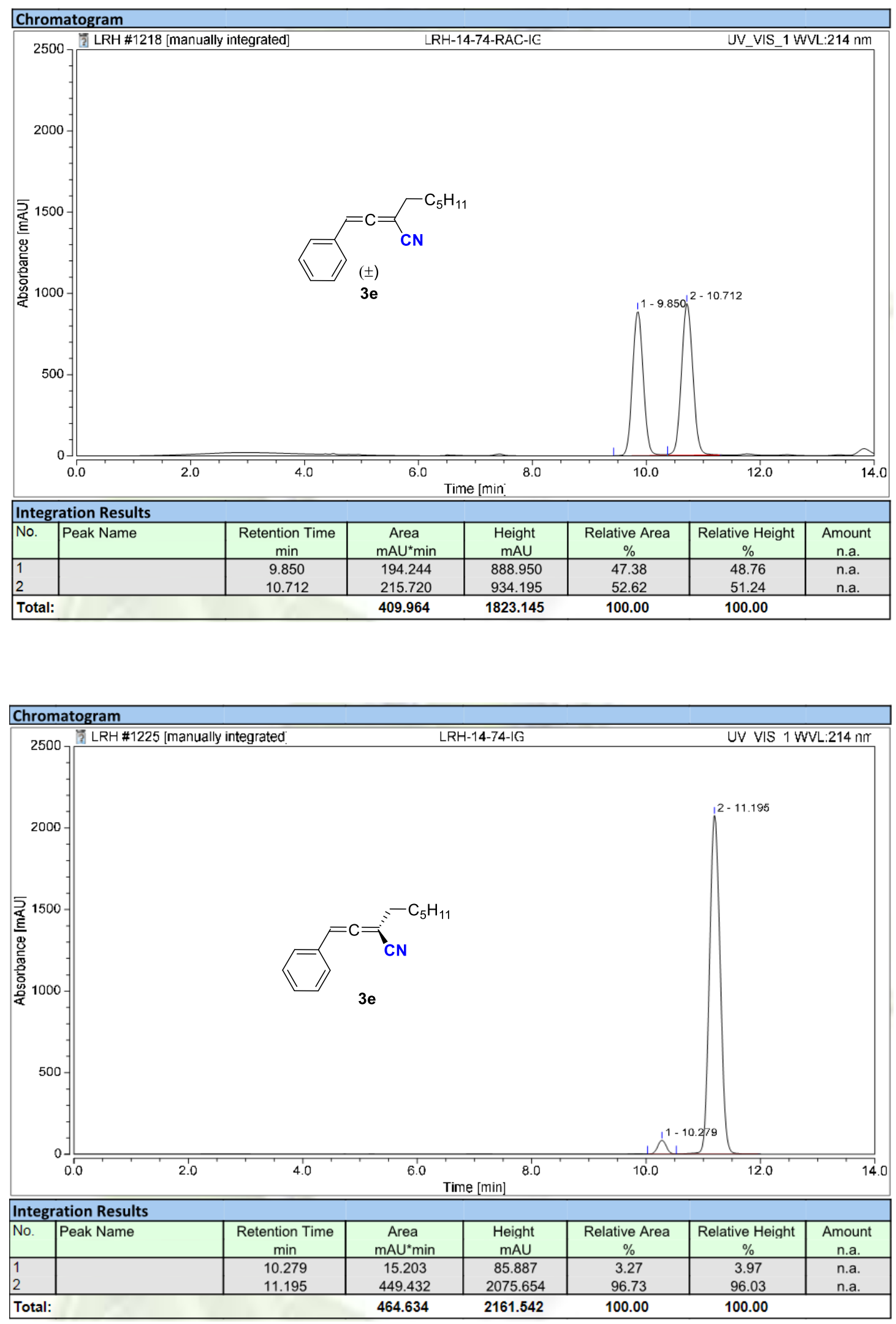

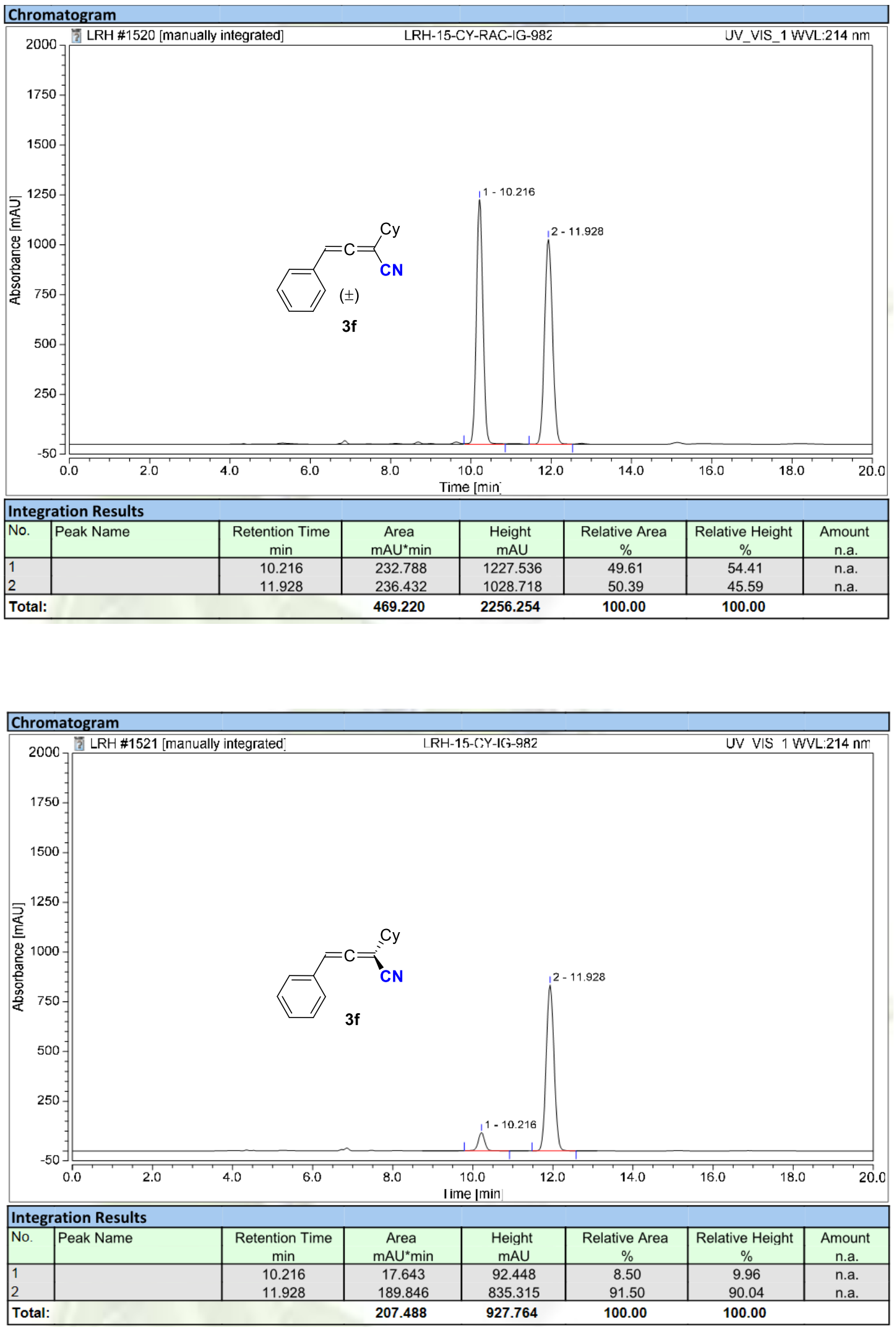

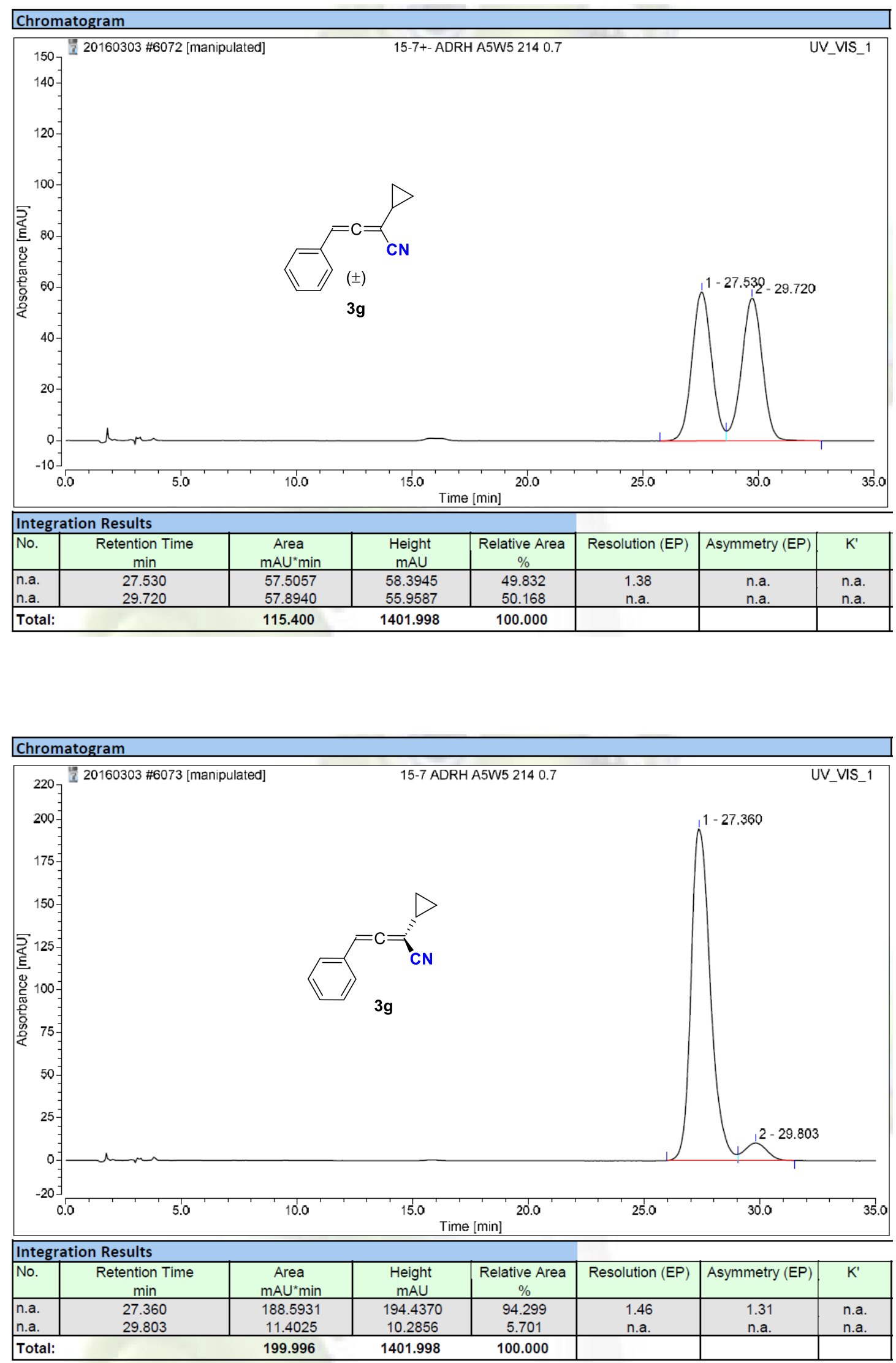

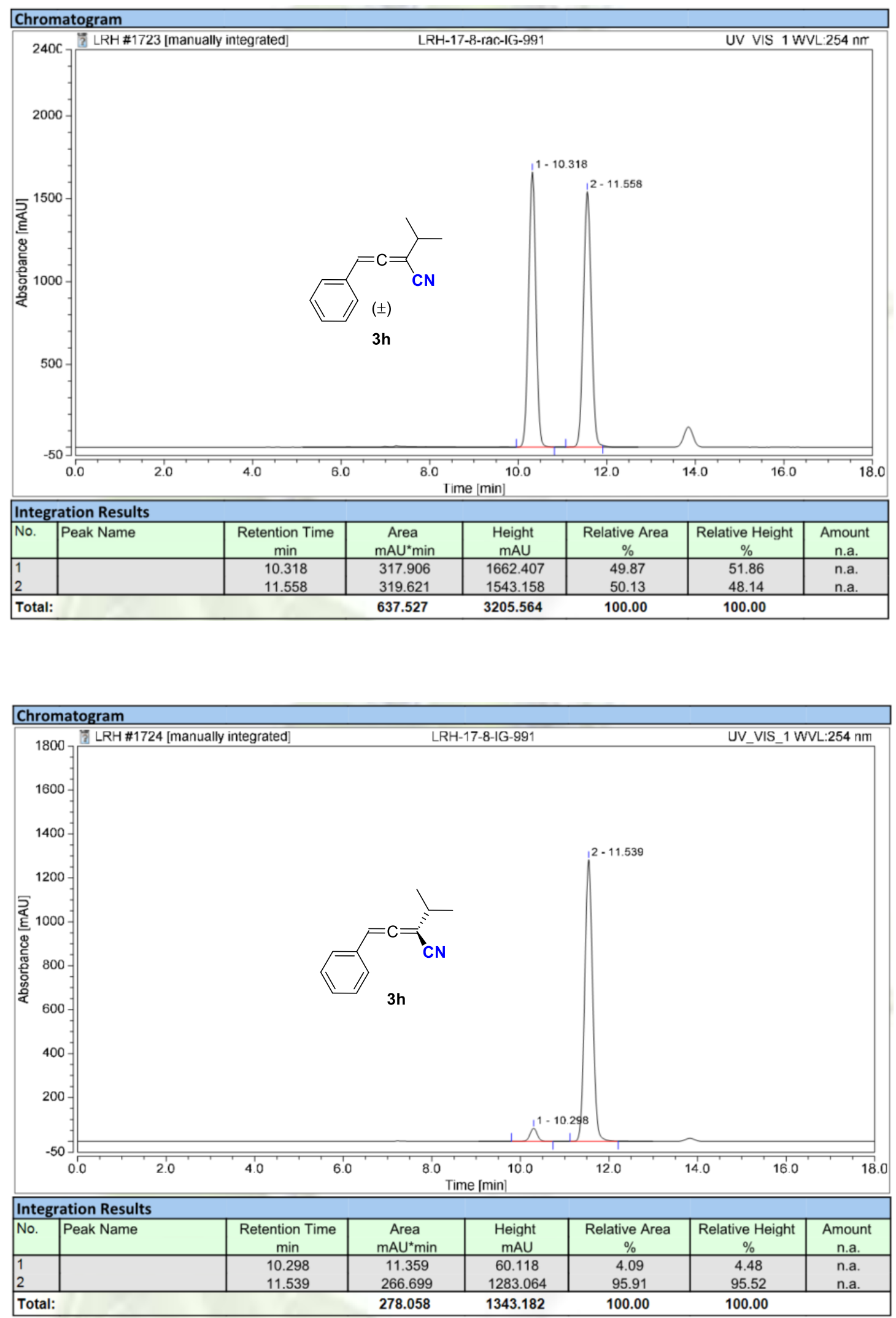

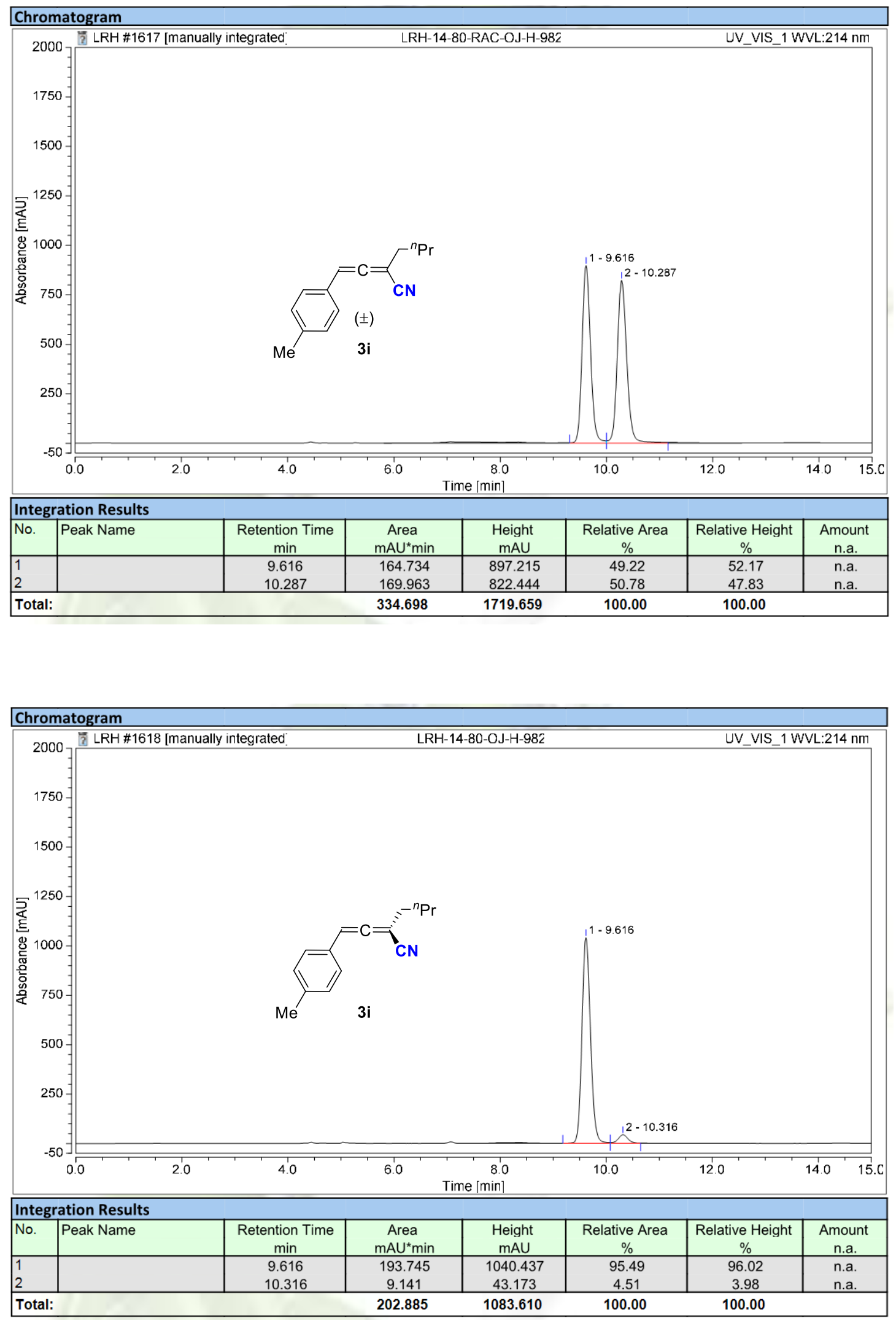


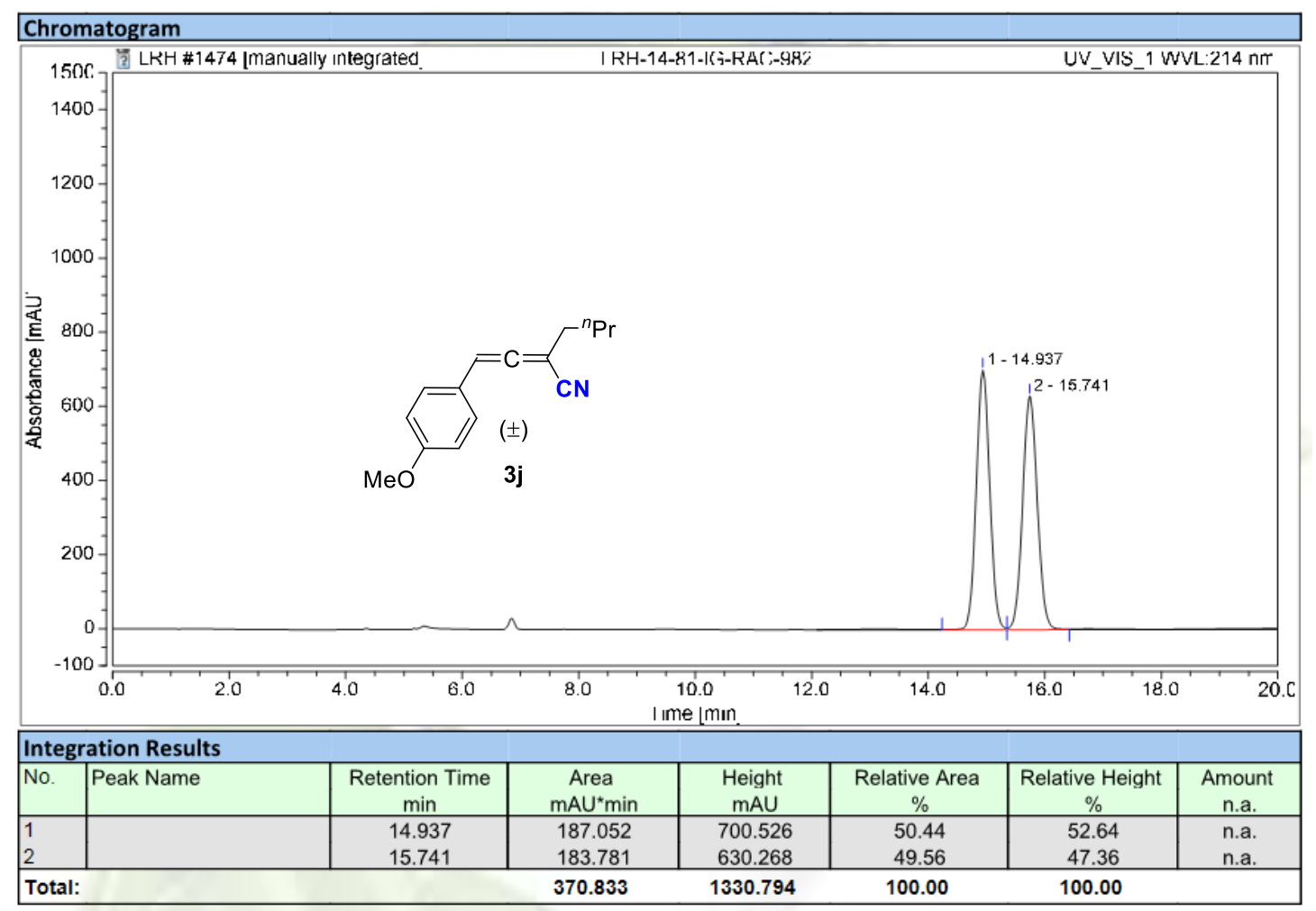

\section{Chromatogram}

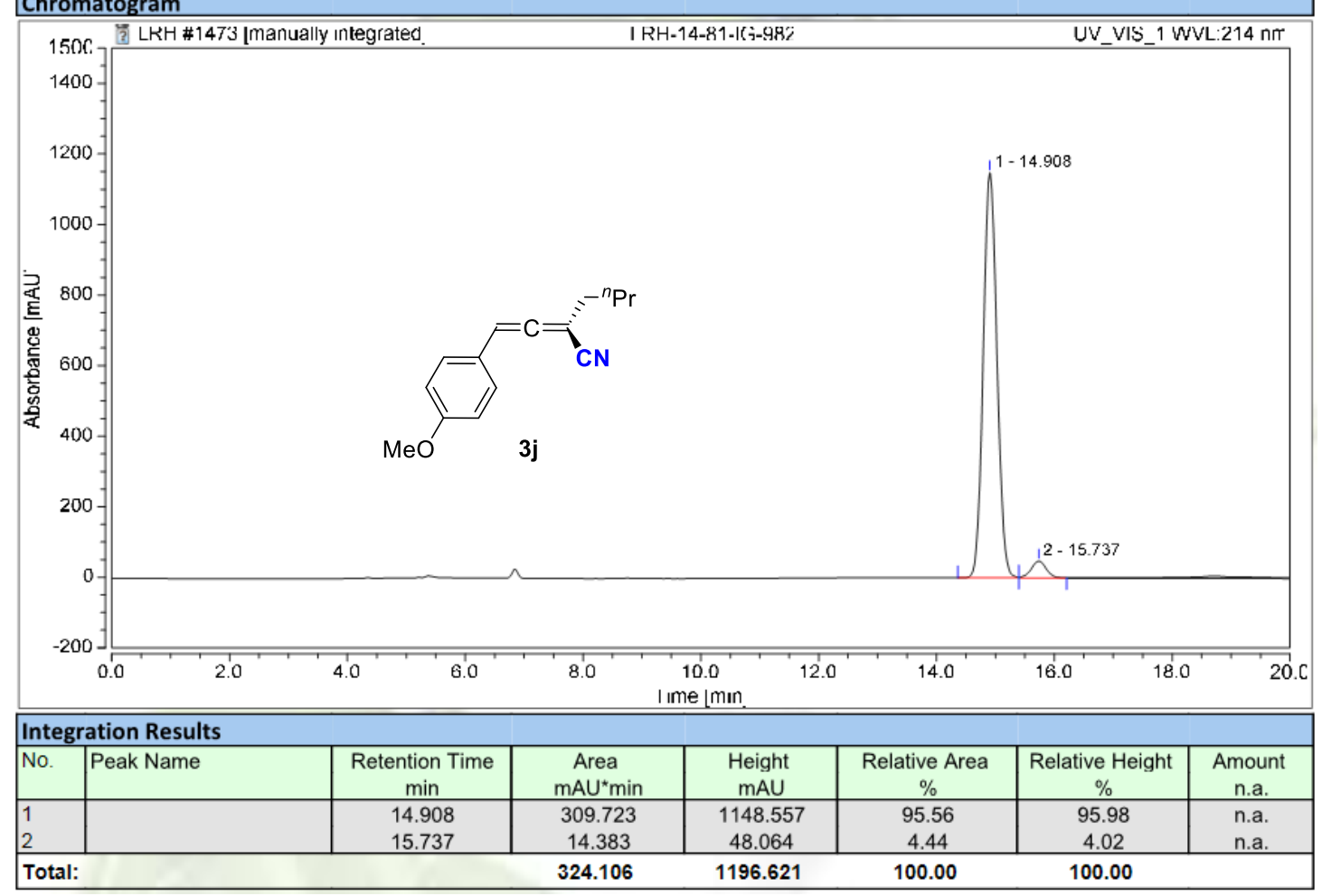



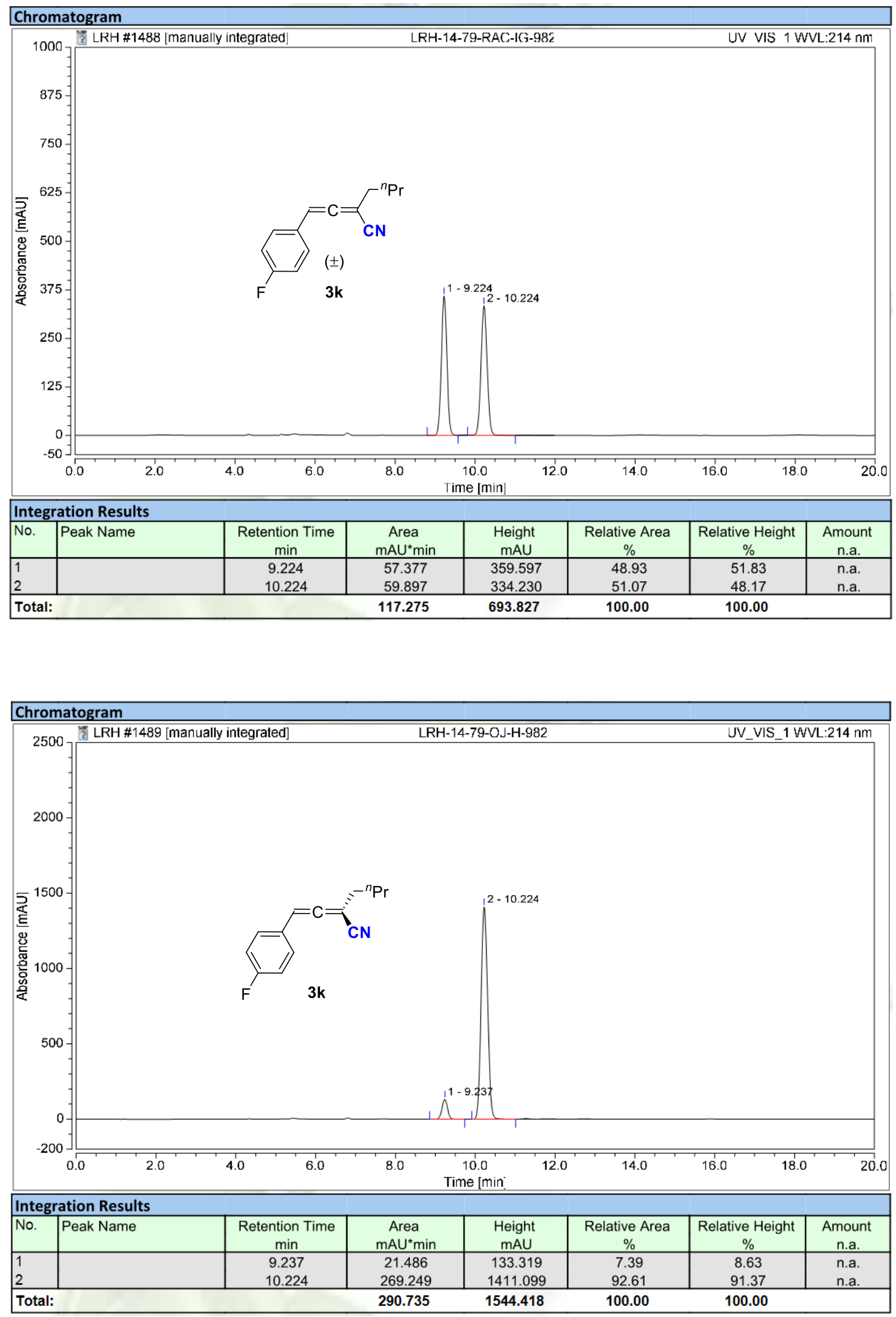

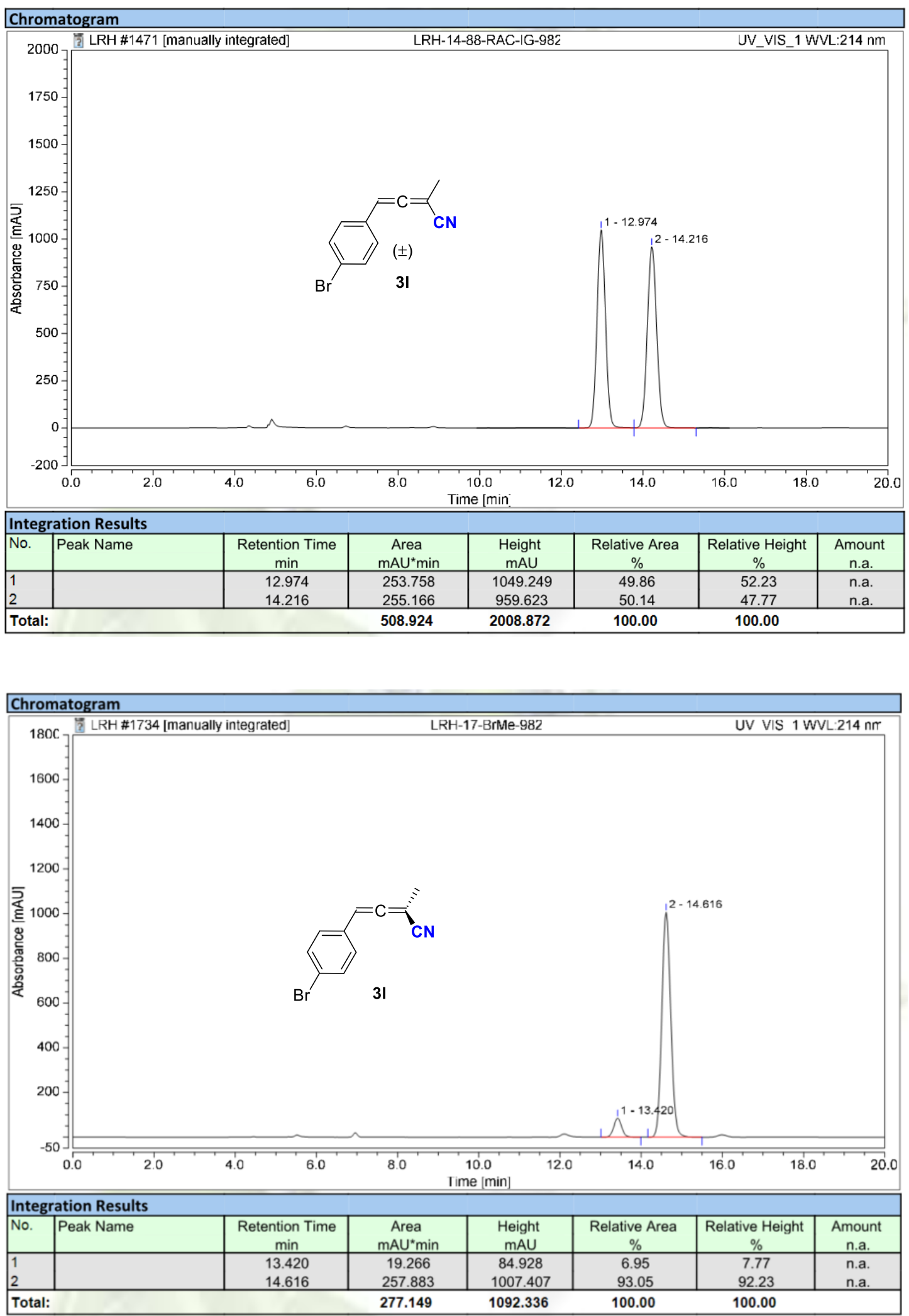

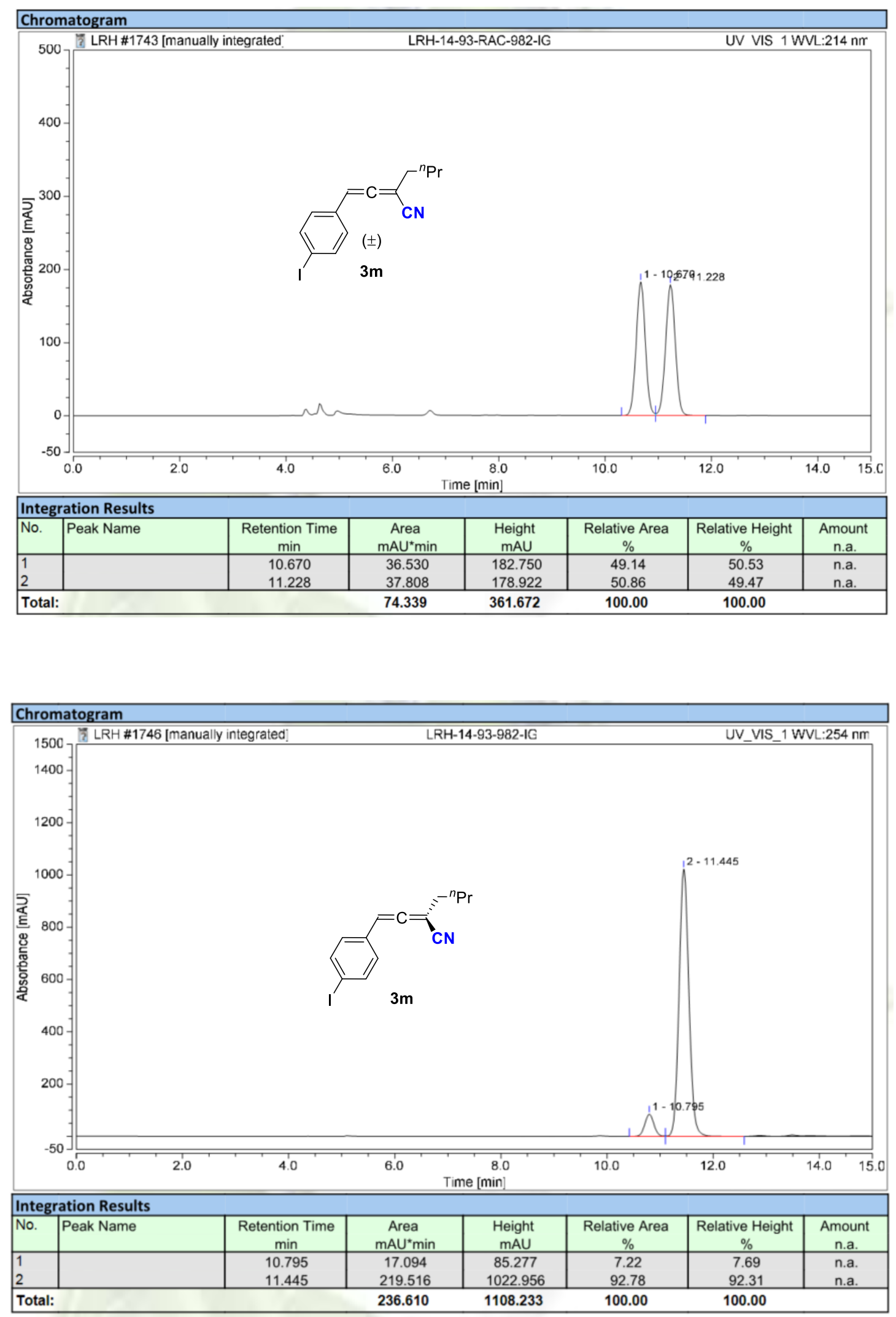

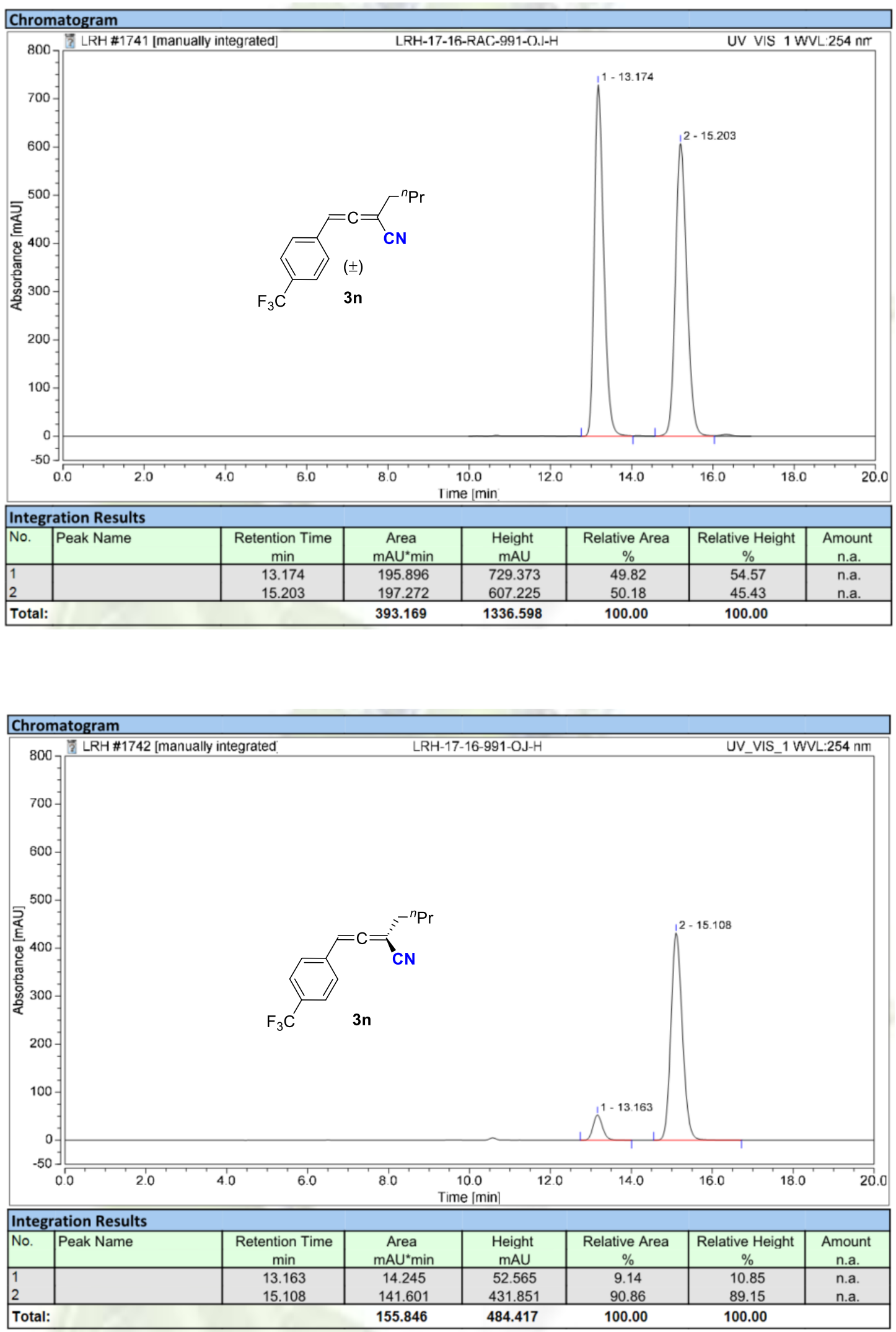

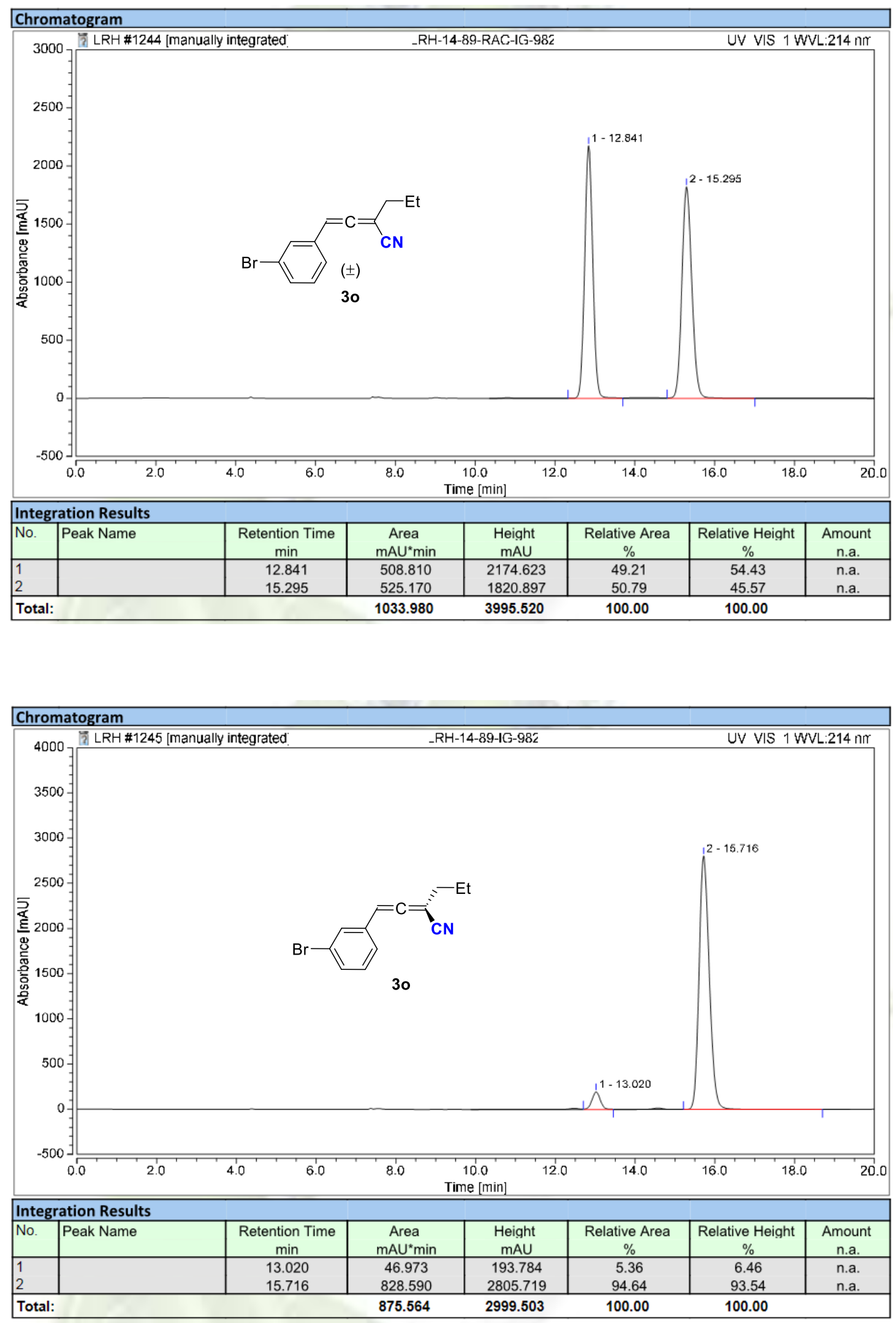

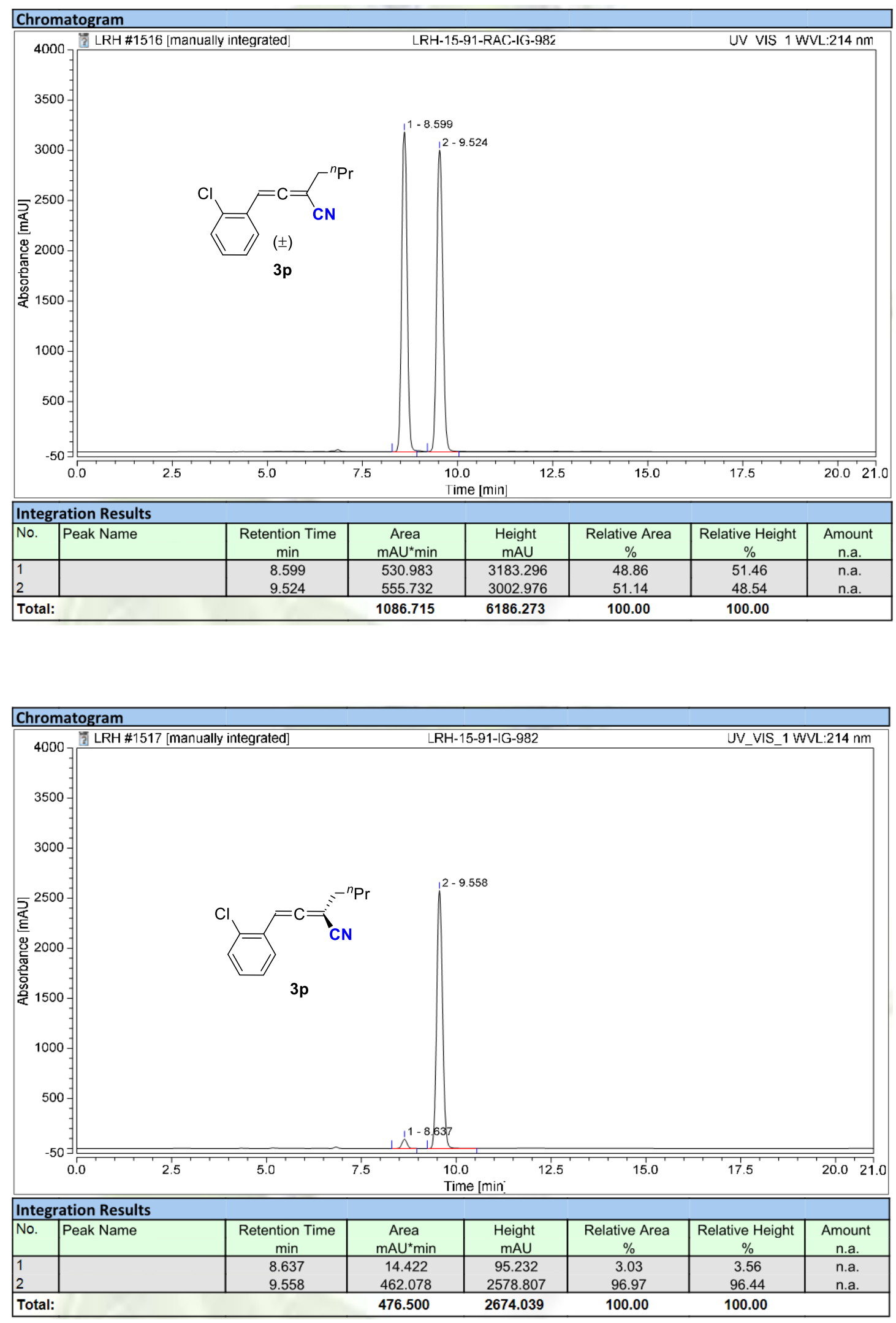

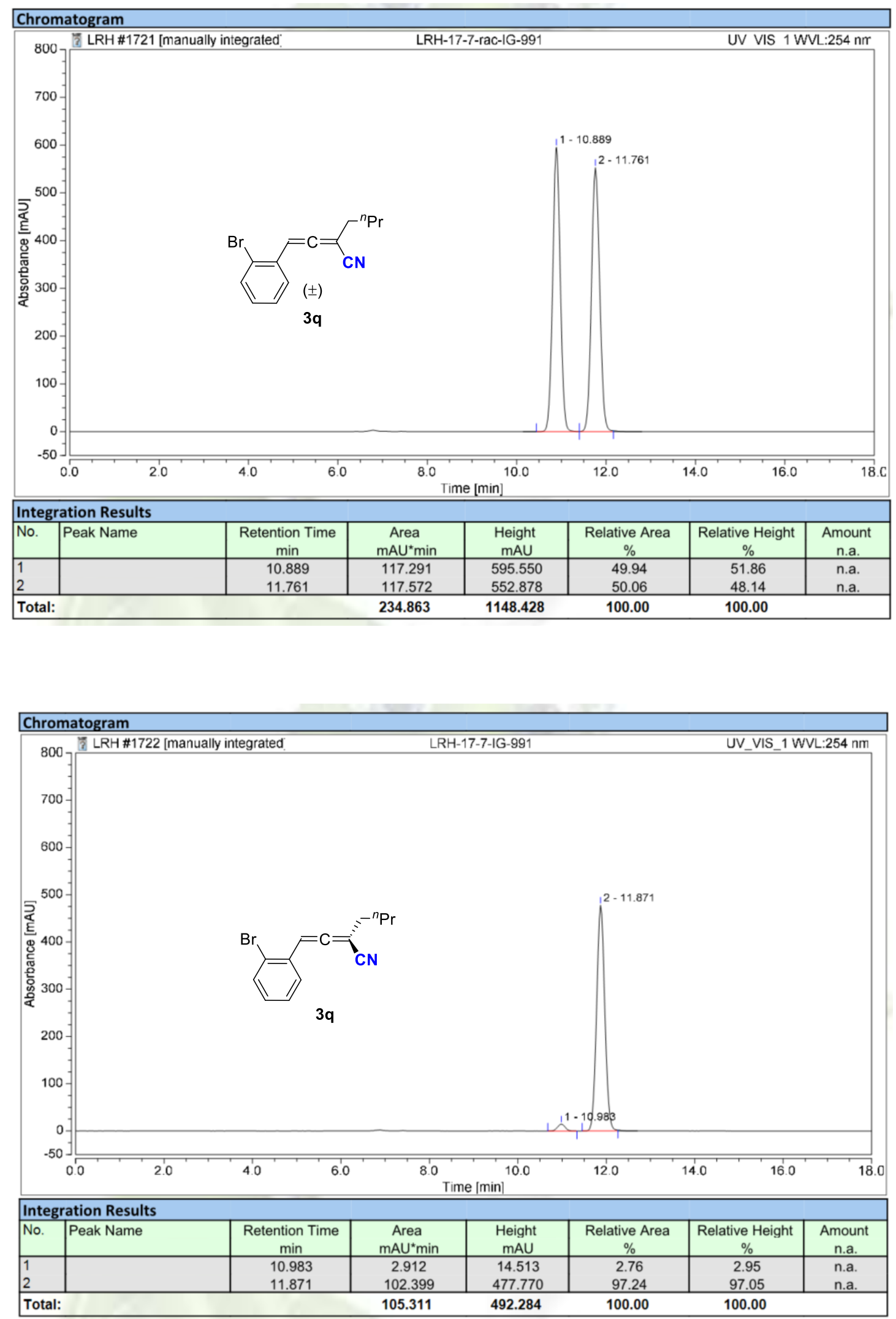

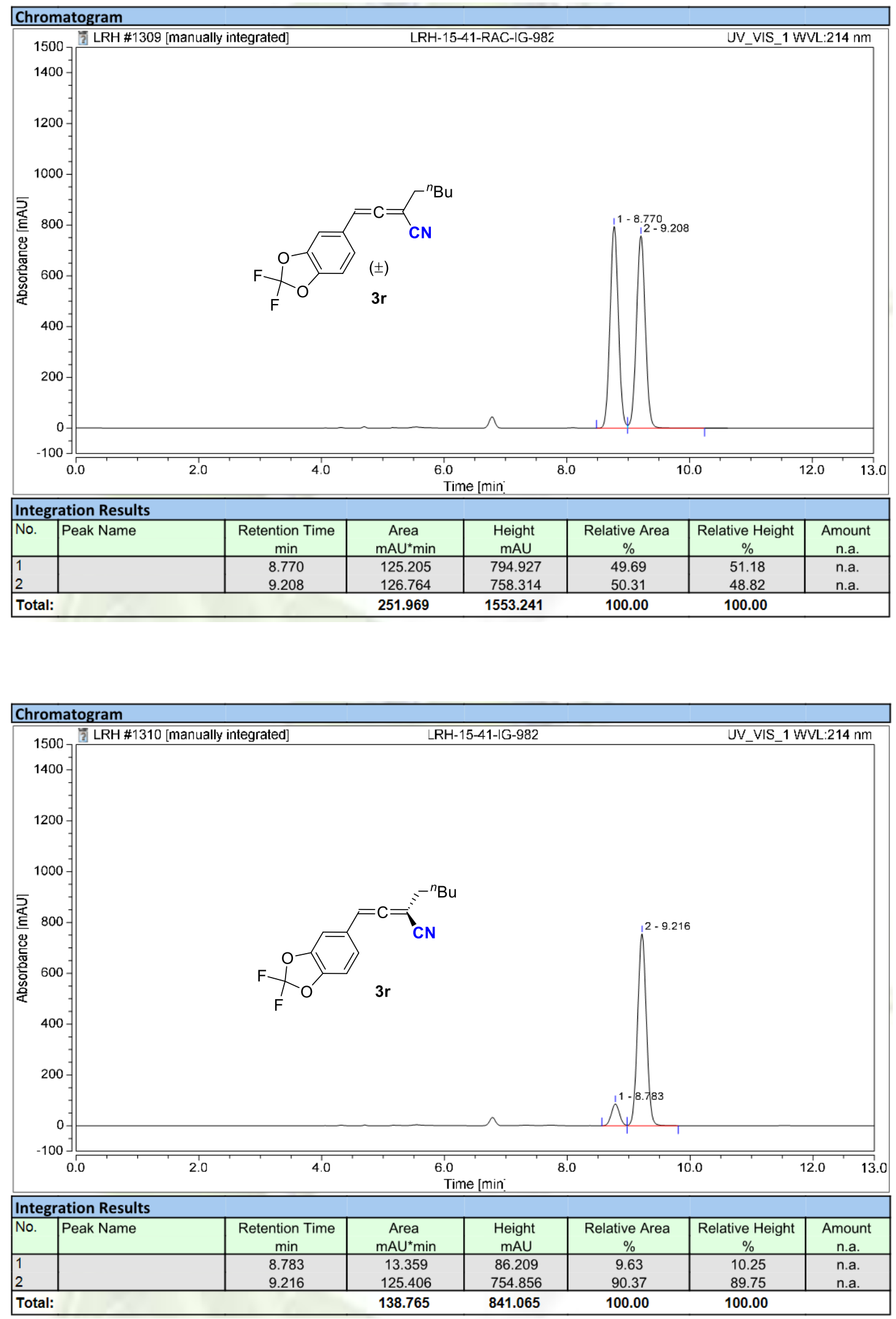

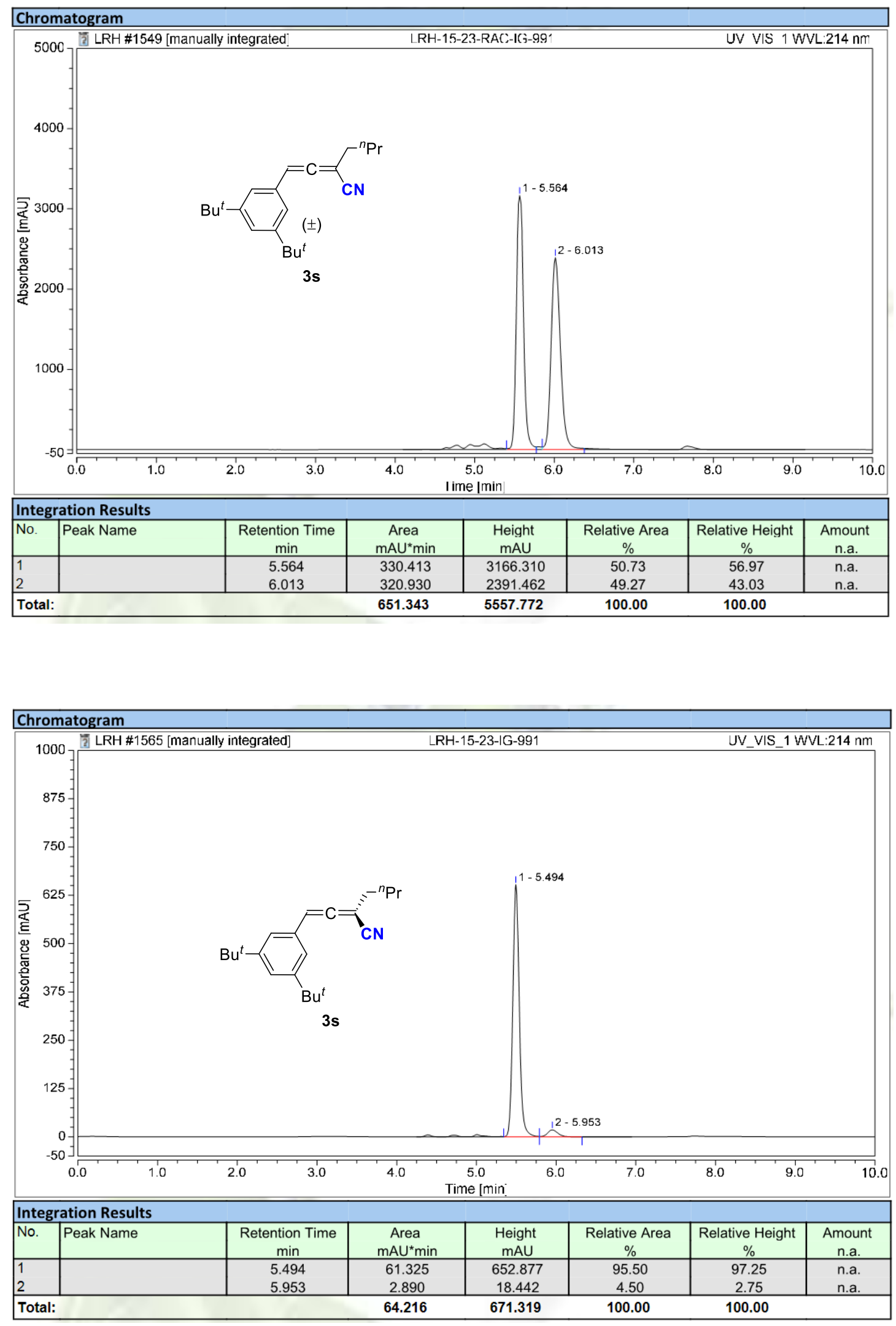

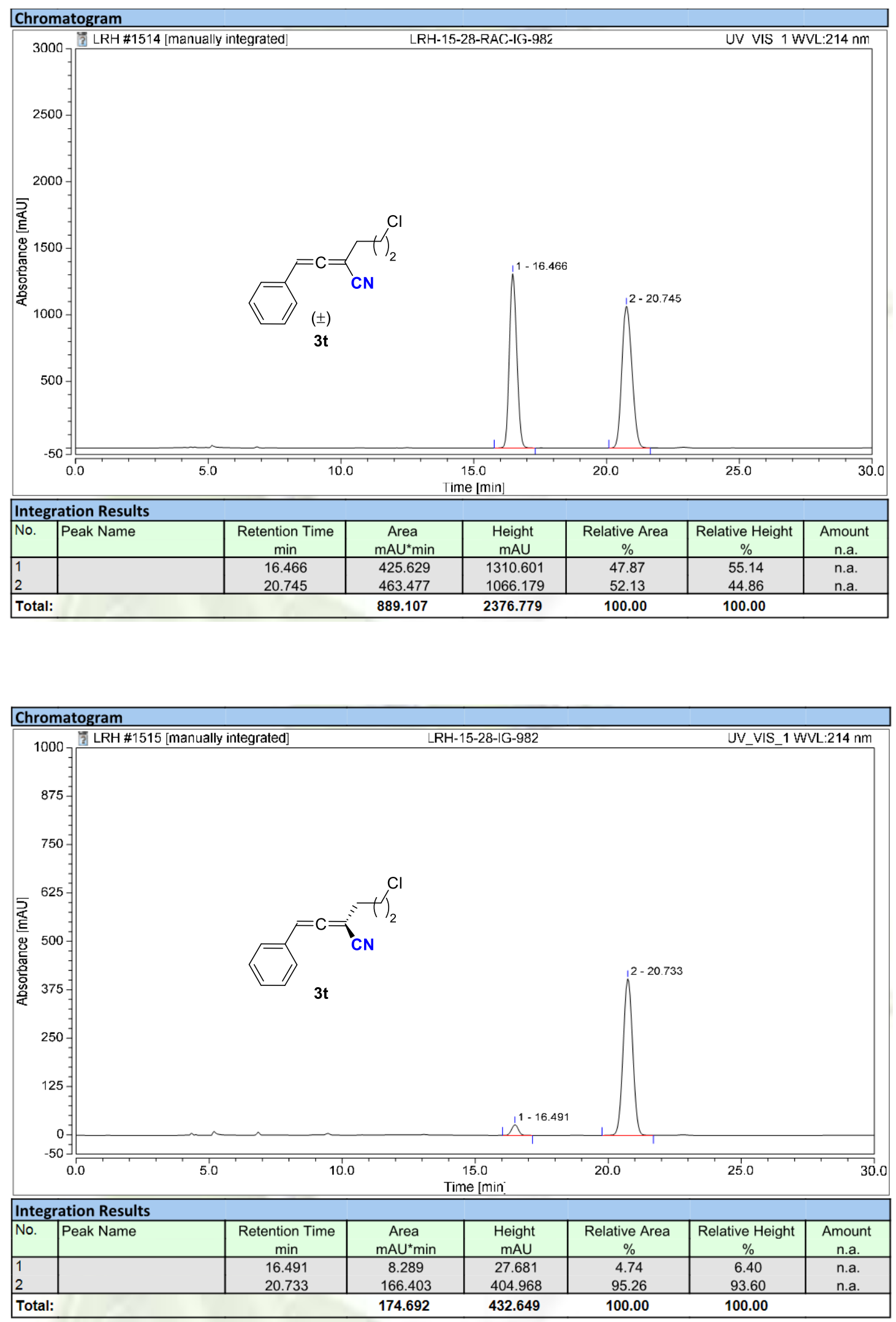

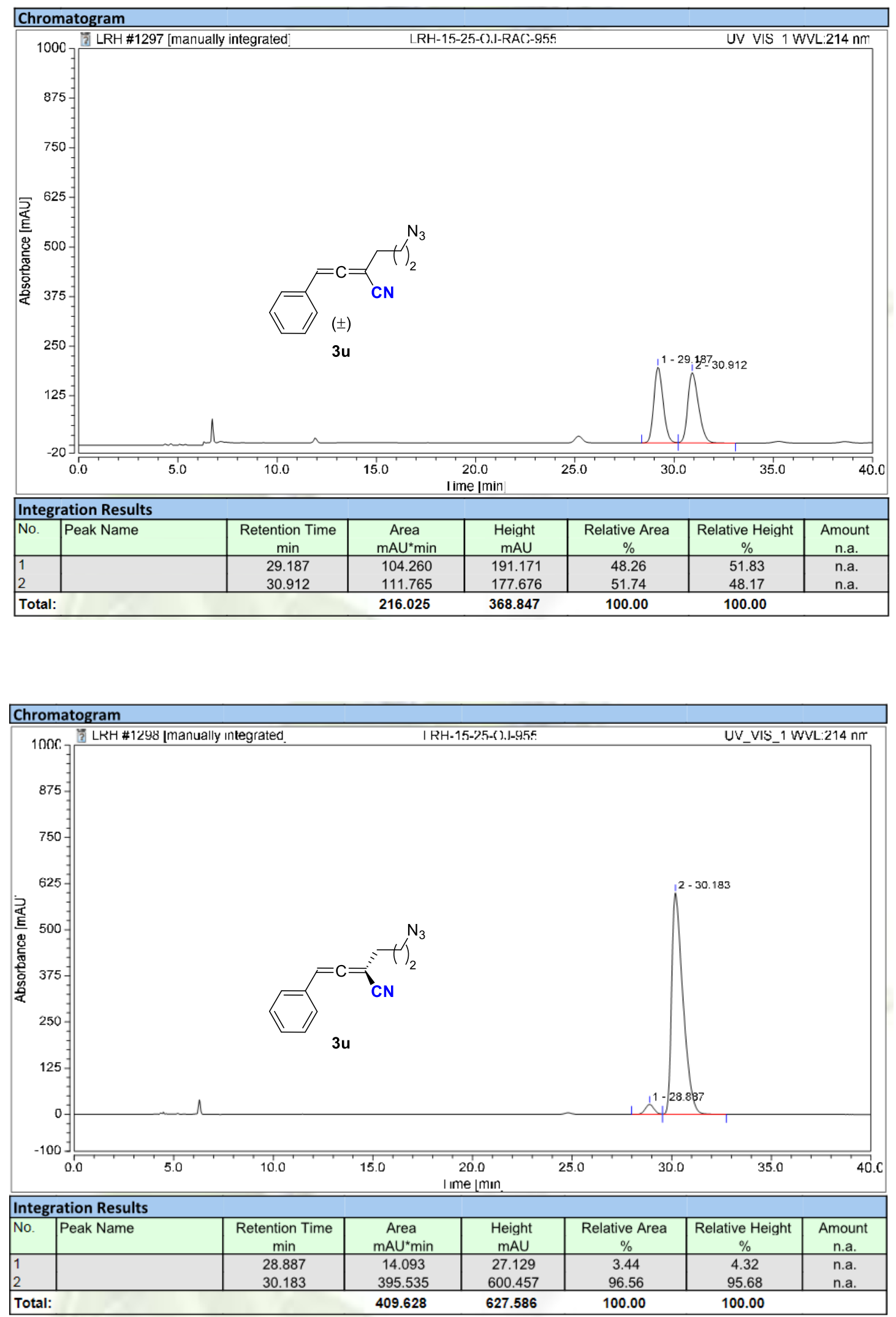

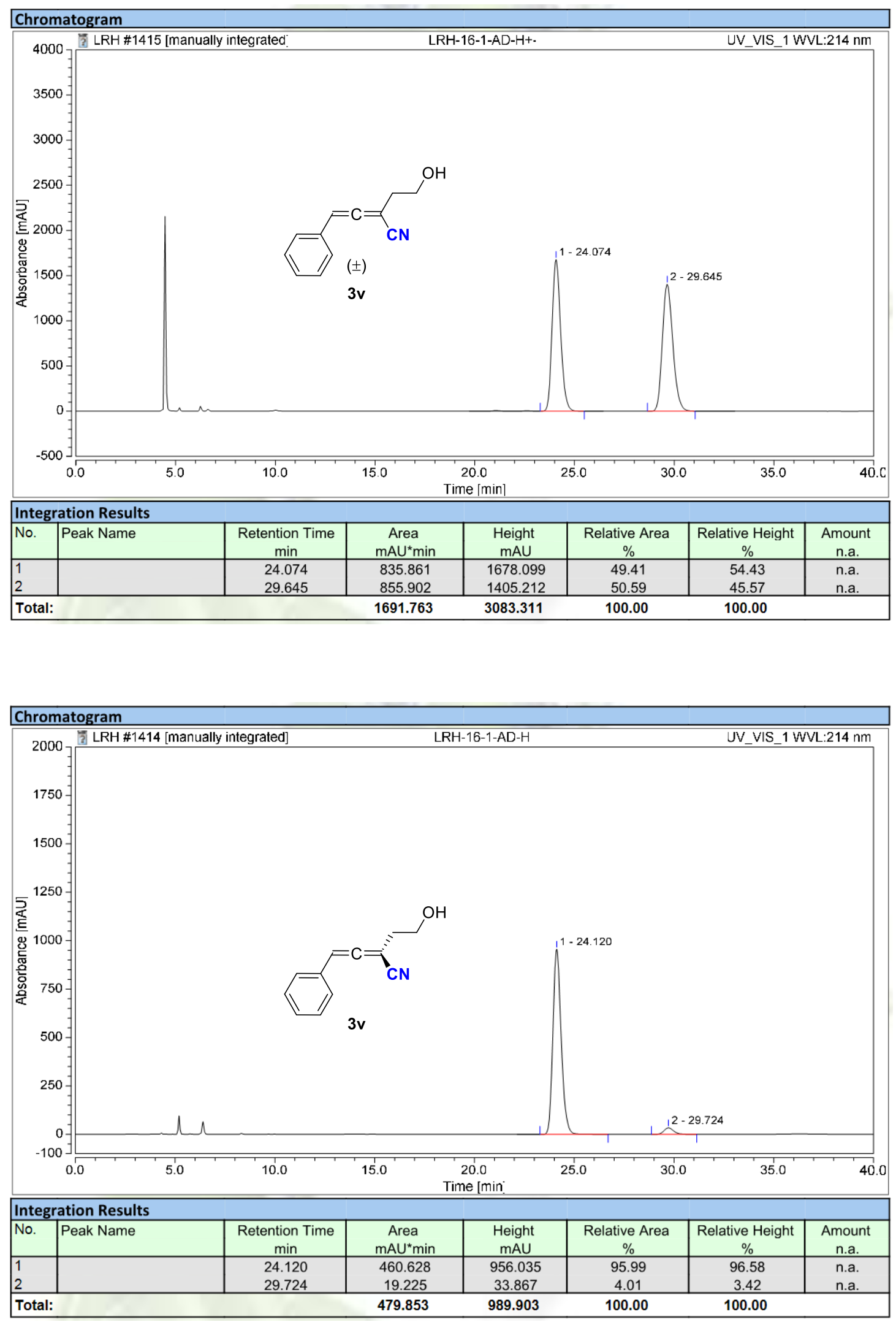

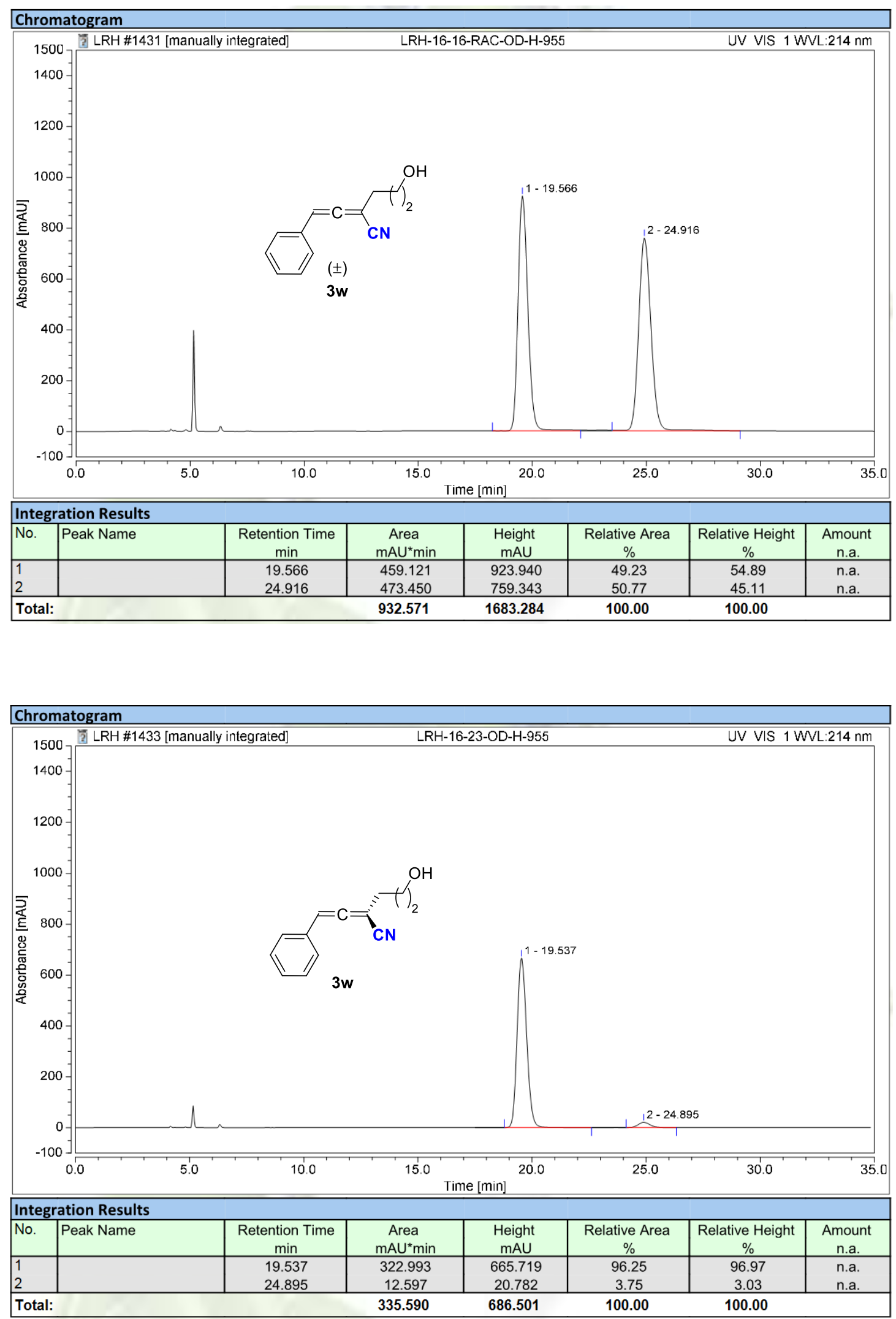

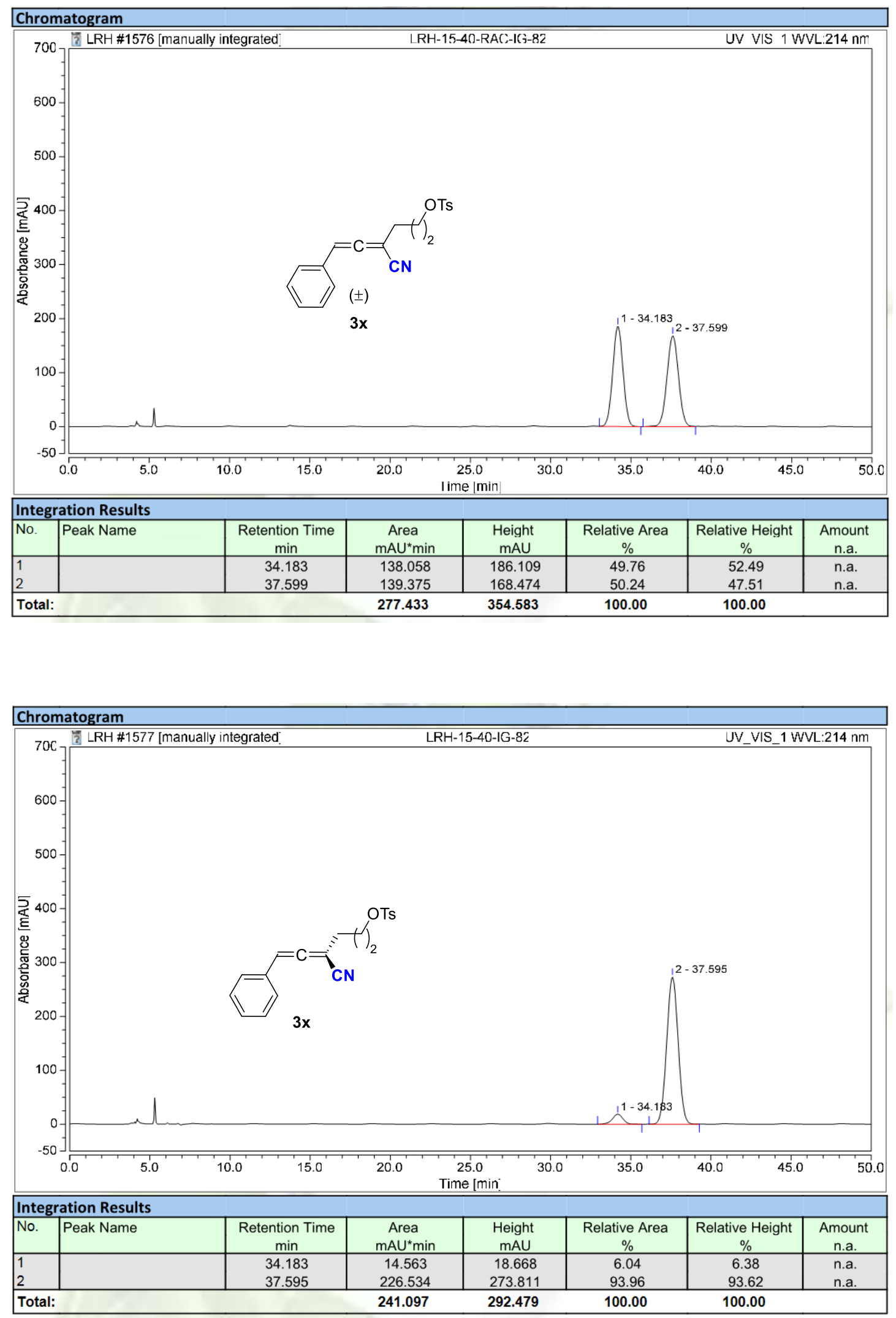

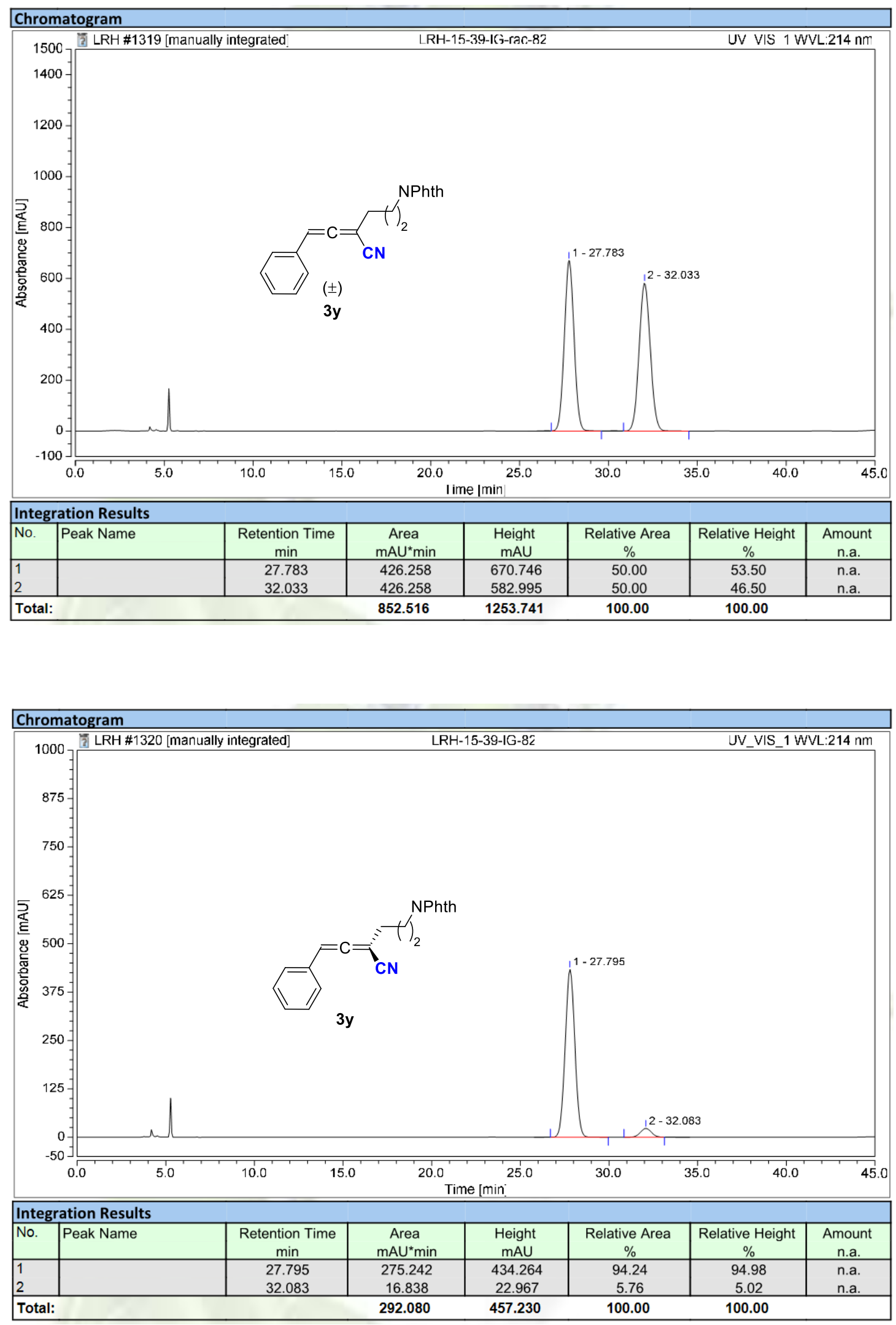

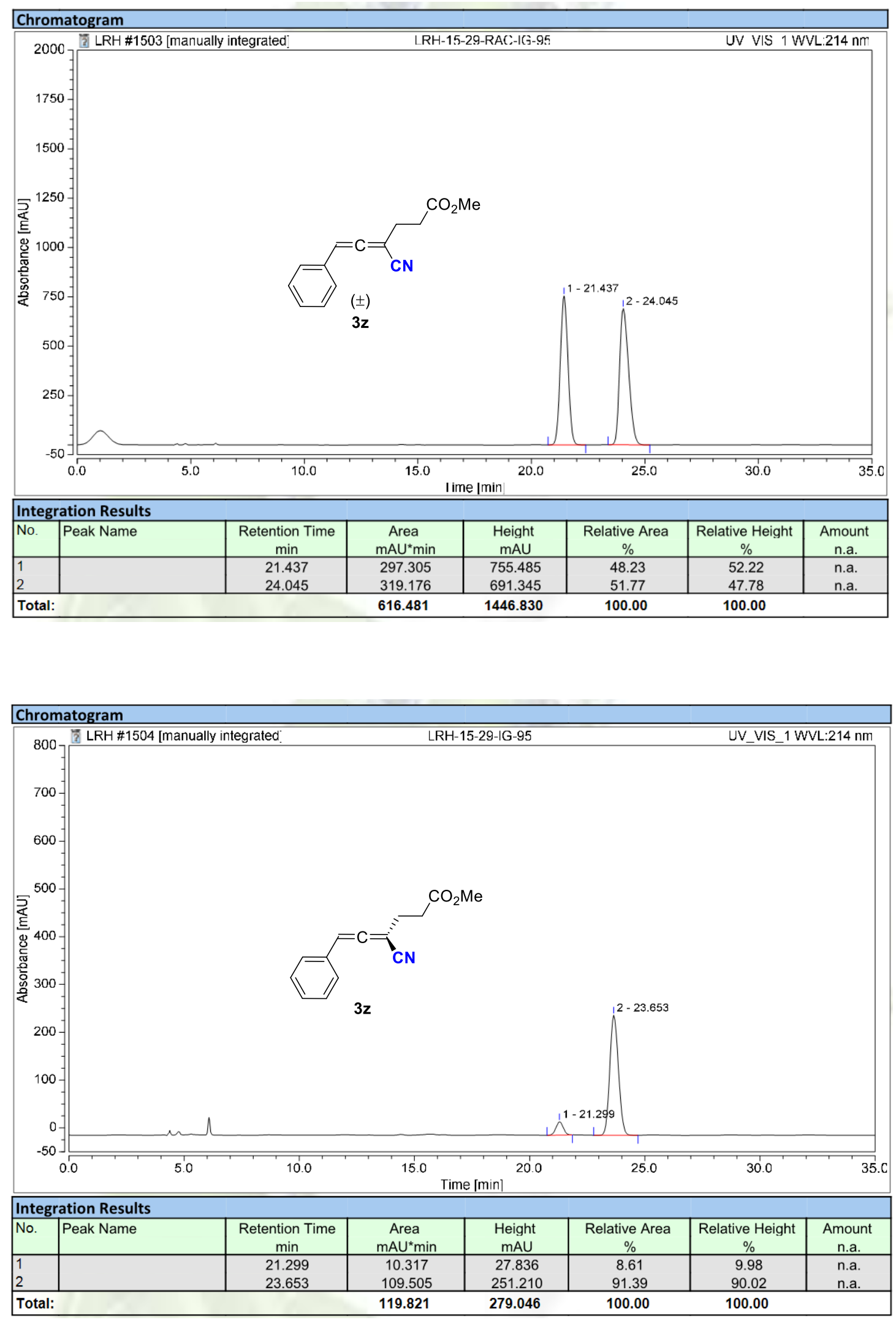

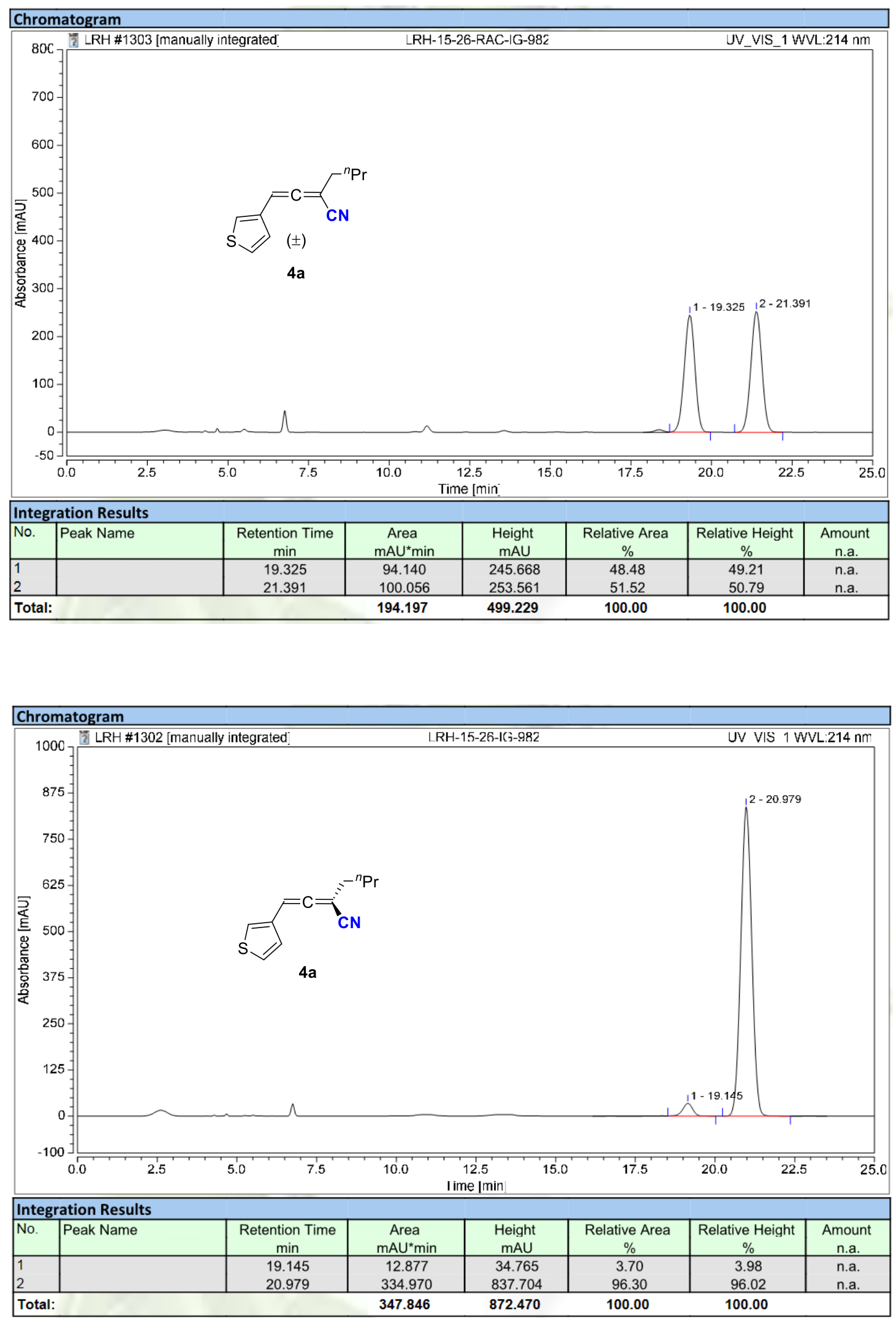

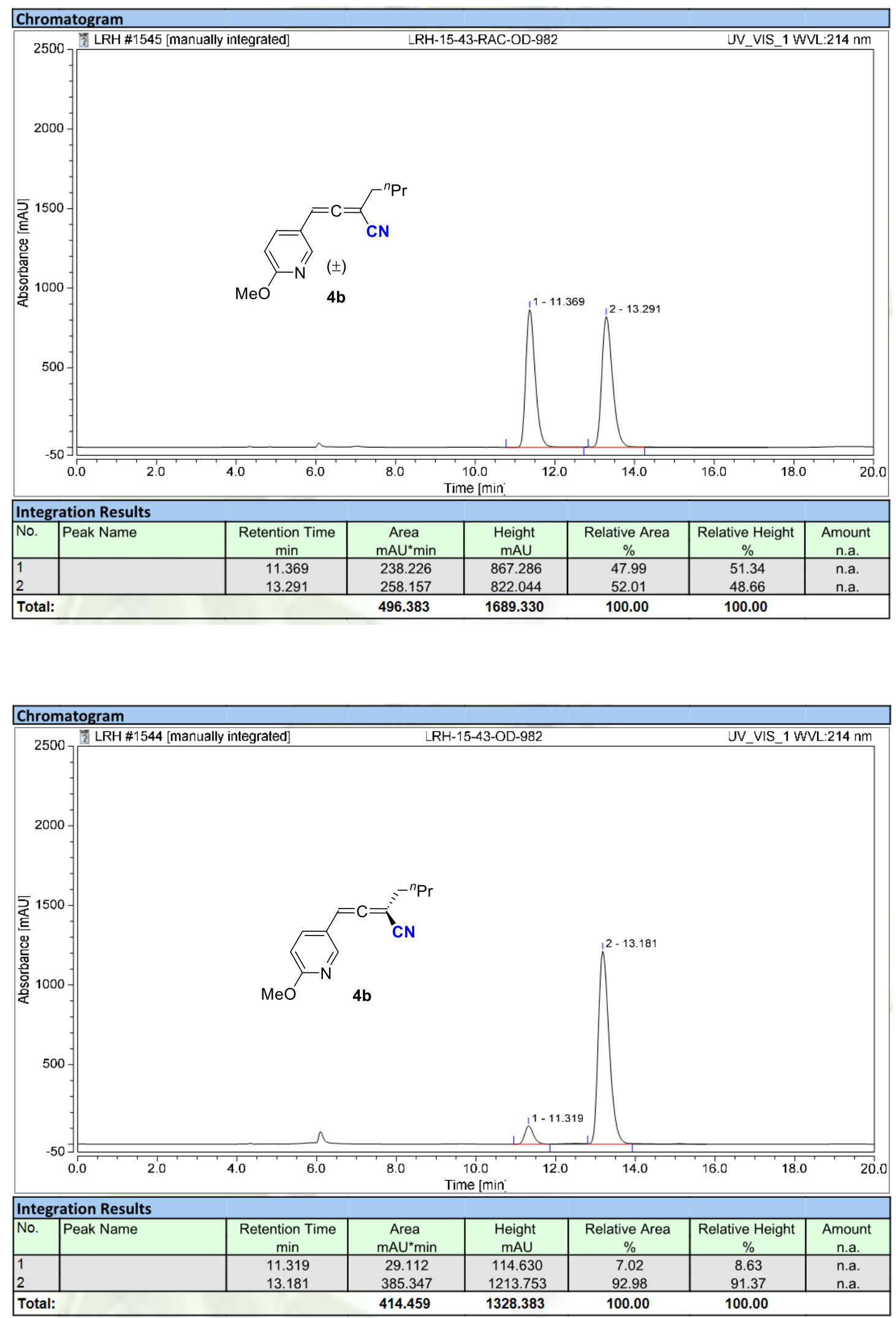

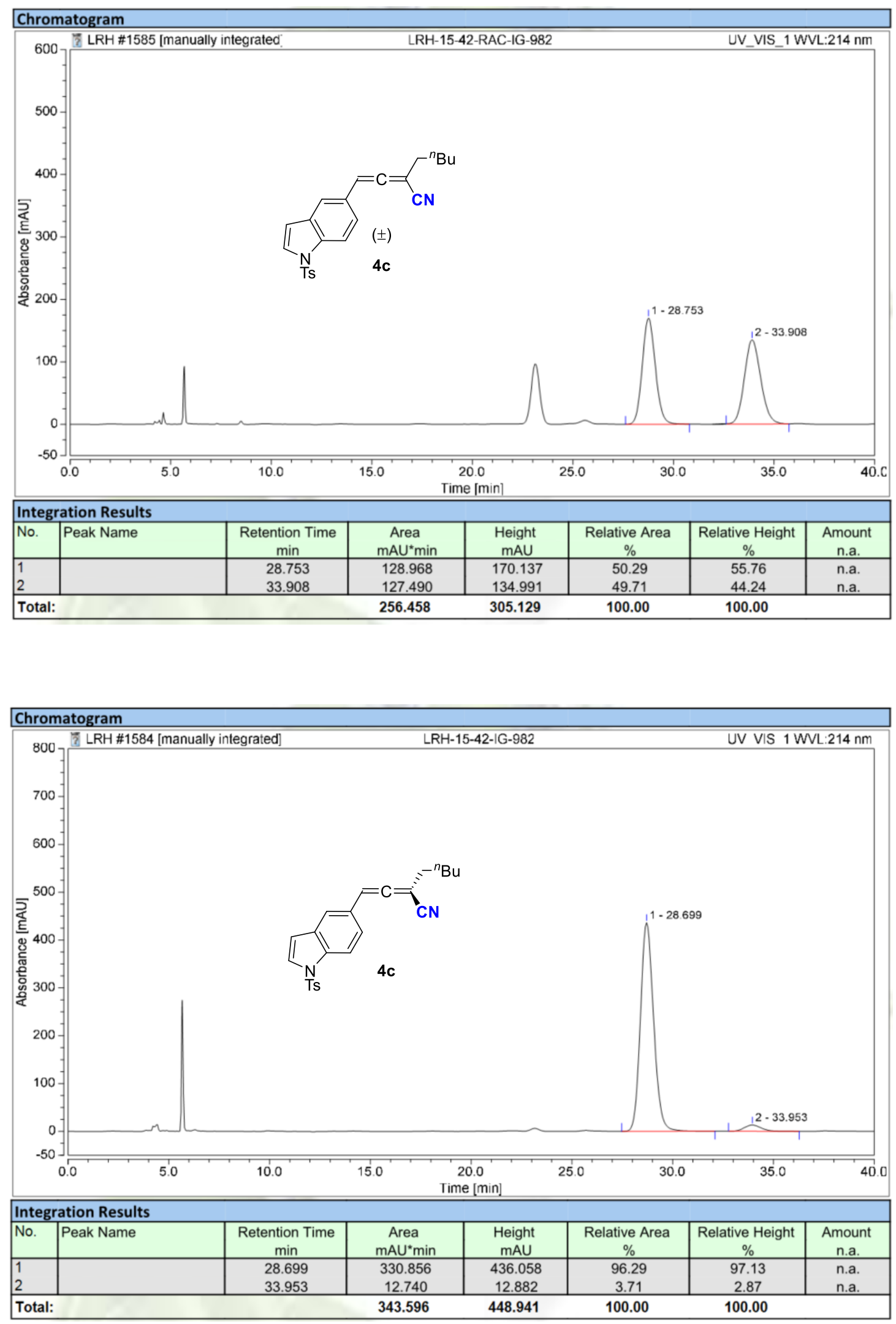

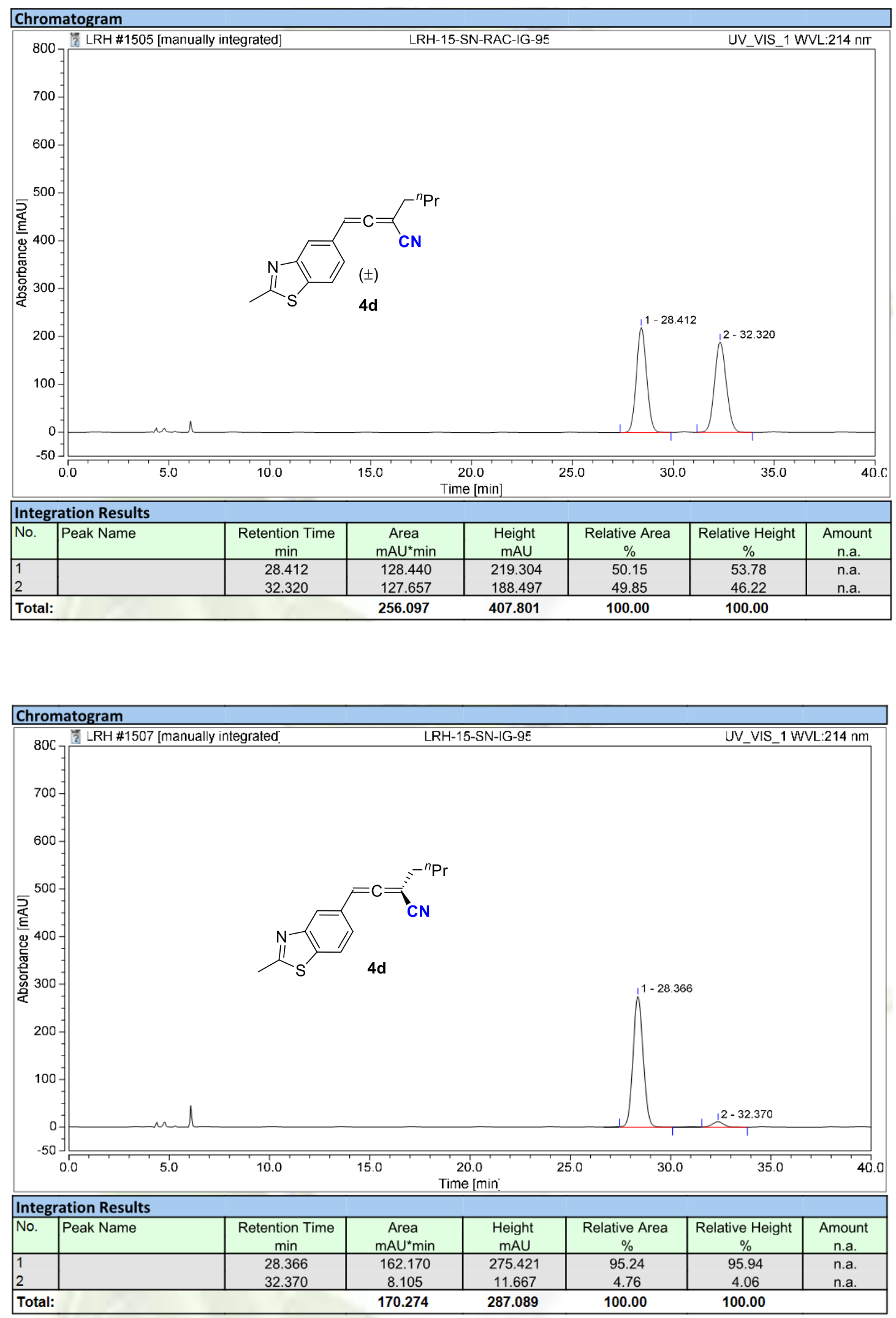

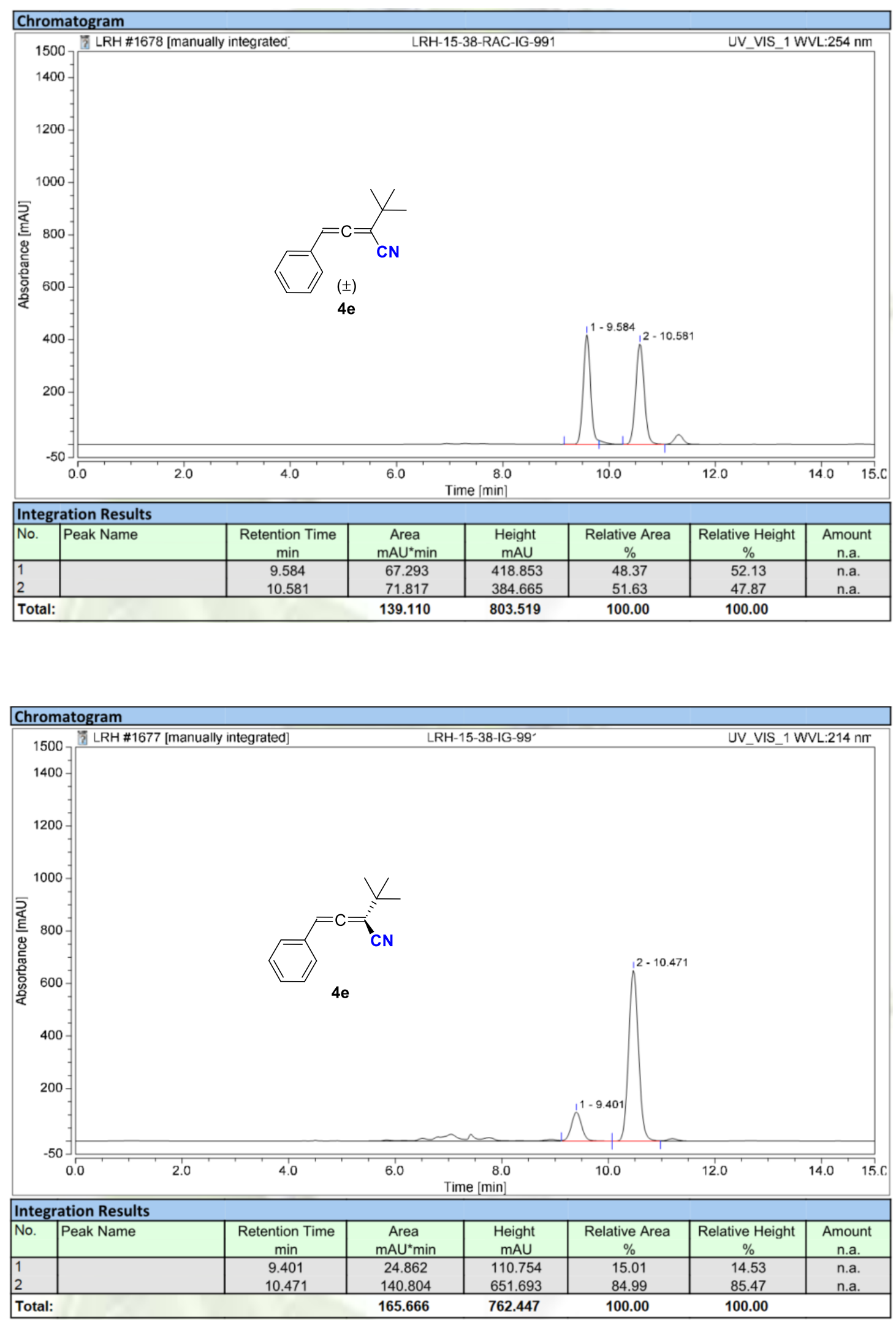

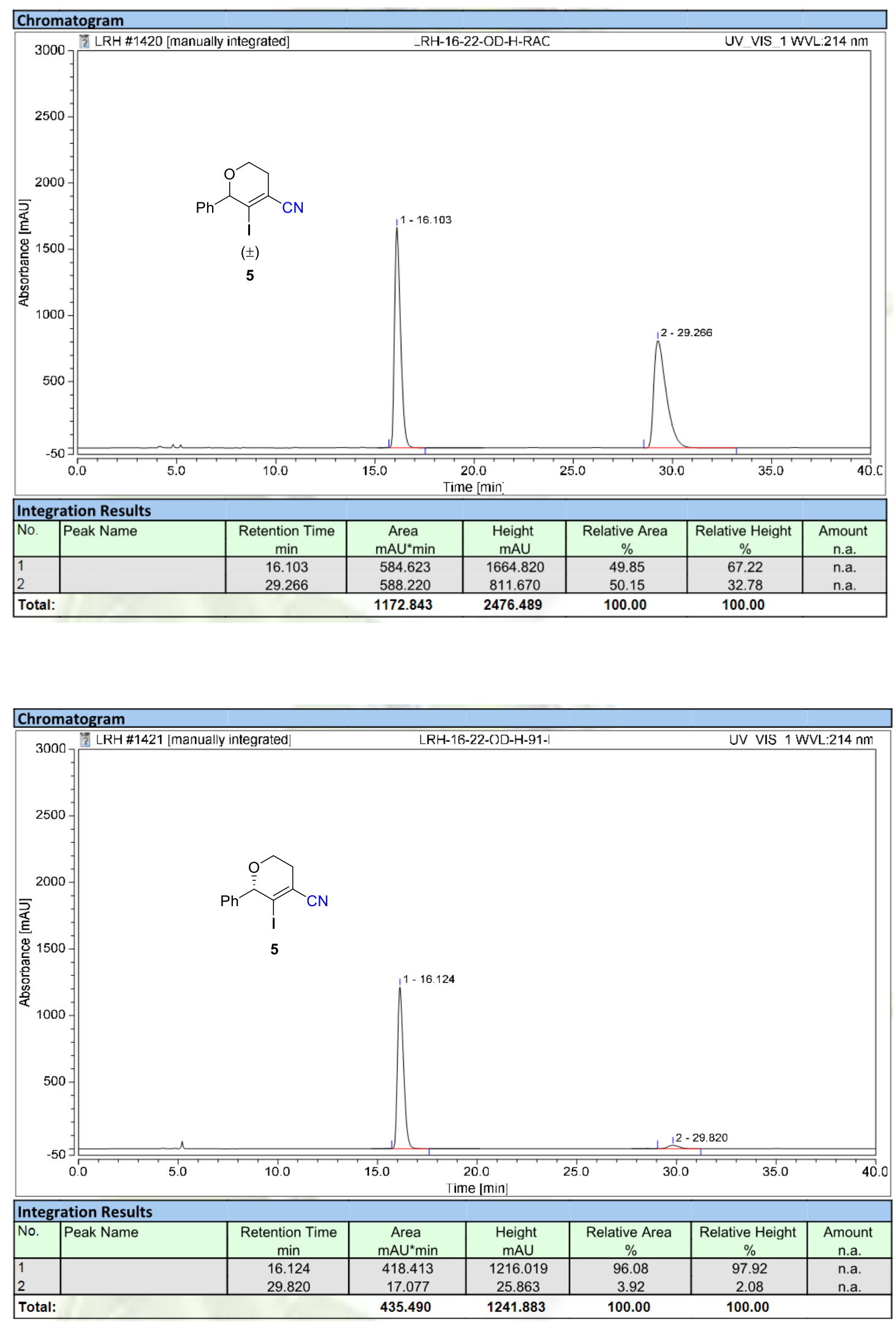

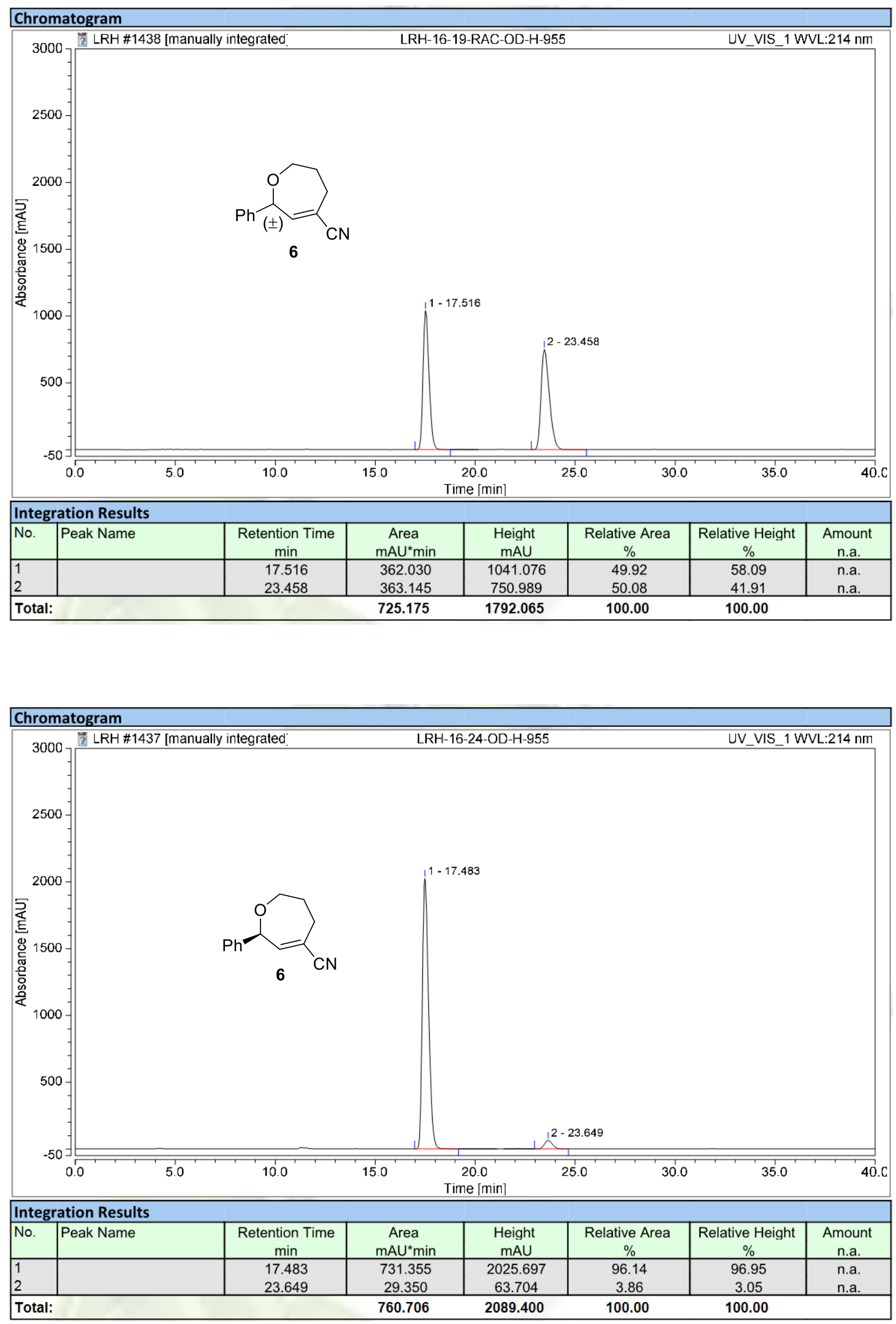

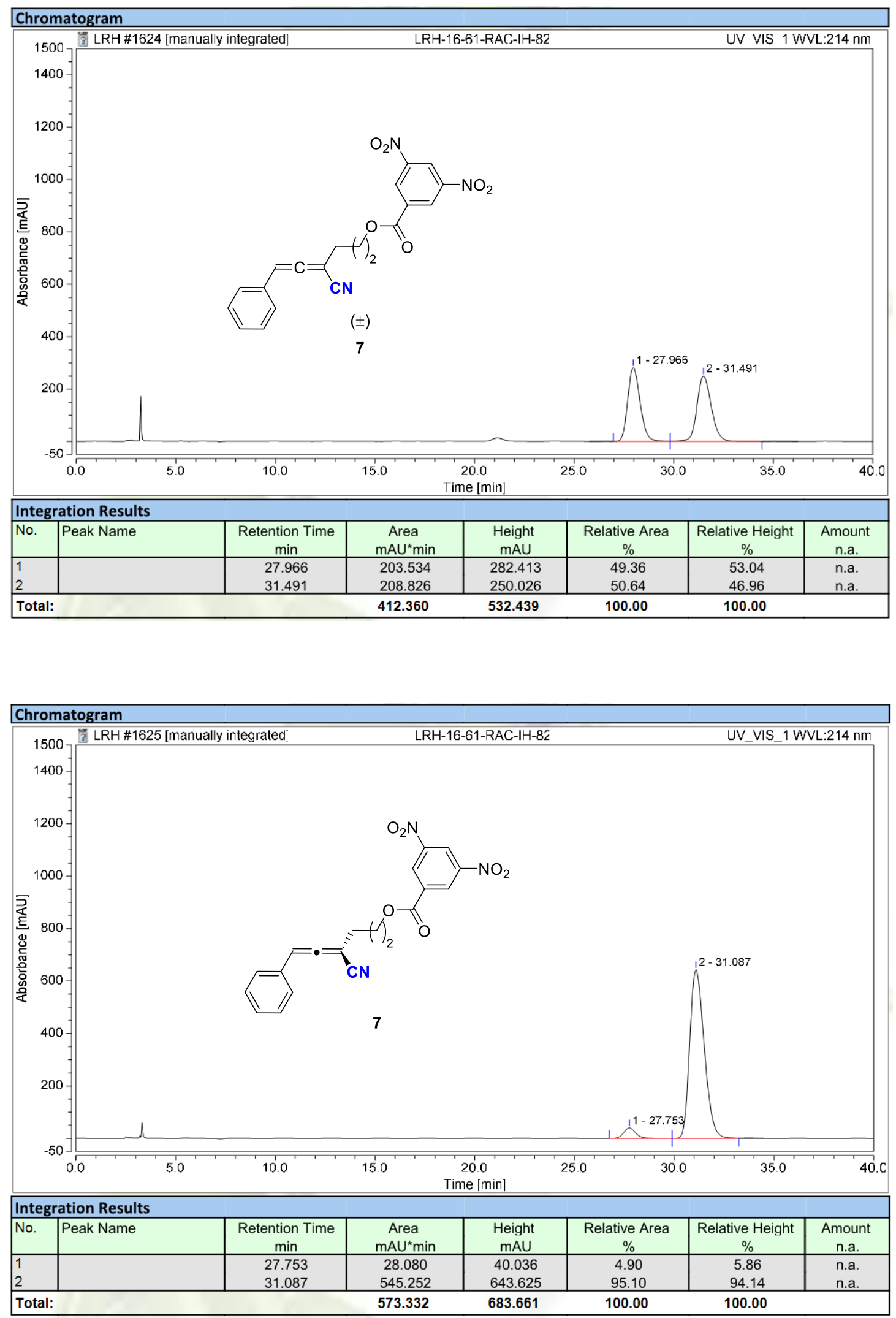

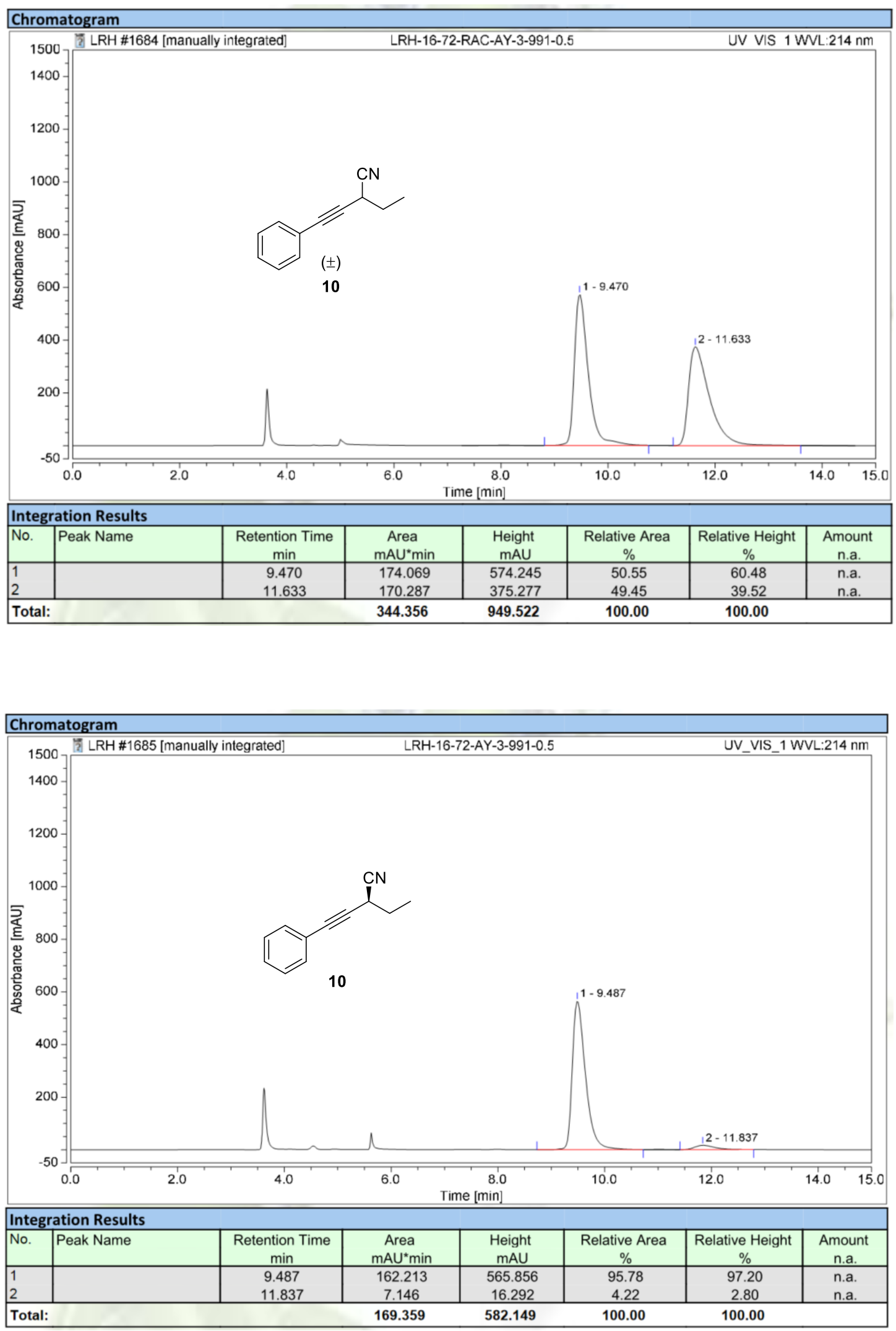

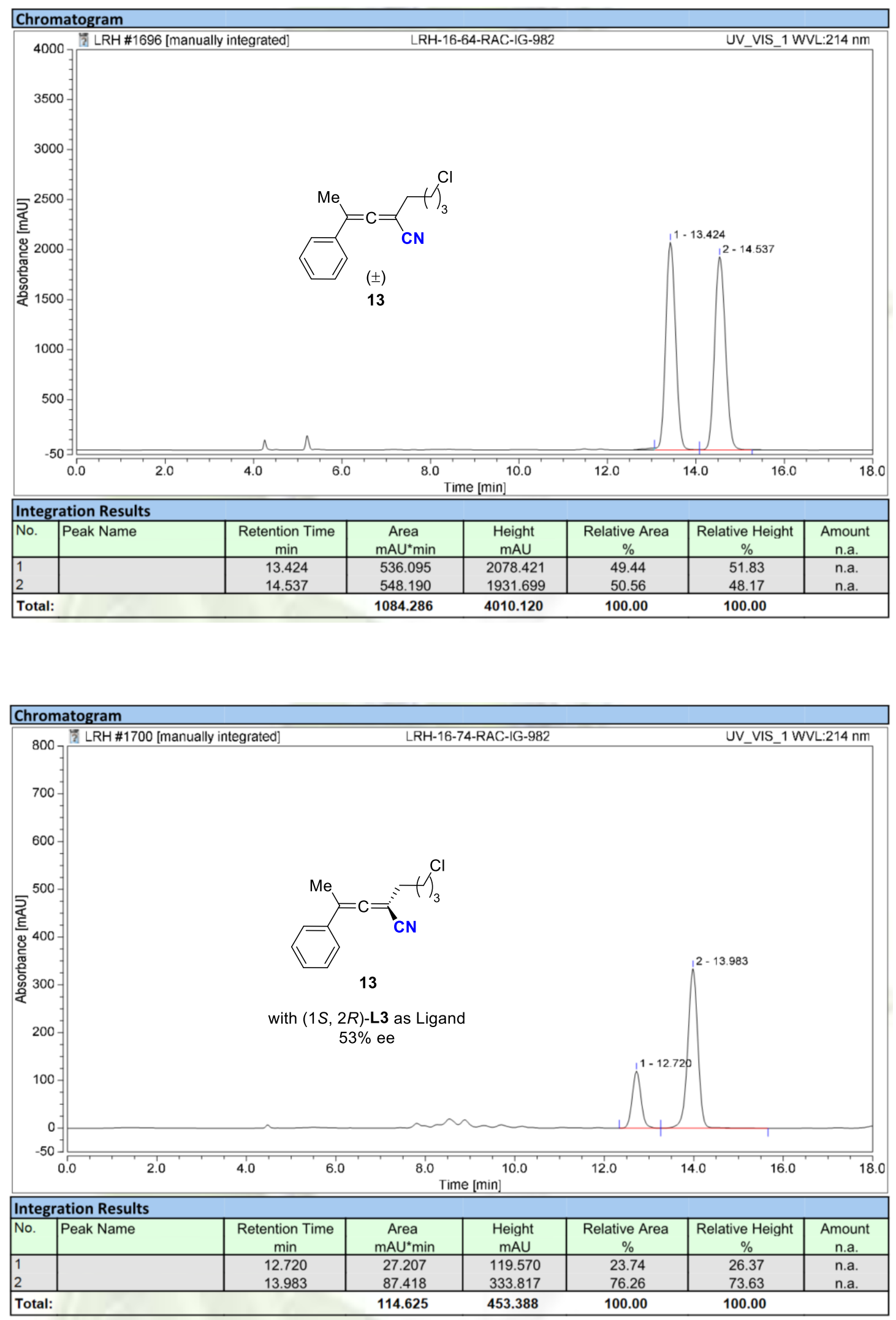


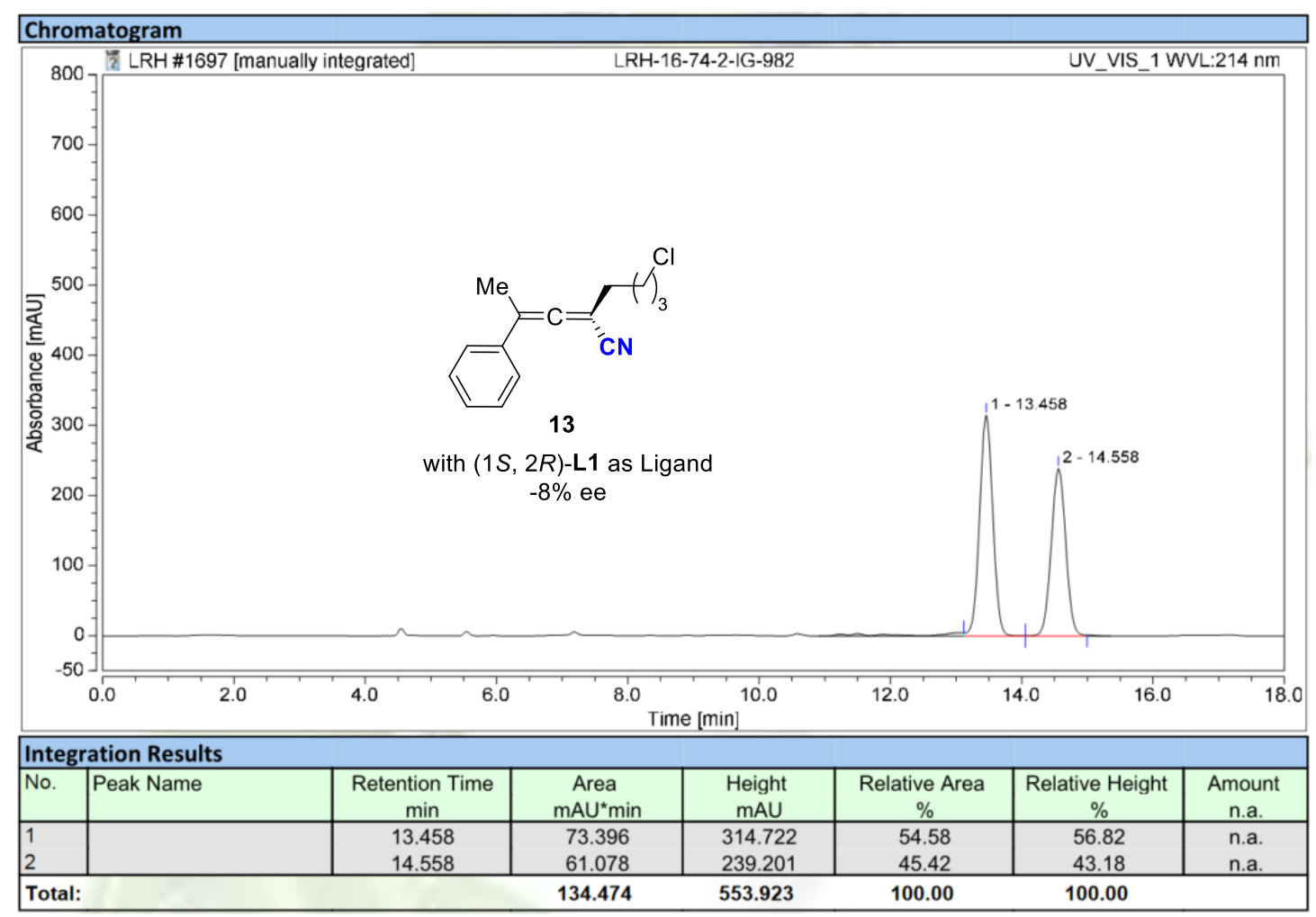

